\title{
Nucleophilic (Radio)Fluorination of Redox-Active Esters via Radical-Polar Crossover Enabled by Photoredox Catalysis
}

Eric Webb, John Park, Erin L. Cole, David J. Donnelly, Samuel Bonacorsi, Jr., William R. Ewing, Abigail Doyle Submitted date: 19/03/2020 - Posted date: 20/03/2020

Licence: CC BY-NC-ND 4.0

Citation information: Webb, Eric; Park, John; Cole, Erin L.; Donnelly, David J.; Bonacorsi, Jr., Samuel; Ewing, William R.; et al. (2020): Nucleophilic (Radio)Fluorination of Redox-Active Esters via Radical-Polar Crossover Enabled by Photoredox Catalysis. ChemRxiv. Preprint. https://doi.org/10.26434/chemrxiv.12009483.v1

We report a redox-neutral method for nucleophilic fluorination of $\mathrm{N}$-hydroxyphthalimide esters using an Ir photocatalyst under visible light irradiation. The method provides access to a broad range of aliphatic fluorides, including primary, secondary, and tertiary benzylic fluorides as well as unactivated tertiary fluorides, that are typically inaccessible by nucleophilic fluorination due to competing elimination. In addition, we show that the decarboxylative fluorination conditions are readily adapted to radiofluorination with $\left[{ }^{18} \mathrm{~F}\right] \mathrm{KF}$. We propose that the reactions proceed by two electron transfers between the Ir catalyst and redox-active ester substrate to afford a carbocation intermediate that undergoes subsequent trapping by fluoride. Examples of trapping with $\mathrm{O}$ - and $\mathrm{C}$-centered nucleophiles and deoxyfluorination via $\mathrm{N}$-hydroxyphthalimidoyl oxalates are also presented, suggesting that this approach may offer a general blueprint for affecting redox-neutral SN1 substitutions under mild conditions.

File list (2)

EWWJBP_JACSManuscript_Final.pdf (1.04 MiB) view on ChemRxiv - download file 


\title{
Nucleophilic (Radio)Fluorination of Redox-Active Esters via Radi- cal-Polar Crossover Enabled by Photoredox Catalysis
}

\author{
Eric W. Webb,${ }^{\dagger}$ John B. Park, ${ }^{\dagger}$ Erin L. Cole,${ }^{\dagger}$ David J. Donnelly, ${ }^{\dagger}$ Samuel J. Bonacorsi,${ }^{\dagger}$ William R. \\ Ewing, ${ }^{\S}$ and Abigail G. Doyle*† \\ 'Department of Chemistry, Princeton University, Princeton, New Jersey 08544, United States \\ tDiscovery Chemistry Platforms, PET Radiochemical Synthesis, Bristol-Myers Squibb Research and Development, P.O. Box \\ 4000, Princeton, New Jersey 08543, United States
}

${ }^{\S}$ Discovery Chemistry, Bristol-Myers Squibb, P.O. Box 5400, Princeton, New Jersey 08543-5400, United States

\begin{abstract}
We report a redox-neutral method for nucleophilic fluorination of $N$-hydroxyphthalimide esters using an Ir photocatalyst under visible light irradiation. The method provides access to a broad range of aliphatic fluorides, including primary, secondary, and tertiary benzylic fluorides as well as unactivated tertiary fluorides, that are typically inaccessible by nucleophilic fluorination due to competing elimination. In addition, we show that the decarboxylative fluorination conditions are readily adapted to radiofluorination with $\left[{ }^{18} \mathrm{~F}\right] \mathrm{KF}$. We propose that the reactions proceed by two electron transfers between the Ir catalyst and redox-active ester substrate to afford a carbocation intermediate that undergoes subsequent trapping by fluoride. Examples of trapping with O- and Ccentered nucleophiles and deoxyfluorination via $N$-hydroxyphthalimidoyl oxalates are also presented, suggesting that this approach may offer a general blueprint for affecting redox-neutral $\mathrm{S}_{\mathrm{N}} 1$ substitutions under mild conditions.
\end{abstract}

\section{INTRODUCTION}

Aliphatic organofluorine compounds are important structural motifs in pharmaceuticals, agrochemicals, and materials, conferring valuable biological and physical properties. ${ }^{1}$ This motif is also prominently featured in positron emission tomography (PET) radiotracers. ${ }^{1 \text { a }}$ Consequently, the identification of mild methods for late-stage introduction of fluorine has been a longstanding goal, with distinct strategies arising using nucleophilic and electrophilic fluorine sources. ${ }^{2}$ For reasons of cost, functional group compatibility, and translation to radiofluorination, researchers have sought mild methods for the preparation of fluoroalkyl groups using nucleophilic fluoride. ${ }^{1,2}$ In most of these methods, $\mathrm{Csp}^{3}-\mathrm{F}$ bond formation follows a bimolecular nucleophilic substitution pathway ( $\mathrm{S}_{\mathrm{N}} 2$ mechanism) and is thus limited to the preparation of activated or unhindered aliphatic fluorides (Scheme 1A). ${ }^{3}$ Indeed, the typical restrictions on substrate scope for bimolecular nucleophilic substitution reactions are even more acute for fluoride due to its low nucleophilicity and high Brønsted basicity, leading to competitive elimination. ${ }^{4,5}$

Stepwise nucleophilic fluorination reactions that proceed through a carbocation intermediate $\left(\mathrm{S}_{\mathrm{N}} 1\right.$ mechanism) provide a complementary strategy to bimolecular alkyl fluoride synthesis, enabling access to unactivated and hindered aliphatic fluorides. However, the generation of carbocation intermediates typically requires harsh Brønsted ${ }^{6}$ or Lewis acidic conditions ${ }^{7}$ that show poor functional group tolerance and lead to elimination and rearrangement pathways (Scheme 1B). To overcome these limitations, researchers have recently explored new strategies for carbocation generation under non-acidic conditions. The Knowles group introduced a methodology to access carbocation
Scheme 1. Nucleophilic fluorination

A. Bimolecular nucleophilic substitution $\left(\mathrm{S}_{\mathrm{N}} 2\right)$ with fluoride

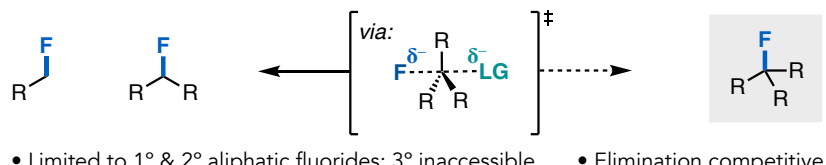

- Limited to $1^{\circ} \& 2^{\circ}$ aliphatic fluorides; $3^{\circ}$ inaccessible - Elimination competitive

B. Lewis or Brønsted acid-facilitated $\mathrm{S}_{\mathrm{N}} 1$

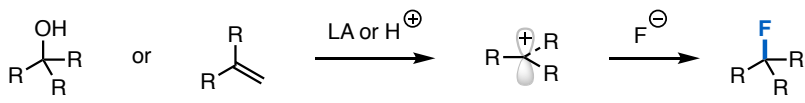

- Caustic reagents preclude late-stage functionalization - Access to $3^{\circ}$ fluorides

C. Photocatalyzed $\mathrm{S}_{\mathrm{N}} 1$ via mesolytic cleavage (Knowles)

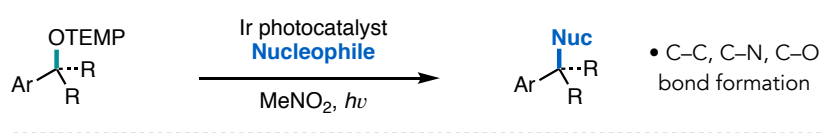

D. Electrochemical oxidation $\mathrm{S}_{\mathrm{N}} 1$ (Baran)

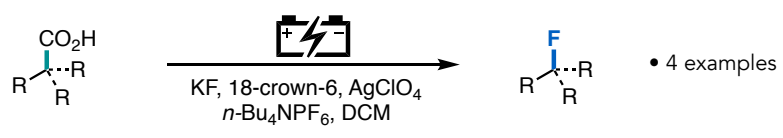

E. This work: Redox-neutral, radical-polar crossover $\mathrm{S}_{\mathrm{N}} 1$ substitution

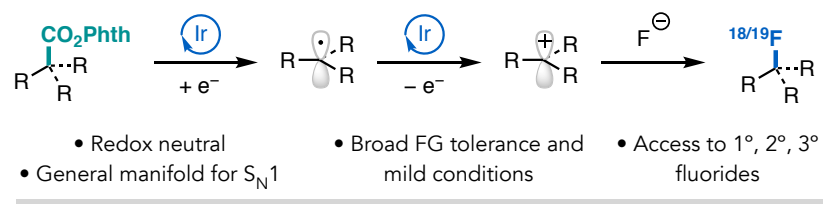


intermediates via mesolytic cleavage following the oxidation of TEMPO-derived alkoxyamine substrates; ${ }^{8}$ although a wide range of nucleophiles are compatible with this approach, the method was not shown to work with fluoride and the substrates can be challenging to access (Scheme 1C). Just recently, the Baran lab reported an electrochemical approach to carbocation generation from readily available carboxylic acids (Scheme 1D). ${ }^{9}$ The method affords access to a broad range of hindered ethers from alcohol nucleophiles and four examples of alkylfluorides from KF. Although this report has significantly advanced the state of the art, the requirement for oxidizing conditions places limits on the substrate scope and the method was not shown to be amenable to radiofluorination. This report was closely followed by the disclosure of a photocatalytic decarboxylative ether synthesis by Ohmiya, Nagao, and coworkers. ${ }^{10}$ Yet further discovery and development of complementary methods for carbocation generation are necessary to enable broad access to hindered and unactivated aliphatic fluorides, including for late-stage fluorination and radiofluorination, as well as expanding the repertoire for additional synthetic applications.

In this context, we sought to develop a redox-neutral method for carbocation generation via radical-polar crossover $^{10,11}$ from $N$-hydroxyphthalimide esters (Scheme 1E). We selected redox active esters as a substrate class since numerous research groups have recently reported their use as precursors to alkyl radicals via single-electron reduction and decarboxylation with release of a non-nucleophilic leaving group. ${ }^{12}$ We hypothesized that upon single electron reduction of the substrate by the excited state of a suitable photoredox catalyst, the resulting radical could be oxidized to the corresponding carbocation by the oxidized photocatalyst and subsequently trapped with fluoride. ${ }^{13}$ Given the similar trends in stability between radical and carbocation species, ${ }^{14}$ we anticipated that highly substituted aliphatic substrates should be particularly amenable to both radical and carbocation generation in a radical-polar crossover, thereby expanding the scope of aliphatic fluorides available via nucleophilic fluorination. This approach would also provide a useful complement to radical methodologies for decarboxylative fluorination that require oxidizing electrophilic fluorine sources ${ }^{15}$ or the combination of a nucleophilic fluoride source with a stoichiometric oxidant, as described by the Groves lab. ${ }^{16}$

Here we report a redox-neutral decarboxylative nucleophilic fluorination that delivers primary, secondary, and tertiary benzylic fluorides and unactivated tertiary fluorides with broad functional group tolerance. We also describe mechanistic experiments that provide evidence for both radical and carbocation intermediates; in so doing, we present applications to the construction of sterically congested ethers and $\mathrm{C}-\mathrm{C}$ bonds, establishing the generality of the strategy as a blueprint for affecting redox-neutral $S_{N} 1$ substitutions. Finally, we show that the method is amenable to ${ }^{18} \mathrm{~F}$-radiofluorination.

\section{RESULTS AND DISCUSSION}

Optimization. We initiated our studies with the $N$-hydroxyphthalimide ester 1 derived from naproxen, as naproxen's electron-rich arene would likely be incompatible with decarboxylative fluorination conditions that utilize electrophilic fluorine sources or stoichiometric oxidants. ${ }^{15 \mathrm{~b}}$ Subjecting 1 to irradiation with $34 \mathrm{~W}$ blue LEDs in the presence of $1 \mathrm{~mol} \% \operatorname{Ir}(\mathrm{dF}-\mathrm{ppy})_{3} 3$ and 3 equiv. of $\mathrm{Et}_{3} \mathrm{~N} \bullet 3 \mathrm{HF}$ delivered benzylic fluoride 2 in almost quantitative yield (Table 1, Entry 1). Fluorination does not proceed in the absence of light or photocatalyst, resulting in recovery of starting material (Table 1, Entries 2 and 3). More reducing or oxidizing iridium photocatalysts were competent in the reaction, as was the organic photocatalyst $4 \mathrm{CzIPN}$, all displaying high yet diminished reactivity compared to photocatalyst 3 (Table 1, Entries 4-6). While DCM was found to be the optimal solvent for the reaction, fluorination proceeded with modest to good yield in tetrahydrofuran and acetonitrile, solvents that are commonly used in radiofluorination (Table 1, Entries 7 and 8). Only trace product was observed using KF/HFIP $(1,1,1,3,3,3 \text {-hexafluoroisopropanol })^{17}$ instead of $\mathrm{Et}_{3} \mathrm{~N} \bullet 3 \mathrm{HF}$ and no product was observed using KF in the absence of HFIP (Table 1, Entries 9 and 10). Although impractical from a preparative standpoint, this result provides support for possible translation to radiofluorination since $\left[{ }^{18} \mathrm{~F}\right] \mathrm{KF}$ is the most common reagent for ${ }^{18} \mathrm{~F}$-radiochemistry (vida infra). ${ }^{18}$ Finally, we found that the reaction could be readily scaled to 4 mmoles (Table 1, Entry 11) and photocatalyst loading could be reduced to 0.0625 mol\% with minimal impact on the reaction efficiency (Table 1, Entry 12). This speaks to the practicality of the method, and the ability to use such low photocatalyst loading holds important mechanistic implications (vide infra).

Table 1. Optimization of Reaction Conditions. ${ }^{a, b}$

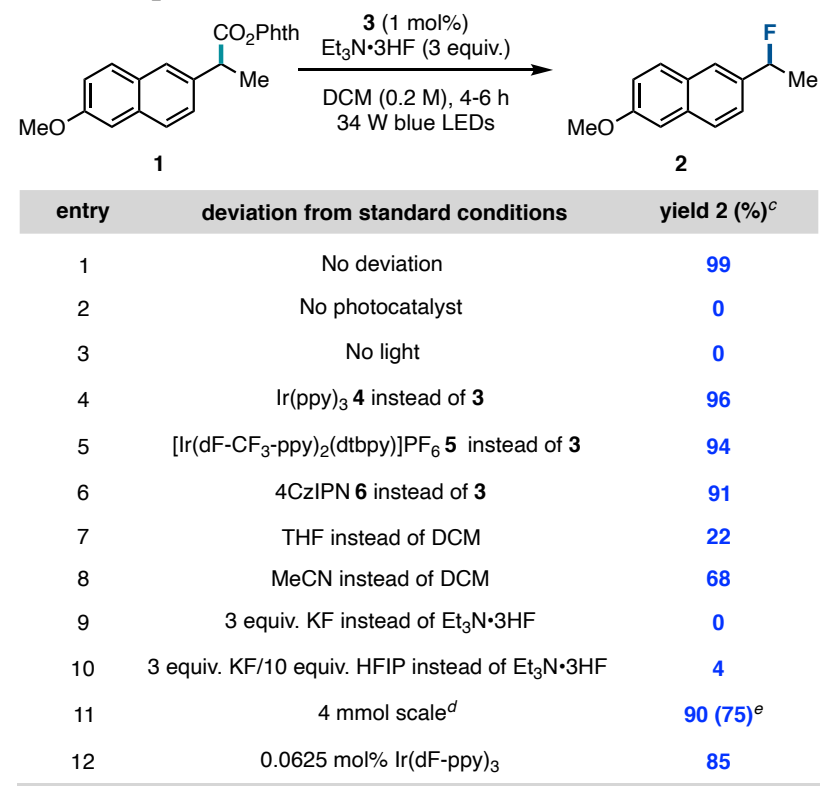

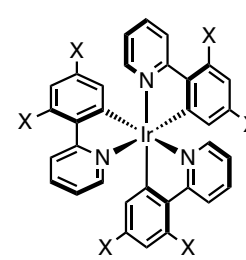

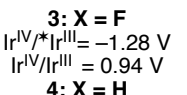

$\mathrm{Ir}^{\mathrm{IV}} / \mathrm{Ir}^{\mathrm{III}}=0.94 \mathrm{~V}$
$4: \mathbf{X}=\mathbf{H}$

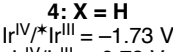

$\mathrm{Ir}^{\mathrm{IV} / \mathrm{Ir}} \mathrm{III}=0.78 \mathrm{~V}$

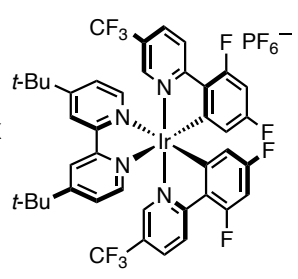

$$
\begin{aligned}
& \left.\mathrm{Ir}^{\mathrm{IV} /{ }^{*}}\right|^{\mathrm{IIII}}=-0.89 \mathrm{~V} \\
& \mathrm{Ir}^{\mathrm{IV} /} / \mathrm{Ir}^{\mathrm{III}}=1.69 \mathrm{~V}
\end{aligned}
$$

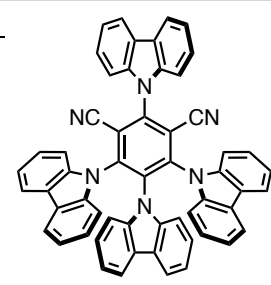

6 $\mathrm{PC}^{+} / \mathrm{PC}=1.43 \mathrm{~V}$
${ }^{a} 0.2 \mathrm{mmol}$ scale. ${ }^{b}$ All potentials given are versus a saturated calomel electrode (SCE) and taken from ref. 19. ${ }^{c}$ Yields determined by ${ }^{19} \mathrm{~F}-\mathrm{NMR}$ using 1-fluoronaphthalene as an external standard. ${ }^{d}$ General conditions except 0.4 $\mathrm{M}, 1.5$ equiv. $\mathrm{Et}_{3} \mathrm{~N} \bullet 3 \mathrm{HF}$. ${ }^{e}$ Isolated yield. 
Table 2. Substrate Scope for Photocatalytic Decarboxylative Fluorination. ${ }^{a}$

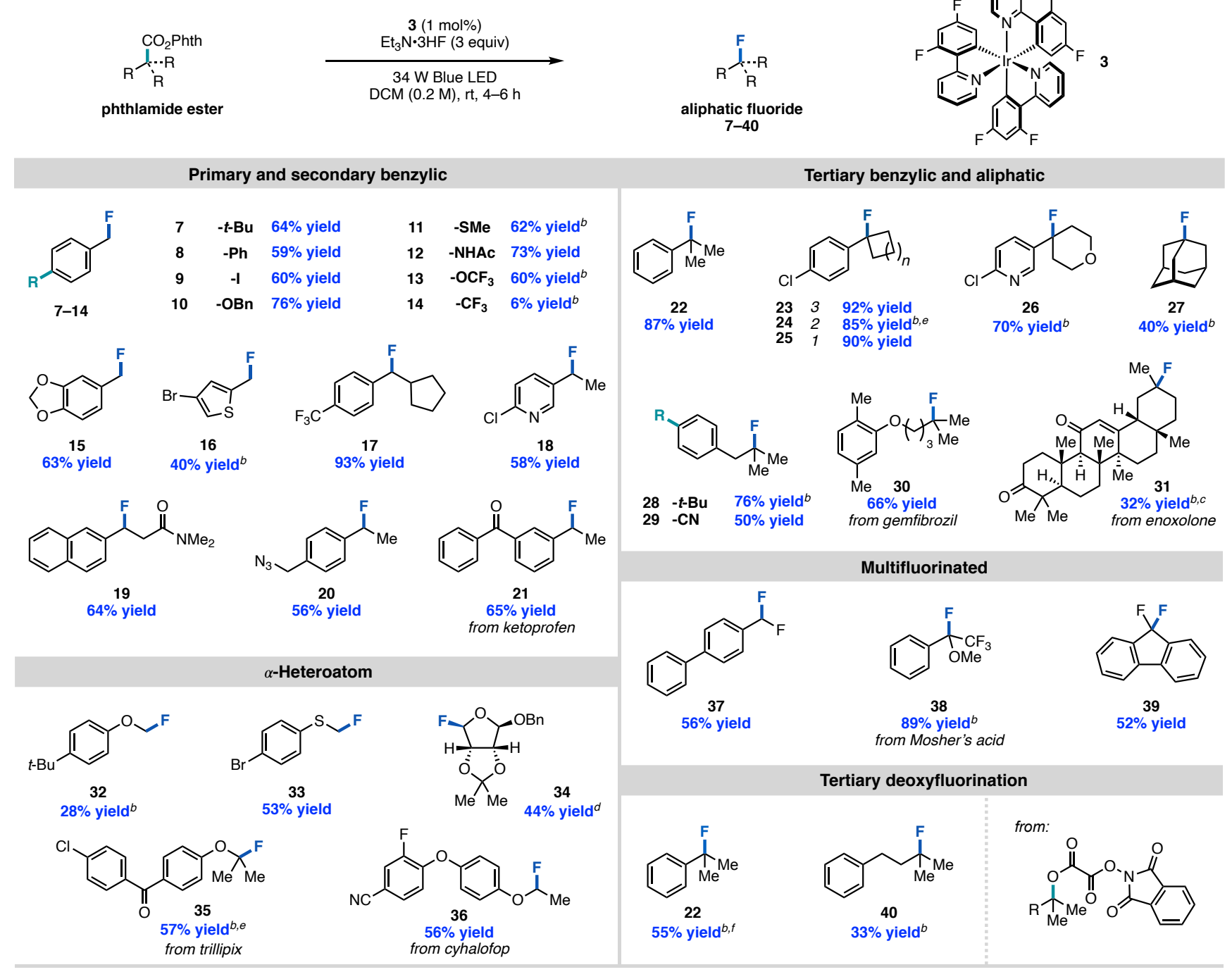

${ }^{a}$ Isolated yields of an average of two runs on $0.5-1.0 \mathrm{mmol} \mathrm{scale.}{ }^{b}$ Yield determined by ${ }^{19} \mathrm{~F}$-NMR using 1 -fluoronaphthalene as an external standard. ${ }^{c} 1.3: 1$ d.r. ${ }^{d}>20: 1$ d.r. ${ }^{e}$ Product unstable. ${ }^{f}$ Yield corrected for starting material contamination with residual alcohol.

Scope Elucidation. With optimized conditions in hand, we evaluated the scope of the transformation. We found that a variety of primary benzylic fluorides could be obtained in good to excellent yields (Table 2, 7-13, 15, and 16). Several substrates bearing electron-rich functionality otherwise susceptible to oxidation under previously reported decarboxylative fluorination conditions were tolerated, ${ }^{15 \mathrm{~b}}$ delivering dioxole $\mathbf{1 5}$, benzyl-protected phenol 10, and thioether 11. Fluorinated products bearing medicinally-relevant amides and trifluoromethoxy groups were generated in good yield (12 and 13). Whereas these electronrich and electron-neutral primary benzylic phthalimide esters were competent substrates, electron-deficient substrates afforded benzylic fluorides in low yield at high conversion (14), presumably because single electron reduction and decarboxylation to the carbon-centered radical is facile but oxidation of the radical to the cation is disfavored due to the electron-withdrawing substituent. ${ }^{12}$ As expected on the basis of this hypothesis, we found that replacing the primary benzylic substrate with a secondary substrate bearing the same substitution pattern restored reactivity, with fluorinated product $\mathbf{1 7}$ obtained in $93 \%$ yield.
A common limitation in nucleophilic fluorination methods that deliver secondary benzylic fluorides is elimination to styrene byproducts. ${ }^{3 \mathrm{~d}, \mathrm{f}}$ For all of the secondary substrates examined in Table 2 , less than $5 \%$ elimination was observed, a testament to the mildness of the conditions. Indeed, even product 19 was obtained in high yield with minimal elimination despite the presence of a $\beta$-carbonyl functional group. By comparison, access to $\beta$-fluoro carbonyl derivatives by deoxyfluorination has presented a major challenge to date due to competing elimination. $^{3 \mathrm{~d}, \mathrm{f}}$ Several handles for subsequent transition metal-mediated coupling were tolerated, including aryl iodides $\mathbf{9}$, bromides $\mathbf{1 6}$ and 33, and chlorides $\mathbf{1 8}$ and 23-26. This tolerance for easily reduced functionality can even be extended to azide-containing product 20, which was generated in $56 \%$ yield and offers a reactive handle for subsequent "click" chemistry that is widely used in bioconjugation. ${ }^{21}$ Additionally, basic heterocycles and heteroaromatic groups otherwise susceptible to oxidation or Minisci chemistry underwent decarboxylative fluorination in good yields (18 and 26).

In contrast to typical methods for nucleophilic fluorination, we found that access to benzylic and unactivated tertiary fluorides 
is possible. For example, acyclic $\mathbf{2 2}$ as well as tertiary benzylic fluorides embedded within carbocyclic and heterocyclic ring systems are generated in 70-92\% yield, as in the cases of $\mathbf{2 3}-$ 26. Likewise, both cyclic and acyclic unactivated tertiary fluorides could be obtained (27-31). Whereas neighboring group participation may be operative in the generation of the homobenzylic tertiary fluorides $\mathbf{2 8}$ and $\mathbf{2 9}$, it does not appear to be necessary given the success of the cyano-substituted homobenzylic fluoride $\mathbf{2 9}$ and fluorides $\mathbf{2 7}$ and $\mathbf{3 1}$ that do not possess a proximal nucleophilic residue. Notably, we were able to extend this protocol to the fluorination of gemfibrozil 30 in $66 \%$ yield.

Fluoroether and fluorothioether functionality has been shown to confer unique and valuable properties to biologically active small molecules. ${ }^{22} \mathrm{We}$ found that the redox-neutral decarboxylative nucleophilic fluorination also delivers $\alpha$-oxy- and $\alpha$-thioether motifs in modest to good yield (32 and 33). As a demonstration of the viability of the method for late-stage derivatization, fluorinated ribose $\mathbf{3 4}$, trillipix-derivative $\mathbf{3 5}$, and the herbicide cyhalofop-derived 36 were all readily accommodated. Likewise, application of the optimal conditions to the preparation of difluoromethyl and perfluorinated groups was success$\mathrm{ful}^{23}$ as in the cases of $\mathbf{3 7}$ and $\mathbf{3 8}$, and permitted the synthesis of difluorofluorene 39 , a motif featured in the hepatitis $\mathrm{C}$ drug ledipasvir.

Fluorine incorporation is commonly used as a bioisostere for several functionalities including $\mathrm{C}-\mathrm{OH}$ and $\mathrm{C}-\mathrm{H}$ bonds. ${ }^{1 \mathrm{~b}} \mathrm{In}$ this regard, the conversion of abundant alcohols into the corresponding alkyl fluorides via deoxyfluorination represents an attractive synthetic disconnection. However, deoxyfluorinations of tertiary alcohols to access tertiary fluorides are typically unsuccessful. ${ }^{24}$ MacMillan and co-workers have recently reported a deoxyfluorination of oxalate half-esters to access tertiary fluorides. ${ }^{25}$ However, the method uses an electrophilic fluorine source. Since tert-alkyl $N$-hydroxyphthalimidoyl oxalates are similar in redox potential to $N$-hydroxyphthalimide esters, we hypothesized that these may be amenable to the catalytic nucleophilic fluorination strategy outlined herein. ${ }^{26}$ Indeed, we were pleased to find that tertiary fluorides $\mathbf{2 2}$ and $\mathbf{4 0}$ could be obtained in $55 \%$ and $33 \%$ yield from tert-alkyl $N$-hydroxyphthalimidoyl oxalate esters under otherwise identical conditions. Since these substrates are readily available from alcohols, the method represents a complementary approach to nucleophilic deoxyfluorination which is typically limited to primary and secondary alcohols. ${ }^{3 \mathrm{a}-\mathrm{f}}$

Mechanistic Investigations. We propose that excited $3\left(E_{1 / 2}^{*}=\right.$ -1.28 vs. $\mathrm{SCE}^{19 \mathrm{a}}$ ) undergoes single electron transfer (SET) with the $N$-hydroxyphthalimide ester $\mathbf{A}\left(\sim-1.3 \mathrm{~V}\right.$ vs $\left.\mathrm{SCE}^{20}\right)$ (Figure 2). Fragmentation of the resulting phthalimide ester radical anion and subsequent extrusion of carbon dioxide generates carbon-centered radical B. Radical intermediate $\mathbf{B}\left(E_{1 / 2}^{o x}=<\right.$ $0.73 \mathrm{~V}$ vs. SCE for $1^{\circ}$ benzylic, $E_{1 / 2}^{o x}=0.09 \mathrm{~V}$ vs. SCE for tertiary aliphatic $\left.{ }^{13 \mathrm{~b}}\right)$ is then oxidized by photocatalyst $\mathbf{3}^{+}\left(\mathrm{Ir}^{\mathrm{IV}} / \mathrm{Ir}^{\mathrm{III}}\right.$ $E_{1 / 2}=0.94 \mathrm{~V}$ vs. SCE ${ }^{19 a}$ ), turning over the photocatalyst and furnishing carbocation $\mathbf{C}$. Finally, this carbocation is trapped by the fluoride source to furnish the desired alkyl fluoride $\mathbf{D}$.

A number of experimental observations provide support for the proposed mechanism. Stern-Volmer quenching analysis of the individual components of the reaction mixture indicates that the phthalimide ester quenches the excited state photocatalyst with an observed $\mathrm{K}_{\mathrm{SV}}$ of $4.7 \times 10^{9} \mathrm{M}^{-1} \mathrm{~s}^{-1}$, which is similar to the quenching rate of the reaction mixture. ${ }^{27}$
Figure 2. Mechanistic Proposal.

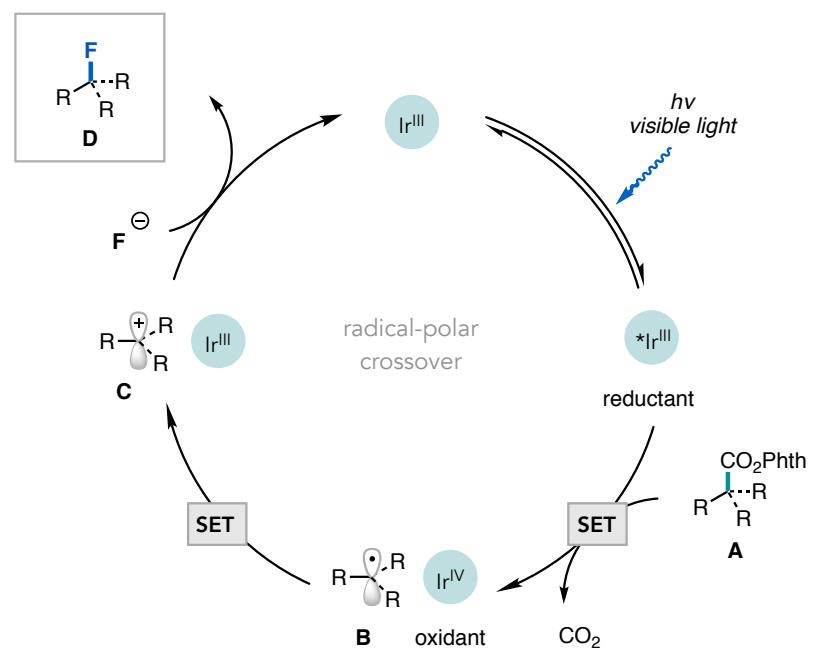

Figure 3. Radical and Cation Trapping. ${ }^{a}$

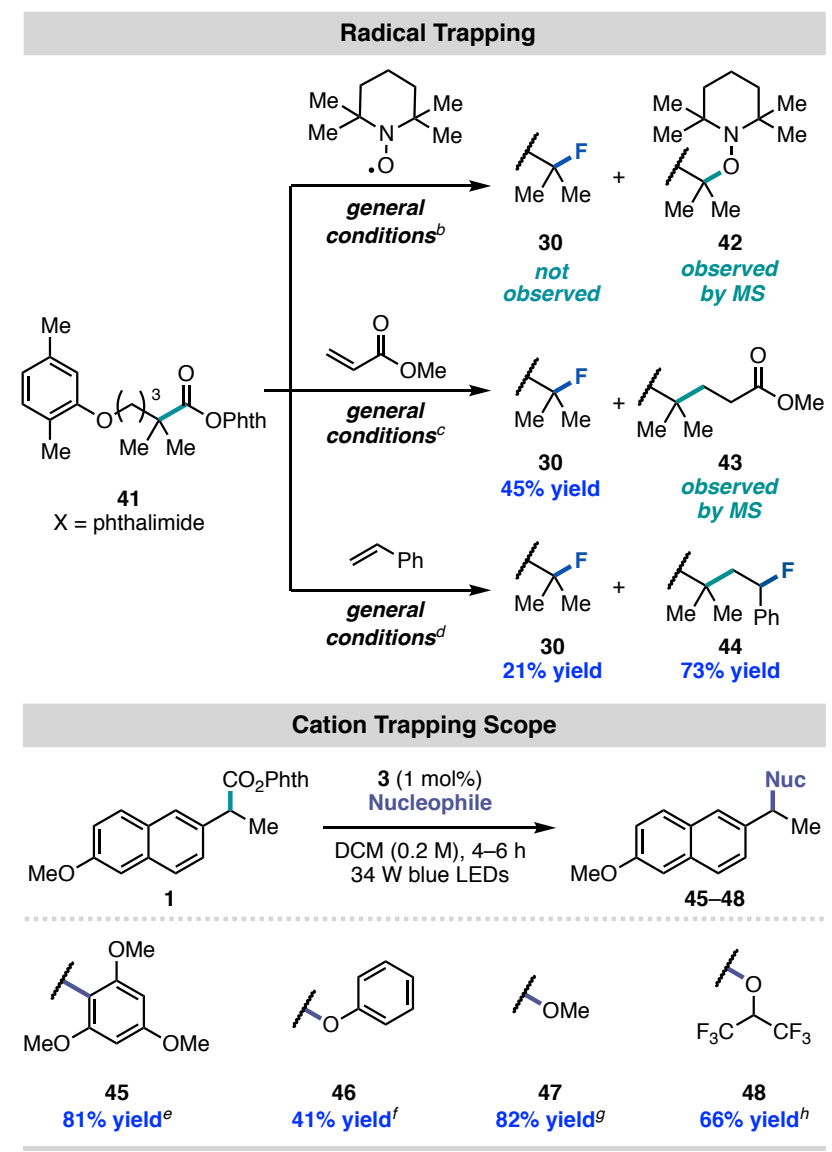

${ }^{a}$ General conditions: $1 \mathrm{~mol} \% \operatorname{Ir}(\mathrm{dF}-\mathrm{ppy})_{3}, 0.2 \mathrm{mmol}$ 41, 3 equiv. Et $3 \mathrm{~N} \bullet 3 \mathrm{HF}$. ${ }^{b} 1.5$ equiv. of TEMPO. ${ }^{c} 1.5$ equiv. of methyl acrylate. ${ }^{d} 1.5$ equiv. styrene. ${ }^{e}$ 3 equiv. of trimethoxybenzene and 0.3 equiv. $\mathrm{Et}_{3} \mathrm{~N} \bullet 3 \mathrm{HF} .{ }^{f} 5$ equiv. phenol and 0.3 equiv. $\mathrm{Et}_{3} \mathrm{~N} \bullet 3 \mathrm{HF}$. ${ }^{g} 6$ equiv. methanol and 0.3 equiv. of $\mathrm{Et} 3 \mathrm{~N} \bullet 3 \mathrm{HF}$. ${ }^{h} 5$ equiv. of HFIP and run for $24 \mathrm{hrs;} ;{ }^{19} \mathrm{~F}$-NMR yield vs external 1 -fluoronapthalene.

The quantum yield of this fluorination reaction is 0.37 , indicating that chain mechanisms are unlikely or inefficient. ${ }^{27,28}$ This result, combined with the observation that the fluorinations proceed with high reaction efficiency at extremely low photocatalyst loadings ( $0.0625 \%$ of $\mathbf{3}$ in Table 1, Entry 12$)$, suggests that the reaction is unimolecular in photocatalyst. 
Subjecting tertiary phthalimide ester $\mathbf{4 1}$ to the fluorination conditions in the presence of several known radical traps provides evidence for the intermediacy of a radical. For example, addition of TEMPO (2,2,6,6-tetramethyl-1-piperidinyloxy) to the reaction resulted in complete inhibition of fluorination, with concomitant detection of TEMPO adduct 42 (Figure 3 ). In the presence of methyl acrylate, radical addition product 43 was observed, along with a diminished yield of tertiary fluoride $\mathbf{3 0}$ $\left(66 \%\right.$ isolated vs. $45 \%$ by ${ }^{19} \mathrm{~F}-\mathrm{NMR}$ in the presence of methyl acrylate). However, fluoride is not incorporated into 43, presumably because radical oxidation adjacent to the ester carbonyl is unfavorable. On the other hand, conducting the fluorination reaction in the presence of 1.5 equiv. styrene afforded a new fluorinated product 44 in addition to the direct fluorination product 30. 44 is most likely generated via addition of radical B to the styrene followed by oxidation of the resulting benzylic radical and trapping with fluoride. Evaluation of a series of electronically differentiated styrene substrates, and the outcome of the reaction with methyl acrylate, provide evidence against an alternative pathway wherein radical oxidation to cation $\mathbf{C}$ precedes olefin addition. ${ }^{27}$

As a test for the intermediacy of a carbocation, we investigated whether other polar nucleophiles could be used in the decarboxylative substitution reaction. Notably, we found that $\mathrm{C}$ - and $\mathrm{O}$ centered nucleophiles were competent with only minor changes to the reaction parameters (Table $3,45-\mathbf{4 8}$ ) ${ }^{29}$ For example, trimethoxybenzene underwent addition to generate benzhydryl $\mathbf{4 5}$ in $81 \%$ yield. This reaction represents a Lewis-acid-free Friedel-Crafts substitution using abundant carboxylic acid precursors in place of alkyl halide substrates. Likewise, several alcohol nucleophiles delivered ether products 46-48 in good yield. The broad tolerance for a range of nucleophiles, including sterically hindered and poorly nucleophilic species, implicates the intermediacy of a carbocation and demonstrates the generality of the strategy to effect a range of challenging substitution reactions under remarkably mild conditions.

Radiochemistry. Aliphatic ${ }^{18}$ F-radiolabelled PET tracers are almost exclusively prepared by nucleophilic substitution of alkyl sulfonates with $\left[{ }^{18} \mathrm{~F}\right] \mathrm{KF}$ in the presence of a phase transfer reagent Kryptofix 2.2.2 $\left(\mathrm{K}_{222}\right){ }^{18}$ As such, access to high specific activity radiolabeled targets bearing unactivated secondary or tertiary fluorides remains a critical challenge. Moreover, the harsh conditions necessary for substitution of even primary or activated secondary substrates $\left(>100{ }^{\circ} \mathrm{C}\right.$ and high basicity) are often not suitable for late-stage radiofluorination and often lead to inseparable olefin byproducts. ${ }^{18} \mathrm{We}$ anticipated that successful translation of the photocatalytic decarboxylative nucleophilic fluorination method would therefore enable access to previously challenging or impossible to prepare radiotracers.

To translate our method, we elected to pursue the decarboxylative radiofluorination in acetonitrile to avoid any potential clinical issues with dichloromethane. Testing the radiofluorinations in $\mathrm{CH}_{3} \mathrm{CN}$ using $\left[{ }^{18} \mathrm{~F}\right] \mathrm{KF} / \mathrm{K}_{222}$ as fluoride source, we found that the previously optimized photocatalyst $\mathbf{3}$ was no longer the most effective, with $\operatorname{Ir}(\mathrm{F}-\mathrm{ppy})_{3}$ instead affording the highest radiochemical incorporation (Table 3, Entries 1 and 4-7). ${ }^{27}$ No radiofluorination was observed in the absence of light or photocatalyst as determined by radio-TLC or radio-HPLC (Table 3, Entries 2 and 3). While we had previously found that HFIP was necessary for achieving fluorination using $\left[{ }^{19} \mathrm{~F}\right] \mathrm{KF}$ under the "cold" conditions (Table 1, Entries 10 and 11), the addition of HFIP proved detrimental in the radiochemical system (Table 3,
Entry 9). Under the optimal conditions, the reaction is particularly fast, furnishing $\left[{ }^{18} \mathbf{F}\right] 2$ in $62 \%$ radiochemical conversion (RCC) within 2 minutes of irradiation (Table 3, Entry 8). The radiofluorination was also scalable $(1850-3700 \mathrm{MBq})$, permitting isolation of sufficient quantities of $\left[{ }^{\mathbf{1 8}} \mathbf{F}\right] \mathbf{2}$ to determine its molar activity $(33.7 \pm 18.9 \mathrm{MBq} / \mathrm{nmol})$, which is on par with other no-carrier added nucleophilic fluorination protocols. ${ }^{17,18,30}$ Notably, these radiochemical reactions were conducted in a 3Dprinted apparatus that permits automated handling of radioactivity with irradiation taking place from the bottom of the vials. $^{27}$

Table 3. Development of Radiochemical Protocol. ${ }^{a}$

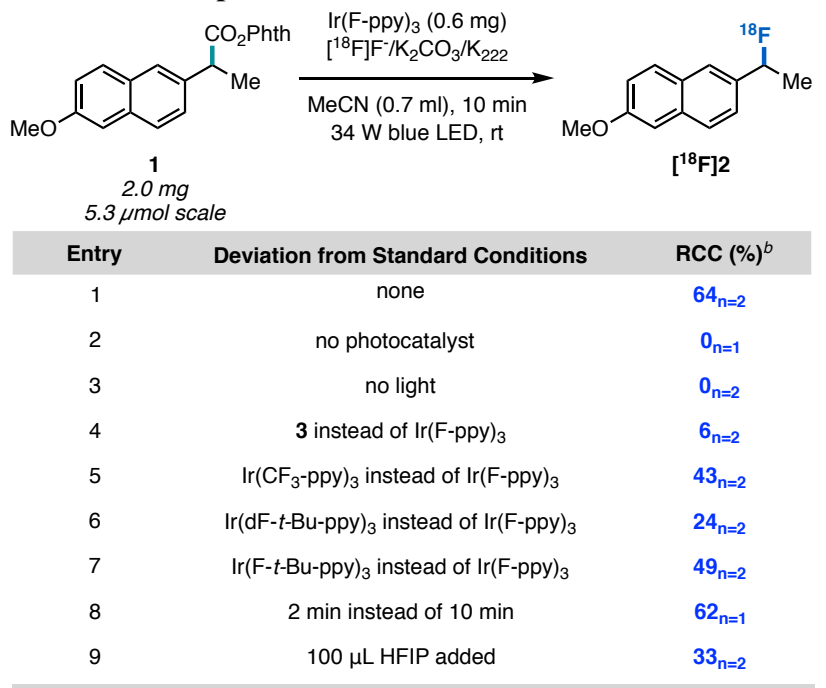

${ }^{a}$ Typical reaction conditions: $5.3 \mu \mathrm{mol} \mathbf{1}, 16 \mathrm{~mol} \%$ photocatalyst, $0.7 \mathrm{~mL}$ $\mathrm{MeCN}$, and $\left[{ }^{18} \mathrm{~F}\right] \mathrm{F}^{-} / \mathrm{K}_{2} \mathrm{CO}_{3} / \mathrm{K}_{222}\left(\sim 370 \mathrm{Mbq}\right.$ of activity per reaction). ${ }^{b} \mathrm{RCC}$ was determined by radio-TLC with number of replicates noted.

Table 4. Scope of Photocatalyzed Radiofluorination.

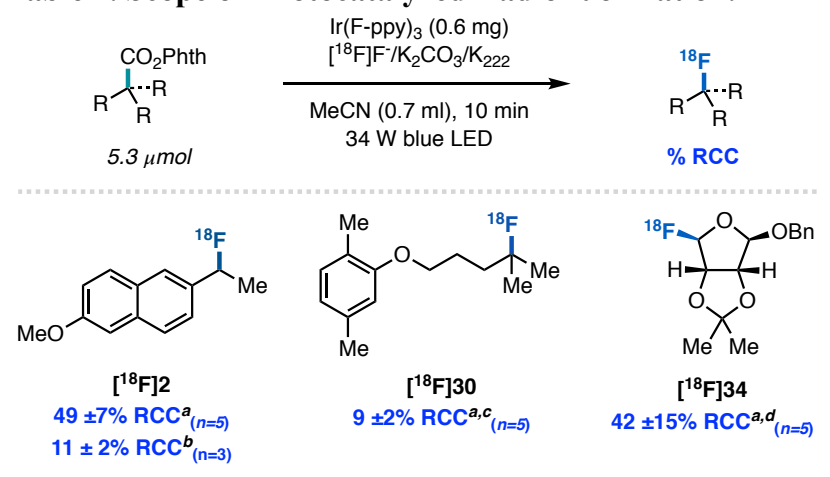

${ }^{a}$ Typical reaction conditions: $5.3 \mu \mathrm{mol} 1,0.6 \mathrm{mg} \mathrm{Ir}(\mathrm{F}-\mathrm{ppy})_{3}, 0.7 \mathrm{~mL} \mathrm{MeCN}$, and $\left[{ }^{18} \mathrm{~F}_{\mathrm{F}} / \mathrm{K}_{2} \mathrm{CO}_{3} / \mathrm{K}_{222}(\sim 370 \mathrm{MBq}\right.$ of activity per reaction). RCC was determined by radio-TLC with number of replicates noted. ${ }^{b}$ Reactions were conducted with $1850-3700 \mathrm{MBq}$ of activity per reaction). ${ }^{c}$ Reactions conducted using photocatalyst $3{ }^{d}$ Product identity confirmed via TLC.

Access to $\left[{ }^{18} \mathbf{F}\right] 2$ is significant since its synthesis by $\mathrm{S}_{\mathrm{N}} 2$ displacement with $\left[{ }^{18} \mathrm{~F}\right] \mathrm{KF}$ would likely be plagued by rapid formation of elimination byproduct. We also briefly explored application of the radiofluorination conditions to the radiosynthesis of other fluorinated motifs that are challenging to access using conventional methods. For example, the radiofluorination protocol is amenable to the installation of a tertiary fluoride: $\left[{ }^{18} \mathbf{F}\right] 30$ derived from gemfibrozil was obtained in $9 \pm 2 \%$ RCC. Furthermore, we found that ribose analogue $\left[{ }^{18} \mathbf{F}\right] 34$ could be 
prepared in $42 \%$ RCC. In this case, the sulfonate precursor readily decomposes at room temperature, severely limiting access to radiolabeled ribose analogues by conventional substitution reactions. ${ }^{31}$

\section{CONCLUSION}

We have developed a photocatalytic method for nucleophilic fluorination of $N$-hydroxyphthalimide esters that exploits the redox activity of radicals as a route to carbocation formation. The approach generates a variety of useful fluorinated motifs under mild conditions and is compatible with functional groups that challenge other synthetic methods using both nucleophilic and electrophilic fluorine sources, such as access to tertiary aliphatic fluorides and tolerance to electron-rich functionality. Moreover, translation of the method to a radiochemical protocol was possible, enabling radiofluorination of derivatives of bioactive molecules. We present a preliminary demonstration of the generality of this approach to redox-neutral $\mathrm{S}_{\mathrm{N}} 1$-like substitutions in extensions to a new substrate class, such as deoxyfluorination of tertiary $N$-hydroxyphthalimidoyl oxalates, and to new nucleophiles, as in the construction of sterically congested ethers and $\mathrm{C}-\mathrm{C}$ bonds.

\section{ASSOCIATED CONTENT}

\section{Supporting Information}

The Supporting Information is available free of charge on the ACS Publications website.

Experimental procedures and characterization and spectral data for new compounds (PDF)

\section{AUTHOR INFORMATION}

\section{Corresponding Author \\ *agdoyle@princeton.edu}

\section{ORCID}

Abigail G. Doyle: 0000-0002-6641-0833

\section{Notes}

The authors declare no competing financial interest.

\section{ACKNOWLEDGMENT}

Financial support was generously provided by NSF (CHE1565983) and Bristol-Myers Squibb. We thank Dhar Murali (BMS) for helpful suggestions, Erin E. Gomez (BMS) for radiochemical and analytical expertise, and Daniel Batella (BMS) for construction of the apparatus to allow radiochemical irradiation. István Pelzer, Kenneth Conover, are John Eng are acknowledged for analytical aid. We thank Laura K. G. Ackermann, Meredith A. Borden, and Talia J. Steiman for helpful discussions.

\section{REFERENCES}

(1) (a) Gillis, E. P.; Eastman, K. J.; Hill, M. D.; Donnelly, D. J.; Meanwell, N. A. Applications of Fluorine in Medicinal Chemistry. J. Med. Chem. 2015, 58, 8315. (b) Jeschke, P. The Unique Role of Fluorine in the Design of Active Ingredients for Modern Crop Protection. ChemBioChem 2004, 5 , 570. (c) Hagmann, W. K. The Many Roles for Fluorine in Medicinal Chemistry. J. Med. Chem. 2008, 51, 4359.

(2) (a) Liang, T.; Neumann, C. N.; Ritter, T. Introduction of Fluorine and Fluorine-Containing Functional Groups. Angew. Chem., Int. Ed. 2013, 52, 8214. (b) Neumann, C. N.; Ritter, T. Late-Stage Fluorination: Fancy Novelty or Useful Tool? Angew. Chem., Int. Ed. 2015, 54, 3216.
(3) Deoxyfluorination with DAST: (a) Middleton, W. J. New Fluorinating Reagents. Dialkylaminosulfur Fluorides. J. Org. Chem. 1975, 40, 574. (b) Markovskij, L. N.; Pashinnik, V. E.; Kirsanov, A. V. Application of Dialkylaminosulfur Trifluorides in the Synthesis of Fluoroorganic Compounds. Synthesis 1973, 12, 787. Deoxyfluorination with Phenofluor/Alkylfluor: (c) Tang, P.; Wang, W.; Ritter, T. Deoxyfluorination of Phenols. J. Am. Chem. Soc. 2011, 133, 11482. (d) Sladojevich, F.; Arlow, S. I.; Tang, P.; Ritter, T. Late-Stage Deoxyfluorination of Alcohols with Phenofluor. J. Am. Chem. Soc. 2013, 135, 2470. (e) Goldberg, N. W.; Shen, X.; Li, J.; Ritter, T. AlkylFluor: Deoxyfluorination of Alcohols. Org. Lett. 2016, 18, 6102. Deoxyfluorination with Pyfluor: (f) Nielsen, M. K.; Ugaz, C. R.; Li, W.; Doyle, A. G. PyFluor: A Low-Cost, Stable, and Selective Deoxyfluorination Reagent. J. Am. Chem. Soc. 2015, 137, 9571. Fluorination with hypervalent iodine reagents: (g) Hara, S.; Nakahigashi, J.; Ishi-i, K.; Sawaguchi, M.; Sakai, H.; Fukuhara, T.; Yoneda, N. Difluorination of Alkenes with Iodotoluene Difluoride. Synlett 1998, 2, 495. (h) Chen, H.; Kaga, A.; Chiba, S. AntiSelective Aminofluorination of Alkenes with Amidines Mediated by Hypervalent Iodine(III) Reagents. Org. Biomol. Chem. 2016, 14, 5481. (i) Kong, W.; Feige, P.; De Haro, T.; Nevado, C. Regio- and Enantioselective Aminofluorination of Alkenes. Angew. Chem., Int. Ed. 2013, 52, 2469. (j) Mennie, K. M.; Banik, S. M.; Reichert, E. C.; Jacobsen, E. N. Catalytic Diastereo- and Enantioselective Fluoroamination of Alkenes. J. Am. Chem. Soc. 2018, 140, 4797. (k) Banik, S. M.; Medley, J. W.; Jacobsen, E. N. Catalytic, Asymmetric Difluorination of Alkenes to Generate Difluoromethylated Stereocenters. Science 2016, 353, 51. (1) Banik, S. M.; Medley, J. W.; Jacobsen, E. N. Catalytic, Diastereoselective 1,2-Di Fluorination of Alkenes. J. Am. Chem. Soc. 2016, 138, 5000. Fluorination via ion-binding catalysis: (m) Pupo, G.; Ibba, F.; Ascough, D. M. H. H.; Vicini, A. C.; Ricci, P.; Christensen, K. E.; Pfeifer, L.; Morphy, J. R.; Brown, J. M.; Paton, R. S.; Gouverneur, V. Asymmetric Nucleophilic Fluorination under Hydrogen Bonding Phase-Transfer Catalysis. Science 2018, 360, 638. (n) Pupo, G.; Vicini, A. C.; Ascough, D. M. H.; Ibba, F.; Christensen, K. E.; Thompson, A. L.; Brown, J. M.; Paton, R. S.; Gouverneur, V. Hydrogen Bonding Phase-Transfer Catalysis with Potassium Fluoride: Enantioselective Synthesis of $\beta$-Fluoroamines. J. Am. Chem. Soc. 2019, 141, 2878. (o) Pfeifer, L.; Engle, K. M.; Pidgeon, G. W.; Sparkes, H. A.; Thompson, A. L.; Brown, J. M.; Gouverneur, V. Hydrogen-Bonded Homoleptic Fluoride-Diarylurea Complexes: Structure, Reactivity, and Coordinating Power. J. Am. Chem. Soc. 2016, 138, 13314.

(4) (a) Liang, S.; Hammond, G. B.; Xu, B. Hydrogen Bonding: Regulator for Nucleophilic Fluorination. Chem. - A Eur. J. 2017, 23, 17850. (b) Engle, K. M.; Pfeifer, L.; Pidgeon, G. W.; Giuffredi, G. T.; Thompson, A. L.; Paton, R. S.; Brown, J. M.; Gouverneur, V. Coordination Diversity in Hydrogen-Bonded Homoleptic Fluoride-Alcohol Complexes Modulates Reactivity. Chem. Sci. 2015, 6, 5293. (c) Nolte, C.; Ammer, J.; Mayr, H. Nucleofugality and Nucleophilicity of Fluoride in Protic Solvents. J. Org. Chem. 2012, 77, 3325. (d) Landini, D.; Maia, A.; Rampoldi, A. Dramatic Effect of the Specific Solvation on the Reactivity of Quaternary Ammonium Fluorides and Poly(Hydrogen Fluorides), $(\mathrm{HF})_{n} \bullet \mathrm{F}^{-}$, in Media of Low Polarity. J. Org. Chem. 1989, 54, 328. (e) Lee, J.-W.; Oliveira, M. T.; Jang, H. Bin; Lee, S.; Chi, D. Y.; Kim, D. W.; Song, C. E. Hydrogen-Bond Promoted Nucleophilic Fluorination: Concept, Mechanism and Applications in Positron Emission Tomography. Chem. Soc. Rev. 2016, 45, 4638.

(5) Liotta, C. L.; Harris, H. P. The Chemistry of "Naked" Anions. I. Reactions of the 18-Crown-6 Complex of Potassium Fluoride with Organic Substrates in Aprotic Organic Solvents. J. Am. Chem. Soc. 1974, 96, 2250

(6) (a) Olah, G. A.; Nojima, M.; Kerekes, I. Hydrofluorination of Alkenes, Cyclopropane, and Alkynes with Poly-Hydrogen Fluoride/Pyridine (Trialkylamine)Reagents. Synthesis. 1973, 1973, 779. (b) Olah, G. A.; Welch, J. T.; Vankar, Y. D.; Nojima, M.; Kerekes, I.; Olah, J. A. Pyridinium Poly(Hydrogen Fluoride) (30\% Pyridine-70\% Hydrogen Fluoride): A Convenient Reagent for Organic Fluorination Reactions. J. Org. Chem. 1979, 44, 3872. (c) Lu, Z.; Zeng, X.; Hammond, G. B.; Xu, B. Widely Applicable Hydrofluorination of Alkenes via Bifunctional Activation of Hydrogen Fluoride. J. Am. Chem. Soc. 2017, 139, 18202. (d) Bertrand, X.; Paquin, J.-F. Direct Hydrofluorination of Methallyl Alkenes Using a Methanesulfonic Acid/Triethylamine Trihydrofluoride Combination. Org. Lett. 2019, 21, 9459.

(7) Silver mediated fluorination: (a) Khotavivattana, T.; Verhoog, S.; Tredwell, M.; Pfeifer, L.; Calderwood, S.; Wheelhouse, K.; Leecollier, T.; Gouverneur, V. ${ }^{18} \mathrm{~F}$-Labeling of Aryl-SCF $3,-\mathrm{OCF}_{3}$ and $-\mathrm{OCHF}_{2}$ with $\left[{ }^{18} \mathrm{~F}\right]$ Fluoride Angew. Chem., Int. Ed. 2015, 54, 9991. (b) Verhoog, S.; Pfeifer, L.; Khotavivattana, T.; Calderwood, S.; Collier, T. L.; Wheelhouse, K.; Tredwell, M.; Gouverneur, V. Silver-Mediated ${ }^{18} \mathrm{~F}$-Labeling of Aryl-CF 3 and Aryl-CHF 2 with ${ }^{18}$ F-Fluoride Synlett 2016, 27, 25. Boron trifluoride 
mediated fluorination and references therein: (c) Cresswell, A. J.; Davies, S. G.; Roberts, P. M.; Thomson, J. E. Beyond the Balz-Schiemann Reaction: The Utility of Tetrafluoroborates and Boron Trifluoride as Nucleophilic Fluoride Sources. Chem. Rev. 2015, 115, 566.

(8) Zhu, Q.; Gentry, E. C.; Knowles, R. R. Catalytic Carbocation Generation Enabled by the Mesolytic Cleavage of Alkoxyamine Radical Cations Angew. Chem., Int. Ed. 2016, 55, 9969.

(9) Xiang, J.; Shang, M.; Kawamata, Y.; Lundberg, H.; Reisberg, S. H.; Chen, M.; Mykhailiuk, P.; Beutner, G.; Collins, M. R.; Davies, A.; Del Bel, M.; Gallego, G. M.; Spangler, J. E.; Starr, J.; Yang, S.; Blackmond, D. G.; Baran, P. S. Hindered Dialkyl Ether Synthesis with Electrogenerated Carbocations. Nature 2019, 573, 398.

(10) Shibutani, S.; Kodo, T.; Takeda, M.; Nagao, K.; Tokunaga, N.; Sasaki, Y.; Ohmiya, H. Organophotoredox-Catalyzed Decarboxylative $\mathrm{C}\left(\mathrm{sp}^{3}\right)-\mathrm{O}$ Bond Formation. J. Am. Chem. Soc 2020, 142, 1211 .

(11) Atom transfer radical addition (ATRA) commonly invokes radical oxidation by a photocatalyst: (a) Wallentin, C. J.; Nguyen, J. D.; Finkbeiner, P.; Stephenson, C. R. J. Visible Light-Mediated Atom Transfer Radical Addition via Oxidative and Reductive Quenching of Photocatalysts. $J$. Am. Chem. Soc. 2012, 134, 8875. (b) Tlahuext-Aca, A.; Garza-Sanchez, R. A.; Glorius, F. Multicomponent Oxyalkylation of Styrenes Enabled by Hydrogen-Bond-Assisted Photoinduced Electron Transfer. Angew. Chem., Int. Ed. 2017, 56 (13), 3708. For a review of ATRA: (c) Courant, T.; Masson, G. Recent Progress in Visible-Light Photoredox-Catalyzed Intermolecular 1,2-Difunctionalization of Double Bonds via an ATRA-Type Mechanism. J. Org. Chem. 2016, 81, 6945. ATRA-based fluorination: (d) Deng, W.; Feng, W.; Li, Y.; Bao, H. Merging Visible-Light Photocatalysis and Transition-Metal Catalysis in Three-Component Alkyl-Fluorination of Olefins with a Fluoride Ion. Org. Lett. 2018, 20, 4245. For radical-polar crossover to form anionic species: (e) Donabauer, K.; Maity, M.; Berger, A. L.; Huff, G. S.; Crespi, S.; König, B. Photocatalytic Carbanion Generation-Benzylation of Aliphatic Aldehydes to Secondary Alcohols. Chem. Sci. 2019, 10 (19), 5162.

(12) For a recent review of transformations using redox active esters, see: Murarka, S. $N$-(Acyloxy)phthalimides as Redox-Active Esters in CrossCoupling Reactions. Adv. Synth. Catal. 2018, 360, 1735

(13) (a) Wayner, D. D. M.; Houmam, A. Redox Properties of Free Radicals. Acta Chem. Scand, 1998, 52, 377. (b) Wayner, D. D. M.; McPhee, D. J.; Griller, D. Oxidation and Reduction Potentials of Transient Free Radicals. J. Am. Chem. Soc. 1988, 110, 132.

(14) Anslyn, E.; Dougherty, D. A. Modern physical organic chemistry; University Science Books, 2006.

(15) Silver-mediated decarboxylative fluorination with Selectfluor $\circledast$ : (a) Yin, F.; Wang, Z.; Li, Z.; Li, C. Silver-Catalyzed Decarboxylative Fluorination of Aliphatic Carboxylic Acids in Aqueous Solution. J. Am. Chem. Soc. 2012, 134, 10401. Photomediated decarboxylative fluorination with Selectfluor ${ }^{\circledR}$ and NFSI: (b) Leung, J. C. T.; Chatalova-Sazepin, C.; West, J. G.; Rueda-Becerril, M.; Paquin, J. F.; Sammis, G. M. Photo-fluorodecarboxylation of 2-Aryloxy and 2-Aryl Carboxylic Acids Angew. Chem., Int. Ed. 2012, 51, 10804. Photoredox-mediated decarboxylative fluorination: (c) Rueda-Becerril, M.; Mahé, O.; Drouin, M.; Majewski, M. B.; West, J. G.; Wolf, M. O.; Sammis, G. M.; Paquin, J. F. Direct C-F Bond Formation Using Photoredox Catalysis J. Am. Chem. Soc. 2014, 136, 2637. (d) Leung, J. C. T.; Sammis, G. M. Radical Decarboxylative Fluorination of Aryloxyacetic Acids Using N-Fluorobenzenesulfonimide and a Photosensitizer Eur. J. Org. Chem. 2015, 2015, 2197. (e) Ventre, S.; Petronijevic, F. R.; Macmillan, D. W. C. Decarboxylative Fluorination of Aliphatic Carboxylic Acids via Photoredox Catalysis. J. Am. Chem. Soc. 2015, 137, 5654. For multifluorinated motifs, see: (f) Chatalova-Sazepin, C.; Binayeva, M.; Epifanov, M.; Zhang, W.; Foth, P.; Amador, C.; Jagdeo, M.; Boswell, B. R.; Sammis, G. M. Xenon Difluoride Mediated Fluorodecarboxylations for the Syntheses of Di- and Trifluoromethoxyarene. Org. Lett. 2016, 18, 4570. (g) Mizuta, S.; Stenhagen, I. S. R.; O’Duill, M.; Wolstenhulme, J.; Kirjavainen, A. K.; Forsback, S. J.; Tredwell, M.; Sandford, G.; Moore, P. R.; Huiban, M.; Luthra, S. K.; Passchier, J.; Solin, O.; Gouverneur, V. Catalytic Decarboxylative Fluorination for the Synthesis of Tri- and Difluoromethyl Arenes. Org. Lett. 2013, 15, 2648. (h) Wang, Z.; Guo, C. Y.; Yang, C.; Chen, J. P. Ag-Catalyzed Chemoselective Decarboxylative Mono- and gem- Difluorination of Malonic Acid Derivatives. J. Am. Chem. Soc. 2019, $141,5617$.
(16) (a) Huang, X.; Liu, W.; Hooker, J. M.; Groves, J. T. Targeted Fluorination with the Fluoride Ion by Manganese-Catalyzed Decarboxylation. $A n-$ gew. Chem., Int. Ed. 2015, 54, 5241. For a recent application of the methodology to the synthesis of multifluorinated motifs: (b) Sap, J. B. I.; Wilson, T. C.; Kee, C. W.; Straathof, N. J. W.; Ende, C. W. am; Mukherjee, P.; Zhang, L.; Genicot, C.; Gouverneur, V. Synthesis of ${ }^{18} \mathrm{~F}$-Difluoromethylarenes Using Aryl Boronic Acids, Ethyl Bromofluoroacetate and ${ }^{18}$ F]Fluoride. Chem. Sci. 2019, 10, 3237.

(17) Gray, E. E.; Nielsen, M. K.; Choquette, K. A.; Kalow, J. A.; Graham, T. J. A.; Doyle, A. G. Nucleophilic (Radio)Fluorination of $\alpha$-Diazocarbonyl Compounds Enabled by Copper-Catalyzed H-F Insertion. J. Am. Chem. Soc. 2016, 138, 10802.

(18) Ametamey, S. M.; Honer, M.; Schubiger, P. A. Molecular Imaging with PET. Chem. Rev. 2008, 108, 1501.

(19) (a) Teegardin, K.; Day, J. I.; Chan, J.; Weaver, J. Advances in Photocatalysis: A Microreview of Visible Light Mediated Ruthenium and Iridium Catalyzed Organic Transformations. Org. Process Res. Dev. 2016, 20, 1156. (b) Speckmeier, E.; Fischer, T. G.; Zeitler, K. A Toolbox Approach to Construct Broadly Applicable Metal-Free Catalysts for Photoredox Chemistry: Deliberate Tuning of Redox Potentials and Importance of Halogens in Donor-Acceptor Cyanoarenes. J. Am. Chem. Soc. 2018, 140 (45), 15353

(20) Okada, K.; Okamoto, K.; Morita, N.; Okubo, K.; Oda, M. Photosensitized Decarboxylative Michael Addition through N-(Acyloxy)Phthalimides via an Electron-Transfer Mechanism. J. Am. Chem. Soc. 1991, 113, 9401.

(21) Boutureira, O.; Bernardes, G. J. L. Advances in Chemical Protein Modification. Chem. Rev. 2015, 115, 2174.

(22) Shimizu, M.; Hiyama, T. Modern Synthetic Methods for Fluorine-Substituted Target Molecules. Angew. Chem. Int. Ed. 2005, 44, 214.

(23) Zafrani, Y.; Yeffet, D.; Sod-Moriah, G.; Berliner, A.; Amir, D.; Marciano, D.; Gershonov, E.; Saphier, S. Difluoromethyl Bioisostere: Examining the "Lipophilic Hydrogen Bond Donor" Concept. J. Med. Chem. 2017, 60 (2), 797-804

(24) Nielsen, M. K.; Ahneman, D. T.; Riera, O.; Doyle, A. G. Deoxyfluorination with Sulfonyl Fluorides: Navigating Reaction Space with Machine Learning. J. Am. Chem. Soc. 2018, 140 (15), 5004.

(25) González-Esguevillas, M.; Miró, J.; Jeffrey, J. L.; MacMillan, D. W. C. Photoredox-catalyzed Deoxyfluorination of Activated Alcohols with Selectfluor. Tetrahedron 2019, 75, 4222.

(26) (a) Lackner, G. L.; Quasdorf, K. W.; Overman, L. E. J. Am. Chem. Soc. 2013, 135, 15342. (b) Lackner, G. L.; Quasdorf, K. W.; Pratsch, G.; Overman, L. E. J. Org. Chem. 2015, 80, 6012.

(27) See supplementary information for additional details and discussion.

(28) Cismesia, M. A.; Yoon, T. P. Characterizing Chain Processes in Visible Light Photoredox Catalysis. Chem. Sci. 2015, 6, 5426.

(29) An acidic additive was required in order to observe reactivity with these alternative nucleophiles. No conversion was observed without $\mathrm{Et}_{3} \mathrm{~N} \bullet 3 \mathrm{HF}$ except in the case of sufficiently acidic HFIP

(30) (a) Zlatopolskiy, B. D.; Zischler, J.; Krapf, P.; Zarrad, F.; Urusova, E. A.; Kordys, E.; Endepols, H.; Neumaier, B. Copper-Mediated Aromatic Radiofluorination Revisited : Efficient Production of PET Tracers on a Preparative Scale. Chem. - A Eur. J. 2015, 21, 5972. (b) Jacobson, O.; Kiesewetter, D. O.; Chen, X. Fluorine-18 Radiochemistry, Labeling Strategies and Synthetic Routes. Bioconjug. Chem. 2015, 26, 1 .

(31) D'Angelo, K. A.; Taylor, M. S. Borinic Acid Catalyzed Stereo- and Regioselective Couplings of Glycosyl Methanesulfonates. J. Am. Chem. Soc. 2016, 138, 11058 . 
Insert Table of Contents artwork here

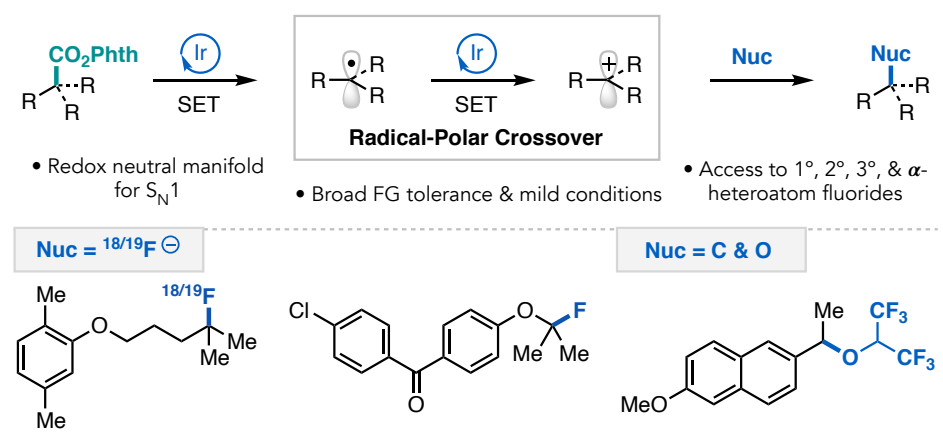




\section{Supporting Information}

\section{Nucleophilic (Radio)Fluorination of Redox-Active Esters via Radical-Polar Crossover Enabled by Photoredox Catalysis}

Eric W. Webb, John B. Park, Erin L. Cole, David J. Donnelly, Samuel J. Bonacorsi, and Abigail G. Doyle

Department of Chemistry, Frick Chemical Laboratory, Princeton University, Princeton, New Jersey 08554, USA

Discovery Chemistry Platforms, PET Radiochemical Synthesis, Bristol-Myers Squibb Research and Development, P.O. Box 4000, Princeton, New Jersey 08543, United States

Discovery Chemistry, Bristol-Myers Squibb, P.O. Box 5400, Princeton, New Jersey 085435400, United States

Email: agdoyle@princeton.edu 


\section{Table of Contents}

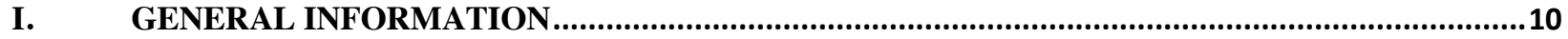

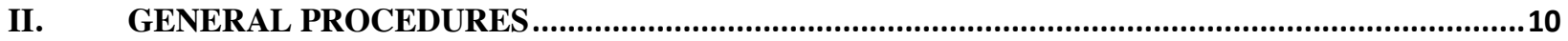

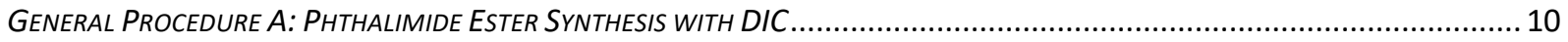

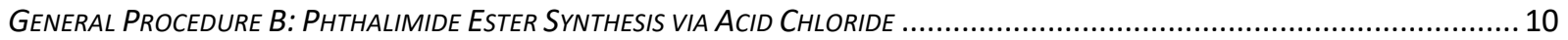

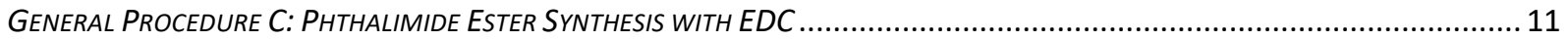

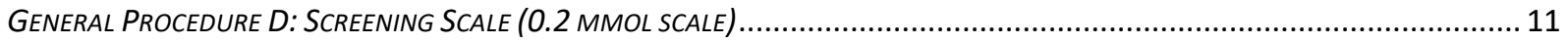

GENERAL PROCEDURE E: ISOLATION SCALE (O.6 MMOL SCALE) ............................................................................... 11

FIGURE S1: SET-UP FOR SCREENING AND ISOLATION SCALE FLUORINATION REACTIONS. ................................................ 12

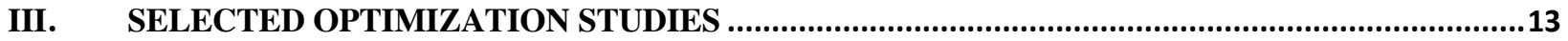

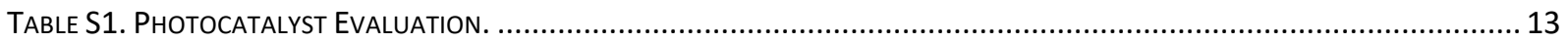

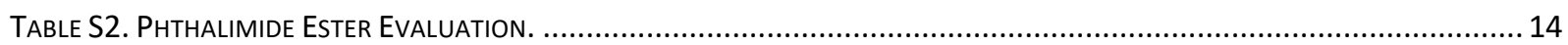

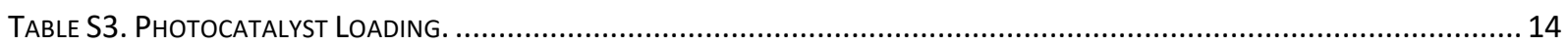

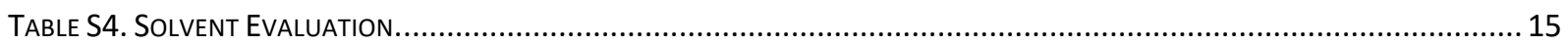

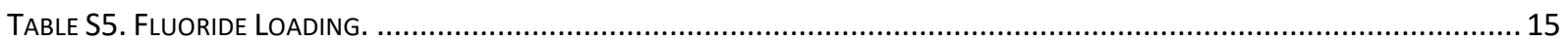

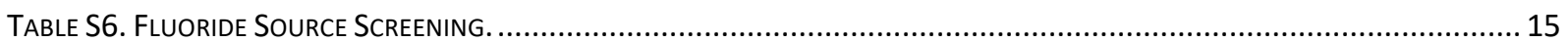

IV. SYNTHESIS AND CHARACTERIZATION OF PHTHALIMIDE ESTERS AND OXALATES .....16

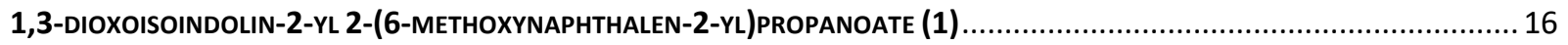

4,5,6,7-TETRACHLORO-1,3-DIOXOISOINDOLIN-2-YL 2-(6-METHOXYNAPHTHALEN-2-YL)PROPANOATE (S1) ........................... 16

1,3-DIOXOISOINDOLIN-2-YL 2-(4-(TERT-BUTYL)PHENYL)ACETATE (S2) ..................................................................... 17

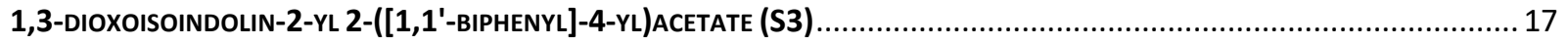

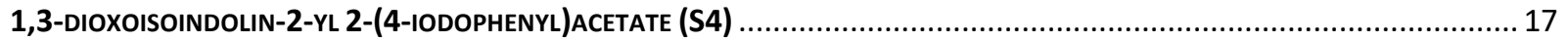

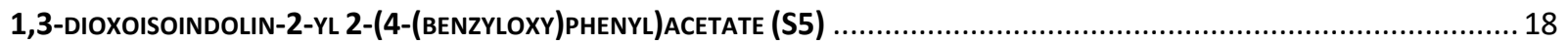

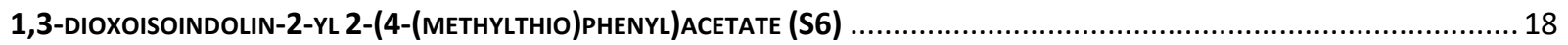

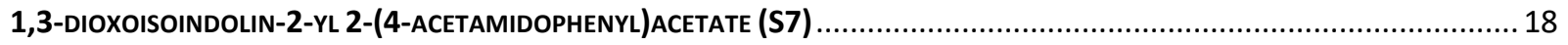

1,3-DIOXOISOINDOLIN-2-YL 2-(4-(TRIFLUOROMETHOXY)PHENYL)ACETATE (S8) .......................................................... 19

1,3-DIOXOISOINDOLIN-2-YL 2-(4-(TRIFLUOROMETHYL)PHENYL)ACETATE (S9) ……............................................... 19

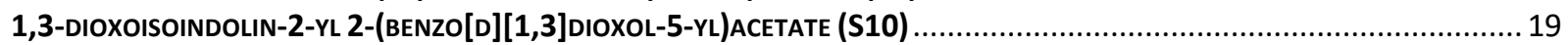

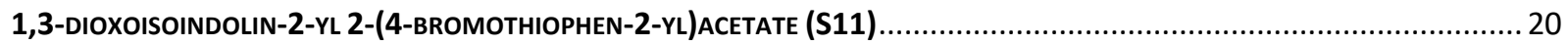

1,3-DIOXOISOINDOLIN-2-YL 2-CYCLOPENTYL-2-(4-(TRIFLUOROMETHYL)PHENYL)ACETATE (S12) ......................................... 20

1,3-DIOXOISOINDOLIN-2-YL 2-(6-CHLOROPYRIDIN-3-YL)PROPANOATE (S13) ........................................................ 22

1,3-DIOXOISOINDOLIN-2-YL 4-(DIMETHYLAMINO)-2-(NAPHTHALEN-2-YL)-4-OXOBUTANOATE (S14) ................................. 22

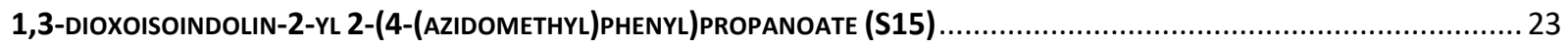

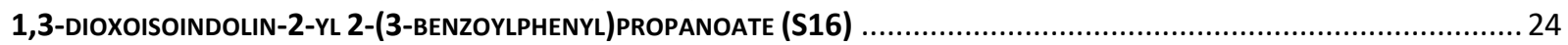

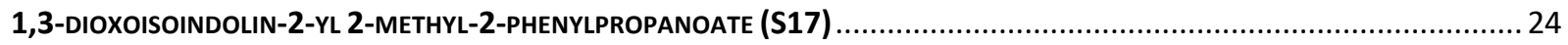

1,3-DIOXOISOINDOLIN-2-YL 1-(4-CHLOROPHENYL)CYCLOHEXANE-1-CARBOXYLATE (S18) ................................................ 25

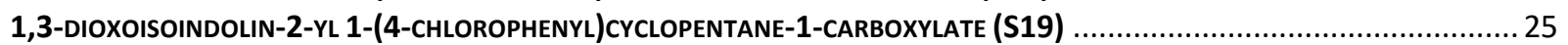

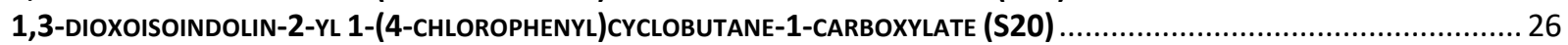

1,3-DIOXOISOINDOLIN-2-YL4-(6-CHLOROPYRIDIN-3-YL)TETRAHYDRO-2H-PYRAN-4-CARBOXYLATE (S21) ............................ 26

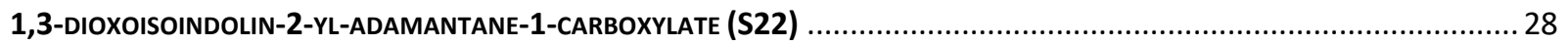

1,3-DIOXOISOINDOLIN-2-YL 3-(4-(TERT-BUTYL)PHENYL)-2,2-DIMETHYLPROPANOATE (S23) ............................................ 28

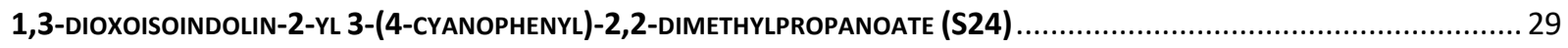

1,3-DIOXOISOINDOLIN-2-YL 5-(2,5-DIMETHYLPHENOXY)-2,2-DIMETHYLPENTANOATE (41) ........................................... 30

1,3-DIOXOISOINDOLIN-2-YL (2S,4AS,6AS,6BR,12AS,12B R,14BR)-2,4A,6A,6B,9,9,12A-HEPTAMETHYL-10,13-DIOXO-

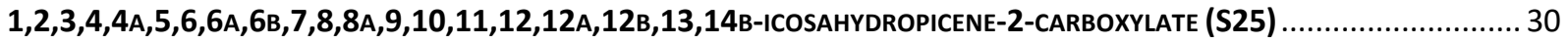

1,3-DIOXOISOINDOLIN-2-YL 2-(4-(TERT-BUTYL)PHENOXY)ACETATE (S26) ................................................................ 31

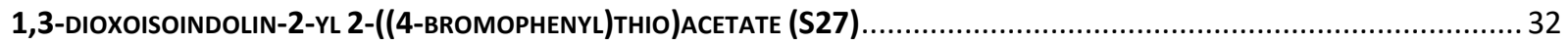

1,3-DIOXOISOINDOLIN-2-YL 2-(4-(4-CHLOROBENZOYL)PHENOXY)-2-METHYLPROPANOATE (S29) ....................................... 34 
1,3-DIOXOISOINDOLIN-2-YL 2-(4-(4-CYANO-2-FLUOROPHENOXY)PHENOXY)PROPANOATE (S30)...................................... 34

1,3-DIOXOISOINDOLIN-2-YL 2-([1,1'-BIPHENYL]-4-YL)-2-FLUOROACETATE (S31) ....................................................... 35

1,3-DIOXOISOINDOLIN-2-YL 3,3,3-TRIFLUORO-2-METHOXY-2-PHENYLPROPANOATE (S32) ............................................. 36

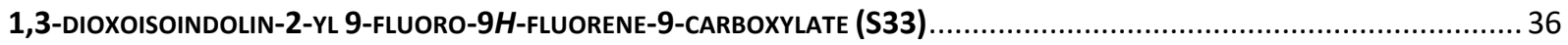

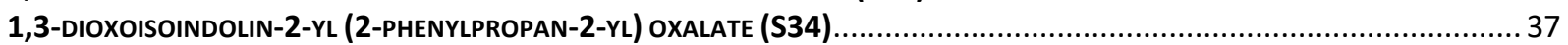

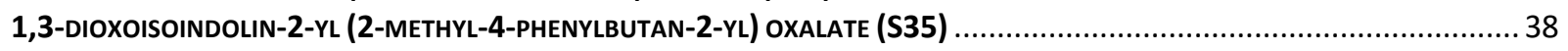

V. ISOLATION AND CHARACTERIZATION OF PRODUCT COMPOUNDS ...............................39

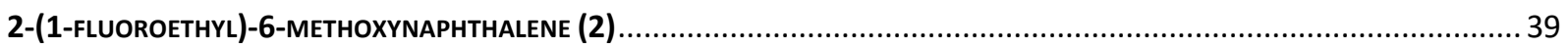

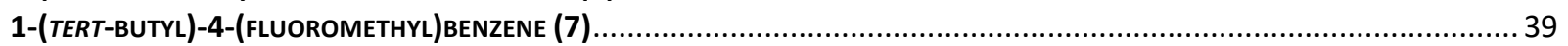

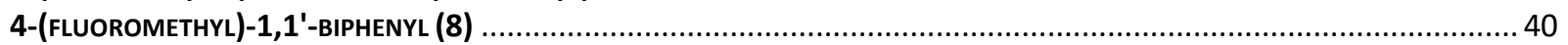

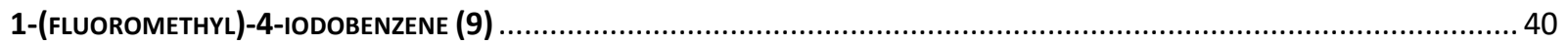

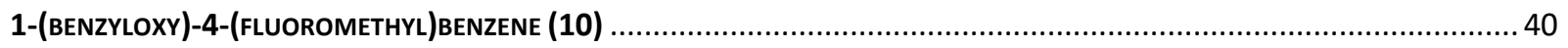

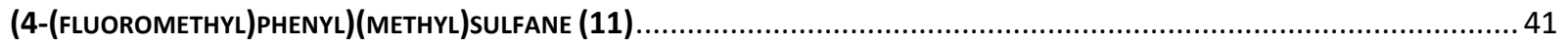

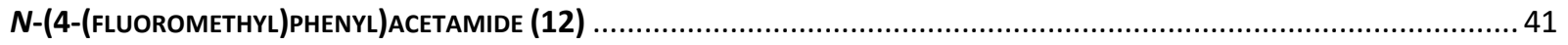

1-(FLUOROMETHYL)-4-(TRIFLUOROMETHOXY)BENZENE (13) ................................................................................ 42

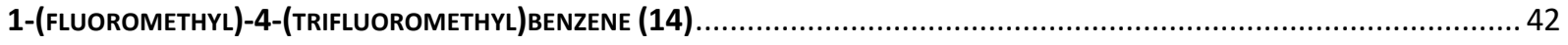

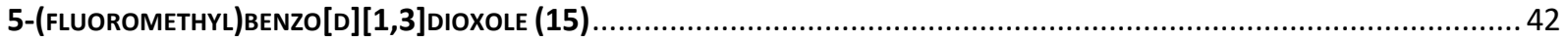

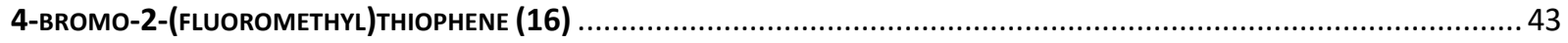

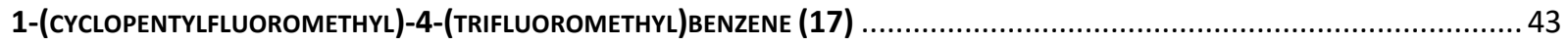

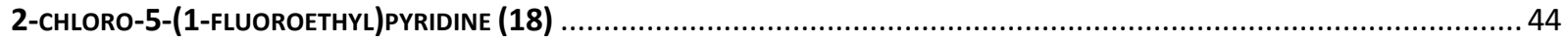

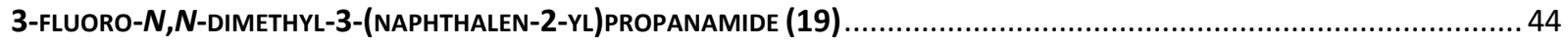

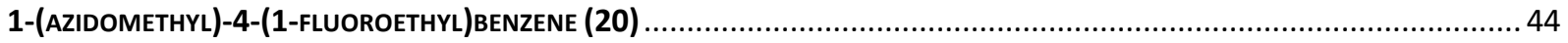

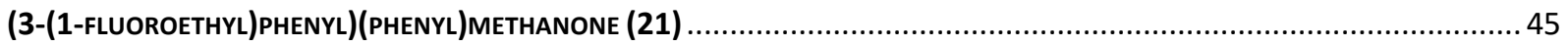

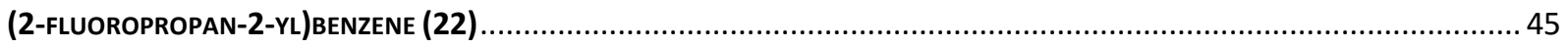

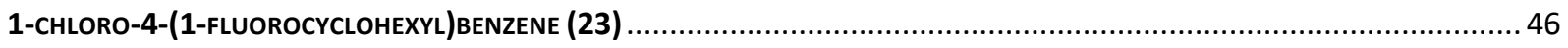

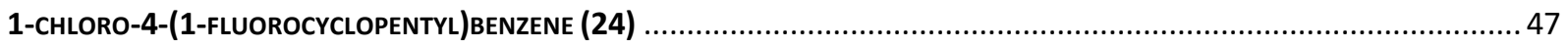

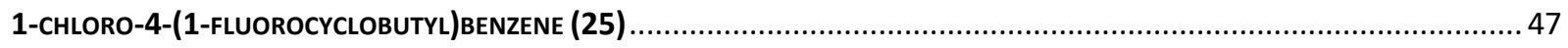

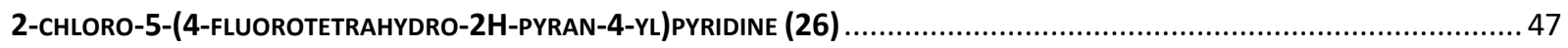

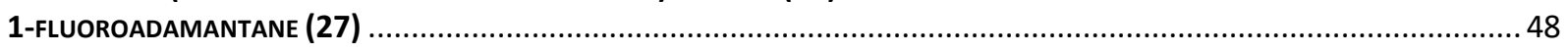

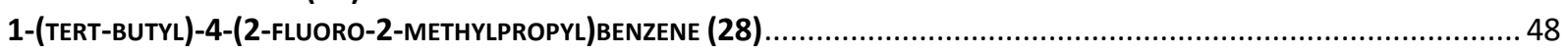

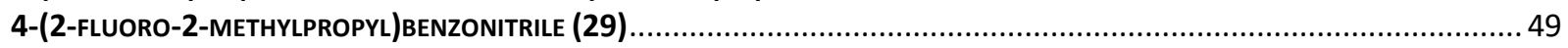

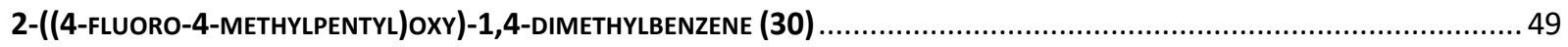

(6AR,6BS,8AS,11S,12AR,14AR,14BS)-11-FLUORO-4,4,6A,6B,8A,11,14B-HEPTAMETHYL-

1,2,4,4A,5,6,6A,6B,7,8,8A,9,10,11,12,12A,14A,14B-OCTADECAHYDROPICENE-3,14-DIONE (31) ................................ 49

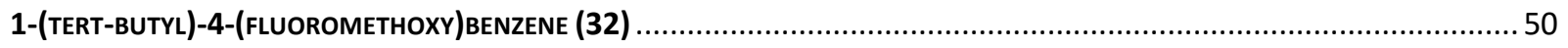

(4-BROMOPHENYL)(FLUOROMETHYL)SULFANE (33) .................................................................................... 51

(3AS,4S,6S,6AR)-4-(BENZYLOXY)-6-FLUORO-2,2-DIMETHYLTETRAHYDROFURO[3,4-D][1,3]DIOXOLE (34) ......................... 51

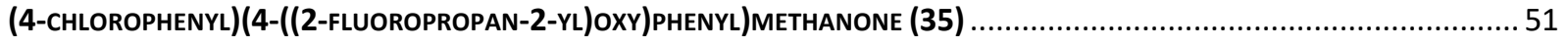

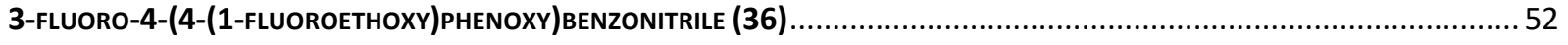

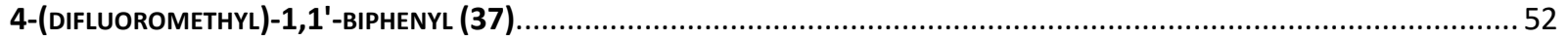

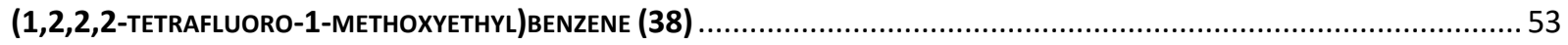

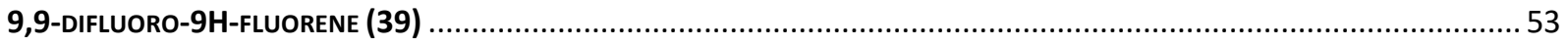

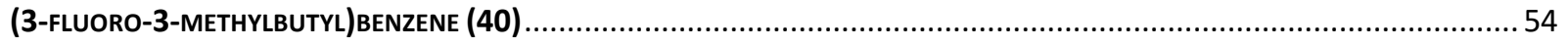

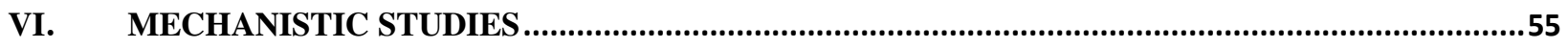

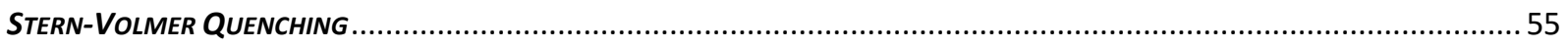

Table S7. Fluorescence Quenching with Triethylamine $3 H F$................................................................... 55

Table S8. Fluorescence Quenching with Phthalimide Ester ................................................................. 56

Table S9. Fluorescence Quenching with Reaction Mixture (1 and Triethylamine $\cdot 3 H F)$................................ 57

Figure S2. Compiled Fluorescence Quenching Graphs........................................................................ 57

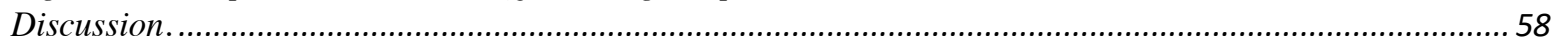

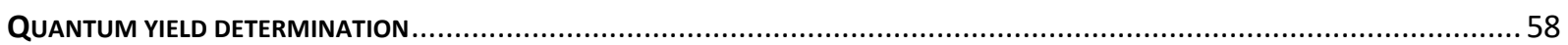




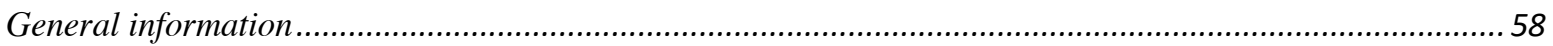

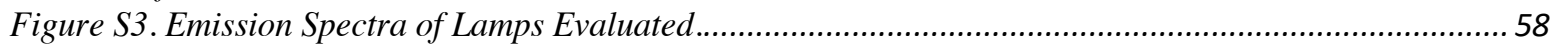

Calibration of light source using potassium ferrioxalate ............................................................................ 59

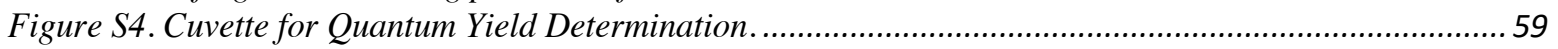

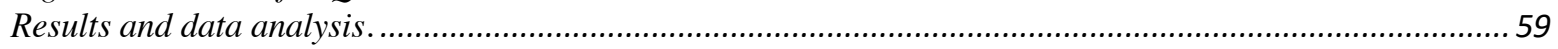

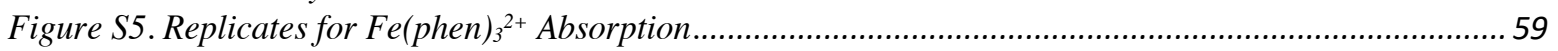

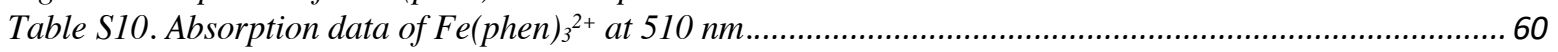

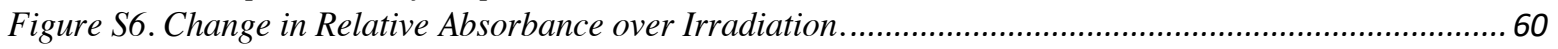

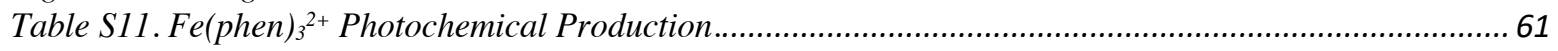

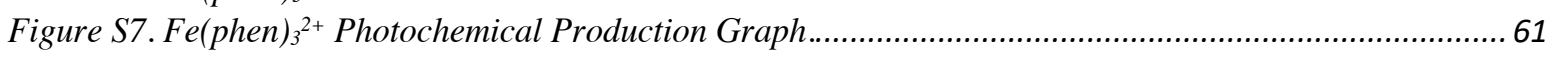

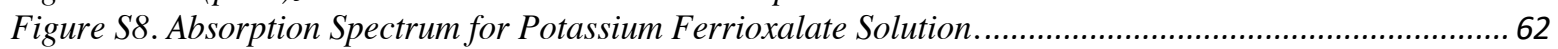

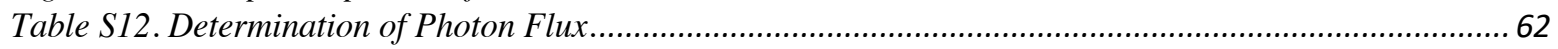

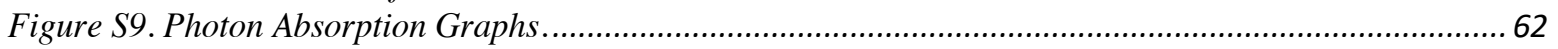

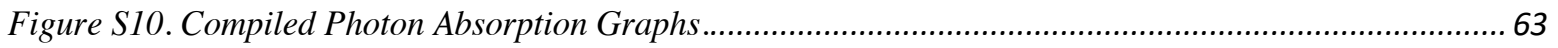

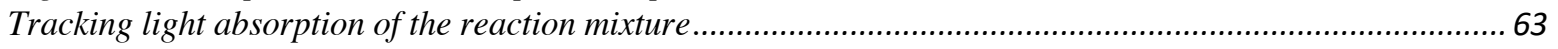

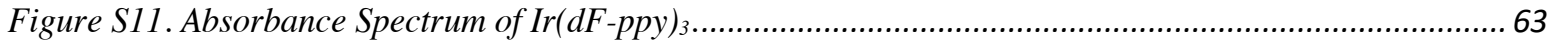

Figure S12. Absorbance Spectrum of 1 with Added Triethylamine $3 H F$..................................................... 64

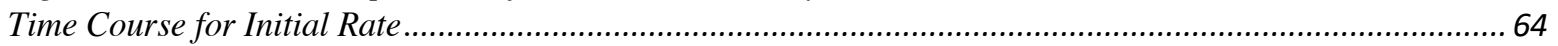

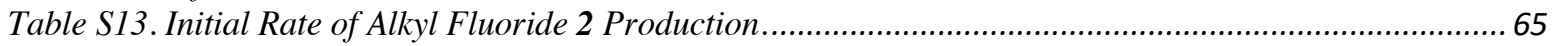

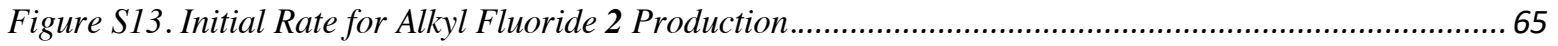

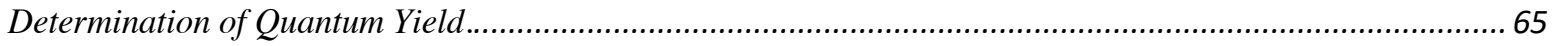

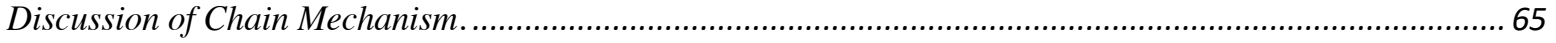

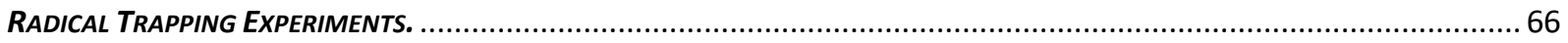

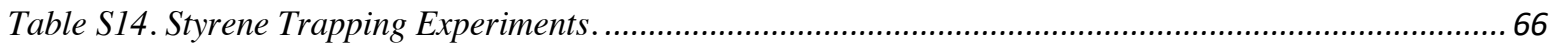

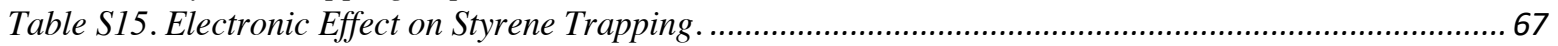

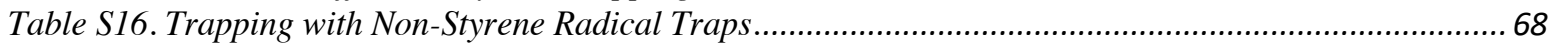

1-((5-(2,5-DIMETHYLPHENOXY)-2-METHYLPENTAN-2-YL)OXY)-2,2,6,6-TETRAMETHYLPIPERIDINE (42) ................................6 68

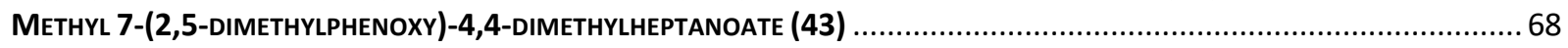

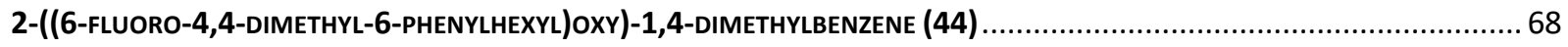

2-((6-FLUORO-4,4-DIMETHYL-6-PHENYLHEXYL)OXY)-1,4-DIMETHYLBENZENE (S36) ........................................................69

4-(6-(2,5-DIMETHYLPHENOXY)-1-FLUORO-3,3-DIMETHYLHEXYL) BENZONITRILE (S37) ................................................69

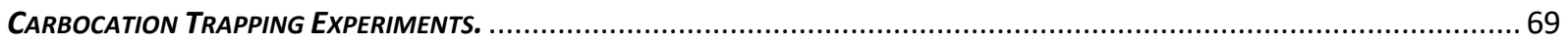

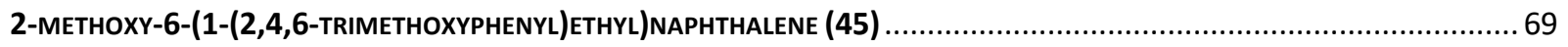

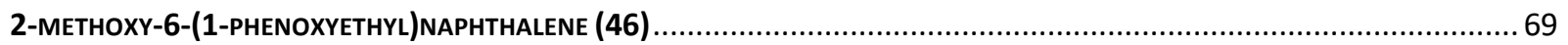

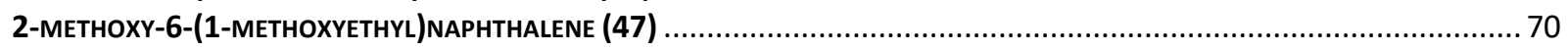

2-(1-((1,1,1,3,3,3-HEXAFLUOROPROPAN-2-YL)OXY)ETHYL)-6-METHOXYNAPHTHALENE (48) ....................................... 70

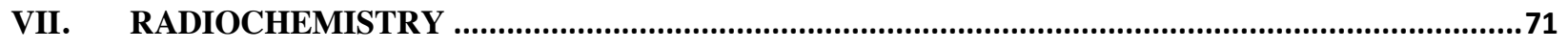

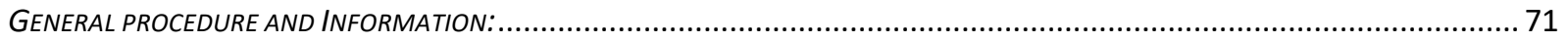

Figure S14. Photocatalytic Radiochemical Fluorination Setup and ApParatus. ................................................ 71

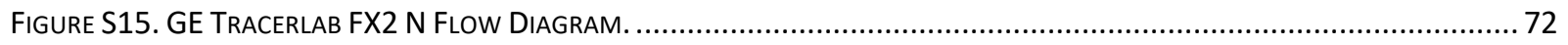

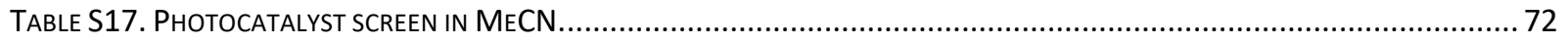

TABLE S18. DeTERMINATION Of OPTIMAL RADIOCHEMICAL PhotocatALYST. ................................................................. 73

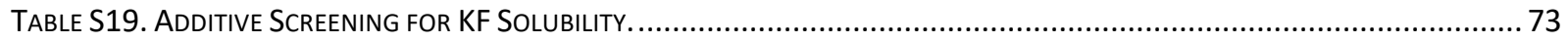

TABLE S20. VARIABLE LOADING OF HFIP AS AN ADDITIVE IN RADIOCHEMISTRY. ......................................................... 74

FIGURE S16. AVERAGE RCC FOR VARIABLE LOADING OF HFIP AdDITIVE................................................................... 74

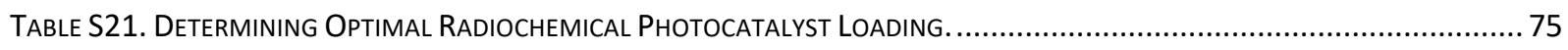

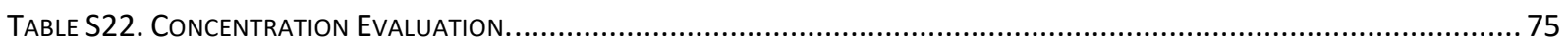

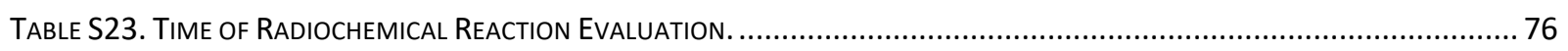

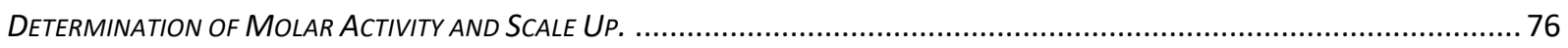

TABLE S24. MolaR ACtIVITY CALIBRATION CURVE. ........................................................................................ 77

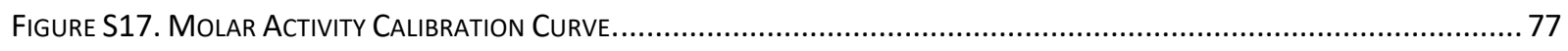

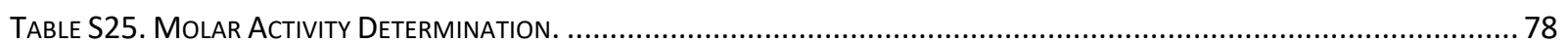


EVALUATION OF RADIOCHEMICAL SCOPE

2-(1-(FLUORO-18 $\left.{ }^{18}\right)$ ETHYL)-6-METHOXYNAPHTHALENE ([ [18 F]2)

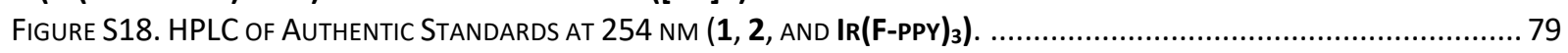

Figure S19. PosiRAM-HPLC OF CRUde ReAction Mixture With 2-(1-(FLUORO- ${ }^{18}$ F)ethyL)-6-Methoxynaphthalene $\left(\left[{ }^{18} \mathrm{~F}\right] 2\right)$.

Figure S20. HPLC OF CRUde ReAction MixtURE WITH 2-(1-(FLUORO- $\left.{ }^{-18} \mathrm{~F}\right)$ ETHYL)-6-METHOXYNAPHTHALENE $\left(\left[{ }^{18} \mathrm{~F}\right] 2\right)$ AT 254 NM.

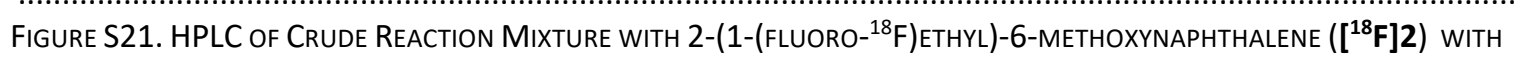

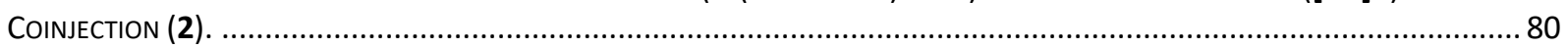

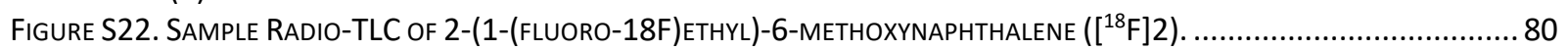

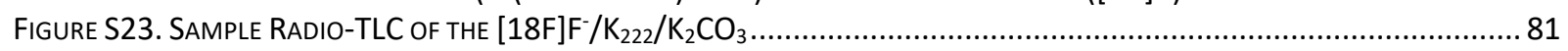

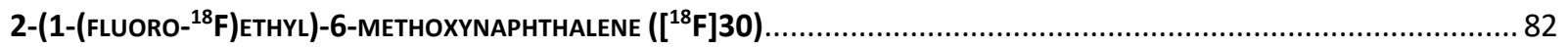

TABLE S27. REPLICATES FOR GENERATION OF 2-((4-(FLUORO- $\left.{ }^{18} F\right)$-4-METHYLPENTYL)OXY)-1,4-DIMETHYLBENZENE $\left(\left[{ }^{18} \mathrm{~F}\right] 30\right) \ldots \ldots . .83$

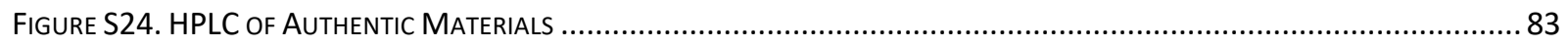

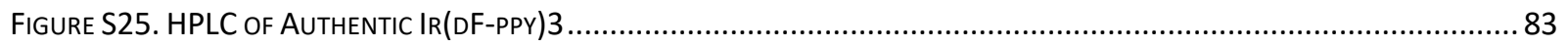

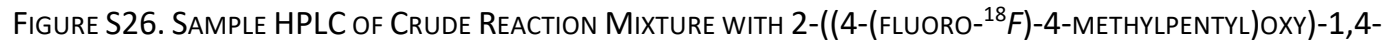

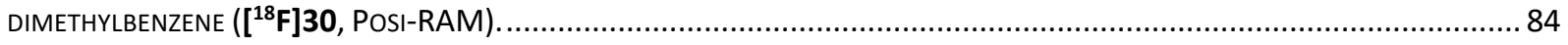

FigURE S27. SAMPLE HPLC OF CRUDE 2-((4-(FLUORO- $\left.{ }^{18} F\right)-4$-METHYLPENTYL)OXY)-1,4-DIMETHYLBENZENE $\left(\left[{ }^{18} \mathrm{~F}\right] 30,254\right.$ NM). .84

FigURE S28. SAMPLE HPLC OF CRUDE 2-((4-(FLUORO- $\left.{ }^{18} F\right)$-4-METHYLPENTYL)OXY)-1,4-DIMETHYLBENZENE $\left(\left[{ }^{18} \mathrm{~F}\right] 30\right)$ WITH 2-((4-

FLUORO-4-METHYLPENTYL)OXY)-1,4-DIMETHYLBENZENE (30) CoINJECTION (254 NM).................................................... 84

FIGURE S29. SAMPLE RADIO-TLC OF 2-((4-(FLUORO-18F)-4-METHYLPENTYL)OXY)-1,4-DIMETHYLBENZENE ([18 F]2).................. 85

(3AR,4R,6R,6AS)-4-(BENZYLOXY)-6-(FLUORO- ${ }^{18} F$ )-2,2-DIMETHYLTETRAHYDROFURO[3,4-D][1,3]DIOXOLE $\left(\left[{ }^{18} \mathrm{~F}\right] 34\right) \ldots \ldots \ldots \ldots . .85$

TABLE S28. RePLICATES FOR GeNERATION OF (3AR, $4 R, 6 R, 6$ AS)-4-(BENZYLOXY)-6-(FLUORO- $\left.{ }^{18} F\right)-2,2-$

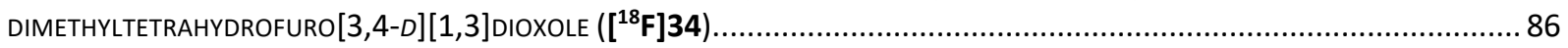

FIGURE S30. SAMPLE RADIO-TLC OF 2-((4-(FLUORO-18F)-4-METHYLPENTYL)OXY)-1,4-DIMETHYLBENZENE $\left(\left[{ }^{18} \mathrm{~F}\right] 34\right)$. ..............8 86

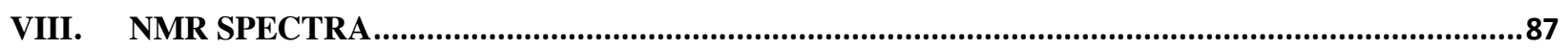

$500 \mathrm{MHz}{ }^{1} \mathrm{H}_{-N M R}$ OF 1,3-DIOXOISOINDOLIN-2-YL 2-(6-METHOXYNAPHTHALEN-2-YL)PROPANOATE (1) IN CDCL3....................88

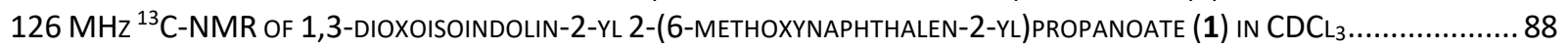

$500 \mathrm{MHz}{ }^{1} \mathrm{H}$-NMR OF 4,5,6,7-TETRACHLORO-1,3-DIOXOISOINDOLIN-2-YL 2-(6-METHOXYNAPHTHALEN-2-YL)PROPANOATE (S1) IN

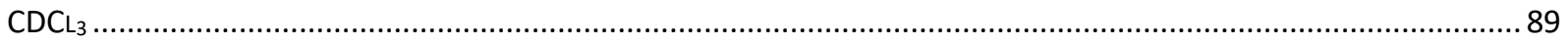

$126 \mathrm{MHZ}^{13} \mathrm{C}$-NMR OF 4,5,6,7-TETRACHLORO-1,3-DIOXOISOINDOLIN-2-YL 2-(6-METHOXYNAPHTHALEN-2-YL)PROPANOATE (S1) IN

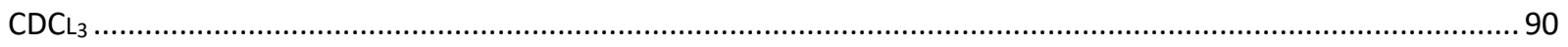

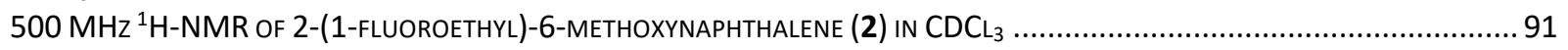

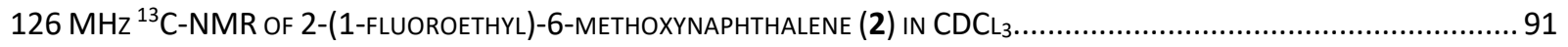

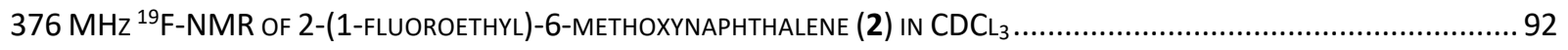

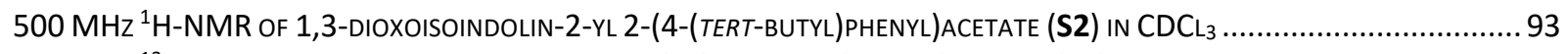

$126 \mathrm{MHz}{ }^{13} \mathrm{C}-\mathrm{NMR}$ OF 1,3-DIOXOISOINDOLIN-2-YL 2-(4-(TERT-BUTYL)PHENYL)ACETATE (S2) IN CDCL3 ..............................93

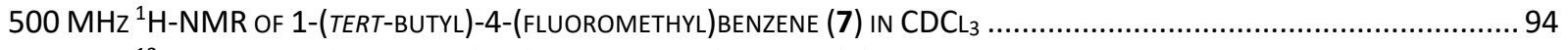

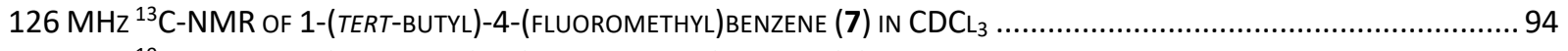

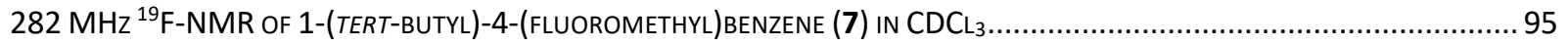

$400 \mathrm{MHz}{ }^{1} \mathrm{H}-\mathrm{NMR}$ OF 1,3-DIOXOISOINDOLIN-2-YL 2-([1,1'-BIPHENYL]-4-YL)ACETATE (S3) IN CDCL3 ................................ 95

$101 \mathrm{MHz}^{13} \mathrm{C}$-NMR OF 1,3-DIOXOISOINDOLIN-2-YL 2-([1,1'-BIPHENYL]-4-YL)ACETATE (S3) IN CDCL3 .................................96

$500 \mathrm{MHz}{ }^{1} \mathrm{H}-N M R$ OF 4 -(FLUOROMETHYL)-1,1'-BIPHENYL (8) IN CDCL

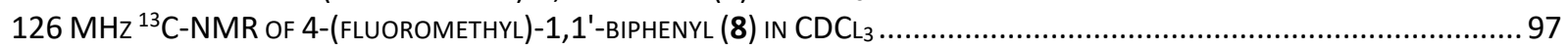

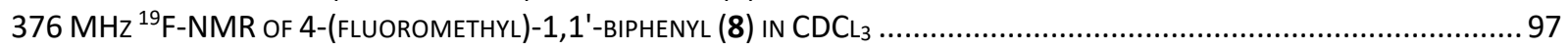

$500 \mathrm{MHz}{ }^{1} \mathrm{H}-\mathrm{NMR}$ OF 1,3-DIOXOISOINDOLIN-2-YL 2-(4-IODOPHENYL)ACETATE (S4) IN CDCL3 …....................................... 98

$126 \mathrm{MHZ}^{13} \mathrm{C}$-NMR OF 1,3-DIOXOISOINDOLIN-2-YL 2-(4-IODOPHENYL)ACETATE (S4) IN CDCL 3 ........................................ 98

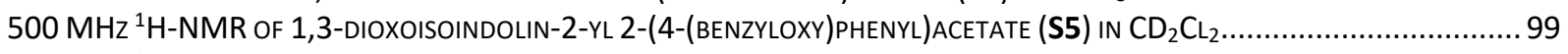

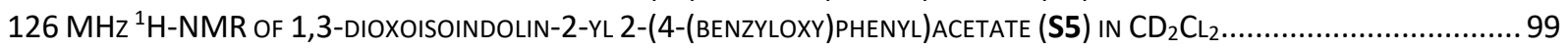

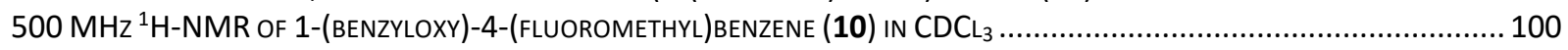

$126 \mathrm{MHZ} 1 \mathrm{H}-\mathrm{NMR}$ OF 1-(BENZYLOXY)-4-(FLUOROMETHYL) BENZENE (10) IN CDCL3 ................................................... 100

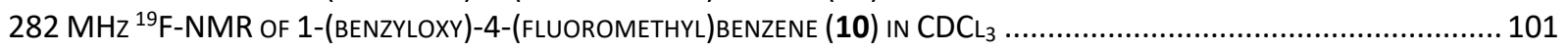


$500 \mathrm{MHz}{ }^{1} \mathrm{H}$-NMR OF 1,3-DIOXOISOINDOLIN-2-YL 2-(4-(METHYLTHIO)PHENYL)ACETATE (S6) IN $\mathrm{CD}_{2} \mathrm{CL}_{2} \ldots \ldots \ldots \ldots \ldots \ldots \ldots \ldots . . . . . . . . . . . .101$

$126 \mathrm{MHz}^{13} \mathrm{C}$-NMR OF 1,3-DIOXOISOINDOLIN-2-YL 2-(4-(METHYLTHIO)PHENYL)ACETATE (S6) IN CD $\mathrm{CL}_{2} \mathrm{CL}_{2} \ldots \ldots \ldots \ldots \ldots \ldots \ldots \ldots . . . . . . . . . . .102$

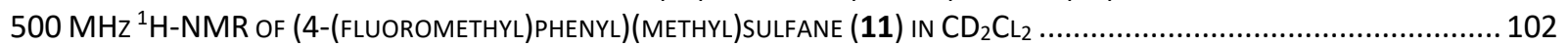

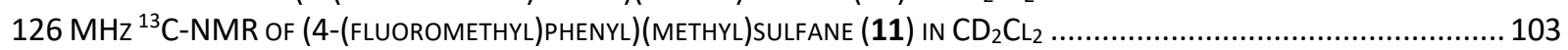

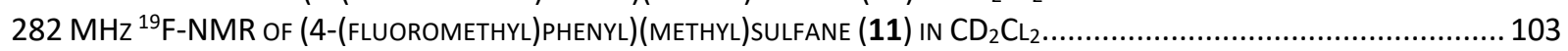

$500 \mathrm{MHz}{ }^{1} \mathrm{H}-\mathrm{NMR}$ OF 1,3-DIOXOISOINDOLIN-2-YL 2-(4-ACETAMIDOPHENYL)ACETATE (S7) IN CDCL $\ldots$................................ 104

$126 \mathrm{MHz}{ }^{13} \mathrm{C}$-NMR OF 1,3-DIOXOISOINDOLIN-2-YL 2-(4-ACETAMIDOPHENYL)ACETATE (S7) IN CDCL3 ................................ 104

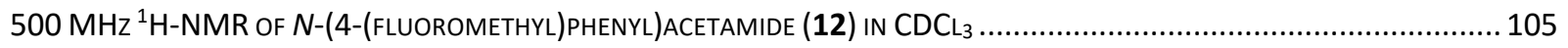

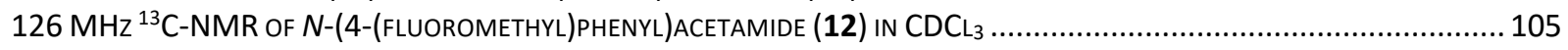

$376 \mathrm{MHz}{ }^{19} \mathrm{~F}$-NMR OF N-(4-(FLUOROMETHYL)PHENYL)ACETAMIDE (12) IN CDCL

$500 \mathrm{MHz}{ }^{1} \mathrm{H}$-NMR OF 1,3-DIOXOISOINDOLIN-2-YL 2-(4-(TRIFLUOROMETHOXY)PHENYL)ACETATE (S8) IN CDCL _................... 106

$126 \mathrm{MHz}{ }^{13} \mathrm{C}$-NMR OF 1,3-DIOXOISOINDOLIN-2-YL 2-(4-(TRIFLUOROMETHOXY)PHENYL)ACETATE (S8) IN CDCL

$282 \mathrm{MHz}{ }^{19} \mathrm{~F}-\mathrm{NMR}$ OF 1,3-DIOXOISOINDOLIN-2-YL 2-(4-(TRIFLUOROMETHOXY)PHENYL)ACETATE (S8) IN CDCL3 ................... 107

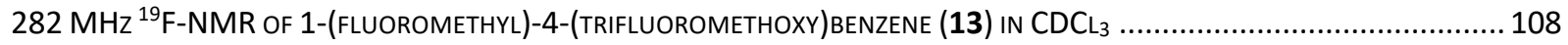

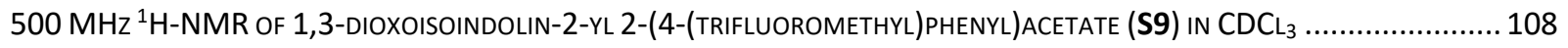

$126 \mathrm{MHz}^{13} \mathrm{C}$-NMR OF 1,3-DIOXOISOINDOLIN-2-YL 2-(4-(TRIFLUOROMETHYL)PHENYL)ACETATE (S9) IN CDCL _.................... 109

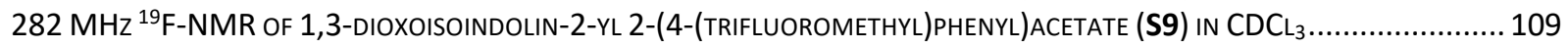

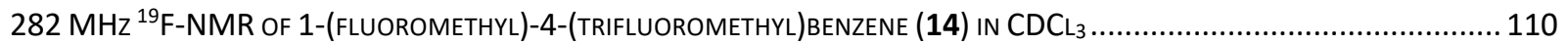

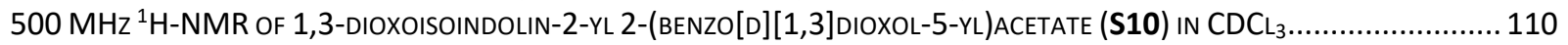

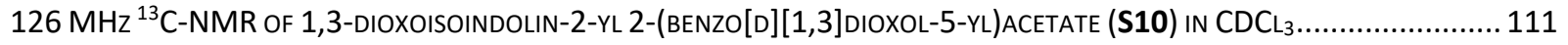

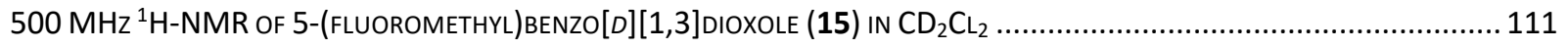

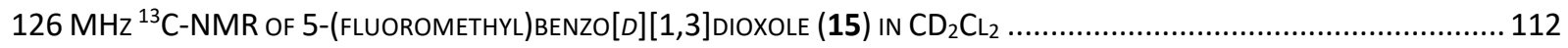

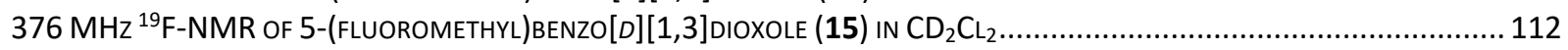

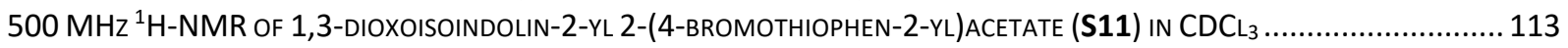

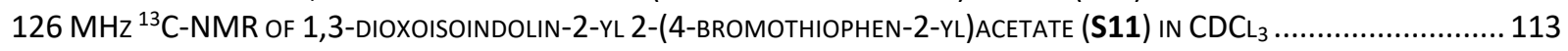

$282 \mathrm{MHz}{ }^{19}$ F-NMR OF 4-BROMO-2-(FLUOROMETHYL)THIOPHENE (16) IN CDCL

$500 \mathrm{MHZ}^{1}{ }^{1}$ H-NMR OF 1,3-DIOXOISOINDOLIN-2-YL 2-CYCLOPENTYL-2-(4-(TRIFLUOROMETHYL)PHENYL)ACETATE (S12) IN CDCL3114

$126 \mathrm{MHZ}^{13}{ }^{13}$-NMR OF 1,3-DIOXOISOINDOLIN-2-YL 2-CYCLOPENTYL-2-(4-(TRIFLUOROMETHYL)PHENYL)ACETATE (S12) IN CDCL 3

$282 \mathrm{MHZ}^{19}$ F-NMR OF 1,3-DIOXOISOINDOLIN-2-YL 2-CYCLOPENTYL-2-(4-(TRIFLUOROMETHYL)PHENYL)ACETATE (S12) IN CDCL3

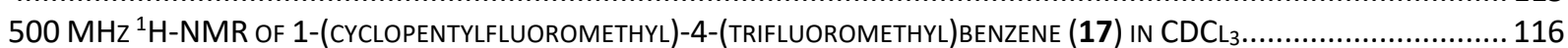

$126 \mathrm{MHz}{ }^{13} \mathrm{C}$-NMR OF 1-(CYCLOPENTYLFLUOROMETHYL)-4-(TRIFLUOROMETHYL)BENZENE (17) IN CDCL $3 . \ldots \ldots \ldots \ldots \ldots \ldots \ldots \ldots . . . . . . . . . . .116$

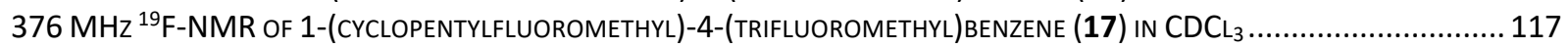

$500 \mathrm{MHz}{ }^{1} \mathrm{H}$-NMR OF 1,3-DIOXOISOINDOLIN-2-YL 2-(6-CHLOROPYRIDIN-3-YL)PROPANOATE (S13) IN $\mathrm{CD}_{2} \mathrm{CL}_{2} \ldots \ldots \ldots \ldots \ldots \ldots \ldots . . . .117$

$126 \mathrm{MHz}{ }^{13} \mathrm{C}$-NMR OF 1,3-DIOXOISOINDOLIN-2-YL 2-(6-CHLOROPYRIDIN-3-YL)PROPANOATE (S13) IN CD ${ }_{2} \mathrm{CL}_{2} \ldots \ldots \ldots \ldots \ldots \ldots \ldots . . . . . . .118$

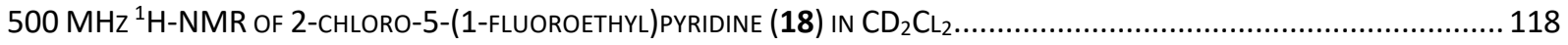

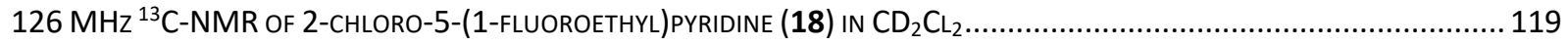

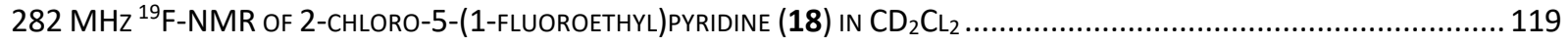

$500 \mathrm{MHz}{ }^{1} \mathrm{H}$-NMR of 1,3-DIOXOISOINDOLIN-2-YL 4-(DIMETHYLAMINO)-2-(NAPHTHALEN-2-YL)-4-OXOBUTANOATE (S15) IN CDCL3

$500 \mathrm{MHZ}^{1} \mathrm{H}-\mathrm{NMR}$ OF (t)-3-FLUORO-N N-DIMETHYL-3-(NAPHTHALEN-2-YL)PROPENAMIDE (19) IN CDCL

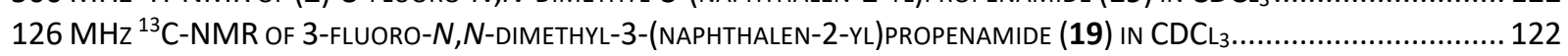

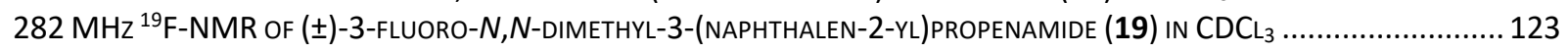

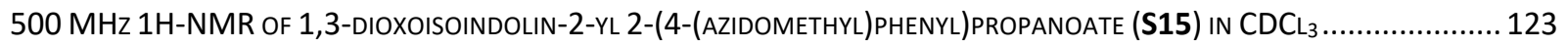

$126 \mathrm{MHz}{ }^{13} \mathrm{C}$-NMR OF 1,3-DIOXOISOINDOLIN-2-YL 2-(4-(AZIDOMETHYL)PHENYL)PROPANOATE (S15) IN CDCL_................... 124

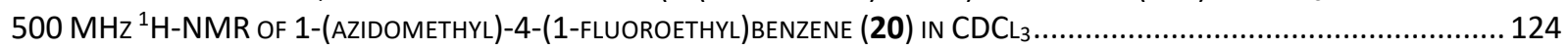

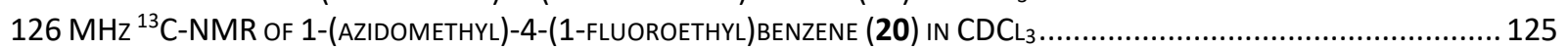

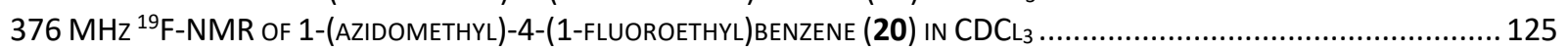

$500 \mathrm{MHZ}^{1}{ }^{\mathrm{H}-N M R}$ OF 1,3-DIOXOISOINDOLIN-2-YL 2-(3-BENZOYLPHENYL)PROPANOATE (S16) IN CDCL _........................... 126

$126 \mathrm{MHz}^{13} \mathrm{C}$-NMR OF 1,3-DIOXOISOINDOLIN-2-YL 2-(3-BENZOYLPHENYL)PROPANOATE (S16) IN CDCL $3 \ldots \ldots \ldots \ldots \ldots \ldots \ldots \ldots . . . . . . . . . . .126$

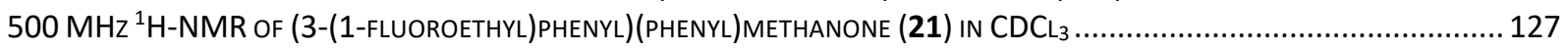

$126 \mathrm{MHz}^{13} \mathrm{C}$-NMR OF (3-(1-FLUOROETHYL)PHENYL)(PHENYL)METHANONE (21) IN CDCL 


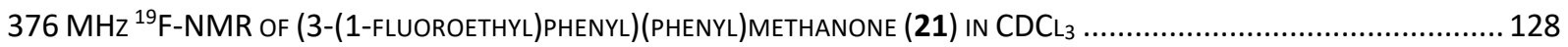

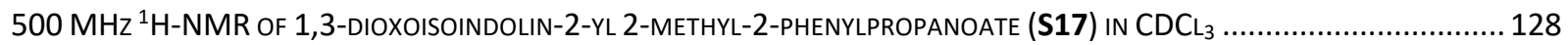

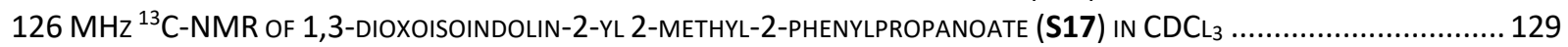

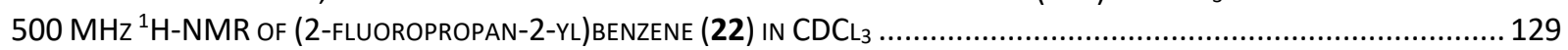

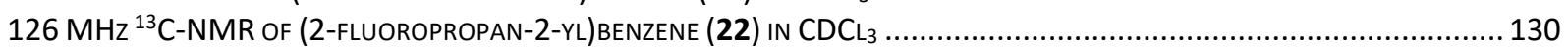

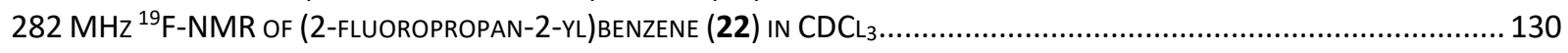

$500 \mathrm{MHz}{ }^{1} \mathrm{H}$-NMR OF 1,3-DIOXOISOINDOLIN-2-YL 1-(4-CHLOROPHENYL)CYCLOHEXANE-1-CARBOXYLATE (S18) IN CDCL 3 ......... 131

$126 \mathrm{MHz}^{13} \mathrm{C}$-NMR OF 1,3-DIOXOISOINDOLIN-2-YL 1-(4-CHLOROPHENYL)CYCLOHEXANE-1-CARBOXYLATE (S18) IN CDCL ....... 131

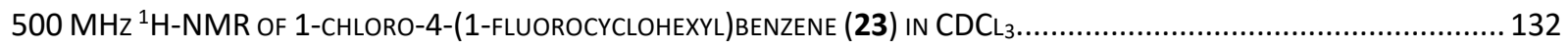

$126 \mathrm{MHz}{ }^{13} \mathrm{C}$-NMR OF 1-CHLORO-4-(1-FLUOROCYCLOHEXYL)BENZENE (23) IN CDCL

$376 \mathrm{MHz}{ }^{19} \mathrm{~F}-\mathrm{NMR}$ OF 1-CHLORO-4-(1-FLUOROCYCLOHEXYL)BENZENE (23) IN CDCL

$500 \mathrm{MHz}{ }^{1} \mathrm{H}-\mathrm{NMR}$ OF 1,3-DIOXOISOINDOLIN-2-YL 1-(4-CHLOROPHENYL) CYCLOPENTANE-1-CARBOXYLATE (S19) IN CDCL $\mathrm{CL}_{3} \ldots . .133$

$126 \mathrm{MHz}^{13} \mathrm{C}$-NMR OF 1,3-DIOXOISOINDOLIN-2-YL 1-(4-CHLOROPHENYL)CYCLOPENTANE-1-CARBOXYLATE (S19) IN CDCL $3 . . . .134$

$470 \mathrm{MHz}{ }^{19} \mathrm{~F}-\mathrm{NMR}$ OF 1-CHLORO-4-(1-FLUOROCYCLOPENTYL)BENZENE (24) IN CDCL

$500 \mathrm{MHz}{ }^{1} \mathrm{H}$-NMR OF 1,3-DIOXOISOINDOLIN-2-YL 1-(4-CHLOROPHENYL)CYCLOBUTANE-1-CARBOXYLATE (S20) IN CDCL3........ 135

$126 \mathrm{MHZ}^{13} \mathrm{C}$-NMR OF 1,3-DIOXOISOINDOLIN-2-YL 1-(4-CHLOROPHENYL)CYCLOBUTANE-1-CARBOXYLATE (S20) IN CDCL $3 . . . . . .136$

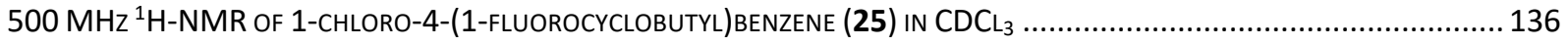

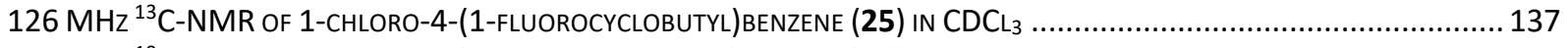

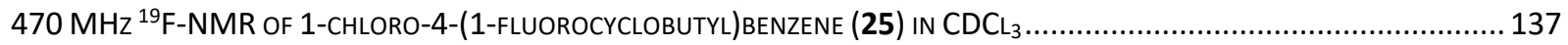

$400 \mathrm{MHZ}^{1} \mathrm{H}$-NMR OF 1,3-DIOXOISOINDOLIN-2-YL 4-(6-CHLOROPYRIDIN-3-YL)TETRAHYDRO-2H-PYRAN-4-CARBOXYLATE (S21) IN

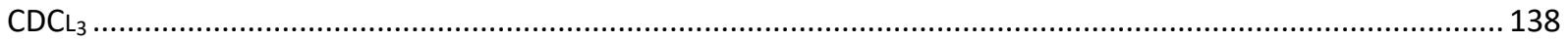

$126 \mathrm{MHz}^{13} \mathrm{C}$-NMR OF 1,3-DIOXOISOINDOLIN-2-YL 4-(6-CHLOROPYRIDIN-3-YL)TETRAHYDRO-2H-PYRAN-4-CARBOXYLATE (S21) IN

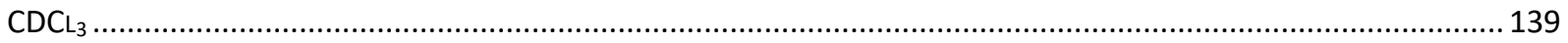

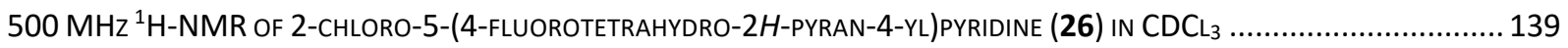

$282 \mathrm{MHz}{ }^{19} \mathrm{~F}$-NMR OF 2-CHLORO-5-(4-FLUOROTETRAHYDRO-2H-PYRAN-4-YL)PYRIDINE (26) IN CDCL $3 \ldots \ldots \ldots . . . . . . . . . . . . . . . . . .140$

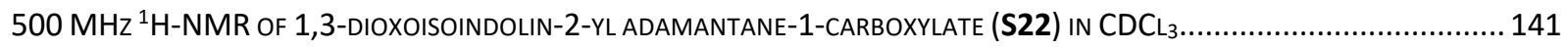

$126 \mathrm{MHz}^{13} \mathrm{C}$-NMR OF 1,3-DIOXOISOINDOLIN-2-YL ADAMANTANE-1-CARBOXYLATE (S22) IN CDCL3.................................. 141

$500 \mathrm{MHZ}^{1} \mathrm{H}$-NMR OF 1,3-DIOXOISOINDOLIN-2-YL 3-(4-(TERT-BUTYL)PHENYL)-2,2-DIMETHYLPROPANOATE (S23) IN CDCL3.... 142

$126 \mathrm{MHZ}^{13} \mathrm{C}$-NMR OF 1,3-DIOXOISOINDOLIN-2-YL 3-(4-(TERT-BUTYL)PHENYL)-2,2-DIMETHYLPROPANOATE (S23) IN CDCL ... 142

$500 \mathrm{MHZ} 1 \mathrm{H}-\mathrm{NMR}$ OF 1-(TERT-BUTYL)-4-(2-FLUORO-2-METHYLPROPYL)BENZENE (28) IN CDCL3 .................................... 143

$126 \mathrm{MHZ}^{13} \mathrm{C}$-NMR OF 1-(TERT-BUTYL)-4-(2-FLUORO-2-METHYLPROPYL)BENZENE (28) IN CDCL3 ..................................... 143

$376 \mathrm{MHz}{ }^{19} \mathrm{~F}-\mathrm{NMR}$ OF 1-(TERT-BUTYL)-4-(2-FLUORO-2-METHYLPROPYL)BENZENE (28) IN CDCL3 ..................................... 144

$500 \mathrm{MHZ} 1 \mathrm{H}$-NMR OF 1,3-DIOXOISOINDOLIN-2-YL 3-(4-CYANOPHENYL)-2,2-DIMETHYLPROPANOATE (S24) IN CDCL3 ........... 144

$126 \mathrm{MHz}{ }^{13} \mathrm{C}$-NMR OF 1,3-DIOXOISOINDOLIN-2-YL 3-(4-CYANOPHENYL)-2,2-DIMETHYLPROPANOATE (S24) IN CDCL3............ 145

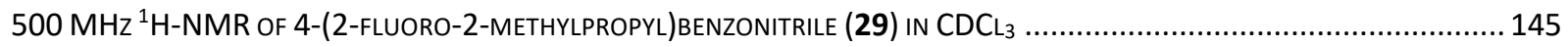

$126 \mathrm{MHz}{ }^{13}$ C-NMR OF 4-(2-FLUORO-2-METHYLPROPYL)BENZONITRILE (29) IN CDCL_................................................. 146

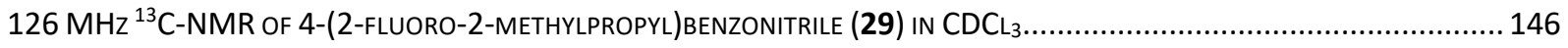

$500 \mathrm{MHZ}^{1} \mathrm{H}$-NMR OF 1,3-DIOXOISOINDOLIN-2-YL 5-(2,5-DIMETHYLPHENOXY)-2,2-DIMETHYLPENTANOATE (41) IN CDCL $\ldots . . .147$

$126 \mathrm{MHZ}^{13} \mathrm{C}$-NMR OF 1,3-DIOXOISOINDOLIN-2-YL 5-(2,5-DIMETHYLPHENOXY)-2,2-DIMETHYLPENTANOATE (41) IN CDCL $\ldots .147$

$500 \mathrm{MHZ}^{1} \mathrm{H}$-NMR OF 2-((4-FLUORO-4-METHYLPENTYL)OXY)-1,4-DIMETHYLBENZENE (30) IN CDCL $3 \ldots \ldots \ldots \ldots \ldots \ldots \ldots \ldots \ldots \ldots \ldots . . . . . . . . . . . . . .148$

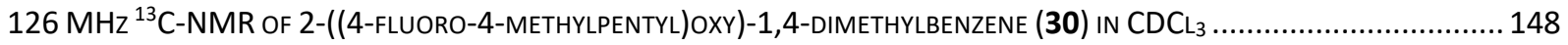

$376 \mathrm{MHz}{ }^{19} \mathrm{~F}$-NMR OF 2-((4-FLUORO-4-METHYLPENTYL)OXY)-1,4-DIMETHYLBENZENE (30) IN CDCL 3 ................................ 149

$500 \mathrm{MHz}{ }^{1} \mathrm{H}-\mathrm{NMR}$ OF 1,3-DIOXOISOINDOLIN-2-YL (2S,4AS,6AS,6B $\left.R, 12 \mathrm{AS}, 12 \mathrm{~B} R, 14 \mathrm{~B} R\right)$-2,4A, 6A,6B, 9,9,12A-HEPTAMETHYL-

10,13-DIOXO-1,2,3,4,4A,5,6,6A,6B,7,8,8A,9,10,11,12,12A,12B,13,14B-ICOSAHYDROPICENE-2-CARBOXYLATE (S25) IN CDCL 3

150

$126 \mathrm{MHz}{ }^{13} \mathrm{C}$-NMR OF 1,3-DIOXOISOINDOLIN-2-YL (2S,4AS,6AS,6BR,12AS,12B R,14B R)-2,4A,6A,6B,9,9,12A-HEPTAMETHYL-

10,13-DIOXO-1,2,3,4,4A,5,6,6A,6B,7,8,8A,9,10,11,12,12A,12B,13,14B-ICOSAHYDROPICENE-2-CARBOXYLATE (S25) IN CDCL 3

$500 \mathrm{MHz}{ }^{1} \mathrm{H}-\mathrm{NMR}$ OF $(4 \mathrm{~A} R, 6 \mathrm{~A} R, 6 \mathrm{~B} S, 8 \mathrm{~A} S, 12 \mathrm{~A} R, 14 \mathrm{~A} R, 14 \mathrm{~B} S)$-11-FLUORO-4,4,6A,6B,8A,11,14B-HEPTAMETHYL-

1,2,4,4A ,5,6,6A,6B, 7,8,8A,9,10,11,12,12A,14A,14B-OCTADECAHYDROPICENE-3,14-DIONE (31) IN CDCL 3 ...................... 152

$126 \mathrm{MHz}{ }^{13} \mathrm{C}-\mathrm{NMR}$ OF $(4 \mathrm{~A} R, 6 \mathrm{~A} R, 6 \mathrm{~B} S, 8 \mathrm{~A} S, 12 \mathrm{~A} R, 14 \mathrm{~A} R, 14 \mathrm{~B} S)$-11-FLUORO-4,4,6A,6B,8A,11,14B-HEPTAMETHYL-

1,2,4,4A,5,6,6A,6B,7,8,8A,9,10,11,12,12A,14A,14B-OCTADECAHYDROPICENE-3,14-DIONE (31) IN CDCL3 ..... 153 
$282 \mathrm{MHz}{ }^{19} \mathrm{~F}-\mathrm{NMR}$ OF (4AR,6AR,6BS,8AS,12AR,14AR,14BS)-11-FLUORO-4,4,6A,6B,8A, 11,14B-HEPTAMETHYL-

1,2,4,4A,5,6,6A,6B, 7,8,8A,9,10,11,12,12A,14A,14B-OCTADECAHYDROPICENE-3,14-DIONE (31) IN CDCL3 ..................... 154

$500 \mathrm{MHz}{ }^{1} \mathrm{H}-\mathrm{NMR}$ OF 1,3-DIOXOISOINDOLIN-2-YL 2-(4-(TERT-BUTYL)PHENOXY)ACETATE (S26) IN CDCL $3 \ldots \ldots \ldots \ldots \ldots . . . . . . . . . . . .154$

$126 \mathrm{MHz}{ }^{13} \mathrm{C}$-NMR OF 1,3-DIOXOISOINDOLIN-2-YL 2-(4-(TERT-BUTYL)PHENOXY)ACETATE (S26) IN CDCL3 .......................... 155

$126 \mathrm{MHz}{ }^{13} \mathrm{C}$-NMR OF 1,3-DIOXOISOINDOLIN-2-YL 2-(4-(TERT-BUTYL)PHENOXY)ACETATE (32) IN CDCL3........................... 155

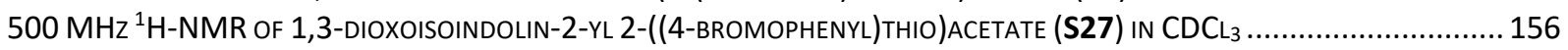

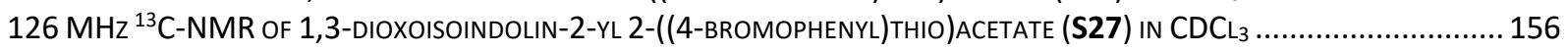

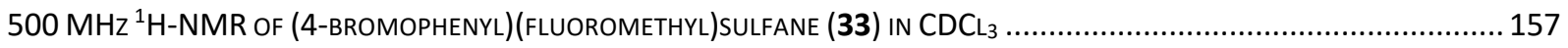

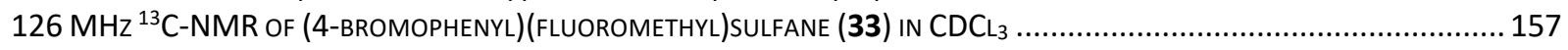

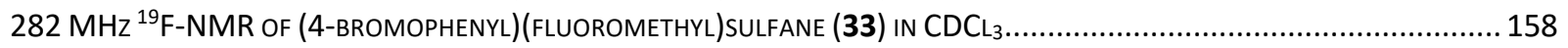

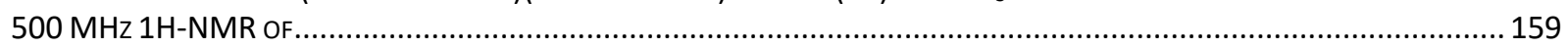

$126 \mathrm{MHz}{ }^{13} \mathrm{C}-\mathrm{NMR}$ OF 1,3-DIOXOISOINDOLIN-2-YL (3AS,4S,6R,6AR)-6-(BENZYLOXY)-2,2-DIMETHYLTETRAHYDROFURO[3,4-

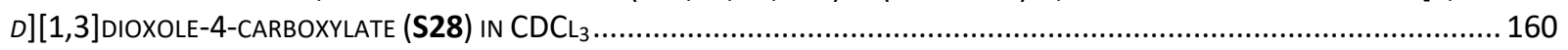

$500 \mathrm{MHz}{ }^{1} \mathrm{H}-\mathrm{NMR}$ OF (3AR,4R,6R,6AS)-4-(BENZYLOXY)-6-FLUORO-2,2-DIMETHYLTETRAHYDROFURO[3,4-D][1,3]DIOXOLE (34)

IN $\mathrm{CDCL}_{3}$

161

$126 \mathrm{MHz}{ }^{13} \mathrm{C}-\mathrm{NMR}$ of $(3 \mathrm{~A} R, 4 R, 6 R, 6 \mathrm{AS})$-4-(BENZYLOXY)-6-FLUORO-2,2-DIMETHYLTETRAHYDROFURO[3,4-D][1,3]DIOXOLE (34)

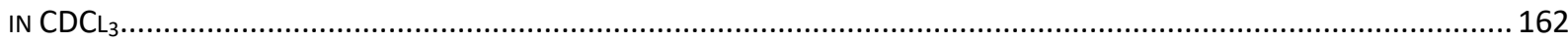

$470 \mathrm{MHz}{ }^{19} \mathrm{~F}-\mathrm{NMR}$ OF (3AR,4R,6R,6AS)-4-(BENZYLOXY)-6-FLUORO-2,2-DIMETHYLTETRAHYDROFURO[3,4-D][1,3]DIOXOLE (34)

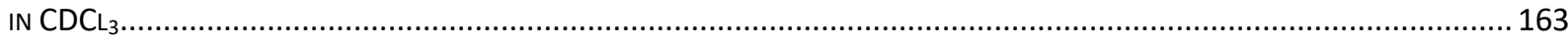

$500 \mathrm{MHz}{ }^{1} \mathrm{H}-\mathrm{NMR}$ OF 1,3-DIOXOISOINDOLIN-2-YL 2-(4-(4-CHLOROBENZOYL)PHENOXY)-2-METHYLPROPANOATE (S29) IN CDCL 3

164

$126 \mathrm{MHz}{ }^{13}$ C-NMR OF 1,3-DIOXOISOINDOLIN-2-YL 2-(4-(4-CHLOROBENZOYL)PHENOXY)-2-METHYLPROPANOATE (S29) IN CDCL 3

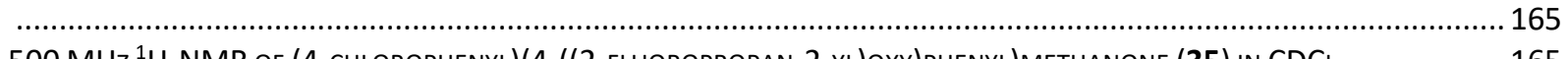

$500 \mathrm{MHZ}{ }^{1} \mathrm{H}-\mathrm{NMR}$ OF (4-CHLOROPHENYL)(4-((2-FLUOROPROPAN-2-YL)OXY)PHENYL)METHANONE (35) IN CDCL $\mathrm{C}_{3} \ldots \ldots . . . . . . . . . .165$

$126 \mathrm{MHz}{ }^{13} \mathrm{C}-\mathrm{NMR}$ OF (4-CHLOROPHENYL)(4-((2-FLUOROPROPAN-2-YL)OXY)PHENYL)METHANONE (35) IN CDCL $3 \ldots . . . . . . . . . . .166$

$470 \mathrm{MHZ}{ }^{19} \mathrm{~F}-\mathrm{NMR}$ OF (4-CHLOROPHENYL)(4-((2-FLUOROPROPAN-2-YL)OXY)PHENYL)METHANONE (35) IN CDCL3 ................ 166

$500 \mathrm{MHz}{ }^{1} \mathrm{H}-\mathrm{NMR}$ OF 1,3-DIOXOISOINDOLIN-2-YL 2-(4-(4-CYANO-2-FLUOROPHENOXY)PHENOXY)PROPANOATE (S30) IN CDCL3

$126 \mathrm{MHz}{ }^{13} \mathrm{C}-\mathrm{NMR}$ OF 1,3-DIOXOISOINDOLIN-2-YL 2-(4-(4-CYANO-2-FLUOROPHENOXY)PHENOXY)PROPANOATE (S30) IN CDCL3

$282 \mathrm{MHz}{ }^{19}$ F-NMR OF 1,3-DIOXOISOINDOLIN-2-YL 2-(4-(4-CYANO-2-FLUOROPHENOXY)PHENOXY)PROPANOATE (S30) IN CDCL3

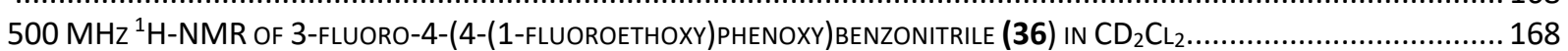

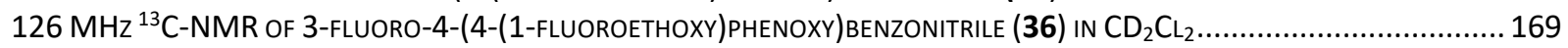

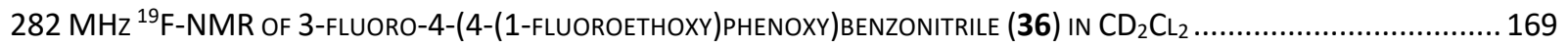

$500 \mathrm{MHz}{ }^{1} \mathrm{H}-\mathrm{NMR}$ 1,3-DIOXOISOINDOLIN-2-YL 2-([1,1'-BIPHENYL]-4-YL)-2-FLUOROACETATE (S31) IN CDCL 3 ................... 170

$126 \mathrm{MHz}{ }^{13} \mathrm{C}$-NMR 1,3-DIOXOISOINDOLIN-2-YL 2-([1,1'-BIPHENYL]-4-YL)-2-FLUOROACETATE (S31) IN CDCL $3 \ldots \ldots . . . . . . . . . . .170$

$282 \mathrm{MHz}{ }^{19} \mathrm{~F}-\mathrm{NMR} 1$,3-DIOXOISOINDOLIN-2-YL 2-([1,1'-BIPHENYL]-4-YL)-2-FLUOROACETATE (S31) IN CDCL3................... 171

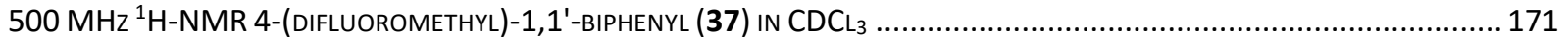

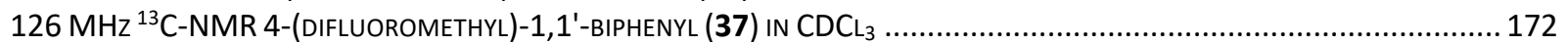

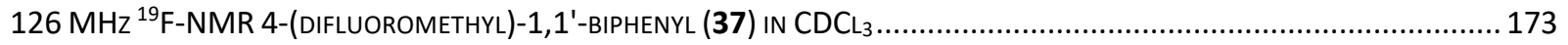

$500 \mathrm{MHz}{ }^{1} \mathrm{H}-\mathrm{NMR}$ OF 1,3-DIOXOISOINDOLIN-2-YL 3,3,3-TRIFLUORO-2-METHOXY-2-PHENYLPROPANOATE (S32) IN CDCL $\ldots . . . .173$

$282 \mathrm{MHz}{ }^{19} \mathrm{~F}$-NMR OF 1,3-DIOXOISOINDOLIN-2-YL 3,3,3-TRIFLUORO-2-METHOXY-2-PHENYLPROPANOATE (S32) IN CDCL $\mathrm{CL}_{3} \ldots . .174$

$500 \mathrm{MHz}{ }^{1} \mathrm{H}-\mathrm{NMR}$ OF 1,3-DIOXOISOINDOLIN-2-YL 9-FLUORO-9H-FLUORENE-9-CARBOXYLATE (S33) IN CDCL $3 \ldots . . . . . . . . . . . . . . .175$

$126 \mathrm{MHz}{ }^{13} \mathrm{C}-N M R$ OF 1,3-DIOXOISOINDOLIN-2-YL 9-FLUORO-9H-FLUORENE-9-CARBOXYLATE (S33) IN CDCL $3 \ldots \ldots \ldots \ldots . . . . . .175$

$282 \mathrm{MHz}{ }^{19} \mathrm{~F}-\mathrm{NMR}$ 1,3-DIOXOISOINDOLIN-2-YL 9-FLUORO-9H-FLUORENE-9-CARBOXYLATE (S33) IN CDCL $3 \ldots \ldots \ldots \ldots \ldots \ldots \ldots . . . . . . . . . . . .176$

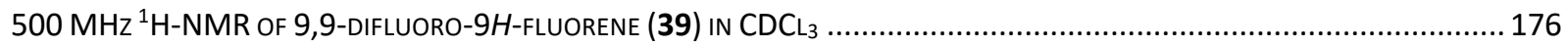

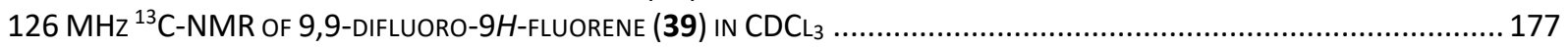

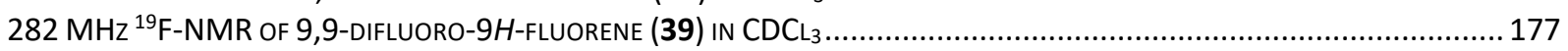

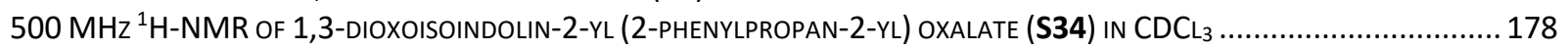

$126 \mathrm{MHz}{ }^{13} \mathrm{C}$-NMR OF 1,3-DIOXOISOINDOLIN-2-YL (2-PHENYLPROPAN-2-YL) OXALATE (S34) IN CDCL $3 \ldots \ldots \ldots \ldots \ldots \ldots \ldots . . . . . . . . . . . . . .178$

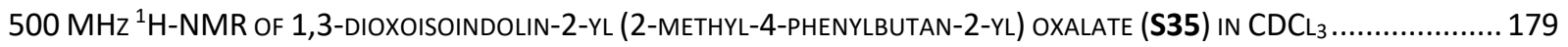

$126 \mathrm{MHz}{ }^{13} \mathrm{C}$-NMR OF 1,3-DIOXOISOINDOLIN-2-YL (2-METHYL-4-PHENYLBUTAN-2-YL) OXALATE (S35) IN CDCL3 ................. 179 


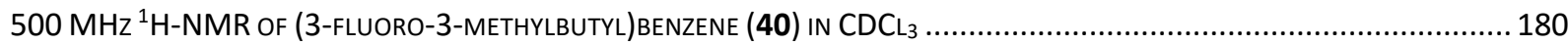

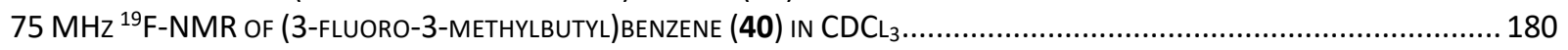

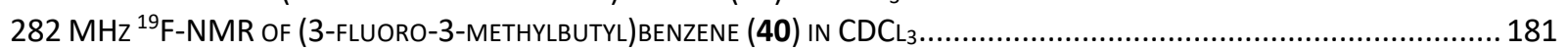

$500 \mathrm{MHz}{ }^{1} \mathrm{H}-\mathrm{NMR}$ OF 2-METHOXY-6-(1-(2,4,6-TRIMETHOXYPHENYL)ETHYL)NAPHTHALENE (45) IN CDCL 3 .........................181

$126 \mathrm{MHz}{ }^{13} \mathrm{C}$-NMR OF 2-METHOXY-6-(1-(2,4,6-TRIMETHOXYPHENYL)ETHYL)NAPHTHALENE (45) IN CDCL

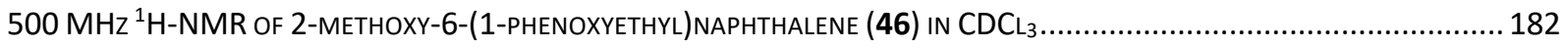

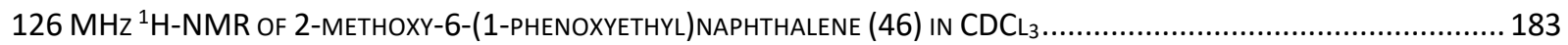

$500 \mathrm{MHz}{ }^{1} \mathrm{H}-\mathrm{NMR}$ OF 2-METHOXY-6-(1-METHOXYETHYL)NAPHTHALENE (47) IN CDCL 3 ........................................... 183

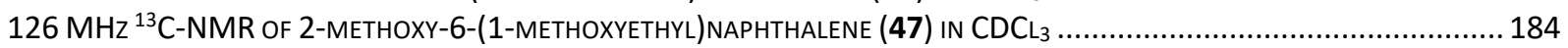

$500 \mathrm{MHz}{ }^{1} \mathrm{H}-\mathrm{NMR}$ OF 2-(1-((1,1,1,3,3,3-HEXAFLUOROPROPAN-2-YL)OXY)ETHYL)-6-METHOXYNAPHTHALENE (48) IN CDCL $3 . .184$

$126 \mathrm{MHz}{ }^{13} \mathrm{C}-\mathrm{NMR}$ OF 2-(1-((1,1,1,3,3,3-HEXAFLUOROPROPAN-2-YL)OXY)ETHYL)-6-METHOXYNAPHTHALENE (48) IN CDCL3. 185

$282 \mathrm{MHz}{ }^{19}$ F-NMR OF 2-(1-((1,1,1,3,3,3-HEXAFLUOROPROPAN-2-YL)OXY)ETHYL)-6-METHOXYNAPHTHALENE (48) IN CDCL . 185

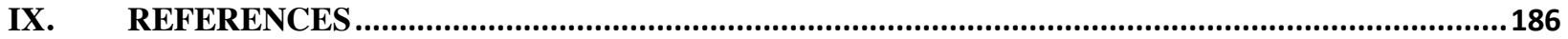




\section{General Information}

General Procedures. Unless otherwise noted, reactions were performed with exclusion of air and moisture. Solvent was freshly distilled/degassed prior to use unless otherwise noted. Organic solutions were concentrated under reduced pressure using a rotary evaporator $\left(25{ }^{\circ} \mathrm{C},<50\right.$ torr $)$. Automated column chromatography was performed using silica gel cartridges on a Biotage SP4 (40-53 $\mu \mathrm{m}, 60 \AA$ Å). SiliaFlash ${ }^{\circ}$ Irregular silica gel P60 (40-63 $\left.\mu \mathrm{m}, 230-400 \mathrm{mesh}\right)$ was used as received. Preparatory thin layer chromatography was conducted on SiliaPlate TLC Glass Backed TLC Extra Hard Layer, $60 \AA$ A, $20 \mathrm{~cm}$ x $20 \mathrm{~cm}, 1000 \mu \mathrm{m}$ thickness.

Materials. Commercial reagents were purchased from Sigma Aldrich, VWR, Fisher, Oakwood, or Strem, and used as received. Dichloromethane (DCM), tetrahydrofuran (THF), acetonitrile (MeCN), dimethylformamide (DMF), dimethyl sulfoxide (DMSO), toluene and dioxane were dried by passing through activated alumina columns and sparged with nitrogen before use. Photocatalysts used were purchased from Stem or Sigma Aldrich and were used as received and stored on the benchtop. Phthalimide esters were prepared according to general procedures and stored on the benchtop. Triethylamine trihydrofluoride was obtained from Sigma Aldrich and was stored on the benchtop.

Instrumentation. Proton nuclear magnetic resonance $\left({ }^{1} \mathrm{H}-\mathrm{NMR}\right)$ spectra were recorded on a Bruker 500 AVANCE spectrometer $(500 \mathrm{mHz})$, a Bruker NanoBay 300 spectrometer $(300 \mathrm{mHz})$, or Varian Unity Inova (400 MHz). Proton chemical shifts are reported in parts per million downfield from tetramethylsilane and referenced to residual protium in the NMR solvent $\left(\mathrm{CDCl}_{3}=\delta 7.26 \mathrm{ppm}, \mathrm{CD}_{3} \mathrm{CN}=\delta 1.94 \mathrm{ppm}, \mathrm{CD}_{2} \mathrm{Cl}_{2}=\delta 5.32 \mathrm{ppm}\right)$. Carbon nuclear magnetic resonance $\left({ }^{13} \mathrm{C}-\mathrm{NMR}\right)$ spectra were recorded on a Bruker 500 AVANCE spectrometer (126 MHz) or a Varian Inova spectrometer $(100 \mathrm{mHz})$. Chemical shifts for carbon are reported in parts per million downfield from tetramethylsilane and are referenced to the carbon resonances of the solvent residual peak $\left(\mathrm{CDCl}_{3}=\delta 77.16 \mathrm{ppm} \mathrm{CD}_{2} \mathrm{Cl}_{2}=\delta 53.84\right.$ ppm, $\left.\mathrm{CD}_{3} \mathrm{CN}=\delta 1.32 \mathrm{ppm}\right)$. ${ }^{1} \mathrm{H}$-decoupled ${ }^{19} \mathrm{~F}$-NMR spectra $(282 \mathrm{MHz}$ ) were recorded on a Bruker NB 300 spectrometer (used for yield determination versus external standard); ${ }^{1} \mathrm{H}$-coupled ${ }^{19} \mathrm{~F}-\mathrm{NMR}$ spectra were recorded on a Bruker 500 AVANCE spectrometer $(470 \mathrm{mHz})$, a Bruker NanoBay 300 spectrometer (282 mHz), or Varian Unity Inova (376 MHz); chemical shifts are reported in parts per million. NMR data are represented as follows: chemical shift $(\delta \mathrm{ppm})$, multiplicity $(\mathrm{s}=$ singlet, $\mathrm{d}=$ doublet, $\mathrm{t}=$ triplet, $\mathrm{q}=$ quartet, $\mathrm{m}=$ multiplet; higher order splitting is specifically noted), coupling constant in Hertz $(\mathrm{Hz})$, integration. High-resolution mass spectra were obtained on an Agilent 6220 using electrospray ionization time-of-flight (ESI-TOF) or on an Agilent 7200 GC-QTOF. FT-IR spectra were recorded on a Perkin-Elmer Spectrum 100 or on a Thermo Nicolet 6700 and are reported in terms of frequency of absorption $\left(\mathrm{cm}^{-1}\right)$. UV-Vis spectra were recorded on an Agilent Cary $60 \mathrm{UV}$-VIS Spectrometer. Fluorescence measurements were obtained on an Agilent Cary Eclipse fluorescence spectrophotometer.

Lamps utilized were 34 watt Kessil H150 Blue. For the emission spectrum of Kessil H150 Blue, see Shields, et. al. ${ }^{1}$ For quantum yield determination, a Kessil PR160-440 nm lamp was utilized and set to 50\% power as the emission spectrum was more monochromatic compared to the Kessil H150 Blue.

\section{General Procedures}

General Procedure A: Phthalimide Ester Synthesis with DIC

This general procedure was adapted from Weix, et. al..$^{2}$ An oven-dried flask with a stir bar was charged with carboxylic acid (4.00 mmol, 1 equiv.), followed by $N$-hydroxyphthalimide (978 mg, $6.00 \mathrm{mmol}$, 1.5 equiv.), and 4dimethylaminopyridine (DMAP, $24.40 .2 \mathrm{mmol}, 0.05$ equiv.). The flask was septa capped and placed under $\mathrm{N}_{2}$. The flask was charged with THF (20 mL) followed by $N, N$ '-diisopropylcarbodiimide (DIC, $0.87 \mathrm{~mL}, 5.6 \mathrm{mmol}, 1.4 \mathrm{equiv}$.) and stirred overnight. Then, the reaction mixture was filtered through a glass frit. The filtered solids were rinsed three times with EtOAc or DCM $(40 \mathrm{~mL})$. The combined filtrates were concentrated via rotary evaporation to yield an orange or yellow solid residue. The residue was subjected to column chromatography. The resulting phthalimide ester was dried overnight under vacuum and subsequently characterized.

\section{General Procedure B: Phthalimide Ester Synthesis via Acid Chloride}

This general procedure was adapted from Fu, et. al. ${ }^{3}$ An oven-dried flask with a stir bar was charged with carboxylic acid (4.00 mmol, 1 equiv.). The flask was septa capped and placed under $\mathrm{N}_{2}$. The flask was charged with $20 \mathrm{~mL}$ of DCM and 5 drops of DMF ( $10 \mu \mathrm{L}$, catalytic). Oxalyl chloride $(0.60 \mathrm{~mL}, 7.1 \mathrm{mmol}, 1.75$ equiv. $)$ was added dropwise with a vent needle in place to allow for gas evolution. The reaction mixture typically went from heterogenous to homogenous, if the carboxylic acid was insoluble, as the reaction proceeded. After gas evolution had stopped (30 min 
to $2 \mathrm{hr}$ ), the reaction mixture was concentrated via rotary evaporation to remove any remaining oxalyl chloride. Following concentration, the flask containing the crude acid chloride was charged with $N$-hydroxyphthalimide (685 $\mathrm{mg}, 4.20 \mathrm{mmol}$., 1.05 equiv.). The flask was placed under $\mathrm{N}_{2}$ and charged with $20 \mathrm{~mL}$ of anhydrous DCM. The mixture was stirred and triethylamine $(0.580 \mathrm{~mL}, 4.20 \mathrm{mmol}, 1.05$ equiv) was added dropwise via syringe and additional gas evolution was observed. The reaction was usually slightly red/orange, indicative of deprotonated $\mathrm{N}$ hydroxyphthalimide. The reaction mixture was stirred overnight at room temperature. The reaction mixture was then concentrated via rotary evaporation. The resulting residue was taken up in DCM and subjected to column chromatography. The resulting phthalimide ester was dried overnight under vacuum and subsequently characterized.

General Procedure C: Phthalimide Ester Synthesis with EDC

An oven-dried $50 \mathrm{~mL}$ flask, equipped with a stir bar, was charged with the appropriate carboxylic acid (4.00 mmol, 1.00 equiv.), $N$-hydroxyphthalimide ( $717 \mathrm{mg}, 4.40 \mathrm{mmol}, 1.10$ equiv.), DMAP (24.4 mg, $0.200 \mathrm{mmol}, 0.0500$ equiv.), and $N$-(3-Dimethylaminopropyl)- $N$ '-ethylcarbodiimide hydrochloride (EDC $\bullet \mathrm{HCl}, 805 \mathrm{mg}, 4.20 \mathrm{mmol}, 1.05$ equiv.). The flask was placed under nitrogen and charged with $20 \mathrm{~mL}$ of THF. The reaction mixture was stirred for 6-12 hours. Upon completion, the reaction mixture was poured into water and the aqueous layer was extracted 3 times with DCM $(60 \mathrm{~mL})$. The organics were washed with sat. $\mathrm{NaHCO}_{3}$ and then water. The organics were dried over sodium sulfate, filtered, and then concentrated via rotary evaporation. The resulting residue was subjected to column chromatography. The resulting phthalimide ester was dried overnight under vacuum and subsequently characterized.

General Procedure D: Screening Scale (0.2 mmol scale)

An oven-dried 1-dram vial with a stir bar was charged with $1.5 \mathrm{mg}$ of $\operatorname{Ir}(\mathrm{dF}-\mathrm{ppy})_{3}(1.5 \mathrm{mg}, 0.0020 \mathrm{mmol}, 0.010$ equiv. $)$ and appropriate phthalimide ester $(0.200 \mathrm{mmol}, 1$ equiv.). The vial was then charged with triethylamine $3 \mathrm{HF}(97.8$ uL, $0.600 \mathrm{mmol}, 3.00$ equiv.). The vial was septa capped and wrapped with labeled electrical tape. The vial was frozen in a $-78{ }^{\circ} \mathrm{C}$ bath and subjected to 3 cycles of evacuation/backfill with nitrogen via spaghetti line. Following this, the vial was placed under $\mathrm{N}_{2}$ and charged with degassed DCM $(1.00 \mathrm{~mL})$ via syringe. The spaghetti line was removed, and the vial was placed $3 \mathrm{~cm}$ in front of a $34 \mathrm{~W}$ H150 Blue Kessil Lamp with 2 fans for cooling. Usually gas evolution was observable. The vials were irradiated for 4-6 hours approximately. Upon completion, the vials were charged with $26.0 \mathrm{uL}$ of 1 -fluoronaphthalene $\left(0.201 \mathrm{mmol}, 1.00\right.$ equiv.) and subjected to analysis by ${ }^{1} \mathrm{H}$-decoupled, ${ }^{19} \mathrm{~F}-\mathrm{NMR}$.

General Procedure E: Isolation scale (0.6 mmol scale)

An oven-dried 2-dram vial with a stir bar was charged with of $\operatorname{Ir}(\mathrm{dF}-\mathrm{ppy})_{3}(4.6 \mathrm{mg}, 0.006 \mathrm{mmol}, 0.01$ equiv.) and appropriate phthalimide ester $(0.600 \mathrm{mmol}, 1$ equiv.). The vial was then charged with triethylamine $3 \mathrm{HF}(3 \mathrm{x} 97.8 \mathrm{uL}$, $1.8 \mathrm{mmol}, 3.00$ equiv.). The vial was placed in a $-78^{\circ} \mathrm{C}$ bath and subjected to 3 cycles of evacuation/backfill via spaghetti line. Following this, the vial was placed under $\mathrm{N}_{2}$ and charged with degassed DCM $(3.00 \mathrm{~mL})$ via syringe. The spaghetti line was removed, and the vial was placed $3 \mathrm{~cm}$ in front of a 34 W H150 Blue Kessil Lamp with 2 fans for cooling. Usually gas evolution was observable. The vials were irradiated for 4-6 hours. Upon completion, the vials were loaded onto silica gel for column chromatography or filtered through a silica/cotton plug and concentrated before being subjected to preparatory TLC. 
Figure S1: Set-up for Screening and Isolation Scale Fluorination Reactions.

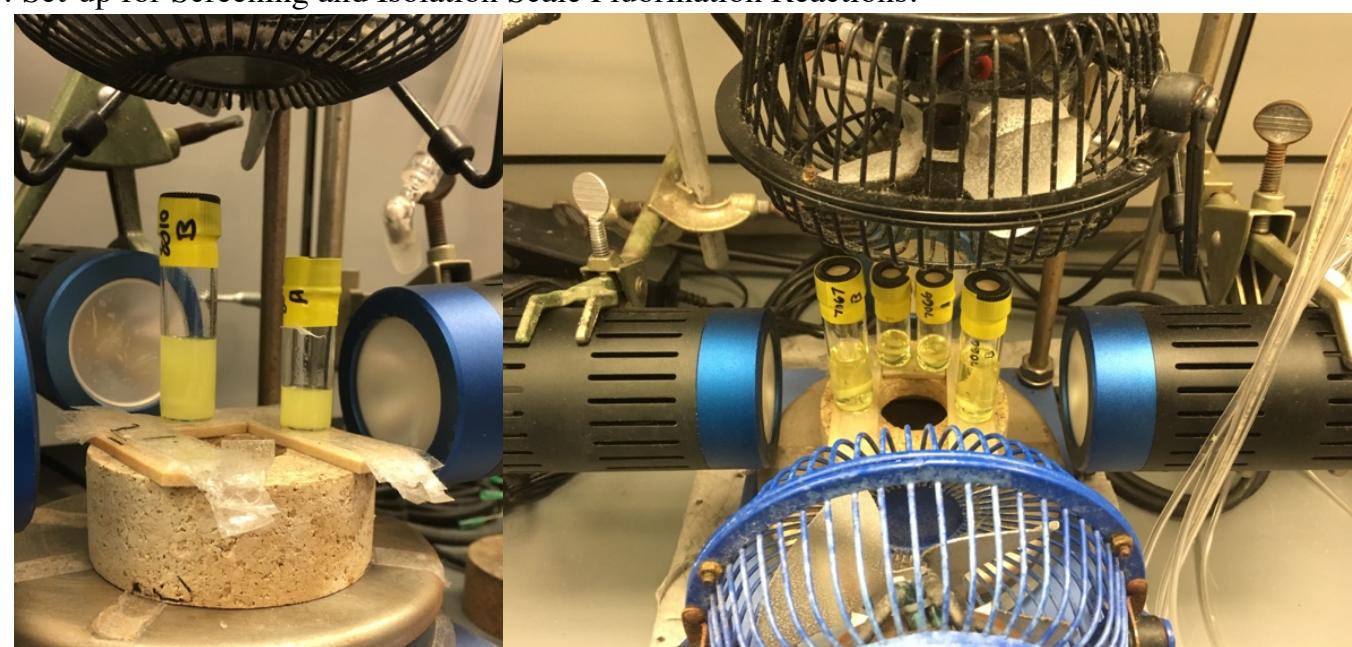

Left: Isolation (left) and screening scale (right) in photocatalysis set up. Right: Screening-multiple vials per lamp, isolation-one vial per lamp. 
III. Selected Optimization Studies

Table S1. Photocatalyst Evaluation.

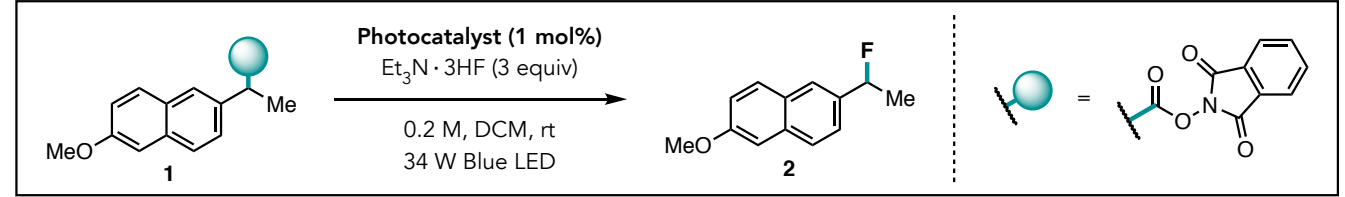

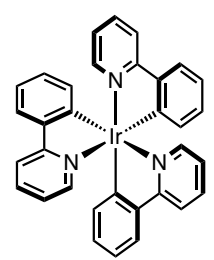

$\underset{96 \% \text { yield }}{\operatorname{Ir}(\mathbf{p p y})_{3}}$

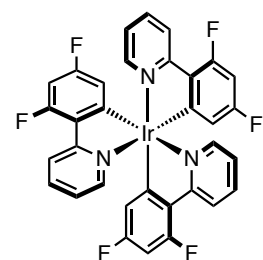

Ir(dF-ppy)
$99 \%$ yield

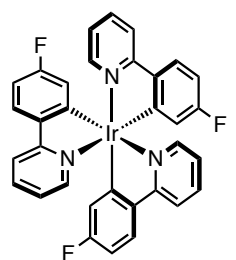

Ir(F-ppy)

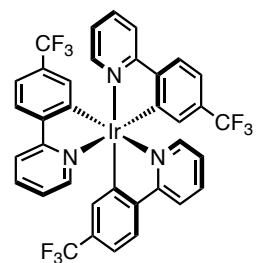

$\operatorname{Ir}\left(\mathrm{CF}_{3}-\text { ppy }\right)_{3}$
$99 \%$ yield

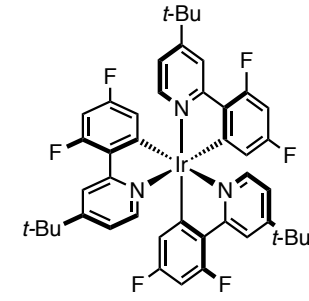

$\operatorname{Ir}(\mathrm{dF}-\mathrm{t}$-Bu-ppy) $99 \%$ yield

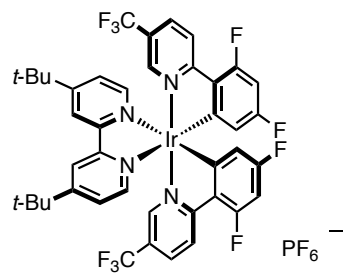

$\left[\operatorname{Ir}\left(\mathrm{dF}-\mathrm{CF}_{3^{-}}-\mathrm{ppy}\right)_{2(}(\mathrm{dtbpy})\right] \mathrm{PF}_{6}$

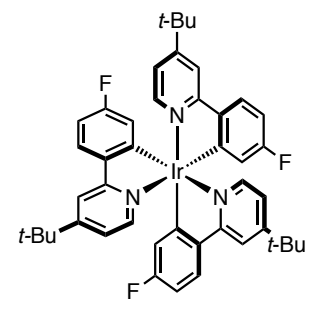

$\operatorname{Ir}(\mathrm{F}-t \text {-Bu-ppy })_{3}$ $98 \%$ yield

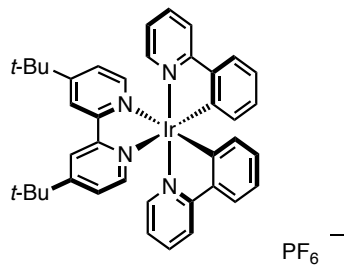

[Ir(ppy)(dtbpy)]PF 6

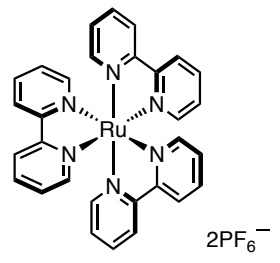

$\mathrm{Ru}(\mathrm{bpy})_{3}\left(\mathrm{PF}_{6}\right)_{2}$ $2 \%$ yield

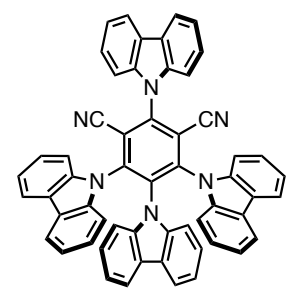

4CzIPN
$91 \%$ yield

$0.2 \mathrm{mmol}$ scale, Determined via ${ }^{19} \mathrm{~F}-\mathrm{NMR}$ vs. external 1 -fluoronaphthalene

Note: With poorer performing substrates, the photocatalysts delivered a wider range in effectiveness; in those cases, $\operatorname{Ir}(\mathrm{dF}-\text { ppy })_{3}$ performed best. 
Table S2. Phthalimide Ester Evaluation.

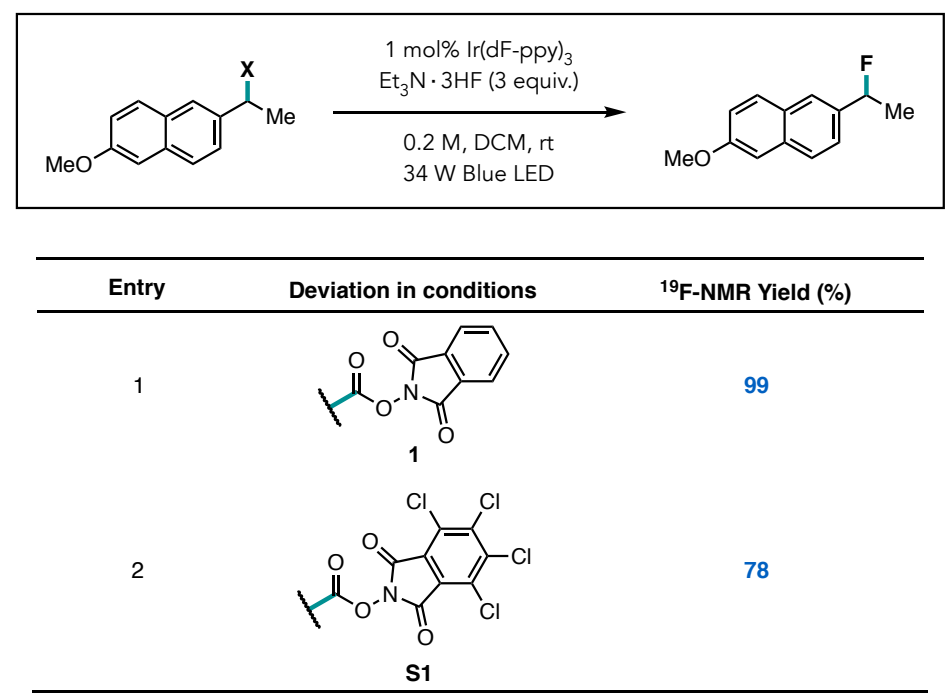

Table S3. Photocatalyst Loading.

\begin{tabular}{|c|c|c|c|}
\hline $\mathrm{MeO}^{-}$ & $\begin{array}{c}\mathrm{X} \text { mol\% Ir(dF-ppy) } \\
\mathrm{Et}_{3} \mathrm{~N} \cdot 3 \mathrm{HF}(3 \text { equiv.) } \\
0.2 \mathrm{M}, \mathrm{DCM}, \mathrm{rt} \\
34 \mathrm{~W} \text { Blue LED }\end{array}$ & $\mathbf{F}$ & 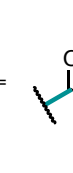 \\
\hline Entry & Deviation in conditions & ${ }^{19}$ F-NMR Yield (\%) & TON \\
\hline 1 & None-1 mol\% Ir(dF-ppy $)_{3}$ & 94 & 94 \\
\hline 2 & $0.5 \mathrm{~mol} \% \operatorname{Ir}(\mathrm{dF}-\mathrm{ppy})_{3}$ & 91 & 182 \\
\hline 3 & 0.25 mol\% $\operatorname{Ir}(\mathrm{dF}-\mathrm{ppy})_{3}$ & 89 & 356 \\
\hline 4 & $0.125 \mathrm{~mol} \% \operatorname{Ir}(\mathrm{dF}-\mathrm{ppy})_{3}$ & 84 & 672 \\
\hline 5 & $0.0625 \mathrm{~mol} \% \operatorname{Ir}(\mathrm{dF}-\mathrm{ppy})_{3}$ & 85 & 1360 \\
\hline 6 & $0.01 \mathrm{~mol} \% \operatorname{Ir}(\mathrm{dF}-\mathrm{ppy})_{3}{ }^{\mathrm{b}}$ & 46 & 4600 \\
\hline
\end{tabular}

ayields determined by ${ }^{19} \mathrm{~F}$-NMR vs external 1-fluoronaphthalene

bIncomplete conversion observed at 4 hours 
Table S4. Solvent Evaluation.

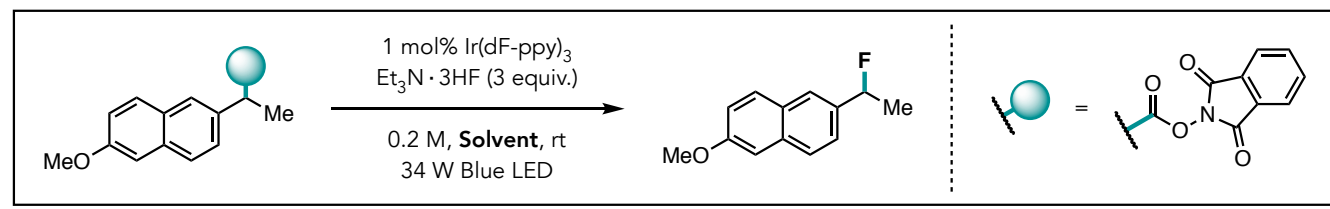

\begin{tabular}{ccc}
\hline Entry & Deviation in conditions & ${ }^{{ }^{9} \text { F-NMR Yield (\%) }}{ }^{\mathbf{a}}$ \\
\hline 1 & DCM & 98 \\
2 & Dioxane & 24 \\
3 & DMF & 7 \\
4 & DMSO & 2 \\
5 & MeCN & 68 \\
6 & THF & 22 \\
\hline
\end{tabular}

ayields determined by ${ }^{19} \mathrm{~F}-\mathrm{NMR}$ vs external 1-fluoronaphthalene

Table S5. Fluoride Loading.

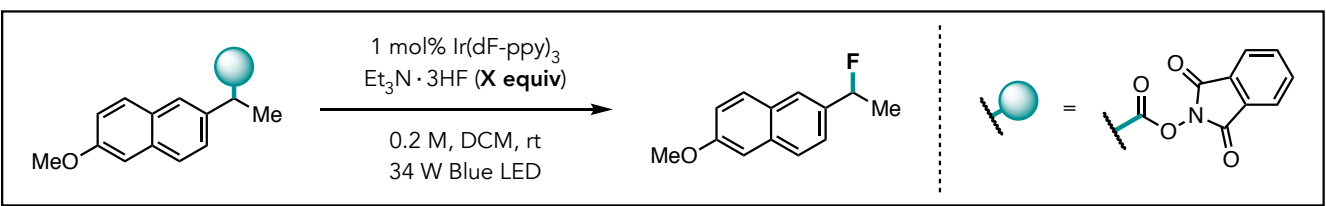

\begin{tabular}{|c|c|c|}
\hline Entry & Deviation in conditions & ${ }^{19}$ F-NMR Yield $(\%)^{a}$ \\
\hline 1 & None $\left(3\right.$ eq. $\mathrm{Et}_{3} \mathrm{~N} \cdot 3 \mathrm{HF}$ ) & 101 \\
\hline 2 & 2 eq. $\mathrm{Et}_{3} \mathrm{~N} \cdot 3 \mathrm{HF}$ & 99 \\
\hline 3 & 1 eq. $\mathrm{Et}_{3} \mathrm{~N} \cdot 3 \mathrm{HF}$ & 87 \\
\hline 4 & 0.75 eq. $\mathrm{Et}_{3} \mathrm{~N} \cdot 3 \mathrm{HF}$ & 81 \\
\hline 5 & 0.5 eq. $\mathrm{Et}_{3} \mathrm{~N} \cdot 3 \mathrm{HF}$ & 67 \\
\hline 6 & 0.25 eq. $\mathrm{Et}_{3} \mathrm{~N} \cdot 3 \mathrm{HF}$ & $56^{*}$ \\
\hline
\end{tabular}

*relative to fluoride, $42 \%$ relative to phthalimide ester

aYields determined by ${ }^{19} \mathrm{~F}-\mathrm{NMR}$ vs external 1 -fluoronaphthalene

Table S6. Fluoride Source Screening.

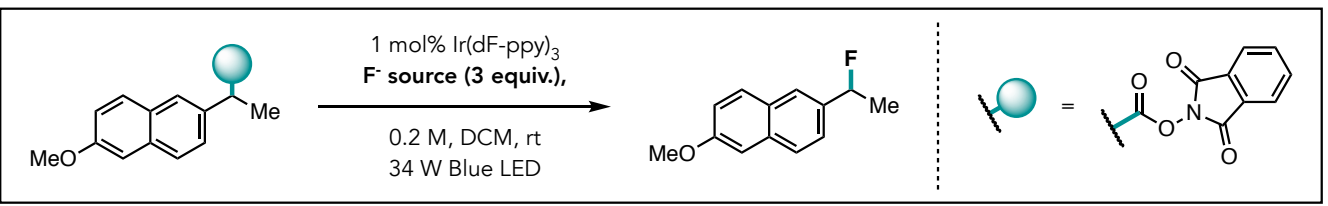

\begin{tabular}{ccc}
\hline Entry & Deviation in conditions & ${ }^{19} \mathrm{~F}-\mathrm{NMR}$ Yield (\%) ${ }^{\mathbf{a}}$ \\
\hline 1 & $\mathrm{Et}_{3} \mathrm{~N} \cdot 3 \mathrm{HF}$ & 93 \\
2 & $\mathrm{KF}$ & 0 \\
3 & $\mathrm{KF}+\mathrm{HFIP}(10$ equiv) & 4 \\
4 & $\mathrm{KF}+18-$ crown-6 (1 equiv) & Trace \\
\hline
\end{tabular}

aYields determined by ${ }^{19} \mathrm{~F}-\mathrm{NMR}$ vs external 1-fluoronaphthalene 


\section{Synthesis and Characterization of Phthalimide Esters and Oxalates}

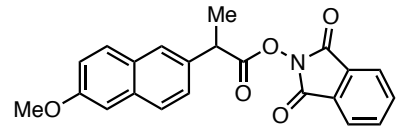

1,3-dioxoisoindolin-2-yl 2-(6-methoxynaphthalen-2-yl)propanoate (1)

According to general procedure $\mathrm{A}$, to an oven dried 50-The $\mathrm{mL}$ flask with a stir bar was added 2-(6-Methoxy-2naphthyl)propionic acid (926.8 mg, $4.00 \mathrm{mmol}, 1.00$ equiv.), $N$-hydroxyphthalimide (992.6 mg, $6.08 \mathrm{mmol}, 1.5$ equiv.), and DMAP (23.8 mg, $0.195 \mathrm{mmol}, 0.05$ equiv.). The flask was septa capped and placed under $\mathrm{N}_{2}$. The flask was then charged with $20 \mathrm{~mL}$ of anhydrous THF followed by DIC $(0.87 \mathrm{~mL}, 5.58 \mathrm{mmol}, 1.4$ equiv.). The flask was stirred overnight. The reaction started an orange color that faded to a pale yellow color as the reaction proceeded. Upon completion, the reaction mixture was filtered through a frit. The filtered solids were washed with EtOAc 3 times. The combined filtrate was concentrated via rotary evaporation to yield a yellow residue. The residue was taken up in EtOAc and subjected to column chromatography (10 to 30\% EtOAc in hexanes). This afforded 1,3-dioxoisoindolin2-yl 2-(6-methoxynaphthalen-2-yl)propanoate as a white solid (1.3093 g, $3.488 \mathrm{mmol}, 87 \%)$.

1H NMR (500 MHz, Chloroform- $d$ ): $\delta 7.86(\mathrm{dd}, J=5.6,3.1 \mathrm{~Hz}, 2 \mathrm{H}), 7.82-7.73(\mathrm{~m}, 5 \mathrm{H}), 7.49(\mathrm{dd}, J=8.4,2.0 \mathrm{~Hz}$, $1 \mathrm{H}), 7.23-7.08(\mathrm{~m}, 2 \mathrm{H}), 4.26(\mathrm{q}, J=7.1 \mathrm{~Hz}, 1 \mathrm{H}), 3.92(\mathrm{~s}, 3 \mathrm{H}), 1.75(\mathrm{~d}, J=7.2 \mathrm{~Hz}, 3 \mathrm{H})$.

${ }^{13}$ C NMR (126 MHz, Chloroform-d): $\delta 171.07,158.03,134.87,134.14,133.59,129.59,129.09,127.69,126.52$, 126.04, 124.08, 119.35, 105.79, 55.48, 43.09, 19.17. (2 carbon peaks are obscured by overlap)

HRMS: (ESI-TOF) calculated for $\mathrm{C}_{22} \mathrm{H}_{18} \mathrm{NO}_{5}{ }^{+}\left([\mathrm{M}+\mathrm{H}]^{+}\right): 376.11795$, found 376.11723 .

FTIR (thin film, cm$\left.^{-1}\right): v 2942(\mathrm{w}), 1809(\mathrm{~m}), 1783(\mathrm{~m}), 1738(\mathrm{~s}), 1632(\mathrm{~m}), 1607(\mathrm{~m}), 1504(\mathrm{w}), 1488(\mathrm{~m}), 1463$ (m), $1419(\mathrm{w}), 1368(\mathrm{~m}), 1276(\mathrm{~m}), 1260(\mathrm{~m}), 1239(\mathrm{~m}), 1216(\mathrm{~m}), 1184(\mathrm{~m}), 1164(\mathrm{~m}), 1138(\mathrm{~s}), 1080(\mathrm{~m}), 1039(\mathrm{~s})$, 1013 (s), 1001 (s), 964 (m), $926(\mathrm{~m}), 896(\mathrm{~m}), 878(\mathrm{~m}), 850(\mathrm{~s}), 821(\mathrm{~m}), 783(\mathrm{~m}), 757(\mathrm{~m}), 693(\mathrm{~s}), 660(\mathrm{~m})$.

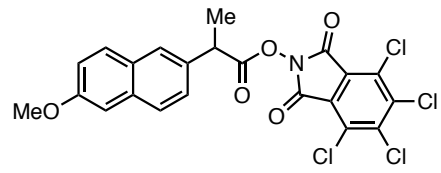

4,5,6,7-tetrachloro-1,3-dioxoisoindolin-2-yl 2-(6-methoxynaphthalen-2-yl)propanoate (S1)

Synthesized according to general procedure A with 2-(6-Methoxy-2-naphthyl)propionic acid (936.8 mg, $4.09 \mathrm{mmol}$, 1 equiv), $N$-hydroxytetrachlorophthalimide (1,743.6 mg, $5.79 \mathrm{mmol}, 1.5$ equiv), DMAP (28.5 mg, $0.2 \mathrm{mmol}, 0.05$ equiv) and DIC ( $0.87 \mathrm{~mL}, 5.58 \mathrm{mmol}, 1.4$ equiv). Purified over silica with flash chromatography (10 to 50\% DCM in hexanes) and fractions containing product were isolated in a flask and subjected to recrystallization with hot DCM. $\mathrm{MeOH}$ was added and the flask was cooled for several hours. A fluorescent yellow solid was observed to precipitate and the solid was isolated on a frit. The solid was transferred to a vial and dried overnight in vacuo to afford the title compound as a flocculant, fluorescent yellow solid $(1.3314 \mathrm{~g}, 2.595 \mathrm{mmol}, 64 \%)$.

${ }^{1}$ H NMR (500 MHz, Chloroform- $d$ ): $\delta 7.84-7.70(\mathrm{~m}, 3 \mathrm{H}), 7.46(\mathrm{dd}, \mathrm{J}=8.4,1.9 \mathrm{~Hz}, 1 \mathrm{H}), 7.16(\mathrm{dd}, \mathrm{J}=8.8,2.5 \mathrm{~Hz}$, $1 \mathrm{H}), 7.13(\mathrm{~d}, \mathrm{~J}=2.5 \mathrm{~Hz}, 1 \mathrm{H}), 4.26(\mathrm{q}, \mathrm{J}=7.2 \mathrm{~Hz}, 1 \mathrm{H}), 3.92(\mathrm{~s}, 3 \mathrm{H}), 1.74(\mathrm{~d}, \mathrm{~J}=7.2 \mathrm{~Hz}, 3 \mathrm{H})$.

${ }^{13}$ C NMR (126 MHz, Chloroform-d): $\delta$ 170.47, 157.97, 141.00, 134.06, 132.98, 130.43, 129.45, 128.92, 127.69, $126.40,125.75,124.69,119.30,105.64,55.36,42.87,18.86$. (1 carbon peak is obscured by overlap)

HRMS: (ESI-TOF) calculated for $\mathrm{C}_{22} \mathrm{H}_{14}{ }^{35} \mathrm{Cl}_{4} \mathrm{NO}_{5}{ }^{+}\left([\mathrm{M}+\mathrm{H}]^{+}\right)$: 511.96206 , found 511.96121 .

Calculated for $\mathrm{C}_{22} \mathrm{H}_{14}{ }^{35} \mathrm{Cl}_{3}{ }^{37} \mathrm{ClNO}_{5}{ }^{+}\left([\mathrm{M}+\mathrm{H}]^{+}\right): 513.95937$, found 513.95783 .

Calculated for $\mathrm{C}_{22} \mathrm{H}_{14}{ }^{35} \mathrm{Cl}_{2}{ }^{37} \mathrm{Cl}_{2} \mathrm{NO}_{5}{ }^{+}\left([\mathrm{M}+\mathrm{H}]^{+}\right)$: 515.95684, found 515.95721.

FTIR (thin film, cm$\left.^{-1}\right): v 2939(\mathrm{w}), 1821(\mathrm{w}), 1795(\mathrm{~m}), 1743(\mathrm{~s}), 1631(\mathrm{w}), 1604(\mathrm{~m}), 1502(\mathrm{w}), 1485(\mathrm{w}), 1461(\mathrm{w})$, $1392(\mathrm{w}), 1378(\mathrm{~s}), 1362(\mathrm{~m}), 1350(\mathrm{~m}), 1301(\mathrm{~m}), 1262(\mathrm{~m}), 1217(\mathrm{~m}), 1197(\mathrm{~m}), 1155(\mathrm{~s}), 1124(\mathrm{w}), 1101(\mathrm{w}), 1078$ (m), $1046(\mathrm{~s}), 1031(\mathrm{~s}), 997(\mathrm{~s}), 958(\mathrm{w}), 926(\mathrm{w}), 883(\mathrm{~m}), 856(\mathrm{~s}), 847(\mathrm{~s}), 810(\mathrm{~s}), 791(\mathrm{~m}), 774(\mathrm{w}), 746(\mathrm{~m}), 729$ (s), $712(\mathrm{~s}), 684(\mathrm{~m}), 661(\mathrm{w}), 650(\mathrm{w}), 619(\mathrm{~m}), 588(\mathrm{w}), 579(\mathrm{w}), 555(\mathrm{w}), 474(\mathrm{~s}), 440(\mathrm{~m}), 405(\mathrm{w})$.

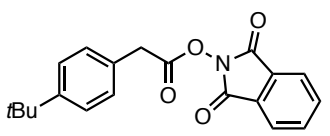




\section{1,3-dioxoisoindolin-2-yl 2-(4-(tert-butyl)phenyl)acetate (S2)}

Synthesized according to general procedure A with 2-(4-(tert-butyl)phenyl)acetic acid (754.8 $\mathrm{mg}, 3.93 \mathrm{mmol}, 1$ equiv.), $N$-hydroxyphthalimide (964.4 mg, $6.00 \mathrm{mmol}, 1.5$ equiv.), DMAP (23.0 mg, $0.21 \mathrm{mmol}, 0.05$ equiv.) and DIC $(0.87 \mathrm{~mL}, 5.58 \mathrm{mmol}, 1.4$ equiv.). Purified over silica with flash chromatography (10 to $20 \%$ EtOAc in hexanes) to afford the title compound as a white solid (1.2663 g, $3.753 \mathrm{mmol}, 96 \%)$.

${ }^{1}$ H NMR (500 MHz, Chloroform- $d$ ): $\delta 7.89(\mathrm{dd}, J=5.5,3.1 \mathrm{~Hz}, 2 \mathrm{H}), 7.79(\mathrm{dd}, J=5.5,3.1 \mathrm{~Hz}, 2 \mathrm{H}), 7.40(\mathrm{~d}, J=8.3$ $\mathrm{Hz}, 2 \mathrm{H}), 7.32$ (d, $J=8.4 \mathrm{~Hz}, 2 \mathrm{H}), 3.97$ (s, 2H), 1.32 (s, 9H).

13C NMR (126 MHz, Chloroform-d): $\delta 167.99,162.03,150.84,134.93,129.07,129.05,128.59,125.98,124.14$, 37.31, 34.69, 31.45.

HRMS: (ESI-TOF) calculated for $\mathrm{C}_{20} \mathrm{H}_{19} \mathrm{NNaO}_{4}^{+}\left(\left[\mathrm{M}+\mathrm{Na}^{+}\right]^{+}\right)$: 360.12063 , found 360.12069 .

FTIR (thin film, cm$\left.^{-1}\right): v 2959(\mathrm{~m}), 1817(\mathrm{~m}), 1788(\mathrm{~m}), 1742(\mathrm{~s}), 1617(\mathrm{w}), 1608(\mathrm{w}), 1466(\mathrm{~m}), 1414(\mathrm{~m}), 1360$ (m), $1331(\mathrm{~m}), 1311(\mathrm{w}), 1291(\mathrm{w}), 1266(\mathrm{~m}), 1214(\mathrm{~m}), 1184(\mathrm{~s}), 1170(\mathrm{~m}), 1142(\mathrm{~m}), 1121(\mathrm{~m}), 1061(\mathrm{~s}), 1024(\mathrm{~m})$, $1013(\mathrm{~m}), 972(\mathrm{~s}), 957$ (m), $935(\mathrm{~m}), 923(\mathrm{~m}), 877$ (s), $843(\mathrm{~m}), 824$ (s), $815(\mathrm{~s}), 789(\mathrm{~s}), 784(\mathrm{~s}), 770(\mathrm{w}), 724(\mathrm{~m})$, $713(\mathrm{~m}), 696(\mathrm{~s}), 619(\mathrm{w}), 579(\mathrm{~m}), 553(\mathrm{~s}), 518(\mathrm{~s}), 494(\mathrm{~m}), 486(\mathrm{~m}), 424(\mathrm{w}), 403(\mathrm{w})$.

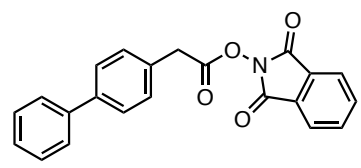

\section{1,3-dioxoisoindolin-2-yl 2-([1,1'-biphenyl]-4-yl)acetate (S3)}

Synthesized according to general procedure A with 4-biphenylacetic acid $(845.9 \mathrm{mg}, 3.99 \mathrm{mmol}, 1$ equiv.), $\mathrm{N}$ hydroxyphthalimide (978.3 mg, $6.00 \mathrm{mmol}, 1.5$ equiv.), DMAP (30.4 mg, $0.25 \mathrm{mmol}, 0.05$ equiv.) and DIC (0.87 $\mathrm{mL}, 5.58 \mathrm{mmol}, 1.4$ equiv.). Purified over silica with flash chromatography (10 to 30\% EtOAc in hexanes) to afford the title compound as a white solid $(1.3390 \mathrm{~g}, 3.747 \mathrm{mmol}, 94 \%)$.

1H NMR (400 MHz, Chloroform- $d$ ): $\delta 7.89(\mathrm{dd}, J=5.5,3.1 \mathrm{~Hz}, 2 \mathrm{H}), 7.79(\mathrm{dd}, J=5.5,3.1 \mathrm{~Hz}, 2 \mathrm{H}), 7.66-7.57$ (m, 4H), $7.53-7.41(\mathrm{~m}, 4 \mathrm{H}), 7.35(\mathrm{t}, J=7.4,1.3 \mathrm{~Hz}, 1 \mathrm{H}), 4.05(\mathrm{~s}, 2 \mathrm{H})$.

${ }^{13}$ C NMR (101 MHz, Chloroform-d): $\delta$ 167.82, 161.99, 140.92, 140.73, 134.95, 130.64, 129.86, 129.07, 128.93 , 127.76, 127.56, 127.27, 124.16, 37.51.

HRMS: (ESI-TOF) calculated for $\mathrm{C}_{22} \mathrm{H}_{16} \mathrm{NO}_{4}{ }^{+}\left([\mathrm{M}+\mathrm{H}]^{+}\right): 358.10738$, found 358.10682.

FTIR (thin film, cm$\left.^{-1}\right): v 3033(\mathrm{w}), 1817(\mathrm{~m}), 1807(\mathrm{~m}), 1786(\mathrm{~m}), 1738(\mathrm{~s}), 1611(\mathrm{~m}), 1567(\mathrm{~m}), 1524(\mathrm{w}), 1488$ (m), $1469(\mathrm{~m}), 1412(\mathrm{~m}), 1360(\mathrm{~m}), 1292(\mathrm{~m}), 1213(\mathrm{~m}), 1189(\mathrm{~m}), 1158(\mathrm{~m}), 1128(\mathrm{~m}), 1101(\mathrm{~m}), 1081(\mathrm{~m}), 1063$ (s), $1018(\mathrm{~m}), 1007(\mathrm{~m}), 970(\mathrm{~m}), 897(\mathrm{~m}), 876(\mathrm{~m}), 839(\mathrm{~m}), 814(\mathrm{~m}), 793(\mathrm{~m}), 770(\mathrm{~m}), 748(\mathrm{~s}), 731(\mathrm{~m}), 690(\mathrm{~s})$

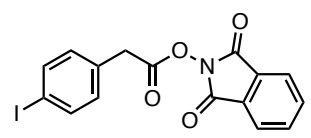

1,3-dioxoisoindolin-2-yl 2-(4-iodophenyl)acetate (S4)

Synthesized according to general procedure A with 4-iodophenylacetic acid (1.313 g, $5.00 \mathrm{mmol}, 1$ equiv.), $N$ hydroxyphthalimide (1.227 g, $7.5 \mathrm{mmol}, 1.5$ equiv.), DMAP (34.3 $\mathrm{mg}, 0.28 \mathrm{mmol}, 0.05$ equiv.) and DIC (1.1 mL, $7.06 \mathrm{mmol}, 1.4$ equiv.). Purified over silica with flash chromatography (10 to 30\% EtOAc in hexanes) to afford the title compound as a white solid (1.7556 g, $4.312 \mathrm{mmol}, 86 \%)$.

${ }^{1}$ H NMR (500 MHz, Chloroform- $d$ ): $\delta 7.96-7.86(\mathrm{~m}, 2 \mathrm{H}), 7.80(\mathrm{dd}, \mathrm{J}=5.2,2.8 \mathrm{~Hz}, 2 \mathrm{H}), 7.71(\mathrm{~d}, \mathrm{~J}=8.0 \mathrm{~Hz}, 2 \mathrm{H})$, $7.14(\mathrm{~d}, \mathrm{~J}=8.3 \mathrm{~Hz}, 2 \mathrm{H}), 3.94(\mathrm{~s}, 2 \mathrm{H})$.

${ }^{13}$ C NMR (126 MHz, Chloroform-d): $\delta$ 167.34, 161.90, 138.14, 135.01, 131.37, 131.27, 128.99, 124.19, 93.69, 37.40 .

HRMS: (ESI-TOF) calculated for $\mathrm{C}_{16} \mathrm{H}_{10} \mathrm{INO}_{4}{ }^{+}\left([\mathrm{M}+\mathrm{H}]^{+}\right)$: 407.97273 , found 407.97266 .

FTIR (thin film, cm$\left.^{-1}\right): v 2938(\mathrm{w}), 1848(\mathrm{w}), 1814(\mathrm{w}), 1785(\mathrm{~m}), 1744(\mathrm{~s}), 1485(\mathrm{~m}), 1463(\mathrm{~m}), 1407$ (m), 1354 (m), 1337 (m), $1186(\mathrm{~m}), 1131$ (m), 1108 (m), 1070 (s), 1059 (s), 1007 (m), 969 (m), 919 (m), 895 (m), 877 (s), 825 (m), $808(\mathrm{~m}), 786(\mathrm{~s}), 742(\mathrm{~m}), 714(\mathrm{~m}), 694(\mathrm{~s})$. 


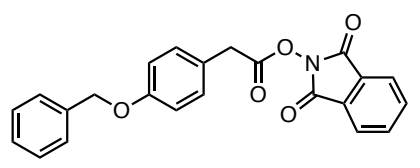

1,3-dioxoisoindolin-2-yl 2-(4-(benzyloxy)phenyl)acetate (S5)

Synthesized according to general procedure A with 2-(4-(benzyloxy)phenyl)acetic acid ( $0.88 \mathrm{~g}, 3.63 \mathrm{mmol}, 1$ equiv.), $N$-hydroxyphthalimide (0.926 g, $5.67 \mathrm{mmol}, 1.5$ equiv.), DMAP $(23.1 \mathrm{mg}, 0.2 \mathrm{mmol}, 0.05$ equiv.) and DIC $(0.7 \mathrm{~mL}$, $4.5 \mathrm{mmol}, 1.4$ equiv.). Purified over silica with flash chromatography (10 to $30 \%$ EtOAc in hexanes) to afford the title compound as a white solid $(1.1466 \mathrm{~g}, 2.96 \mathrm{mmol}, 82 \%)$.

${ }^{1}$ H NMR (500 MHz, Methylene Chloride-d 2 ): $\delta 7.88(\mathrm{dd}, J=5.5,3.1 \mathrm{~Hz}, 2 \mathrm{H}), 7.81(\mathrm{dd}, J=5.5,3.1 \mathrm{~Hz}, 2 \mathrm{H}), 7.44$ $(\mathrm{d}, J=7.3 \mathrm{~Hz}, 2 \mathrm{H}), 7.39(\mathrm{t}, J=7.4 \mathrm{~Hz}, 2 \mathrm{H}), 7.36-7.27(\mathrm{~m}, 3 \mathrm{H}), 6.99(\mathrm{~d}, J=8.7 \mathrm{~Hz}, 2 \mathrm{H}), 5.07(\mathrm{~s}, 2 \mathrm{H}), 3.94(\mathrm{~s}, 2 \mathrm{H})$. ${ }^{13}$ C NMR (126 MHz, Methylene Chloride-d ${ }_{2}$ ): $\delta$ 168.66, 162.37, 158.97, 137.56, 135.43, 130.98, 129.36, 129.06, 128.50, 128.10, 124.56, 124.41, 115.60, 70.52, 37.36.

HRMS: (ESI-TOF) calculated for $\mathrm{C}_{23} \mathrm{H}_{17} \mathrm{NO}_{5}{ }^{+}\left([\mathrm{M}+\mathrm{H}]^{+}\right)$: 387.11067 , found 387.11076.

FTIR (thin film, cm$\left.^{-1}\right): v 3033(\mathrm{w}), 2934(\mathrm{w}), 1812(\mathrm{w}), 1785(\mathrm{~s}), 1739(\mathrm{~s}), 1612(\mathrm{w}), 1581(\mathrm{w}), 1514(\mathrm{~s}), 1465(\mathrm{~m})$, 1450 (w), 1424 (w), 1390 (w), 1362 (w), 1346 (w), 1314 (w), 1272 (w), 1257 (w), 1234 (s), 1181 (m), 1160 (w), 1104 (s), $1078(\mathrm{~m}), 1039(\mathrm{~m}), 1026(\mathrm{~m}), 1011(\mathrm{w}), 975(\mathrm{~m}), 953(\mathrm{~m}), 928(\mathrm{w}), 909(\mathrm{w}), 875(\mathrm{~s}), 860(\mathrm{w}), 829(\mathrm{~m}), 808(\mathrm{~m})$, 784 (s), 765 (m), 733 (s), 693 (s), 598 (w), 581 (w), $519(\mathrm{~s}), 503$ (m), 488 (w), $476(\mathrm{w}), 455(\mathrm{w}), 408(\mathrm{w})$.

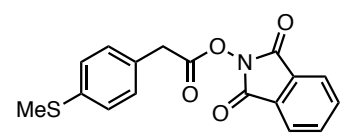

\section{1,3-dioxoisoindolin-2-yl 2-(4-(methylthio)phenyl)acetate (S6)}

Synthesized according to general procedure A with 2-(4-(methylthio)phenyl)acetic acid $(0.546 \mathrm{~g}, 3.00 \mathrm{mmol}, 1$ equiv.), $N$-hydroxyphthalimide ( $0.749 \mathrm{~g}, 4.59 \mathrm{mmol}, 1.5$ equiv.), DMAP (20.6 mg, $0.2 \mathrm{mmol}, 0.05$ equiv.) and DIC ( $0.7 \mathrm{~mL}, 4.5 \mathrm{mmol}, 1.4$ equiv). Purified over silica with flash chromatography (10 to $30 \%$ EtOAc in hexanes) to afford the title compound as a yellow solid $(0.941 \mathrm{~g}, 2.88 \mathrm{mmol}, 96 \%)$.

${ }^{1}$ H NMR (500 MHz, Methylene Chloride-d2): $\delta 7.88(\mathrm{dd}, \mathrm{J}=5.5,3.1 \mathrm{~Hz}, 2 \mathrm{H}), 7.81(\mathrm{dd}, \mathrm{J}=5.6,3.1 \mathrm{~Hz}, 1 \mathrm{H}), 7.31$ $(\mathrm{d}, \mathrm{J}=8.4 \mathrm{~Hz}, 2 \mathrm{H}), 7.27$ (d, J = 8.4 Hz, 2H), 3.96 (s, 2H), 2.49 (s, 3H).

13C NMR (126 MHz, Methylene Chloride-d2): $\delta$ 168.38, 162.34, 139.03, 135.45, 130.27, 129.34, 128.84, 127.09 , $124.43,37.65,15.94$.

HRMS: (ESI-TOF) calculated for $\mathrm{C}_{17} \mathrm{H}_{13} \mathrm{NO}_{4} \mathrm{~S}^{+}\left([\mathrm{M}+\mathrm{H}]^{+}\right): 327.05653$, found 327.05595 .

FTIR (thin film, cm$\left.^{-1}\right): v 2916(\mathrm{w}), 1805(\mathrm{w}), 1781(\mathrm{~m}), 1738(\mathrm{~s}), 1606(\mathrm{w}), 1515(\mathrm{w}), 1493(\mathrm{~m}), 1465(\mathrm{~m}), 1435$ (w), $1410(\mathrm{w}), 1372(\mathrm{~m}), 1349(\mathrm{~m}), 1327(\mathrm{~m}), 1274(\mathrm{w}), 1244(\mathrm{w}), 1212(\mathrm{~m}), 1183(\mathrm{~m}), 1124(\mathrm{~m}), 1103(\mathrm{~s}), 1077(\mathrm{~s})$, $1012(\mathrm{~m}), 971(\mathrm{~s}), 924(\mathrm{~m}), 876(\mathrm{~s}), 861(\mathrm{~m}), 800(\mathrm{~m}), 787(\mathrm{~m}), 754(\mathrm{~s}), 712(\mathrm{~m}), 693(\mathrm{~s}), 596(\mathrm{~m}), 566(\mathrm{~m}), 518(\mathrm{~m})$, $503(\mathrm{~s}), 478(\mathrm{~m})$.

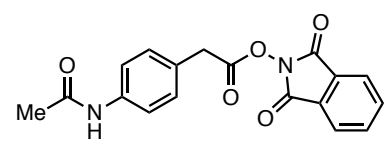

\section{1,3-dioxoisoindolin-2-yl 2-(4-acetamidophenyl)acetate (S7)}

Synthesized according to general procedure $\mathrm{C}$ with 2-(4-acetamidophenyl)acetic acid (771.8 $\mathrm{mg}, 3.99 \mathrm{mmol}, 1$ equiv.), $N$-hydroxyphthalimide (725.1 mg, $4.45 \mathrm{mmol}, 1.12$ equiv.), DMAP (25.5 mg, $0.21 \mathrm{mmol}, 0.05$ equiv.) and $\mathrm{EDC} \cdot \mathrm{HCl}$ ( $812.9 \mathrm{mg}, 4.24 \mathrm{mmol}, 1.06$ equiv.). Purified over silica with flash chromatography (30\% acetone in hexanes) to afford the title compound as a cream colored solid $(1.1943 \mathrm{~g}, 3.530 \mathrm{mmol}, 88 \%)$.

Note: use of oxalyl chloride conditions (general procedure B) resulted in generation of the imidoyl phthalimide ester from the amide and use of DIC conditions (general procedure A) resulted in a difficult separation from $N, N^{\prime}$ diisopropyl urea.

1H NMR (500 MHz, Chloroform- $d$ ): $\delta 7.88(\mathrm{dd}, J=5.5,3.1 \mathrm{~Hz}, 2 \mathrm{H}), 7.79(\mathrm{dd}, J=5.5,3.1 \mathrm{~Hz}, 2 \mathrm{H}), 7.51(\mathrm{~d}, J=8.2$ $\mathrm{Hz}, 2 \mathrm{H}), 7.33$ (d, J = 8.2 Hz, 2H), 7.22 (broad s, 1H), 3.95 (s, 2H), 2.17 (s, 3H). 
${ }^{13}$ C NMR (126 MHz, Chloroform-d): $\delta 168.39,167.81,161.97,137.65,134.96,130.10,129.01,127.38,124.16$, $120.24,37.26,24.79$.

HRMS: (ESI-TOF) calculated for $\mathrm{C}_{18} \mathrm{H}_{15} \mathrm{~N}_{2} \mathrm{O}_{5}{ }^{+}\left([\mathrm{M}+\mathrm{H}]^{+}\right): 339.09755$, found 339.09730 .

FTIR (thin film, cm$\left.^{-1}\right): v 3120(\mathrm{w}), 3045(\mathrm{w}), 2923(\mathrm{w}), 2852(\mathrm{w}), 1809(\mathrm{w}), 1783(\mathrm{~m}), 1736(\mathrm{~s}), 1692(\mathrm{~m}), 1642(\mathrm{~m})$, $1600(\mathrm{~m}), 1537(\mathrm{~m}), 1514(\mathrm{~m}), 1467(\mathrm{~m}), 1415(\mathrm{~m}), 1373(\mathrm{~m}), 1358(\mathrm{~m}), 1326(\mathrm{~m}), 1286(\mathrm{~m}), 1263(\mathrm{~m}), 1220(\mathrm{~m})$, $1184(\mathrm{~m}), 1127(\mathrm{~m}), 1082(\mathrm{~s}), 1023(\mathrm{~m}), 1015(\mathrm{~m}), 975(\mathrm{~s}), 951(\mathrm{~m}), 934(\mathrm{~m}), 877(\mathrm{~s}), 815(\mathrm{~m}), 789(\mathrm{~s}), 767(\mathrm{~m}), 715$ (m), $696(\mathrm{~s}), 640(\mathrm{~m}), 609(\mathrm{~m}), 584(\mathrm{~m}), 517(\mathrm{~s}), 506(\mathrm{~s}), 488(\mathrm{~m}), 411(\mathrm{w}), 404(\mathrm{~m})$.

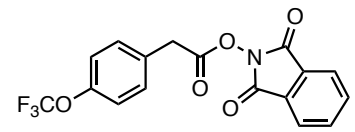

1,3-dioxoisoindolin-2-yl 2-(4-(trifluoromethoxy)phenyl)acetate (S8)

Synthesized according to general procedure A with 4-(trifluoromethoxy)phenylacetic acid $(2.139 \mathrm{~g}, 9.72 \mathrm{mmol}, 1$ equiv.), $N$-hydroxyphthalimide ( $2.510 \mathrm{~g}, 15.4 \mathrm{mmol}, 1.5$ equiv.), DMAP (67.8 $\mathrm{mg}, 0.55 \mathrm{mmol}, 0.05$ equiv.) and DIC ( $2.3 \mathrm{~mL}, 14.8 \mathrm{mmol}, 1.4$ equiv.). Purified over silica with flash chromatography (10 to 30\% EtOAc in hexanes) to afford the title compound as a yellow solid $(3.4854 \mathrm{~g}, 9.542 \mathrm{mmol}, 95 \%)$.

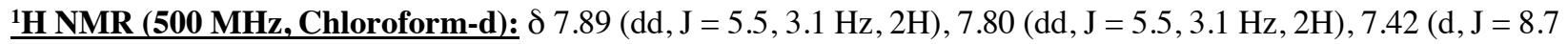
$\mathrm{Hz}, 2 \mathrm{H}), 7.23(\mathrm{~d}, \mathrm{~J}=8.1 \mathrm{~Hz}, 2 \mathrm{H}), 4.01(\mathrm{~s}, 2 \mathrm{H})$.

${ }^{13}$ C NMR (126 MHz, Chloroform-d): $\delta 167.48,161.92,148.96,135.04,130.94,130.29,128.94,124.21,121.48$, 120.54 (q, J = 257.4 Hz), 37.09 .

${ }^{19}$ F NMR (282 MHz, Chloroform-d): $\delta-57.85$ (s, 3F).

HRMS: (ESI-TOF) calculated for $\mathrm{C}_{17} \mathrm{H}_{10} \mathrm{~F}_{3} \mathrm{NO}_{5}{ }^{+}\left([\mathrm{M}+\mathrm{Na}]^{+}\right)$: 365.05111 , found 365.05174 .

FTIR (thin film, cm-1): $v 2973$ (w), 2931 (w), 1814 (m), 1787 (m), 1745 (s), 1611 (w), 1596 (w), 1510 (m), 1469 (w), $1420(\mathrm{w}), 1371(\mathrm{w}), 1357(\mathrm{w}), 1314(\mathrm{w}), 1220(\mathrm{~s}), 1166(\mathrm{~s}), 1150(\mathrm{~s}), 1101(\mathrm{~s}), 1081(\mathrm{~s}), 1015(\mathrm{~m}), 977(\mathrm{~m}), 956$ (m), $926(\mathrm{~m}), 877(\mathrm{~m}), 794(\mathrm{~m}), 777(\mathrm{~m}), 764(\mathrm{~m}), 691(\mathrm{~s}), 654(\mathrm{~m}), 591(\mathrm{~m}), 517(\mathrm{~m}), 481(\mathrm{~m})$.

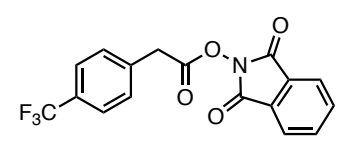

1,3-dioxoisoindolin-2-yl 2-(4-(trifluoromethyl)phenyl)acetate (S9)

Synthesized according to general procedure A with 4-(trifluoromethyl)phenylacetic acid $(2.008 \mathrm{~g}, 9.84 \mathrm{mmol}, 1$ equiv.), $N$-hydroxyphthalimide ( $2.400 \mathrm{~g}, 14.7 \mathrm{mmol}, 1.5$ equiv.), DMAP (62.5 mg, $0.51 \mathrm{mmol}, 0.05$ equiv.) and DIC ( $2.3 \mathrm{~mL}, 14.8 \mathrm{mmol}, 1.4$ equiv.). Purified over silica with flash chromatography (10 to 30\% EtOAc in hexanes) to afford the title compound as a yellow-white solid $(3.253 \mathrm{~g}, 9.314 \mathrm{mmol}, 93 \%)$.

${ }^{1}$ H NMR (500 MHz, Chloroform-d): $\delta 7.89(\mathrm{dd}, \mathrm{J}=5.5,3.1 \mathrm{~Hz}, 2 \mathrm{H}), 7.80(\mathrm{dd}, \mathrm{J}=5.5,3.1 \mathrm{~Hz}, 2 \mathrm{H}), 7.65(\mathrm{~d}, \mathrm{~J}=8.0$ $\mathrm{Hz}, 2 \mathrm{H}), 7.52(\mathrm{~d}, \mathrm{~J}=7.9 \mathrm{~Hz}, 2 \mathrm{H}), 4.06(\mathrm{~s}, 2 \mathrm{H})$.

${ }^{13}$ C NMR (126 MHz, Chloroform-d): $\delta 167.18,161.88,135.59$ (q, $\left.J=1.5 \mathrm{~Hz}\right), 135.05,130.35$ (q, $\left.J=32.7 \mathrm{~Hz}\right)$, $129.88,128.95,125.99(\mathrm{q}, J=3.8 \mathrm{~Hz}), 124.11(\mathrm{q} J=272.1 \mathrm{~Hz}), 37.60$.

19F NMR (282 MHz, Chloroform-d): $\delta$-62.67 (s, 3F)

HRMS: (ESI-TOF) calculated for $\mathrm{C}_{17} \mathrm{H}_{10} \mathrm{~F}_{3} \mathrm{NO}_{4}{ }^{+}\left([\mathrm{M}+\mathrm{H}]^{+}\right): 349.05619$, found 349.05637

FTIR (thin film, cm$\left.^{-1}\right): v 2972(\mathrm{w}), 2935(\mathrm{w}), 1814(\mathrm{w}), 1786(\mathrm{w}), 1743(\mathrm{~s}), 1616(\mathrm{w}), 1468(\mathrm{w}), 1420(\mathrm{w}), 1372(\mathrm{w})$, $1360(\mathrm{w}), 1323(\mathrm{~m}), 1244(\mathrm{w}), 1198(\mathrm{w}), 1185(\mathrm{w}), 1173(\mathrm{~m}), 1158(\mathrm{~m}), 1134(\mathrm{~m}), 1104(\mathrm{~s}), 1064$ (s), $1015(\mathrm{~m}), 978$ $(\mathrm{m}), 960(\mathrm{~m}), 940(\mathrm{~m}), 877(\mathrm{~m}), 841(\mathrm{w}), 805(\mathrm{~m}), 775(\mathrm{~m}), 753(\mathrm{w}), 718(\mathrm{w}), 692(\mathrm{~s}), 669(\mathrm{~m}), 634(\mathrm{w}), 595(\mathrm{~m}), 518$ (m), $497(\mathrm{~m}), 482(\mathrm{w}), 413(\mathrm{w}), 405(\mathrm{w})$.

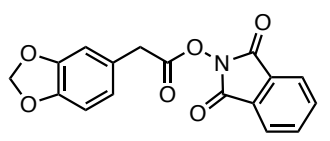

1,3-dioxoisoindolin-2-yl 2-(benzo[d][1,3]dioxol-5-yl)acetate (S10)

Synthesized according to general procedure A with 3,4-(methylenedioxy)phenylacetic acid (723.8 mg, $4.02 \mathrm{mmol}, 1$ equiv.), $N$-hydroxyphthalimide ( $967.1 \mathrm{mg}, 6.00 \mathrm{mmol}, 1.5$ equiv.), DMAP (25.1 mg, $0.21 \mathrm{mmol}, 0.05$ equiv.) and DIC 
(0.87 mL, $5.58 \mathrm{mmol}, 1.4$ equiv.). Purified over silica with flash chromatography (10 to 30\% EtOAc in hexanes) to afford the title compound as a cream-colored solid $(1.2579 \mathrm{~g}, 3.867 \mathrm{mmol}, 96 \%)$.

1H NMR (500 MHz, Chloroform- $d$ ): $\delta 7.91(\mathrm{dd}, \mathrm{J}=5.5,3.1 \mathrm{~Hz}, 2 \mathrm{H}), 7.81(\mathrm{dd}, \mathrm{J}=5.5,3.1 \mathrm{~Hz}, 2 \mathrm{H}), 6.90(\mathrm{~d}, \mathrm{~J}=1.7$ $\mathrm{Hz}, 1 \mathrm{H}), 6.87-6.80(\mathrm{~m}, 2 \mathrm{H}), 5.99(\mathrm{~s}, 2 \mathrm{H}), 3.93(\mathrm{~s}, 2 \mathrm{H})$.

${ }^{13}$ C NMR (126 MHz, Chloroform-d): $\delta$ 167.87, 161.96, 148.16, 147.43, 134.94, 129.03, 125.05, 124.14, 122.83, 109.86, 108.67, 101.36, 37.51.

HRMS: (ESI-TOF) calculated for $\mathrm{C}_{17} \mathrm{H}_{12} \mathrm{NO}_{6}{ }^{+}\left([\mathrm{M}+\mathrm{H}]^{+}\right)$: 326.06591 , found 326.06529 .

FTIR (thin film, cm$\left.^{-1}\right): ~ v 2916(\mathrm{w}), 1816(\mathrm{~m}), 1788(\mathrm{~m}), 1742(\mathrm{~s}), 1610(\mathrm{w}), 1506(\mathrm{~m}), 1495(\mathrm{~m}), 1468(\mathrm{~m}), 1449$ (m), $1406(\mathrm{w}), 1358(\mathrm{~m}), 1335(\mathrm{~m}), 1290(\mathrm{w}), 1278(\mathrm{w}), 1246(\mathrm{~s}), 1187(\mathrm{~m}), 1159(\mathrm{~m}), 1138(\mathrm{~m}), 1065(\mathrm{~s}), 1034(\mathrm{~s})$, $1015(\mathrm{~m}), 970(\mathrm{~m}), 946(\mathrm{~m}), 925(\mathrm{~m}), 891(\mathrm{~m}), 878(\mathrm{~s}), 854(\mathrm{~m}), 819(\mathrm{~m}), 810(\mathrm{~m}), 792(\mathrm{~m}), 780(\mathrm{~m}), 743(\mathrm{~m}), 720$ (m), 695 (s).

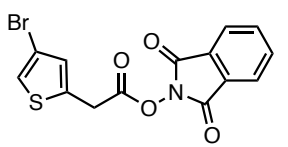

\section{1,3-dioxoisoindolin-2-yl 2-(4-bromothiophen-2-yl)acetate (S11)}

Synthesized according to general procedure A with 4-bromothiopheneacetic acid ( $884.7 \mathrm{mg}, 4.00 \mathrm{mmol}, 1 \mathrm{equiv}$.), $\mathrm{N}$ hydroxyphthalimide ( $912.5 \mathrm{mg}, 5.59 \mathrm{mmol}, 1.5$ equiv.), DMAP (20.6 mg, $0.17 \mathrm{mmol}, 0.05$ equiv.) and DIC ( $0.87 \mathrm{~mL}$, $5.58 \mathrm{mmol}, 1.4$ equiv.). Purified over silica with flash chromatography (10 to $25 \%$ EtOAc in hexanes) to afford the title compound as a yellow-orange solid (1.2774 g, $3.488 \mathrm{mmol}, 87 \%)$.

${ }^{1}$ H NMR (500 MHz, Chloroform- $d$ ): $\delta 7.93(\mathrm{dd}, \mathrm{J}=5.5,3.1 \mathrm{~Hz}, 2 \mathrm{H}), 7.83(\mathrm{dd}, \mathrm{J}=5.5,3.1 \mathrm{~Hz}, 2 \mathrm{H}), 7.21$ (d, J = 1.4 $\mathrm{Hz}, 1 \mathrm{H}), 7.07(\mathrm{~d}, \mathrm{~J}=1.3 \mathrm{~Hz}, 1 \mathrm{H}), 4.20(\mathrm{~s}, 2 \mathrm{H})$.

${ }^{13}$ C NMR (126 MHz, Chloroform-d): $\delta$ 166.32, 161.76, 135.07, 133.57, 130.62, 128.94, 124.26, 123.28, 109.78 , 32.13 .

HRMS: (ESI-TOF) calculated for $\mathrm{C}_{14} \mathrm{H}_{9} \mathrm{BrNO}_{4} \mathrm{~S}^{+}\left([\mathrm{M}+\mathrm{H}]^{+}\right)$: 365.94392 , found 365.94249 .

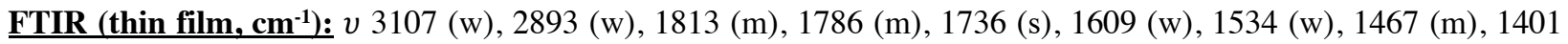
(m), 1359 (m), 1315 (m), 1289 (w), 1234 (w), 1185 (m), 1156 (m), 1144 (w), 1131 (m), 1067 (s), 1014 (m), 967 (s), $929(\mathrm{~m}), 896(\mathrm{~m}), 876(\mathrm{~s}), 837(\mathrm{~m}), 820(\mathrm{~m}), 794(\mathrm{~s}), 734(\mathrm{~s}), 697(\mathrm{~s})$.

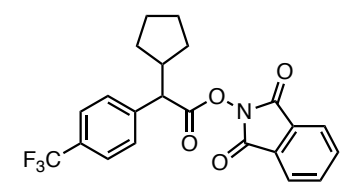

\section{1,3-dioxoisoindolin-2-yl 2-cyclopentyl-2-(4-(trifluoromethyl)phenyl)acetate (S12)}
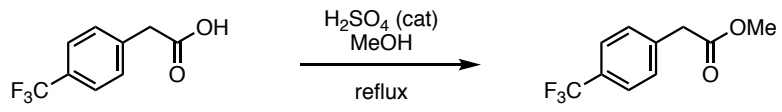

A 200-mL flask was charged with 2-(4-(trifluormethyl)phenylacetic acid (4.1077 g, 20.12 mmol, 1 equiv.) followed by $40 \mathrm{~mL}$ of $\mathrm{MeOH}$. Concentrated sulfuric acid $(5 \mathrm{~mL})$ was added slowly. The flask was fitted with a reflux condenser and heated at reflux overnight. After refluxing overnight, the reaction mixture was neutralized using saturated sodium carbonated. After neutralization, the reaction mixture was transferred to a separatory funnel and extracted 4 times with EtOAc. The combined organic extracts were dried over magnesium sulfate, filtered, and then concentrated via rotary evaporation to a slightly yellow oily residue. The residue was subjected to column chromatography using $15-25 \%$ EtOAc/hexanes. Methyl 2-(4-(trifluoromethyl)phenyl)acetate was obtained (3.9034 g, $17.89 \mathrm{mmol}, 89 \%)$ as a slightly yellow oil.

1H NMR (500 MHz, Chloroform- $d$ ): $\delta 7.59$ (d, $J=8.1 \mathrm{~Hz}, 2 \mathrm{H}), 7.40(\mathrm{~d}, J=8.0 \mathrm{~Hz}, 2 \mathrm{H}), 3.71(\mathrm{~s}, 3 \mathrm{H}), 3.69$ (s, 2H). 13C NMR (126 MHz, Chloroform-d): $\delta$ 171.37, 138.02, 129.83, $129.63(\mathrm{q}, J=32.4 \mathrm{~Hz}), 125.66$ (q, $J=3.7 \mathrm{~Hz})$, 124.25 (q, $J=272.0 \mathrm{~Hz}), 52.41,41.01$.

19F NMR (376 MHz, Chloroform- $d$ ): $\delta-62.60(\mathrm{~s}, 3 \mathrm{~F})$. 


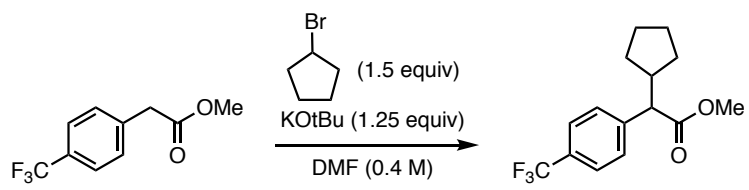

To an oven dried $50 \mathrm{~mL}$ flask was added $\mathrm{KO} t \mathrm{Bu}(1.1234 \mathrm{~g}, 10 \mathrm{mmol}, 1.25$ equiv.). The flask was placed under nitrogen and then charged with $15 \mathrm{~mL}$ of DMF and cooled to $0^{\circ} \mathrm{C}$. Methyl 2-cyclopentyl-2-(4-(trifluoromethyl)phenyl)acetate ( $1.7509 \mathrm{~g}, 8.03 \mathrm{mmol}, 1$ equiv.) was added as a solution in $4 \mathrm{~mL}$ of DMF. The reaction mixture turned orange-red as the solution was stirred for 40 minutes. Then cyclopentyl bromide (1.2 mL, 12 mmol, 1.5 equiv.) was added via syringe. Sodium bromide was observed to precipitate. After 3 hours, the reaction mixture was neutralized with saturated ammonium chloride and transferred to a separatory funnel. The organics were extracted with EtOAc 3 times and then were dried over magnesium sulfate and concentrated. The resulting oily residue was purified over silica with flash chromatography (3 to 5\% EtOAc in hexanes) to afford methyl 2-cyclopentyl-2-(4(trifluoromethyl)phenyl)acetate as an oil (2.0656 g, $7.125 \mathrm{mmol}, 90 \%)$.

1H NMR (400 MHz, Chloroform- $d)$ : $\delta 7.57(\mathrm{~d}, J=8.6 \mathrm{~Hz}, 2 \mathrm{H}), 7.46(\mathrm{~d}, J=8.1 \mathrm{~Hz}, 2 \mathrm{H}), 3.66(\mathrm{~s}, 3 \mathrm{H}), 3.35(\mathrm{~d}, J=$ $11.1 \mathrm{~Hz}, 1 \mathrm{H}), 2.55(\mathrm{dtt}, J=11.0,8.8,7.2 \mathrm{~Hz}, 1 \mathrm{H}), 2.00-1.83(\mathrm{~m}, 1 \mathrm{H}), 1.74-1.55(\mathrm{~m}, 3 \mathrm{H}), 1.53-1.36(\mathrm{~m}, 2 \mathrm{H}), 1.27$ $(\mathrm{dq}, J=12.7,8.4 \mathrm{~Hz}, 1 \mathrm{H}), 1.06-0.92(\mathrm{~m}, 1 \mathrm{H})$.

${ }^{13}$ C NMR (126 MHz, Chloroform- $\left.d\right)$ : $\delta 173.93,143.06,129.62(\mathrm{q}, J=32.5 \mathrm{~Hz}), 128.77,125.58(\mathrm{q}, J=3.8 \mathrm{~Hz})$, $124.29(\mathrm{q}, J=272.0 \mathrm{~Hz}), 57.65,52.16,43.73,31.64,30.84,25.19,24.93$.

19F NMR (376 MHz, Chloroform- $d)$ : $\delta-62.53$ (s, 3F).

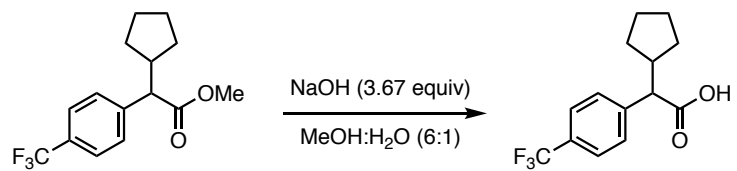

To a flask containing methyl 2-cyclopentyl-2-(4-(trifluoromethyl)phenyl)acetate (2.0656 g, $6.88 \mathrm{mmol}, 1$ equiv.) was added sodium hydroxide (1.01 g, $25 \mathrm{mmol}, 3.67$ equiv), $30 \mathrm{~mL}$ of $\mathrm{MeOH}$ and $5 \mathrm{~mL}$ of water. The flask was stirred overnight at room temperature. After stirring overnight, the reaction mixture was decanted to a separatory funnel and washed with ether. The ether wash was discarded. The aqueous layer was acidified using concentrated $\mathrm{HCl}$ to $\mathrm{pH} 2$. After acidifying, the organics were extracted 4 times with ether. The organics were dried over magnesium sulfate and then concentrated via rotary evaporation and dried under vacuum to afford 2-cyclopentyl-2-(4(trifluoromethyl)phenyl)acetic acid as a crystalline white solid (1.9217 g, $7.058 \mathrm{mmol}, 98 \%)$.

1H NMR (500 MHz, Chloroform- $d)$ : $\delta 7.58(\mathrm{~d}, J=8.1 \mathrm{~Hz}, 2 \mathrm{H}), 7.48(\mathrm{~d}, J=8.1 \mathrm{~Hz}, 2 \mathrm{H}), 3.36(\mathrm{~d}, J=11.1 \mathrm{~Hz}, 1 \mathrm{H})$, 2.54 (dddd, $J=16.0,11.2,8.8,7.3 \mathrm{~Hz}, 1 \mathrm{H}), 1.97(\mathrm{dtd}, J=12.3,7.4,4.5 \mathrm{~Hz}, 1 \mathrm{H}), 1.74-1.66(\mathrm{~m}, 1 \mathrm{H}), 1.66-1.55$ (m, $2 \mathrm{H}), 1.53-1.38(\mathrm{~m}, 2 \mathrm{H}), 1.33(\mathrm{~m}, 1 \mathrm{H}), 0.98(\mathrm{~m}, 1 \mathrm{H})$.

${ }^{13}$ C NMR (126 MHz, Chloroform- $\left.d\right)$ : $\delta 178.93,142.29,129.75(\mathrm{q}, J=32.5 \mathrm{~Hz}), 128.76,125.53(\mathrm{q}, J=3.7 \mathrm{~Hz})$, $124.09(\mathrm{q}, J=272.0 \mathrm{~Hz}), 57.43,43.21,31.50,30.69,25.08,24.78$.

19F NMR (376 MHz, Chloroform-d): $\delta-62.58(\mathrm{~s}, 3 \mathrm{~F})$.

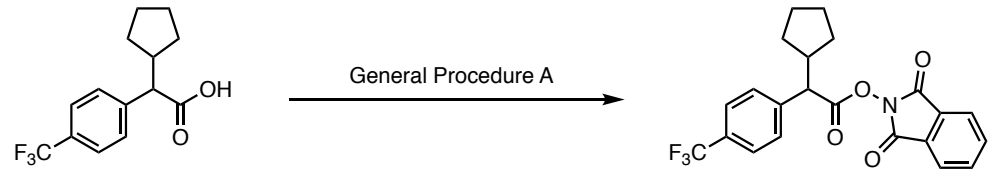

Synthesized according to general procedure A with 2-cyclopentyl-2-(4-(trifluoromethyl)phenyl)acetic acid (1.9217 g, 7 mmol, 1 equiv.), $N$-hydroxyphthalimide ( $1.7118 \mathrm{~g}, 10.5 \mathrm{mmol}, 1.5$ equiv.), DMAP (48.9 mg, $0.35 \mathrm{mmol}, 0.05$ equiv.) and DIC (0.87 mL, $5.58 \mathrm{mmol}, 0.8$ equiv.). Purified over silica with flash chromatography (5 to $25 \%$ EtOAc in hexanes) to afford the title compound as a white solid $(2.7877 \mathrm{~g}, 6.68 \mathrm{mmol}, 95 \%)$.

1H NMR (500 MHz, Chloroform- $d$ ): $\delta 7.86(\mathrm{dd}, J=5.6,3.1 \mathrm{~Hz}, 2 \mathrm{H}), 7.78(\mathrm{dd}, J=5.6,3.1 \mathrm{~Hz}, 2 \mathrm{H}), 7.64(\mathrm{~d}, J=8.1$ $\mathrm{Hz}, 2 \mathrm{H}), 7.54(\mathrm{~d}, J=8.0 \mathrm{~Hz}, 2 \mathrm{H}), 3.75(\mathrm{~d}, J=10.7 \mathrm{~Hz}, 1 \mathrm{H}), 2.67-2.52(\mathrm{~m}, 1 \mathrm{H}), 2.18-2.09(\mathrm{~m}, 1 \mathrm{H}), 1.82-1.71(\mathrm{~m}$, $1 \mathrm{H}), 1.71-1.60(\mathrm{~m}, 2 \mathrm{H}), 1.58-1.47(\mathrm{~m}, 3 \mathrm{H}), 1.18-1.06(\mathrm{~m}, 1 \mathrm{H})$.

13C NMR (126 MHz, Chloroform- $d)$ : $\delta$ 169.69, 161.94, $140.82(\mathrm{~d}, J=1.4 \mathrm{~Hz}), 134.96,130.28(\mathrm{q}, J=32.6 \mathrm{~Hz})$, 129.02, 128.90, $125.91(\mathrm{q}, J=3.7 \mathrm{~Hz}), 124.17(\mathrm{q}, J=272.0 \mathrm{~Hz}), 124.13,54.42,44.12,31.48,31.01,25.22,24.86$. 
${ }^{19}$ F NMR (282 MHz, Chloroform- $d$ ) $\delta-62.58$ (s, 3F).

HRMS: (ESI-TOF) calculated for $\mathrm{C}_{22} \mathrm{H}_{19} \mathrm{~F}_{3} \mathrm{NO}_{4}{ }^{+}\left([\mathrm{M}+\mathrm{H}]^{+}\right)$: 418.12607, found 418.12569.

FTIR (thin film, cm$\left.^{-1}\right): v 2959(\mathrm{w}), 2879(\mathrm{w}), 1804(\mathrm{w}), 1780(\mathrm{~m}), 1743(\mathrm{~s}), 1619(\mathrm{w}), 1466(\mathrm{w}), 1454(\mathrm{w}), 1421(\mathrm{w})$, $1354(\mathrm{~m}), 1323(\mathrm{~s}), 1290(\mathrm{w}), 1184(\mathrm{~m}), 1164(\mathrm{~s}), 1135(\mathrm{~s}), 1120(\mathrm{~s}), 1109(\mathrm{~s}), 1081(\mathrm{~m}), 1059(\mathrm{~s}), 1019(\mathrm{~s}), 966(\mathrm{~s})$, $906(\mathrm{~m}), 873(\mathrm{~s}), 836(\mathrm{~m}), 787(\mathrm{~m}), 724(\mathrm{w}), 694(\mathrm{~s}), 658(\mathrm{~m}), 636(\mathrm{w}), 603(\mathrm{~m}), 517(\mathrm{~s}), 495(\mathrm{w}), 401(\mathrm{w})$.

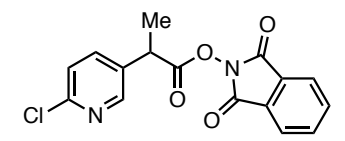

1,3-dioxoisoindolin-2-yl 2-(6-chloropyridin-3-yl)propanoate (S13)

2-(6-chloropyridin-3-yl)propanoic acid was prepared according to Liu, et. al. ${ }^{4}$ Prepared according to general procedure A with 2-(6-chloropyridin-3-yl)propanoic acid (2.275 g, $12.3 \mathrm{mmol}, 1$ equiv.), $N$-hydroxyphthalimide (1.5985 g, 9.8 mmol, 0.8 equiv.), DMAP (70.6 mg, $0.62 \mathrm{mmol}, 0.05$ equiv.) and DIC ( $2.2 \mathrm{~mL}, 14 \mathrm{mmol}, 1.4$ equiv.). Purified over silica with flash chromatography (30 to 50\% EtOAc in hexanes) to afford the title compound as a white solid with some $N$-hydroxyphthalimide as an impurity. The solid was subjected to recrystallization from diethyl ether to afford the title compound $(1.4539 \mathrm{~g}, 35.9 \%)$.

$\underline{\left.{ }^{1} \text { H NMR (500 MHz, Methylene Chloride- } \boldsymbol{d}_{2}\right):} \delta 8.43(\mathrm{~s}, 1 \mathrm{H}), 7.95-7.72(\mathrm{~m}, 5 \mathrm{H}), 7.38(\mathrm{~d}, J=8.9 \mathrm{~Hz}, 1 \mathrm{H}), 4.19(\mathrm{q}$, $J=6.7,6.1 \mathrm{~Hz}, 1 \mathrm{H}), 1.68(\mathrm{~d}, J=7.7 \mathrm{~Hz}, 3 \mathrm{H})$.

${ }^{13}$ C NMR (126 MHz, Methylene Chloride- $\left.\boldsymbol{d}_{2}\right):$ o $170.64,162.19,151.35,149.62,138.51,135.52,133.81,129.18$, 124.97, 124.44, 40.46, 19.07 .

HRMS: (ESI-TOF) calculated for $\mathrm{C}_{16} \mathrm{H}_{12} \mathrm{ClN}_{2} \mathrm{O}_{4} \mathrm{~S}^{+}\left([\mathrm{M}+\mathrm{H}]^{+}\right)$: 331.04801 , found 331.04770 .

FTIR (thin film, cm$\left.^{-1}\right): v 3515(\mathrm{w}), 2994(\mathrm{w}), 2360(\mathrm{w}), 2340(\mathrm{w}), 1810(\mathrm{~m}), 1782(\mathrm{~s}), 1737(\mathrm{~s}), 1609(\mathrm{~m}), 1585$ $(\mathrm{m}), 1564(\mathrm{~m}), 1457(\mathrm{~s}), 1394(\mathrm{~m}), 1356(\mathrm{~s}), 1331(\mathrm{~m}), 1289(\mathrm{~m}), 1185(\mathrm{~s}), 1169(\mathrm{~s}), 1157(\mathrm{~m}), 1138(\mathrm{~s}), 1103$ $(\mathrm{s}), 1080(\mathrm{~s}), 1049(\mathrm{~s}), 1024(\mathrm{~s}), 1016(\mathrm{~s}), 981(\mathrm{~s}), 957(\mathrm{~s}), 875(\mathrm{~s}), 844(\mathrm{~s}), 808(\mathrm{~m}), 790(\mathrm{~s}), 774(\mathrm{~s}), 727(\mathrm{~m})$, $693(\mathrm{~s}), 630(\mathrm{~m}), 601(\mathrm{~m}), 588(\mathrm{~m}), 519(\mathrm{~s}), 472(\mathrm{~m}), 457(\mathrm{~m}), 420(\mathrm{~m})$.

This matched literature characterization. ${ }^{4}$

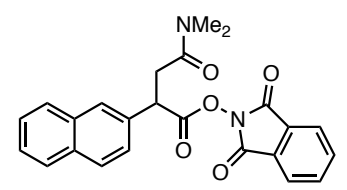

1,3-dioxoisoindolin-2-yl 4-(dimethylamino)-2-(naphthalen-2-yl)-4-oxobutanoate (S14)
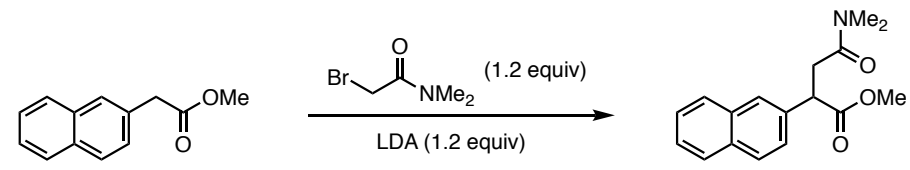

A 50-mL oven-dried flask with a stir bar was placed under nitrogen. The flask was charged with diisopropylamine $\left(1.4 \mathrm{~mL}, 9.9 \mathrm{mmol}, 1.2\right.$ equiv.) and $10 \mathrm{~mL}$ of THF. The flask was cooled to $-78^{\circ} \mathrm{C} . \mathrm{n}-\mathrm{BuLi}(3.60 \mathrm{~mL}, 2.5 \mathrm{M}$ in hexanes, 1.08 equiv.) was added via syringe. The reaction mixture was stirred for 10 minutes. Then, methyl 2-(naphthalene-2yl)acetate (1.6613 g, $8.3 \mathrm{mmol}, 1$ equiv.) was added as a solution in $20 \mathrm{~mL}$ of THF. The reaction turned a fluorescent yellow/orange and was stirred for 15 minutes. Dimethyl bromoacetamide $(1.1 \mathrm{~mL}, 10 \mathrm{mmol}, 1.2$ equiv.) was then added via syringe and the reaction was allowed to stir overnight and warm to room temperature. The reaction mixture faded to a cream color as the reaction proceeded. The reaction mixture was quenched with saturated ammonium chloride. The organics were extracted 4 times with EtOAc. The combined organic extracts were dried over magnesium sulfate, filtered, and concentrated via rotary evaporation to yield a cream solid. Purified over silica with flash chromatography (15 to $85 \%$ EtOAc in hexanes) to afford methyl 4-(dimethylamino)-2-(naphthalen-2-yl)-4oxobutanoate was obtained as a white solid $(2.1303 \mathrm{~g}, 7.47 \mathrm{mmol}, 90 \%)$.

${ }^{1}$ H NMR (500 MHz, Chloroform- $d$ ): $\delta 7.81(\mathrm{~d}, J=8.4 \mathrm{~Hz}, 3 \mathrm{H}), 7.76(\mathrm{~d}, J=1.8 \mathrm{~Hz}, 1 \mathrm{H}), 7.50-7.42(\mathrm{~m}, 3 \mathrm{H}), 4.36$ (dd, $J=10.7,4.1 \mathrm{~Hz}, 1 \mathrm{H}), 3.69$ (s, 3H), $3.35(\mathrm{dd}, J=16.4,10.7 \mathrm{~Hz}, 1 \mathrm{H}), 3.00$ (s, 3H), 2.95 (s, 3H), 2.65 (dd, $J=16.4$, $4.0 \mathrm{~Hz}, 1 \mathrm{H})$.

${ }^{13}$ C NMR (126 MHz, Chloroform-d): $\delta$ 174.29, 170.56, 135.95, 133.46, 132.68, 128.62, 127.82, 127.66, 126.61, 126.35, 126.03, 125.84, 52.37, 47.51, 37.76, 37.04, 35.49. 


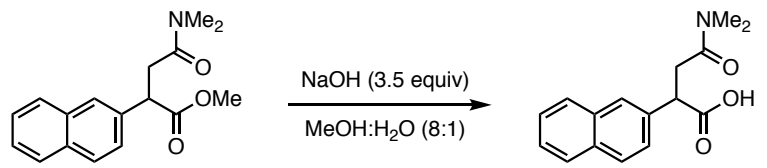

A 100-mL flask was charged with $\mathrm{NaOH}$ (1.05 g, 26 mmol, 3.5 equiv.) and methyl 4-(dimethylamino)-2-(naphthalen2-yl)-4-oxobutanoate (2.1114 g, $7.4 \mathrm{mmol}, 1$ equiv.). The flask was charged with $40 \mathrm{~mL}$ of $\mathrm{MeOH}$ and $5 \mathrm{~mL}$ of water. The reaction mixture was stirred at room temperature overnight. The reaction mixture was acidified to $\mathrm{pH} 2 \mathrm{using}$ conc. $\mathrm{HCl}$. The organics were extracted 4 times with EtOAc. The organics were dried over magnesium sulfate, filtered, concentrated via rotary evaporation, and dried under vacuum to afford 4-(dimethylamino)-2-(naphthalen-2-yl)-4oxobutanoic acid as a white solid (1.8017 g, $6.64 \mathrm{mmol}, 90 \%)$.

1H NMR (400 MHz, Chloroform- $d)$ : $\delta 7.85-7.78(\mathrm{~m}, 4 \mathrm{H}), 7.52-7.43(\mathrm{~m}, 3 \mathrm{H}), 4.40(\mathrm{dd}, J=10.5,3.8 \mathrm{~Hz}, 1 \mathrm{H})$, $3.32(\mathrm{dd}, J=16.5,10.5 \mathrm{~Hz}, 1 \mathrm{H}), 2.99$ (s, 3H), 2.92 (s, 3H), 2.72 (dd, $J=16.5,3.8 \mathrm{~Hz}, 1 \mathrm{H})$.

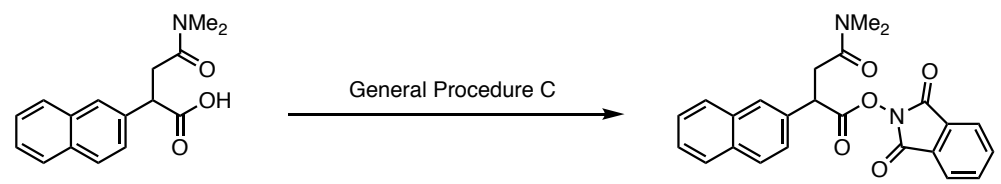

Prepared according to general procedure $\mathrm{C}$ with 4-(dimethylamino)-2-(naphthalen-2-yl)-4-oxobutanoic acid (1.3531 g, 5 mmol, 1 equiv.), $N$-hydroxyphthalimide (0.9019 g, $5.5 \mathrm{mmol}, 1.1$ equiv.), DMAP (33.6 mg, $0.25 \mathrm{mmol}, 0.05$ equiv.), $\mathrm{EDC} \cdot \mathrm{HCl}(1.0024 \mathrm{~g}, 5.25 \mathrm{mmol}, 1.05$ equiv.) and $25 \mathrm{~mL}$ THF. Purified over silica with flash chromatography ( 25 to $85 \%$ EtOAc in hexanes) to afford the title compound as a white solid (0.9696 g, $2.328 \mathrm{mmol}, 73 \%$ ).

1H NMR (500 MHz, Chloroform- $d$ ): $\delta 7.94-7.82(\mathrm{~m}, 6 \mathrm{H}), 7.75(\mathrm{dd}, J=5.5,3.1 \mathrm{~Hz}, 2 \mathrm{H}), 7.54(\mathrm{dd}, J=8.5,1.9 \mathrm{~Hz}$, $1 \mathrm{H}), 7.51-7.48(\mathrm{~m}, 2 \mathrm{H}), 4.82(\mathrm{dd}, J=9.0,5.3 \mathrm{~Hz}, 1 \mathrm{H}), 3.34(\mathrm{dd}, J=16.4,9.0 \mathrm{~Hz}, 1 \mathrm{H}), 2.97(\mathrm{~s}, 3 \mathrm{H}), 2.96$ (s, $3 \mathrm{H})$, $2.91(\mathrm{dd}, J=16.4,5.4 \mathrm{~Hz}, 1 \mathrm{H})$.

${ }^{13}$ C NMR (126 MHz, Chloroform-d): $\delta$ 170.33, 169.32, 161.64, 134.64, 133.89, 133.51, 132.93, 129.00, 128.87, 128.07, 127.73, 127.33, 126.45, 126.31, 125.62, 123.91, 45.39, 37.96, 37.03, 35.64.

HRMS: (ESI-TOF) calculated for $\mathrm{C}_{24} \mathrm{H}_{21} \mathrm{~N}_{2} \mathrm{O}_{5}{ }^{+}\left([\mathrm{M}+\mathrm{H}]^{+}\right): 417.14450$, found 417.14362 .

FTIR (thin film, cm-1): $v 3048$ (w), 2931 (w), 1802 (w), 1777 (m), 1738 (s), 1650 (s), 1549 (w), 1510 (w), 1500 (w), $1467(\mathrm{~m}), 1453(\mathrm{w}), 1417(\mathrm{w}), 1400(\mathrm{~m}), 1351(\mathrm{~m}), 1292(\mathrm{w}), 1262(\mathrm{w}), 1238(\mathrm{w}), 1216(\mathrm{w}), 1205(\mathrm{w}), 1185(\mathrm{~m})$, $1159(\mathrm{w}), 1146(\mathrm{w}), 1122(\mathrm{~m}), 1090(\mathrm{~m}), 1049(\mathrm{~m}), 1014(\mathrm{w}), 988(\mathrm{~m}), 963(\mathrm{~m}), 929(\mathrm{w}), 895(\mathrm{w}), 875(\mathrm{~m}), 848(\mathrm{~m})$, $837(\mathrm{~m}), 818(\mathrm{~m}), 788(\mathrm{~m}), 767(\mathrm{w}), 753(\mathrm{~m}), 735(\mathrm{w}), 705(\mathrm{~m}), 698(\mathrm{~s}), 681(\mathrm{w}), 663(\mathrm{~m}), 642(\mathrm{~m}), 622(\mathrm{w}), 611(\mathrm{w})$, $571(\mathrm{w}), 518(\mathrm{~m}), 506(\mathrm{~m}), 482(\mathrm{~m}), 426(\mathrm{w}), 411(\mathrm{w}), 404(\mathrm{w})$.

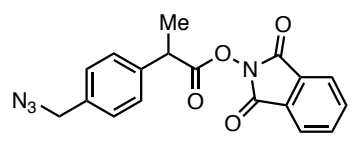

\section{1,3-dioxoisoindolin-2-yl 2-(4-(azidomethyl)phenyl)propanoate (S15)}

Safety note: This procedure results in the production of hydrazoic acid which represents a potential explosion hazard. During workup and acidifying, it is important to use sufficient quantities of water to generate only dilute, aqueous solutions of hydrazoic acid and to be cognizant of the toxicity of $\mathrm{HN}_{3}$.

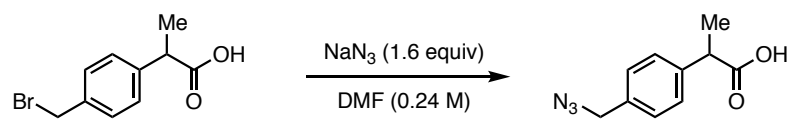

According to Büll, et. al..$^{5}$ a 50-mL oven-dried flask was charged with 2-(4-bromomethyl)phenyl)propionic acid ( $1.4673 \mathrm{~g}, 6 \mathrm{mmol}, 1$ equiv.) and sodium azide ( $0.6129 \mathrm{~g}, 9.4 \mathrm{mmol}, 1.6$ equiv.). The flask was placed under nitrogen and then charged with $25 \mathrm{~mL}$ of DMF. The reaction mixture was overnight at room temperature. After stirring the reaction was decanted to a separatory funnel, diluted with water and then with $1 \mathrm{~N} \mathrm{HCl}$ and extracted 4 times using EtOAc. The combined organics were dried over magnesium sulfate, filtered, and concentrated via rotary evaporation. Purified over silica with flash chromatography (20 to $40 \%$ EtOAc in hexanes) to afford 2-(4(azidomethyl)phenyl)propanoic acid as a slightly yellow solid (1.1951 g, $5.82 \mathrm{mmol}, 96 \%)$. 
${ }^{1}$ H NMR (500 MHz, Chloroform-d): $\delta 7.35(\mathrm{~d}, J=8.2 \mathrm{~Hz}, 2 \mathrm{H}), 7.29(\mathrm{~d}, J=8.2 \mathrm{~Hz}, 2 \mathrm{H}), 4.33(\mathrm{~s}, 2 \mathrm{H}), 3.76(\mathrm{q}, J=$ $7.2 \mathrm{~Hz}, 1 \mathrm{H}), 1.52(\mathrm{~d}, J=7.2 \mathrm{~Hz}, 3 \mathrm{H})$.

${ }^{13}$ C NMR (126 MHz, Chloroform-d): $\delta$ 178.94, 140.04, 134.79, 128.68, 128.25, 54.58, 44.98, 18.28.
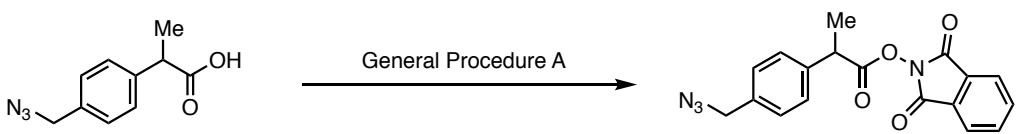

Prepared according to general procedure A with 2-(4-(azidomethyl)phenyl)propanoic acid (1.1951 g, $5.8 \mathrm{mmol}, 1$ equiv.), $N$-hydroxyphthalimide ( $1.4517 \mathrm{~g}, 9 \mathrm{mmol}, 1.5$ equiv.), DMAP (35.8 $\mathrm{mg}, 0.3 \mathrm{mmol}, 0.05$ equiv.) and DIC (1.5 $\mathrm{mL}, 8.4 \mathrm{mmol}, 1.4$ equiv.). Purified over silica with flash chromatography (10 to $20 \%$ EtOAc in hexnaes) to afford the title compound as a white solid (1.8688 g, $5.33 \mathrm{mmol}, 92 \%)$.

${ }^{1}$ H NMR (500 MHz, Chloroform- $d$ ): $\delta 7.87(\mathrm{dd}, J=5.5,3.1 \mathrm{~Hz}, 2 \mathrm{H}), 7.78(\mathrm{dd}, J=5.5,3.1 \mathrm{~Hz}, 2 \mathrm{H}), 7.43(\mathrm{~d}, J=8.2$ $\mathrm{Hz}, 2 \mathrm{H}), 7.36(\mathrm{~d}, J=8.2 \mathrm{~Hz}, 2 \mathrm{H}), 4.36(\mathrm{~s}, 2 \mathrm{H}), 4.14(\mathrm{q}, J=7.2 \mathrm{~Hz}, 1 \mathrm{H}), 1.68(\mathrm{~d}, J=7.1 \mathrm{~Hz}, 3 \mathrm{H})$.

${ }^{13}$ C NMR (126 MHz, Chloroform-d): $\delta$ 170.75, 138.60, 135.22, 134.92, 129.05, 128.86, 128.24, 124.11, 54.57, 42.84, 19.09 .

HRMS: (ESI-TOF) calculated for $\mathrm{C}_{18} \mathrm{H}_{15} \mathrm{~N}_{2} \mathrm{O}_{4}{ }^{+}\left(\left[\mathrm{M}+\mathrm{H}-\mathrm{N}_{2}\right]^{+}\right)$: 323.10263 , found 323.10241 .

FTIR (thin film, cm$^{-1}$ ): $v 2981$ (w), 2123 (w), 2089 (m), 2030 (w), 2017 (w), 1992 (w), 1967 (w), 1943 (w), 1918 (w), 1869 (w), 1844 (w), 1830 (w), 1802 (m), 1781 (m), 1741 (s), 1685 (w), 1653 (w), $1636(w), 1606(w), 1510(w)$, 1467 (w), 1454 (w), 1437 (w), 1419 (w), 1358 (m), 1239 (m), 1213 (w), $1201(\mathrm{w}), 1186(\mathrm{~m}), 1169(\mathrm{~m}), 1124(\mathrm{~m})$, $1103(\mathrm{~m}), 1080(\mathrm{~m}), 1049(\mathrm{~s}), 1021(\mathrm{~m}), 1002(\mathrm{~m}), 985(\mathrm{w}), 957(\mathrm{~m}), 876(\mathrm{~s}), 856(\mathrm{~m}), 824(\mathrm{w}), 788(\mathrm{w}), 774(\mathrm{~m}), 724$ (m), $693(\mathrm{~s}), 610(\mathrm{w}), 588(\mathrm{w}), 570(\mathrm{~m}), 552(\mathrm{w}), 536(\mathrm{w}), 518(\mathrm{~m}), 481(\mathrm{w}), 448(\mathrm{w}), 403(\mathrm{w})$.

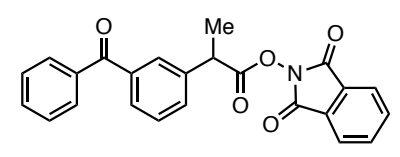

1,3-dioxoisoindolin-2-yl 2-(3-benzoylphenyl)propanoate (S16)

Synthesized according to general procedure A with 2-(3-benzoylphenyl)propanoic acid (1.263 g, $5 \mathrm{mmol}, 1$ equiv.), $N$-hydroxyphthalimide ( $1.179 \mathrm{~g}, 7.5 \mathrm{mmol}, 1.5$ equiv), DMAP (40.3 mg, $0.25 \mathrm{mmol}, 0.05$ equiv.) and DIC (950 uL, 7 mmol, 1.4 equiv.). Purified over silica with flash chromatography (10 to 30\% EtOAc in hexanes) to afford the title compound as a white solid (1.657 g, $4.15 \mathrm{mmol}, 83 \%)$.

1H NMR (500 MHz, Chloroform-d): $\delta 7.88(\mathrm{dd}, J=5.5,3.1 \mathrm{~Hz}, 2 \mathrm{H}), 7.87-7.83(\mathrm{~m}, 3 \mathrm{H}), 7.79(\mathrm{dt}, J=5.5,3.1 \mathrm{~Hz}$, $2 \mathrm{H}), 7.77(\mathrm{dt}, J=7.7,1.4 \mathrm{~Hz}, 1 \mathrm{H}), 7.67(\mathrm{dt}, J=7.7,1.5 \mathrm{~Hz}, 1 \mathrm{H}), 7.60(\mathrm{tt}, 7.5,1.3 \mathrm{~Hz}, 1 \mathrm{H}), 7.53(\mathrm{t}, J=7.8 \mathrm{~Hz}, 1 \mathrm{H})$, $7.50(\mathrm{t}, J=7.7 \mathrm{~Hz}, 2 \mathrm{H}), 4.20(\mathrm{q}, J=7.2 \mathrm{~Hz}, 1 \mathrm{H}), 1.71(\mathrm{~d}, J=7.1 \mathrm{~Hz}, 3 \mathrm{H})$.

${ }^{13}$ C NMR (126 MHz, Chloroform-d): $\delta$ 196.44, 170.57, 138.74, 138.39, 137.47, 134.95, 132.72, 131.67, 130.35, $129.79,129.46,129.13,129.05,128.52,124.13,43.00,19.04$. (1 carbon peak obscured by overlap)

HRMS: (ESI-TOF) calculated for $\mathrm{C}_{24} \mathrm{H}_{18} \mathrm{NO}_{5}{ }^{+}\left([\mathrm{M}+\mathrm{H}]^{+}\right): 400.11795$, found 400.11798 .

FTIR (thin film, cm $\left.^{-1}\right): v 3488(\mathrm{w}), 3382(\mathrm{w}), 3064(\mathrm{w}), 2926(\mathrm{w}), 1811(\mathrm{w}), 1783(\mathrm{~m}), 1735(\mathrm{~s}), 1646(\mathrm{~m}), 1621(\mathrm{~m})$, $1597(\mathrm{~m}), 1488(\mathrm{w}), 1469(\mathrm{~m}), 1447(\mathrm{~m}), 1437(\mathrm{~m}), 1365(\mathrm{~m}), 1350(\mathrm{~m}), 1313(\mathrm{~m}), 1284(\mathrm{~s}), 1258(\mathrm{~m}), 1232(\mathrm{~m})$, 1185 (s), 1122 (s), 1080 (s), 1045 (s), 1014 (m), 993 (s), 971 (s), 954 (s), 924 (m), 876 (s), 853 (s), 820 (s), 804 (m), $792(\mathrm{~m}), 783(\mathrm{~m}), 777(\mathrm{~m}), 753(\mathrm{~m}), 722(\mathrm{~m}), 709(\mathrm{~s}), 694(\mathrm{~s}), 687(\mathrm{~s})$.

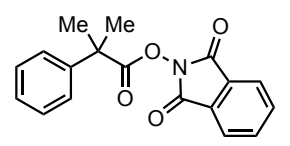

1,3-dioxoisoindolin-2-yl 2-methyl-2-phenylpropanoate (S17)

Prepared according to general procedure A with 2-phenylisobutyric acid (1.6978 g, $10.4 \mathrm{mmol}, 1$ equiv.), $\mathrm{N}$ hydroxyphthalimide ( $2.380 \mathrm{~g}, 15 \mathrm{mmol}, 1.5$ equiv.), DMAP (66.3 mg, $0.5 \mathrm{mmol}, 0.05$ equiv.) and DIC (2.0 mL, 14 mmol, 1.4 equiv.). Purified over silica with flash chromatography (10 to 30\% EtOAc in hexanes) to afford the title compound (2.7783 g, $8.98 \mathrm{mmol}, 87 \%)$ 
1H NMR (500 MHz, Chloroform- $d$ ): $\delta 7.87(\mathrm{dd}, J=5.5,3.1 \mathrm{~Hz}, 1 \mathrm{H}), 7.78(\mathrm{dd}, J=5.5,3.1 \mathrm{~Hz}, 1 \mathrm{H}), 7.51-7.49$ (m, $1 \mathrm{H}), 7.42(\mathrm{dd}, J=8.6,7.0 \mathrm{~Hz}, 1 \mathrm{H}), 7.34-7.30(\mathrm{~m}, 1 \mathrm{H}), 1.79(\mathrm{~s}, 6 \mathrm{H})$.

${ }^{13}$ C NMR (126 MHz, Chloroform-d): $\delta$ 173.37, 162.10, 142.71, 134.83, 129.15, 128.81, 127.57, 125.93, 124.02 , 46.49, 26.99 .

HRMS: (ESI-TOF) calculated for $\mathrm{C}_{18} \mathrm{H}_{16} \mathrm{NO}_{4}{ }^{+}\left([\mathrm{M}+\mathrm{H}]^{+}\right)$: 310.10738 , found 310.1075 .

FTIR (thin film, cm$\left.^{-1}\right): v 3060(\mathrm{w}), 3006(\mathrm{w}), 2977$ (w), 2934 (w), $1803(\mathrm{w}), 1772(\mathrm{~s}), 1740$ (s), $1599(\mathrm{w}), 1494(\mathrm{w})$, $1469(\mathrm{~m}), 1447$ (w), 1392 (w), 1369 (m), 1358 (m), 1332 (w), 1295 (w), $1234(\mathrm{w}), 1220(\mathrm{w}), 1187(\mathrm{~s}), 1159(\mathrm{w}), 1132$ (m), $1109(\mathrm{~m}), 1081(\mathrm{w}), 1055(\mathrm{~s}), 1030(\mathrm{~m}), 1017(\mathrm{~m}), 1008(\mathrm{~s}), 965$ (s), $947(\mathrm{~m}), 931(\mathrm{~m}), 915(\mathrm{w}), 874(\mathrm{~s}), 850(\mathrm{~s})$, $788(\mathrm{~s}), 775(\mathrm{~s}), 725(\mathrm{~s}), 696(\mathrm{~s}), 599(\mathrm{~s}), 543(\mathrm{~m}), 517(\mathrm{~s}), 484(\mathrm{~m}), 419(\mathrm{~m})$.

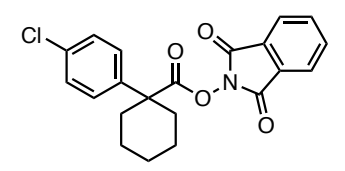

1,3-dioxoisoindolin-2-yl 1-(4-chlorophenyl)cyclohexane-1-carboxylate (S18)

Prepared according to general procedure A with 1-(4-chlorophenyl)cyclohexane-1-carboxylic acid (1.1887 g, 5.00 mmol, 1 equiv.), $N$-hydroxyphthalimide (1.2407 g, $7.50 \mathrm{mmol}, 1.5$ equiv.), DMAP (30.7 mg, $0.25 \mathrm{mmol}, 0.05$ equiv.) and DIC (1.1 mL, $7 \mathrm{mmol}, 1.4$ equiv.). Purified over silica with flash chromatography (5 to $25 \%$ EtOAc in hexanes) to afford the title compound $(1.7094 \mathrm{~g}, 4.45 \mathrm{mmol}, 89 \%)$.

1H NMR (500 MHz, Chloroform- $d$ ): $\delta 7.86(\mathrm{dd}, J=5.6,3.1 \mathrm{~Hz}, 2 \mathrm{H}), 7.78(\mathrm{dd}, J=5.6,3.1 \mathrm{~Hz}, 2 \mathrm{H}), 7.46(\mathrm{~d}, J=8.1$ $\mathrm{Hz}, 2 \mathrm{H}), 7.39(\mathrm{~d}, J=8.4 \mathrm{~Hz}, 2 \mathrm{H}), 2.61(\mathrm{~d}, J=12.9 \mathrm{~Hz}, 2 \mathrm{H}), 1.79(\mathrm{~m}, 7 \mathrm{H}), 1.33(\mathrm{~d}, J=12.9 \mathrm{~Hz}, 1 \mathrm{H})$.

${ }^{13}$ C NMR (126 MHz, Chloroform-d): $\delta$ 171.47, 162.17, 140.77, 134.88, 133.61, 129.13, 129.02, 127.64, 124.03, $50.83,35.40,25.44,23.48$.

HRMS: (ESI-TOF) calculated for $\mathrm{C}_{21} \mathrm{H}_{19} \mathrm{Cl}^{35} \mathrm{NO}_{4}{ }^{+}\left([\mathrm{M}+\mathrm{H}]^{+}\right)$: 384.09971, found 384.09910.

(ESI-TOF) calculated for $\mathrm{C}_{21} \mathrm{H}_{19} \mathrm{Cl}^{37} \mathrm{NO}_{4}{ }^{+}\left([\mathrm{M}+\mathrm{H}]^{+}\right): 386.09763$, found 386.09678 .

FTIR (thin film, cm-1): $v 2932(\mathrm{w}), 2864(\mathrm{w}), 1806(\mathrm{w}), 1783(\mathrm{~m}), 1740(\mathrm{~s}), 1591(\mathrm{w}), 1492(\mathrm{~m}), 1467(\mathrm{~m}), 1457$ (w), $1402(\mathrm{w}), 1373(\mathrm{~m}), 1356(\mathrm{~m}), 1282(\mathrm{w}), 1186(\mathrm{~m}), 1169(\mathrm{~m}), 1125(\mathrm{~m}), 1092(\mathrm{~m}), 1074(\mathrm{~m}), 1057(\mathrm{~s}), 1033(\mathrm{~s})$, $1009(\mathrm{~m}), 971(\mathrm{~s}), 924(\mathrm{~m}), 908(\mathrm{~m}), 897(\mathrm{~m}), 874(\mathrm{~s}), 845(\mathrm{~m}), 827(\mathrm{~s}), 787(\mathrm{~m}), 745(\mathrm{~m}), 717(\mathrm{~m}), 697(\mathrm{~s}), 611(\mathrm{w})$, $571(\mathrm{w}), 538(\mathrm{w}), 519(\mathrm{~s}), 461(\mathrm{w}), 443(\mathrm{~m})$.

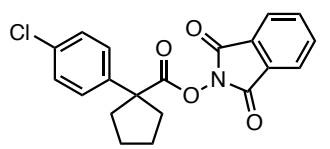

1,3-dioxoisoindolin-2-yl 1-(4-chlorophenyl)cyclopentane-1-carboxylate (S19)

Prepared according to general procedure A with 1-(4-chlorophenyl)cyclopentane-1-carboxylic acid (1.1270 g, 5 mmol, 1 equiv.), $N$-hydroxyphthalimide (1.2052 g, $7.5 \mathrm{mmol}, 1.5$ equiv.), DMAP (32.2 mg, $0.25 \mathrm{mmol}, 0.05$ equiv.) and DIC (1.1 mL, $7 \mathrm{mmol}, 1.4$ equiv.). Purified over silica with flash chromatography (5 to $25 \%$ EtOAc in hexanes) to afford the title compound $(1.7766 \mathrm{~g}, 4.80 \mathrm{mmol}, 96 \%)$.

1H NMR (500 MHz, Chloroform- $d$ ): $\delta \delta 7.85(\mathrm{dd}, J=5.5,3.1 \mathrm{~Hz}, 2 \mathrm{H}), 7.77(\mathrm{dd}, J=5.5,3.0 \mathrm{~Hz}, 2 \mathrm{H}), 7.42(\mathrm{~d}, J=$ $8.7 \mathrm{~Hz}, 2 \mathrm{H}), 7.37(\mathrm{~d}, J=8.7 \mathrm{~Hz}, 2 \mathrm{H}), 2.80-2.74(\mathrm{~m}, 2 \mathrm{H}), 2.12-2.05(\mathrm{~m}, 2 \mathrm{H}), 1.97-1.81(\mathrm{~m}, 4 \mathrm{H})$.

${ }^{13}$ C NMR (126 MHz, Chloroform-d): $\delta$ 172.35, 162.09, 139.77, 134.87, 133.55, 129.10, 128.91, 128.40, 124.02, $57.68,37.25,23.89$.

HRMS: (ESI-TOF) calculated for $\mathrm{C}_{20} \mathrm{H}_{16} \mathrm{Cl}^{35} \mathrm{NNaO}_{4}{ }^{+}\left([\mathrm{M}+\mathrm{Na}]^{+}\right): 392.06601$, found 392.06581 .

(ESI-TOF) calculated for $\mathrm{C}_{20} \mathrm{H}_{16} \mathrm{Cl}^{37} \mathrm{NNaO}_{4}{ }^{+}\left([\mathrm{M}+\mathrm{Na}]^{+}\right)$: 394.06387, found 394.06230 .

FTIR (thin film, $\left.\mathbf{c m}^{-1}\right): v 2977$ (w), 2875 (w), 1803 (m), 1781 (m), 1737 (s), 1609 (w), 1490 (m), 1466 (m), 1453 (m), $1402(\mathrm{w}), 1361(\mathrm{~m}), 1300(\mathrm{w}), 1186(\mathrm{~m}), 1170(\mathrm{w}), 1133(\mathrm{~m}), 1104(\mathrm{w}), 1068(\mathrm{~s}), 1031(\mathrm{~m}), 1014(\mathrm{~m}), 982(\mathrm{~s})$, $966(\mathrm{~m}), 952(\mathrm{~m}), 895(\mathrm{w}), 874(\mathrm{~s}), 829(\mathrm{~s}), 785(\mathrm{~s}), 749(\mathrm{~m}), 717(\mathrm{~m}), 696(\mathrm{~s}), 632(\mathrm{w}), 607(\mathrm{~m}), 584(\mathrm{~m}), 515(\mathrm{~s})$, 481 (m), 441 (m), 404 (m).

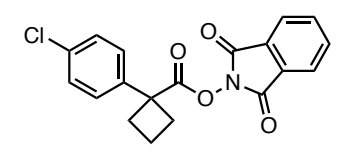




\section{1,3-dioxoisoindolin-2-yl 1-(4-chlorophenyl)cyclobutane-1-carboxylate (S20)}

Prepared according to general procedure A with 1-(4-chlorophenyl)cyclobutane-1-carboxylic acid (1.0394 g, $5 \mathrm{mmol}$, 1 equiv.), $N$-hydroxyphthalimide ( $1.2175 \mathrm{~g}, 7.5 \mathrm{mmol}, 1.5$ equiv.), DMAP (31.4 mg, $0.25 \mathrm{mmol}, 0.05$ equiv.) and DIC ( $1.1 \mathrm{~mL}, 7 \mathrm{mmol}, 1.4$ equiv.). Purified over silica with flash chromatography (10 to $25 \%$ EtOAc in hexanes) to afford the title compound (1.6953 g, $4.77 \mathrm{mmol}, 97 \%)$.

1H NMR (500 MHz, Chloroform- $d$ ): $\delta 7.86(\mathrm{dd}, \mathrm{J}=5.5,3.1 \mathrm{~Hz}, 2 \mathrm{H}), 7.77(\mathrm{dd}, \mathrm{J}=5.5,3.1 \mathrm{~Hz}, 2 \mathrm{H}), 7.40-7.35$ (m, 4H), 3.06 (tdd, J = 9.0, 6.2, 3.8 Hz, 2H), 2.65 (tdd, J = 9.3, 7.8, $2.8 \mathrm{~Hz}, 2 \mathrm{H}$ ), 2.26 (dddd, J = 16.8, 11.5, 9.1, 7.8 Hz, $1 \mathrm{H}), 2.02(\mathrm{dtt}, \mathrm{J}=11.6,9.2,5.2 \mathrm{~Hz}, 1 \mathrm{H})$.

${ }^{13}$ C NMR (126 MHz, Chloroform-d): $\delta$ 171.96, 162.05, 139.80, 134.88, 133.51, 129.11, 128.97, 128.04, 124.05, $50.61,32.81,16.86$

HRMS: (ESI-TOF) calculated for $\mathrm{C}_{19} \mathrm{H}_{14} \mathrm{Cl}^{35} \mathrm{NNaO}_{4}{ }^{+}\left([\mathrm{M}+\mathrm{Na}]^{+}\right)$: 378.05036, found 378.05014.

(ESI-TOF) calculated for $\mathrm{C}_{19} \mathrm{H}_{14} \mathrm{Cl}^{37} \mathrm{NNaO}_{4}{ }^{+}\left([\mathrm{M}+\mathrm{Na}]^{+}\right): 380.04816$, found 380.04645 .

FTIR (thin film, $\left.\mathbf{c m}^{-1}\right): v 2947$ (w), $1802(\mathrm{w}), 1773$ (s), 1737 (s), $1492(\mathrm{~m}), 1466(\mathrm{~m}), 1400$ (w), 1367 (m), 1247 (w), $1184(\mathrm{~m}), 1169(\mathrm{~s}), 1125(\mathrm{~m}), 1112(\mathrm{~m}), 1093(\mathrm{~m}), 1074(\mathrm{~s}), 1053(\mathrm{~m}), 1021(\mathrm{~s}), 1010(\mathrm{~s}), 959$ (s), $874(\mathrm{~s}), 833(\mathrm{~s})$, $776(\mathrm{~m}), 719(\mathrm{~m}), 709(\mathrm{~m}), 692(\mathrm{~s}), 598(\mathrm{~m}), 536(\mathrm{~m}), 516(\mathrm{~s}), 493(\mathrm{~m}), 435(\mathrm{~m}), 407(\mathrm{~m})$.

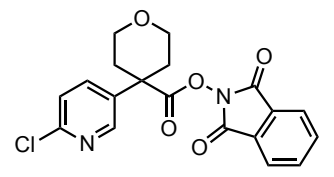

\section{1,3-dioxoisoindolin-2-yl4-(6-chloropyridin-3-yl)tetrahydro-2H-pyran-4-carboxylate (S21)}<smiles>O=C(O)Cc1ccc(Cl)nc1</smiles>

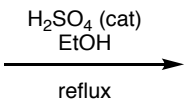<smiles>CCOC(=O)Cc1ccc(Cl)nc1</smiles>

To a 100-mL flask with a stir bar was added 6-chloro-3-pyridineacetic acid (1.127 g, 6.6 mmol, 1 equiv). Then, 15 $\mathrm{mL}$ ethanol and $1.6 \mathrm{~mL}$ sulfuric acid were added. A reflux condenser was then attached to the flask and the flask was heated in an oil bath at $95^{\circ} \mathrm{C}$ until reflux was observed. The reaction was left stirring for 16 hours overnight, then cooled to room temperature. Then the reaction mixture was poured into a separatory funnel, diluted with $30 \mathrm{~mL}$ diethyl ether and washed 2 times with $\mathrm{NaHCO}_{3}(2 \times 15 \mathrm{~mL})$. The organic layer was then washed with brine and dried over sodium sulfate. Concentration under vacuum afforded a brown oil which was purified over silica with flash chromatography (15 to 30\% EtOAc in hexanes) to afford ethyl 2-(6-chloropyridin-3-yl)acetate (0.999 g, $5.00 \mathrm{mmol}$, $76 \%)$ as a colorless oil.

${ }^{1}$ H NMR (500 MHz, Chloroform- $d$ ): $\delta 8.29(\mathrm{dd}, J=2.5,0.8 \mathrm{~Hz}, 1 \mathrm{H}), 7.62(\mathrm{dd}, J=8.2,2.5 \mathrm{~Hz}, 1 \mathrm{H}), 7.30(\mathrm{dd}, J=$ $8.2,0.7 \mathrm{~Hz}, 1 \mathrm{H}), 4.16(\mathrm{q}, J=7.1 \mathrm{~Hz}, 2 \mathrm{H}), 3.60(\mathrm{~s}, 2 \mathrm{H}), 1.26(\mathrm{t}, J=7.1 \mathrm{~Hz}, 3 \mathrm{H})$.

${ }^{13}$ C NMR (126 MHz, Chloroform- $d$ ): $\delta$ 170.39, 150.47, 150.23, 139.83, 128.88, 124.22, 61.53, 37.77, 14.26.

HRMS: (EI) calculated for $\mathrm{C}_{9} \mathrm{H}_{10} \mathrm{ClNO}_{2}{ }^{+}\left(\left[\mathrm{M}^{*+}\right]\right): 199.03946$, found 199.03954 .

FTIR (thin film, cm$\left.^{-1}\right): v 2982(\mathrm{w}), 1729(\mathrm{~s}), 1588(\mathrm{w}), 1567(\mathrm{w}), 1460(\mathrm{~s}), 1417(\mathrm{w}), 1385(\mathrm{~m}), 1368(\mathrm{w}), 1334(\mathrm{w})$, $1290(\mathrm{w}), 1229(\mathrm{~m}), 1159(\mathrm{~s}), 1139(\mathrm{~s}), 1101(\mathrm{~s}), 1023(\mathrm{~s}), 945(\mathrm{w}), 879(\mathrm{w}), 818(\mathrm{~m}), 747(\mathrm{w}), 671(\mathrm{w}), 632(\mathrm{w}), 579$ (w), $506(\mathrm{w}), 453(\mathrm{w}), 414(\mathrm{w})$.<smiles>CCOC(=O)Cc1ccc(Cl)nc1</smiles>
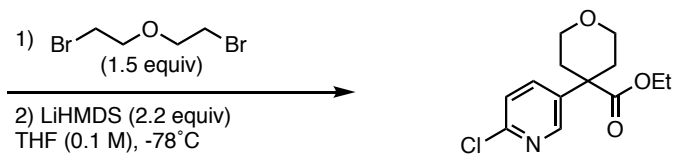

To a flame-dried 100-mL flask with a stir bar under $\mathrm{N}_{2}$ was added LiHMDS (11.0 mL of a $1.0 \mathrm{M}$ solution in THF, $11.0 \mathrm{mmol}, 2.2$ equiv.). The flask was placed into a dry ice/acetone bath and $30 \mathrm{~mL}$ of THF was added. In a separate vial with a septa cap under $\mathrm{N}_{2}$ was added ethyl 2-(6-chloropyridin-3-yl)acetate $(0.917 \mathrm{~g}, 5 \mathrm{mmol}, 1 \mathrm{equiv}$.) and bis(2bromoethyl)ether (910 uL, $7.5 \mathrm{mmol}, 1.5$ equiv.). These were dissolved in $5 \mathrm{~mL}$ of anhydrous THF and added dropwise to the LiHMDS solution over 20 minutes with vigorous stirring. The vial was rinsed with an additional 10 $\mathrm{mL}$ of THF and the yellow reaction was allowed to stir and warm up to room temperature overnight. The red reaction mixture was poured into a separatory funnel and quenched with saturated $\mathrm{NH}_{4} \mathrm{Cl}$. The aqueous layer was extracted with EtOAc $(3 \times 20 \mathrm{~mL})$ and the combined organic layers were rinsed with brine, dried over $\mathrm{Na}_{2} \mathrm{SO}_{4}$ and concentrated 
under vacuum. The crude oil was purified over silica with flash chromatography (40 to $80 \%$ EtOAc in hexanes) to afford a light yellow oil (1.1805 g, $4.38 \mathrm{mmol}, 95 \%$ yield).

1H NMR (500 MHz, Chloroform- $d$ ): $\delta 8.41(\mathrm{~d}, J=2.7 \mathrm{~Hz}, 1 \mathrm{H}), 7.65(\mathrm{dd}, J=8.5,2.7 \mathrm{~Hz}, 1 \mathrm{H}), 7.31(\mathrm{dd}, J=8.4,0.7$ $\mathrm{Hz}, 1 \mathrm{H}), 4.16(\mathrm{q}, J=7.1 \mathrm{~Hz}, 2 \mathrm{H}), 3.94(\mathrm{dt}, J=12.1,3.8 \mathrm{~Hz}, 2 \mathrm{H}), 3.56(\mathrm{td}, J=11.7,2.0 \mathrm{~Hz}, 2 \mathrm{H}), 2.53(\mathrm{dd}, J=13.7$, $2.6 \mathrm{~Hz}, 2 \mathrm{H}), 1.95(\mathrm{ddd}, J=13.4,11.2,4.4 \mathrm{~Hz}, 2 \mathrm{H}), 1.20(\mathrm{t}, J=7.1 \mathrm{~Hz}, 3 \mathrm{H})$.

HRMS: (ESI-TOF) calculated for $\mathrm{C}_{13} \mathrm{H}_{16} \mathrm{ClNO}^{+}\left([\mathrm{M}+\mathrm{H}]^{+}\right): 270.08915$, found 270.0892 .

FTIR (thin film, cm$\left.^{-1}\right): v 2962(\mathrm{w}), 2934(\mathrm{w}), 2854(\mathrm{w}), 1724(\mathrm{~s}), 1581(\mathrm{w}), 1562(\mathrm{w}), 1463(\mathrm{~m}), 1389(\mathrm{w}), 1370(\mathrm{w})$, $1294(\mathrm{~m}), 1220(\mathrm{~m}), 1195(\mathrm{~s}), 1129(\mathrm{~s}), 1103$ (s), 1047 (m), 1019 (s), 947 (w), 915 (w), 860 (w), $836(\mathrm{w}), 813(\mathrm{w})$, $768(\mathrm{w}), 742(\mathrm{~m}), 633(\mathrm{w}), 558(\mathrm{w}), 450(\mathrm{w}), 412(\mathrm{w})$.
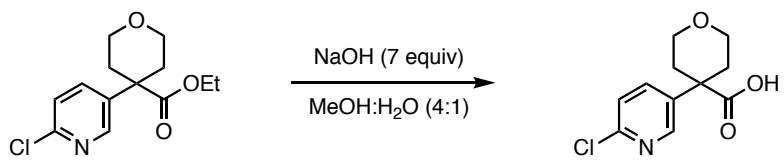

To an oven-dried 50-mL round bottom flask with a stir bar was added ethyl 4-(6-chloropyridin-3-yl)tetrahydro- $2 \mathrm{H}$ pyran-4-carboxylate $(1.179 \mathrm{~g}, 4.4 \mathrm{mmol}, 1$ equiv.) and $20 \mathrm{~mL}$ methanol. The mixture was stirred and $5 \mathrm{~mL}$ of a $6 \mathrm{M}$ $\mathrm{NaOH}$ solution was added in one portion. Then, $1 \mathrm{~mL}$ water was added, and the reaction was stirred and monitored by TLC. After 16 hours, the reaction was concentrated under reduced pressure to a crude oil and then acidified with 6 $\mathrm{M} \mathrm{HCl}$ until the mixture was $\mathrm{pH} 1$. The mixture was then transferred to a separatory funnel and diluted with ether. The aqueous layer was washed with ether $(2 \times 50 \mathrm{~mL})$ and the combined organic layers were washed with water and brine and dried over $\mathrm{Na}_{2} \mathrm{SO}_{4}$. The ether was then evaporated under reduced pressure to yield a crude white solid which was purified by column chromatography (30 to $60 \%$ EtOAc in hexanes) to afford the title compound as a white solid (0.8031 g, $3.32 \mathrm{mmol}, 76 \%)$.

1H NMR (500 MHz, Chloroform- $d$ ): $\delta 8.49(\mathrm{dd}, J=2.7,0.8 \mathrm{~Hz}, 1 \mathrm{H}), 7.73(\mathrm{dd}, J=8.5,2.7 \mathrm{~Hz}, 1 \mathrm{H}), 7.37(\mathrm{dd}, J=$ $8.4,0.7 \mathrm{~Hz}, 1 \mathrm{H}), 3.98(\mathrm{~d}, J=12.1 \mathrm{~Hz}, 2 \mathrm{H}), 3.66(\mathrm{ddd}, J=12.3,11.1,2.1 \mathrm{~Hz}, 2 \mathrm{H}), 2.58(\mathrm{dd}, J=13.7,2.7 \mathrm{~Hz}, 1 \mathrm{H})$, 2.02 (ddd, $J=13.5,11.1,4.3 \mathrm{~Hz}, 2 \mathrm{H})$.

${ }^{13}$ C NMR (126 MHz, Chloroform- $d$ ): $\delta$ 176.72, 150.94, 147.88, 136.85, 136.55, 124.51, 65.31, 46.87, 33.81 .

HRMS: (ESI-TOF) calculated for $\mathrm{C}_{11} \mathrm{H}_{12} \mathrm{ClNO}_{3}{ }^{+}\left([\mathrm{M}+\mathrm{H}]^{+}\right)$: 242.05785 , found 242.0577 .

FTIR (thin film, cm$^{-1}$ ): $v 2967$ (w), 2923 (w), 2847 (w), 2629 (w), 1688 (m), 1579 (w), 1565 (w), 1460 (w), 1443 (w), $1428(\mathrm{w}), 1401(\mathrm{w}), 1392(\mathrm{w}), 1374(\mathrm{w}), 1351(\mathrm{w}), 1298(\mathrm{~m}), 1260(\mathrm{w}), 1246(\mathrm{~m}), 1236(\mathrm{~m}), 1203(\mathrm{~m}), 1160(\mathrm{w})$, $1136(\mathrm{~m}), 1101(\mathrm{~s}), 1047(\mathrm{w}), 1036(\mathrm{w}), 1020(\mathrm{~m}), 954(\mathrm{w}), 938(\mathrm{w}), 911(\mathrm{~m}), 895(\mathrm{~m}), 850(\mathrm{w}), 827(\mathrm{~m}), 772(\mathrm{~m})$, $759(\mathrm{w}), 739(\mathrm{~m}), 687(\mathrm{w}), 632(\mathrm{w}), 582(\mathrm{w}), 551(\mathrm{~m}), 511(\mathrm{w}), 466(\mathrm{w}), 448(\mathrm{~m}), 413(\mathrm{~m})$.
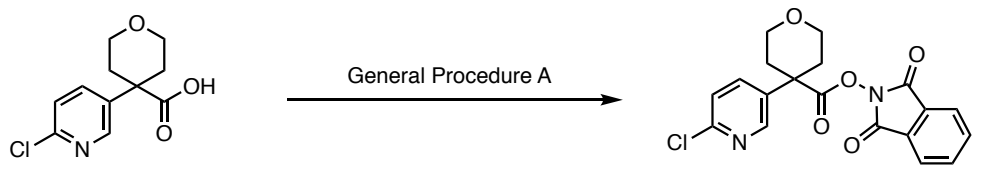

Synthesized according to general procedure A with 4-(6-chloropyridin-3-yl)tetrahydro-2H-pyran-4-carboxylic acid (0.738 g, 3 mmol, 1 equiv.), $N$-hydroxyphthalimide ( $743.7 \mathrm{mg}, 4.5 \mathrm{mmol}, 1.5$ equiv.), DMAP (32.1 mg, $0.15 \mathrm{mmol}$, 0.05 equiv) and DIC ( $650 \mathrm{uL}, 4.2 \mathrm{mmol}, 1.4$ equiv). Purified over silica with flash chromatography (15 to $60 \%$ EtOAc in hexanes) to afford the title compound as a white solid (0.2774 g, $0.717 \mathrm{mmol}, 23 \%)$

${ }^{1}$ H NMR (400 MHz, Chloroform- $d$ ): $\delta 8.55(\mathrm{dd}, J=2.8,0.8 \mathrm{~Hz}, 1 \mathrm{H}), 7.88(\mathrm{dd}, J=5.5,3.1 \mathrm{~Hz}, 2 \mathrm{H}), 7.85-7.77$ (m, $3 \mathrm{H}), 7.42(\mathrm{dd}, J=8.5,0.8 \mathrm{~Hz}, 1 \mathrm{H}), 4.07(\mathrm{ddd}, J=12.3,4.4,2.7 \mathrm{~Hz}, 2 \mathrm{H}), 3.83(\mathrm{td}, J=11.8,1.9 \mathrm{~Hz}, 1 \mathrm{H}), 2.65(\mathrm{dd}, J$ $=13.7,2.3 \mathrm{~Hz}, 2 \mathrm{H}), 2.18$ (ddd, $J=13.6,11.5,4.5 \mathrm{~Hz}, 2 \mathrm{H})$.

${ }^{13}$ C NMR (126 MHz, Chloroform-d): $\delta$ 170.19, 161.84, 151.42, 147.52, 136.94, 135.64, 135.13, 128.92, 124.67 , 124.25, 77.16, 65.14, 47.46, 34.61.

HRMS: (ESI-TOF) calculated for $\mathrm{C}_{19} \mathrm{H}_{16} \mathrm{ClNO}_{5}{ }^{+}\left([\mathrm{M}+\mathrm{H}]^{+}\right)$: 387.07423 , found 387.0747 .

FTIR (thin film, cm$\left.^{-1}\right): v 3057(\mathrm{w}), 2967$ (w), 2934 (w), $2864(\mathrm{w}), 1801(\mathrm{w}), 1775(\mathrm{~m}), 1737(\mathrm{~s}), 1605(\mathrm{w}), 1584(\mathrm{w})$, $1563(\mathrm{w}), 1469(\mathrm{~m}), 1436(\mathrm{w}), 1428(\mathrm{w}), 1392(\mathrm{w}), 1353(\mathrm{~m}), 1328(\mathrm{w}), 1294(\mathrm{w}), 1245(\mathrm{w}), 1222(\mathrm{w}), 1185(\mathrm{~m})$, 1154 (w), 1141 (m), 1104 (s), 1073 (s), 1029 (s), 1017 (s), $963(\mathrm{~m}), 930(\mathrm{~m}), 914(\mathrm{w}), 889(\mathrm{w}), 873(\mathrm{~m}), 850(\mathrm{~m}), 841$ $(\mathrm{s}), 812(\mathrm{w}), 784(\mathrm{~m}), 755(\mathrm{~s}), 739(\mathrm{~m}), 695(\mathrm{~s}), 635(\mathrm{~m}), 607(\mathrm{~m}), 582(\mathrm{~m}), 552(\mathrm{~m}), 536(\mathrm{w}), 516(\mathrm{~s}), 478(\mathrm{w}), 449$ (m), $436(\mathrm{~m}), 412(\mathrm{~m}), 403(\mathrm{~m})$. 


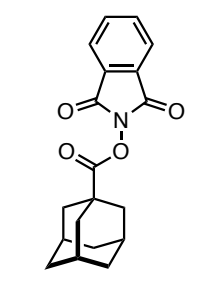

1,3-dioxoisoindolin-2-yl-adamantane-1-carboxylate (S22)

Synthesized according to general procedure A with 1-adamantanecarboxylic acid ( $0.9711 \mathrm{~g}, 5.3 \mathrm{mmol}, 1$ equiv.), $\mathrm{N}$ hydroxyphthalimide ( $1.230 \mathrm{~g}, 7.5 \mathrm{mmol}, 1.5$ equiv), DMAP $(45.7 \mathrm{mg}, 0.3 \mathrm{mmol}, 0.05$ equiv) and DIC (1.1 mL, 8.4 mmol, 1.4 equiv). Purified over silica with flash chromatography (10 to 30\% EtOAc in hexanes) to afford the title compound as a white solid (1.4662 $\mathrm{g}, 4.51 \mathrm{mmol}, 84 \%)$.

1H NMR (500 MHz, Chloroform- $d$ ): $\delta 7.88(\mathrm{dd}, J=5.5,3.1 \mathrm{~Hz}, 2 \mathrm{H}), 7.78(\mathrm{dd}, J=5.5,3.1 \mathrm{~Hz}, 2 \mathrm{H}), 2.14(\mathrm{~d}, J=3.0$ $\mathrm{Hz}, 6 \mathrm{H}), 2.12-2.09(\mathrm{t}, J=3.1 \mathrm{~Hz} 3 \mathrm{H}), 1.78(\mathrm{t}, J=3.1 \mathrm{~Hz}, 6 \mathrm{H})$.

13C NMR (126 MHz, Chloroform-d): $\delta$ 173.41, 162.31, 134.76, 129.21, 123.99, 40.65, 38.58, 36.33, 27.77.

This matches previous literature characterization. ${ }^{6}$

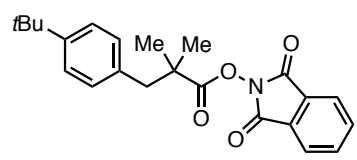

1,3-dioxoisoindolin-2-yl 3-(4-(tert-butyl)phenyl)-2,2-dimethylpropanoate (S23)

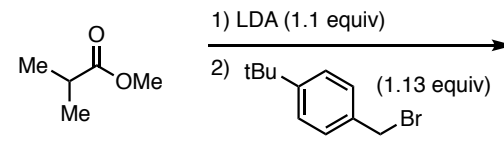

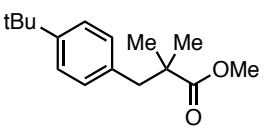

To an oven-dried 50-mL flask under nitrogen was added diisopropylamine ( $1.25 \mathrm{~mL}, 9 \mathrm{mmol}, 1.1$ equiv.) and $10 \mathrm{~mL}$ of THF. The flask was cooled to $-78{ }^{\circ} \mathrm{C}$ and then charged with $n$-BuLi in hexanes $(3.35 \mathrm{~mL}$ of a $2.5 \mathrm{M}$ solution, 8.4 mmol, 1.05 equiv.). The reaction mixture was stirred for 5 minutes to generate LDA. Subsequently, methyl isobutyrate $(0.92 \mathrm{~mL}, 8 \mathrm{mmol}, 1$ equiv.) was added via syringe. The reaction mixture was stirred for 20 minutes to generate the enolate at $-78^{\circ} \mathrm{C}$. 1 -(bromomethyl)-4-(tert-butyl)benzene $(1.65 \mathrm{~mL}, 9 \mathrm{mmol}, 1.13$ equiv) was added in $10 \mathrm{~mL}$ of THF. The reaction mixture was stirred overnight and allowed to warm to room temperature. After quenching with saturated ammonium chloride, the reaction mixture was extracted 4 times with ethyl acetate. The combined organics were dried over sodium sulfate and concentrated via rotary evaporation resulting in a yellow, oily residue. The resulting ester was carried on without further characterization.

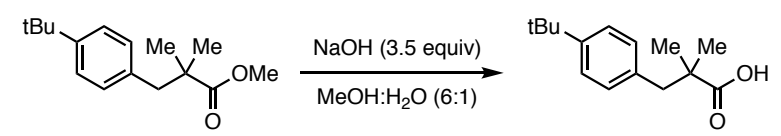

To the flask containing the crude methyl 3-(4-(tert-butyl)phenyl)-2,2-dimethylpropanoate was added sodium hydroxide $(1.1 \mathrm{~g}, 27.5 \mathrm{mmol}, 3.43$ equiv.) as well as $5 \mathrm{~mL}$ of water and $30 \mathrm{~mL}$ of $\mathrm{MeOH}$. The reaction mixture was stirred for $24 \mathrm{~h}$ at room temperature. Afterwards the reaction mixture was washed with ether and the ether wash was discarded. The reaction mixture was acidified to $\mathrm{pH} 2$ using conc. $\mathrm{HCl}$. The reaction mixture was then extracted 3 times using ether. The organics were combined, dried over sodium sulfate, and concentrated via rotary evaporation to yield 3-(4-(tert-butyl)phenyl)-2,2-dimethylpropanoic acid as a white solid (1.4434 g, $6.16 \mathrm{mmol}, 77 \%$ over 2 steps).

${ }^{1}$ H NMR (500 MHz, Chloroform- $d$ ): $\delta 7.29$ (d, $\left.J=7.5 \mathrm{~Hz}, 2 \mathrm{H}\right), 7.10(\mathrm{~d}, J=7.8 \mathrm{~Hz}, 2 \mathrm{H}), 2.87$ (s, 2H), 1.31 (s, 9H), $1.21(\mathrm{~s}, 6 \mathrm{H})$.

${ }^{13}$ C NMR (126 MHz, Chloroform- $d$ ): $\delta 183.58,149.42,134.57,130.08,125.07,45.43,43.49,34.52,31.53,24.83$.
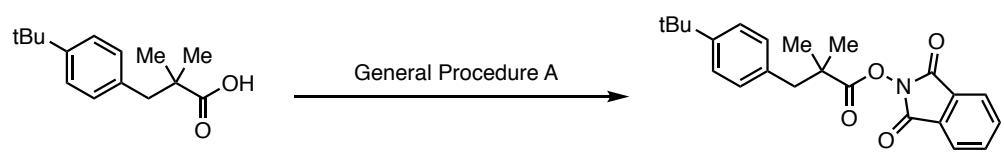
Synthesized according to general procedure A with 3-(4-(tert-butyl)phenyl)-2,2-dimethylpropanoic acid (1.4434 g, 6 mmol, 1 equiv.), $N$-hydroxyphthalimide ( $1.5108 \mathrm{~g}, 9.3 \mathrm{mmol}, 1.5$ equiv.), DMAP (42.2 mg, $0.3 \mathrm{mmol}, 0.05$ equiv.) and DIC (1.35 mL, $8.4 \mathrm{mmol}, 1.4$ equiv.). Purified over silica with flash chromatography (15 to $25 \%$ EtOAc in hexanes) to afford the title compound as a white solid (2.1696 g, $5.72 \mathrm{mmol}, 93 \%)$.

1H NMR (500 MHz, Chloroform- $d$ ): $\delta 7.90(\mathrm{dd}, J=5.5,3.1 \mathrm{~Hz}, 2 \mathrm{H}), 7.80(\mathrm{dd}, J=5.5,3.1 \mathrm{~Hz}, 2 \mathrm{H}), 7.34(\mathrm{~d}, J=8.3$ $\mathrm{Hz}, 2 \mathrm{H}), 7.24(\mathrm{~d}, J=8.2 \mathrm{~Hz}, 2 \mathrm{H}), 3.06(\mathrm{~s}, 2 \mathrm{H}), 1.38(\mathrm{~s}, 6 \mathrm{H}), 1.31(\mathrm{~s}, 9 \mathrm{H})$.

${ }^{13}$ C NMR (126 MHz, Chloroform-d): $\delta$ 173.94, 162.26, 149.71, 134.83, 133.52, 130.25, 129.21, 125.25, 124.04, 45.23, 43.28, 34.57, 31.53, 25.01 .

HRMS: (ESI-TOF) calculated for $\mathrm{C}_{23} \mathrm{H}_{26} \mathrm{NO}_{4}{ }^{+}\left([\mathrm{M}+\mathrm{H}]^{+}\right): 380.18563$, found 380.18575 .

FTIR (thin film, $\left.\mathbf{c m}^{-1}\right): v 2960(\mathrm{w}), 2861(\mathrm{w}), 1807(\mathrm{w}), 1781(\mathrm{~m}), 1739(\mathrm{~s}), 1607(\mathrm{w}), 1519(\mathrm{w}), 1463(\mathrm{~m}), 1450(\mathrm{w})$, $1383(\mathrm{~m}), 1358(\mathrm{~m}), 1300(\mathrm{w}), 1269(\mathrm{w}), 1184(\mathrm{~m}), 1168(\mathrm{w}), 1147(\mathrm{~m}), 1134(\mathrm{~m}), 1079(\mathrm{~m}), 1054(\mathrm{~s}), 1006(\mathrm{~m}), 977$ (m), $957(\mathrm{~m}), 942(\mathrm{~m}), 900(\mathrm{w}), 879(\mathrm{~s}), 864(\mathrm{w}), 842(\mathrm{~m}), 802(\mathrm{~m}), 781(\mathrm{~m}), 755(\mathrm{w}), 708(\mathrm{~m}), 693(\mathrm{~s}), 645(\mathrm{w}), 627$ (m), $591(\mathrm{~m}), 562(\mathrm{~m}), 531(\mathrm{w}), 516(\mathrm{~m}), 449(\mathrm{w})$.

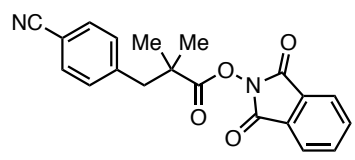

1,3-dioxoisoindolin-2-yl 3-(4-cyanophenyl)-2,2-dimethylpropanoate (S24)

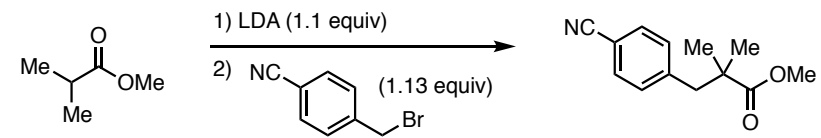

To an oven-dried 50-mL flask under nitrogen was added diisopropylamine (1.25 mL, $9.00 \mathrm{mmol}, 1.1$ equiv.) and 10 $\mathrm{mL}$ of THF. The flask was cooled to $-78^{\circ} \mathrm{C}$ and then charged with $n$-BuLi in hexanes $(3.35 \mathrm{~mL}$ of a $2.5 \mathrm{M}$ solution, $8.4 \mathrm{mmol}, 1.05$ equiv.). The reaction mixture was stirred for 5 minutes to generate LDA. Subsequently, methyl isobutyrate $(0.93 \mathrm{~mL}, 8 \mathrm{mmol}, 1$ equiv.) was added via syringe. The reaction mixture was stirred for 20 minutes to generate the enolate at $-78^{\circ} \mathrm{C}$. 4-(bromomethyl)benzonitrile $(1.765 \mathrm{~g}, 9 \mathrm{mmol}, 1.13$ equiv.) was added in $10 \mathrm{~mL}$ of THF. The reaction mixture was stirred overnight and allowed to warm to room temperature. After quenching with saturated ammonium chloride, the reaction mixture was extracted 4 times with ethyl acetate. The combined organics were dried over sodium sulfate and concentrated via rotary evaporation resulting in a yellow, oily residue. The resulting ester was carried on without further characterization.

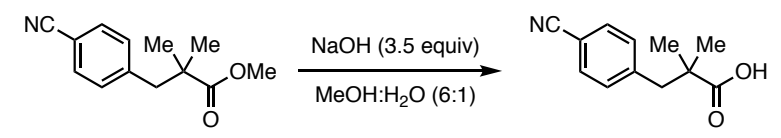

To the flask containing the crude methyl 3-(4-cyanophenyl)-2,2-dimethylpropanoate was added sodium hydroxide $(0.97 \mathrm{~g}, 24.3 \mathrm{mmol}, 3.0$ equiv.) as well as $5 \mathrm{~mL}$ of water and $30 \mathrm{~mL}$ of $\mathrm{MeOH}$. The reaction mixture was stirred for $24 \mathrm{~h}$ at room temperature. Afterwards the reaction mixture was washed with ether and the ether wash was discarded. The reaction mixture was acidified to $\mathrm{pH} 2$ using conc. $\mathrm{HCl}$. The reaction mixture was then extracted 3 times using ether. The organics were combined, dried over sodium sulfate, and concentrated via rotary evaporation to yield 3-(4cyanophenyl)-2,2-dimethylpropanoic acid was obtained as a white solid (1.2108 g, $5.96 \mathrm{mmol}, 75 \%$ over 2 steps).

${ }^{1}$ H NMR (500 MHz, Chloroform-d): $\delta 7.57$ (d, J=7.8 Hz, 2H), 7.28 (d, $\left.8.3 \mathrm{~Hz}, 2 \mathrm{H}\right), 2.95$ (s, 2H), 1.22 (s, 6H). ${ }^{13}$ C NMR (126 MHz, Chloroform-d): $\delta$ 182.47, 143.32, 132.03, 131.14, 119.01, 110.80, 45.87, 43.51, 24.97.

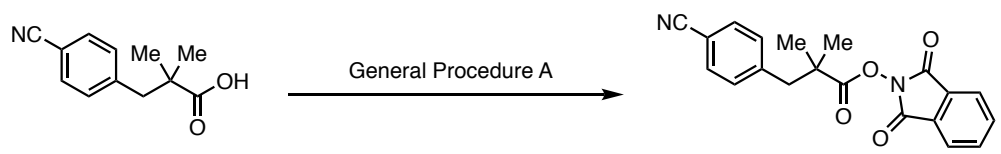

Synthesized according to general procedure A with 3-(4-cyanophenyl)-2,2-dimethylpropanoic acid (1.2108 g, $6 \mathrm{mmol}$, 1 equiv.), $N$-hydroxyphthalimide ( $1.4579 \mathrm{~g}, 9.3 \mathrm{mmol}, 1.5$ equiv.), DMAP (40.3 mg, $0.3 \mathrm{mmol}, 0.05$ equiv.) and DIC $(1.3 \mathrm{~mL}, 8.4 \mathrm{mmol}, 1.4$ equiv.). Purified over silica with flash chromatography (10 to $25 \%$ EtOAc in hexanes) to afford the title compound as a white solid $(1.9452 \mathrm{~g}, 5.58 \mathrm{mmol}, 94 \%)$. 
1H NMR (500 MHz, Chloroform- $d$ ): $\delta 7.90(\mathrm{dd}, J=5.5,3.1 \mathrm{~Hz}, 2 \mathrm{H}), 7.81(\mathrm{dd}, J=5.5,3.0 \mathrm{~Hz}, 2 \mathrm{H}), 7.63(\mathrm{~d}, J=8.1$ $\mathrm{Hz}, 2 \mathrm{H}), 7.45(\mathrm{~d}, J=8.0 \mathrm{~Hz}, 2 \mathrm{H}), 3.13(\mathrm{~s}, 2 \mathrm{H}), 1.40(\mathrm{~s}, 6 \mathrm{H})$.

${ }^{13}$ C NMR (126 MHz, Chloroform-d): $\delta$ 173.10, 162.14, 142.19, 134.98, 132.24, 131.30, 129.07, 124.14, 119.04 , $111.11,46.03,43.34,25.27$.

HRMS: (ESI-TOF) calculated for $\mathrm{C}_{20} \mathrm{H}_{17} \mathrm{~N}_{2} \mathrm{O}_{5}{ }^{+}\left([\mathrm{M}+\mathrm{H}]^{+}\right)$: 349.11828 , found 349.11746 .

FTIR (thin film, cm$\left.^{-1}\right): v 2992(\mathrm{w}), 2227(\mathrm{w}), 1803(\mathrm{~m}), 1779(\mathrm{~m}), 1738(\mathrm{~s}), 1608(\mathrm{w}), 1509(\mathrm{w}), 1465(\mathrm{~m}), 1417$ (w), 1390 (w), $1371(\mathrm{~m}), 1357(\mathrm{~m}), 1286(\mathrm{w}), 1184(\mathrm{~m}), 1132(\mathrm{~m}), 1080(\mathrm{~m}), 1042(\mathrm{~s}), 1013(\mathrm{~s}), 978(\mathrm{~m}), 963(\mathrm{~m})$, $946(\mathrm{w}), 902(\mathrm{w}), 877$ (s), $867(\mathrm{~m}), 844(\mathrm{~m}), 828(\mathrm{~m}), 803(\mathrm{~m}), 785$ (m), $758(\mathrm{w}), 732(\mathrm{w}), 710(\mathrm{w}), 690(\mathrm{~s}), 648(\mathrm{w})$, $625(\mathrm{w}), 588(\mathrm{~m}), 555(\mathrm{~m}), 530(\mathrm{w}), 516(\mathrm{~m}), 444(\mathrm{w})$.

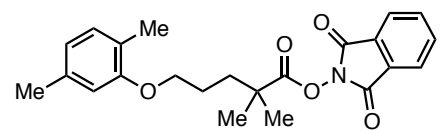

1,3-dioxoisoindolin-2-yl 5-(2,5-dimethylphenoxy)-2,2-dimethylpentanoate (41)

Synthesized according to general procedure A with 5-(2,5-dimethylphenoxy)-2,2-dimethylpentanoic acid (1.0135 g, $4.0 \mathrm{mmol}, 1$ equiv.), $N$-hydroxyphthalimide ( $0.9755 \mathrm{~g}, 6.0 \mathrm{mmol}, 1.5$ equiv.), DMAP ( $21.3 \mathrm{mg}, 0.2 \mathrm{mmol}, 0.05$ equiv.) and DIC ( $0.87 \mathrm{~mL}, 5.6 \mathrm{mmol}, 1.4$ equiv). Purified over silica with flash chromatography (10 to $30 \%$ EtOAc in hexanes) to afford the title compound as a white solid $(1.5616 \mathrm{~g}, 3.95 \mathrm{mmol}, 98 \%)$.

1H NMR (500 MHz, Chloroform- $d$ ): $\delta 7.89(\mathrm{dd}, \mathrm{J}=5.4,3.1 \mathrm{~Hz}, 2 \mathrm{H}), 7.79(\mathrm{dd}, \mathrm{J}=5.5,3.1 \mathrm{~Hz}, 2 \mathrm{H}), 7.00(\mathrm{~d}, \mathrm{~J}=7.3$ $\mathrm{Hz}, 1 \mathrm{H}), 6.66(\mathrm{~m}, 2 \mathrm{H}), 4.01(\mathrm{t}, \mathrm{J}=4.9 \mathrm{~Hz}, 2 \mathrm{H}), 2.31(\mathrm{~s}, 3 \mathrm{H}), 2.19(\mathrm{~s}, 3 \mathrm{H}), 2.00-1.91(\mathrm{~m}, 4 \mathrm{H}), 1.45(\mathrm{~s}, 6 \mathrm{H})$.

${ }^{13}$ C NMR (126 MHz, Chloroform- $d$ ): $\delta$ 173.79, 162.12, 156.97, 136.50, 134.70, 130.27, 129.07, 123.90, 123.62 , $120.69,111.99,67.73,41.98,37.41,25.15,25.00,21.42,15.81$.

HRMS: (ESI-TOF) calculated for $\mathrm{C}_{23} \mathrm{H}_{25} \mathrm{NNaO}_{5}^{+}\left([\mathrm{M}+\mathrm{Na}]^{+}\right): 418.16249$, found 418.16148 .

FTIR (thin film, cm$\left.^{-1}\right): v 3004(\mathrm{w}), 2875(\mathrm{w}), 1849(\mathrm{w}), 1804(\mathrm{~m}), 1778(\mathrm{~s}), 1740(\mathrm{~s}), 1616(\mathrm{~m}), 1585(\mathrm{~m}), 1510(\mathrm{~m})$, $1469(\mathrm{~m}), 1414(\mathrm{~m}), 1397(\mathrm{~m}), 1373(\mathrm{~m}), 1312(\mathrm{~m}), 1276(\mathrm{~m}), 1264(\mathrm{~s}), 1210(\mathrm{~m}), 1187(\mathrm{~m}), 1158(\mathrm{~m}), 1130(\mathrm{~s}), 1080$ (m), $1038(\mathrm{~s}), 1000(\mathrm{~m}), 969(\mathrm{~s}), 947(\mathrm{~m}), 931(\mathrm{~m}), 877(\mathrm{~s}), 856(\mathrm{~m}), 837(\mathrm{~m}), 813(\mathrm{~m}), 803(\mathrm{~s}), 786(\mathrm{~s}), 766(\mathrm{~m}), 754$ (m), $704(\mathrm{~s}), 693(\mathrm{~s})$.

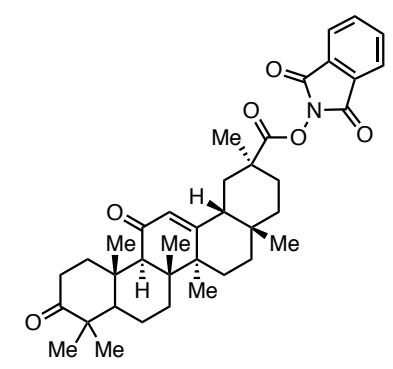

1,3-dioxoisoindolin-2-yl (2S,4a $S, 6 \mathrm{a} S, 6 \mathrm{~b} R, 12 \mathrm{a} S, 12 \mathrm{~b} R, 14 \mathrm{~b} R)-2,4 \mathrm{a}, 6 \mathrm{a}, 6 \mathrm{~b}, 9,9,12 \mathrm{a}-\mathrm{heptamethyl-10,13-dioxo-}$

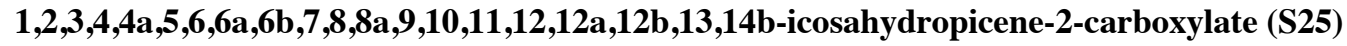
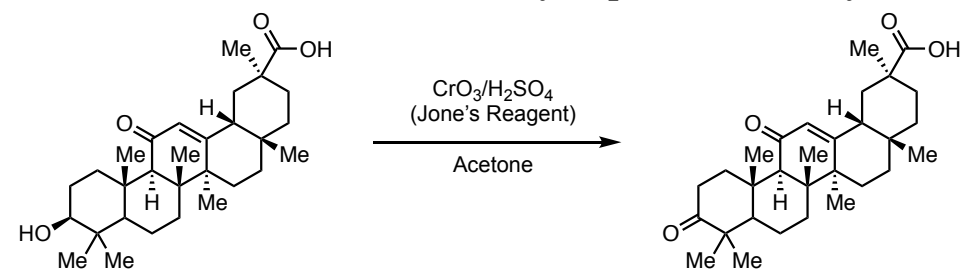

Enoxolone was oxidized according to the procedure from Subba Rao, et. al. ${ }^{7}$ To a $500-\mathrm{mL}$ round bottom flask with a stir bar was added $2.38 \mathrm{~g}$ enoxolone $\left(2.38 \mathrm{~g}, 5 \mathrm{mmol}, 1\right.$ equiv.) and $100 \mathrm{~mL}$ dry acetone. The flask was cooled to $0^{\circ} \mathrm{C}$ and Jones Reagent ( $3.0 \mathrm{~mL}$ of $2 \mathrm{M}$ solution, Sigma, $6 \mathrm{mmol}, 1.2$ equiv) was added over 5 minutes until the brown color persisted. The mixture was stirred further for 40 minutes. 2-Propanol $(8 \mathrm{~mL})$ was then added to quench the reaction and the reaction mixture was filtered over a frit and the filtered solids were washed thoroughly with dichloromethane. The filtrate was concentrated to a viscous oil and then diluted to $200 \mathrm{~mL}$ with dichloromethane and poured into a separatory funnel. The organic layer was washed with water $(2 \times 100 \mathrm{~mL})$, brine and then dried with 
sodium sulfate. The solution was concentrated to yield an off-white solid which was used directly for phthalimide ester synthesis.

1H NMR (500 MHz, Chloroform- $d$ ): $\delta 5.75(\mathrm{~s}, 1 \mathrm{H}), 2.96(\mathrm{dq}, J=11.0,4.0,3.5 \mathrm{~Hz}, 1 \mathrm{H}), 2.64$ (ddd, $J=15.7,11.3$, $7.1 \mathrm{~Hz}, 1 \mathrm{H}), 2.45(\mathrm{~s}, 1 \mathrm{H}), 2.36(\mathrm{ddd}, J=15.8,6.5,4.0 \mathrm{~Hz}, 1 \mathrm{H}), 2.21(\mathrm{dd}, J=13.3,2.3 \mathrm{~Hz} 1 \mathrm{H}), 2.09-1.98(\mathrm{~m}, 2 \mathrm{H})$, $1.94(\mathrm{ddd}, J=13.7,4.4,2.7 \mathrm{~Hz}, 1 \mathrm{H}), 1.86(\mathrm{td}, J=13.6,4.6 \mathrm{~Hz}, 1 \mathrm{H}), 1.70(\mathrm{td}, J=12.1,5.5 \mathrm{~Hz}, 1 \mathrm{H}), 1.63(\mathrm{t}, J=13.5$ $\mathrm{Hz}, 1 \mathrm{H}), 1.60-1.50(\mathrm{~m}, 1 \mathrm{H}), 1.44$ (ddt, $J=18.0,14.5,7.2 \mathrm{~Hz}, 3 \mathrm{H}), 1.38(\mathrm{~s}, 3 \mathrm{H}), 1.36-1.29(\mathrm{~m}, 2 \mathrm{H}), 1.27(\mathrm{~s}, 3 \mathrm{H})$, $1.23(\mathrm{~s}, 3 \mathrm{H}), 1.22-1.19(\mathrm{~m}, 1 \mathrm{H}), 1.17(\mathrm{~s}, 3 \mathrm{H}), 1.10(\mathrm{~s}, 3 \mathrm{H}), 1.07(\mathrm{~s}, 3 \mathrm{H}), 1.05-1.03(\mathrm{~m}, 1 \mathrm{H}), 0.86(\mathrm{~s}, 3 \mathrm{H})$.

${ }^{13}$ C NMR (126 MHz, Chloroform- $d$ ): $\delta$ 2 217.40, 199.82, 181.35, 169.94, 128.57, 61.20, 55.59, 48.39, 47.94, 45.43, $43.93,43.46,41.06,39.88,37.83,36.85,34.39,32.27,32.03,31.05,28.72,28.57,26.67,26.52,23.50,21.60,18.93$, $18.68,15.80$.

HRMS: (ESI-TOF) calculated for $\mathrm{C}_{30} \mathrm{H}_{45} \mathrm{O}_{4}{ }^{+}\left([\mathrm{M}+\mathrm{H}]^{+}\right)$: 469.33124 , found 469.3317 .

FTIR (thin film, cm$\left.^{-1}\right): v 3305$ (w), $2964(\mathrm{w}), 2947$ (w), $2873(\mathrm{w}), 2848(\mathrm{w}), 2360(\mathrm{w}), 2340(\mathrm{w}), 2185(\mathrm{w}), 2050$ (w), $2026(\mathrm{w}), 1978(\mathrm{w}), 1726(\mathrm{~s}), 1681(\mathrm{~m}), 1643(\mathrm{~s}), 1617(\mathrm{w}), 1444(\mathrm{w}), 1385(\mathrm{~m}), 1362(\mathrm{w}), 1347(\mathrm{w}), 1328(\mathrm{w})$, $1279(\mathrm{w}), 1250(\mathrm{w}), 1205(\mathrm{~m}), 1193(\mathrm{w}), 1175(\mathrm{w}), 1142(\mathrm{~m}), 1110(\mathrm{w}), 1086(\mathrm{~m}), 1071(\mathrm{w}), 1049(\mathrm{w}), 1028(\mathrm{w})$, $989(\mathrm{w}), 982(\mathrm{w}), 948(\mathrm{w}), 885(\mathrm{w}), 873(\mathrm{w}), 822(\mathrm{w}), 779(\mathrm{~m}), 747(\mathrm{w}), 727(\mathrm{w}), 692(\mathrm{w}), 669(\mathrm{~m}), 622(\mathrm{w}), 605$ (w), $584(\mathrm{~m}), 560(\mathrm{w}), 537(\mathrm{w}), 479(\mathrm{w}), 461(\mathrm{w}), 418(\mathrm{w}), 403(\mathrm{w})$.
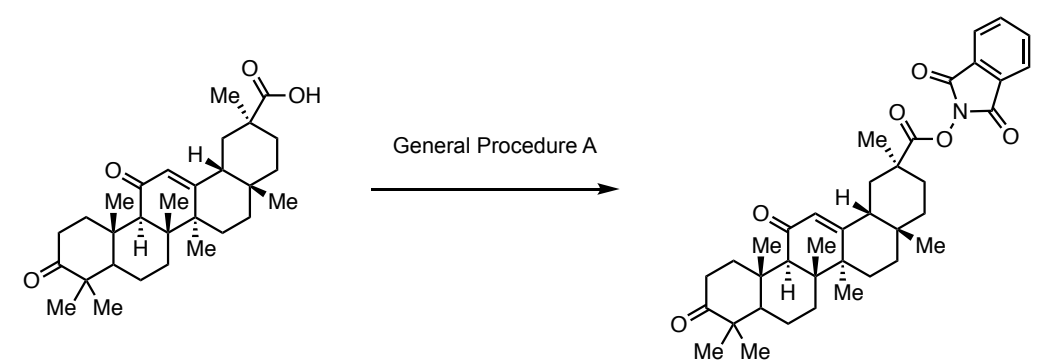

Synthesized according to general procedure A with the acid from the previous step (0.9062 g, 1.9 mmol, 1 equiv.), $N$ hydroxyphthalimide ( $0.469 \mathrm{~g}, 3 \mathrm{mmol}, 1.5$ equiv.), DMAP ( $16.9 \mathrm{mg}, 0.1 \mathrm{mmol}, 0.05$ equiv.) and DIC ( $0.5 \mathrm{~mL}, 2.7$ mmol, 1.4 equiv.). Purified over silica with flash chromatography (10 to 30\% EtOAc in hexanes) to afford the title compound as a white solid $(1.1255 \mathrm{~g}, 1.83 \mathrm{mmol}, 95 \%)$.

${ }^{1}$ H NMR (500 MHz, Chloroform- $d$ ): $\delta 7.88(\mathrm{dd}, J=5.4,3.1 \mathrm{~Hz}, 2 \mathrm{H}), 7.79(\mathrm{dd}, J=5.5,3.1 \mathrm{~Hz}, 2 \mathrm{H}), 5.80(\mathrm{~s}, 1 \mathrm{H})$, $2.96(\mathrm{ddd}, J=13.7,7.1,4.0 \mathrm{~Hz}, 1 \mathrm{H}), 2.63(\mathrm{ddd}, J=15.8,11.2,7.1 \mathrm{~Hz}, 1 \mathrm{H}), 2.48(\mathrm{dd}, J=13.7,2.6 \mathrm{~Hz}, 1 \mathrm{H}), 2.43$ (s, $1 \mathrm{H}), 2.35(\mathrm{ddd}, J=15.8,6.5,4.0 \mathrm{~Hz}, 1 \mathrm{H}), 2.14(\mathrm{dq}, J=13.3,3.7,3.0 \mathrm{~Hz}, 1 \mathrm{H}), 2.06(\operatorname{td}, J=13.7,4.3 \mathrm{~Hz}, 1 \mathrm{H}), 1.89$ $(\mathrm{td}, J=13.7,4.6 \mathrm{~Hz}, 1 \mathrm{H}), 1.79(\mathrm{t}, J=13.7 \mathrm{~Hz}, 1 \mathrm{H}), 1.73-1.53(\mathrm{~m}, 4 \mathrm{H}), 1.52-1.45(\mathrm{~m}, 1 \mathrm{H}), 1.44(\mathrm{~s}, 3 \mathrm{H}), 1.43-$ $1.39(\mathrm{~m}, 1 \mathrm{H}), 1.39(\mathrm{~s}, 3 \mathrm{H}), 1.30(\mathrm{dd}, J=10.9,3.5 \mathrm{~Hz}, 1 \mathrm{H}), 1.26(\mathrm{~s}, 3 \mathrm{H}), 1.22(\mathrm{~m}, 1 \mathrm{H}), 1.18(\mathrm{~s}, 3 \mathrm{H}), 1.10(\mathrm{~s}, 3 \mathrm{H}), 1.08$ $(\mathrm{m}, 1 \mathrm{H}), 1.06(\mathrm{~s}, 3 \mathrm{H}), 0.92(\mathrm{~s}, 3 \mathrm{H})$.

${ }^{13}$ C NMR (126 MHz, Chloroform-d): $\delta$ 217.39, 199.48, 172.73, 169.04, 162.23, 134.88, 129.15, 128.90, 124.07, $61.20,55.57,47.92,47.88,45.32,44.03,43.36,41.42,39.88,37.37,36.85,34.38,32.26,31.99,31.54,28.52$, 28.08 , $26.68,26.57,26.53,23.44,21.57,18.93,18.67,15.81$.

HRMS: (ESI-TOF) calculated for $\mathrm{C}_{38} \mathrm{H}_{48} \mathrm{NO}_{6}{ }^{+}\left([\mathrm{M}+\mathrm{H}]^{+}\right): 614.34761$, found 614.3478 .

FTIR (thin film, cm $\left.^{-1}\right): v 3305$ (w), 2964 (w), 2947 (w), 2873 (w), 2848 (w), 2360 (w), 2340 (w), 2185 (w), 2050 (w), $2026(\mathrm{w}), 1978(\mathrm{w}), 1726(\mathrm{~s}), 1681(\mathrm{~m}), 1643(\mathrm{~s}), 1617(\mathrm{w}), 1444(\mathrm{w}), 1385(\mathrm{~m}), 1362(\mathrm{w}), 1347(\mathrm{w}), 1328(\mathrm{w})$, $1279(\mathrm{w}), 1250(\mathrm{w}), 1205(\mathrm{~m}), 1193(\mathrm{w}), 1175(\mathrm{w}), 1142(\mathrm{~m}), 1110(\mathrm{w}), 1086(\mathrm{~m}), 1071(\mathrm{w}), 1049(\mathrm{w}), 1028(\mathrm{w})$, $989(\mathrm{w}), 982(\mathrm{w}), 948(\mathrm{w}), 885(\mathrm{w}), 873(\mathrm{w}), 822(\mathrm{w}), 779(\mathrm{~m}), 747(\mathrm{w}), 727(\mathrm{w}), 692(\mathrm{w}), 669(\mathrm{~m}), 622(\mathrm{w}), 605$ (w), $584(\mathrm{~m}), 560(\mathrm{w}), 537(\mathrm{w}), 479(\mathrm{w}), 461(\mathrm{w}), 418(\mathrm{w}), 403(\mathrm{w})$.

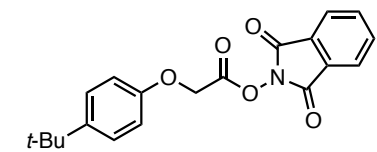

1,3-dioxoisoindolin-2-yl 2-(4-(tert-butyl)phenoxy)acetate (S26)

Synthesized according to general procedure A with 2-(4-(tert-butyl)phenoxy)acetic acid $(0.8435 \mathrm{~g}, 4.0 \mathrm{mmol}, 1$ equiv.), $N$-hydroxyphthalimide ( $0.9814 \mathrm{~g}, 6.0 \mathrm{mmol}, 1.5$ equiv.), DMAP ( $35.0 \mathrm{mg}, 0.2 \mathrm{mmol}, 0.05$ equiv) and DIC 
( $0.9 \mathrm{~mL}, 5.4 \mathrm{mmol}, 1.4$ equiv.). Purified over silica with flash chromatography (5 to $25 \%$ EtOAc in hexanes) to afford the title compound as a white solid $(1.0869 \mathrm{~g}, 3.08 \mathrm{mmol}, 76 \%)$.

1H NMR (500 MHz, Chloroform- $d$ ): $\delta 7.91(\mathrm{dd}, J=5.4,3.1 \mathrm{~Hz}, 2 \mathrm{H}), 7.81(\mathrm{dd}, J=5.5,3.1 \mathrm{~Hz}, 2 \mathrm{H}), 7.35(\mathrm{~d}, J=8.9$ $\mathrm{Hz}, 2 \mathrm{H}), 6.93(\mathrm{~d}, J=8.9 \mathrm{~Hz}, 2 \mathrm{H}), 5.02(\mathrm{~s}, 2 \mathrm{H}), 1.30(\mathrm{~s}, 9 \mathrm{H})$.

${ }^{13}$ C NMR (126 MHz, Chloroform-d): $\delta$ 165.88, 161.69, 155.24, 145.33, 135.08, 128.93, 126.69, 124.28, 114.46, $63.73,34.33,31.60$.

HRMS: (ESI-TOF) calculated for $\mathrm{C}_{20} \mathrm{H}_{19} \mathrm{NNaO}_{5}{ }^{+}\left([\mathrm{M}+\mathrm{Na}]^{+}\right): 376.11554$, found 376.11562 .

FTIR (thin film, cm$\left.^{-1}\right): v 2946(\mathrm{w}), 2858(\mathrm{w}), 1844(\mathrm{~m}), 1795(\mathrm{~m}), 1737(\mathrm{~s}), 1607(\mathrm{~m}), 1582(\mathrm{w}), 1511(\mathrm{~s}), 1479(\mathrm{w})$, $1467(\mathrm{~m}), 1434(\mathrm{~m}), 1411(\mathrm{w}), 1392(\mathrm{w}), 1364(\mathrm{~m}), 1298(\mathrm{~m}), 1245(\mathrm{~s}), 1187(\mathrm{~m}), 1159(\mathrm{w}), 1138(\mathrm{~m}), 1118(\mathrm{~m})$, $1103(\mathrm{~m}), 1067(\mathrm{~s}), 1014(\mathrm{~m}), 967(\mathrm{~s}), 913(\mathrm{~s}), 875(\mathrm{~s}), 848(\mathrm{~m}), 824(\mathrm{~s}), 804(\mathrm{~m}), 787(\mathrm{~s}), 727(\mathrm{w}), 696(\mathrm{~s}), 669(\mathrm{~m})$, $602(\mathrm{~m}), 575(\mathrm{~m}), 550(\mathrm{~s}), 518(\mathrm{~s}), 472(\mathrm{~m}), 443(\mathrm{w})$.

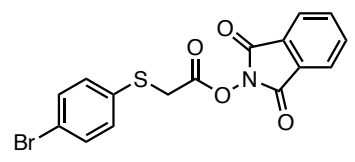

1,3-dioxoisoindolin-2-yl 2-((4-bromophenyl)thio)acetate (S27)
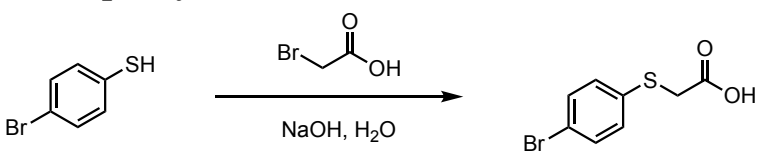

According to literature procedure, ${ }^{8}$ to a $100 \mathrm{~mL}$ flask with a stir bar was added 4-bromothiophenol $(1.9917 \mathrm{~g}, 10.5$ mmol, 1 equiv.), sodium hydroxide (1.63 g, $40.8 \mathrm{mmol}, 4$ equiv.), and 2-bromoacetic acid (2.04 g, $14.68 \mathrm{mmol}, 1.4$ equiv.). The flask was then charged with $30 \mathrm{~mL}$ of water and allowed to stir overnight at room temperature. The reaction mixture was acidified using $1 \mathrm{~N} \mathrm{HCl}$. The reaction mixture was decanted to a separatory funnel and extracted 3 times with ether. The combined ethereal extracts were dried, filtered, and concentrated to produce a slightly yellow solid residue. Purified over silica with flash chromatography (25 to $100 \%$ EtOAc in hexanes) to afford the title compound as a white solid $(2.2446 \mathrm{~g}, 9.08 \mathrm{mmol}, 94 \%)$.

${ }^{1}$ H NMR (500 MHz, Chloroform-d): $\delta 7.44$ (d, $\left.J=8.5 \mathrm{~Hz}, 2 \mathrm{H}\right), 7.29$ (d, $\left.J=8.6 \mathrm{~Hz}, 2 \mathrm{H}\right), 3.65$ (s, 2H). ${ }^{13}$ C NMR (126 MHz, Chloroform-d): $\delta$ 175.14, 133.58, 132.30, 131.73, 121.49, 36.49.

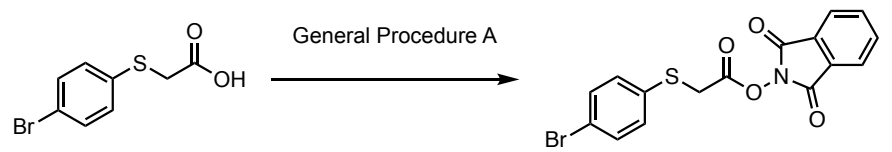

Synthesized according to general procedure A with the acid from the previous step ( $0.9851 \mathrm{~g}, 4$ mmol, 1 equiv), Nhydroxyphthalimide ( $0.9947 \mathrm{~g}, 6 \mathrm{mmol}, 1.5$ equiv), DMAP (34.2 mg, $0.2 \mathrm{mmol}, 0.05$ equiv) and DIC (0.9 mL, 5.4 mmol, 1.4 equiv). Purified over silica with flash chromatography (5 to $25 \%$ EtOAc in hexanes) to afford the title compound as a white solid $(1.2215 \mathrm{~g}, 3.11 \mathrm{mmol}, 78 \%)$.

1H NMR (500 MHz, Chloroform- $d$ ): $\delta 7.89(\mathrm{dd}, J=5.4,3.1 \mathrm{~Hz}, 2 \mathrm{H}), 7.80(\mathrm{dd}, J=5.5,3.1 \mathrm{~Hz}, 2 \mathrm{H}), 7.49(\mathrm{~d}, J=$ $8.7 \mathrm{~Hz}, 2 \mathrm{H}), 7.45(\mathrm{~d}, J=8.6 \mathrm{~Hz}, 2 \mathrm{H}), 3.86(\mathrm{~s}, 2 \mathrm{H})$.

${ }^{13}$ C NMR (126 MHz, Chloroform-d): $\delta 166.20,161.71,135.05,133.80,132.61,132.37,128.93,124.24,122.88$, 34.66.

HRMS: (ESI-TOF) calculated for $\mathrm{C}_{16} \mathrm{H}_{11} \mathrm{BrNO}_{4} \mathrm{~S}^{+}\left([\mathrm{M}+\mathrm{H}]^{+}\right)$: 391.95867, found 391.95748 .

FTIR (thin film, cm-1): $v 3095$ (w), 2938 (w), 1818 (m), 1789 (m), 1739 (s), 1609 (w), 1475 (m), 1466 (m), 1365 $(\mathrm{m}), 1352(\mathrm{~m}), 1325(\mathrm{~m}), 1282(\mathrm{~m}), 1185(\mathrm{~m}), 1118(\mathrm{~m}), 1092(\mathrm{~m}), 1059(\mathrm{~s}), 1015(\mathrm{~m}), 1002(\mathrm{~s}), 964(\mathrm{~s}), 867(\mathrm{~s}), 808$ (s), $781(\mathrm{~s}), 725(\mathrm{~m}), 692(\mathrm{~s}), 601(\mathrm{~s}), 550(\mathrm{~m}), 518(\mathrm{~s}), 489(\mathrm{~s}), 475(\mathrm{~s}), 408(\mathrm{~m})$.<smiles>CC1(C)OC(C(=O)ON2C(=O)c3ccccc3C2=O)OC(O)C1O</smiles> 
1,3-dioxoisoindolin-2-yl carboxylate (S28)
$(3 \mathrm{a} S, 4 S, 6 R, 6 \mathrm{a} R)$-6-(benzyloxy)-2,2-dimethyltetrahydrofuro[3,4- $d][1,3]$ dioxole-4-

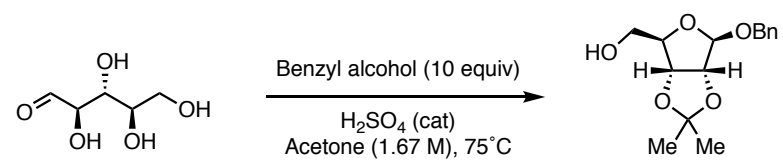

According to literature method, ${ }^{9}$ to a 100- $\mathrm{mL}$ flask with a stir bar was added D-ribose (3.0115 g, $20 \mathrm{mmol}, 1$ equiv.), benzyl alcohol ( $20 \mathrm{~mL}, 190 \mathrm{mmol}, 10$ equiv.), and $12.0 \mathrm{~mL}$ of acetone. Sulfuric acid (6 drops) was added and the reaction mixture was heated to $75^{\circ} \mathrm{C}$ for 5 hours. The reaction was cooled to room temperature and then neutralized by the addition of triethylamine until basic. Volatiles were removed via rotary evaporation. The resulting yellow solution was washed with water, dried over sodium sulfate, filtered, and concentrated. The resulting solution was concentrated via vacuum distillation to remove as much benzyl alcohol as possible. Upon cooling, a brown solid precipitated. Purified over silica with flash chromatography (5 to $40 \%$ EtOAc in hexanes) to yield $((3 \mathrm{a} R, 4 R, 6 R, 6 \mathrm{a} R)$ 6-(benzyloxy)-2,2-dimethyltetrahydrofuro[3,4- $d][1,3]$ dioxol-4-yl)methanol as a white solid (1.4447 g, 26\% yield).

Note: Washing and/or sonication of the white solid with hexanes was important to remove all benzyl alcohol prior to subsequent oxidation to the carboxylic acid.

1H NMR (500 MHz, Chloroform- $d)$ : $\delta 7.39-7.30(\mathrm{~m}, 5 \mathrm{H}), 5.18(\mathrm{~s}, 1 \mathrm{H}), 4.86(\mathrm{~d}, J=5.9 \mathrm{~Hz}, 1 \mathrm{H}), 4.77(\mathrm{~d}, J=11.6$ $\mathrm{Hz}, 1 \mathrm{H}), 4.67(\mathrm{~d}, J=5.9 \mathrm{~Hz}, 1 \mathrm{H}), 4.58(\mathrm{~d}, J=11.6 \mathrm{~Hz}, 1 \mathrm{H}), 4.46(\mathrm{t}, J=3.0 \mathrm{~Hz}, 1 \mathrm{H}), 3.72(\mathrm{dd}, J=12.6,2.3 \mathrm{~Hz}, 1 \mathrm{H})$, $3.64(\mathrm{dt}, J=11.6,5.9 \mathrm{~Hz}, 1 \mathrm{H}), 3.13(\mathrm{~d}, J=10.2 \mathrm{~Hz}, 1 \mathrm{H}), 1.48(\mathrm{~s}, 3 \mathrm{H}), 1.32(\mathrm{~s}, 3 \mathrm{H})$.

${ }^{13}$ C NMR (126 MHz, Chloroform- $d$ ): $\delta$ 136.46, 128.83, 128.43, 128.37, 112.28, 108.19, 88.63, 86.15, 81.65, $70.35,64.22,26.48,24.80$.

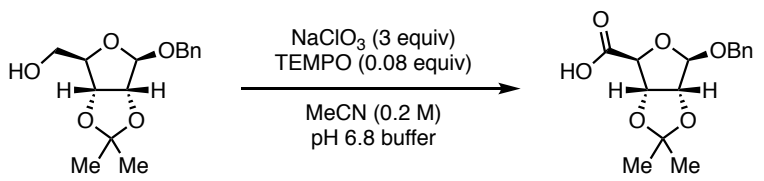

According to literature conditions,$^{10}$ to a $100-\mathrm{mL}$ flask equipped with a stir bar was ((3a $\left.R, 4 R, 6 R, 6 \mathrm{a} R\right)$-6-(benzyloxy)2,2-dimethyltetrahydrofuro[3,4- $d][1,3]$ dioxol-4-yl)methanol (1.4016 g, $5 \mathrm{mmol}, 1$ equiv), sodium chlorite (1.3820 g, $15 \mathrm{mmol}, 3$ equiv), and TEMPO (62.1 mg, $0.4 \mathrm{mmol}, 0.08$ equiv). The flask was charged with $20 \mathrm{~mL}$ of phosphate buffer $\left(6.7 \mathrm{M}, 6.8 \mathrm{pH}\right.$ sodium phosphate buffer) and $25 \mathrm{~mL}$ of MeCN. The reaction mixture was heated to $35^{\circ} \mathrm{C}$ and stirred. Sodium hypochlorite $(0.4 \mathrm{~mL}$ of a $12 \%$ solution) was added dropwise over 2 hours. The reaction mixture was stirred overnight at $35^{\circ} \mathrm{C}$. The reaction mixture was poured into a $10 \%$ sodium hydroxide solution and extracted with ether. The organics were separated and discarded. The aqueous layer was carefully acidified using $1 \mathrm{~N} \mathrm{HCl}$ to $\mathrm{pH} 4$. The reaction mixture was then extracted 3 times wither ether. The combined organics were separated, dried over sodium sulfate, filtered, and then concentrated to produce a yellow oil. Purified over silica with flash chromatography to afford (3aS,4S,6R,6aR)-6-(benzyloxy)-2,2-dimethyltetrahydrofuro[3,4- $d][1,3]$ dioxole-4-carboxylic acid as a clear oil that solidified to a white solid upon standing $(0.7245 \mathrm{~g}, 2.46 \mathrm{mmol}, 49 \%)$.

1H NMR (500 MHz, Chloroform- $d$ ): $\delta 7.40-7.30(\mathrm{~m}, 5 \mathrm{H}), 5.29(\mathrm{~d}, J=1.8 \mathrm{~Hz}, 1 \mathrm{H}), 5.23(\mathrm{~d}, J=5.9 \mathrm{~Hz}, 1 \mathrm{H}), 4.87$ $(\mathrm{dd}, J=11.7,1.7 \mathrm{~Hz}, 1 \mathrm{H}), 4.73(\mathrm{~s}, 1 \mathrm{H}), 4.68(\mathrm{dd}, J=5.9,1.8 \mathrm{~Hz}, 1 \mathrm{H}), 4.59(\mathrm{dd}, J=11.6,1.7 \mathrm{~Hz}, 1 \mathrm{H}), 1.51(\mathrm{~s}, 3 \mathrm{H})$, $1.35(\mathrm{~s}, 3 \mathrm{H})$.

${ }^{13}$ C NMR (126 MHz, Chloroform-d): $\delta$ 173.67, 136.68, 128.70, 128.25, 128.20, 113.12, 107.64, 84.50, 83.99, 82.34, $70.10,26.44,25.02$.

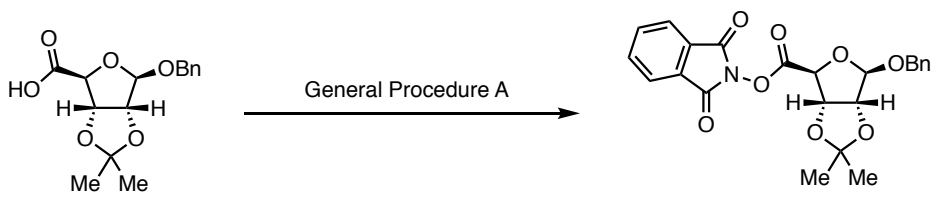

Synthesized according to general procedure A with the acid from the previous step ( $0.7245 \mathrm{~g}, 2.46 \mathrm{mmol}, 1$ equiv.), $N$-hydroxyphthalimide ( $0.6002 \mathrm{~g}, 3.8 \mathrm{mmol}, 1.5$ equiv.), DMAP $(25.7 \mathrm{mg}, 0.21 \mathrm{mmol}, 0.085$ equiv.) and DIC (0.6 $\mathrm{mL}, 1.87 \mathrm{mmol}, 1.6$ equiv). Purified over silica with flash chromatography (10 to $25 \%$ EtOAc in hexanes) to afford the title compound as a sticky white solid $(0.8261 \mathrm{~g}, 1.88 \mathrm{mmol}, 76 \%)$. 
$\underline{\left.{ }^{1} \text { H NMR (400 MHz, Chloroform- } d\right):} \delta 7.90(\mathrm{dd}, J=5.5,3.1 \mathrm{~Hz}, 2 \mathrm{H}), 7.80(\mathrm{dd}, J=5.5,3.1 \mathrm{~Hz}, 2 \mathrm{H}), 7.36-7.26(\mathrm{~m}$, $5 \mathrm{H}), 5.41(\mathrm{dd}, J=5.8,1.3 \mathrm{~Hz}, 1 \mathrm{H}), 5.28(\mathrm{~s}, 1 \mathrm{H}), 5.07-5.06(\mathrm{~m}, 1 \mathrm{H}), 4.96(\mathrm{~d}, J=12.4 \mathrm{~Hz}, 1 \mathrm{H}), 4.75-4.72(\mathrm{~m}, 1 \mathrm{H})$, $4.60(\mathrm{~d}, J=12.4 \mathrm{~Hz}, 1 \mathrm{H}), 1.51(\mathrm{~s}, 3 \mathrm{H}), 1.35(\mathrm{~s}, 3 \mathrm{H})$.

${ }^{13}$ C NMR (126 MHz, Chloroform- $d$ ): $\delta$ 166.92, 161.62, 137.03, 135.01, 129.02, 128.59, 128.57, 127.99, 124.22, $113.46,106.81,84.55,82.65,82.62,69.57,26.56,25.18$.

HRMS: (ESI-TOF) calculated for $\mathrm{C}_{23} \mathrm{H}_{22} \mathrm{NO}_{8}{ }^{+}\left([\mathrm{M}+\mathrm{H}]^{+}\right): 440.13399$, found 440.13329 .

FTIR (thin film, cm$^{-1}$ ): $v 2354$ (m), 2340 (w), 2057 (w), 2047 (w), 2030 (w), 2017 (w), 2009 (w), 1992 (w), 1968 (w), $1813(\mathrm{w}), 1790(\mathrm{w}), 1741(\mathrm{~s}), 1700(\mathrm{w}), 1685(\mathrm{w}), 1653(\mathrm{w}), 1636(\mathrm{w}), 1466(\mathrm{w}), 1457(\mathrm{w}), 1373(\mathrm{w}), 1210(\mathrm{w})$, $1186(\mathrm{w}), 1158(\mathrm{w}), 1132(\mathrm{w}), 1078(\mathrm{~m}), 1042(\mathrm{~m}), 965(\mathrm{~m}), 938(\mathrm{~m}), 904(\mathrm{~m}), 875(\mathrm{~m}), 862(\mathrm{~m}), 783(\mathrm{w}), 738(\mathrm{~m})$, $693(\mathrm{~s}), 603(\mathrm{w}), 516(\mathrm{~m}), 457(\mathrm{w}), 436(\mathrm{w}), 419(\mathrm{w}), 407(\mathrm{w})$.

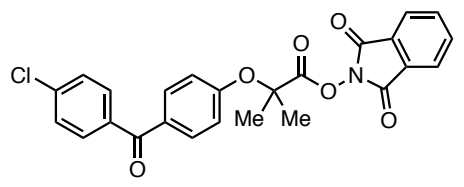

1,3-dioxoisoindolin-2-yl 2-(4-(4-chlorobenzoyl)phenoxy)-2-methylpropanoate (S29)

Prepared according to general procedure B with fenofibric acid (2-[4-(4-Chlorobenzoyl)phenoxy]-2-methylpropionic acid, $1.2509 \mathrm{~g}, 3.92 \mathrm{mmol}, 1$ equiv.), DMF (5 drops, catalytic), and oxalyl chloride (0.6 mL, $7.1 \mathrm{mmol}, 1.75$ equiv.) followed by $N$-hydroxyphthalimide ( $0.6795 \mathrm{~g}, 4.17 \mathrm{mmol}, 1.06$ equiv.) and triethylamine $(0.6 \mathrm{~mL}, 4.30 \mathrm{mmol}, 1.1$ equiv.). Purified via column chromatography (15-25\% EtOAc/hexanes). This afforded the title compound as a white solid $(1.4510 \mathrm{~g}, 3.13 \mathrm{mmol}, 80 \%)$.

1H NMR (500 MHz, Chloroform- $d$ ): $\delta 7.90(\mathrm{dd}, \mathrm{J}=5.5,3.1 \mathrm{~Hz}, 2 \mathrm{H}), 7.86-7.79(\mathrm{~m}, 4 \mathrm{H}), 7.74(\mathrm{~d}, \mathrm{~J}=8.5 \mathrm{~Hz}, 2 \mathrm{H})$, $7.46(\mathrm{~d}, \mathrm{~J}=8.6 \mathrm{~Hz}, 2 \mathrm{H}), 7.12(\mathrm{~d}, \mathrm{~J}=8.8 \mathrm{~Hz}, 2 \mathrm{H}), 1.88(\mathrm{~s}, 6 \mathrm{H})$.

${ }^{13}$ C NMR (126 MHz, Chloroform-d): $\delta$ 194.45, 170.50, 161.86, 158.71, 138.62, 136.44, 135.10, 132.24, 131.60, $131.41,129.01,128.73,124.25,118.65,78.77,25.87$.

HRMS: (ESI-TOF) calculated for $\mathrm{C}_{25} \mathrm{H}_{19} \mathrm{ClNO}_{6}{ }^{+}\left([\mathrm{M}+\mathrm{H}]^{+}\right): 464.08954$, found 464.08910 .

FTIR (thin film, $\left.\mathbf{~ c m}^{-1}\right): v 3017.57(\mathrm{w}), 1840.21(\mathrm{w}), 1811.43(\mathrm{~m}), 1784.61(\mathrm{~s}), 1744.45(\mathrm{~s}), 1659.00(\mathrm{~s}), 1597.77(\mathrm{~s})$, $1588.11(\mathrm{~s}), 1501.55(\mathrm{~m}), 1486.74(\mathrm{~m}), 1467.20(\mathrm{~m}), 1455.57(\mathrm{~m}), 1398.41(\mathrm{~m}), 1388.08(\mathrm{~m}), 1352.14(\mathrm{~m}), 1317.38$ (s), $1299.68(\mathrm{~s}), 1286.12(\mathrm{~m}), 1272.64(\mathrm{~s}), 1234.47(\mathrm{~s}), 1202.19(\mathrm{~m}), 1184.23(\mathrm{~s}), 1169.78(\mathrm{~s}), 1147.78(\mathrm{~s}), 1128.67$ (s), $1088.98(\mathrm{~s}), 1080.00(\mathrm{~s}), 1046.00(\mathrm{~s}), 1013.51(\mathrm{~s}), 961.51(\mathrm{~s}), 928.90(\mathrm{~s}), 907.65(\mathrm{~s}), 876.72(\mathrm{~s}), 854.82(\mathrm{~s}), 830.17$ (s), $783.67(\mathrm{~s}), 761.21(\mathrm{~s}), 748.91(\mathrm{~s}), 738.14(\mathrm{~m}), 722.95(\mathrm{~m}), 709.20(\mathrm{~s}), 692.92(\mathrm{~s}), 675.74(\mathrm{~s})$.<smiles>Cc1ccccc1C(=O)ONC(=O)C(C)Oc1ccc(Oc2ccc(N)cc2F)cc1</smiles>

1,3-dioxoisoindolin-2-yl 2-(4-(4-cyano-2-fluorophenoxy)phenoxy)propanoate (S30)<smiles>CC(Oc1ccc(Oc2ccc(N=[W])cc2F)cc1)C(=O)O</smiles>

To a 100-mL flask with a stir bar was added cyhalofop butyl (butyl (R)-2-[4-(4-cyano-2fluorophenoxy)phenoxy]propionate, $1.027 \mathrm{~g}, 2.8 \mathrm{mmol}, 1$ equiv.) and $14 \mathrm{~mL}$ methanol $(0.2 \mathrm{M})$. Then $1.5 \mathrm{~mL} 6 \mathrm{M}$ $\mathrm{NaOH}$ (3.2 equiv.) was added in one portion and the reaction was stirred at room temperature overnight. The reaction mixture was then concentrated and extracted with $20 \mathrm{~mL}$ of ether three times. The aqueous layer was then acidified to $\mathrm{pH} 1$ and extracted with $30 \mathrm{~mL}$ DCM three times. The organic layers were washed with water, brine and dried over $\mathrm{Na}_{2} \mathrm{SO}_{4}$ and concentrated to yield a cloudy-white solid. The solid was then purified by column chromatography with $10 \%-20 \% \mathrm{MeOH}: \mathrm{DCM}$ to afford quantitative yield of ().

1H NMR (500 MHz, Chloroform- $d$ ): $\delta 7.46(\mathrm{dd}, J=10.1,2.0 \mathrm{~Hz}, 1 \mathrm{H}), 7.35(\mathrm{dt}, J=8.5,1.7 \mathrm{~Hz}, 1 \mathrm{H}), 7.02(\mathrm{~d}, J=$ $9.1 \mathrm{~Hz}, 2 \mathrm{H}), 6.94(\mathrm{~d}, J=9.1 \mathrm{~Hz}, 2 \mathrm{H}), 6.89(\mathrm{t}, J=8.3 \mathrm{~Hz}, 1 \mathrm{H}), 4.80(\mathrm{q}, J=6.8 \mathrm{~Hz}, 1 \mathrm{H}), 1.69(\mathrm{~d}, J=6.8 \mathrm{~Hz}, 3 \mathrm{H})$. 19F NMR (282 MHz, Chloroform- $d)$ : $\delta-129.84(\mathrm{t}, J=9.3 \mathrm{~Hz})$. 


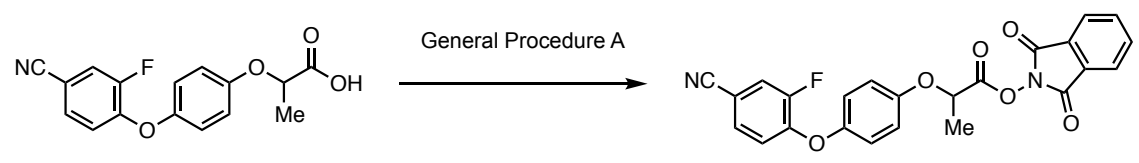

Synthesized according to general procedure A with the acid from the previous step (1.00 g, $3.32 \mathrm{mmol}, 1$ equiv.), $N$ hydroxyphthalimide ( $0.7356 \mathrm{~g}, 4.5 \mathrm{mmol}, 1.36$ equiv), DMAP $(18.6 \mathrm{mg}, 0.15 \mathrm{mmol}, 0.048$ equiv) and DIC ( $0.66 \mathrm{~mL}$, $4.26 \mathrm{mmol}, 1.28$ equiv.). The reaction mixture turned golden orange over the reaction period ( $72 \mathrm{hrs}$ ). Purified over silica with flash chromatography (10 to $30 \%$ EtOAc in hexanes) to afford the title compound as a white solid ( 0.6535 g, $1.46 \mathrm{mmol} 49 \%)$.

1H NMR (500 MHz, Chloroform- $d$ ): $\delta 7.89(\mathrm{dd}, J=5.5,3.1 \mathrm{~Hz}, 2 \mathrm{H}), 7.81(\mathrm{dd}, J=5.5,3.0 \mathrm{~Hz}, 2 \mathrm{H}), 7.46(\mathrm{dd}, J=$ $10.1,2.0 \mathrm{~Hz}, 1 \mathrm{H}), 7.35(\mathrm{dt}, J=8.6,1.6 \mathrm{~Hz}, 1 \mathrm{H}), 7.06(\mathrm{~d}, J=1.7 \mathrm{~Hz}, 4 \mathrm{H}), 6.91(\mathrm{t}, J=8.3 \mathrm{~Hz}, 1 \mathrm{H}), 5.09(\mathrm{q}, J=6.8 \mathrm{~Hz}$, $1 \mathrm{H}), 1.89(\mathrm{~d}, J=6.8 \mathrm{~Hz}, 3 \mathrm{H})$.

${ }^{13}$ C NMR (126 MHz, Chloroform- $\left.d\right): \delta 168.64,161.69,154.53,152.51(\mathrm{~d}, J=252.0 \mathrm{~Hz}), 150.59(\mathrm{~d}, J=10.6 \mathrm{~Hz})$, $149.32,135.13,129.55(\mathrm{~d}, J=3.9 \mathrm{~Hz}), 128.91,124.27,121.41,120.70(\mathrm{~d}, J=21.2 \mathrm{~Hz}), 118.84,117.87(\mathrm{~d}, J=2.6$ $\mathrm{Hz}), 117.20,106.19(\mathrm{~d}, J=8.1 \mathrm{~Hz}), 71.77,19.08$.

${ }^{19}$ F NMR (282 MHz, $\left.\mathbf{C D C l}_{3}\right): \delta-130.00(\mathrm{t}, J=9.4 \mathrm{~Hz})$.

HRMS: (ESI-TOF) calculated for $\mathrm{C}_{24} \mathrm{H}_{15} \mathrm{FN}_{2} \mathrm{NaO}_{6}{ }^{+}\left([\mathrm{M}+\mathrm{Na}]^{+}\right): 469.08064$, found 469.0803 .

FTIR (thin film, $\left.\mathbf{c m}^{-1}\right): v 2232(\mathrm{w}), 1819(\mathrm{w}), 1788(\mathrm{~m}), 1740(\mathrm{~s}), 1614(\mathrm{w}), 1584(\mathrm{w}), 1496(\mathrm{~s}), 1467$ (m), $1424(\mathrm{w})$, $1358(\mathrm{w}), 1282(\mathrm{~s}), 1243(\mathrm{~m}), 1218(\mathrm{~s}), 1185(\mathrm{~s}), 1114(\mathrm{~s}), 1069$ (s), 1037 (s), 962 (s), 945 (s), 875 (s), 841 (s), 803 (m), $785(\mathrm{~s}), 693(\mathrm{~s}), 611(\mathrm{~s}), 565(\mathrm{~m}), 535(\mathrm{~m}), 517(\mathrm{~s}), 460(\mathrm{~m}), 409(\mathrm{~m})$.

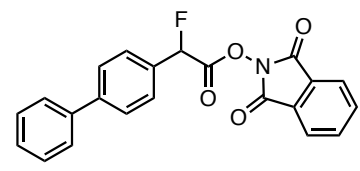

\section{1,3-dioxoisoindolin-2-yl 2-([1,1'-biphenyl]-4-yl)-2-fluoroacetate (S31)}<smiles>O=C(O)Cc1ccc(-c2ccccc2)cc1</smiles>
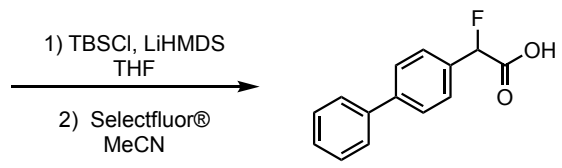

2-([1,1'-biphenyl]-4-yl)-2-fluoroacetic acid was prepared according to the procedure from Gouverneur. ${ }^{11}$ To an ovendried 100-mL flask equipped with a stir bar was added 4-biphenylacetic acid (2.1984 g, $10.36 \mathrm{mmol}, 1$ equiv.) and $3.77 \mathrm{~g}$ TBSCl (3.77 g, $25 \mathrm{mmol}, 2.4$ equiv.). The flask was septa capped and put under vacuum and refilled with nitrogen three times. THF $\left(10 \mathrm{~mL}\right.$, anhydrous) was added to the flask and the solution was stirred at $0^{\circ} \mathrm{C}$. Then, LiHMDS ( $22 \mathrm{~mL}, 1 \mathrm{M}$ solution in THF, $22 \mathrm{mmol}$ ) was added dropwise over 20 minutes and the reaction mixture turned from cloudy yellow to a clear brown solution overnight. The resulting solution was concentrated in vacuo and the crude bis-silylketenacetal was dissolved in hexanes $(50 \mathrm{~mL})$ and the $\mathrm{LiCl}$ was filtered off. The solid was washed with an additional $50 \mathrm{~mL}$ hexane and the filtrate was concentrated in vacuo. The residue was redissolved in $20 \mathrm{~mL}$ acetonitrile and added dropwise to a solution of Selectfluor $(4.8 \mathrm{~g}, 13.5 \mathrm{mmol}, 1.3$ equiv.) in acetonitrile $(40 \mathrm{~mL})$ at room temperature. The solution was stirred at room temperature overnight. The reaction was then poured into $100 \mathrm{~mL}$ $1 \mathrm{M} \mathrm{HCl}$ and extracted with $100 \mathrm{~mL} \mathrm{Et}_{2} \mathrm{O}$ twice. The combined ether layers were extracted with $60 \mathrm{~mL} 1 \mathrm{M} \mathrm{NaOH}$ twice and the combined basic layers were washed with $200 \mathrm{~mL} \mathrm{Et}_{2} \mathrm{O}$ twice. The basic aqueous layer was acidified to pH 1 with $6 \mathrm{M} \mathrm{HCl}$, then extracted with $200 \mathrm{~mL} \mathrm{Et}_{2} \mathrm{O}$ twice. The combined ether layers were dried over $\mathrm{Na}_{2} \mathrm{SO}_{4}$ and concentrated under reduced pressure. The crude residue was used directly for phthalimide ester synthesis.

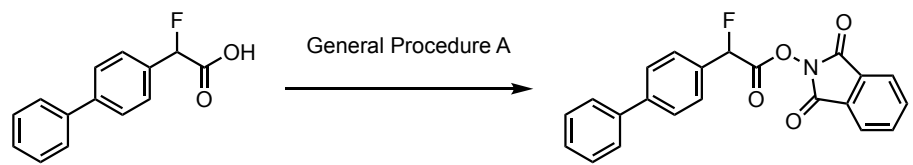

Prepared according to general procedure A, using the crude residue from above, $N$-hydroxyphthalimide $(2.44 \mathrm{~g}, 15.0$ mmol, 1.4 equiv.), DMAP (68.2 mg, $0.56 \mathrm{mmol}, 0.05$ equiv.), and DIC ( $2.0 \mathrm{~mL}, 12.9 \mathrm{~mol}, 1.25$ equiv.). The reaction started turned an orange that faded to a pale yellow as the reaction proceeded. The residue was purified by (10 to $30 \%$ EtOAc in hexanes). This afforded $1.256 \mathrm{~g}$ of 1,3-dioxoisoindolin-2-yl 2-([1,1'-biphenyl]-4-yl)-2-fluoroacetate as an off-yellow solid with some trace impurities. $1.055 \mathrm{~g}$ of the compound was recrystallized from $80 \mathrm{~mL} \mathrm{Et}{ }_{2} \mathrm{O}$ at $50^{\circ} \mathrm{C}$ 
and left in a freezer for 24 hours. The mother liquor was removed and the solid was washed with cold ether and dried to yield the title compound as an off-white solid $(663.5 \mathrm{mg}, 1.77 \mathrm{mmol}, 18 \%$ overall yield).

${ }^{1}$ H NMR (500 MHz, Chloroform- $\left.d\right): \delta 7.90(\mathrm{dd}, J=5.5,3.1 \mathrm{~Hz}, 2 \mathrm{H}), 7.81(\mathrm{dd}, J=5.5,3.1 \mathrm{~Hz}, 2 \mathrm{H}), 7.73(\mathrm{~d}, J=8.3$ $\mathrm{Hz}, 2 \mathrm{H}), 7.69(\mathrm{dd}, J=8.3,1.6 \mathrm{~Hz}, 2 \mathrm{H}), 7.62(\mathrm{dd}, J=8.3,1.3 \mathrm{~Hz}, 2 \mathrm{H}), 7.47(\mathrm{t}, J=7.7 \mathrm{~Hz}, 2 \mathrm{H}), 7.42-7.36(\mathrm{~m}, 1 \mathrm{H})$, $6.24(\mathrm{~d}, J=47.2 \mathrm{~Hz}, 1 \mathrm{H})$.

13 C NMR (126 MHz, Chloroform-d): $\delta 165.29$ (d, $J=29.5 \mathrm{~Hz}), 161.41,143.75$ (d, $J=2.9 \mathrm{~Hz}), 140.22,135.15$, $131.19(\mathrm{~d}, J=19.9 \mathrm{~Hz}), 129.04,128.88,128.27(\mathrm{~d}, J=5.0 \mathrm{~Hz}), 128.04,128.03,127.41,124.32,88.17(\mathrm{~d}, J=189.2$ $\mathrm{Hz})$.

19F NMR (282 MHz, Chloroform- $d$ ): $\delta-179.17(\mathrm{~d}, J=47.6 \mathrm{~Hz})$.

HRMS: (ESI-TOF) calculated for $\mathrm{C}_{22} \mathrm{H}_{14} \mathrm{FNNaO}_{4}{ }^{+}\left([\mathrm{M}+\mathrm{Na}]^{+}\right)$: 398.07991, found 398.0797.

FTIR (thin film, $\left.\mathbf{c m}^{-1}\right): v 3102(\mathrm{w}), 3045(\mathrm{w}), 2361(\mathrm{~m}), 2339(\mathrm{~m}), 1819(\mathrm{w}), 1791(\mathrm{w}), 1742(\mathrm{~m}), 1610(\mathrm{w}), 1487$ (m), $1464(\mathrm{~s}), 1409(\mathrm{~m}), 1358(\mathrm{~m}), 1294(\mathrm{~m}), 1185(\mathrm{~m}), 1171(\mathrm{~m}), 1112(\mathrm{~m}), 1080(\mathrm{w}), 1047(\mathrm{~m}), 1007(\mathrm{w}), 960(\mathrm{~m})$, $931(\mathrm{w}), 873(\mathrm{~m}), 839(\mathrm{w}), 792(\mathrm{~m}), 754(\mathrm{w}), 695(\mathrm{w}), 668(\mathrm{w}), 624(\mathrm{w}), 594(\mathrm{~m}), 551(\mathrm{~m}), 528(\mathrm{~m}), 517(\mathrm{w}), 491(\mathrm{w})$, $436(w), 408(w)$.

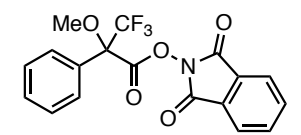

1,3-dioxoisoindolin-2-yl 3,3,3-trifluoro-2-methoxy-2-phenylpropanoate (S32)

Prepared according to general procedure B, using Moshers's acid (3,3,3-trifluoro-2-methoxy-2-phenylpropanoic acid, $0.9667 \mathrm{~g}, 4.13 \mathrm{mmol}, 1$ equiv.), DMF (5 drops), and oxalyl chloride ( $0.6 \mathrm{~mL}, 7.09 \mathrm{mmol}, 1.72$ equivalent), followed by $N$-hydroxyphthalimide $(0.7429 \mathrm{~g}, 4.55 \mathrm{mmol}, 1.10$ equiv. $)$ and triethylamine $(0.6 \mathrm{~mL}, 4.30 \mathrm{mmol}, 1.04$ equiv. $)$ Purified by column chromatrography (10-25\% EtOAc in hexanes). This afforded the title compound as a white solid $(1.1070 \mathrm{~g}, 71 \%, 2.92 \mathrm{mmol})$.

1H NMR (500 MHz, Chloroform- $d$ ): $7.92(\mathrm{dd}, J=5.5,3.1 \mathrm{~Hz}, 2 \mathrm{H}), 7.83(\mathrm{dd}, J=5.5,3.1 \mathrm{~Hz}, 2 \mathrm{H}), 7.75-7.68(\mathrm{~m}$, $2 \mathrm{H}), 7.53-7.46(\mathrm{~m}, 3 \mathrm{H}), 3.82(\mathrm{q}, J=1.4 \mathrm{~Hz}, 3 \mathrm{H})$.

${ }^{13}$ C NMR (126 MHz, Chloroform-d): $\delta$ 163.96, 161.43, 135.22, 131.01, 130.43, 128.90, 128.74, 127.57, 124.35, $122.80(\mathrm{q}, J=289.0 \mathrm{~Hz}), 85.02(\mathrm{q}, J=28.9 \mathrm{~Hz}), 56.66(\mathrm{q}, J=1.5 \mathrm{~Hz})$.

19F NMR (282 MHz, Chloroform- $d$ ): $\delta$-72.31(s, 3F).

HRMS: (ESI-TOF) calculated for $\mathrm{C}_{18} \mathrm{H}_{12} \mathrm{FNNaO}_{5}{ }^{+}\left([\mathrm{M}+\mathrm{Na}]^{+}\right)$: 402.05598, found 402.05513 .

FTIR (thin film, $\left.\mathbf{~ c m}^{-1}\right): v 408.3(\mathrm{~m}), 516.2(\mathrm{~s}), 592.1(\mathrm{~m}), 640.9(\mathrm{~m}), 692.2(\mathrm{~s}), 729.8(\mathrm{~s}), 764.7(\mathrm{~s}), 788.8(\mathrm{~m}), 838.1$ $(\mathrm{m}), 873.4(\mathrm{~s}), 900.7(\mathrm{~m}), 926.1(\mathrm{~m}), 981.8(\mathrm{~s}), 1000.8(\mathrm{~m}), 1016.3(\mathrm{~m}), 1036.0(\mathrm{~m}), 1079.2(\mathrm{~s}), 1117.2(\mathrm{~s}), 1155.6(\mathrm{~s})$, $1167.5(\mathrm{~s}), 1184.0(\mathrm{~s}), 1228.5(\mathrm{~m}), 1267.6(\mathrm{~m}), 1289.8(\mathrm{~m}), 1324.3(\mathrm{w}), 1353.2(\mathrm{~m}), 1452.4(\mathrm{~m}), 1469.3(\mathrm{~m}), 1495.4$ (w), $1608.5(\mathrm{w}), 1738.4(\mathrm{~s}), 1789.3(\mathrm{~m}), 1815.2(\mathrm{~m}), 2850.3(\mathrm{w}), 2956.8(\mathrm{w})$.

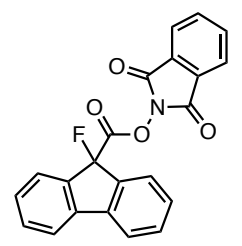

\section{1,3-dioxoisoindolin-2-yl 9-fluoro-9H-fluorene-9-carboxylate (S33)}

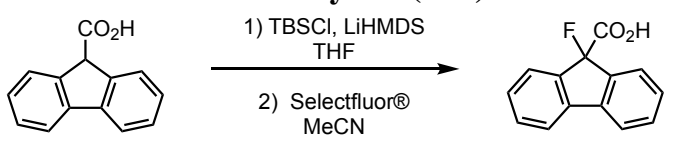

9-fluoro-9H-fluorene-9-carboxylic acid was prepared according to the procedure from Gouverneur. ${ }^{11}$ To an ovendried 100-mL flask with a stir bar was added fluorene-9-carboxylic acid $(2.1628 \mathrm{~g}, 10.3 \mathrm{mmol}, 1$ equiv.) and $3.487 \mathrm{~g}$ $\mathrm{TBSCl}(3.487 \mathrm{~g}, 23.1 \mathrm{mmol})$. The flask was septa capped and put under vacuum and refilled with nitrogen three times. $10 \mathrm{~mL}$ of anhydrous THF was added to the flask and the solution was stirred at $0^{\circ} \mathrm{C}$. Then, $22 \mathrm{~mL}$ of LiHMDS $(1 \mathrm{M}$ solution in THF, $22 \mathrm{mmol}$ ) was added dropwise over 20 minutes and the reaction mixture turned from cloudy yellow to a clear brown solution overnight. The resulting solution was concentrated in vacuo and the crude bis-silylketenacetal 
was dissolved in $50 \mathrm{~mL}$ hexane and the $\mathrm{LiCl}$ was filtered off. The solid was washed with $50 \mathrm{~mL}$ hexane and the filtrate was concentrated under reduced pressure. The residue was dissolved in $20 \mathrm{~mL}$ acetonitrile and added dropwise to a solution of Selectfluor $(4.6 \mathrm{~g}, 13 \mathrm{mmol})$ in acetonitrile $(40 \mathrm{~mL})$ at room temperature. The solution was stirred at room temperature overnight. The reaction was then poured into $100 \mathrm{~mL} 1 \mathrm{M} \mathrm{HCl}$ and extracted with $100 \mathrm{~mL} \mathrm{Et}_{2} \mathrm{O}$ twice. The combined ether layers were extracted with $60 \mathrm{~mL} 1 \mathrm{M} \mathrm{NaOH}$ twice and the combined basic layers were washed with $200 \mathrm{~mL} \mathrm{Et}_{2} \mathrm{O}$ twice. The basic aqueous layer was acidified to $\mathrm{pH} 1$ with $6 \mathrm{M} \mathrm{HCl}$, then extracted with $200 \mathrm{~mL}$ $\mathrm{Et}_{2} \mathrm{O}$ twice. The combined ether layers were dried over $\mathrm{Na}_{2} \mathrm{SO}_{4}$ and concentrated under reduced pressure. The crude residue was used directly for phthalimide ester synthesis.

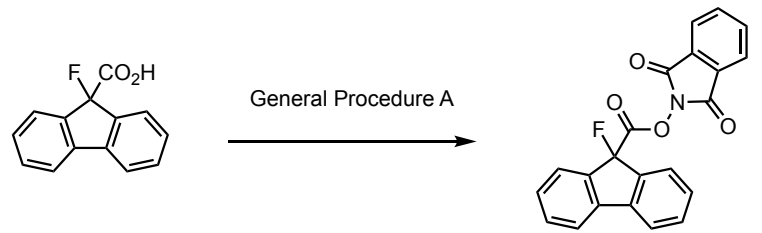

Prepared according to general procedure A, using the crude residue from above, $N$-hydroxyphthalimide (1.64 g, 10.1 mmol, 0.98 equiv.), DMAP (66.8 mg, $0.55 \mathrm{mmol}, 0.05$ equiv.), and DIC (2.2 mL, $15.2 \mathrm{~mol}, 1.4$ equiv.). The reaction started turned an orange that faded to a pale yellow as the reaction proceeded. The residue was purified by ( 10 to $30 \%$ EtOAc in hexanes). This afforded 1,3-dioxoisoindolin-2-yl 9-fluoro-9H-fluorene-9-carboxylate as an off-yellow solid with some trace impurities. The compound was recrystallized from $60 \mathrm{~mL} \mathrm{Et}_{2} \mathrm{O}$ and $5 \mathrm{~mL} \mathrm{DCM}$ at $45^{\circ} \mathrm{C}$ and left in a freezer for 24 hours. Then, $7 \mathrm{~mL}$ hexane was added to the solution to crash out any remaining phthalimide ester. The mother liquor was removed and the solid was washed with cold ether and dried to yield the title compound as a lightyellow solid (660.7 mg, $1.77 \mathrm{mmol}, 18 \%)$.

1H NMR (500 MHz, Chloroform- $d): \delta 7.84(\mathrm{dd}, J=5.5,3.1 \mathrm{~Hz}, 2 \mathrm{H}), 7.79-7.74(\mathrm{~m}, 4 \mathrm{H}), 7.68(\mathrm{~d}, J=7.5 \mathrm{~Hz}, 2 \mathrm{H})$, $7.53(\mathrm{td}, J=7.6,1.4 \mathrm{~Hz}, 2 \mathrm{H}), 7.42(\mathrm{t}, J=7.6 \mathrm{~Hz}, 2 \mathrm{H})$.

${ }^{13}$ C NMR (126 MHz, Chloroform- $\left.d\right)$ : $\delta 166.55(\mathrm{~d}, J=36.2 \mathrm{~Hz}), 161.31,141.43(\mathrm{~d}, J=2.4 \mathrm{~Hz}), 139.24(\mathrm{~d}, J=18.7$ $\mathrm{Hz}), 135.03,131.99(\mathrm{~d}, J=2.1 \mathrm{~Hz}), 129.05$ (d, $J=1.9 \mathrm{~Hz}), 128.87,125.59,124.17,120.91,97.07$ (d, $J=197.0 \mathrm{~Hz})$.

${ }^{19}$ F NMR (282 MHz, CDCl 3 ): $\delta-160.39(\mathrm{~s}, 1 \mathrm{~F})$.

HRMS: (ES-TOF) calculated for $\mathrm{C}_{22} \mathrm{H}_{12} \mathrm{FNO}_{4}{ }^{+}\left(\left[\mathrm{M}^{*}\right]\right): 373.07449$, found 373.07426 .

FTIR (thin film, cm-1): $v 3339$ (w), 3052 (w), 2967 (w), 1817 (s), 1788 (s), 1746 (s), 1653 (w), 1608 (m), 1572 (m), $1488(\mathrm{w}), 1466(\mathrm{~m}), 1451(\mathrm{~s}), 1419(\mathrm{w}), 1360(\mathrm{~m}), 1325(\mathrm{~m}), 1292(\mathrm{w}), 1233(\mathrm{~m}), 1205(\mathrm{~m}), 1187(\mathrm{~m}), 1160(\mathrm{~s}), 1128$ (s), $1106(\mathrm{~m}), 1079(\mathrm{~m}), 1034(\mathrm{~s}), 973(\mathrm{~s}), 950(\mathrm{~s}), 938(\mathrm{~s}), 930(\mathrm{~s}), 873(\mathrm{~s}), 829(\mathrm{~m}), 781(\mathrm{~m}), 764(\mathrm{~s}), 748(\mathrm{~s}), 726(\mathrm{~s})$, 708 (s), 688 (s), 619 (s), 595 (s), 575 (s), 515 (s), 427 (s), 416 (s).

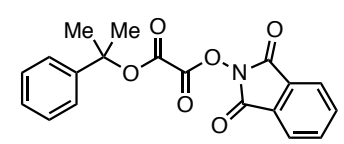

\section{1,3-dioxoisoindolin-2-yl (2-phenylpropan-2-yl) oxalate (S34)}

The phthalimidoyl oxalate was prepared according to literature precedent from Overman. ${ }^{12}$ To an oven-dried $100-\mathrm{mL}$ flask equipped with a stir bar with was added 2-phenyl-2-propanol (430 $\mu \mathrm{L}, 3.07 \mathrm{mmol}, 1$ equiv.), DMAP (43.1 mg, $0.35 \mathrm{mmol}, 0.11$ equiv.), and triethylamine $(0.85 \mathrm{~mL}, 6.10 \mathrm{mmol}, 2.0$ equiv.). Then $50 \mathrm{~mL}$ of a $0.06 \mathrm{M}$ solution of chloro- $N$-phthalimidoyl oxalate in THF (3.0 mmol, 0.98 equiv.) was added dropwise. The resulting mixture was stirred overnight at room temperature. The volatiles were removed under reduced pressure and the crude mixture was dissolved in $25 \mathrm{~mL}$ dichloromethane and transferred to an Erlenmeyer flask containing $400 \mathrm{~mL}$ hexanes. Brown solid crashed out immediately and the solution was filtered over a frit and washed with hexanes $(4 \mathrm{x} 25 \mathrm{~mL})$. The filtrate was concentrated to yield the title compound as an off yellow-white solid $(0.9612 \mathrm{~g}, 2.72 \mathrm{mmol}, 89 \%, 62 \% \mathrm{molar}$ purity contaminated with 2-phenyl-2-propanol).

1H NMR (500 MHz, Chloroform- $d$ ): $\delta 7.93(\mathrm{dd}, J=5.5,3.1 \mathrm{~Hz}, 2 \mathrm{H}), 7.83(\mathrm{dd}, J=5.5,3.1 \mathrm{~Hz}, 2 \mathrm{H}), 7.47(\mathrm{dd}, J=$ $8.4,1.3 \mathrm{~Hz}, 2 \mathrm{H}), 7.41-7.37(\mathrm{~m}, 2 \mathrm{H}), 7.30-7.34(\mathrm{~m}, 1 \mathrm{H}), 1.96(\mathrm{~s}, 6 \mathrm{H})$.

${ }^{13}$ C NMR (126 MHz, Chloroform- $\left.d\right)$ : $\delta \quad 161.06,154.33,152.78,143.41,135.21,128.87,128.78,128.08,124.64$, $124.42,88.26,28.25$. 
FTIR (thin film, cm-1): $v 2956(\mathrm{w}), 2850(\mathrm{w}), 1815(\mathrm{~m}), 1789(\mathrm{~m}), 1738(\mathrm{~s}), 1609(\mathrm{w}), 1495(\mathrm{w}), 1469$ (m), 1452 (m), $1353(\mathrm{~m}), 1324(\mathrm{w}), 1290(\mathrm{~m}), 1268(\mathrm{~m}), 1229(\mathrm{~m}), 1184$ (s), $1168(\mathrm{~s}), 1156(\mathrm{~s}), 1117$ (s), $1079(\mathrm{~s}), 1036(\mathrm{~m})$, $1016(\mathrm{~m}), 1001(\mathrm{~m}), 982(\mathrm{~s}), 926(\mathrm{~m}), 901(\mathrm{~m}), 873(\mathrm{~s}), 838(\mathrm{~m}), 789(\mathrm{~m}), 765(\mathrm{~s}), 730(\mathrm{~s}), 692(\mathrm{~s}), 641(\mathrm{~m}), 592(\mathrm{~m})$, $516(\mathrm{~s}), 408(\mathrm{~m})$.

This phthalimidoyl oxalate proved too unstable to obtain HRMS data via ESI.

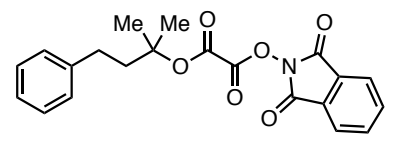

1,3-dioxoisoindolin-2-yl (2-methyl-4-phenylbutan-2-yl) oxalate (S35)

The phthalimidoyl oxalate was prepared according to literature precedent from Overman. ${ }^{12}$ To an oven-dried 100-mL flask with a stir bar was added 2-methyl-4-phenyl-2-butanol (1.00 mL, $5.88 \mathrm{mmol}, 1$ equiv.), DMAP ( $80.5 \mathrm{mg}, 0.66$ mmol, 0.11 equiv.), and triethylamine ( $1.7 \mathrm{~mL}, 12.2 \mathrm{mmol}, 2.1$ equiv.). Then $200 \mathrm{~mL}$ of a $0.06 \mathrm{M}$ solution of chloro$\mathrm{N}$-phthalimidoyl oxalate in THF was added dropwise (12 mmol, 2.0 equiv.). The resulting mixture was stirred overnight at room temperature. The volatiles were removed under reduced pressure and the crude mixture was dissolved in $60 \mathrm{~mL}$ dichloromethane and transferred to an Erlenmeyer flask containing $600 \mathrm{~mL}$ hexanes. A brown precipitate formed immediately and the suspension was filtered through frit. The filtered solids were washed with hexanes $(4 \times 50 \mathrm{~mL})$. The filtrate was concentrated to yield the title compound as a brown-yellow oil $(2.13 \mathrm{~g}, 5.58$ mmol, 95\%).

${ }^{1}$ H NMR (500 MHz, Chloroform- $d$ ): $\delta 7.93(\mathrm{dd}, J=5.5,3.1 \mathrm{~Hz}, 1 \mathrm{H}), 7.83(\mathrm{dd}, J=5.5,3.1 \mathrm{~Hz}, 1 \mathrm{H}), 7.31-7.27$ (m, $2 \mathrm{H}), 7.23-7.17(\mathrm{~m}, 3 \mathrm{H}), 2.77-2.72(\mathrm{~m}, 2 \mathrm{H}), 2.21-2.17(\mathrm{~m}, 2 \mathrm{H}), 1.69(\mathrm{~s}, 6 \mathrm{H})$.

${ }^{13}$ C NMR (126 MHz, Chloroform-d): $\delta 161.07,154.45,153.16,141.36,135.19,128.85,128.64,128.48,126.19$, $124.39,89.41,42.84,30.34,25.74$.

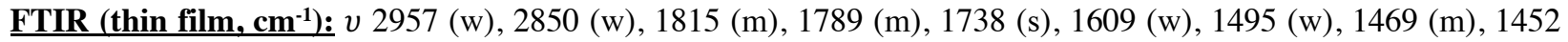
(m), $1353(\mathrm{~m}), 1324(\mathrm{w}), 1290(\mathrm{~m}), 1268(\mathrm{~m}), 1229(\mathrm{~m}), 1184(\mathrm{~s}), 1168(\mathrm{~s}), 1156(\mathrm{~s}), 1117$ (s), $1079(\mathrm{~s}), 1036(\mathrm{~m})$, $1016(\mathrm{~m}), 1001(\mathrm{~m}), 982(\mathrm{~s}), 926(\mathrm{~m}), 901(\mathrm{~m}), 873(\mathrm{~s}), 838(\mathrm{~m}), 789(\mathrm{~m}), 765(\mathrm{~s}), 730(\mathrm{~s}), 692(\mathrm{~s}), 641(\mathrm{~m}), 592(\mathrm{~m})$, $516(\mathrm{~s}), 408(\mathrm{~m})$.

This phthalimidoyl oxalate proved too unstable to obtain HRMS data via ESI. 


\section{Isolation and characterization of product compounds}<smiles>COc1ccc2cc(C(C)F)ccc2c1</smiles>

2-(1-fluoroethyl)-6-methoxynaphthalene (2)

Synthesized according to general procedure $\mathrm{E}$ with 1,3-dioxoisoindolin-2-yl 2-(6-methoxynaphthalen-2-yl)propanoate $\left(0.2276 \mathrm{~g}, 0.606 \mathrm{mmol}, 1\right.$ equiv.), $\operatorname{Ir}(\mathrm{dF}-\mathrm{ppy})_{3}(3.8 \mathrm{mg}, 0.006 \mathrm{mmol}, 0.01$ equiv.), triethylamine trihydrofluoride (293.4 $\mu \mathrm{L}, 1.8 \mathrm{mmol}, 3$ equiv.), and DCM (3.00 mL, 0.2 M). Purified over silica with flash chromatography (10\% ether in pentanes) to afford the title compound as a white solid $(111.3 \mathrm{mg}, 0.545 \mathrm{mmol}, 90 \%)$. A second run with 1,3dioxoisoindolin-2-yl 2-(6-methoxynaphthalen-2-yl)propanoate ( $0.2255 \mathrm{~g}, 0.601 \mathrm{mmol}, 1$ equiv.), $\operatorname{Ir}(\mathrm{dF}-\mathrm{ppy})_{3}(3.6 \mathrm{mg}$, $0.006 \mathrm{mmol}, 0.01$ equiv.), triethylamine trihydrofluoride $(293.4 \mu \mathrm{L}, 1.8 \mathrm{mmol}, 3$ equiv.), and DCM (3 $\mathrm{mL}, 0.2 \mathrm{M})$ afforded $110.0 \mathrm{mg}(90 \%, 0.539 \mathrm{mmol})$.

\section{Scale up protocol:}

Synthesized according to general procedure $\mathrm{E}$ with 1,3-dioxoisoindolin-2-yl 2-(6-methoxynaphthalen-2-yl)propanoate (1.481 g, $3.95 \mathrm{~mol}, 1$ equiv.), $\mathrm{Ir}(\mathrm{dF}-\mathrm{ppy})_{3}(30.6 \mathrm{mg}, 0.04 \mathrm{mmol}, 0.01$ equiv.), triethylamine trihydrofluoride $(978 \mu \mathrm{L}$, $6.0 \mathrm{mmol}, 1.5$ equiv.), and DCM (10.00 mL, $0.4 \mathrm{M})$. Purified over silica with flash chromatography (3\% ether in hexanes) to afford the title compound as a white solid (600.2 $\mathrm{mg}, 2.94 \mathrm{mmol}, 75 \%)$.

1H NMR (500 MHz, Chloroform-d): $\delta 7.77-7.72(\mathrm{~m}, 3 \mathrm{H}), 7.45(\mathrm{dd}, J=8.5,1.8 \mathrm{~Hz}, 1 \mathrm{H}), 7.17$ (dd, $J=8.8,2.5 \mathrm{~Hz}$, $1 \mathrm{H}), 7.14(\mathrm{~d}, J=2.5 \mathrm{~Hz}, 1 \mathrm{H}), 5.76(\mathrm{dq}, J=47.6,6.4 \mathrm{~Hz}, 1 \mathrm{H}), 3.93(\mathrm{~s}, 3 \mathrm{H}), 1.73(\mathrm{dd}, J=23.7,6.4 \mathrm{~Hz}, 3 \mathrm{H})$.

${ }^{13}$ C NMR (126 MHz, Chloroform- $\left.d\right): \delta 158.08,136.61(\mathrm{~d}, J=19.4 \mathrm{~Hz}), 134.55(\mathrm{~d}, J=1.6 \mathrm{~Hz}), 129.72,128.64$, $127.34,124.40(\mathrm{~d}, J=7.7 \mathrm{~Hz}), 123.94(\mathrm{~d}, J=5.4 \mathrm{~Hz}), 119.31,105.79,91.38(\mathrm{~d}, J=166.9 \mathrm{~Hz}), 55.47,22.99(\mathrm{~d}, J=$ $25.5 \mathrm{~Hz})$.

${ }^{19}$ F NMR (376 MHz, Chloroform- $d$ ): $-164.98(\mathrm{dq}, J=47.6,23.6 \mathrm{~Hz})$.

HRMS: (EI-TOF) calculated for $\mathrm{C}_{13} \mathrm{H}_{13} \mathrm{FO}^{+}\left[\mathrm{M}^{\circ}\right]$ : 204.09449, found 204.09542 .

FTIR (thin film, cm$\left.^{-1}\right): v 2962(\mathrm{w}), 2933(\mathrm{w}), 1633(\mathrm{~m}), 1605(\mathrm{~s}), 1505(\mathrm{~m}), 1486(\mathrm{~s}), 1462(\mathrm{~m}), 1391(\mathrm{~m}), 1374(\mathrm{~m})$, $1353(\mathrm{~m}), 1314(\mathrm{~m}), 1266(\mathrm{~m}), 1237(\mathrm{~m}), 1218(\mathrm{~m}), 1196(\mathrm{~m}), 1177$ (s), $1162(\mathrm{~s}), 1063(\mathrm{~m}), 1028(\mathrm{~s}), 993(\mathrm{~m}), 961$ $(\mathrm{m}), 929(\mathrm{~m}), 900(\mathrm{~m}), 870(\mathrm{~m}), 853(\mathrm{~s}), 816(\mathrm{~s}), 751(\mathrm{~m}), 672(\mathrm{~m})$.

\section{1-(tert-butyl)-4-(fluoromethyl)benzene (7)}

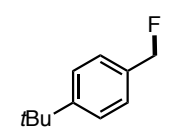

Synthesized according to general procedure E with 1,3-dioxoisoindolin-2-yl 2-(4-(tert-butyl)phenyl)acetate (0.2730 $\mathrm{g}, 0.809 \mathrm{mmol}, 1$ equiv.), $\mathrm{Ir}(\mathrm{dF}-\mathrm{ppy})_{3}(4.2 \mathrm{mg}, 0.008 \mathrm{mmol}, 0.01$ equiv.), triethylamine trihydrofluoride $(391.2 \mu \mathrm{L}$, $2.4 \mathrm{mmol}, 3$ equiv), and DCM (4.00 mL, 0.2 M). Purified by preparatory TLC (10\% ether in pentanes) to afford the title compound as a slightly yellow oil $(89.8 \mathrm{mg}, 0.540 \mathrm{mmol}, 67 \%)$. A second run with 1,3-dioxoisoindolin-2-yl 2(4-(tert-butyl)phenyl)acetate (277.5 mg, $0.823 \mathrm{mmol}, 1$ equiv.), $\mathrm{Ir}(\mathrm{dF}-\mathrm{ppy})_{3}(4.2 \mathrm{mg}, 0.008 \mathrm{mmol}, 0.01 \mathrm{equiv}$.), triethylamine trihydrofluoride (391.2 $\mu \mathrm{L}, 2.4 \mathrm{mmol}, 3$ equiv.), and DCM (4.00 mL, $0.2 \mathrm{M})$ afforded $83.5 \mathrm{mg}(0.502$ $\mathrm{mmol}, 61 \%)$.

${ }^{1}$ H NMR (500 MHz, Chloroform- $d$ ): $\delta 7.43(\mathrm{dd}, J=8.2,1.7 \mathrm{~Hz}, 2 \mathrm{H}), 7.34(\mathrm{dd}, J=8.3,2.0 \mathrm{~Hz}, 2 \mathrm{H}), 5.36(\mathrm{~d}, J=$ $47.5 \mathrm{~Hz}, 2 \mathrm{H}), 1.34(\mathrm{~s}, 9 \mathrm{H})$.

${ }^{13}$ C NMR (126 MHz, Chloroform-d): $\delta 152.10(\mathrm{~d}, J=3.4 \mathrm{~Hz}), 133.34(\mathrm{~d}, J=17.1 \mathrm{~Hz}), 127.77$ (d, $\left.J=5.4 \mathrm{~Hz}\right)$, $125.70,84.71(\mathrm{~d}, J=164.9 \mathrm{~Hz}), 34.81,31.44$.

19F NMR (282 MHz, Chloroform- $d$ ): $\delta$-204.37 ( $\mathrm{t}, J=48.1 \mathrm{~Hz})$.

HRMS: (EI-TOF) calculated for $\mathrm{C}_{11} \mathrm{H}_{15} \mathrm{~F}^{+}\left[\mathrm{M}^{\circ}\right]$ : 166.11523 , found: 166.11604

FTIR (thin film, cm$\left.^{-1}\right): v 2961(\mathrm{~s}), 2902(\mathrm{~m}), 2869(\mathrm{~m}), 1616(\mathrm{w}), 1514(\mathrm{w}), 1465(\mathrm{~m}), 1411(\mathrm{w}), 1394(\mathrm{w}), 1364(\mathrm{~s})$, $1269(\mathrm{~m}), 1223(\mathrm{~m}), 1203(\mathrm{w}), 1108(\mathrm{~m}), 980(\mathrm{~s}), 851(\mathrm{~m}), 833(\mathrm{~s}), 810(\mathrm{~s}), 741(\mathrm{w}), 671(\mathrm{~m}), 572(\mathrm{~m}), 524(\mathrm{~m}), 453$ (w), $429(\mathrm{w}), 418(\mathrm{w}), 405(\mathrm{w})$. 


\section{4-(fluoromethyl)-1,1'-biphenyl (8)}

Synthesized according to general procedure $\mathrm{E}$ with 1,3-dioxoisoindolin-2-yl 2-([1,1'-biphenyl]-4-yl)acetate $(0.2878$ $\mathrm{g}, 0.805 \mathrm{mmol}, 1$ equiv.), Ir(dF-ppy) $3(5.2 \mathrm{mg}, 0.008 \mathrm{mmol}, 0.01$ equiv.), triethylamine trihydrofluoride $(391.2 \mu \mathrm{L}$, $2.4 \mathrm{mmol}, 3$ equiv.), and DCM (4.00 mL, $0.2 \mathrm{M})$. Purified over silica with flash chromatography (10\% ether in pentanes) to afford the title compound as a white solid $(85.7 \mathrm{mg}, 0.460 \mathrm{mmol}, 57 \%)$. A second run with $1,3-$ dioxoisoindolin-2-yl 2-([1,1'-biphenyl]-4-yl)acetate (0.2881 g, $0.806 \mathrm{mmol}, 1$ equiv.), $\operatorname{Ir}(\mathrm{dF}-\mathrm{ppy})_{3}(5.9 \mathrm{mg}, 0.008$ mmol, 0.01 equiv.), triethylamine trihydrofluoride $(391.2 \mu \mathrm{L}, 2.4 \mathrm{mmol}, 3$ equiv.), and DCM (4.00 mL, $0.2 \mathrm{M})$ afforded $81.9 \mathrm{mg}(0.440 \mathrm{mmol}, 55 \%)$.

1H NMR (500 MHz, Chloroform- $d$ ): $\delta 7.63(\mathrm{~d}, J=7.0 \mathrm{~Hz}, 2 \mathrm{H}), 7.60(\mathrm{~d}, J=7.1 \mathrm{~Hz}, 2 \mathrm{H}), 7.47-7.44$ (m, 4H), 7.37 $(\mathrm{t}, 7.4,2.0 \mathrm{~Hz}, 1 \mathrm{H}), 5.43(\mathrm{~d}, J=47.9 \mathrm{~Hz}, 2 \mathrm{H})$.

${ }^{13}$ C NMR (126 MHz, Chloroform- $d$ ): $141.90(\mathrm{~d}, J=3.2 \mathrm{~Hz}), 140.75,135.26(\mathrm{~d}, J=17.0 \mathrm{~Hz}), 128.98,128.22(\mathrm{~d}, J$ $=5.7 \mathrm{~Hz}), 127.69,127.52,127.31,84.56(\mathrm{~d}, J=166.0 \mathrm{~Hz})$.

${ }^{19}$ F NMR (376 MHz, Chloroform- $\left.d\right)$ : $\delta-206.32(\mathrm{t}, J=47.8 \mathrm{~Hz})$.

HRMS: (EI-TOF) calculated for $\mathrm{C}_{13} \mathrm{H}_{11} \mathrm{~F}^{+}\left[\mathrm{M}^{\circ+}\right]$ : 186.08387 , found 186.08393 .

FTIR (thin film, cm$\left.^{-1}\right): v 3057$ (w), 3034 (w), 2954 (w), $2892(\mathrm{w}), 1613(\mathrm{w}), 1566(\mathrm{w}), 1486(\mathrm{~m}), 1465$ (w), 1406 (m), $1376(\mathrm{~m}), 1222(\mathrm{~m}), 1183(\mathrm{w}), 1157(\mathrm{w}), 1125(\mathrm{w}), 1024(\mathrm{w}), 970(\mathrm{~m}), 947(\mathrm{~m}), 846(\mathrm{~m}), 821(\mathrm{~m}), 758(\mathrm{~s}), 730$ (s), $689(\mathrm{~s}), 601(\mathrm{~m}), 548(\mathrm{w}), 489(\mathrm{~m})$.

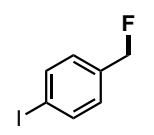

\section{1-(fluoromethyl)-4-iodobenzene (9)}

Synthesized according to general procedure $\mathrm{E}$ with 1,3-dioxoisoindolin-2-yl 2-(4-iodophenyl)acetate ( $0.3270 \mathrm{~g}, 0.803$ mmol, 1 equiv.), $\mathrm{Ir}(\mathrm{dF}-\mathrm{ppy})_{3}(3.5 \mathrm{mg}, 0.005 \mathrm{mmol}, 0.01$ equiv.), triethylamine trihydrofluoride $(391.2 \mu \mathrm{L}, 2.4 \mathrm{mmol}$, 3 equiv.), and DCM (4.00 mL, $0.2 \mathrm{M}$ ). Purified over silica with flash chromatography (5\% ether in hexanes) to afford the title compound as a white solid contaminated with $6 \mathrm{~mol} \%$ of 1,2-bis(4-iodophenyl)ethane (adjusted for bibenzyl, $109.3 \mathrm{mg}, 0.463 \mathrm{mmol}, 58 \%)$. A second run with 1,3-dioxoisoindolin-2-yl 2-(4-iodophenyl)acetate (0.2523 g, 0.620 mmol, 1 equiv.), $\operatorname{Ir}(\mathrm{dF}-\mathrm{ppy})_{3}$ ( $3.1 \mathrm{mg}, 0.004 \mathrm{mmol}, 0.01$ equiv.), triethylamine trihydrofluoride $(293.4 \mu \mathrm{L}, 1.8 \mathrm{mmol}$, 3 equiv.), and DCM (3.00 mL, $0.2 \mathrm{M})$ afforded $90.6 \mathrm{mg}$ (0.384 mmol, 62\% yield, adjusted for 6\% 1,2-bis(4iodophenyl)ethane

contaminant).

${ }^{1}$ H NMR (500 MHz, Chloroform- $d$ ): $\delta 7.73(\mathrm{~d}, J=7.9 \mathrm{~Hz}, 2 \mathrm{H}), 7.12(\mathrm{~d}, J=6.5 \mathrm{~Hz}, 2 \mathrm{H}), 5.32(\mathrm{~d}, J=47.5 \mathrm{~Hz}, 2 \mathrm{H})$. 13 C NMR (126 MHz, Chloroform- $d): \delta 137.88,135.94(\mathrm{~d}, J=17.4 \mathrm{~Hz}), 129.28(\mathrm{~d}, J=6.0 \mathrm{~Hz}), 94.63(\mathrm{~d}, J=3.7$ $\mathrm{Hz}), 83.99(\mathrm{~d}, J=167.4 \mathrm{~Hz})$.

19F NMR (376 MHz, Chloroform- $d$ ): $\delta$-208.94 (t, $J=47.4 \mathrm{~Hz})$.

HRMS: (EI-TOF) calculated for $\mathrm{C}_{7} \mathrm{H}_{6} \mathrm{FI}^{+}\left(\left[\mathrm{M}^{\circ}\right]^{+}\right): 235.94927$, found 235.95047.

Characterization data matched literature. ${ }^{13}$

\section{1-(benzyloxy)-4-(fluoromethyl)benzene (10)}

Synthesized according to general procedure E with 1,3-dioxoisoindolin-2-yl 2-(4-(benzyloxy)phenyl)acetate ( 0.2340 $\mathrm{g}, 0.604 \mathrm{mmol}, 1$ equiv.), $\mathrm{Ir}(\mathrm{dF}-\mathrm{ppy})_{3}(5.1 \mathrm{mg}, 0.006 \mathrm{mmol}, 0.01$ equiv.), triethylamine trihydrofluoride $(293.4 \mu \mathrm{L}$, $1.8 \mathrm{mmol}, 3$ equiv.), and DCM (3.00 mL, 0.2 M). Purified over silica with flash chromatography (10\% ether in pentanes) to afford the title compound as a white solid $(101.1 \mathrm{mg}, 0.468 \mathrm{mmol}, 77 \%)$. A second run with 1,3dioxoisoindolin-2-yl 2-(4-(benzyloxy)phenyl)acetate (0.2358 g, $0.609 \mathrm{mmol}, 1$ equiv.), Ir(dF-ppy) 3 (4.5 mg, 0.006 
mmol, 0.01 equiv.), triethylamine trihydrofluoride (293.4 $\mu \mathrm{L}, 1.8 \mathrm{mmol}, 3$ equiv.), and DCM (3.00 mL, $0.2 \mathrm{M})$ afforded $98.4 \mathrm{mg}(0.455 \mathrm{mmol}, 75 \%$ yield $)$.

Note: This material decomposes in matter of hours to a purple solid if not kept rigorously dry.

'H NMR (500 MHz, Chloroform-d $)$ : $\delta 7.46(\mathrm{~d}, \mathrm{~J}=7.2 \mathrm{~Hz}, 2 \mathrm{H}), 7.42(\mathrm{t}, \mathrm{J}=7.4 \mathrm{~Hz}, 2 \mathrm{H}), 7.38-7.33$ (m, 3H), 7.02 $(\mathrm{d}, \mathrm{J}=8.2 \mathrm{~Hz}, 2 \mathrm{H}), 5.30(\mathrm{~d}, \mathrm{~J}=48.6 \mathrm{~Hz}, 2 \mathrm{H}), 5.11(\mathrm{~s}, 2 \mathrm{H})$.

${ }^{13}$ C NMR (126 MHz, Chloroform- $d$ ): $\delta 159.45(\mathrm{~d}, \mathrm{~J}=3.4 \mathrm{~Hz}), 136.88,130.00(\mathrm{~d}, \mathrm{~J}=5.0 \mathrm{~Hz}), 128.77,128.77$, $128.19,127.59,115.07(\mathrm{~d}, \mathrm{~J}=2.1 \mathrm{~Hz}), 84.67(\mathrm{~d}, \mathrm{~J}=164.5 \mathrm{~Hz}), 70.17$.

${ }^{19}$ F NMR (282 MHz, Chloroform- $\left.d\right)$ : $\delta$ - $199.59(\mathrm{t}, \mathrm{J}=48.6 \mathrm{~Hz})$.

HRMS: (EI-TOF) calculated for $\mathrm{C}_{14} \mathrm{H}_{13} \mathrm{FO}^{+}\left[\mathrm{M}^{*}\right]: 216.09449$, found 216.09451.

FTIR (thin film, cm$\left.^{-1}\right): v 2916(\mathrm{w}), 1805(\mathrm{w}), 1781(\mathrm{~m}), 1738(\mathrm{~s}), 1606(\mathrm{w}), 1515(\mathrm{w}), 1493(\mathrm{~m}), 1465(\mathrm{~m}), 1435$ (w), $1410(\mathrm{w}), 1372(\mathrm{~m}), 1349(\mathrm{~m}), 1327(\mathrm{~m}), 1274(\mathrm{w}), 1244(\mathrm{w}), 1212(\mathrm{~m}), 1183(\mathrm{~m}), 1124(\mathrm{~m}), 1103(\mathrm{~s}), 1077(\mathrm{~s})$, $1012(\mathrm{~m}), 971(\mathrm{~s}), 924(\mathrm{~m}), 876(\mathrm{~s}), 861(\mathrm{~m}), 800(\mathrm{~m}), 787(\mathrm{~m}), 754(\mathrm{~s}), 712(\mathrm{~m}), 693(\mathrm{~s}), 596(\mathrm{~m}), 566(\mathrm{~m}), 518(\mathrm{~m})$, $503(\mathrm{~s}), 478(\mathrm{~m})$.

(4-(fluoromethyl)phenyl)(methyl)sulfane (11)

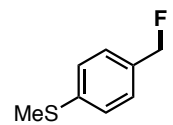

Synthesized according to general procedure $\mathrm{E}$ with 1,3-dioxoisoindolin-2-yl 2-(4-(methylthio)phenyl)acetate (0.333 $\mathrm{g}, 1.02 \mathrm{mmol}, 1$ equiv.), Ir(dF-ppy) $3(7.4 \mathrm{mg}, 0.01 \mathrm{mmol}, 0.01$ equiv.), triethylamine trihydrofluoride ( $489 \mu \mathrm{L}, 3.0$ mmol, 3 equiv.), and DCM (5 mL, $0.2 \mathrm{M})$. Purified over silica with flash chromatography (3\% ether in pentanes) to afford the title compound as a white solid contaminated with $5 \mathrm{~mol} \%$ of 1,2-bis(4-(methylthio)phenyl)ethane (adjusted for bibenzyl, $99.0 \mathrm{mg}, \quad 0.634 \mathrm{mmol}, 63 \%)$. A second run with 1,3-dioxoisoindolin-2-yl 2-(4(methylthio)phenyl)acetate (0.3288 g, $1.00 \mathrm{mmol}, 1$ equiv.), $\operatorname{Ir}(\mathrm{dF}-\mathrm{ppy})_{3}(8.1 \mathrm{mg}, 0.01 \mathrm{mmol}, 0.01$ equiv.), triethylamine trihydrofluoride ( $489 \mu \mathrm{L}, 3.0 \mathrm{mmol}, 3$ equiv.), and DCM $(5.00 \mathrm{~mL}, 0.2 \mathrm{M})$ afforded $93.8 \mathrm{mg}(60 \%$ yield, $0.600 \mathrm{mmol}$, adjusted for $5 \%$ bibenzyl).

1H NMR (500 MHz, Methylene Chloride- $\left.\boldsymbol{d}_{2}\right):$ $\delta 7.31(\mathrm{dd}, J=8.4,2.1 \mathrm{~Hz}, 2 \mathrm{H}), 7.27(\mathrm{dd}, J=7.8,1.1 \mathrm{~Hz}, 2 \mathrm{H}), 5.31$ $(\mathrm{d}, J=48.2 \mathrm{~Hz}, 2 \mathrm{H}), 2.49$ (s, 3H).

${ }^{13}$ C NMR (126 MHz, Methylene Chloride- $\left.d_{2}\right): \delta 140.33(\mathrm{~d}, J=3.8 \mathrm{~Hz}), 133.30(\mathrm{~d}, J=17.0 \mathrm{~Hz}), 128.97(\mathrm{~d}, J=5.5$ $\mathrm{Hz}), 126.67(\mathrm{~d}, J=1.7 \mathrm{~Hz}), 84.94(\mathrm{~d}, J=164.6 \mathrm{~Hz}), 15.82$.

${ }^{19}$ F NMR (282 MHz, Chloroform- $\left.d\right)$ : $\delta-204.39(\mathrm{t}, J=48.0 \mathrm{~Hz})$.

HRMS: (EI-TOF) calculated for $\mathrm{C}_{8} \mathrm{H}_{9} \mathrm{FS}^{+}\left[\mathrm{M}^{\cdot+}\right]$ : 156.04035 , found 156.04705 .

FTIR (thin film, cm$\left.^{-1}\right): v 2921(\mathrm{w}), 1603(\mathrm{~m}), 1495(\mathrm{~s}), 1437(\mathrm{~m}), 1406(\mathrm{~m}), 1375$ (m), 1219 (w), $1093(\mathrm{~m}), 969(\mathrm{~s})$, $837(\mathrm{~m}), 802(\mathrm{~s})$.

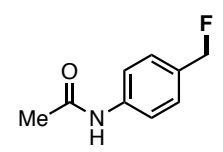

$N$-(4-(fluoromethyl)phenyl)acetamide (12)

Synthesized according to general procedure $\mathrm{E}$ with 1,3-dioxoisoindolin-2-yl 2-(4-acetamidophenyl)acetate $(0.2732 \mathrm{~g}$, $0.808 \mathrm{mmol}, 1$ equiv.), $\mathrm{Ir}(\mathrm{dF}-\mathrm{ppy})_{3}(4.8 \mathrm{mg}, 0.006 \mathrm{mmol}, 0.01$ equiv.), triethylamine trihydrofluoride $(391.2 \mu \mathrm{L}, 2.4$ mmol, 3 equiv.), and DCM (4.00 mL, $0.2 \mathrm{M}$ ). Purified over silica with flash chromatography (20\% acetone in hexanes) to afford the title compound as a cream white solid $(98.7 \mathrm{mg}, 0.590 \mathrm{mmol}, 73 \%)$. A second run with 1,3dioxoisoindolin-2-yl 2-(4-acetamidophenyl)acetate $(0.2730 \mathrm{~g}, 0.807 \mathrm{mmol}, 1$ equiv. $), \operatorname{Ir}(\mathrm{dF}-\mathrm{ppy})_{3}(4.9 \mathrm{mg}, 0.006$ mmol, 0.01 equiv.), triethylamine trihydrofluoride $(391.2 \mu \mathrm{L}, 2.4 \mathrm{mmol}, 3$ equiv.), and $\mathrm{DCM}(4.00 \mathrm{~mL}, 0.2 \mathrm{M})$

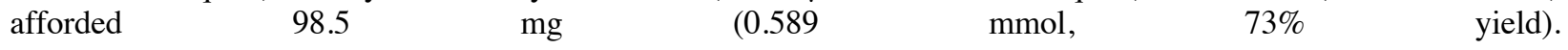

1H NMR (500 MHz, Chloroform- $d)$ : $\delta 7.56(\mathrm{~d}, J=8.1 \mathrm{~Hz}, 2 \mathrm{H}), 7.36(\mathrm{dd}, J=8.5,2.0 \mathrm{~Hz}, 2 \mathrm{H}), 7.33$ (broad s, $1 \mathrm{H})$, $5.35(\mathrm{~d}, J=48.1 \mathrm{~Hz}, 2 \mathrm{H}), 2.21(\mathrm{~s}, 3 \mathrm{H})$.

${ }^{13}$ C NMR (126 MHz, Chloroform- $\left.d\right): ~ \delta 168.64,138.55(\mathrm{~d}, J=3.3 \mathrm{~Hz}), 132.05(\mathrm{~d}, J=17.3 \mathrm{~Hz}), 128.86(\mathrm{~d}, J=5.5$ $\mathrm{Hz}), 119.94(\mathrm{~d}, \mathrm{~J}=1.1 \mathrm{~Hz}), 84.42(\mathrm{~d}, J=165.4 \mathrm{~Hz}), 24.75$. 
${ }^{19}$ F NMR (376 MHz, Chloroform- $\left.d\right)$ : $\delta-203.91(\mathrm{t}, J=48.1 \mathrm{~Hz})$.

HRMS: (ESI-TOF) calculated for $\mathrm{C}_{9} \mathrm{H}_{11} \mathrm{FNO}^{+}[\mathrm{M}+\mathrm{H}]^{+}: 168.08192$, found 168.08186 .

FTIR (thin film, cm-1): $v 3296$ (m), 3262 (m), 3192 (w), 3124 (w), 3042 (w), 2961 (m), 2903 (w), $1666(\mathrm{~s}), 1598$ (s), $1529(\mathrm{~s}), 1512(\mathrm{~s}), 1472(\mathrm{~s}), 1410(\mathrm{~s}), 1367(\mathrm{~s}), 1315(\mathrm{~s}), 1263$ (s), $1218(\mathrm{~s}), 1180(\mathrm{~s}), 1165(\mathrm{~m}), 1118(\mathrm{~m}), 1036$ (m), $1015(\mathrm{~m}), 957(\mathrm{~s}), 939(\mathrm{~s}), 861(\mathrm{~s}), 833(\mathrm{~s}), 799$ (s), $739(\mathrm{~s}), 724$ (s), 649 (s), $632(\mathrm{~s}), 605$ (s), 559 (s), $507(\mathrm{~s}), 431$ (m).

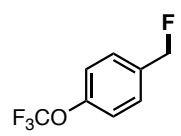

1-(fluoromethyl)-4-(trifluoromethoxy)benzene (13)

Synthesized according to general procedure E with 1,3-dioxoisoindolin-2-yl 2-(4-(trifluoromethoxy)phenyl)acetate $\left(0.2285 \mathrm{~g}, 0.626 \mathrm{mmol}, 1\right.$ equiv.), $\operatorname{Ir}(\mathrm{dF}-\mathrm{ppy})_{3}(5.7 \mathrm{mg}, 0.008 \mathrm{mmol}, 0.01$ equiv.), triethylamine trihydrofluoride (293.4 $\mu \mathrm{L}, 1.8 \mathrm{mmol}, 3$ equiv.), and DCM (3.00 mL, 0.2 M). 1-Fluoronaphthalene (77.5 $\mu \mathrm{L}, 0.600 \mathrm{mmol}, 1$ equiv.) was added, the reaction mixture was stirred, and an aliquot was removed and analyzed by ${ }^{19} \mathrm{~F}-\mathrm{NMR}$ to obtain $64 \%$ yield of the title compound $(0.401 \mathrm{mmol}, 77.8 \mathrm{mg})$. A second run with 1,3-dioxoisoindolin-2-yl 2-(4(trifluoromethoxy)phenyl)acetate ( $0.2227 \mathrm{~g}, 0.610 \mathrm{mmol}, 1$ equiv.), $\mathrm{Ir}(\mathrm{dF}-\mathrm{ppy})_{3}(5.1 \mathrm{mg}, 0.007 \mathrm{mmol}, 0.01$ equiv.), triethylamine trihydrofluoride $\left(293.4 \mu \mathrm{L}, 1.8 \mathrm{mmol}, 3\right.$ equiv.), and DCM $(3.00 \mathrm{~mL}, 0.2 \mathrm{M})$ afforded $56 \%{ }^{19} \mathrm{~F}-\mathrm{NMR}$ yield (relative to external 1-fluoronaphthalene, $0.342 \mathrm{mmol}, 66.4 \mathrm{mg}$ ).

${ }^{19}$ F NMR (282 MHz, Chloroform-d): $\delta$-58.61(s, 3F), -209.57 (t, $\left.J=47.7 \mathrm{~Hz}, 1 \mathrm{~F}\right)$. HRMS: (EI-TOF) calculated for $\mathrm{C}_{8} \mathrm{H}_{6} \mathrm{~F}_{4} \mathrm{O}^{+}\left[\mathrm{M}^{*}\right]: 194.03493$, found 194.03462 .

This matched literature characterization. ${ }^{14}$

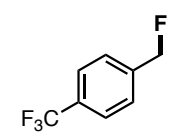

1-(fluoromethyl)-4-(trifluoromethyl)benzene (14)

Synthesized according to general procedure E with 1,3-dioxoisoindolin-2-yl 2-(4-(trifluoromethyl)phenyl)acetate $(0.2132 \mathrm{~g}, 0.610 \mathrm{mmol}, 1$ equiv. $), \operatorname{Ir}(\mathrm{dF}-\mathrm{ppy})_{3}(4.4 \mathrm{mg}, 0.006 \mathrm{mmol}, 0.01$ equiv.), triethylamine trihydrofluoride (293.4 $\mu \mathrm{L}, 1.8 \mathrm{mmol}, 3$ equiv.), and DCM $(3.00 \mathrm{~mL}, 0.2 \mathrm{M})$. Fluorobenzene $(56.5 \mu \mathrm{L}, 0.6 \mathrm{mmol}, 1$ equiv.) was added, the reaction mixture was stirred, and an aliquot was removed and analyzed by ${ }^{19} \mathrm{~F}-\mathrm{NMR}$ to obtain 5\% NMR yield (0.0305 $\mathrm{mmol}, 5.4 \mathrm{mg}$ ). A second run with 1,3-dioxoisoindolin-2-yl 2-(4-(trifluoromethyl)phenyl)acetate $(0.2149 \mathrm{~g}, 0.615$ mmol, 1 equiv.), $\operatorname{Ir}(\mathrm{dF}-\mathrm{ppy})_{3}(4.7 \mathrm{mg}, 0.006 \mathrm{mmol}, 0.01$ equiv.), triethylamine trihydrofluoride $(293.4 \mu \mathrm{L}, 1.8 \mathrm{mmol}$, 3 equiv.), and DCM (3.00 mL, $0.2 \mathrm{M}$ ) afforded $6 \%{ }^{19} \mathrm{~F}-\mathrm{NMR}$ yield (relative to external fluorobenzene, $0.0369 \mathrm{mmol}$, $6.6 \mathrm{mg})$.

19F NMR (282 MHz, Chloroform-d): $\delta$-63.49 (s, 3F), -214.38 (t, $J=47.4 \mathrm{~Hz}, 1 \mathrm{~F}),-63.18$ (s, 3F, 1,2-bis(4(trifluoromethyl)phenyl)ethane side product).

HRMS: (EI-TOF) calculated for $\mathrm{C}_{8} \mathrm{H}_{6} \mathrm{~F}_{4}{ }^{+}\left[\mathrm{M}^{\cdot+}\right]: 178.04001$, found 178.04007 .

\section{5-(fluoromethyl)benzo[d][1,3]dioxole (15)}

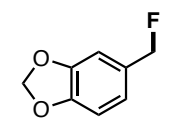

Synthesized according to general procedure E with 1,3-dioxoisoindolin-2-yl 2-(benzo[d][1,3]dioxol-5-yl)acetate $\left(0.3241 \mathrm{~g}, 0.996 \mathrm{mmol}, 1\right.$ equiv.), $\operatorname{Ir}(\mathrm{dF}-\mathrm{ppy})_{3}(5.2 \mathrm{mg}, 0.0068 \mathrm{mmol}, 0.01$ equiv.), triethylamine trihydrofluoride (489 $\mu \mathrm{L}, 3 \mathrm{mmol}, 3$ equiv), and DCM (5.00 mL, 0.2 M). Purified over silica with flash chromatography (5\% ether in pentanes) to afford the title compound as a colorless oil $(93.1 \mathrm{mg}, 0.604 \mathrm{mmol}, 61 \%)$. A second run with 1,3dioxoisoindolin-2-yl 2-(benzo[d][1,3]dioxol-5-yl)acetate (0.3258 g, $1.00 \mathrm{mmol}, 1$ equiv.), Ir(dF-ppy) 3 (5.5 mg, 0.0072 mmol, 0.01 equiv.), triethylamine trihydrofluoride ( $489 \mu \mathrm{L}, 3 \mathrm{mmol}, 3$ equiv.), and DCM $(5.00 \mathrm{~mL}, 0.2 \mathrm{M})$ afforded $103.8 \mathrm{mg}(0.673 \mathrm{mmol}, 65 \%)$. 
1H NMR (500 MHz, Methylene Chloride- $\left.\mathbf{d}_{2}\right): \delta 6.90-6.79(\mathrm{~m}, 3 \mathrm{H}), 5.98(\mathrm{~s}, 2 \mathrm{H}), 5.25(\mathrm{~d}, J=48.4 \mathrm{~Hz}, 2 \mathrm{H})$.

${ }^{13} \mathrm{C}$ NMR (126 MHz, Methylene Chloride- $\left.\boldsymbol{d}_{2}\right)$ : $\delta 148.75(\mathrm{~d}, J=3.4), 148.48(\mathrm{~d}, J=2.0 \mathrm{~Hz}), 130.54(\mathrm{~d}, J=17.0$

$\mathrm{Hz}), 122.67(\mathrm{~d}, J=6.3 \mathrm{~Hz}), 109.09(\mathrm{~d}, J=4.6 \mathrm{~Hz}), 108.67(\mathrm{~d}, J=2.1 \mathrm{~Hz}), 102.02,85.25(\mathrm{~d}, J=164.7 \mathrm{~Hz})$.

${ }^{19}$ F NMR (376 MHz, Methylene Chloride- $\left.d_{2}\right)$ : $\delta-199.76(\mathrm{t}, J=48.4 \mathrm{~Hz})$.

HRMS: (EI-TOF) calculated for $\mathrm{C}_{8} \mathrm{H}_{7} \mathrm{FO}_{2}^{+}\left[\mathrm{M}^{+}\right]$: 154.04246, found 154.04333.

FTIR (thin film, $\left.\mathbf{c m}^{-1}\right): v 2962(\mathrm{w}), 2899(\mathrm{~m}), 2783(\mathrm{w}), 1855(\mathrm{w}), 1609(\mathrm{w}), 1504(\mathrm{~s}), 1491(\mathrm{~s}), 1445(\mathrm{~s}), 1380(\mathrm{~m})$, $1249(\mathrm{~s}), 1198(\mathrm{~m}), 1125(\mathrm{~m}), 1100(\mathrm{~m}), 1037(\mathrm{~m}), 965(\mathrm{~s}), 925$ (s), $862(\mathrm{~s}), 809(\mathrm{~s}), 771(\mathrm{~s}), 727$ (m), $716(\mathrm{~m})$.

\section{4-bromo-2-(fluoromethyl)thiophene (16)}

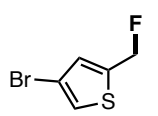

Synthesized according to general procedure E with 1,3-dioxoisoindolin-2-yl 2-(4-bromothiophen-2-yl)acetate (0.2235 $\mathrm{g}, 0.610 \mathrm{mmol}, 1$ equiv. $), \operatorname{Ir}(\mathrm{dF}-\mathrm{ppy})_{3}(3.7 \mathrm{mg}, 0.006 \mathrm{mmol}, 0.01$ equiv. $)$, triethylamine trihydrofluoride $(293.4 \mu \mathrm{L}$, $1.8 \mathrm{mmol}, 3$ equiv.), and DCM (3.00 mL, 0.2 M). 1-Fluoronaphthalene ( $26.0 \mu \mathrm{L}, 0.200 \mathrm{mmol}, 0.33$ equiv.) was added, the reaction mixture was stirred, and an aliquot was removed and analyzed by ${ }^{19} \mathrm{~F}-\mathrm{NMR}$ to obtain $41 \%{ }^{19} \mathrm{~F}-\mathrm{NMR}$ yield $(0.250 \mathrm{mmol}, 48.8 \mathrm{mg})$. A second run with 1,3-dioxoisoindolin-2-yl 2-(4-bromothiophen-2-yl)acetate $(0.2217 \mathrm{~g}, 0.605$ mmol, 1 equiv.), $\operatorname{Ir}(\mathrm{dF}-\mathrm{ppy})_{3}(3.2 \mathrm{mg}, 0.006 \mathrm{mmol}, 0.01$ equiv.), triethylamine trihydrofluoride $(293.4 \mu \mathrm{L}, 1.8 \mathrm{mmol}$, 3 equiv.), and DCM (3.00 mL, $0.2 \mathrm{M})$ afforded $40 \%{ }^{19} \mathrm{~F}-\mathrm{NMR}$ yield $(0.242 \mathrm{mmol}, 47.2 \mathrm{mg})$. Volatility of the of the title compound prevented clean isolation.

${ }^{1}$ H NMR (500 MHz, Chloroform- $\left.d\right): \delta 7.29(\mathrm{~d}, J=1.9 \mathrm{~Hz}, 1 \mathrm{H}), 7.07(\mathrm{~d}, J=2.4 \mathrm{~Hz}, 1 \mathrm{H}), 5.43(\mathrm{~d}, J=48.6 \mathrm{~Hz}$, 2H).

${ }^{13}$ C NMR (126 MHz, Chloroform- $\left.d\right): \delta 144.45,139.15(\mathrm{~d}, J=19.8 \mathrm{~Hz}), 131.12(\mathrm{~d}, J=5.7 \mathrm{~Hz}), 125.02(\mathrm{~d}, J=4.0$

$\mathrm{Hz}), 77.94(\mathrm{~d}, J=169.0 \mathrm{~Hz})$.

19F NMR (282 MHz, Chloroform- $d$ ): $\delta-193.56(\mathrm{t}, J=48.7 \mathrm{~Hz})$.

HRMS: (EI-TOF) calculated for $\mathrm{C}_{4} \mathrm{H}_{4}{ }^{79} \mathrm{BrFS}^{+}\left[\mathrm{M}^{\circ+}\right]$ : 195.91956 , found 193.92077 ; calculated for $\mathrm{C}_{4} \mathrm{H}_{4}{ }^{81} \mathrm{BrFS}^{+}$

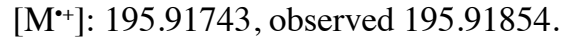

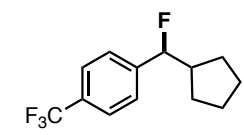

1-(cyclopentylfluoromethyl)-4-(trifluoromethyl)benzene (17)

Synthesized according to general procedure $\mathrm{E}$ with 1,3-dioxoisoindolin-2-yl 2-cyclopentyl-2-(4(trifluoromethyl)phenyl)acetate $\left(0.2588 \mathrm{~g}, 0.620 \mathrm{mmol}, 1\right.$ equiv.), $\operatorname{Ir}(\mathrm{dF}-\mathrm{ppy})_{3}(2.7 \mathrm{mg}, 0.006 \mathrm{mmol}, 0.01$ equiv.), triethylamine trihydrofluoride $(293.4 \mu \mathrm{L}, 1.8 \mathrm{mmol}, 3$ equiv.), and DCM $(3.00 \mathrm{~mL}, 0.2 \mathrm{M})$. Purified over silica with flash chromatography (5\% ether in hexanes) to afford the title compound as a colorless oil $(138.2 \mathrm{mg}, 0.561 \mathrm{mmol}$, 91\%). A second run with 1,3-dioxoisoindolin-2-yl 2-cyclopentyl-2-(4-(trifluoromethyl)phenyl)acetate $(0.2565 \mathrm{~g}$, $0.615 \mathrm{mmol}, 1$ equiv.), $\mathrm{Ir}(\mathrm{dF}-\mathrm{ppy})_{3}(3.3 \mathrm{mg}, 0.006 \mathrm{mmol}, 0.01$ equiv.), triethylamine trihydrofluoride $(293.4 \mu \mathrm{L}, 1.8$ mmol, 3 equiv.), and DCM (3.00 mL, $0.2 \mathrm{M})$ afforded $144.3 \mathrm{mg}$ of the title compound $(0.586 \mathrm{mmol}, 95 \%)$.

${ }^{1}$ H NMR (500 MHz, Chloroform- $d$ ): $\delta 7.63(\mathrm{~d}, J=8.1 \mathrm{~Hz}, 2 \mathrm{H}), 7.44(\mathrm{~d}, J=8.0 \mathrm{~Hz}, 2 \mathrm{H}), 5.27(\mathrm{dd}, J=47.5,7.5 \mathrm{~Hz}$, $1 \mathrm{H}), 2.35(\mathrm{dq}, J=18.5,7.9 \mathrm{~Hz}, 1 \mathrm{H}), 1.80(\mathrm{qd}, J=8.5,7.8,4.2 \mathrm{~Hz}, 1 \mathrm{H}), 1.71-1.61(\mathrm{~m}, 2 \mathrm{H}), 1.61-1.47(\mathrm{~m}, 4 \mathrm{H})$, $1.37-1.24(\mathrm{~m}, 1 \mathrm{H})$.

${ }^{13}$ C NMR (126 MHz, Chloroform- $d$ ): $\delta 144.25$ (dd, $\left.J=20.7,1.4 \mathrm{~Hz}\right), 130.41(\mathrm{qd}, J=32.4,1.8 \mathrm{~Hz}), 126.39(\mathrm{~d}, J=$ $7.0 \mathrm{~Hz}), 125.44(\mathrm{q}, J=3.8 \mathrm{~Hz}), 124.18(\mathrm{q}, J=272.1 \mathrm{~Hz}), 97.18(\mathrm{~d}, J=173.9 \mathrm{~Hz}), 46.10(\mathrm{~d}, J=22.0 \mathrm{~Hz}), 28.70(\mathrm{~d}, J$ $=6.3 \mathrm{~Hz}), 28.48(\mathrm{~d}, J=2.4 \mathrm{~Hz}), 25.57(\mathrm{~d}, J=2.1 \mathrm{~Hz})$.

${ }^{19}$ F NMR (376 MHz, Chloroform- $d$ ): $\delta-62.61$ (s, 3F), -177.39 (dd, $\left.J=47.6,18.4 \mathrm{~Hz}\right)$.

HRMS: (ESI-TOF) calculated for $\mathrm{C}_{13} \mathrm{H}_{14} \mathrm{~F}_{4}+[\mathrm{M}]^{+}: 246.10261$, found 246.10295 .

FTIR (thin film, cm$\left.^{-1}\right): v 2957(\mathrm{w}), 2872(\mathrm{w}), 1622(\mathrm{w}), 1452(\mathrm{w}), 1418(\mathrm{w}), 1323(\mathrm{~s}), 1163(\mathrm{~s}), 1122(\mathrm{~s}), 1066(\mathrm{~s})$, $1018(\mathrm{~s}), 988(\mathrm{~m}), 935(\mathrm{~m}), 836(\mathrm{~s}), 762(\mathrm{~m}), 680$ (w), $605(\mathrm{~m}), 530(\mathrm{w}), 466$ (w), $450(\mathrm{w}), 409(\mathrm{~m})$. 
2-chloro-5-(1-fluoroethyl)pyridine (18)<smiles>CC(F)c1ccc(Cl)nc1</smiles>

Synthesized according to general procedure E with 1,3-dioxoisoindolin-2-yl 2-(6-chloropyridin-3-yl)propanoate (contaminated with trace 1,3-dioxo-2,3-dihydro-1H-inden-2-yl 2-(6-chloropyridin-3-yl)-2-methylpropanoate) $\left(0.1981 \mathrm{~g}, 0.599 \mathrm{mmol}, 1\right.$ equiv.), $\mathrm{Ir}(\mathrm{dF}-\mathrm{ppy})_{3}(4.8 \mathrm{mg}, 0.006 \mathrm{mmol}, 0.01$ equiv.), triethylamine trihydrofluoride (293.4 $\mu \mathrm{L}, 1.8 \mathrm{mmol}, 3$ equiv.), and DCM (3.00 mL, $0.2 \mathrm{M})$. Purified over silica with flash chromatography ( 3 to $5 \%$ ether in hexanes) to afford the title compound as a clear oil $(56.1 \mathrm{mg}, 0.352 \mathrm{mmol}, 59 \%)$ with 2-chloro-5-(2-fluoropropan2-yl)pyridine as an impurity from dimethylation of the 2-chloropyridine-5-acetic acid starting material (3.2 $\mathrm{mg}, 5 \%)$. A second run with 1,3-dioxoisoindolin-2-yl 2-(6-chloropyridin-3-yl)propanoate ( $0.1983 \mathrm{~g}, 0.600 \mathrm{mmol}, 1$ equiv.), $\operatorname{Ir}(\mathrm{dF}-\mathrm{ppy})_{3}(4.3 \mathrm{mg}, 0.006 \mathrm{mmol}, 0.01$ equiv), triethylamine trihydrofluoride ( $293.4 \mu \mathrm{L}, 1.8 \mathrm{mmol}, 3$ equiv), DCM (3 $\mathrm{mL}, 0.2 \mathrm{M})$ afforded $54.6 \mathrm{mg}$ of the title compound ( $0.342 \mathrm{mmol}, 57 \%$ yield) with 2-chloro-5-(2-fluoropropan-2yl)pyridine as an impurity $(3.1 \mathrm{mg}, 5 \%)$.

1H NMR (500 MHz, Methylene Chloride- $\left.d_{2}\right): \delta 8.36(\mathrm{~s}, 1 \mathrm{H}), 7.68(\mathrm{dd}, J=8.4,2.5 \mathrm{~Hz}, 1 \mathrm{H}), 7.36(\mathrm{~d}, J=8.4 \mathrm{~Hz}, 1 \mathrm{H})$, $5.67(\mathrm{dq}, J=47.2,6.5 \mathrm{~Hz}, 2 \mathrm{H}), 1.64(\mathrm{dd}, J=24.1,6.6 \mathrm{~Hz}, 3 \mathrm{H})$.

${ }^{13}$ C NMR (126 MHz, Methylene Chloride- $\left.d_{2}\right): \delta 151.70,147.52(\mathrm{~d}, J=6.9 \mathrm{~Hz}), 136.45(\mathrm{~d}, J=21.8 \mathrm{~Hz}), 136.42(\mathrm{~d}$, $J=6.1 \mathrm{~Hz}), 124.69,88.93(\mathrm{~d}, J=168.6 \mathrm{~Hz}), 22.88(\mathrm{~d}, J=24.7 \mathrm{~Hz})$.

19F NMR (282 MHz, Methylene Chloride- $\boldsymbol{d}_{2}$ ): $\delta$-170.28 (dq, $J=47.7,24.0 \mathrm{~Hz}, 1 \mathrm{~F}$ ). 2-chloro-5-(2-fluoropropan-2yl)pyridine: $\delta-138.73$ (hept, $J=22.1 \mathrm{~Hz}, 1 \mathrm{~F}$ ).

HRMS: (EI-TOF) calculated for $\mathrm{C}_{7} \mathrm{H}_{7} \mathrm{ClFN}\left[\mathrm{M}^{*}\right]$ : 159.02456 , found 159.02435 .

FTIR (thin film, $\left.\mathbf{~ c m}^{-1}\right): 2986$ (w), 2932 (w), 1591 (w), 1568 (w), 1460 (s), 1377 (w), 1327 (n), 1289 (w), 1215 (w), $1141(\mathrm{w}), 1114(\mathrm{~s}), 1069$ (s), 1022 (n), 1005 (n), 931 (w), 882 (n), 834 (n), 795 (n), 742 (n), 666 (w), 632 (w), 549 (w), $521(\mathrm{w}), 433(\mathrm{~m}), 414(\mathrm{~m})$.

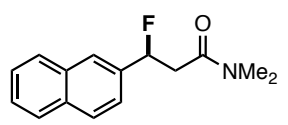

3-fluoro- $N, N$-dimethyl-3-(naphthalen-2-yl)propanamide (19)

Synthesized according to general procedure E with 1,3-dioxoisoindolin-2-yl 4-(dimethylamino)-2-(naphthalen-2-yl)4-oxobutanoate $\left(0.2557 \mathrm{~g}, 0.614 \mathrm{mmol}, 1\right.$ equiv.), $\operatorname{Ir}(\mathrm{dF}-\mathrm{ppy})_{3}(3.1 \mathrm{mg}, 0.006 \mathrm{mmol}, 0.01$ equiv.), triethylamine trihydrofluoride $(293.4 \mu \mathrm{L}, 1.8 \mathrm{mmol}, 3$ equiv.), and DCM (3.00 mL, 0.2 M). Purified over silica with flash chromatography (5\% acetone, $25-50 \%$ EtOAc in hexanes) to afford the title compound as a white solid (90.0 $\mathrm{mg}$, $0.367 \mathrm{mmol}, 60 \%)$. A second run with 1,3-dioxoisoindolin-2-yl 4-(dimethylamino)-2-(naphthalen-2-yl)-4oxobutanoate $\left(0.2556 \mathrm{~g}, 0.6 \mathrm{mmol}, 1\right.$ equiv.), $\operatorname{Ir}(\mathrm{dF}-\mathrm{ppy})_{3}(5.2 \mathrm{mg}, 0.006 \mathrm{mmol}, 0.01$ equiv.), triethylamine trihydrofluoride ( $293.4 \mu \mathrm{L}, 1.8 \mathrm{mmol}, 3$ equiv.), DCM (3.00 mL, $0.2 \mathrm{M})$ afforded $102.5 \mathrm{mg}(0.418 \mathrm{mmol}, 68 \%$ yield).

${ }^{1}$ H NMR (500 MHz, Chloroform-d): $\delta$ 7.89-7.82 (m, 4H), $7.53-7.48(\mathrm{~m}, 3 \mathrm{H}), 6.22$ (ddd, $\left.J=46.6,8.3,4.3 \mathrm{~Hz}, 1 \mathrm{H}\right)$, 3.21 (ddd, $J=15.7,14.4,8.3 \mathrm{~Hz}, 1 \mathrm{H}), 3.00(\mathrm{~s}, 3 \mathrm{H}), 2.98(\mathrm{~s}, 3 \mathrm{H}), 2.81$ (ddd, $J=30.7,15.7,4.4 \mathrm{~Hz}, 1 \mathrm{H})$.

${ }^{13}$ C NMR (126 MHz, Chloroform- $\left.d\right): \delta 169.12(\mathrm{~d}, J=4.8 \mathrm{~Hz}), 137.16(\mathrm{~d}, J=19.7 \mathrm{~Hz}), 133.42,133.18,128.62$, $128.29,127.87,126.57,126.53,124.91(\mathrm{~d}, J=8.1 \mathrm{~Hz}), 123.24(\mathrm{~d}, J=5.9 \mathrm{~Hz}), 91.78(\mathrm{~d}, J=171.0 \mathrm{~Hz}), 41.37(\mathrm{~d}, J=$ $26.5 \mathrm{~Hz}), 37.56,35.70$.

${ }^{19}$ F NMR (282 MHz, Chloroform- $d$ ): $\delta-174.38$ (ddd, $J=45.8,30.5,14.3 \mathrm{~Hz}, 1 \mathrm{~F}$ ).

HRMS: (ESI-TOF) calculated for $\mathrm{C}_{15} \mathrm{H}_{16} \mathrm{FNNaO}^{+}[\mathrm{M}+\mathrm{Na}]^{+}: 268.11081$, found 268.11055 .

FTIR (thin film, cm$\left.^{-1}\right): v 3058(\mathrm{w}), 2925(\mathrm{w}), 1629(\mathrm{~s}), 1578(\mathrm{~m}), 1496(\mathrm{~m}), 1397(\mathrm{~s}), 1348(\mathrm{~m}), 1324(\mathrm{~m}), 1258(\mathrm{~m})$, $1213(\mathrm{w}), 1174(\mathrm{w}), 1159(\mathrm{~m}), 1143(\mathrm{~m}), 1125(\mathrm{~m}), 1059(\mathrm{~m}), 1002(\mathrm{~s}), 967(\mathrm{w}), 954(\mathrm{~m}), 903(\mathrm{~m}), 867(\mathrm{~m}), 849(\mathrm{~m})$, 825 (s), $750(\mathrm{~s}), 737$ (m), 659 (w), 601(w), $570(\mathrm{~m}), 539(\mathrm{w}), 496(\mathrm{w}), 483(\mathrm{~s}), 474(\mathrm{~s}), 448(\mathrm{w}), 413(\mathrm{w})$.

1-(azidomethyl)-4-(1-fluoroethyl)benzene (20)

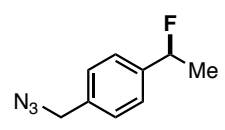

Synthesized according to general procedure E with 1,3-dioxoisoindolin-2-yl 2-(4-(azidomethyl)phenyl)propanoate $\left(0.2133 \mathrm{~g}, 0.609 \mathrm{mmol}, 1\right.$ equiv.), $\mathrm{Ir}(\mathrm{dF}-\mathrm{ppy})_{3}(2.7 \mathrm{mg}, 0.006 \mathrm{mmol}, 0.01$ equiv $)$, triethylamine trihydrofluoride (293.4 
$\mu \mathrm{L}, 1.8 \mathrm{mmol}, 3$ equiv.), and DCM (3.00 mL, 0.2 M). Purified over silica with flash chromatography (5 to $10 \%$ ether in hexanes) followed by preparatory-TLC to afford the title compound as a colorless oil $(63.7 \mathrm{mg}, 0.355 \mathrm{mmol}, 58 \%)$. A second run with 1,3-dioxoisoindolin-2-yl 2-(4-(azidomethyl)phenyl)propanoate ( $0.2145 \mathrm{~g}, 0.612 \mathrm{mmol}, 1$ equiv.), $\operatorname{Ir}(\mathrm{dF}-\mathrm{ppy})_{3}(5.2 \mathrm{mg}, 0.006 \mathrm{mmol}, 0.01$ equiv.), triethylamine trihydrofluoride $(293.4 \mu \mathrm{L}, 1.8 \mathrm{mmol}, 3 \mathrm{equiv}$.$) , and$ DCM (3.00 mL, $0.2 \mathrm{M})$ afforded $58.7 \mathrm{mg}$ of the title compound ( $0.328 \mathrm{mmol}, 54 \%$ yield).

1H NMR (500 MHz, Chloroform- $d$ ): $\delta 7.38(\mathrm{~d}, J=7.8 \mathrm{~Hz}, 2 \mathrm{H}), 7.34(\mathrm{~d}, J=8.0 \mathrm{~Hz}, 2 \mathrm{H}), 5.64(\mathrm{dq}, J=47.6,6.4 \mathrm{~Hz}$, $1 \mathrm{H}), 4.35(\mathrm{~s}, 2 \mathrm{H}), 1.65$ (dd, $J=23.9,6.5 \mathrm{~Hz}, 3 \mathrm{H})$.

13C NMR (126 MHz, Chloroform- $d)$ : $\delta 141.76(\mathrm{~d}, J=19.7 \mathrm{~Hz}), 135.50(\mathrm{~d}, J=2.1 \mathrm{~Hz}), 128.47,125.83(\mathrm{~d}, J=6.7$ $\mathrm{Hz}), 90.73(\mathrm{~d}, J=168.0 \mathrm{~Hz}), 54.58,23.05(\mathrm{~d}, J=25.1 \mathrm{~Hz})$.

19F NMR (376 MHz, Chloroform- $d$ ): $\delta-167.77$ (dq, $J=47.7,23.9)$.

HRMS: (ESI-TOF) calculated for $\mathrm{C}_{9} \mathrm{H}_{11} \mathrm{FN}^{+}\left[\mathrm{M}+\mathrm{H}-\mathrm{N}_{2}\right]^{+}: 152.08700$, found 152.08720 .

FTIR (thin film, cm$\left.^{-1}\right): v 2984(\mathrm{w}), 2931$ (w), 2092 (s), 1515 (w), 1445 (w), 1421 (m), 1376 (m), 1342 (m), 1248 (m), $1214(\mathrm{~m}), 1064(\mathrm{~m}), 1020(\mathrm{~m}), 1005(\mathrm{~m}), 880(\mathrm{~m}), 843(\mathrm{~m}), 815(\mathrm{~m}), 724(\mathrm{~m}), 671(\mathrm{~m}), 544(\mathrm{~m}), 488(\mathrm{w}), 442$ (w).

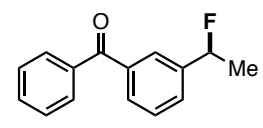

(3-(1-fluoroethyl)phenyl)(phenyl)methanone (21)

Synthesized according to general procedure $\mathrm{E}$ with 1,3-dioxoisoindolin-2-yl 2-(3-benzoylphenyl)propanoate (0.2381 $\mathrm{g}, 0.596 \mathrm{mmol}, 1$ equiv.), $\mathrm{Ir}(\mathrm{dF}-\mathrm{ppy})_{3}(4.7 \mathrm{mg}, 0.006 \mathrm{mmol}, 0.01$ equiv), triethylamine trihydrofluoride $(293.4 \mu \mathrm{L}, 1.8$ mmol, 3 equiv), and DCM (3.00 mL, $0.2 \mathrm{M})$. Purified over silica with flash chromatography (5\% ether in pentanes) to afford the title compound as a clear oil $(91.2 \mathrm{mg}, 0.400 \mathrm{mmol}, 67 \%)$. A second run with 1,3-dioxoisoindolin-2-yl 2-(3-benzoylphenyl)propanoate ( $0.2415 \mathrm{~g}, 0.605 \mathrm{mmol}, 1$ equiv.), $\mathrm{Ir}(\mathrm{dF}-\mathrm{ppy})_{3}(4.7 \mathrm{mg}, 0.006 \mathrm{mmol}, 0.01$ equiv)), triethylamine trihydrofluoride ( $293.4 \mu \mathrm{L}, 1.8 \mathrm{mmol}, 3$ equiv.), and DCM ( $3 \mathrm{~mL}, 0.2 \mathrm{M})$ afforded $87.4 \mathrm{mg}$ of the title compound $(0.383 \mathrm{mmol}, 63 \%$ yield $)$.

1H NMR (500 MHz, Chloroform-d): $\delta 7.83-7.77(\mathrm{~m}, 3 \mathrm{H}), 7.74(\mathrm{dt}, J=7.6,0.9 \mathrm{~Hz}, 1 \mathrm{H}), 7.64-7.57(\mathrm{~m}, 2 \mathrm{H}), 7.50$ $(\mathrm{td}, J=7.6,3.5 \mathrm{~Hz}, 3 \mathrm{H}), 5.70(\mathrm{dq}, J=47.5,6.5 \mathrm{~Hz}, 1 \mathrm{H}), 1.67(\mathrm{dd}, J=24.0,6.5 \mathrm{~Hz}, 3 \mathrm{H})$.

${ }^{13}$ C NMR (126 MHz, Chloroform- $\left.d\right)$ : $\delta 196.60,142.01(\mathrm{~d}, J=20.0 \mathrm{~Hz}), 137.95,137.50,132.74,130.20,130.08$, $129.24(\mathrm{~d}, J=6.7 \mathrm{~Hz}), 128.65,128.50,126.81(\mathrm{~d}, J=6.9 \mathrm{~Hz}), 90.62(\mathrm{~d}, J=168.9 \mathrm{~Hz}), 23.13(\mathrm{~d}, J=25.0 \mathrm{~Hz})$.

${ }^{19}$ F NMR (376 MHz, Chloroform- $d$ ): $\delta-168.56(\mathrm{dq}, J=47.7,23.9 \mathrm{~Hz}, 1 \mathrm{~F})$.

HRMS: (ESI-TOF) calculated for $\mathrm{C}_{15} \mathrm{H}_{12} \mathrm{FO}^{+}([\mathrm{M}+\mathrm{H}])^{+}: 228.09449$, found 228.09466 .

FTIR (thin film, cm$\left.^{-1}\right): 3061$ (w), 2982 (w), 2930 (w), 2360 (w), 1656 (s), 1596 (m), 1483 (w), 1446 (w), 1376 (w), $1343(\mathrm{w}), 1317(\mathrm{~m}), 1271$ (s), $1210(\mathrm{~s}), 1178(\mathrm{w}), 1139$ (w), $1065(\mathrm{~m}), 1023(\mathrm{w}), 1009$ (w), 999 (w), $968(\mathrm{~m}), 955$ $(\mathrm{m}), 910(\mathrm{w}), 885(\mathrm{~m}), 819(\mathrm{~m}), 783(\mathrm{~m}), 719(\mathrm{~s}), 698(\mathrm{~s}), 644(\mathrm{~s}), 615(\mathrm{~m}), 525(\mathrm{w}), 477(\mathrm{w}), 439(\mathrm{w})$.

\section{(2-fluoropropan-2-yl)benzene (22)}

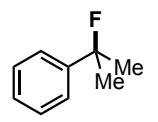

Synthesized according to general procedure $\mathrm{E}$ with 1,3-dioxoisoindolin-2-yl 2-methyl-2-phenylpropanoate (0.1889 g, $0.611 \mathrm{mmol}, 1$ equiv.), $\mathrm{Ir}(\mathrm{dF}-\mathrm{ppy})_{3}(4.3 \mathrm{mg}, 0.006 \mathrm{mmol}, 0.01$ equiv. $)$, triethylamine trihydrofluoride $(293.4 \mu \mathrm{L}, 1.8$ mmol, 3 equiv.), and DCM (3.00 mL, $0.2 \mathrm{M}$ ). Purified over silica with flash chromatography (5 to $10 \%$ ether in hexanes) to afford the title compound as a colorless oil $(70.3 \mathrm{mg}, 0.509 \mathrm{mmol}, 83 \%)$. A second run with 1,3dioxoisoindolin-2-yl 2-methyl-2-phenylpropanoate $\left(0.1883 \mathrm{~g}, 0.609 \mathrm{mmol}, 1\right.$ equiv.), $\mathrm{Ir}(\mathrm{dF}-\mathrm{ppy})_{3}$ (4.3 $\mathrm{mg}, 0.006$ mmol, 0.01 equiv.), triethylamine trihydrofluoride (293.4 $\mu \mathrm{L}, 1.8 \mathrm{mmol}, 3$ equiv.), and DCM (3.00 mL, $0.2 \mathrm{M})$ afforded $76.0 \mathrm{mg}$ of the title compound $(0.550 \mathrm{mmol}, 90 \%$ yield $)$.

1H NMR (500 MHz, Chloroform- $d)$ : $\delta 7.42-7.34(\mathrm{~m}, 3 \mathrm{H}), 7.29(\mathrm{~m}, 1 \mathrm{H}), 7.27(\mathrm{tt}, J=7.0,1.6 \mathrm{~Hz}, 1 \mathrm{H}), 1.70(\mathrm{~d}, J=$ $22.0 \mathrm{~Hz}, 6 \mathrm{H})$.

${ }^{13}$ C NMR (126 MHz, Chloroform- $\left.d\right): \delta 145.97(\mathrm{~d}, J=21.9 \mathrm{~Hz}), 128.39(\mathrm{~d}, J=1.0 \mathrm{~Hz}), 127.37$ (d, $\left.J=1.4 \mathrm{~Hz}\right)$, $123.89(\mathrm{~d}, J=8.9 \mathrm{~Hz}), 112.55,95.80(\mathrm{~d}, J=168.9 \mathrm{~Hz}), 29.44(\mathrm{~d}, J=25.8 \mathrm{~Hz})$. 
${ }^{19}$ F NMR (282 MHz, Chloroform-d): $\delta-137.40$ (hept, $J=21.9 \mathrm{~Hz}$ ).

HRMS: (EI-TOF) calculated for $\mathrm{C}_{9} \mathrm{H}_{11} \mathrm{~F}^{+}\left(\left[\mathrm{M}^{*}\right]^{+}\right)$: 184.04496 , found 184.04548 .

FTIR (thin film, $\mathbf{c m}^{-1}$ ): $v 3030$ (w), 2984 (w), 1604 (w), 1496 (w), 1447 (w), 1383 (w), 1368 (w), 1279 (m), 1203 (w), $1156(\mathrm{w}), 1104(\mathrm{w}), 1074(\mathrm{w}), 1030$ (w), 939 (m), 912 (w), $851(\mathrm{~m}), 762$ (s), 697 (s), 552 (m), $467(\mathrm{w}), 405$ (w). This matched literature characterization. ${ }^{15}$

Synthesized according to general procedure D with 1,3-dioxoisoindolin-2-yl (2-phenylpropan-2-yl) oxalate (71.7 mg of material, $0.177 \mathrm{mmol}, 1$ equiv.), note: this material was determined to contain 38\% 2-phenyl-2-propanol starting material $), \operatorname{Ir}(\mathrm{dF} \text {-ppy })_{3}(1.7 \mathrm{mg}, 0.002 \mathrm{mmol}, 0.01$ equiv.), triethylamine trihydrofluoride $(97.8 \mu \mathrm{L}, 0.6 \mathrm{mmol}, 3 \mathrm{equiv}$. $)$, and DCM (1.00 mL, 0.2 M). 1-Fluoronaphthalene (26.0 uL, $0.200 \mathrm{mmol}, 1$ equiv.) was added, the reaction mixture was stirred, and an aliquot was removed and analyzed by ${ }^{19} \mathrm{~F}-\mathrm{NMR}$ to obtain $55 \%{ }^{19} \mathrm{~F}-\mathrm{NMR}$ yield of the title compound $(0.0980 \mathrm{mmol}, 13.5 \mathrm{mg})$. A second run with 1,3-dioxoisoindolin-2-yl (2-phenylpropan-2-yl) oxalate (71.1 mg, 0.176 mmol, 1 equiv.), Ir(dF-ppy) 3 (1.5 mg, $0.002 \mathrm{mmol}, 0.01$ equiv.), triethylamine trihydrofluoride $(97.8 \mu \mathrm{L}, 0.6 \mathrm{mmol}$, 3 equiv.), and DCM (1.00 mL, $0.2 \mathrm{M})$ afforded 54\% ${ }^{19} \mathrm{~F}-\mathrm{NMR}$ yield of the title compound (0.0940 mmol, $\left.13.0 \mathrm{mg}\right)$.

${ }^{19}$ F NMR (282 MHz, Methylene Chloride- $\left.\boldsymbol{d}_{2}\right)$ : $\delta-137.10$ (heptet, $\left.J=22.0 \mathrm{~Hz}, 1 \mathrm{~F}\right)$.

Sample calculations for yield determination:

$$
\begin{gathered}
\text { Material mass }=M W_{\text {oxalate }} \text { Moles } \text { oxalate }_{\text {ox }}+\text { Mole Fraction } \text { Impurity } \text { MW }_{\text {Impurity }} \text { Moles }_{\text {oxalate }} \\
71.7 \mathrm{mg}=353.33 \frac{\mathrm{g}}{\mathrm{mol}} X_{\text {oxalate }}+0.38 \times 136.19 \frac{\mathrm{g}}{\mathrm{mol}} X_{\text {oxalate }} \\
71.7 \mathrm{mg}=405.08 \frac{\mathrm{g}}{\mathrm{mol}} X_{\text {oxalate }} \\
0.177 \mathrm{mmol}=X_{\text {oxalate }}
\end{gathered}
$$

19F-NMR integration relative to 1-fluoronaphthalene as above is 1:0.49.

$$
\begin{gathered}
\text { Yield }=\frac{\text { Actual mmol }}{\text { Theoretical mmol }}=\frac{0.200 \mathrm{mmol} \times 0.49}{0.177 \mathrm{mmol}} \\
\text { Yield }=\frac{0.0980 \mathrm{mmol}}{0.177 \mathrm{mmol}}=55 \% \text { yield }
\end{gathered}
$$

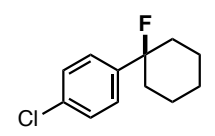

1-chloro-4-(1-fluorocyclohexyl)benzene (23)

Synthesized according to general procedure E with 1,3-dioxoisoindolin-2-yl 1-(4-chlorophenyl)cyclohexane-1carboxylate $(0.2352 \mathrm{~g}, 0.613 \mathrm{mmol}, 1$ equiv. $), \operatorname{Ir}(\mathrm{dF}-\mathrm{ppy})_{3}(2.7 \mathrm{mg}, 0.006 \mathrm{mmol}, 0.01$ equiv.), triethylamine trihydrofluoride (293.4 $\mu \mathrm{L}, 1.8 \mathrm{mmol}, 3$ equiv.), and DCM (3.00 mL, $0.2 \mathrm{M})$. Purified over silica with flash chromatography ( 5 to $10 \%$ ether in hexanes) to afford the title compound as a colorless oil with $4 \%$ contamination with the styrene side product $(114.8 \mathrm{mg}, 0.540 \mathrm{mmol}, 88 \%)$. A second run with 1,3-dioxoisoindolin-2-yl 1-(4chlorophenyl)cyclohexane-1-carboxylate $(0.2331 \mathrm{~g}, 0.607 \mathrm{mmol}, 1$ equiv.), Ir(dF-ppy) 3 (3.2 mg, $0.006 \mathrm{mmol}, 0.01$ equiv.), triethylamine trihydrofluoride (293.4 uL, $1.8 \mathrm{mmol}, 3$ equiv.), and DCM (3.00 mL, $0.2 \mathrm{M})$ afforded $123.0 \mathrm{mg}$ of the title compound $(0.578 \mathrm{mmol}, 95 \%$ yield $)$ contaminated with $3 \%$ of the styrenal side product present.

Note: the alkyl fluoride undergoes complete conversion to the corresponding styrene overnight at room temperature in $\mathrm{CDCl}_{3}$.

1H NMR (500 MHz, Chloroform- $d$ ): $\delta 7.34(\mathrm{~s}, 4 \mathrm{H}), 2.07-1.97(\mathrm{~m}, 2 \mathrm{H}), 1.87-1.66(\mathrm{~m}, 7 \mathrm{H}), 1.33(\mathrm{~m}, 1 \mathrm{H})$.

${ }^{13}$ C NMR (126 MHz, Chloroform-d): $\delta 144.61(\mathrm{~d}, J=22.3 \mathrm{~Hz}), 133.13(\mathrm{~d}, J=1.9 \mathrm{~Hz}), 128.48(\mathrm{~d}, J=0.9 \mathrm{~Hz})$, $125.60(\mathrm{~d}, J=9.4 \mathrm{~Hz}), 95.85(\mathrm{~d}, J=174.3 \mathrm{~Hz}), 37.24(\mathrm{~d}, J=23.8 \mathrm{~Hz}), 25.00,22.01(\mathrm{~d}, J=1.6 \mathrm{~Hz})$.

19F NMR (376 MHz, Chloroform- $d$ ): $\delta$ - 158.55 (broad s, $1 \mathrm{~F})$.

HRMS: (ESI-TOF) calculated for $\mathrm{C}_{12} \mathrm{H}_{14}{ }^{35} \mathrm{ClF}^{+}\left[\mathrm{M}^{*}\right]^{+}:$: 212.07626, found 212.07627;

(ESI-TOF) calculated for $\mathrm{C}_{12} \mathrm{H}_{14}{ }^{37} \mathrm{ClF}^{+}\left[\mathrm{M}^{*}\right]^{+}: 214.07354$, found 214.07364 . 
FTIR (thin film, cm-1): $v 2933$ (s), 2854 (m), 1489 (m), 1449 (m), 1437 (m), 1427 (m), 1398 (m), 1365 (w), 1351 (w), $1339(\mathrm{w}), 1258(\mathrm{w}), 1220(\mathrm{w}), 1149(\mathrm{w}), 1139$ (w), $1103(\mathrm{w}), 1091(\mathrm{~s}), 1034(\mathrm{~m}), 1009(\mathrm{~s}), 946(\mathrm{~s}), 924(\mathrm{~m}), 907$ (s), $841(\mathrm{~s}), 830(\mathrm{~m}), 817(\mathrm{~s}), 769(\mathrm{~m}), 734(\mathrm{~s}), 714(\mathrm{~m}), 677(\mathrm{~m}), 630(\mathrm{w}), 535(\mathrm{~s}), 516(\mathrm{~m}), 481(\mathrm{~s}), 449(\mathrm{~m}), 412(\mathrm{w})$.

1-chloro-4-(1-fluorocyclopentyl)benzene (24)

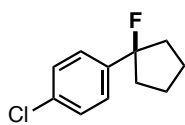

Synthesized according to general procedure E with 1,3-dioxoisoindolin-2-yl 1-(4-chlorophenyl)cyclopentane-1carboxylate $\left(0.2266 \mathrm{~g}, 0.613 \mathrm{mmol}, 1\right.$ equiv.), $\operatorname{Ir}(\mathrm{dF}-\mathrm{ppy})_{3}(2.9 \mathrm{mg}, 0.006 \mathrm{mmol}, 0.01$ equiv.), triethylamine trihydrofluoride $(293.4 \mu \mathrm{L}, 1.8 \mathrm{mmol}, 3$ equiv.), and DCM (3.00 mL, 0.2 M). The product was not isolable as it decomposed in less than 5 minutes when neat. 1-Fluoronaphthalene ( $77.5 \mu \mathrm{L}, 0.600 \mathrm{mmol}, 1$ equiv.) was added, the reaction mixture was stirred, and an aliquot was removed and analyzed by ${ }^{19} \mathrm{~F}-\mathrm{NMR}$ to obtain $85 \%{ }^{19} \mathrm{~F}-\mathrm{NMR}$ yield $(0.521 \mathrm{mmol}, 103.5 \mathrm{mg})$. A second run with 1,3-dioxoisoindolin-2-yl 1-(4-chlorophenyl)cyclopentane-1-carboxylate (0.2263 g, $0.612 \mathrm{mmol}, 1$ equiv.), Ir(dF-ppy) 3 (3.0 mg, $0.006 \mathrm{mmol}, 0.01$ equiv.), triethylamine trihydrofluoride (293.4 $\mu \mathrm{L}, 1.8 \mathrm{mmol}, 3$ equiv), and DCM (3.00 mL, 0.2 M) afforded 85\% NMR yield by ${ }^{19} \mathrm{~F}-\mathrm{NMR}$ (relative to external 1 fluoronaphthalene, $0.520 \mathrm{mmol}, 103.3 \mathrm{mg})$.

${ }^{19}$ F NMR (470 MHz, Chloroform-d): $\delta-141.30(\mathrm{tt}, J=37.4,21.0 \mathrm{~Hz})$.

HRMS: (EI-TOF) calculated for $\mathrm{C}_{10} \mathrm{H}_{10}{ }^{35} \mathrm{ClF}^{+}\left(\left[\mathrm{M}^{*}\right]^{+}\right)$: 198.06061 , found 198.06093 . (EI-TOF) calculated for $\mathrm{C}_{10} \mathrm{H}_{10}{ }^{37} \mathrm{ClF}^{+}\left(\left[\mathrm{M}^{*}\right]^{+}\right)$: 200.05785 , found 200.05799 .

\section{1-chloro-4-(1-fluorocyclobutyl)benzene (25)}

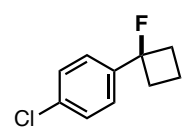

Synthesized according to general procedure E with 1,3-dioxoisoindolin-2-yl 1-(4-chlorophenyl)cyclobutane-1carboxylate $(0.2150 \mathrm{~g}, 0.604 \mathrm{mmol}, 1$ equiv. $), \operatorname{Ir}(\mathrm{dF}-\mathrm{ppy})_{3}(2.1 \mathrm{mg}, 0.006 \mathrm{mmol}, 0.01$ equiv.), triethylamine trihydrofluoride $(293.4 \mu \mathrm{L}, 1.8 \mathrm{mmol}, 3$ equiv.), DCM (3.00 mL, 0.2 M). Purified over silica with flash chromatography (5 to $10 \%$ ether in hexanes) to afford the title compound as a colorless oil (101.2 $\mathrm{mg}, 0.548 \mathrm{mmol}$, $91 \%)$. A second run with 1,3-dioxoisoindolin-2-yl 1-(4-chlorophenyl)cyclobutane-1-carboxylate (0.2264 g, 0.636 mmol, 1 equiv. $)$, $\operatorname{Ir}(\mathrm{dF}-\mathrm{ppy})_{3}(2.8 \mathrm{mg}, 0.006 \mathrm{mmol}, 0.01$ equiv. $)$, triethylamine trihydrofluoride $(293.4 \mu \mathrm{L}, 1.8 \mathrm{mmol}$, 3 equiv.), and DCM (3.00 mL, $0.2 \mathrm{M})$ afforded $105.2 \mathrm{mg}$ of the title compound $(0.570 \mathrm{mmol}, 89 \%$ yield $)$.

1H NMR (500 MHz, Chloroform-d): $\delta 7.43(\mathrm{~d}, J=8.4 \mathrm{~Hz}, 2 \mathrm{H}), 7.39$ (d, $J=8.5 \mathrm{~Hz}, 2 \mathrm{H}), 2.68$ (dddd, $J=21.3,13.4$, $10.1,7.7 \mathrm{~Hz}, 2 \mathrm{H}), 2.55(\mathrm{dddd}, J=15.1,13.5,8.7,5.0 \mathrm{~Hz}, 2 \mathrm{H}), 2.11$ (dddt, $J=15.1,10.1,7.9,5.0 \mathrm{~Hz}, 1 \mathrm{H}), 1.82-1.70$ (m, 1H).

13C NMR (126 MHz, Chloroform-d): $\delta 140.87$ (d, $J=23.1 \mathrm{~Hz}), 133.88(\mathrm{~d}, J=2.7 \mathrm{~Hz}), 128.68,126.23(\mathrm{~d}, J=7.2$ $\mathrm{Hz}), 97.15(\mathrm{~d}, J=207.1 \mathrm{~Hz}), 35.32(\mathrm{~d}, J=23.3 \mathrm{~Hz}), 12.50(\mathrm{~d}, J=9.7 \mathrm{~Hz})$.

${ }^{19}$ F NMR (470 MHz, Chloroform-d): $\delta-127.11(\mathrm{tt}, J=21.8,15.0 \mathrm{~Hz})$.

HRMS: (EI-TOF) calculated for $\mathrm{C}_{10} \mathrm{H}_{10}{ }^{35} \mathrm{ClF}^{+}\left(\left[\mathrm{M}^{*}\right]^{+}\right)$: 184.04496 , found 184.04548 .

(EI-TOF) calculated for $\mathrm{C}_{10} \mathrm{H}_{10}{ }^{37} \mathrm{ClF}^{+}\left(\left[\mathrm{M}^{*}\right]^{+}\right)$: 186.04217 , found 186.04258 .

FTIR (thin film, cm$\left.^{-1}\right): ~ v 2995$ (w), 2946 (w), 1601 (w), 1494 (s), 1459 (w), 1419 (w), 1400 (w), 1306 (m), 1247 (m), 1134 (m), 1093 (s), 1063 (m), 1013 (s), 942 (w), 880 (w), 827 (s), 735 (w), 717 (w), 631 (w), 536 (s), 446 (m), 404 (w).

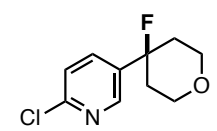

2-chloro-5-(4-fluorotetrahydro-2H-pyran-4-yl)pyridine (26)

Synthesized according to general procedure D with 1,3-dioxoisoindolin-2-yl 2-(6-chloropyridin-3-yl)propanoate (0.0781 g, $0.202 \mathrm{mmol}, 1$ equiv.), $\mathrm{Ir}\left(\mathrm{dF}\right.$-ppy) ${ }_{3}(2.0 \mathrm{mg}, 0.002 \mathrm{mmol}, 0.01$ equiv.), triethylamine trihydrofluoride (97.8 $\mu \mathrm{L}, 0.6 \mathrm{mmol}, 3$ equiv), and DCM (1.00 mL, $0.2 \mathrm{M})$. 1-Fluoronaphthalene (26.0 $\mu \mathrm{L}, 0.200 \mathrm{mmol}, 1$ equiv.) was was added, the reaction mixture was stirred, and an aliquot was removed and analyzed by ${ }^{19} \mathrm{~F}-\mathrm{NMR}$ to obtain ${ }^{72 \%}{ }^{19} \mathrm{~F}-$ 
NMR yield (0.145 mmol, $31.4 \mathrm{mg}$ ). A second run with 1,3-dioxoisoindolin-2-yl 2-(6-chloropyridin-3-yl)propanoate $\left(0.0776 \mathrm{~g}, 0.201 \mathrm{mmol}, 1\right.$ equiv.), $\mathrm{Ir}(\mathrm{dF}-\mathrm{ppy})_{3}(1.8 \mathrm{mg}, 0.002 \mathrm{mmol}, 0.01$ equiv.), triethylamine trihydrofluoride (97.8 $\mu \mathrm{L}, 0.6 \mathrm{mmol}, 3$ equiv.), and DCM (1.00 mL, $0.2 \mathrm{M}$ ) afforded $68 \%{ }^{19} \mathrm{~F}-\mathrm{NMR}$ yield (relative to external 1fluoronaphthalene, $0.136 \mathrm{mmol}, 29.4 \mathrm{mg}$ ). Purification was accomplished via preparatory TLC using 30\% EtOAc in hexanes ( $2 \%$ triethylamine additive).

1H NMR (500 MHz, Chloroform- $d$ ): $\delta 8.42(\mathrm{~d}, J=2.6 \mathrm{~Hz}, 1 \mathrm{H}), 7.68(\mathrm{dd}, J=8.3,2.6 \mathrm{~Hz}, 1 \mathrm{H}), 7.35(\mathrm{~d}, J=8.4 \mathrm{~Hz}$, $1 \mathrm{H}), 3.96(\mathrm{dd}, J=12.0,5.7 \mathrm{~Hz}, 2 \mathrm{H}), 3.87(\mathrm{td}, J=12.0,2.0 \mathrm{~Hz}, 2 \mathrm{H}), 2.12(\mathrm{dddd}, J=40.0,14.0,12.4,5.6 \mathrm{~Hz}, 2 \mathrm{H})$, $1.95-1.90(\mathrm{~m}, 2 \mathrm{H})$.

${ }^{13}$ C NMR (126 MHz, Chloroform- $d$ ): $\delta 151.17(\mathrm{~d}, J=1.9 \mathrm{~Hz}), 146.03(\mathrm{~d}, J=9.5 \mathrm{~Hz}), 138.60(\mathrm{~d}, J=22.4 \mathrm{~Hz})$, $135.00(\mathrm{~d}, J=9.1 \mathrm{~Hz}), 124.20(\mathrm{~d}, J=1.2 \mathrm{~Hz}), 92.31(\mathrm{~d}, J=176.1 \mathrm{~Hz}), 63.52,37.07(\mathrm{~d}, J=22.7 \mathrm{~Hz})$.

${ }^{19}$ F NMR (282 MHz, Chloroform- $d$ ): $\delta-161.91$ (tt, $\left.J=39.2,10.4 \mathrm{~Hz}, 1 \mathrm{~F}\right)$.

HRMS: (ESI-TOF) calculated for $\mathrm{C}_{10} \mathrm{H}_{11} \mathrm{ClFNO}\left[\mathrm{M}^{*}\right]^{+}: 215.05077$, found 215.05114.

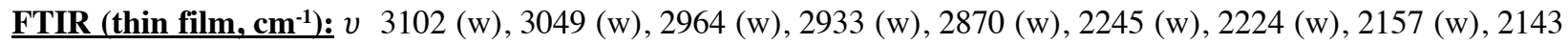
(w), $2023(\mathrm{w}), 1588(\mathrm{w}), 1566(\mathrm{w}), 1459(\mathrm{~m}), 1419(\mathrm{w}), 1386(\mathrm{w}), 1373(\mathrm{w}), 1357(\mathrm{w}), 1295(\mathrm{w}), 1243(\mathrm{w}), 1218(\mathrm{w})$, $1158(\mathrm{w}), 1125$ (w), 1101 (s), $1043(\mathrm{w}), 1016(\mathrm{~m}), 1003(\mathrm{w}), 939$ (w), $920(\mathrm{w}), 847$ (w), $840(\mathrm{~m}), 753(\mathrm{w}), 743(\mathrm{w})$, $697(w), 632(w), 561(w), 554(w), 484(w), 462(w), 438(w), 417(w), 411(w)$.

1-fluoroadamantane (27)

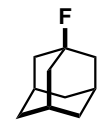

Synthesized according to general procedure E with 1,3-dioxoisoindolin-2-yl-adamantane-1-carboxylate $(0.1975 \mathrm{~g}$, $0.607 \mathrm{mmol}, 1$ equiv.), $\mathrm{Ir}(\mathrm{dF}-\mathrm{ppy})_{3}(4.5 \mathrm{mg}, 0.006 \mathrm{mmol}, 0.01$ equiv.), triethylamine trihydrofluoride $(293.4 \mu \mathrm{L}, 1.8$ mmol, 3 equiv.), and DCM (3.00 mL, $0.2 \mathrm{M})$. 1-Fluoronaphthalene ( $26 \mu \mathrm{L}, 0.2 \mathrm{mmol}, 0.33$ equiv.) was added, the reaction mixture was stirred, and an aliquot was removed and analyzed by ${ }^{19} \mathrm{~F}-\mathrm{NMR}$ to obtain $36 \%{ }^{19} \mathrm{~F}$-NMR yield $(0.219 \mathrm{mmol}, 33.7 \mathrm{mg})$. A second run with 1,3-dioxoisoindolin-2-yl-adamantane-1-carboxylate $(0.2806 \mathrm{~g}, \mathrm{XX} \mathrm{mmol}$, 1 equiv.), Ir(dF-ppy $)_{3}(3.9 \mathrm{mg}, 0.006 \mathrm{mmol}, 0.01$ equiv), triethylamine trihydrofluoride ( $293.4 \mu \mathrm{L}, 1.8 \mathrm{mmol}, 3$ equiv), DCM (3 mL, $0.2 \mathrm{M})$ afforded $40 \%$ NMR yield.

199 NMR (282 MHz, Chloroform- $d$ ): $\delta$-128.48 (s, 1F).

HRMS: (EI-TOF) calculated for $\mathrm{C}_{10} \mathrm{H}_{15} \mathrm{~F}^{+}\left[\left(\mathrm{M}^{*}\right)^{+}\right]$: found 154.11523; observed 154.11554.

Characterization matched literature characterization. ${ }^{16}$

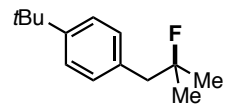

1-(tert-butyl)-4-(2-fluoro-2-methylpropyl)benzene (28)

Synthesized according to general procedure E with 1,3-dioxoisoindolin-2-yl 3-(4-(tert-butyl)phenyl)-2,2dimethylpropanoate $(0.3081 \mathrm{~g}, 0.812 \mathrm{mmol}, 1$ equiv. $), \operatorname{Ir}(\mathrm{dF}-\mathrm{ppy})_{3}(3.0 \mathrm{mg}, 0.006 \mathrm{mmol}, 0.01$ equiv.), triethylamine trihydrofluoride (391.2 $\mu \mathrm{L}, 2.4 \mathrm{mmol}, 3$ equiv.), and DCM (4.00 mL, 0.2 M). 1-Fluoronaphthalene (103.3 $\mu \mathrm{L}, 0.800$ mmol, 1.0 equiv.) was added, the reaction mixture was stirred, and an aliquot was removed and analyzed by ${ }^{19} \mathrm{~F}-\mathrm{NMR}$ to obtain $78 \%{ }^{19} \mathrm{~F}-\mathrm{NMR}$ yield $(0.633 \mathrm{mmol}, 131.9 \mathrm{mg})$. A second run with 1,3-dioxoisoindolin-2-yl 3-(4-(tertbutyl)phenyl)-2,2-dimethylpropanoate $\left(0.3061 \mathrm{~g}, 0.807 \mathrm{mmol}, 1\right.$ equiv.), $\mathrm{Ir}(\mathrm{dF}-\mathrm{ppy})_{3}$ (3.1 mg, $0.006 \mathrm{mmol}, 0.01$ equiv.), triethylamine trihydrofluoride ( $391.2 \mu \mathrm{L}, 2.4 \mathrm{mmol}, 3$ equiv.), and DCM (4.00 mL, $0.2 \mathrm{M})$ afforded $74 \%{ }^{19} \mathrm{~F}-\mathrm{NMR}$ yield of the title compound $(0.597 \mathrm{mmol}, 124.4 \mathrm{mg})$.

${ }^{1}$ H NMR (500 MHz, Chloroform- $d$ ): $\delta 7.31(\mathrm{~d}, J=8.3 \mathrm{~Hz}, 2 \mathrm{H}), 7.14(\mathrm{~d}, J=7.9 \mathrm{~Hz}, 2 \mathrm{H}), 2.88(\mathrm{~d}, J=20.4 \mathrm{~Hz}, 2 \mathrm{H})$, $1.34(\mathrm{~d}, J=21.2 \mathrm{~Hz}, 6 \mathrm{H}), 1.31(\mathrm{~s}, 9 \mathrm{H})$.

${ }^{13}$ C NMR (126 MHz, Chloroform- $\left.d\right): \delta 149.44,134.04(\mathrm{~d}, J=4.3 \mathrm{~Hz}), 130.16,125.13,95.61(\mathrm{~d}, J=167.6 \mathrm{~Hz})$, $47.22(\mathrm{~d}, J=22.9 \mathrm{~Hz}), 34.53,31.53,26.76(\mathrm{~d}, J=24.4 \mathrm{~Hz})$.

19F NMR (376 MHz, Chloroform- $d$ ): $\delta-136.72$ (triplet of heptets, $J=21.2,20.4 \mathrm{~Hz}, 1 \mathrm{~F}$ ).

HRMS: (ESI-TOF) calculated for $\mathrm{C}_{14} \mathrm{H}_{21} \mathrm{~F}^{+}\left[\mathrm{M}^{*}\right]^{+}: 208.16218$, found 208.16195 . 
4-(2-fluoro-2-methylpropyl)benzonitrile (29)

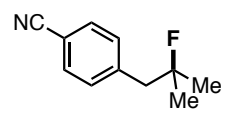

Synthesized according to general procedure E with 1,3-dioxoisoindolin-2-yl 3-(4-cyanophenyl)-2,2dimethylpropanoate $\left(0.2813 \mathrm{~g}, 0.808 \mathrm{mmol}, 1\right.$ equiv.), $\mathrm{Ir}(\mathrm{dF}-\mathrm{ppy})_{3}(4.0 \mathrm{mg}, 0.008 \mathrm{mmol}, 0.01$ equiv.), triethylamine trihydrofluoride $(391.2 \mu \mathrm{L}, 2.4 \mathrm{mmol}, 3$ equiv.), and DCM $(4.00 \mathrm{~mL}, 0.2 \mathrm{M})$. Purified over silica with flash chromatography ( $10 \%$ ether in pentanes) to afford the title compound as a colorless oil $(73.1 \mathrm{mg}, 0.412 \mathrm{mmol}, 51 \%)$. A second run with 1,3-dioxoisoindolin-2-yl 3-(4-cyanophenyl)-2,2-dimethylpropanoate (0.2806 g, $0.805 \mathrm{mmol}, 1$ equiv), $\operatorname{Ir}(\mathrm{dF}-\mathrm{ppy})_{3}(3.9 \mathrm{mg}, 0.008 \mathrm{mmol}, 0.01$ equiv.), triethylamine trihydrofluoride (391.2 $\mu \mathrm{L}, 2.4 \mathrm{mmol}, 3$ equiv.), and DCM (4.00 mL, $0.2 \mathrm{M})$ afforded $70.3 \mathrm{mg}$ of the title compound $(0.397 \mathrm{mmol}, 49 \%)$.

1H NMR (500 MHz, Chloroform- $d$ ): $\delta 7.59$ (d, $J=8.3 \mathrm{~Hz}, 2 \mathrm{H}), 7.33(\mathrm{~d}, J=7.9 \mathrm{~Hz}, 2 \mathrm{H}), 2.95(\mathrm{~d}, J=21.5 \mathrm{~Hz}, 2 \mathrm{H})$, $1.33(\mathrm{~d}, J=21.2 \mathrm{~Hz}, 6 \mathrm{H})$.

${ }^{13}$ C NMR (126 MHz, Chloroform- $\left.d\right)$ : $\delta 142.65(\mathrm{~d}, J=2.1 \mathrm{~Hz}), 132.00,131.27(\mathrm{~d}, J=1.6 \mathrm{~Hz}), 119.07,110.69,94.76$ $(\mathrm{d}, J=169.8 \mathrm{~Hz}), 47.60(\mathrm{~d}, J=22.5 \mathrm{~Hz}), 26.80(\mathrm{~d}, J=24.4 \mathrm{~Hz})$.

19F NMR (376 MHz, Chloroform- $d$ ): $\delta-138.36$ (triplet of heptets, $J=21.5,21.2 \mathrm{~Hz}, 1 \mathrm{~F}$ ).

HRMS: (ESI-TOF) calculated for $\mathrm{C}_{11} \mathrm{H}_{13} \mathrm{FN}^{+}(\mathrm{M}+\mathrm{H})^{+}: 178.10265$, found 178.10261.

FTIR (thin film, cm$\left.^{-1}\right): v 2983(\mathrm{~m}), 2935(\mathrm{w}), 2227(\mathrm{~s}), 1610(\mathrm{~m}), 1507(\mathrm{~m}), 1459(\mathrm{w}), 1417(\mathrm{w}), 1386(\mathrm{~m}), 1374(\mathrm{~s})$, 1334 (w), 1261 (m), 1224 (m), 1188 (m), 1140 (m), 1126 (s), 1022 (w), 974 (w), 894 (m), 869 (s), 850 (s), 823 (s), $762(\mathrm{~s}), 744(\mathrm{w}), 695(\mathrm{w}), 569(\mathrm{~s}), 534(\mathrm{~m}), 515(\mathrm{w}), 467(\mathrm{~m}), 444(\mathrm{w}), 428(\mathrm{w})$.

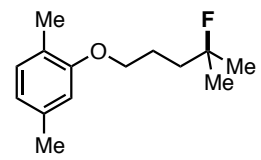

2-((4-fluoro-4-methylpentyl)oxy)-1,4-dimethylbenzene (30)

Synthesized according to general procedure E with 1,3-dioxoisoindolin-2-yl 5-(2,5-dimethylphenoxy)-2,2dimethylpentanoate $\left(0.2449 \mathrm{~g}, 0.619 \mathrm{mmol}, 1\right.$ equiv.), $\mathrm{Ir}(\mathrm{dF}-\mathrm{ppy})_{3}(3.3 \mathrm{mg}, 0.006 \mathrm{mmol}, 0.01$ equiv.), triethylamine trihydrofluoride ( $293.4 \mu \mathrm{L}, 1.8 \mathrm{mmol}, 3$ equiv.), and DCM $(4.00 \mathrm{~mL}, 0.2 \mathrm{M})$. Purified over silica on a 1000-micron preparatory plate (10\% ether in pentanes) to afford the title compound as a colorless oil ( $86.9 \mathrm{mg}, 0.387 \mathrm{mmol}, 63 \%)$. A second run with 1,3-dioxoisoindolin-2-yl 5-(2,5-dimethylphenoxy)-2,2-dimethylpentanoate (0.2394 g, $0.605 \mathrm{mmol}$, 1 equiv.), $\mathrm{Ir}(\mathrm{dF}-\mathrm{ppy})_{3}(2.7 \mathrm{mg}, 0.006 \mathrm{mmol}, 0.01$ equiv.), triethylamine trihydrofluoride $(293.4 \mu \mathrm{L}, 1.8 \mathrm{mmol}, 3$ equiv.), and DCM (4.00 mL, $0.2 \mathrm{M})$ afforded $93.4 \mathrm{mg}$ of the title compound (0.416 mmol, 69\%).

1H NMR (500 MHz, Chloroform-d): $\delta 7.02(\mathrm{~d}, J=7.4 \mathrm{~Hz}, 1 \mathrm{H}), 6.67(\mathrm{~d}, J=7.3 \mathrm{~Hz}, 1 \mathrm{H}), 6.64(\mathrm{~s}, 1 \mathrm{H}), 3.97(\mathrm{t}, J=$ $6.1 \mathrm{~Hz}, 2 \mathrm{H}), 2.32(\mathrm{~s}, 3 \mathrm{H}), 2.19(\mathrm{~s}, 3 \mathrm{H}), 1.96-1.89(\mathrm{~m}, 2 \mathrm{H}), 1.86-1.77(\mathrm{~m}, 2 \mathrm{H}), 1.40(\mathrm{~d}, J=21.4 \mathrm{~Hz}, 6 \mathrm{H})$.

${ }^{13}$ C NMR (126 MHz, Chloroform- $d$ ): $\delta$ 157.07, 136.63, 130.45, 123.72, 120.86, 112.12, 95.63 (d, $\left.J=165.2 \mathrm{~Hz}\right)$, $67.95,38.12(\mathrm{~d}, J=23.1 \mathrm{~Hz}), 26.83(\mathrm{~d}, J=24.8 \mathrm{~Hz}), 24.33(\mathrm{~d}, J=5.0 \mathrm{~Hz}), 21.55,15.91$.

19F NMR (376 MHz, Chloroform-d): $\delta$-138.52 (triplet of hept, $J=20.8,20.6 \mathrm{~Hz}, 1 \mathrm{~F}$ ).

HRMS: (ESI-TOF) calculated for $\mathrm{C}_{14} \mathrm{H}_{22} \mathrm{FO}^{+}(\mathrm{M}+\mathrm{H})^{+}: 225.16492$, found 225.16498 .

FTIR (thin film, cm$\left.^{-1}\right): ~ v 2979(\mathrm{~m}), 2923(\mathrm{~m}), 2868(\mathrm{w}), 1615(\mathrm{~m}), 1585(\mathrm{~m}), 1558(\mathrm{w}), 1540(\mathrm{w}), 1510(\mathrm{~s}), 1459$ (w), $1414(\mathrm{~m}), 1385(\mathrm{~m}), 1373(\mathrm{~m}), 1309(\mathrm{~m}), 1285(\mathrm{~m}), 1264(\mathrm{~s}), 1217(\mathrm{~m}), 1190(\mathrm{w}), 1157(\mathrm{~s}), 1129(\mathrm{~s}), 1046(\mathrm{~s})$, $1001(\mathrm{~m}), 967(\mathrm{w}), 948(\mathrm{w}), 923(\mathrm{w}), 869(\mathrm{~m}), 843(\mathrm{w}), 802(\mathrm{~s}), 762(\mathrm{~m}), 715(\mathrm{w}), 604(\mathrm{w}), 587(\mathrm{~m}), 542(\mathrm{w}), 447$ (m), $401(\mathrm{w})$.

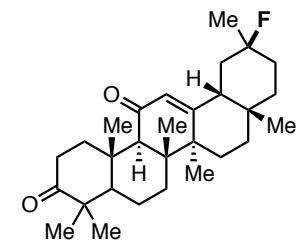

(6aR,6bS,8aS,11S,12aR,14aR,14bS)-11-fluoro-4,4,6a,6b,8a,11,14b-heptamethyl1,2,4,4a,5,6,6a,6b,7,8,8a,9,10,11,12,12a,14a,14b-octadecahydropicene-3,14-dione (31) 
Synthesized according to general procedure D with 1,3-dioxoisoindolin-2-yl $(2 S, 4 \mathrm{a} S, 6 \mathrm{a} S, 6 \mathrm{~b} R, 12 \mathrm{a} S, 12 \mathrm{~b} R, 14 \mathrm{~b} R)$ 2,4a,6a,6b,9,9,12a-heptamethyl-10,13-dioxo-1,2,3,4,4a ,5,6,6a,6b,7,8,8a,9,10,11,12,12a,12b,13,14b-

icosahydropicene-2-carboxylate $\left(0.1225 \mathrm{~g}, 0.200 \mathrm{mmol}, 1\right.$ equiv.), $\mathrm{Ir}(\mathrm{dF}-\mathrm{ppy})_{3}(2.0 \mathrm{mg}, 0.002 \mathrm{mmol}, 0.01$ equiv.), triethylamine trihydrofluoride ( $97.8 \mu \mathrm{L}, 0.6 \mathrm{mmol}, 3$ equiv.), and DCM (1.00 mL, 0.2 M). 1-Fluoronaphthalene (26.0 $\mu \mathrm{L}, 0.200 \mathrm{mmol}, 1$ equiv.) was added, the reaction mixture was stirred, and an aliquot was removed and analyzed by ${ }^{19} \mathrm{~F}-\mathrm{NMR}$ to obtain $32 \%{ }^{19} \mathrm{~F}-\mathrm{NMR}$ yield of both diastereomers (1.14:1 d.r., $0.0639 \mathrm{mmol}, 28.3 \mathrm{mg}$ ). A second run with 1,3-dioxoisoindolin-2-yl $\quad(2 S, 4 \mathrm{a} S, 6 \mathrm{a} S, 6 \mathrm{~b} R, 12 \mathrm{a} S, 12 \mathrm{~b} R, 14 \mathrm{~b} R)-2,4 \mathrm{a}, 6 \mathrm{a}, 6 \mathrm{~b}, 9,9,12 \mathrm{a}$-heptamethyl-10,13-dioxo$1,2,3,4,4 \mathrm{a}, 5,6,6 \mathrm{a}, 6 \mathrm{~b}, 7,8,8 \mathrm{a}, 9,10,11,12,12 \mathrm{a}, 12 \mathrm{~b}, 13,14 \mathrm{~b}$-icosahydropicene-2-carboxylate $(0.1225 \mathrm{~g}, 0.200 \mathrm{mmol}, 1$ equiv.), $\operatorname{Ir}(\mathrm{dF}-\mathrm{ppy})_{3}(1.6 \mathrm{mg}, 0.002 \mathrm{mmol}, 0.01$ equiv.), triethylamine trihydrofluoride ( $97.8 \mu \mathrm{L}, 0.6 \mathrm{mmol}, 3$ equiv.), and DCM (1.00 mL, 0.2 M) afforded 32\% NMR yield (1.14:1 d.r., $0.0639 \mathrm{mmol}, 28.3 \mathrm{mg}$ ).

1 H NMR (500 MHz, Chloroform- $d$ ): (both diastereomers) $\delta 5.68$ (s, 1H, major diastereomer), 5.63 (s, 1H, minor diastereomer), $2.99-2.90(\mathrm{~m}, 1 \mathrm{H}$, both diasteromers overlap), $2.67-2.58(\mathrm{~m}, 1 \mathrm{H}$, both diastereomers overlap), 2.44 (s, $1 \mathrm{H}$, minor diastereomer), $2.42(\mathrm{~s}, 1 \mathrm{H}$, major diastereomer), $2.402 .32(\mathrm{~m}, 2 \mathrm{H}$, both diastereomers overlap), 2.23 $2.11(\mathrm{~m}, 1 \mathrm{H}$, both diastereomers overlap), $2.05(\mathrm{dd}, J=13.5,4.2 \mathrm{~Hz}, 1 \mathrm{H}$, minor diastereomer), 1.98 (td, $J=13.5,4.3$ $\mathrm{Hz}, 1 \mathrm{H}$, major diastereomer), $1.90-1.81$ (m, 2H. both diastereomers overlap), $1.80-1.61(\mathrm{~m}, 4 \mathrm{H}$, both diastereomers overlap), $1.60-1.49(\mathrm{~m}, 4 \mathrm{H}$, both diastereomers overlap), $1.48-1.42(\mathrm{~m}, 2 \mathrm{H}$, both diastereomers overlap), $1.42-$ $1.37(\mathrm{~m}, 4 \mathrm{H}$, both diastereomers overlap), $1.35-1.33(\mathrm{~m}, 3 \mathrm{H}$, both diastereomers overlap), $1.32-1.22$ (m, 7H, both diastereomers overlap), $1.18(\mathrm{~s}, 3 \mathrm{H}$, major diastereomer), $1.17(\mathrm{~s}, 3 \mathrm{H}$, minor diastereomer), 1.10 (s, $7 \mathrm{H}$, minor diastereomer), 1.06 (s, 7H, major diastereomer), 0.89 (s, 3H, major diastereomer), 0.88 (s, 3H, minor diastereomer).

${ }^{13}$ C NMR (126 MHz, Chloroform- $d$ ): (both diastereomers) $\delta$ 217.29, 217.24, 199.54, 199.48, 169.23, 168.28, $128.50,128.46,95.58(\mathrm{~d}, J=310.1 \mathrm{~Hz}), 94.23(\mathrm{~d}, J=310.9 \mathrm{~Hz}), 61.23,61.16,55.57,55.43,49.42(\mathrm{~d}, J=11.0 \mathrm{~Hz})$, 47.93, 47.87, 46.39 (d, $J=0.75 \mathrm{~Hz}), 45.39,45.36,43.52,43.45,43.01$ (d, $J=20.9 \mathrm{~Hz}), 42.09$ (d, $J=22.6 \mathrm{~Hz}), 39.91$, $39.82,38.19(\mathrm{~d}, J=10.9 \mathrm{~Hz}), 36.84,36.80,35.33(\mathrm{~d}, J=2.1 \mathrm{~Hz}), 34.37,34.32,33.14(\mathrm{~d}, J=20.7 \mathrm{~Hz}), 32.68(\mathrm{~d}, J=$ $1.7 \mathrm{~Hz}), 32.25,32.21(\mathrm{~d}, J=2.4 \mathrm{~Hz}) \mathrm{j}, 32.07,31.75,29.85,28.24,28.10(\mathrm{~d}, J=3.2 \mathrm{~Hz}), 27.78(\mathrm{~d}, J=25.0 \mathrm{~Hz}), 26.60$, $26.59,26.51,26.49,26.47,26.05(\mathrm{~d}, J=1.2 \mathrm{~Hz}), 23.65,23.46,23.21(\mathrm{~d}, J=26.0 \mathrm{~Hz}), 21.56,21.55,18.93(\mathrm{~d}), 18.68$, $18.68,15.82,15.80$.

19F NMR (282 MHz, Chloroform- $d$ ): $\delta-124.71$ (qt, $J=22.8,13.5 \mathrm{~Hz}, 1 \mathrm{~F}$, minor diastereomer), $-154.69--155.24$ ( $\mathrm{m}, 1 \mathrm{~F}$, major diastereomer).

HRMS: (ESI-TOF) calculated for $\mathrm{C}_{29} \mathrm{H}_{42} \mathrm{FO}_{2}{ }^{+}\left(\mathrm{M}^{*}-\mathrm{HF}\right)^{+}: 422.31793$, found 422.31814.

FTIR (thin film, cm$\left.^{-1}\right): v 2976$ (m), 2250 (w), 2208 (w), 2192 (w), 2174 (w), 2159 (w), 2013 (w), 1983 (w), 1969 (w), $1954(\mathrm{w}), 1702(\mathrm{w}), 1658(\mathrm{~m}), 1460(\mathrm{w}), 1386(\mathrm{~m}), 1272(\mathrm{w}), 1206(\mathrm{w}), 1111(\mathrm{w}), 913(\mathrm{~s}), 870(\mathrm{w}), 736(\mathrm{~s}), 648$ (w), $487(w), 444(w), 430(w), \quad 419(w), 408(w)$.

\section{1-(tert-butyl)-4-(fluoromethoxy)benzene (32)}

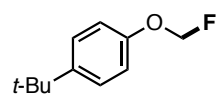

Synthesized according to general procedure $\mathrm{E}$ with 1,3-dioxoisoindolin-2-yl 2-(4-(tert-butyl)phenoxy)acetate (0.2194 $\mathrm{g}, 0.621 \mathrm{mmol}, 1$ equiv. $), \operatorname{Ir}(\mathrm{dF}-\mathrm{ppy})_{3}(2.3 \mathrm{mg}, 0.006 \mathrm{mmol}, 0.01$ equiv.), triethylamine trihydrofluoride $(293.4 \mu \mathrm{L}$, $1.8 \mathrm{mmol}, 3$ equiv.), and DCM (3.00 mL, $0.2 \mathrm{M})$. 1-Fluoronaphthalene (77.50 $\mu \mathrm{L}, 0.600 \mathrm{mmol}, 1.00$ equiv.) was added, the reaction mixture was stirred, and an aliquot was removed and analyzed by ${ }^{19} \mathrm{~F}-\mathrm{NMR}$ to obtain $26 \%{ }^{19} \mathrm{~F}$ NMR yield $(0.161 \mathrm{mmol}, 29.4 \mathrm{mg})$. A second run with 1,3-dioxoisoindolin-2-yl 2-(4-(tert-butyl)phenoxy)acetate (0.2213 g, $0.626 \mathrm{mmol}, 1$ equiv.), $\mathrm{Ir}(\mathrm{dF}-\mathrm{ppy})_{3}(3.4 \mathrm{mg}, 0.006 \mathrm{mmol}, 0.01$ equiv .), triethylamine trihydrofluoride (293.4 $\mu \mathrm{L}, 1.8 \mathrm{mmol}, 3$ equiv), and DCM (3.00 mL, $0.2 \mathrm{M})$ afforded $29 \%{ }^{19} \mathrm{~F}-\mathrm{NMR}$ yield $(0.182 \mathrm{mmol}, 33.1 \mathrm{mg})$. Attempted isolation and purification by flash chromatography or preparatory TLC showed an inseparable and unidentifiable impurity.

1H NMR (500 MHz, Chloroform- $d$ ): $\delta 7.36(\mathrm{dd}, J=8.7,1.8 \mathrm{~Hz}, 2 \mathrm{H}), 7.03(\mathrm{dd}, J=8.8,1.9 \mathrm{~Hz}, 2 \mathrm{H}), 5.70(\mathrm{~d}, J=$ $54.9 \mathrm{~Hz}, 2 \mathrm{H}), 1.32(\mathrm{~s}, 9 \mathrm{H})$.

${ }^{19}$ F NMR (376 MHz, Chloroform- $\left.d\right)$ : $\delta-147.96(\mathrm{t}, J=55.0 \mathrm{~Hz}, 1 \mathrm{~F})$.

HRMS: (EI-TOF) calculated for $\mathrm{C}_{11} \mathrm{H}_{15} \mathrm{FO}^{+}\left(\mathrm{M}^{*}\right)^{+}:$182.11014, found 182.10960 .

This matches literature characterization. ${ }^{17}$ 
(4-bromophenyl)(fluoromethyl)sulfane (33)

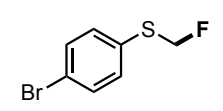

Synthesized according to general procedure E with 1,3-dioxoisoindolin-2-yl 2-((4-bromophenyl)thio)acetate $(0.2386$ $\mathrm{g}, 0.608 \mathrm{mmol}, 1$ equiv.), Ir(dF-ppy) ${ }_{3}(4.3 \mathrm{mg}, 0.006 \mathrm{mmol}, 0.01$ equiv.), triethylamine trihydrofluoride $(293.4 \mu \mathrm{L}$, $1.8 \mathrm{mmol}, 3$ equiv.), and DCM (3.00 mL, $0.2 \mathrm{M})$. Purified over silica with flash chromatography (100\% hexanes) to afford the title compound as a whitish oil $(74.2 \mathrm{mg}, 0.336 \mathrm{mmol}, 55 \%)$ with a dimer (1,2-bis((4bromophenyl)thio)ethane) impurity $(7.1 \mathrm{mg}, 5 \%)$. A second run with 1,3-dioxoisoindolin-2-yl 2-((4bromophenyl)thio)acetate $\left(0.2398 \mathrm{~g}, 0.611 \mathrm{mmol}, 1\right.$ equiv.), $\mathrm{Ir}(\mathrm{dF}-\mathrm{ppy})_{3}$ (5.6 $\mathrm{mg}, 0.006 \mathrm{mmol}, 0.01$ equiv.), triethylamine trihydrofluoride ( $293.4 \mu \mathrm{L}, 1.8 \mathrm{mmol}, 3$ equiv.), and DCM $(3.00 \mathrm{~mL}, 0.2 \mathrm{M})$ afforded $67.9 \mathrm{mg}$ of the title compound $(0.307 \mathrm{mmol}, 50 \%)$ with dimer impurity (6.5 mg, 5\%, 1,2-bis((4-bromophenyl)thio)ethane).

1H NMR (500 MHz, Chloroform- $d$ ): (5\% dimer impurity denoted with *) $\delta 7.47(\mathrm{~d}, J=8.5 \mathrm{~Hz}, 2 \mathrm{H})$, *7.39 (d, $J=$ $8.6 \mathrm{~Hz}, 4 \mathrm{H}), 7.36(\mathrm{~d}, J=8.5 \mathrm{~Hz}, 2 \mathrm{H}), * 7.12(\mathrm{~d}, J=8.6 \mathrm{~Hz}, 4 \mathrm{H}), 5.70(\mathrm{~d}, J=52.7 \mathrm{~Hz}, 2 \mathrm{H}), * 2.47(\mathrm{~s}, 4 \mathrm{H})$.

${ }^{13}$ C NMR (126 MHz, Chloroform- $d$ ): (5\% dimer impurity denoted with *) $\delta 133.53(\mathrm{~d}, J=2.9 \mathrm{~Hz}), 132.45,132.37$ $(\mathrm{d}, J=2.3 \mathrm{~Hz}), * 131.93, * 128.25,122.25(\mathrm{~d}, J=0.9 \mathrm{~Hz}), 88.40(\mathrm{~d}, J=217.2 \mathrm{~Hz}), * 16.07$.

${ }^{19}$ F NMR (282 MHz, Chloroform- $\left.d\right)$ : $\delta-182.27(\mathrm{t}, J=52.7 \mathrm{~Hz}, 1 \mathrm{~F})$.

HRMS: (ESI-TOF) calculated for $\mathrm{C}_{7} \mathrm{H}_{6} \mathrm{BrFS}^{+}\left[\left(\mathrm{M}^{*}\right)^{+}\right]$: 219.93521 , found 219.93539.

FTIR (thin film, cm$\left.^{-1}\right): v 2939(\mathrm{w}), 1565(\mathrm{w}), 1473(\mathrm{~s}), 1427(\mathrm{w}), 1387(\mathrm{~m}), 1320(\mathrm{~m}), 1230(\mathrm{w}), 1199(\mathrm{w}), 1091(\mathrm{~s})$, $1068(\mathrm{~s}), 1008(\mathrm{~s}), 954(\mathrm{~s}), 807(\mathrm{~s}), 736(\mathrm{~s}), 719(\mathrm{~s}), 501(\mathrm{~m}), 478(\mathrm{~s}), 426(\mathrm{w})$.

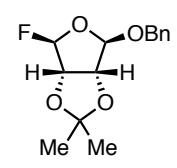

(3aS,4S,6S,6aR)-4-(benzyloxy)-6-fluoro-2,2-dimethyltetrahydrofuro[3,4-d][1,3]dioxole (34)

Synthesized according to general procedure E with 1,3-dioxoisoindolin-2-yl (3aS,4S,6R,6aR)-6-(benzyloxy)-2,2dimethyltetrahydrofuro[3,4- $d][1,3]$ dioxole-4-carboxylate $(0.2628 \mathrm{~g}, 0.598 \mathrm{mmol}, 1$ equiv.), Ir(dF-ppy) 3 (3.6 mg, $0.006 \mathrm{mmol}, 0.01$ equiv.), triethylamine trihydrofluoride ( $293.4 \mu \mathrm{L}, 1.8 \mathrm{mmol}, 3$ equiv.), and DCM (3.00 mL, $0.2 \mathrm{M})$. Purified over silica with flash chromatography (10\% ether in pentanes) to afford the title compound as a white solid (68.3 mg, $0.255 \mathrm{mmol}, 42 \%,>20: 1$ d.r.). A second run with 1,3-dioxoisoindolin-2-yl (3aS,4S,6R,6aR)-6-(benzyloxy)2,2-dimethyltetrahydrofuro[3,4- $d][1,3]$ dioxole-4-carboxylate $(0.2665 \mathrm{~g}, 0.606 \mathrm{mmol}, 1 \text { equiv.), Ir(dF-ppy) })_{3}(3.5 \mathrm{mg}$, $0.006 \mathrm{mmol}, 0.01$ equiv.), triethylamine trihydrofluoride (293.4 $\mu \mathrm{L}, 1.8 \mathrm{mmol}, 3$ equiv.), and DCM (3.00 mL, $0.2 \mathrm{M})$ afforded $73.3 \mathrm{mg}$ of the title compound $(0.273 \mathrm{mmol}, 46 \%$ yield, $>20: 1 \mathrm{dr})$.

1H NMR (500 MHz, Chloroform- $d$ ): $\delta 7.39-7.28(\mathrm{~m}, 5 \mathrm{H}), 5.84(\mathrm{~d}, J=60.5 \mathrm{~Hz}, 1 \mathrm{H}), 5.33(\mathrm{~d}, J=2.8 \mathrm{~Hz}, 1 \mathrm{H})$, $4.87(\mathrm{t}, J=6.0 \mathrm{~Hz}, 1 \mathrm{H}), 4.83(\mathrm{~d}, J=11.6 \mathrm{~Hz}, 1 \mathrm{H}), 4.75(\mathrm{~d}, J=5.7 \mathrm{~Hz}, 1 \mathrm{H}), 4.52(\mathrm{~d}, J=11.5 \mathrm{~Hz}, 1 \mathrm{H}), 1.44(\mathrm{~s}, 3 \mathrm{H})$, $1.31(\mathrm{~s}, 3 \mathrm{H})$.

${ }^{13}$ C NMR (126 MHz, Chloroform- $d$ ): $\delta$ 136.75, 128.64, 128.48, 128.16, $116.04(\mathrm{~d}, J=227.0 \mathrm{~Hz}), 113.07$ (d, $J=$ $1.2 \mathrm{~Hz}), 109.20(\mathrm{~d}, J=1.9 \mathrm{~Hz}), 84.18(\mathrm{~d}, J=39.8 \mathrm{~Hz}), 83.58,69.56,26.29,24.90$.

${ }^{19}$ F NMR (470 MHz, Chloroform- $d$ ): $\delta-119.26$ (ddd, $\left.J=60.4,6.4,2.8 \mathrm{~Hz}, 1 \mathrm{~F}\right)$.

HRMS: (EI-TOF) calculated for $\mathrm{C}_{13} \mathrm{H}_{14} \mathrm{FO}_{4}{ }^{+}\left(\left[\mathrm{M}-\mathrm{CH}_{3}\right]^{*}\right)^{+}: 253.08706$, found 253.08760 .

FTIR (thin film, cm$\left.^{-1}\right): v 2992(\mathrm{w}), 2980(\mathrm{w}), 2957$ (w), 2937 (w), $1455(\mathrm{w}), 1379(\mathrm{~m}), 1355(\mathrm{~m}), 1276(\mathrm{w}), 1245$ (w), $1209(\mathrm{~m}), 1159(\mathrm{~m}), 1123(\mathrm{~m}), 1088$ (s), 1057 (s), 1018 (s), 996 (s), 967 (s), 934 (s), 863 (s), 840 (m), 815 (s), $786(\mathrm{~m}), 758(\mathrm{~s}), 701(\mathrm{~s}), 653(\mathrm{w}), 636(\mathrm{~m}), 563(\mathrm{~m}), 513(\mathrm{~m}), 504(\mathrm{~m}), 443(\mathrm{~m}), 428(\mathrm{w})$.

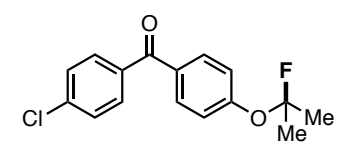

(4-chlorophenyl)(4-((2-fluoropropan-2-yl)oxy)phenyl)methanone (35)

Synthesized according to general procedure E with 1,3-dioxoisoindolin-2-yl 2-(4-(4-chlorobenzoyl)phenoxy)-2methylpropanoate $(0.2811 \mathrm{~g}, 0.606 \mathrm{mmol}, 1$ equiv. $), \operatorname{Ir}(\mathrm{dF}-\mathrm{ppy})_{3}(2.7 \mathrm{mg}, 0.006 \mathrm{mmol}, 0.01$ equiv.), triethylamine 
trihydrofluoride ( $293.4 \mu \mathrm{L}, 1.8 \mathrm{mmol}, 3$ equiv.), and DCM (3.00 mL, 0.2 M). 1-Fluoronaphthalene (77.50 $\mu \mathrm{L}, 0.600$ mmol, 1.00 equiv.) was added, the reaction mixture was stirred, and an aliquot was removed and analyzed by ${ }^{19} \mathrm{~F}-$ NMR to obtain $56 \%{ }^{19} \mathrm{~F}-\mathrm{NMR}$ yield $(0.339 \mathrm{mmol}, 99.3 \mathrm{mg})$. A second run with 1,3-dioxoisoindolin-2-yl 2-(4-(4chlorobenzoyl)phenoxy)-2-methylpropanoate $\left(0.2788 \mathrm{~g}, 0.601 \mathrm{mmol}, 1\right.$ equiv.), $\mathrm{Ir}(\mathrm{dF}-\mathrm{ppy})_{3}$ (2.1 mg, $0.006 \mathrm{mmol}$, 0.01 equiv.), triethylamine trihydrofluoride ( $293.4 \mu \mathrm{L}, 1.8 \mathrm{mmol}, 3$ equiv.), and DCM (3.00 mL, $0.2 \mathrm{M})$ afforded $57 \%$ ${ }^{19} \mathrm{~F}-\mathrm{NMR}$ yield of the title compound (relative to 1 -fluoronaphthalene, $0.343 \mathrm{mmol}, 100.3 \mathrm{mg}$ ).

Note: this compound rapidly decomposes from a flocculant white solid (purification over silica via flash chromatography, $10 \%$ ether in hexanes) to a brown oily residue upon workup and purification (approx. 30 minutes at room temperature). This decomposition affords acetone as observed by ${ }^{1} \mathrm{H}-\mathrm{NMR}$.

1H NMR (500 MHz, Chloroform-d): $\delta 7.77(\mathrm{~d}, J=8.7 \mathrm{~Hz}, 2 \mathrm{H}), 7.74(\mathrm{~d}, J=8.5 \mathrm{~Hz}, 2 \mathrm{H}), 7.46(\mathrm{~d}, J=8.4 \mathrm{~Hz}, 2 \mathrm{H})$, $7.23(\mathrm{~d}, J=8.6 \mathrm{~Hz}, 2 \mathrm{H}), 1.66(\mathrm{~d}, J=17.4 \mathrm{~Hz}, 6 \mathrm{H})$.

${ }^{13}$ C NMR (126 MHz, Chloroform- $d$ ): $\delta 194.51,157.73$ (d, $\left.J=1.9 \mathrm{~Hz}\right), 138.72,136.07,132.84,131.75,131.31$, $128.65,121.46(\mathrm{~d}, J=3.2 \mathrm{~Hz}), 115.69(\mathrm{~d}, J=220.9 \mathrm{~Hz}), 25.64(\mathrm{~d}, J=29.2 \mathrm{~Hz})$.

19F NMR (470 MHz, Chloroform- $d$ ): $\delta-89.95$ (hept, $\mathrm{J}=17.3 \mathrm{~Hz}, 1 \mathrm{~F}$ ).

HRMS: (ESI-TOF) calculated for $\mathrm{C}_{25} \mathrm{H}_{19} \mathrm{ClNO}_{6}{ }^{+}\left([\mathrm{M}+\mathrm{H}]^{+}\right)$: 464.08954, found 464.08910.

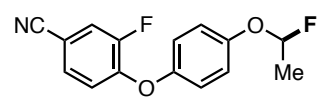

3-fluoro-4-(4-(1-fluoroethoxy)phenoxy)benzonitrile (36)

Synthesized according to general procedure E with 1,3-dioxoisoindolin-2-yl 2-(4-(4-cyano-2fluorophenoxy)phenoxy)propanoate ( $0.2691 \mathrm{~g}, 0.603 \mathrm{mmol}, 1$ equiv.), $\mathrm{Ir}(\mathrm{dF}-\mathrm{ppy})_{3}(4.6 \mathrm{mg}, 0.006 \mathrm{mmol}, 0.01 \mathrm{equiv}$.), triethylamine trihydrofluoride ( $293.4 \mu \mathrm{L}, 1.8 \mathrm{mmol}, 3$ equiv.), and DCM (3.00 mL, $0.2 \mathrm{M})$. Purified over silica via flash chromatography ( 0 to $5 \%$ ether in hexanes) to afford the title compound as a clear oil $(96.8 \mathrm{mg}, 0.352 \mathrm{mmol}$, $58 \%)$. A second run with 1,3-dioxoisoindolin-2-yl 2-(4-(4-cyano-2-fluorophenoxy)phenoxy)propanoate $(0.2363 \mathrm{~g}$, $0.529 \mathrm{mmol}, 1$ equiv. $), \operatorname{Ir}(\mathrm{dF}-\mathrm{ppy})_{3}(5.0 \mathrm{mg}, 0.006 \mathrm{mmol}, 0.01$ equiv. $)$, triethylamine trihydrofluoride $(293.4 \mu \mathrm{L}, 1.8$ mmol, 3 equiv.), and DCM (3.00 mL, $0.2 \mathrm{M})$ afforded $77.8 \mathrm{mg}$ of the title compound $(0.283 \mathrm{mmol}, 53 \%)$.

1H NMR (500 MHz, Methylene Chloride- $\boldsymbol{d}_{2}$ ): $\delta 7.47(\mathrm{dd}, J=10.4,2.0 \mathrm{~Hz}, 1 \mathrm{H}), 7.37(\mathrm{dt}, J=8.6,1.7 \mathrm{~Hz}, 1 \mathrm{H}), 7.11$ $(\mathrm{d}, J=9.1 \mathrm{~Hz}, 2 \mathrm{H}), 7.04(\mathrm{~d}, J=9.1 \mathrm{~Hz}, 2 \mathrm{H}), 6.91(\mathrm{t}, J=8.4 \mathrm{~Hz}, 1 \mathrm{H}), 5.95(\mathrm{dq}, J=62.8,4.9 \mathrm{~Hz}, 1 \mathrm{H}), 1.65(\mathrm{dd}, J=$ $20.0,4.9 \mathrm{~Hz}, 3 \mathrm{H})$.

${ }^{13}$ C NMR (126 MHz, Methylene Chloride- $\boldsymbol{d}_{2}$ ): $\delta 154.17(\mathrm{~d}, J=2.6 \mathrm{~Hz}), 152.99$ (d, $\left.J=251.0 \mathrm{~Hz}\right), 150.60,150.59$ $(\mathrm{d}, J=10.7 \mathrm{~Hz}), 130.08(\mathrm{~d}, J=3.9 \mathrm{~Hz}), 121.43,121.14(\mathrm{~d}, J=21.4 \mathrm{~Hz}), 119.44(\mathrm{~d}, J=1.9 \mathrm{~Hz}), 119.17(\mathrm{~d}, J=1.6$ $\mathrm{Hz}), 118.11(\mathrm{~d}, J=2.6 \mathrm{~Hz}), 108.87(\mathrm{~d}, J=216.6 \mathrm{~Hz}), 106.91(\mathrm{~d}, J=8.2 \mathrm{~Hz}), 21.40(\mathrm{~d}, J=24.9 \mathrm{~Hz})$.

${ }^{19}$ F NMR (282 MHz, Methylene Chloride- $d_{2}$ ): $\delta-116.18$ (dq, $\left.J=63.0,19.9 \mathrm{~Hz}, 1 \mathrm{~F}\right),-130.76(\mathrm{t}, J=9.2 \mathrm{~Hz}, 1 \mathrm{~F})$.

HRMS: (EI-TOF) calculated for $\mathrm{C}_{15} \mathrm{H}_{12} \mathrm{~F}_{2} \mathrm{NO}_{2}{ }^{+}([\mathrm{M}+\mathrm{H}])^{+}: 275.07524$, found 275.07593

FTIR (thin film, $\left.\mathbf{c m}^{-1}\right): v 3076$ (w), $3001(\mathrm{w}), 2943(\mathrm{w}), 2232(\mathrm{w}), 1722(\mathrm{w}), 1614(\mathrm{w}), 1584(\mathrm{w}), 1495$ (s), 1446

(w), $1422(\mathrm{w}), 1402(\mathrm{~s}), 1366(\mathrm{w}), 1280(\mathrm{~s}), 1214(\mathrm{~s}), 1190(\mathrm{~s}), 1147$ (m), $1117(\mathrm{~s}), 1071(\mathrm{~s}), 1011$ (w), $944(\mathrm{~s}), 845$

$(\mathrm{s}), 800(\mathrm{~s}), 763(\mathrm{w}), 737(\mathrm{w}), 710(\mathrm{w}), 625(\mathrm{w}), 613(\mathrm{~m}), 578(\mathrm{w}), 536(\mathrm{~m}), 475(\mathrm{w}), 452(\mathrm{w}), 426(\mathrm{w})$.

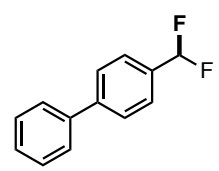

4-(difluoromethyl)-1,1'-biphenyl (37)

Synthesized according to general procedure E with 1,3-dioxoisoindolin-2-yl 2-([1,1'-biphenyl]-4-yl)-2-fluoroacetate $(0.2282 \mathrm{~g}, 0.608 \mathrm{mmol}, 1$ equiv. $), \operatorname{Ir}(\mathrm{dF}-\mathrm{ppy})_{3}(4.1 \mathrm{mg}, 0.006 \mathrm{mmol}, 0.01$ equiv.), triethylamine trihydrofluoride (293.4 $\mu \mathrm{L}, 1.8 \mathrm{mmol}, 3$ equiv.), and DCM $(3.00 \mathrm{~mL}, 0.2 \mathrm{M})$. Purified over silica via flash chromatography (5\% ether in hexanes) to afford the title compound as a white solid $(69.9 \mathrm{mg}, 0.342 \mathrm{mmol}, 56 \%)$. A second run with 1,3dioxoisoindolin-2-yl 2-([1,1'-biphenyl]-4-yl)-2-fluoroacetate $(0.2251 \mathrm{~g}, 0.600 \mathrm{mmol}, 1$ equiv.), $\operatorname{Ir}(\mathrm{dF}-\mathrm{ppy}) 3$ (4.6 mg, 
$0.006 \mathrm{mmol}, 0.01$ equiv.), triethylamine trihydrofluoride ( $293.4 \mu \mathrm{L}, 1.8 \mathrm{mmol}, 3$ equiv.), and DCM (3.00 mL, $0.2 \mathrm{M})$ afforded $75.1 \mathrm{mg}$ of the title compound $(0.368 \mathrm{mmol}, 61 \%)$.

1H NMR (500 MHz, Chloroform- $d): \delta 7.68(\mathrm{~d}, J=8.0 \mathrm{~Hz}, 2 \mathrm{H}), 7.59(\mathrm{t}, J=7.8 \mathrm{~Hz}, 4 \mathrm{H}), 7.47(\mathrm{t}, J=7.7 \mathrm{~Hz}, 2 \mathrm{H})$, $7.39(\mathrm{t}, J=7.3 \mathrm{~Hz}, 1 \mathrm{H}), 6.70(\mathrm{t}, J=56.5 \mathrm{~Hz}, 1 \mathrm{H})$.

${ }^{13}$ C NMR (126 MHz, Chloroform- $\left.d\right): \delta 143.85(\mathrm{t}, J=2.0 \mathrm{~Hz}), 140.33,133.34(\mathrm{t}, J=22.4 \mathrm{~Hz}), 129.06,128.05$, $127.59,127.40,126.17(\mathrm{t}, J=6.0 \mathrm{~Hz}), 114.89(\mathrm{t}, J=238.5 \mathrm{~Hz})$.

${ }^{19}$ F NMR (376 MHz, Chloroform- $\left.d\right)$ : $\delta-110.39(\mathrm{~d}, J=56.7 \mathrm{~Hz}, 2 \mathrm{~F})$.

HRMS: (EI-TOF) calculated for $\mathrm{C}_{15} \mathrm{H}_{12} \mathrm{~F}_{2} \mathrm{NO}_{2}{ }^{+}\left(\left[\mathrm{M}^{*}\right]^{+}\right): 204.07451$, found 204.07426 .

FTIR (thin film, cm$\left.^{-1}\right): v 3059(\mathrm{~m}), 3037(\mathrm{~m}), 2361(\mathrm{~m}), 2334(\mathrm{w}), 1806(\mathrm{~s}), 1613(\mathrm{~m}), 1570(\mathrm{~m}), 1486(\mathrm{~m}), 1411$ $(\mathrm{m}), 1376(\mathrm{~s}), 1314(\mathrm{~m}), 1257(\mathrm{~m}), 1224(\mathrm{~s}), 1200(\mathrm{~s}), 1182(\mathrm{~m}), 1125(\mathrm{~m}), 1071(\mathrm{~s}), 1019(\mathrm{~s}), 1004(\mathrm{~s}), 953(\mathrm{~s}), 836$ (s), $765(\mathrm{~s}), 738(\mathrm{~s}), 689(\mathrm{~s}), 647(\mathrm{~s}), 635(\mathrm{~s}), 559(\mathrm{~s}), 506(\mathrm{~m}), 416(\mathrm{~m})$.

(1,2,2,2-tetrafluoro-1-methoxyethyl)benzene (38)

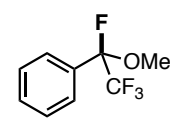

Synthesized according to general procedure E with 1,3-dioxoisoindolin-2-yl 3,3,3-trifluoro-2-methoxy-2phenylpropanoate $\left(0.2317 \mathrm{~g}, 0.611 \mathrm{mmol}, 1\right.$ equiv.), $\mathrm{Ir}(\mathrm{dF}-\mathrm{ppy})_{3}(3.3 \mathrm{mg}, 0.006 \mathrm{mmol}, 0.01$ equiv.), triethylamine trihydrofluoride (293.4 $\mu \mathrm{L}, 1.8 \mathrm{mmol}, 3$ equiv.), and DCM (3.00 mL, 0.2 M). 1-Fluoronaphthalene (77.5 $\mu \mathrm{L}, 0.600$ mmol, 1.00 equiv.) was added, the reaction mixture was stirred, and an aliquot was removed and analyzed by ${ }^{19} \mathrm{~F}-$ NMR to obtain $89 \%{ }^{19} \mathrm{~F}-\mathrm{NMR}$ yield $(0.544 \mathrm{mmol}, 113.2 \mathrm{mg})$. A second run with 1,3-dioxoisoindolin-2-yl 3,3,3trifluoro-2-methoxy-2-phenylpropanoate $\left(0.2342 \mathrm{~g}, 0.617 \mathrm{mmol}, 1\right.$ equiv.), $\operatorname{Ir}(\mathrm{dF}-\mathrm{ppy})_{3}(4.2 \mathrm{mg}, 0.006 \mathrm{mmol}, 0.01$ equiv.), triethylamine trihydrofluoride ( $293.4 \mu \mathrm{L}, 1.8 \mathrm{mmol}, 3$ equiv.), and $\mathrm{DCM}(3.00 \mathrm{~mL}, 0.2 \mathrm{M})$ afforded $88 \%{ }^{19} \mathrm{~F}-$ NMR yield of the title compound (relative to 1-fluoronaphthalene, $0.543 \mathrm{mmol}, 113.1 \mathrm{mg}$ ).

19F NMR (282 MHz, Chlorform- $d$ ): $\delta-83.68(\mathrm{~d}, J=3.7 \mathrm{~Hz}, 3 \mathrm{~F}),-134.00(\mathrm{q}, J=3.9 \mathrm{~Hz}, 1 \mathrm{~F})$

HRMS: (EI-TOF) calculated for $\mathrm{C}_{9} \mathrm{H}_{8} \mathrm{~F}_{4} \mathrm{NO}^{+}\left(\left[\mathrm{M}^{*}\right]^{+}\right)$: 208.05058, found 208.05119 .

This matched literature characaterization. ${ }^{18}$

Lit. ${ }^{1} \mathrm{H}$ NMR: $\delta 7.51(\mathrm{~s}, 5 \mathrm{H}), 3.83(\mathrm{~s}, 3 \mathrm{H})$.

Lit. ${ }^{19}$ F NMR: $\delta-83.8(\mathrm{~d}, J=3.52 \mathrm{~Hz}, 3 \mathrm{~F}),-134.3(\mathrm{q}, J=3.52 \mathrm{~Hz}, 1 \mathrm{~F})$.

9,9-difluoro-9H-fluorene (39)

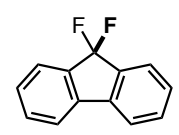

Synthesized according to general procedure E with 1,3-dioxoisoindolin-2-yl 9-fluoro- $9 H$-fluorene-9-carboxylate $\left(0.2283 \mathrm{~g}, 0.612 \mathrm{mmol}, 1\right.$ equiv.), $\mathrm{Ir}(\mathrm{dF}-\mathrm{ppy})_{3}(4.9 \mathrm{mg}, 0.006 \mathrm{mmol}, 0.01$ equiv.), triethylamine trihydrofluoride (293.4 $\mu \mathrm{L}, 1.8 \mathrm{mmol}, 3$ equiv.), and DCM (3.00 mL, 0.2 M). Purified over silica via flash chromatography (3-5\% ether in hexanes) to afford the title compound as a yellow solid $(68.9 \mathrm{mg}, 0.341 \mathrm{mmol}, 56 \%)$. A second run with $1,3-$ dioxoisoindolin-2-yl 9-fluoro-9H-fluorene-9-carboxylate $\left(0.2245 \mathrm{~g}, 0.601 \mathrm{mmol}, 1\right.$ equiv.), $\operatorname{Ir}(\mathrm{dF}-\mathrm{ppy})_{3}$ (5.2 $\mathrm{mg}$, $0.006 \mathrm{mmol}, 0.01$ equiv.), triethylamine trihydrofluoride ( $293.4 \mu \mathrm{L}, 1.8 \mathrm{mmol}, 3$ equiv.), and DCM (3.00 mL, $0.2 \mathrm{M})$ afforded $57.7 \mathrm{mg}$ of the title compound $(0.285 \mathrm{mmol}, 48 \%)$.

$\underline{1}$ H NMR (500 MHz, Methylene Chloride- $\left.\boldsymbol{d}_{2}\right): \delta 7.63(\mathrm{dd}, J=7.2,1.8 \mathrm{~Hz}, 2 \mathrm{H}), 7.58(\mathrm{dd}, J=7.6,1.1 \mathrm{~Hz}, 2 \mathrm{H}), 7.47$ $(\mathrm{td}, J=7.6,1.0 \mathrm{~Hz}, 2 \mathrm{H}), 7.34(\mathrm{td}, J=7.5,1.0 \mathrm{~Hz}, 2 \mathrm{H})$.

${ }^{13}$ C NMR (126 MHz, Chloroform- $\left.d\right): \delta 139.57(\mathrm{t}, J=5.0 \mathrm{~Hz}), 138.05(\mathrm{t}, J=25.1 \mathrm{~Hz}), 132.16(\mathrm{t}, J=1.1 \mathrm{~Hz}), 128.91$ $(\mathrm{t}, J=1.4 \mathrm{~Hz}), 123.91,123.35(\mathrm{~d}, J=242.6 \mathrm{~Hz}), 120.50$.

${ }^{19} \mathrm{~F}$ NMR (282 MHz, Methylene Chloride- $\left.d_{2}\right): \delta-111.54$ (s, 2F).

HRMS: (EI-TOF) calculated for $\mathrm{C}_{13} \mathrm{H}_{8} \mathrm{~F}_{2}{ }^{+}\left(\left[\mathrm{M}^{*}\right]^{+}\right): 202.05886$, found 202.05986.

FTIR (thin film, $\left.\mathbf{c m}^{-1}\right): v 3077(\mathrm{w}), 2920(\mathrm{w}), 2320(\mathrm{w}), 1962(\mathrm{w}), 1923(\mathrm{w}), 1886(\mathrm{w}), 1815(\mathrm{w}), 1610(\mathrm{~m}), 1589$ (m), $1491(\mathrm{~m}), 1452(\mathrm{~s}), 1310(\mathrm{~m}), 1292(\mathrm{~m}), 1256(\mathrm{~s}), 1208(\mathrm{~s}), 1164(\mathrm{~s}), 1115(\mathrm{~m}), 1048(\mathrm{~s}), 1031(\mathrm{~s}), 983(\mathrm{~s}), 938$ $(\mathrm{s}), 869(\mathrm{~m}), 765(\mathrm{~s}), 727(\mathrm{~s}), 653(\mathrm{~s}), 617(\mathrm{~s}), 585(\mathrm{~s}), 509(\mathrm{~m}), 422(\mathrm{~s}), 416(\mathrm{~s})$. 


\section{(3-fluoro-3-methylbutyl)benzene (40)}

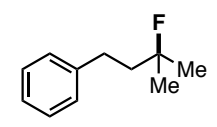

Synthesized according to general procedure E with 1,3-dioxoisoindolin-2-yl (2-methyl-4-phenylbutan-2-yl) oxalate $(0.2257 \mathrm{~g}, 0.592 \mathrm{mmol}, 1$ equiv. $), \operatorname{Ir}(\mathrm{dF}-\mathrm{ppy})_{3}(4.6 \mathrm{mg}, 0.006 \mathrm{mmol}, 0.01$ equiv.), triethylamine trihydrofluoride (293.4 $\mu \mathrm{L}, 1.8 \mathrm{mmol}, 3$ equiv.), and DCM (3.00 mL, 0.2 M). 1-Fluoronaphthalene ( $26.0 \mu \mathrm{L}, 0.200 \mathrm{mmol}, 0.33$ equiv.) was added, the reaction mixture was stirred, and an aliquot was removed and analyzed by ${ }^{19} \mathrm{~F}-\mathrm{NMR}$ to obtain $33 \%{ }^{19} \mathrm{~F}$ NMR yield $(0.136 \mathrm{mmol}, 22.6 \mathrm{mg})$.

${ }^{1}$ H NMR (300 MHz, Chloroform-d): $\delta 7.34-7.27$ (m, 2H), $7.22-7.17(\mathrm{~m}, 3 \mathrm{H}), 2.77-2.70(\mathrm{~m}, 2 \mathrm{H}), 2.00-1.87$ $(\mathrm{dm}, J=19.3 \mathrm{~Hz}, 2 \mathrm{H}), 1.42(\mathrm{~d}, J=21.4 \mathrm{~Hz}, 6 \mathrm{H})$.

${ }^{13}$ C NMR (75 MHz, Chloroform- $d$ ): $\delta$ 142.19, 128.58, 128.43, 126.00, $95.43(\mathrm{~d}, J=165.6 \mathrm{~Hz}), 43.50(\mathrm{~d}, J=22.9$ $\mathrm{Hz}), 30.42(\mathrm{~d}, J=5.2 \mathrm{~Hz}), 26.84(\mathrm{~d}, J=24.8 \mathrm{~Hz})$.

19F NMR (282 MHz, Chloroform- $d$ ): $\delta-138.83$ (hept of triplets, $J=21.4,19.3 \mathrm{~Hz}, 1 \mathrm{~F}$ ).

HRMS: (EI-TOF) calculated for $\mathrm{C}_{11} \mathrm{H}_{15} \mathrm{~F}^{+}\left(\left[\mathrm{M}^{*}\right]^{+}\right): 166.11523$, found 166.11517 .

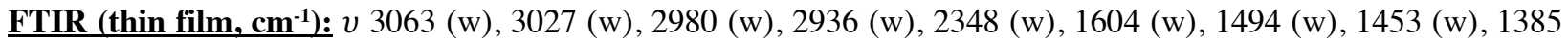
(w), $1373(\mathrm{~m}), 1253(\mathrm{w}), 1211(\mathrm{w}), 1181(\mathrm{w}), 1138(\mathrm{w}), 1073(\mathrm{w}), 1031(\mathrm{w}), 884(\mathrm{w}), 826(\mathrm{w}), 766(\mathrm{w}), 739(\mathrm{~m}), 697$ (s), $591(\mathrm{w}), 518(\mathrm{w}), 496(\mathrm{w}), 457(\mathrm{w}), 417(\mathrm{w}), 407(\mathrm{w})$.

Characterization matched literature precedent. ${ }^{19}$ 


\section{Mechanistic Studies}

\section{Stern-Volmer Quenching}

Fluorescence measurements were obtained using septa-capped UV-Quartz cuvettes (10 mm pathlength, 4 polished windows) obtained from Starna Cells (Cat\#: 3-Q-10-GL 14-S). Excitation was performed at 400 nm; fluorescence spectra were obtained from 420-600 nm.

In a nitrogen filled glovebox, using deaerated materials (triethylamine $3 \mathrm{HF}$ was sparged under nitrogen for $1 \mathrm{~h}$ prior to entering the glovebox; anhydrous DCM was obtained from a solvent purification system and sparged for $1 \mathrm{~h}$ prior to entering the glovebox), the following solutions were prepared:

\section{Photocatalyst solution (0.0002 M):}

To an oven dried scintillation vial with a stir bar was added $\operatorname{Ir}(\mathrm{dF}-\mathrm{ppy})_{3}\left(1.70 \mathrm{mg}, 2.23 \times 10^{-3} \mathrm{mmol}\right)$. This was diluted with $10.8 \mathrm{~mL}$ of DCM and stirred until completely dissolved, producing a $2.06 \times 10^{-4} \mathrm{M}$ solution of $\operatorname{Ir}(\mathrm{dF}-\mathrm{ppy})_{3}$.

Phthalimide ester solution (0.004 M):

To an oven dried scintillation vial, equipped with a stir bar, was added naproxen phthalimide ester 1 (16.2 mg, 4.32x 10-

${ }^{2} \mathrm{mmol}$ ). This was diluted in $10.8 \mathrm{~mL}$ of DCM and stirred until completely dissolved, producing a colorless, $4.00 \mathrm{x} 10^{-}$

${ }^{3} \mathrm{M}$ solution of phthalimide ester $\mathbf{1}$.

Triethylamine $3 \mathrm{HF}$ solution $(0.004 \mathrm{M})$ :

To an oven dried scintillation vial, equipped with a stir bar was added $10.8 \mathrm{~mL}$ of DCM. followed by of Triethylamine $3 \mathrm{HF}\left(7.0 \mu \mathrm{L}, 4.3 \times 10^{-2} \mathrm{mmol}\right)$ was then added and stirred to produce a $4.0 \times 10^{-3} \mathrm{M}$ solution of triethylamine $3 \mathrm{HF}$.

Following preparation of these solutions, the solutions were allocated to the cuvettes and fluorescence quenching was determined with individual quenchers triethylamine $3 \mathrm{HF}$ and phthalimide ester, as well as the reaction mixture:

Table S7. Fluorescence Quenching with Triethylamine·3HF.

\begin{tabular}{|c|c|c|c|c|c|c|c|}
\hline \multirow[b]{2}{*}{$\begin{array}{l}\text { Concentration in } \\
\text { Quencher (mM) }\end{array}$} & \multicolumn{2}{|c|}{ Triethylamine $\cdot 3 \mathrm{HF}$} & \multicolumn{2}{|c|}{$\operatorname{lr}(\mathrm{dF}-\mathrm{ppy})_{3}$} & \multirow{2}{*}{$\frac{\text { DCM }}{\substack{\text { DCM aliquot } \\
(\mu \mathrm{L})}}$} & \multicolumn{2}{|c|}{ Fluorescence } \\
\hline & $\begin{array}{l}\text { Stock solution } \\
\text { aliquot }(\mu \mathrm{L})\end{array}$ & $\begin{array}{l}\text { Stock solution } \\
\text { conc. (M) }\end{array}$ & $\begin{array}{l}\text { Stock solution } \\
\text { aliquot }(\mu \mathrm{L})\end{array}$ & $\begin{array}{l}\text { Stock solution } \\
\text { conc. (M) }\end{array}$ & & $\begin{array}{l}\text { Measured intensity } \\
\text { at } 480 \mathrm{~nm} \text { (a.u.) }\end{array}$ & $\begin{array}{c}\mathrm{I}_{0} / \mathrm{l} \text { at } \\
480 \mathrm{~nm}\end{array}$ \\
\hline 0 & 0 & 0.004 & 200 & 0.0002 & 1900 & 657.4334717 & 1 \\
\hline 0.200 & 100 & 0.004 & 200 & 0.0002 & 1800 & 644.2330322 & 1.02049016 \\
\hline 0.400 & 200 & 0.004 & 200 & 0.0002 & 1700 & 660.0245361 & 0.99607429 \\
\hline 0.600 & 300 & 0.004 & 200 & 0.0002 & 1600 & 662.6502686 & 0.99212738 \\
\hline 0.800 & 400 & 0.004 & 200 & 0.0002 & 1500 & 630.7843628 & 1.04224757 \\
\hline \multirow[t]{5}{*}{1.00} & 500 & 0.004 & 200 & 0.0002 & 1400 & 617.6901245 & 1.06434189 \\
\hline & & & & & & Slope: & 54.719 \\
\hline & & & & & & Intercept: & 0.992 \\
\hline & & & & & & $R^{2}:$ & 0.489 \\
\hline & & & & & & RMSE: & 0.023 \\
\hline
\end{tabular}


Table S8. Fluorescence Quenching with Phthalimide Ester.

\begin{tabular}{|c|c|c|c|c|c|c|c|c|}
\hline \multirow[b]{2}{*}{$\begin{array}{l}\text { Concentration in } \\
\text { Quencher (mM) }\end{array}$} & \multicolumn{2}{|c|}{ Naproxen Phthalimide Ester } & \multicolumn{3}{|c|}{$\operatorname{Ir}(\mathrm{dF}-\mathrm{ppy})_{3}$} & \multirow{2}{*}{$\begin{array}{c}\text { DCM } \\
\begin{array}{c}\text { DCM aliquot } \\
(\mu \mathrm{L})\end{array}\end{array}$} & & \\
\hline & $\begin{array}{c}\text { Stock solution } \\
\text { aliquot }(\mu \mathrm{L})\end{array}$ & $\begin{array}{l}\text { Stock solution } \\
\text { conc. (M) }\end{array}$ & $\begin{array}{l}\text { Stock solution } \\
\text { aliquot }(\mu \mathrm{L})\end{array}$ & \multicolumn{2}{|c|}{$\begin{array}{l}\text { Stock solution } \\
\text { conc. (M) }\end{array}$} & & & \\
\hline 0 & 0 & 0.004 & 200 & \multicolumn{2}{|c|}{0.0002} & 1900 & & \\
\hline 0.200 & 100 & 0.004 & 200 & \multicolumn{2}{|c|}{0.0002} & 1800 & & \\
\hline 0.400 & 200 & 0.004 & 200 & \multicolumn{2}{|c|}{0.0002} & 1700 & & \\
\hline 0.600 & 300 & 0.004 & 200 & \multicolumn{2}{|c|}{0.0002} & 1600 & & \\
\hline 0.800 & 400 & 0.004 & 200 & \multicolumn{2}{|c|}{0.0002} & 1500 & & \\
\hline \multirow[t]{2}{*}{1.00} & 500 & 0.004 & 200 & \multicolumn{2}{|c|}{0.0002} & 1400 & & \\
\hline & \multicolumn{2}{|c|}{ Fluorescence-Run 1} & \multicolumn{4}{|c|}{ Fluorescence-Run 2} & \multicolumn{2}{|c|}{ Fluorescence-Run 3} \\
\hline $\begin{array}{l}\text { Concentration in } \\
\text { Quencher (mM) }\end{array}$ & $\begin{array}{l}\text { Measured intensity } \\
\text { at } 480 \mathrm{~nm} \text { (a.u.) }\end{array}$ & $\begin{array}{c}\mathrm{I}_{0} / \mathrm{l} \text { at } \\
480 \mathrm{~nm}\end{array}$ & $\begin{array}{r}\text { Measured in } \\
\text { at } 480 \mathrm{~nm}\end{array}$ & $\begin{array}{l}\text { nsity } \\
\text { u.) }\end{array}$ & & $\begin{array}{c}\mathrm{I}_{0} / \mathrm{I} \text { at } \\
480 \mathrm{~nm}\end{array}$ & $\begin{array}{l}\text { Measured intensity } \\
\text { at } 480 \mathrm{~nm} \text { (a.u.) }\end{array}$ & $\begin{array}{c}\mathrm{I}_{0} / \mathrm{l} \text { at } \\
480 \mathrm{~nm}\end{array}$ \\
\hline 0 & 645.4230957 & 1 & 614.459 & & & 1 & 614.6759033 & 1 \\
\hline 0.200 & 245.3461304 & 2.63066344 & 228.971 & & & 2.69231512 & 221.5214081 & 2.77479233 \\
\hline 0.400 & 163.0663605 & 3.95803950 & 145.791 & & & 4.2999474 & 149.3206024 & 4.11648422 \\
\hline 0.600 & 121.1813278 & 5.32609361 & 115.776 & & & 5.47978551 & 115.0957718 & 5.34056025 \\
\hline 0.800 & 94.12566376 & 6.85703633 & $a^{a}$ & & & - & 88.39720917 & 6.95356685 \\
\hline \multirow[t]{4}{*}{1.00} & 79.78944397 & 8.08907875 & $75.6037064,7$ & 903778 & 8.394 & $45959,8.17668722$ & 74.44662476 & 8.25659867 \\
\hline & Slope: & 7070.4 & & & & 7216.1 & & 7149.1 \\
\hline & Intercept: & 1.1083 & & & & 1.1538 & & 1.1658 \\
\hline & $R^{2}:$ & 0.998 & & & & 0.998 & & 0.998 \\
\hline
\end{tabular}

an this replication, the $1.00 \mathrm{mM}$ concentration data point was inadvertently performed twice, and the $0.8 \mathrm{mM}$ loading was skipped. 
Table S9. Fluorescence Quenching with Reaction Mixture (1 and Triethylamine·3HF).

\begin{tabular}{|c|c|c|c|c|c|c|c|}
\hline \multirow[b]{2}{*}{$\begin{array}{l}\text { Concentration in } \\
\text { Quencher (mM) }\end{array}$} & \multicolumn{2}{|c|}{ Naproxen Phthalimide Ester } & \multicolumn{2}{|c|}{ Triethylamine $\bullet 3 \mathrm{HF}$} & \multicolumn{2}{|c|}{$\operatorname{Ir(dF-ppy)})_{3}$} & \multirow{2}{*}{$\begin{array}{c}\text { DCM } \\
\begin{array}{c}\text { DCM aliquot } \\
(\mu \mathrm{L})\end{array}\end{array}$} \\
\hline & $\begin{array}{l}\text { Stock solution } \\
\text { aliquot }(\mu \mathrm{L})\end{array}$ & $\begin{array}{l}\text { Stock solution } \\
\text { conc. (M) }\end{array}$ & $\begin{array}{c}\text { Stock solution } \\
\text { aliquot }(\mu \mathrm{L})\end{array}$ & $\begin{array}{l}\text { Stock solution } \\
\text { conc. (M) }\end{array}$ & $\begin{array}{l}\text { Stock solution } \\
\text { aliquot }(\mu \mathrm{L})\end{array}$ & $\begin{array}{l}\text { Stock solution } \\
\text { conc. (M) }\end{array}$ & \\
\hline 0 & 0 & 0.004 & 0 & 0.004 & 200 & 0.0002 & 1800 \\
\hline 0.200 & 100 & 0.004 & 100 & 0.004 & 200 & 0.0002 & 1600 \\
\hline 0.400 & 200 & 0.004 & 200 & 0.004 & 200 & 0.0002 & 1400 \\
\hline 0.600 & 300 & 0.004 & 300 & 0.004 & 200 & 0.0002 & 1200 \\
\hline 0.800 & 400 & 0.004 & 400 & 0.004 & 200 & 0.0002 & 1000 \\
\hline \multirow[t]{2}{*}{1.00} & 500 & 0.004 & 500 & 0.004 & 200 & 0.0002 & 800 \\
\hline & \multicolumn{2}{|c|}{ Fluorescence-Run 1} & \multicolumn{2}{|c|}{ Fluorescence-Run 2} & & & \\
\hline $\begin{array}{l}\text { Concentration in } \\
\text { Quencher (mM) }\end{array}$ & $\begin{array}{l}\text { Measured intensity } \\
\text { at } 480 \mathrm{~nm} \text { (a.u.) }\end{array}$ & $\begin{array}{c}\mathrm{I}_{0} / \mathrm{l} \text { at } \\
480 \mathrm{~nm}\end{array}$ & $\begin{array}{l}\text { Measured intensity } \\
\text { at } 480 \mathrm{~nm} \text { (a.u.) }\end{array}$ & $\begin{array}{c}\mathrm{I}_{0} / 1 \mathrm{at} \\
480 \mathrm{~nm}\end{array}$ & & & \\
\hline 0 & 623.660390 & 1 & 625.824768 & 1 & & & \\
\hline 0.200 & 236.922119 & 2.63234324 & 241.048889 & 2.5625659 & & & \\
\hline 0.400 & 154.681686 & 4.03189514 & 150.061050 & 4.17046773 & & & \\
\hline 0.600 & 110.838532 & 5.62674668 & 111.429939 & 5.61630718 & & & \\
\hline 0.800 & 89.1032562 & 6.99929908 & 87.2636719 & 7.17165293 & & & \\
\hline \multirow[t]{4}{*}{1.00} & 718322296 & 8.68217989 & 77.6254883 & 8.06210411 & & & \\
\hline & Slope: & 7586.7 & & 7211.8 & & & \\
\hline & Intercept: & 1.0354 & & 1.1636 & & & \\
\hline & $R^{2}:$ & 0.999 & & 0.994 & & & \\
\hline
\end{tabular}

Figure S2. Compiled Fluorescence Quenching Graphs.

\section{Stern-Volmer Fluorescence Quenching}

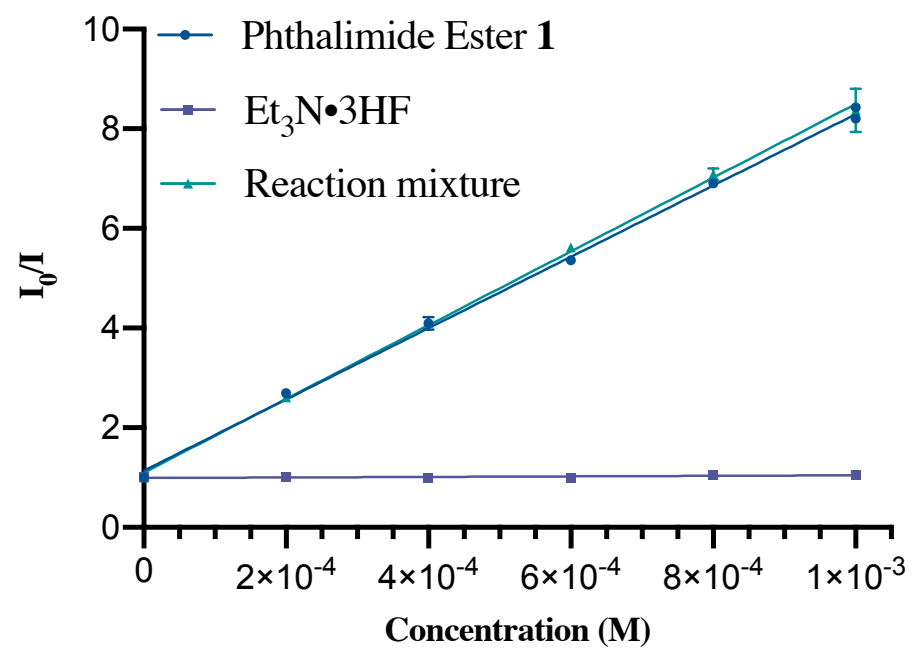

Note: Error bars are not observable on all points as they are smaller than the size of the points represented. 


\section{Discussion}

Based on the observed quenching and the close match between individual quencher and reaction mixture quenching, the naproxen phthalimide ester (1) interacts with the photocatalyst preferentially to triethylamine $3 \mathrm{HF}$. As triethylamine-3HF is a reductive quencher, while phthalimide esters are oxidative quenchers, the observed interaction is indicative of oxidative quenching of the photocatalyst, providing further mechanistic support for the proposed strategy. The Stern-Volmer quenching rate constant is $4.7 \times 10^{9} \mathrm{M}^{-1} \mathrm{~s}^{-1}$ (based on a literature lifetime ${ }^{20}$ for $\operatorname{Ir}(\mathrm{dF}-\mathrm{ppy})_{3}$, $\tau=1570 \mathrm{~ns})$. This approaches the bimolecular diffusion rate limit $\left(10^{10} \mathrm{M}^{-1} \mathrm{~s}^{-1}\right)$. Furthermore, no significant increase in quenching rate was observed in the presence of triethylamine $3 \mathrm{HF}$ indicating that a hydrogen bonding interaction was unlikely. ${ }^{21}$ However, the extremely low concentrations employed for Stern-Volmer analysis make it difficult to observe evidence of interaction between a potential hydrogen-bond donor and a phthalimide ester hydrogen-bond acceptor. This is particularly noteworthy as an acidic additive is necessary for conversion and reactivity (vide infra).

\section{Quantum yield determination}

The quantum yield was determined using an adaptation of procedures reported by Yoon ${ }^{22}$ and Knowles. ${ }^{23}$

\section{General information}

Potassium ferrioxalate $\left(\mathrm{K}_{3} \mathrm{Fe}\left(\mathrm{C}_{2} \mathrm{O}_{4}\right)_{3} \cdot 3 \mathrm{H}_{2} \mathrm{O}\right)$ was obtained from Alfa Aesar and used as received. Concentrated sulfuric acid (17.8 M) was obtained from VWR and used as received. A Kessil PR-160 $440 \mathrm{~nm}$ lamp was used at 50\% power.

Figure S3. Emission Spectra of Lamps Evaluated.

\section{Kessil PR-160 Emission}

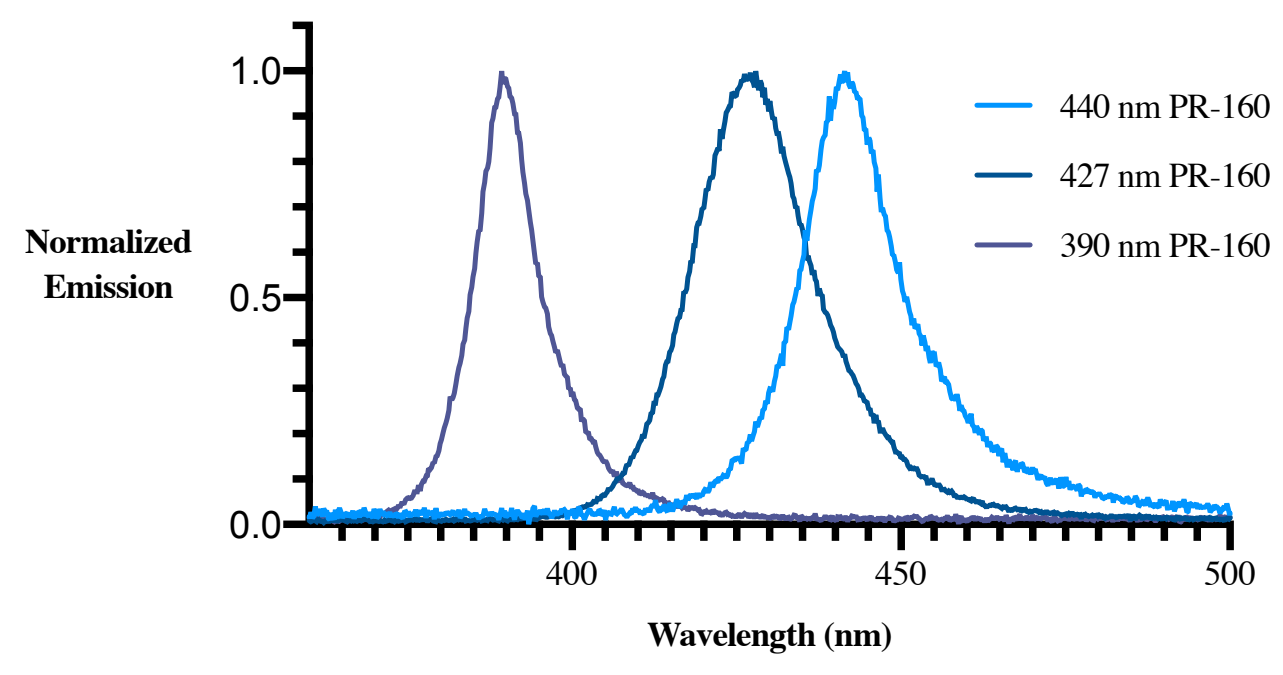

The following solutions were prepared:

Developer solution (0.5 M buffered sulfuric acid, $0.056 \mathrm{M}$ phenanthroline):

To a $250-\mathrm{mL}$ volumetric flask was added $56.23 \mathrm{~g}(0.4132 \mathrm{~mol})$ of sodium acetate trihydrate (Aldrich) and $2.5023 \mathrm{~g}$ $(0.01389 \mathrm{~mol})$ of 1,10-phenanthroline (Aldrich). This was diluted with deionized water and $7.02 \mathrm{~mL}$ of concentrated sulfuric acid $(17.8 \mathrm{M})$ was added. Following this, the solution was diluted to $250 \mathrm{~mL}$ using deionized water. This solution was stored in the dark to slow degradation.

0.05 M sulfuric acid stock solution:

To a $100-\mathrm{mL}$ volumetric flask was added $90 \mathrm{~mL}$ of deionized water followed by $0.280 \mathrm{~mL}$ of concentrated sulfuric acid $(17.8 \mathrm{M})$. The solution was then diluted to $100 \mathrm{~mL}$ with deionized water.

\subsection{Ferrioxalate solution:}


To a 25 -mL volumetric flask was added $1.8432 \mathrm{~g}$ of potassium ferrioxalate $\left(\left[\mathrm{K}_{3} \mathrm{Fe}\left(\mathrm{C}_{2} \mathrm{O}_{4}\right)_{3} \bullet 3 \mathrm{H}_{2} \mathrm{O}\right]\right.$, $\mathrm{mw}=491.25 \mathrm{~g} / \mathrm{mol}$, $3.7521 \mathrm{mmol}$ ). Note: The potassium ferrioxalate was weighed as fast as possible and solutions were kept in the dark to minimize the amount of background decomposition. The volumetric flask was wrapped in aluminum foil and diluted with approx. $20 \mathrm{~mL}$ of $0.05 \mathrm{M}$ sulfuric acid stock solution (see above). Following this, the volumetric flask was sonicated and shaken to dissolve all of the potassium ferrioxalate solid. During this, the flask was kept wrapped in aluminum foil to prevent any incident light and was visualized in a darkened room to check for complete solvation of the green solid. Following complete dissolution of the solid, the solution was diluted to $25 \mathrm{~mL}$ with $0.05 \mathrm{M}$ sulfuric acid solution.

Calibration of light source using potassium ferrioxalate.

Using black, opaque electrical tape, a $1 \mathrm{~cm}$ by $1 \mathrm{~cm}$ quartz cuvette was taped on 2 sides to prevent side incident light and then partially taped across the front face to produce a constant aperture for photon flux through the cuvette. The cuvette was placed a fixed distance (approx. $3 \mathrm{~cm}$ ) from the lamp. The cuvette was equipped with a stir bar stirred at $500 \mathrm{rpm}$. The vial was charged with $3 \mathrm{~mL}$ of $0.15 \mathrm{M}$ potassium ferrioxalate solution as prepared above. A $20 \mathrm{uL}$ aliquot of the solution was taken and diluted into $5 \mathrm{~mL}$ of developer solution. The developer solution vial with the aliquot was immediately wrapped with aluminum foil and set aside to let the iron ligate with phenanthroline to produce $\mathrm{Fe}$ (phen) $)_{3}{ }^{2+}$ as a red-orange solution. The cuvette was then irradiated using the Kessil PR-160 lamp (440 nm) at 50\% power. Aliquots $(20 \mathrm{uL})$ were taken at 20 second intervals. Immediately following the dilution of each aliquot into 5 $\mathrm{mL}$ of developer solution, the vial containing the developer solution and aliquot was wrapped in aluminum foil to prevent additional incidence of light. After all of the aliquot/developer solutions were allowed to develop for 45 minutes to $1 \mathrm{hr}$ and become red-orange while wrapped, the solutions were sequentially unwrapped from aluminum foil and quickly transferred to cuvettes. Absorbance spectra were obtained between 460 and $560 \mathrm{~nm}$. The absorbance at $510 \mathrm{~nm}$ was used to determine the quantity and rate of production of $\mathrm{Fe}(\mathrm{phen})_{3}{ }^{2+}\left(\varepsilon_{510 \mathrm{~nm}}=11,100 \mathrm{M}^{-1} \mathrm{~cm}^{-1}\right)$. This process was repeated in triplicate.

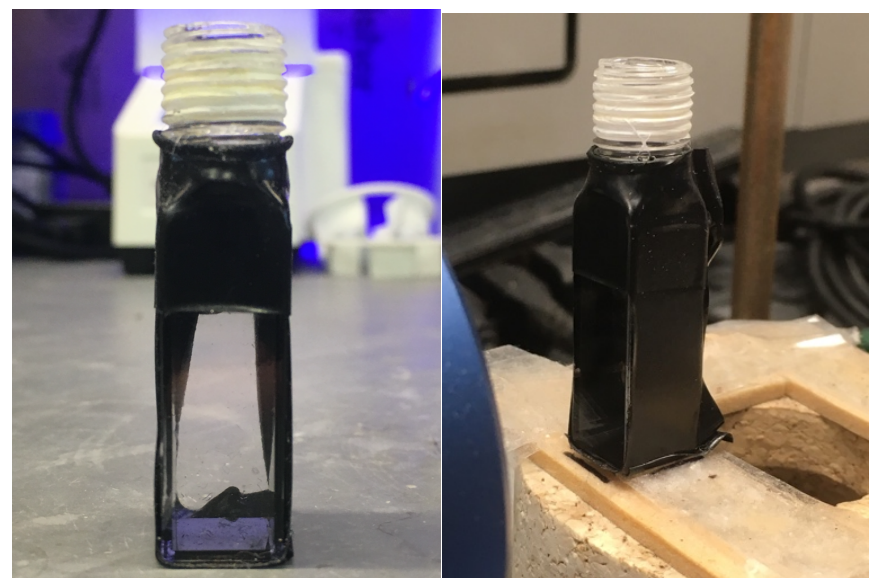

Figure S4. Cuvette for Quantum Yield Determination. The face of the cuvette $(1 \mathrm{~cm}$ pathlength) towards the lamp has tape only above a certain threshold (approximately $2.5 \mathrm{~cm}$ so that several $20-75 \mu \mathrm{L}$ aliquots could be taken).

Results and data analysis.

Figure S5. Replicates for Fe(phen) $)_{3}^{2+}$ Absorption.

Absorbance-Run 1

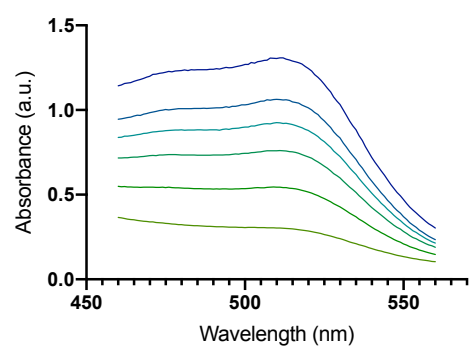

Absorbance-Run 2

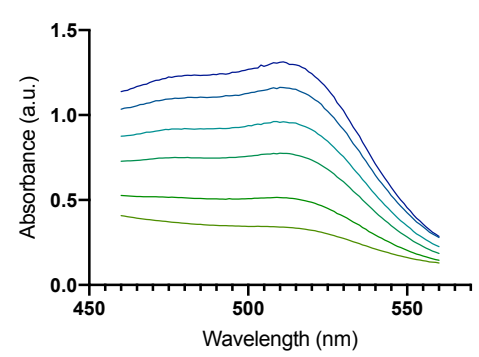

Absorbance-Run 3

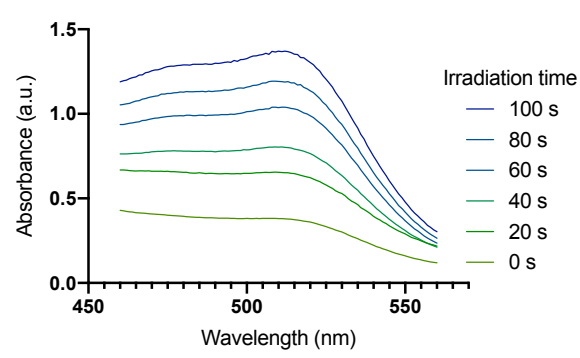


Table S10. Absorption data of $\mathrm{Fe}(\mathrm{phen})_{3^{2+}}$ at $510 \mathrm{~nm}$.

\begin{tabular}{|c|c|c|c|c|c|c|}
\hline \multirow[b]{2}{*}{$\begin{array}{c}\text { Time of } \\
\text { Irradiation (s) }\end{array}$} & \multicolumn{2}{|c|}{ Run 1} & \multicolumn{2}{|c|}{ Run 2} & \multicolumn{2}{|c|}{ Run 3} \\
\hline & $\begin{array}{c}\text { Measured } \\
\text { Absorbance (a.u.) }\end{array}$ & $\begin{array}{l}\Delta \text { Absorbance } \\
\text { (a.u.) }\end{array}$ & $\begin{array}{c}\text { Measured } \\
\text { Absorbance (a.u.) }\end{array}$ & $\begin{array}{c}\Delta \text { Absorbance } \\
\text { (a.u.) }\end{array}$ & $\begin{array}{c}\text { Measured } \\
\text { Absorbance (a.u.) }\end{array}$ & $\begin{array}{c}\Delta \text { Absorbance } \\
\text { (a.u.) }\end{array}$ \\
\hline 0 & 0.303585678 & 0 & 0.341238111 & 0 & 0.381247968 & 0 \\
\hline 20 & 0.543958962 & 0.240373284 & 0.513885617 & 0.172647506 & 0.654968739 & 0.273620771 \\
\hline 40 & 0.759392679 & 0.455807 & 0.775179505 & 0.430556942 & 0.802574098 & 0.421326131 \\
\hline 60 & 0.92545116 & 0.621865481 & 0.957174957 & 0.615936846 & 1.037086844 & 0.655838877 \\
\hline 80 & 1.062799573 & 0.759213895 & 1.162686944 & 0.821448833 & 1.192298889 & 0.811050922 \\
\hline 100 & 1.30434072 & 1.000755042 & 1.310974479 & 0.969736368 & 1.370225549 & 0.988977582 \\
\hline $\begin{array}{l}\text { Linear regression } \\
\qquad R^{2}\end{array}$ & - & 0.990 & - & 0.995 & - & 0.988 \\
\hline
\end{tabular}

Figure S6. Change in Relative Absorbance over Irradiation.

\section{$\Delta$ Absorbance over Irradiation Time}

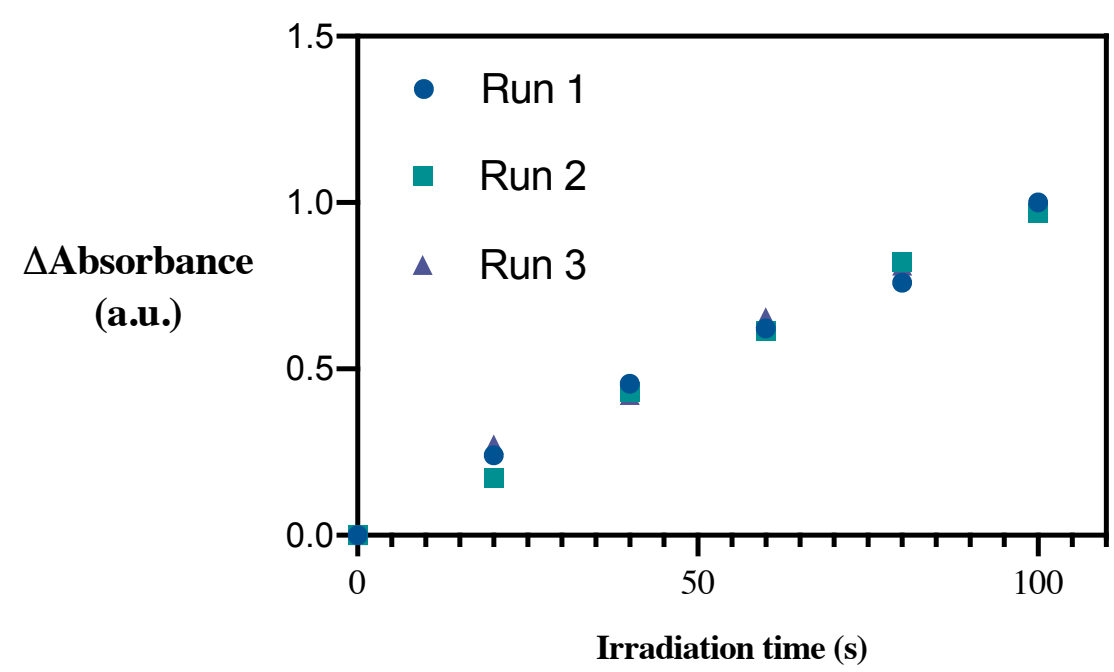

From this, the number of iron ions produced by ferrioxalate photodegradation may be determined:

$$
\text { moles } \mathrm{Fe}^{2+}=\frac{\Delta A b s_{510 \mathrm{~nm}} V_{1} V_{3}}{\varepsilon_{510 \mathrm{~nm}} l V_{2}}
$$

$\Delta A b s=$ difference in absorbance at $510 \mathrm{~nm}$ between sample and 'blank'

$l=$ path length of cuvette $(1 \mathrm{~cm})$

$\varepsilon_{510 \mathrm{~nm}}=$ extinction coefficient of $\mathrm{Fe}(\text { phen })_{3}{ }^{2+}$ complex at $510 \mathrm{~nm}\left(\varepsilon=11,100 \mathrm{M}^{-1} \mathrm{~cm}^{-1}\right)$

$V_{l}=$ total volume of irradiated solution ( $3 \mathrm{~mL}$ at start and decreased with each aliquot)

$V_{2}=$ volume of aliquot taken from $V_{l}(20 \mathrm{uL})$

$V_{3}=$ the volume that $V_{2}$ is diluted into $(5 \mathrm{~mL})$ 
Table S11. Fe(phen $)_{3}^{2+}$ Photochemical Production.

\begin{tabular}{cccc}
\hline $\begin{array}{c}\text { Time of } \\
\text { Irradiation (s) }\end{array}$ & $\begin{array}{c}\text { Run 1 } \\
\mathrm{Fe}^{2+} \text { produced } \\
\left(\times 10^{-5} \mathrm{~mol}\right)\end{array}$ & $\begin{array}{c}\text { Run 2 } \\
\mathrm{Fe}^{2+} \text { produced } \\
\left(\times 10^{-5} \mathrm{~mol}\right)\end{array}$ & $\begin{array}{c}\text { Run 3 } \\
\mathrm{Fe}^{2+} \text { produced } \\
\left(\times 10^{-5} \mathrm{~mol}\right)\end{array}$ \\
\hline 0 & 0 & 0 & 0 \\
20 & 1.61332 & 1.15876 & 1.83646 \\
40 & 3.03871 & 2.87038 & 2.80884 \\
60 & 4.11776 & 4.07850 & 4.34272 \\
80 & 4.99303 & 5.40232 & 5.33394 \\
100 & 6.53646 & 6.33386 & 6.45954 \\
\hline
\end{tabular}

Figure S7. Fe(phen) $)^{2+}$ Photochemical Production Graph.

\section{$\mathrm{Fe}(\text { phen })_{3}{ }^{2+}$ Photochemical Production}

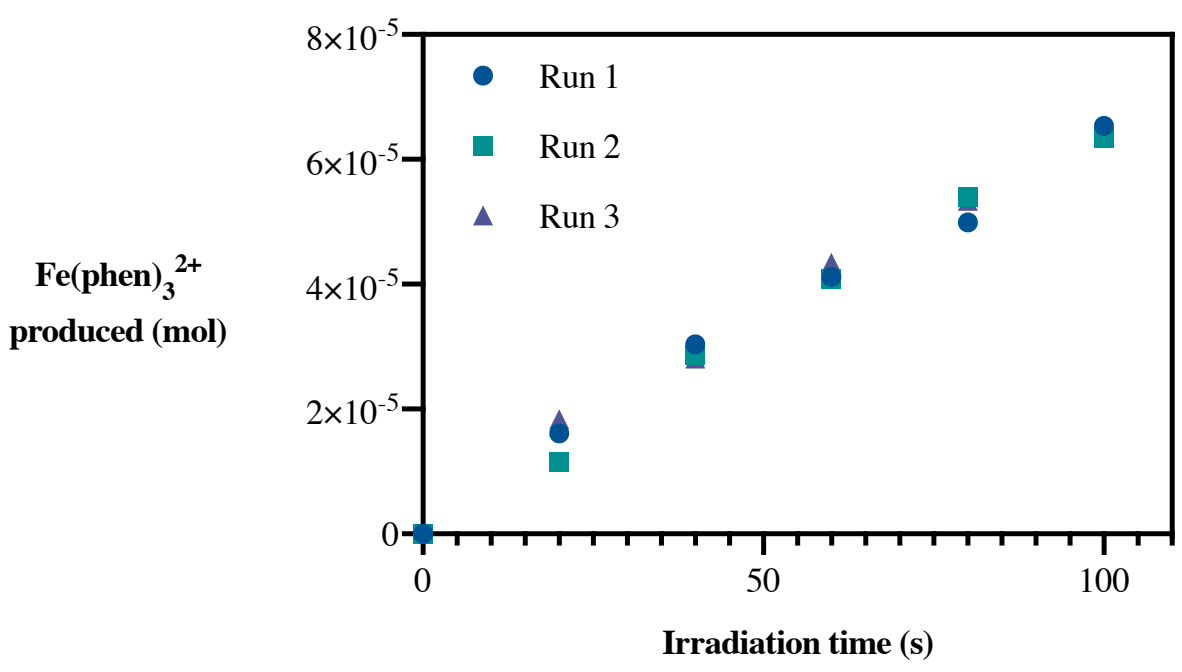

From these iron-phenanthroline complex production data, the total number of photons to pass through the cuvette can be determined as a function of time.

$$
\text { photons absorbed }=\frac{\text { mol of } F e^{2+}}{\phi_{436 \mathrm{~nm}} x F}
$$

Eq. S2

$\phi_{436 n m}=$ quantum efficiency for wavelength and concentration (1.012 at $0.15 \mathrm{M}$ ferrioxalate solution ${ }^{24}$ ) $F=$ fraction of light absorbed by the ferrioxalate solution (absorbance of a $0.15 \mathrm{M}$ potassium ferrioxalate solution is 2.8184 a.u. at $440 \mathrm{~nm}$ (from Absorption spectrum), implying that $\mathrm{F}=0.9985 \sim 1$ at $440 \mathrm{~nm}$ )

$$
\begin{gathered}
F=1-10^{- \text {Absorbance }_{440} \mathrm{~nm}} \\
F=1-10^{-2.8184} \\
F=0.9985
\end{gathered}
$$


Figure S8. Absorption Spectrum for Potassium Ferrioxalate Solution.

\section{UV-Vis Absorption Spectrum of $\mathrm{K}_{3} \mathrm{Fe}\left(\mathrm{C}_{2} \mathrm{O}_{4}\right)_{3}(0.15 \mathrm{M})$}

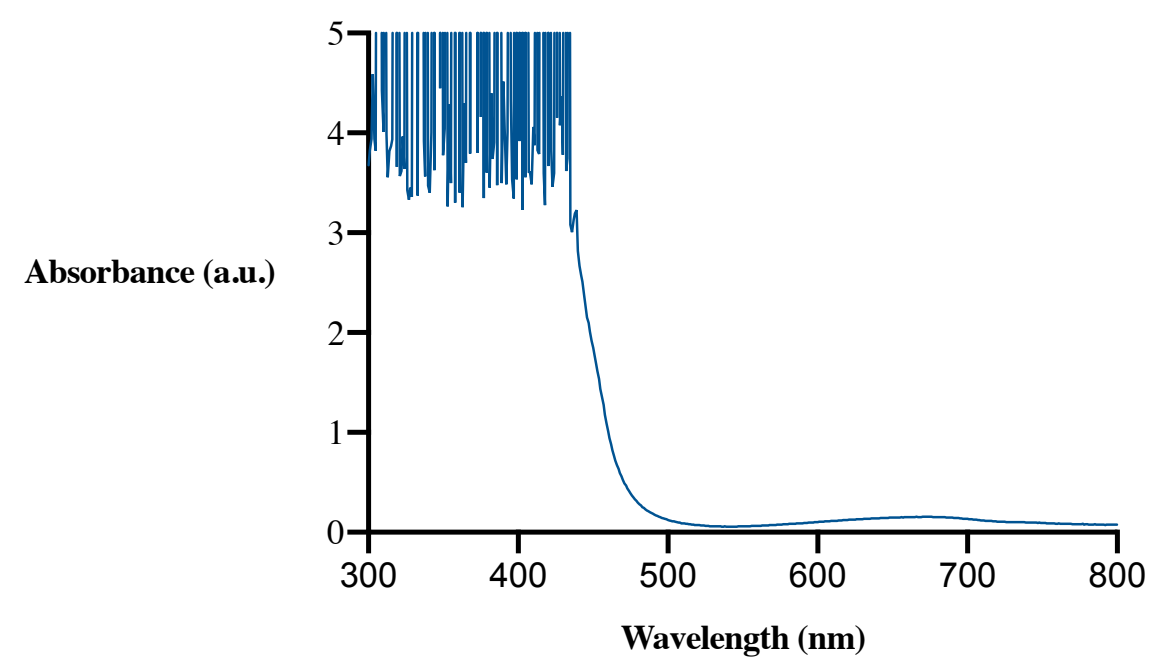

Table S12. Determination of Photon Flux.

\begin{tabular}{cccc}
\hline $\begin{array}{c}\text { Time of } \\
\text { Irradiation (s) }\end{array}$ & $\begin{array}{c}\text { Run 1 } \\
\text { Photons absorbed } \\
\left(\times 10^{-5} \text { einstein }\right)\end{array}$ & $\begin{array}{c}\text { Run 2 } \\
\text { Photons absorbed } \\
\left(\times 10^{-5} \text { einstein }\right)\end{array}$ & $\begin{array}{c}\text { Run 3 } \\
\text { Photons absorbed } \\
\left(\times 10^{-5} \text { einstein }\right)\end{array}$ \\
\hline 0 & 0 & 0 & 0 \\
20 & 1.59685 & 1.14693 & 1.81772 \\
40 & 3.0077 & 2.84108 & 2.78017 \\
60 & 4.07573 & 4.03687 & 4.29839 \\
80 & 4.94207 & 5.34718 & 5.27950 \\
100 & 6.46975 & 6.26922 & 6.39361 \\
\hline $\begin{array}{c}\text { Linear Regression } \\
\text { fit slope }\left(\mathrm{x} 10^{-7}\right)\end{array}$ & $6.54 \pm 0.18$ & $6.52 \pm 0.13$ & $6.67 \pm 0.19$ \\
$\mathrm{R}^{2}$ & 0.987 & 0.994 & 0.985 \\
\hline $\begin{array}{c}\text { Photon flux }\left(\times 10^{-7}\right. \\
\text { einsteins/s) }\end{array}$ & $6.54 \pm 0.18$ & $6.52 \pm 0.13$ & $6.67 \pm 0.19$ \\
\hline
\end{tabular}

Figure S9. Photon Absorption Graphs.

Photon Absorption over Time (Replicate 1)

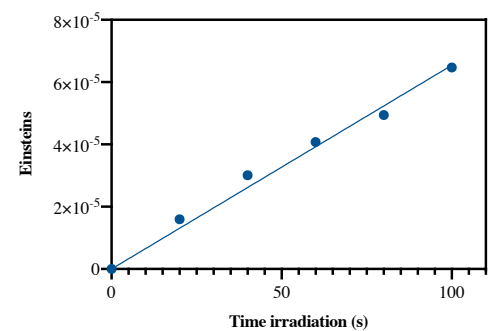

Photon Absorption over Time (Replicate 2)

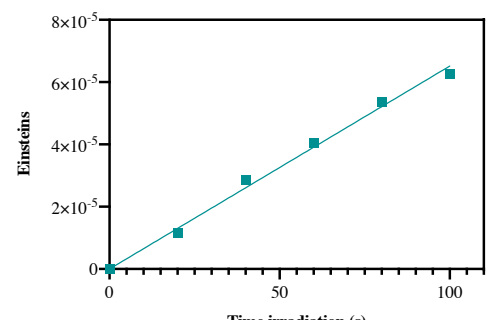

Time irradiation (s)
Photon Absorption over Time (Replicate 3)

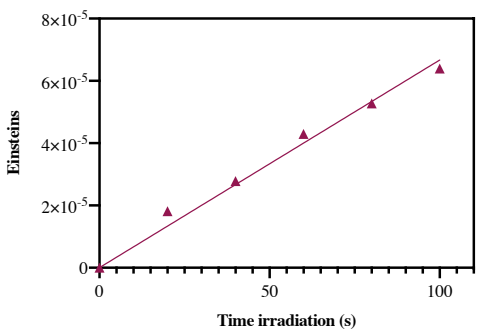


Figure S10. Compiled Photon Absorption Graphs.

\section{Photon Absorption over Time}

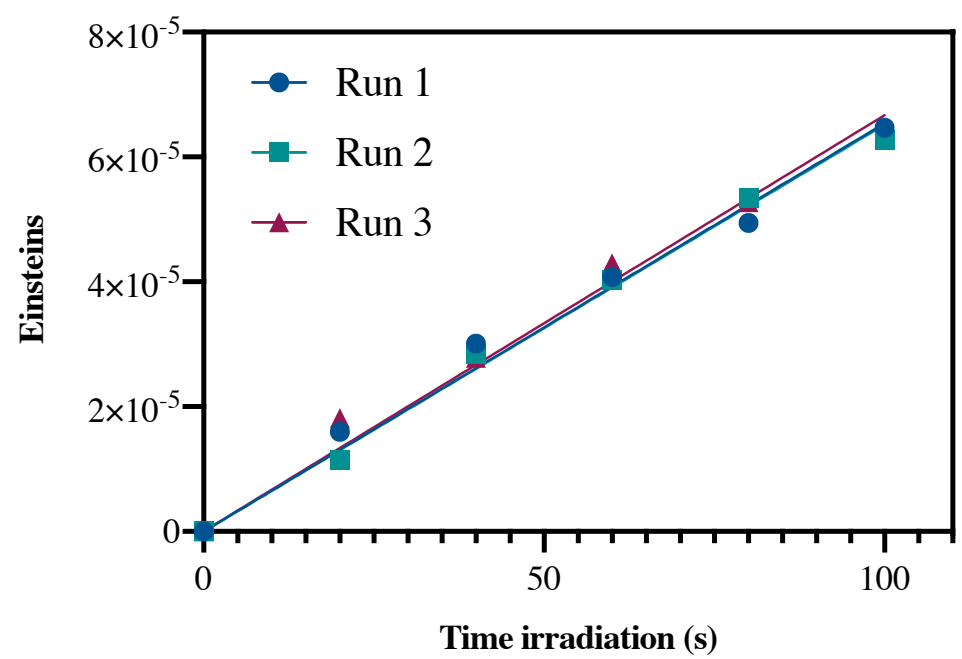

The average photon flux is therefore $6.58 \pm 0.29 \times 10^{-7}$ Einstein s${ }^{-1}$.

Tracking light absorption of the reaction mixture.

The absorption spectrum of the photocatalyst was determined at catalytically relevant concentrations $(0.002 \mathrm{M})$.

Figure S11. Absorbance Spectrum of Ir(dF-ppy $)_{3}$.

\section{UV-Vis Absorbance Spectrum of $\operatorname{Ir}(\mathrm{dF}-\mathrm{ppy})_{3}$}

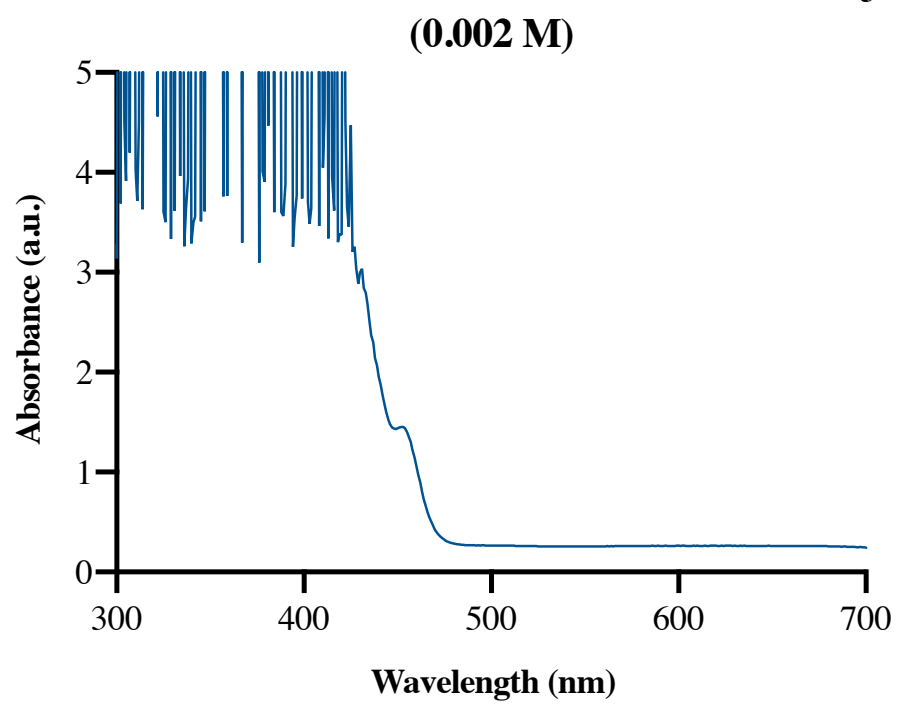

Based on the absorption spectrum $\left(\mathrm{Abs}_{440 \mathrm{~nm}}=1.9578\right.$ a.u. $)$, more than $98 \%$ of light at $440 \mathrm{~nm}$ was absorbed by the photocatalyst:

$$
\begin{aligned}
& F=1-10^{- \text {Absorbance }_{440} \mathrm{~nm}} \\
& F=1-10^{-1.9578} \\
& F=0.989
\end{aligned}
$$


Using this, it was inferred that essentially all photon flux determined by actinometry was absorbed by the photocatalyst and negligible light passed through the cuvette unabsorbed or was absorbed by phthalimide ester $\mathbf{1}$.

Notably, the phthalimide ester did not absorb significantly in the visible region at reaction concentrations. Moreover, no bathochromic shift was observed in the presence of a potential hydrogen bond donor like $\mathrm{Et}_{3} \mathrm{~N} \bullet 3 \mathrm{HF}$. Further, the lack of reactivity in the absence of a photocatalyst is indicative that the reaction is photocatalyst mediated (see Figure $1)$.

Figure S12. Absorbance Spectrum of 1 with Added Triethylamine·3HF.

\section{UV-Vis Absorbance Spectrum of Phtalimide Ester 1}

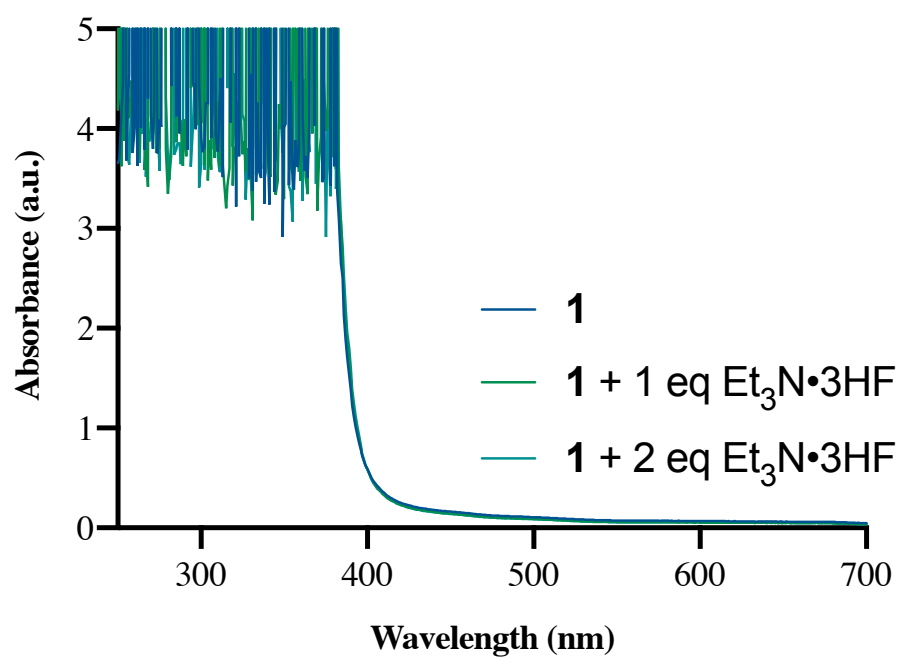

Time Course for Initial Rate.

The initial rate of the reaction was determined in duplicate:

To an oven dried scintillation vial with a stir bar was added $\operatorname{Ir}(\mathrm{dF}-\mathrm{ppy})_{3}(16.3 \mathrm{mg}, 0.0214 \mathrm{mmol}, 0.01$ eq. $)$, 2fluorobiphenyl (120.3 mg, $0.6986 \mathrm{mmol}, 0.3322$ eq.), and phthalimide ester 1 ( $789.3 \mathrm{mg}, 2.103 \mathrm{mmol}, 1$ eq.). The vial was charged with $1.00 \mathrm{~mL}$ of triethylamine $3 \mathrm{HF}$ (6.13 mol, $2.92 \mathrm{eq}$.). The vial was frozen in a $-78{ }^{\circ} \mathrm{C}$ bath and subjected to three cycles of evacuation/backfill with nitrogen. Following this, the vial was removed and charged with $10.5 \mathrm{~mL}$ of dry, sparged DCM. The vial was stirred to dissolve all of the solids. Aliquots $(3.00 \mathrm{~mL})$ were taken from the vial and added to the cuvette (taped with electrical tape and located as during calibration) which was capped with a septa cap and under nitrogen via spaghetti line during the transfer. After the transfer, an aliquot (approximately 75 $\mu \mathrm{L}$ ) was taken from the cuvette, diluted in approximately $400 \mathrm{uL}$ of $\mathrm{CDCl}_{3}$ and subjected to ${ }^{1} \mathrm{H}$-decoupled, ${ }^{19} \mathrm{~F}$-NMR analysis to verify no product formation. Subsequently, the cuvette was irradiated using the calibrated Kessil PR160 $440 \mathrm{~nm}$ (vide supra). Aliquots ( $\sim 75 \mu \mathrm{L})$ were taken at 1-minute intervals, diluted in $\sim 400 \mathrm{uL} \mathrm{of} \mathrm{CDCl}_{3}$, and subjected to ${ }^{1} \mathrm{H}$-decoupled, ${ }^{19} \mathrm{~F}-\mathrm{NMR}$ analysis to determine production rate of alkyl fluoride $\mathbf{2}$. 
Table S13. Initial Rate of Alkyl Fluoride 2 Production.

\begin{tabular}{ccc}
\hline $\begin{array}{c}\text { Time of } \\
\text { Irradiation (s) }\end{array}$ & $\begin{array}{c}\text { Run 1 } \\
\text { Product yield } \\
\left(\times 10^{-5} \mathrm{mmol}\right)\end{array}$ & $\begin{array}{c}\text { Run 2 } \\
\text { Product yield } \\
\left(\times 10^{-5} \mathrm{mmol}\right)\end{array}$ \\
\hline 0 & 0 & 0 \\
60 & 0.998 & 1.20 \\
120 & 2.59 & 2.79 \\
180 & 3.99 & 4.59 \\
240 & 5.59 & 5.79 \\
300 & 7.58 & 7.78 \\
\hline $\begin{array}{c}\text { Linear regression } \\
\text { fit slope }\left(\times 10^{-7}\right)\end{array}$ & $2.377 \pm 0.075$ & $2.504 \pm 0.052$ \\
$\mathrm{R}^{2}$ & 0.986 & 0.994 \\
\hline
\end{tabular}

Figure S13. Initial Rate for Alkyl Fluoride 2 Production.

Alkyl Fluoride 2 Initial Production Rate

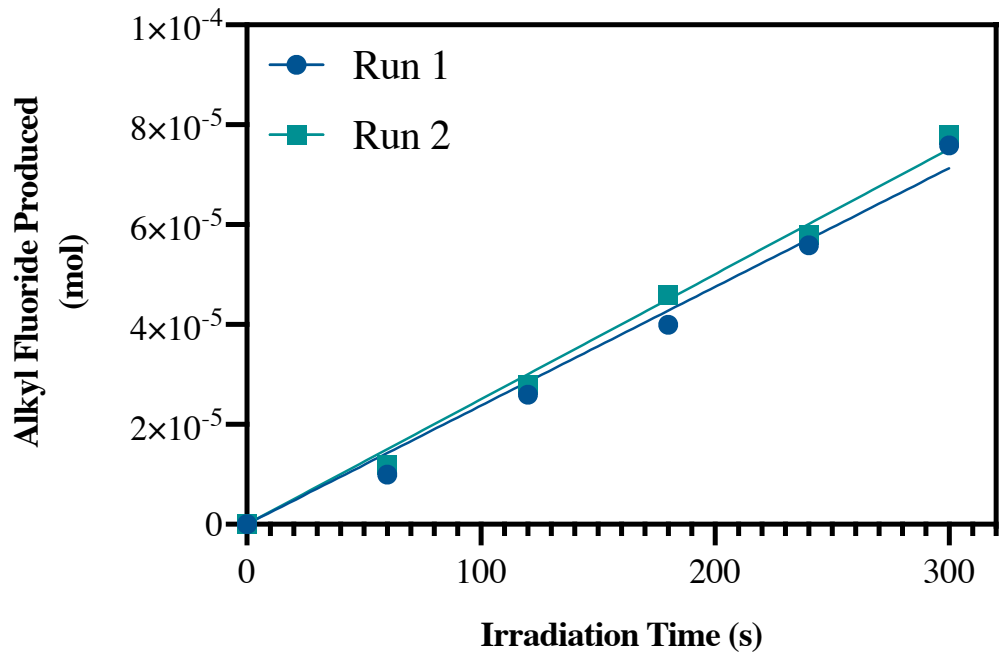

Determination of Quantum Yield.

Using the average initial rate of alkyl fluoride production of $2.441 \times 10^{-7} \mathrm{~mol} / \mathrm{s}$, the quantum yield was determined:

$$
\text { Quantum yield }=\frac{\text { Product rate }}{\text { Photon flux rate }}=\frac{2.441 \times 10^{-7} \mathrm{~mol} / \mathrm{s}}{6.58 \times 10^{-7} \text { einstein } / \mathrm{s}}=0.37
$$

This is indicative that the alkyl fluorination is likely not proceeding via a chain mechanism.

Discussion of Chain Mechanism.

Scheme S1. Potential Chain Mechanism Pathway. 


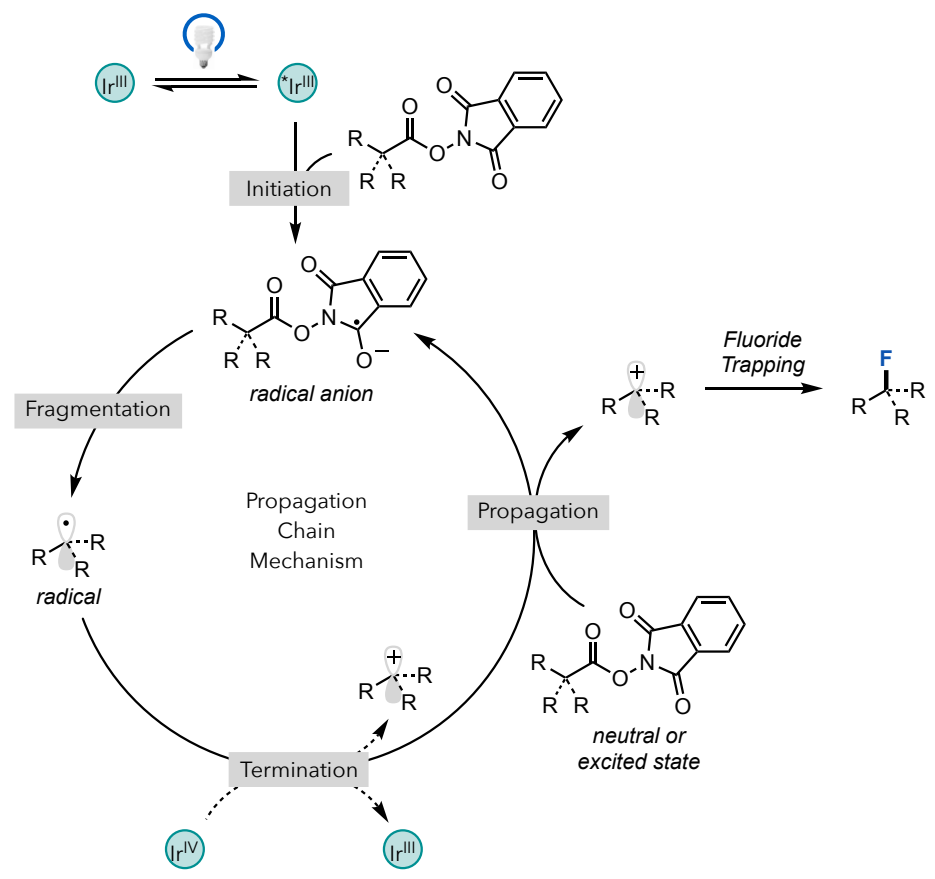

As the photocatalyst absorbs almost all of the light-the photocatalyst has significantly higher extinction coefficients in the visible region compared to the phthalimide ester substrate (see absorbance spectra of $\mathbf{1}$ and $\operatorname{Ir}(\mathrm{dF} \text {-ppy) })_{3}$--there is likely only a very small fraction of excited phthalimide ester substrate at any given time. This makes an excited state phthalimide ester unlikely to propagate a chain mechanism despite the greater concentration of the phthalimide ester substrate. An energy transfer mechanism to produce an excited state phthalimide ester is unlikely as the emission spectrum of $\operatorname{Ir}(\mathrm{dF}-\mathrm{ppy})_{3}$ (emission $>450 \mathrm{~nm}$ ) does not overlap with the absorption spectrum of the phthalimide ester (only absorbance $<400 \mathrm{~nm}$ ).

A ground state chain propagation mechanism is also unlikely on the basis of redox potentials. The literature potential for the reduction of $N$-hydroxyphthalimide esters is reported to be $\mathrm{E}_{\text {red,peak }}=-1.28 \mathrm{~V}$ vs. SCE in MeCN. ${ }^{25} \mathrm{The}$ literature oxidation potentials for benzylic and tertiary radicals meanwhile are reported to be $0.73 \mathrm{~V}$ and $0.09 \mathrm{~V}$ vs SCE, respectively. ${ }^{26,27}$ Therefore, reduction of the redox active ester by the radical species is thermodynamically disfavored by approximately 1.3-2.0 V. While there could be a significant shift in the reduction potential of the redox active ester to more positive potentials due to the presence of a hydrogen bond donor (triethylamine· $3 \mathrm{HF}$ ), no absorption shift was observed by UV-Vis spectroscopy (see above, Figure S11) nor was a significant increase in quenching rate observed in the presence of triethylamine-3HF (see Stern-Volmer quenching experiments in Tables S8, S9, and Figure S1).

\section{Radical Trapping Experiments.}

To determine the presence of radicals in this reaction, a variety of radical traps were evaluated with minor modifications. Following general procedure D, each radical trap was added prior to evacuation/backfill with nitrogen in the indicated amount.

Table S14. Styrene Trapping Experiments. 


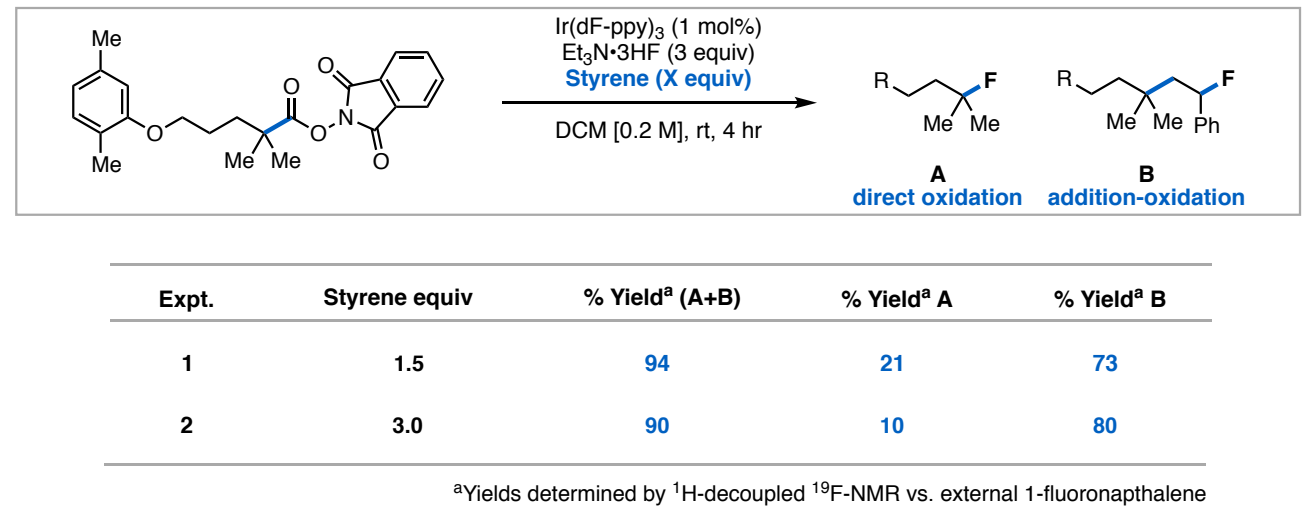

Styrene is a well-known and utilized trap for radicals. However, styrene could also act as a competitive nucleophile. If a tertiary carbocation was formed, styrene may outcompete fluoride as a nucleophile for the initial carbocation and then fluoride may add to the resulting benzylic cation. While it is unlikely for styrene to be a competitive nucleophile for one carbocation over another, to probe whether styrene was acting as a nucleophile we varied the electronics of the styrene by using the para -OMe and - $\mathrm{CN}$ species. We anticipated that the more electron rich alkene would be more likely to serve as a nucleophile leading to a higher ratio of benzylic to tertiary fluoride. Conversely, with the electron poor alkene, we anticipated that the ratio would shift from the benzylic towards the tertiary fluoride. However, within error, we did not observe a significant difference in the relative ratios of product formation by varying the electronics of the styrene (Table S15). Therefore, we concluded that the styrene is more likely acting as a radical trap than as a nucleophile.

Table S15. Electronic Effect on Styrene Trapping.

\begin{tabular}{|c|c|c|c|c|c|c|c|}
\hline $\mathrm{Me}$ & $\mathrm{Me} \mathrm{Me}$ & 0 & \multicolumn{2}{|c|}{$\begin{array}{c}\mathrm{Ir}(\mathrm{dF}-\mathrm{ppy})_{3}(1 \text { mol\%) } \\
\mathrm{Et}_{3} \mathrm{~N} \cdot 3 \mathrm{HF}(3 \text { equiv) } \\
p-\mathrm{X}-\mathrm{Styrene}(2 \text { equiv) }\end{array}$} & direct os & \multicolumn{2}{|c|}{ ation addition-oxidation } \\
\hline Expt. & p-X-Styrene & Hammett $\left(\sigma_{\mathrm{p}}\right)$ & $\sigma_{\mathrm{p}}^{+}$ & $\sigma_{\mathrm{p}}^{-}$ & $\%$ Yield $^{\mathrm{a}}(\mathrm{A}+\mathrm{B})$ & $\%$ Yield $^{\mathrm{a}} \mathbf{A}$ & $\%$ Yield $^{\mathrm{a}} \mathrm{B}$ \\
\hline 1 & $p-\mathrm{CN}$ & 0.66 & 0.66 & 1.00 & 64 & 4 & 60 \\
\hline 2 & $p-H$ & 0.00 & 0.00 & 0.00 & 85 & 17 & 68 \\
\hline 3 & $p$-OMe & -0.27 & -0.78 & -0.26 & 64 & 8 & 56 \\
\hline
\end{tabular}

As the styrene trapping may be occurring via a cationic trapping mechanism, we also conducted the reaction using methyl acrylate (as a non-nucleophilic olefin) and TEMPO (2,2,6,6-Tetramethyl-1-piperidinyloxy) as radical traps. 
Table S16. Trapping with Non-Styrene Radical Traps.

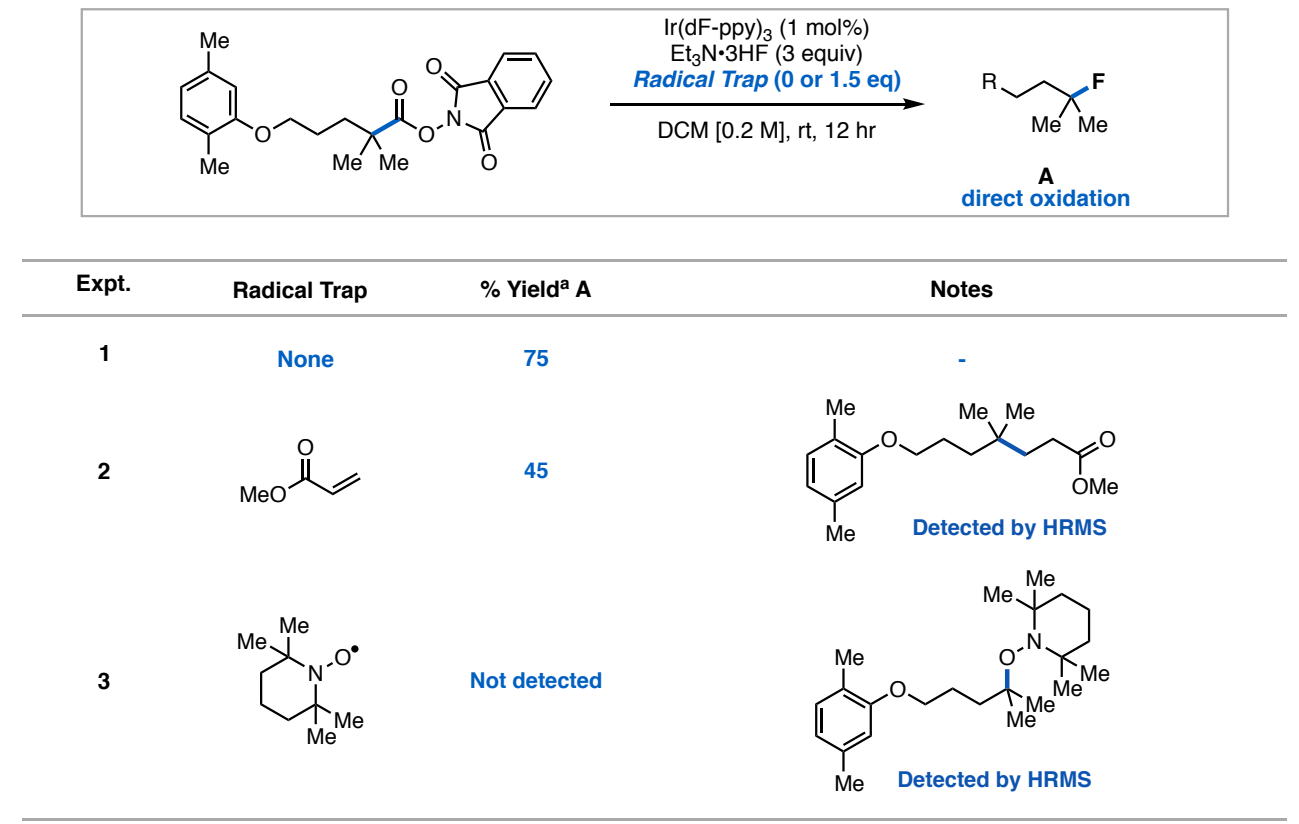

aYields determined by ${ }^{1} \mathrm{H}$-decoupled ${ }^{19} \mathrm{~F}$-NMR vs. external 1-fluoronapthalene

As the reaction efficiency is significantly diminished or even completely quenched in the presence of these radical traps, it was inferred that radicals were intermediates in the formation of the alkyl fluorides. Moreover, detection of radical trap adducts lends additional evidence that alkyl radicals are present.

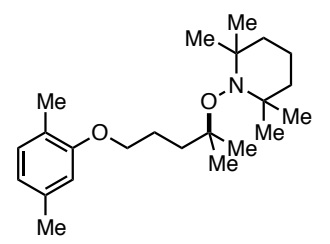

1-((5-(2,5-dimethylphenoxy)-2-methylpentan-2-yl)oxy)-2,2,6,6-tetramethylpiperidine (42)

HRMS: (ESI-TOF) calculated for $\mathrm{C}_{23} \mathrm{H}_{40} \mathrm{NO}_{2}{ }^{+}\left([\mathrm{M}+\mathrm{H}]^{+}\right)$: 362.30536, found 362.3057.

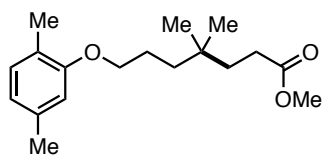

Methyl 7-(2,5-dimethylphenoxy)-4,4-dimethylheptanoate (43)

HRMS: (EI-TOF) calculated for $\mathrm{C}_{18} \mathrm{H}_{28} \mathrm{O}_{3}{ }^{+}\left[\mathrm{M}^{*}\right]$ : 292.20330, found 292.20396.<smiles>Cc1ccc(C)c(OCCCC(C)(C)CC(C)(C)c2ccccc2)c1</smiles>

2-((6-fluoro-4,4-dimethyl-6-phenylhexyl)oxy)-1,4-dimethylbenzene (44)

${ }^{19}$ F NMR (282 MHz, Chloroform-d): $\delta-171.30$ (ddd, $J=48.8,40.5,18.9$ ).

HRMS: (EI-TOF) calculated for $\mathrm{C}_{22} \mathrm{H}_{29} \mathrm{FO}^{+}\left[\mathrm{M}^{\bullet+}\right]$ : 328.21970 , found 328.22000 . 


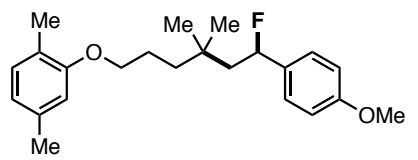

2-((6-fluoro-4,4-dimethyl-6-phenylhexyl)oxy)-1,4-dimethylbenzene (S36)

19F NMR (282 MHz, Chloroform- $d$ ): $\delta-165.57$ (ddd, J=48.6, 38.8, $18.5 \mathrm{~Hz}$ ).

HRMS: (EI-TOF) calculated for $\mathrm{C}_{23} \mathrm{H}_{31} \mathrm{FO}_{2}{ }^{+}\left[(\mathrm{M}-\mathrm{HF})^{\bullet+}\right]$ : 338.22403, found 338.22511.

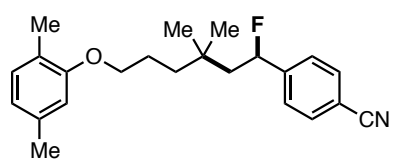

4-(6-(2,5-dimethylphenoxy)-1-fluoro-3,3-dimethylhexyl)benzonitrile (S37)

19F NMR (282 MHz, Chloroform- $d$ ): $\delta-176.82$ (ddd, J=49.2, 42.0, $19.2 \mathrm{~Hz}$ ).

HRMS: (ESI-TOF) calculated for $\mathrm{C}_{23} \mathrm{H}_{28} \mathrm{FNO}^{+}\left[\mathrm{M}^{\bullet+}\right]$ : 353.21494, found 353.21565.

\section{Carbocation Trapping Experiments.}

To probe for evidence of a carbocation, a variety of nucleophiles were evaluated using small modifications to general procedure $\mathrm{E}$ and $\mathrm{F}$ with catalytic quantities of triethylamine trihydrofluoride. Intriguingly, we observed no conversion in the absence of triethylamine trihydrofluoride acid. This is likely due to the necessity of a Lewis- or Brönsted acid to interact with the phthalimide radical anion to prevent back electron transfer (BET) from the radical anion to the now oxidized photocatalyst $\left(\mathrm{Ir}^{\mathrm{IV}}\right)$ and thereby facilitate irreversible fragmentation.

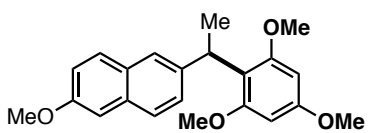

2-methoxy-6-(1-(2,4,6-trimethoxyphenyl)ethyl)naphthalene (45)

Synthesized according to general procedure E with minor alteration, with 1,3-dioxoisoindolin-2-yl 2-(6methoxynaphthalen-2-yl)propanoate $\left(230.0 \mathrm{mg}, 0.613 \mathrm{mmol}, 1\right.$ equiv.), $\mathrm{Ir}(\mathrm{dF}-\mathrm{ppy})_{3}$ (3.6 mg, $0.0047 \mathrm{mmol}, 0.007$ equiv.), 1,3,5-trimethoxybenzene ( $307.3 \mathrm{mg}, 1.83 \mathrm{mmol}, 3$ equiv.), catalytic triethylamine trihydrofluoride $(10.0 \mu \mathrm{L}$, $0.061 \mathrm{mmol}, 0.1$ equiv.), and DCM (3.00 mL, 0.2 M). Purified over silica with flash chromatography (0-15\% EtOAc in hexanes) to afford the title compound as a white solid $(0.1774 \mathrm{~g}, 0.503 \mathrm{mmol}, 82 \%)$.

1H NMR (500 MHz, Chloroform- $d$ ): $\delta 7.70-7.64(\mathrm{~m}, 2 \mathrm{H}), 7.57(\mathrm{~d}, J=8.5 \mathrm{~Hz}, 1 \mathrm{H}), 7.36(\mathrm{dd}, J=8.5,1.9 \mathrm{~Hz}, 1 \mathrm{H})$, $7.11-7.07$ (m, 2H), 6.14 (s, 2H), $4.87(\mathrm{q}, J=7.2 \mathrm{~Hz}, 1 \mathrm{H}), 3.90$ (s, 3H), 3.80 (s, 3H), 3.68 (s, 6H), 1.73 (d, $J=7.2$, $3 \mathrm{H})$.

${ }^{13}$ C NMR (126 MHz, Chloroform- $d$ ): $\delta$ 159.58, 159.20, 156.96, 142.03, 132.66, 129.31, 129.03, 127.64, 125.85 (2 carbon signals overlap), $124.73,118.17,115.95,105.63,91.54,55.91,55.38,33.02,17.91$.

HRMS: (EI-TOF) calculated for $\mathrm{C}_{22} \mathrm{H}_{25} \mathrm{O}_{4}{ }^{+}\left[(\mathrm{M}+\mathrm{H})^{+}\right]$: 353.17474 , found 353.17546 .

FTIR (thin film, cm$\left.^{-1}\right): v 2935(\mathrm{~m}), 2834(\mathrm{~m}), 1602(\mathrm{~s}), 1591(\mathrm{~s}), 1502(\mathrm{~m}), 1490(\mathrm{~m}), 1449(\mathrm{~s}), 1436(\mathrm{~m}), 1413(\mathrm{~m})$, $1389(\mathrm{~m}), 1362(\mathrm{~m}), 1330(\mathrm{~m}), 1260(\mathrm{~m}), 1218(\mathrm{~s}), 1203(\mathrm{~s}), 1192(\mathrm{~s}), 1178(\mathrm{~m}), 1162(\mathrm{~m}), 1150(\mathrm{~s}), 1116(\mathrm{~s}), 1070$ $(\mathrm{m}), 1053(\mathrm{~s}), 1036(\mathrm{~s}), 988(\mathrm{~m}), 952(\mathrm{~m}), 924(\mathrm{~m}), 884(\mathrm{~s}), 854(\mathrm{~s}), 817(\mathrm{~s}), 782(\mathrm{~m}), 768(\mathrm{~m}), 748(\mathrm{~m}), 731(\mathrm{~m}), 703.8$ $(\mathrm{m}), 656(\mathrm{~m}), 632(\mathrm{~m}), 612(\mathrm{~m}), 515(\mathrm{~m}), 501(\mathrm{~m}), 476(\mathrm{~s}), 459(\mathrm{~m})$.

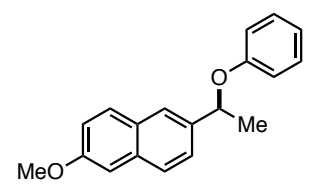

2-methoxy-6-(1-phenoxyethyl)naphthalene (46)

Synthesized according to general procedure E with minor alteration, with 1,3-dioxoisoindolin-2-yl 2-(6methoxynaphthalen-2-yl)propanoate (224.7 mg, $0.600 \mathrm{mmol}, 1$ equiv.), $\operatorname{Ir}(\mathrm{dF}-\mathrm{ppy}) 3$ (4.3 mg, $0.006 \mathrm{mmol}, 0.01$ equiv.), phenol (290.5 mg, $3.00 \mathrm{mmol}, 5$ equiv.), catalytic triethylamine trihydrofluoride (30.0 $\mu \mathrm{L}, 0.183 \mathrm{mmol}, 0.1$ 
equiv.), and DCM (3.00 mL, 0.2 M). Purified over silica with flash chromatography ( $2 \%$ ether in hexanes) followed by preparatory TLC ( $5 \%$ ether in hexanes) to afford the title compound as a clear oil $(66.8 \mathrm{mg}, 0.36 \mathrm{mmol}, 60 \%$ ).

1H NMR (500 MHz, Chloroform- $d): \delta 7.75(\mathrm{~d}, J=1.7 \mathrm{~Hz}, 1 \mathrm{H}), 7.72(\mathrm{t}, J=8.9 \mathrm{~Hz}, 2 \mathrm{H}), 7.49(\mathrm{dd}, J=8.4,1.8 \mathrm{~Hz}$, $1 \mathrm{H}), 7.22-7.18(\mathrm{~m}, 2 \mathrm{H}), 7.15(\mathrm{dd}, J=8.8,2.6 \mathrm{~Hz}, 1 \mathrm{H}), 7.12(\mathrm{~d}, J=2.5 \mathrm{~Hz}, 1 \mathrm{H}), 6.92(\mathrm{dd}, J=8.9,1.1 \mathrm{~Hz}, 2 \mathrm{H}), 6.87$ $(\mathrm{tt}, J=7.3,1.1 \mathrm{~Hz}, 1 \mathrm{H}), 5.45(\mathrm{q}, J=6.4 \mathrm{~Hz}, 1 \mathrm{H}), 3.91(\mathrm{~s}, 3 \mathrm{H}), 1.71(\mathrm{~d}, J=6.5 \mathrm{~Hz}, 3 \mathrm{H})$.

${ }^{13}$ C NMR (126 MHz, Chloroform-d): $\delta$ 158.15, 157.76, 138.56, 134.11, 129.50, 129.43, 128.89, 127.47, 124.35, $120.75,119.09,116.12,105.78,76.19,55.45,24.67$.

HRMS: (EI-TOF) calculated for $\mathrm{C}_{19} \mathrm{H}_{18} \mathrm{O}_{2}{ }^{+}\left(\left[\mathrm{M}^{*}\right]^{+}\right): 278.13013$, found 278.12997.

FTIR (thin film, cm$\left.^{-1}\right): 2978$ (w), 2929 (w), 2360 (w), 2342 (w), 2249 (w), 1634 (w), 1607 (m), 1598 (m), $1586(\mathrm{w})$, $1506(\mathrm{w}), 1484(\mathrm{~s}), 1464(\mathrm{w}), 1417$ (w), 1390 (w), 1373 (w), 1303 (w), 1265 (m), 1234 (s), 1195 (m), $1170(\mathrm{~s}), 1123$ (w), $1081(\mathrm{~m}), 1068(\mathrm{~m}), 1028(\mathrm{~m}), 963(\mathrm{w}), 905(\mathrm{~s}), 852(\mathrm{~m}), 811(\mathrm{w}), 752(\mathrm{~m}), 727(\mathrm{~s}), 690(\mathrm{~s}), 675(\mathrm{w}), 649(\mathrm{w})$, $551(\mathrm{w}), 509(\mathrm{w}), 475(\mathrm{~m}), 455(\mathrm{w}), 420(\mathrm{w})$.

\section{2-methoxy-6-(1-methoxyethyl)naphthalene (47)}

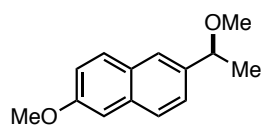

Synthesized according to general procedure E with minor alteration, with 1,3-dioxoisoindolin-2-yl 2-(6methoxynaphthalen-2-yl)propanoate (227.4 mg, $0.606 \mathrm{mmol}, 1$ equiv.), $\mathrm{Ir}(\mathrm{dF}-\mathrm{ppy})_{3}(1.7 \mathrm{mg}, 0.0022 \mathrm{mmol}, 0.0037$ equiv.), methanol ( $150 \mu \mathrm{L}, 3.70 \mathrm{mmol}, 6.1$ equiv.), catalytic triethylamine trihydrofluoride $(5.0 \mu \mathrm{L}, 0.031 \mathrm{mmol}, 0.05$ equiv.), and DCM (3.00 mL, $0.2 \mathrm{M})$. The anhydrous, sparged methanol was added following evacuation and backfill with nitrogen. Purified over silica with flash chromatography (7-15\% EtOAc in hexanes) to afford the title compound as a white solid $(0.1074 \mathrm{~g}, 0.497 \mathrm{mmol}, 81 \%)$.

1H NMR (500 MHz, Chloroform- $d$ ): $\delta 7.74(\mathrm{t}, J=8.8 \mathrm{~Hz}, 2 \mathrm{H}), 7.67(\mathrm{~d}, J=1.6 \mathrm{~Hz}, 1 \mathrm{H}), 7.44(\mathrm{dt}, J=8.5,1.5 \mathrm{~Hz}$, $1 \mathrm{H}), 7.19-7.13(\mathrm{~m}, 2 \mathrm{H}), 4.43(\mathrm{q}, J=6.5 \mathrm{~Hz}, 1 \mathrm{H}), 3.93(\mathrm{~s}, 3 \mathrm{H}), 3.25(\mathrm{~s}, 3 \mathrm{H}), 1.51(\mathrm{~d}, J=6.4 \mathrm{~Hz}, 3 \mathrm{H})$.

13. NMR (126 MHz, Chloroform-d): $\delta$ 157.74, 138.72, 134.28, 129.43, 128.84, 127.33, 125.22, 124.83, 119.01, 105.84, 79.85, 56.58, 55.46, 23.98 .

HRMS: (EI-TOF) calculated for $\mathrm{C}_{14} \mathrm{H}_{16} \mathrm{O}_{2}^{+}\left[\mathrm{M}^{\bullet+}\right]: 216.11448$, found 216.11442 .

FTIR (thin film, cm$\left.^{-1}\right): ~ v 2975(\mathrm{~m}), 2933(\mathrm{~m}), 2855(\mathrm{w}), 2819(\mathrm{w}), 1628(\mathrm{w}), 1602(\mathrm{~m}), 1504(\mathrm{w}), 1481(\mathrm{~m}), 1462$ (m), $1435(\mathrm{~m}), 1416(\mathrm{w}), 1390(\mathrm{~m}), 1369(\mathrm{~m}), 1306(\mathrm{~m}), 1263(\mathrm{~m}), 1233(\mathrm{~m}), 1198(\mathrm{~m}), 1172(\mathrm{~s}), 1161(\mathrm{~s}), 1109(\mathrm{~s})$, 1088 (s), 1058 (s), $1026(\mathrm{~s}), 990(\mathrm{~m}), 960(\mathrm{w}), 925(\mathrm{w}), 890(\mathrm{~m}), 855$ (s), $816(\mathrm{~s}), 763(\mathrm{w}), 750(\mathrm{~m}), 674(\mathrm{~m}), 620(\mathrm{w})$, $539(\mathrm{~m}), 524(\mathrm{w}), 478(\mathrm{~s}), 470(\mathrm{~s}), 416(\mathrm{w})$.<smiles></smiles>

2-(1-((1,1,1,3,3,3-hexafluoropropan-2-yl)oxy)ethyl)-6-methoxynaphthalene (48)

Synthesized according to general procedure D with minor alteration, with 1,3-dioxoisoindolin-2-yl 2-(6methoxynaphthalen-2-yl)propanoate $\left(75.4 \mathrm{mg}, 0.201 \mathrm{mmol}, 1\right.$ equiv.), $\operatorname{Ir}(\mathrm{dF}-\mathrm{ppy})_{3}(1.6 \mathrm{mg}, 0.0022 \mathrm{mmol}, 0.01$ equiv.), HFIP (105 $\mu \mathrm{L}, 1.00 \mathrm{mmol}, 5$ equiv.), and DCM (1.00 mL, $0.2 \mathrm{M})$. After irradiating for $24 \mathrm{~h}$, 1Fluoronaphthalene ( $26.0 \mu \mathrm{L}, 0.200 \mathrm{mmol}, 1$ equiv.) was added and an aliquot was analyzed by ${ }^{19} \mathrm{~F}-\mathrm{NMR}$ to afford $66 \%$ yield $(0.133 \mathrm{mmol}, 46.7 \mathrm{mg})$. Purified over silica with flash chromatography ( $0-10 \% \mathrm{Et}_{2} \mathrm{O}$ in hexanes) to afford the title compound as a white solid.

1H NMR (500 MHz, Chloroform- $d$ ): $\delta 7.79(\mathrm{~d}, J=8.5 \mathrm{~Hz}, 1 \mathrm{H}), 7.75(\mathrm{~d}, J=8.9 \mathrm{~Hz}, 1 \mathrm{H}), 7.67(\mathrm{~d}, J=1.7 \mathrm{~Hz}, 1 \mathrm{H})$, $7.46(\mathrm{dd}, J=8.5,1.7 \mathrm{~Hz}, 1 \mathrm{H}), 7.22-7.12(\mathrm{~m}, 2 \mathrm{H}), 4.97(\mathrm{q}, J=6.4 \mathrm{~Hz}, 1 \mathrm{H}), 4.02($ hept $, J=5.7 \mathrm{~Hz}, 1 \mathrm{H}), 3.94(\mathrm{~s}, 3 \mathrm{H})$, $1.66(\mathrm{~d}, J=6.5 \mathrm{~Hz}, 3 \mathrm{H})$.

${ }^{13}$ C NMR (126 MHz, Chloroform-d): $\delta$ 158.42, 135.04, 134.44, 129.62, 128.50, 127.97, 127.12, 124.81, 122.42 (qq, $J=285.1,2.0 \mathrm{~Hz}), 121.29(\mathrm{qq}, J=282.5,2.8 \mathrm{~Hz}), 119.54,105.91,81.67,72.74$ (hept, $J=32.1 \mathrm{~Hz}), 55.47,23.18$.

${ }^{19}$ F NMR (282 MHz, Chloroform- $\left.d\right)$ : $\delta-72.79(\mathrm{qd}, J=9.2,5.7 \mathrm{~Hz}, 3 \mathrm{~F}),-73.64(\mathrm{qd}, J=9.2,5.6 \mathrm{~Hz}, 3 \mathrm{~F})$.

HRMS: (EI-TOF) calculated for $\mathrm{C}_{16} \mathrm{H}_{14} \mathrm{FO}_{2}{ }^{+}\left[\left(\mathrm{M}^{*}\right)^{+}\right]: 352.08925$ found 352.08959 . 


\section{Radiochemistry}

\section{General procedure and Information:}

Anhydrous acetonitrile was obtained from Aldrich. $\left[{ }^{18} \mathrm{O}\right]-\mathrm{H}_{2} \mathrm{O}$ was obtained from Medical Isotopes Inc. Phthalimide esters were synthesized and then stored in a $-20{ }^{\circ} \mathrm{C}$ fridge. $\left[{ }^{18} \mathrm{~F}\right]$ Fluoride was produced on a General Electric (GE) 800 series PETTrace cyclotron by the nuclear reaction ${ }^{18} \mathrm{O}(p, n)^{18} \mathrm{~F}$ with a $65 \mu \mathrm{A}$ beam for 5 minutes producing $300 \mathrm{mCi}$. $\left[{ }^{18} \mathrm{~F}\right]$ fluoride in $\left[{ }^{18} \mathrm{O}\right]$ water was pushed from the cyclotron target by hydrogen as a $2.3 \mathrm{~mL}$ bolus and delivered to a GE Tracerlab FX2 N synthesis module. The $\left.{ }^{[18} \mathrm{F}\right]$ fluoride was trapped on a Sep-Pak QMA Plus Light cartridge preconditioned with $5.0 \mathrm{~mL}$ of $\mathrm{H}_{2} \mathrm{O}, 5 \mathrm{~mL}$ of $8.4 \%$ sodium bicarbonate, $5 \mathrm{~mL}$ of acetonitrile, and then dried with a stream of compressed air. The $\left[{ }^{18} \mathrm{~F}\right] \mathrm{F}^{-}$was eluted from the QMA cartridge using a $0.1 \mathrm{~mL}$ of potassium carbonate solution $\left(30 \mathrm{mg} / \mathrm{mL}, 3 \mathrm{mg}, 0.022 \mathrm{mmol}\right.$ in $\left.\mathrm{H}_{2} \mathrm{O}\right)$ and 4,7,13,21,24-hexaoxa-1,10-diazabicyclo[8.8.8] hexacosane (10 $\mathrm{mg}, 0.027 \mathrm{mmol}$ ) in $1.5 \mathrm{~mL}$ of $\mathrm{MeCN}$. The resulting $\left[{ }^{18} \mathrm{~F}\right] \mathrm{F} / \mathrm{K}_{222} / \mathrm{K}_{2} \mathrm{CO}_{3}$ solution was azeotropically dried and then cooled, taken up in $5 \mathrm{~mL}$ of $\mathrm{MeCN}$, and transferred to a $10 \mathrm{~mL}$ septa capped scintillation vial. The vial was transferred to a separate hot cell and the $\left[{ }^{18} \mathrm{~F}\right] \mathrm{F}-\mathrm{K}_{222} / \mathrm{K}_{2} \mathrm{CO}_{3}$ solution was taken up in a syringe and transferred in 0.3 or $0.5 \mathrm{~mL}$ aliquots to $\mathrm{N}_{2}$-filled, 1-dram, septa-capped vials (approx. 3 minutes with $\mathrm{N}_{2}$ using a low flow rate of approx. 0.3 $\mathrm{mL} / \mathrm{min}$, with an $18 \mathrm{~g}$ vent needle) precharged with photocatalyst and phthalimide ester $(0.787 \mu$ mol photocatalyst and $5.3 \mu \mathrm{mol}$ of phthalimide ester per reaction vial) in $0.2 \mathrm{~mL}$ or $0.4 \mathrm{~mL}$ of $\mathrm{MeCN}$, in the photochemistry apparatus. This typically charged the vials with $10-20 \mathrm{mCi}$ of activity. After the addition of $\left[{ }^{18} \mathrm{~F}\right] \mathrm{F}^{-}$in $\mathrm{MeCN}$, the vials were irradiated from the bottom for 10 minutes. Note: no stirring was conducted during irradiation. Following irradiation, the amount of activity present in the vial was determined by a Capintec dose calibrator. A $100 \mathrm{uL}$ aliquot was taken for HPLC analysis. A $100 \mathrm{uL}$ aliquot was added to a vial containing authentic ${ }^{19} \mathrm{~F}$-product for co-injection and verification of product identity via HPLC. Finally, a $100 \mathrm{uL}$ aliquot was taken and diluted in $1 \mathrm{~mL}$ of MeCN and this solution was spotted on a silica TLC plate. The TLC plate was developed with 50\% EtOAc/hexanes eluent, and then analyzed using an Eckert \& Ziegler AR-2000 Radio-TLC imaging scanner to determine the radiochemical conversion.

Figure S14. Photocatalytic Radiochemical Fluorination Setup and Apparatus.

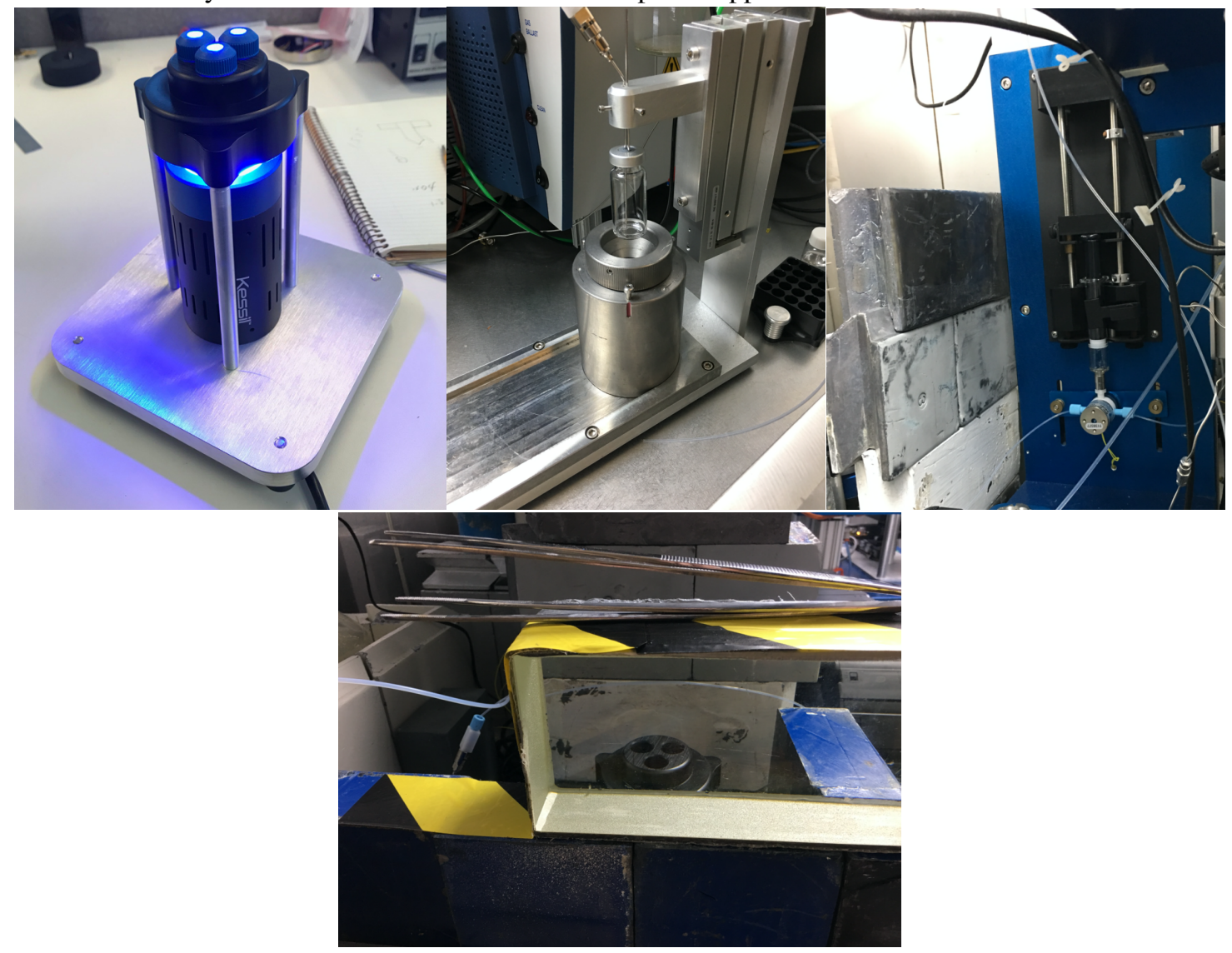


Top left: Photocatalysis set up incorporating a Kessil H150 Blue. Top middle: scintillation vial and tungsten pig used for transfer between hot cells. Top right: automated Hamilton syringe used to dispense $\left[{ }^{18} \mathrm{~F}\right] \mathrm{F}^{-}$in $\mathrm{MeCN}$ solution to sparged vials. Bottom: shielding used during manual manipulations.

Figure S15. GE Tracerlab FX2 N Flow Diagram.

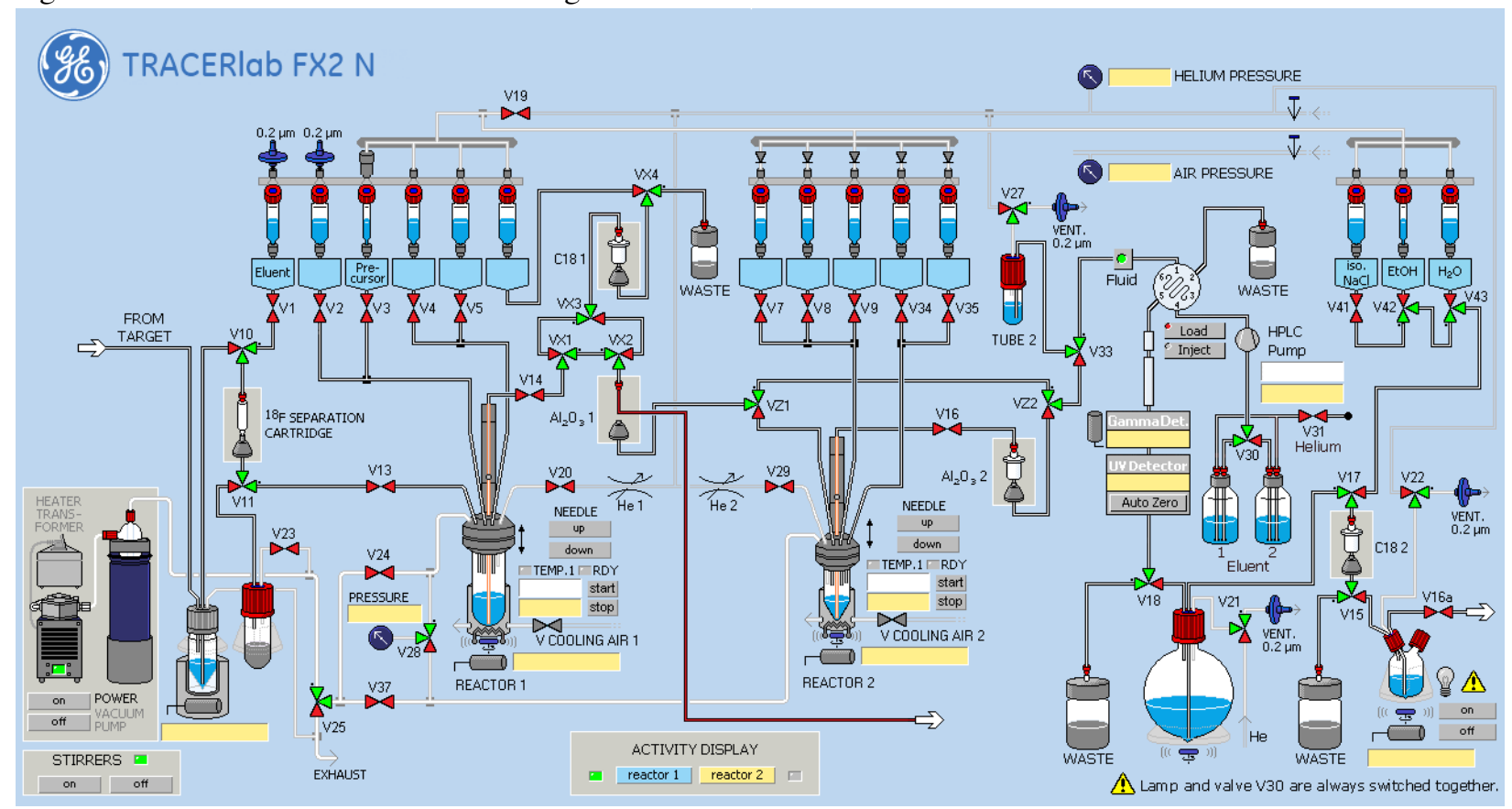

Teflon line to scintillation vial from VX2.

As toxic solvents and reagents present a potential translational challenge to the implementation of methodologies for $\left[{ }^{18} \mathrm{~F}\right] \mathrm{F}^{-}$incorporation, the methylene chloride (DCM) solvent that this system was initially optimized upon was an obstacle for translation. Therefore, to enable translation, we elected to utilize acetonitrile (MeCN). In making this specific solvent change, we observed that there was a significant solvent/photocatalyst interaction effect (Table S17).

Table S17. Photocatalyst screen in MeCN.

\begin{tabular}{|c|c|c|}
\hline & $\begin{array}{l}\begin{array}{l}\text { Photocatalyst ( } 1 \text { mol\%) } \\
\mathrm{Et}_{3} \mathrm{~N} \cdot 3 \mathrm{HF} \text { (3 equiv.) }\end{array} \\
\underset{\begin{array}{c}0.2 \mathrm{M}, \mathrm{MeCN}, \mathrm{rt}, 4 \mathrm{~h} \\
34 \mathrm{~W} \text { Blue LED }\end{array}}{\longrightarrow}\end{array}$ & \\
\hline Entry & Photocatalyst & ${ }^{19} \mathrm{~F}$-NMR Yield (\%) ${ }^{\mathrm{a}, \mathrm{b}}$ \\
\hline 1 & $\operatorname{Ir}(\mathrm{dF}-\mathrm{ppy})_{3}$ & $75 \%$ \\
\hline 2 & $\operatorname{Ir}\left(\mathrm{CF}_{3}-\mathrm{ppy}\right)_{3}$ & $89 \%$ \\
\hline 3 & $\operatorname{Ir}(\mathrm{F}-\mathrm{ppy})_{3}$ & $98 \%$ \\
\hline 4 & $\operatorname{Ir}(\mathrm{ppy})_{3}$ & $64 \%$ \\
\hline 5 & $\operatorname{Ir}(\mathrm{dF}-\mathrm{tBu}-\mathrm{ppy})_{3}$ & $83 \%$ \\
\hline 6 & {$\left[\operatorname{lr}(\text { ppy })_{2}(\mathrm{dtbpy})\right]\left(\mathrm{PF}_{6}\right)$} & $74 \%$ \\
\hline 7 & {$\left[\mathrm{Ir}\left(\mathrm{dF}-\mathrm{CF}_{3}-\mathrm{ppy}\right)_{2}(\mathrm{dtbpy})\right]\left(\mathrm{PF}_{6}\right)$} & $71 \%$ \\
\hline 8 & $\mathrm{Ru}(\mathrm{bpy})_{3}\left(\mathrm{PF}_{6}\right)_{2}$ & $1 \%$ \\
\hline
\end{tabular}

a $0.2 \mathrm{mmol}$ scale reaction conducted according to general procedure $\mathrm{D}$.

b ${ }^{19} \mathrm{~F}-\mathrm{NMR}$ yields determined relative to external 1 -fluoronaphthalene. 
Homoleptic iridium photocatalyst were screened for the radiofluorination as previous reports have observed lowered of specific activity in radiochemical reactions when catalysts with fluoride containing counterions were utilized ${ }^{28,29}$ (e.g. $\mathrm{PF}_{6}^{-}, \mathrm{BF}_{4}^{-}$).

Table S18. Determination of Optimal Radiochemical Photocatalyst.

\begin{tabular}{|c|c|c|c|c|c|c|c|c|c|}
\hline & $\mathrm{Me}$ & $\begin{array}{c}5.3 \mu \mathrm{mol} \\
.0 \mathrm{mg} / \text { reaction }\end{array}$ & & $\begin{array}{c}\begin{array}{c}\text { Photocatalyst (16 mol } \\
{\left[{ }^{18} \mathrm{FJF} / \mathrm{K}_{2} \mathrm{CO}_{3} / \mathrm{K}_{222}\right.}\end{array} \\
\mathrm{MeCN}(0.7 \mathrm{~mL}) \\
34 \mathrm{~W} \text { BLED, } 10 \mathrm{~min}\end{array}$ & & Y & & & \\
\hline Photocatalyst & $\begin{array}{c}\text { Run } \mathbf{1} \\
\text { EOS Activity (mCi) }\end{array}$ & $\begin{array}{c}\text { Run } 1 \\
\text { HPLC RCC (\%) }\end{array}$ & $\begin{array}{c}\text { Run } 1 \\
\text { TLC RCC (\%) }\end{array}$ & $\begin{array}{c}\text { Run } \mathbf{2} \\
\text { EOS Activity (mCi) }\end{array}$ & $\begin{array}{c}\text { Run } 2 \\
\text { HPLC RCC (\%) }\end{array}$ & $\begin{array}{c}\text { Run } 2 \\
\text { TLC RCC (\%) }\end{array}$ & & $\begin{array}{c}\text { Average } \\
\text { IPLC RCC (\%) }\end{array}$ & $\begin{array}{c}\text { Average } \\
\operatorname{TLC} \operatorname{RCC}(\%)\end{array}$ \\
\hline $\operatorname{Ir}(\mathrm{F}-\mathrm{ppy})_{3}$ & 3.94 & 64 & 58 & 9.92 & 39 & 45 & & 52 & 51 \\
\hline $\operatorname{Ir}(\mathrm{dF}-\mathrm{ppy})_{3}$ & 12.43 & 11 & 6 & 12.61 & 10 & 6 & & 11 & 6 \\
\hline $\operatorname{Ir}\left(\mathrm{CF}_{3}-\mathrm{ppy}\right)_{3}$ & 12.72 & 28 & 33 & 8.90 & 41 & 53 & & 35 & 43 \\
\hline $\operatorname{Ir}(\mathrm{dF}-\mathrm{tBu}-\mathrm{ppy})_{3}$ & 11.01 & 25 & 25 & 8.89 & 24 & 23 & & 25 & 24 \\
\hline $\operatorname{Ir}(\mathrm{F}-\mathrm{t} \mathrm{Bu}-\mathrm{ppy})_{3}$ & 12.13 & 41 & 45 & 7.48 & 45 & 53 & & 43 & 49 \\
\hline
\end{tabular}

Table S19. Additive Screening for KF Solubility.

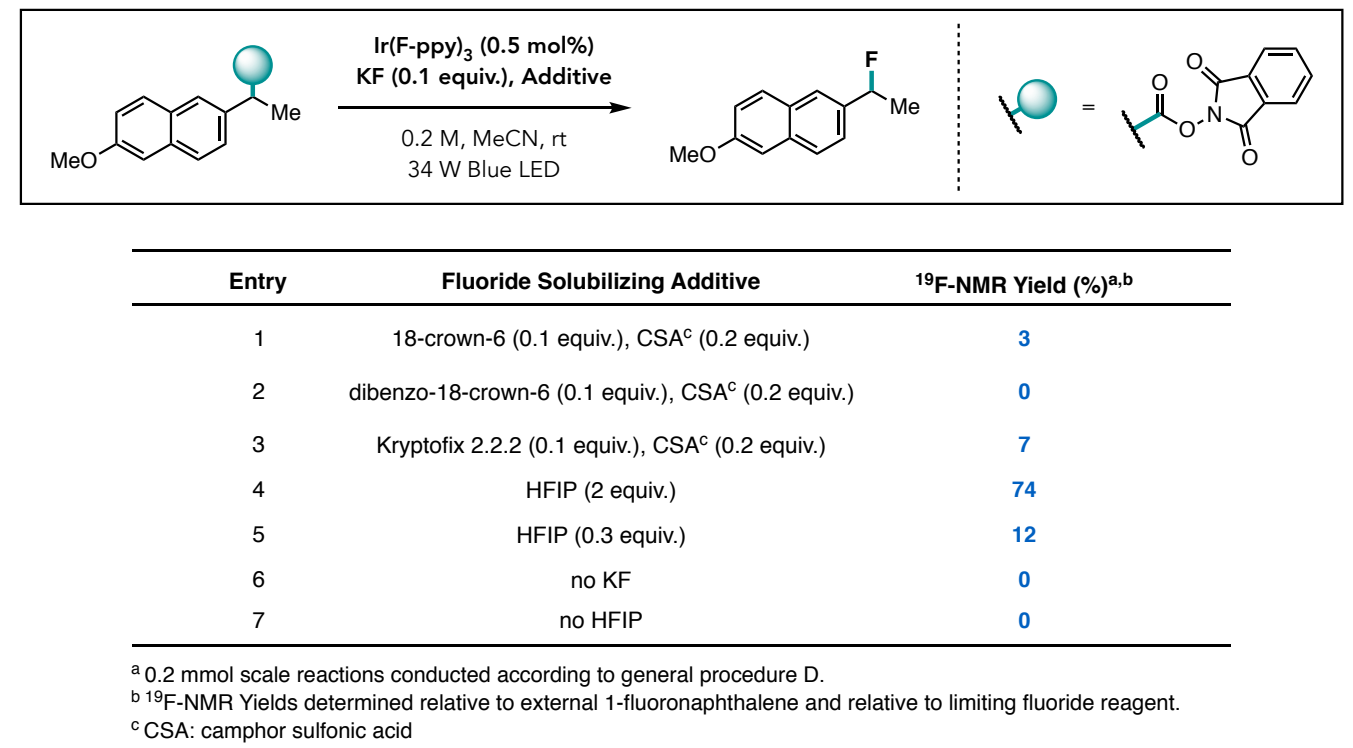

Based off of our previous studies conducted using 1,1,1,3,3,3-hexafluoroisopropanol (HFIP) to produced soluble HFspecies $^{28}$ and Table S18, we initially explored using HFIP and camphor sulfonic acid as acidic, solubilizing additives. Adding HFIP prior to sparging, we found that HFIP was not necessary for reactivity and appeared to hinder radiochemical incorporation (Table S19 and Figure S16). 
Table S20. Variable Loading of HFIP as an Additive in Radiochemistry.

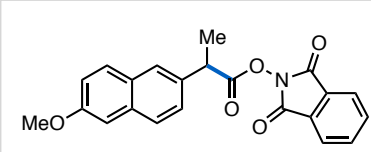

$5.3 \mu \mathrm{mol}$

$2.0 \mathrm{mg} /$ reaction

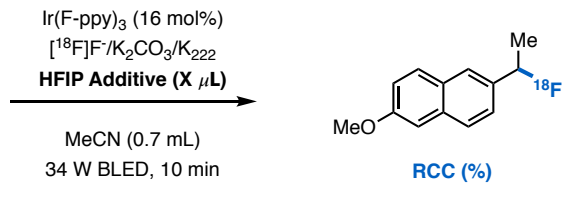

\begin{tabular}{|c|c|c|c|c|c|c|c|c|}
\hline $\begin{array}{l}\text { HFIP Additive } \\
(\mu \mathrm{L})\end{array}$ & $\begin{array}{c}\text { Run } \mathbf{1} \\
\text { EOS Activity (mCi) }\end{array}$ & $\begin{array}{c}\text { Run } 1 \\
\text { HPLC RCC (\%) }\end{array}$ & $\begin{array}{c}\text { Run } 1 \\
\text { TLC RCC (\%) }\end{array}$ & $\begin{array}{c}\text { Run } \mathbf{2} \\
\text { EOS Activity (mCi) }\end{array}$ & $\begin{array}{c}\text { Run } 2 \\
\text { HPLC RCC (\%) }\end{array}$ & $\begin{array}{c}\text { Run } 2 \\
\text { TLC RCC (\%) }\end{array}$ & $\begin{array}{c}\text { Average } \\
\operatorname{HPLC} \text { RCC (\%) }\end{array}$ & $\begin{array}{c}\text { Average } \\
\text { TLC RCC (\%) }\end{array}$ \\
\hline 0 & 7.83 & 64 & 60 & 5.62 & 56 & 67 & 60 & 60 \\
\hline 25 & 5.32 & 42 & 49 & 5.56 & 38 & 31 & 40 & 40 \\
\hline 50 & 10.37 & 41 & 31 & 5.35 & 40 & 37 & 41 & 34 \\
\hline 75 & 1.20 & 42 & 42 & 4.40 & 43 & 37 & 43 & 40 \\
\hline 100 & 5.93 & 34 & 32 & 4.19 & 40 & 33 & 37 & 33 \\
\hline 200 & 8.11 & 27 & 19 & 4.37 & 29 & 21 & 28 & 20 \\
\hline
\end{tabular}

Figure S16. Average RCC for Variable Loading of HFIP Additive.

HFIP Effect (HPLC)

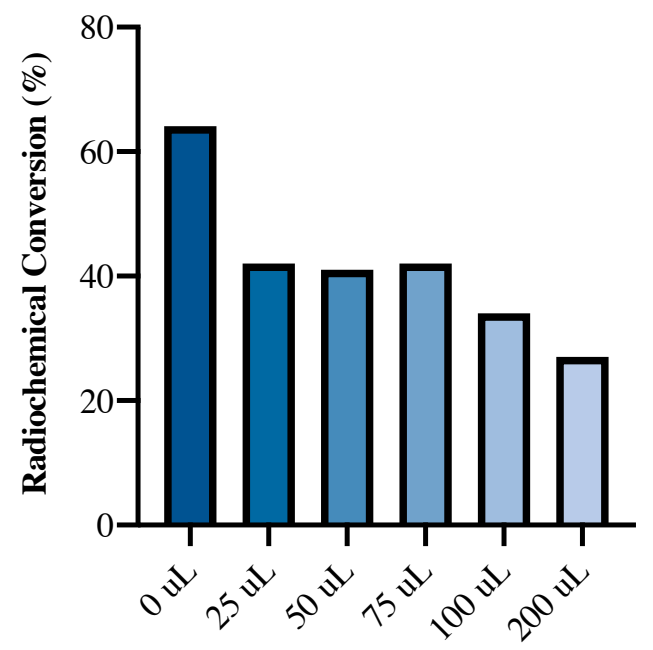

HFIPAdded
HFIP Effect (TLC)

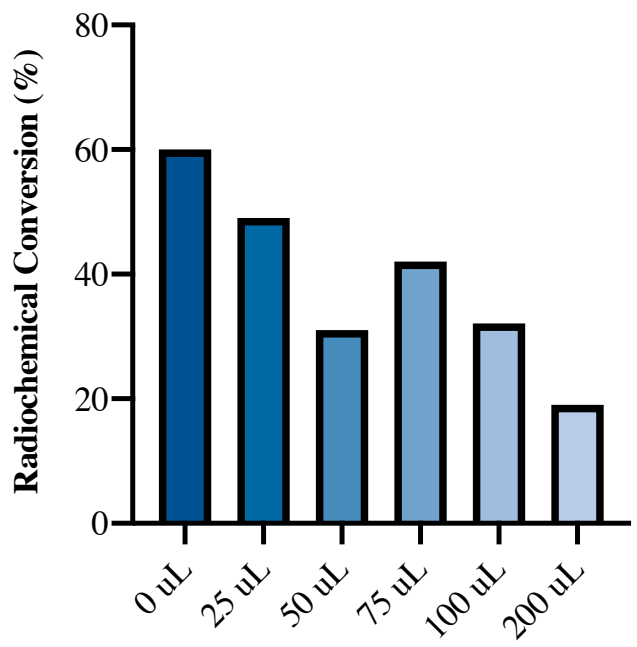

HFIPAdded

Probing the photocatalyst loading on the radiochemical transformation showed an optimal loading between 8 and 16 mol\% relative to phthalimide ester starting material. (A1EF5-034) 
Table S21. Determining Optimal Radiochemical Photocatalyst Loading.

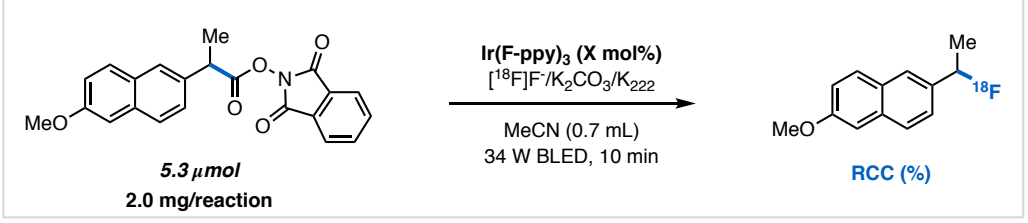

\begin{tabular}{|c|c|c|c|c|c|c|c|c|}
\hline $\begin{array}{l}\text { Photocatalyst } \\
\text { Loading (\%) }\end{array}$ & $\begin{array}{c}\text { Run } \mathbf{1} \\
\text { EOS Activity (mCi) }\end{array}$ & $\begin{array}{c}\text { Run } 1 \\
\text { HPLC RCC (\%) }\end{array}$ & $\begin{array}{c}\text { Run } 1 \\
\text { TLC RCC (\%) }\end{array}$ & $\begin{array}{c}\text { Run } \mathbf{2} \\
\text { EOS Activity (mCi) }\end{array}$ & $\begin{array}{c}\text { Run } 2 \\
\text { HPLC RCC (\%) }\end{array}$ & $\begin{array}{c}\text { Run } 2 \\
\text { TLC RCC (\%) }\end{array}$ & $\begin{array}{c}\text { Average } \\
\operatorname{HPLC} \text { RCC (\%) }\end{array}$ & $\begin{array}{c}\text { Average } \\
\operatorname{TLC} \operatorname{RCC}(\%)\end{array}$ \\
\hline 0 & 5.55 & 0 & 0 & 9.42 & 0 & 0 & 0 & 0 \\
\hline 2 & 15.66 & 16 & 12 & 8.59 & 23 & 34 & 20 & 23 \\
\hline 4 & 13.23 & 25 & 38 & 7.01 & 28 & 36 & 27 & 37 \\
\hline 8 & 10.11 & 32 & 51 & 6.83 & 33 & 33 & 33 & 42 \\
\hline 16 & 12.96 & 28 & 33 & 6.59 & 39 & 40 & 34 & 36 \\
\hline 32 & 0.42 & 38 & 30 & 5.56 & 40 & 30 & 39 & 30 \\
\hline
\end{tabular}

Table S22. Concentration Evaluation.

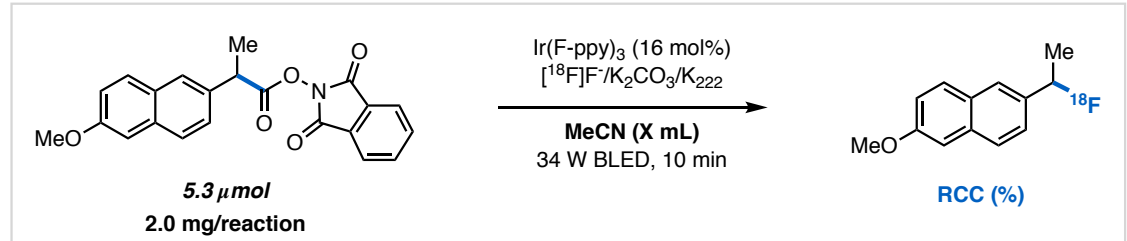

\begin{tabular}{ccccc}
\hline $\begin{array}{c}\text { Total volume } \\
(\mu \mathbf{L})\end{array}$ & $\begin{array}{c}\text { Concentration } \\
(\mathbf{m M})\end{array}$ & $\begin{array}{c}\text { Run 1 } \\
\text { EOS Activity (mCi) }\end{array}$ & $\begin{array}{c}\text { Run 1 } \\
\text { HPLC RCC (\%) }\end{array}$ & $\begin{array}{c}\text { Run 1 } \\
\text { TLC RCC }(\%)\end{array}$ \\
\hline 500 & 10.7 & 9.87 & 53 & 58 \\
700 & 7.61 & 11.04 & 45 & 48 \\
100 & 5.33 & 11.88 & 45 & 52 \\
1200 & 4.44 & 10.24 & 34 & 54 \\
1500 & 3.55 & 10.42 & 34 & 52 \\
2000 & 2.66 & 9.33 & 32 & 52 \\
\hline
\end{tabular}


Table S23. Time of Radiochemical Reaction Evaluation.

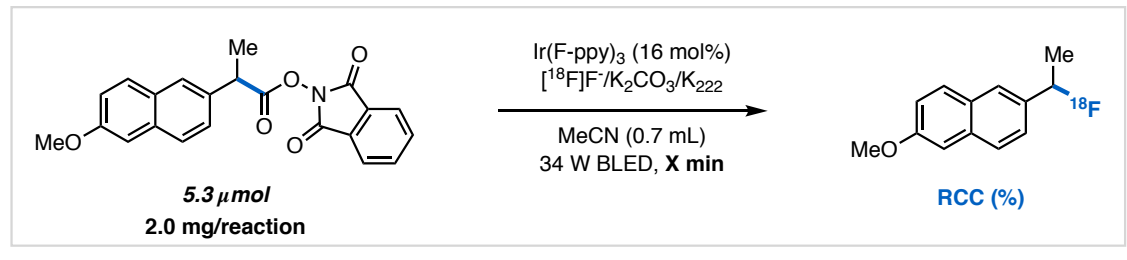

\begin{tabular}{c:ccc}
$\begin{array}{c}\text { Irradiation } \\
\text { Time (min) }\end{array}$ & $\begin{array}{c}\text { Run 1 } \\
\text { EOS Activity (mCi) }\end{array}$ & $\begin{array}{c}\text { Run 1 } \\
\text { HPLC RCC (\%) }\end{array}$ & $\begin{array}{c}\text { Run 1 } \\
\text { TLC RCC (\%) }\end{array}$ \\
\hline 0 & 7.19 & 0 & 0 \\
\hline 2 & 7.56 & 48 & 62 \\
5 & 6.15 & 49 & 71 \\
10 & 10.37 & 45 & 48 \\
15 & 6.65 & 51 & 67 \\
20 & 5.94 & 48 & 61 \\
30 & 3.79 & 45 & 64 \\
\hline
\end{tabular}

Determination of Molar Activity and Scale Up.

To determine the molar activity of $\left[{ }^{18} \mathrm{~F}\right] \mathrm{F}^{-}$incorporation, a UV-calibration curve was developed by serial dilution of a known quantity of $\left[{ }^{19} \mathrm{~F}\right]-2-(1-$ fluoroethyl)-6-methoxynaphthalene an injection onto an Agilent 1200 series HPLC equipped with a UV- and a Lab-Logic Posi-RAM detector.

Determination of number of moles of $\left[{ }^{18} \mathrm{~F} /{ }^{19} \mathrm{~F}\right]-2$-(1-fluoroethyl)-6-methoxynaphthalene by Beer's Law:

$$
\text { Absorbance }=\text { slc }
$$

$$
\begin{gathered}
\varepsilon=\text { molar absorptivity } \\
l=\text { path length } \\
c=\text { concentration }
\end{gathered}
$$

For a constant injection volume, pathlength, and molar absorptivity:

$$
\text { Absorbance } \propto A X
$$

wherein:

$X$ is the number of nanomoles and $A$ is a constant

Utilizing the calibration curve, the constant A may be determined and then used to determine the quantity of $\left[{ }^{18} \mathrm{~F} /{ }^{19} \mathrm{~F}\right]-$ 2-(1-fluoroethyl)-6-methoxynaphthalene. 
Table S24. Molar Activity Calibration Curve.

\begin{tabular}{|c|c|c|c|c|c|}
\hline Dilution & $\begin{array}{l}\text { Final Concentration } \\
\qquad\left(10^{-5} \mathrm{M}\right)\end{array}$ & $\begin{array}{l}\text { Moles Injected } \\
\quad\left(10^{-9} \mathrm{~mol}\right)\end{array}$ & $\begin{array}{c}\text { Absorbance Area } 1 \\
(\mathrm{mAu})\end{array}$ & $\begin{array}{c}\text { Absorbance Area } 2 \\
(\mathrm{mAu})\end{array}$ & $\begin{array}{c}\text { Avg. Absorance Area } \\
(\mathrm{mAu})\end{array}$ \\
\hline $1.3 \mathrm{mg} \mathbf{1}$ in $10.00 \mathrm{~mL}$ (Sol 1) & 63.7 & 15.9 & 3254.1 & 3316.1 & 3285.1 \\
\hline $1000 \mu \mathrm{L}$ Sol 1 to $5.00 \mathrm{~mL}$ & 12.7 & 3.18 & 667.2 & 661.3 & 664.25 \\
\hline $700 \mu \mathrm{L}$ Sol 1 to $5.00 \mathrm{~mL}$ & 8.91 & 2.23 & 460.6 & 463.0 & 461.8 \\
\hline $400 \mu \mathrm{L}$ Sol 1 to $5.00 \mathrm{~mL}$ & 5.09 & 1.27 & 263.9 & 265.5 & 264.7 \\
\hline $100 \mu \mathrm{L}$ Sol 1 to $5.00 \mathrm{~mL}$ & 1.27 & 0.318 & 64.5 & 66.2 & 65.4 \\
\hline \multirow[t]{4}{*}{$100 \mu \mathrm{L}$ Sol 1 to $50.00 \mathrm{~mL}$} & 0.127 & 0.0318 & 5.4 & 5.6 & 5.5 \\
\hline & & & & Slope (mAu/nmol): & 206.6 \\
\hline & & & & Std. Dev. Slope (mAu/nmol): & 0.21 \\
\hline & & & & $R^{2}:$ & 1.00 \\
\hline
\end{tabular}

Figure S17. Molar Activity Calibration Curve.

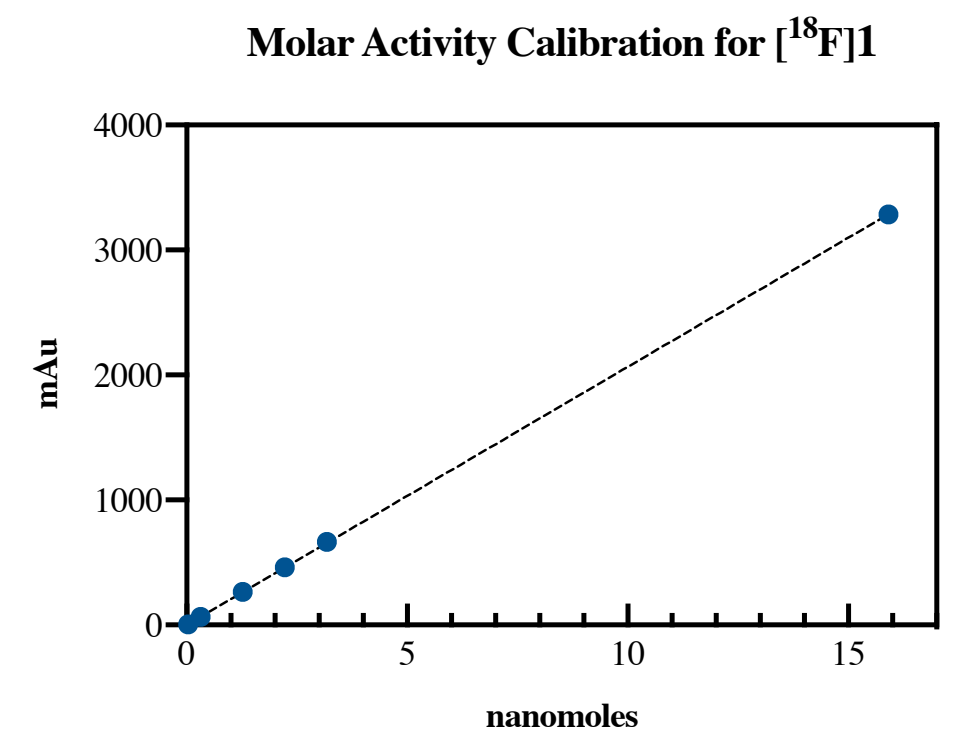

Following the determination of a specific activity curve, scale ups using higher activity (50-100 mCi) were conducted. The $\left[{ }^{18} \mathrm{~F}\right]$-2-(1-fluoroethyl)-6-methoxynaphthalene peak was isolated from the HPLC and the isolated activity was determined as well as the absorbance of the peak. From this the specific activity was determined by dividing the activity by the amount of fluorinated product.

$$
\begin{gathered}
\text { Absorbance }=A X \\
\frac{\text { Absorbance }}{A}=\frac{\text { Absorbance }}{206.6 \frac{\mathrm{mAu}}{\mathrm{nmol}}}=X \mathrm{nmol}
\end{gathered}
$$

$$
\text { Specific Activity }=\frac{\text { Activity }}{X \text { nmol }}
$$


Table S25. Molar Activity Determination.

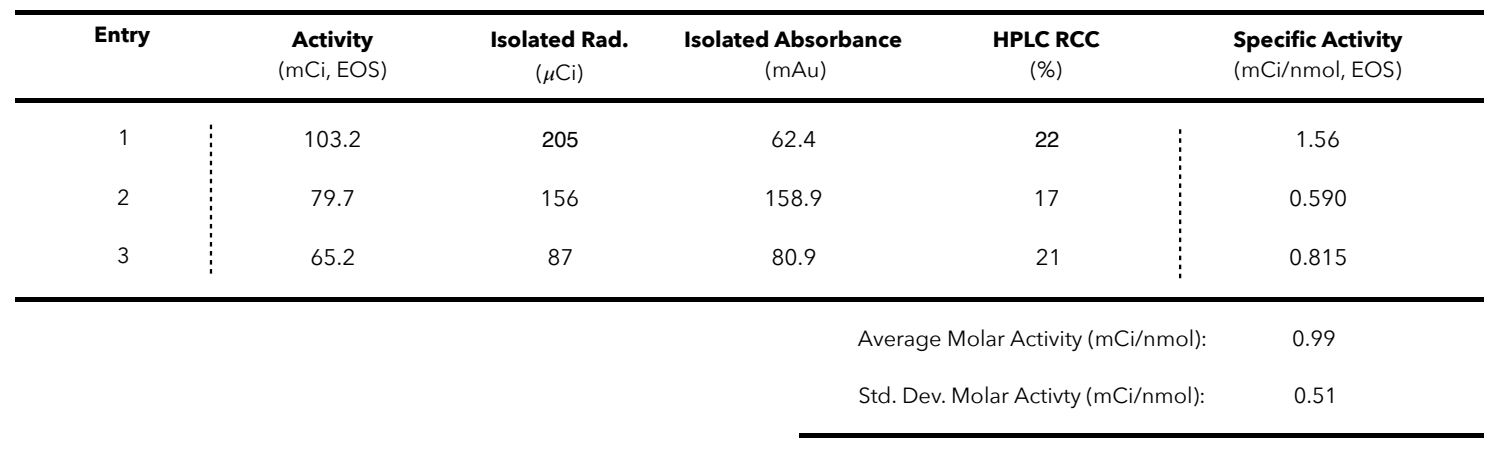

\section{Evaluation of Radiochemical Scope}

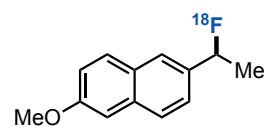

\section{2-(1-(fluoro- $\left.{ }^{18} \mathbf{F}\right)$ ethyl)-6-methoxynaphthalene $\left(\left[{ }^{18} \mathbf{F}\right] 2\right)$}

A vial was charged with 1,3-dioxoisoindolin-2-yl 2-(6-methoxynaphthalen-2-yl)propanoate (14.5 mg, $0.0386 \mathrm{mmol}$, 1 equiv.) and $4.44 \mathrm{mg}$ of $\operatorname{Ir}(\mathrm{F}-\mathrm{ppy})_{3}(4.44 \mathrm{mg}, 0.00582 \mathrm{mmol}, 0.15$ equiv.) $\mathrm{MeCN}(2.8 \mathrm{~mL})$ was added to the vial and used to dissolve all of the solids. Aliquots $(400 \mu \mathrm{L})$ were then removed from the vial and added to 5 separate vials. These vials were septa capped and subjected to the general procedure for radiochemistry. 2-(1-(fluoro- $\left.{ }^{18} \mathrm{~F}\right)$ ethyl)-6methoxynaphthalene was determined to be obtained by coinjection with authentic 2-(1-(fluoro- $\left.{ }^{19} \mathrm{~F}\right)$ ethyl)-6methoxynaphthalene. This procedure was repeated to furnish additional replicates.

Table S26. Replicates for generation of 2-(1-(fluoro- $\left.{ }^{18} \mathrm{~F}\right)$ ethyl)-6-methoxynaphthalene.

\begin{tabular}{cccc}
\hline Entry & Activity at EOS $(\mathrm{mCi})$ & RCC-TLC $(\%, \mathbf{5 0} \%$ EtOAC in hexanes) & RCC-HPLC (\%) \\
\hline 1 & 13.14 & 61 & 63 \\
2 & 16.63 & 49 & 36 \\
3 & 16.10 & 47 & 31 \\
4 & 13.61 & 44 & 29 \\
5 & 11.71 & 44 & 34 \\
\hline 6 & 103.2 & 11 & 22 \\
7 & 79.7 & 13 & 17 \\
8 & 65.2 & 10 & 21 \\
\hline
\end{tabular}


Figure S18. HPLC of Authentic Standards at $254 \mathrm{~nm}\left(\mathbf{1}, \mathbf{2}\right.$, and $\left.\operatorname{Ir}(\mathbf{F}-\mathbf{p p y})_{3}\right)$.

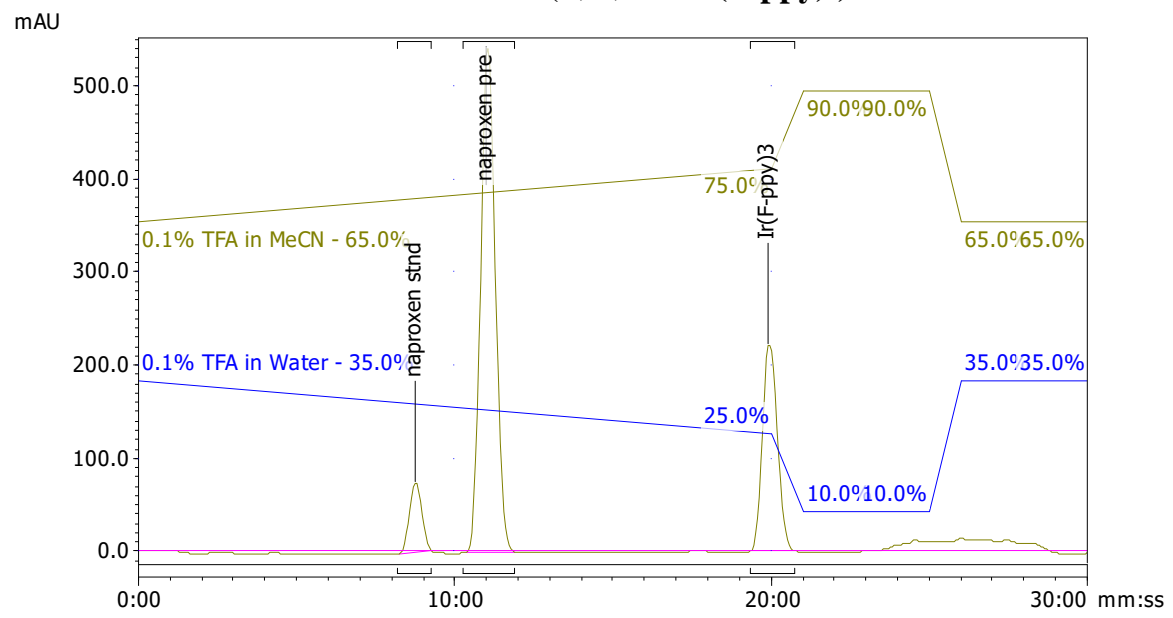

HPLC Column: Zorbax SB-C18, $5 \mu \mathrm{m}, 4.6 x 250 \mathrm{~mm}$. 2-(1-(fluoro- $\left.{ }^{19} \mathrm{~F}\right)$ ethyl)-6-methoxynaphthalene (2), 8:44 min. 1,3-dioxoisoindolin-2-yl 2-(6-methoxynaphthalen-2-yl)propanoate (1), 11:01 min. $\operatorname{Ir}(\mathrm{F}-\mathrm{ppy})_{3}, 19: 55 \mathrm{~min}$.

Figure S19. PosiRam-HPLC of Crude Reaction Mixture with 2-(1-(fluoro- $\left.{ }^{18} \mathrm{~F}\right)$ ethyl)-6-methoxynaphthalene ([$\left.\left.{ }^{18} \mathbf{F}\right] 2\right)$.

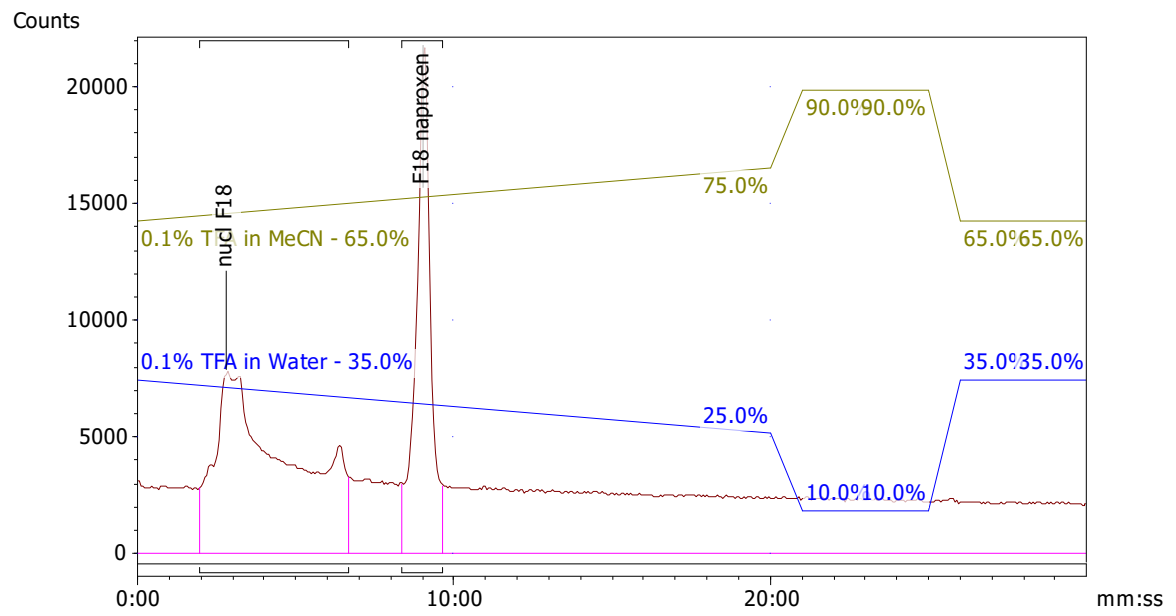


Figure S20. HPLC of Crude Reaction Mixture with 2-(1-(fluoro- ${ }^{18}$ F)ethyl)-6-methoxynaphthalene $\left(\left[{ }^{18} \mathbf{F}\right] 2\right)$ at 254 nm.

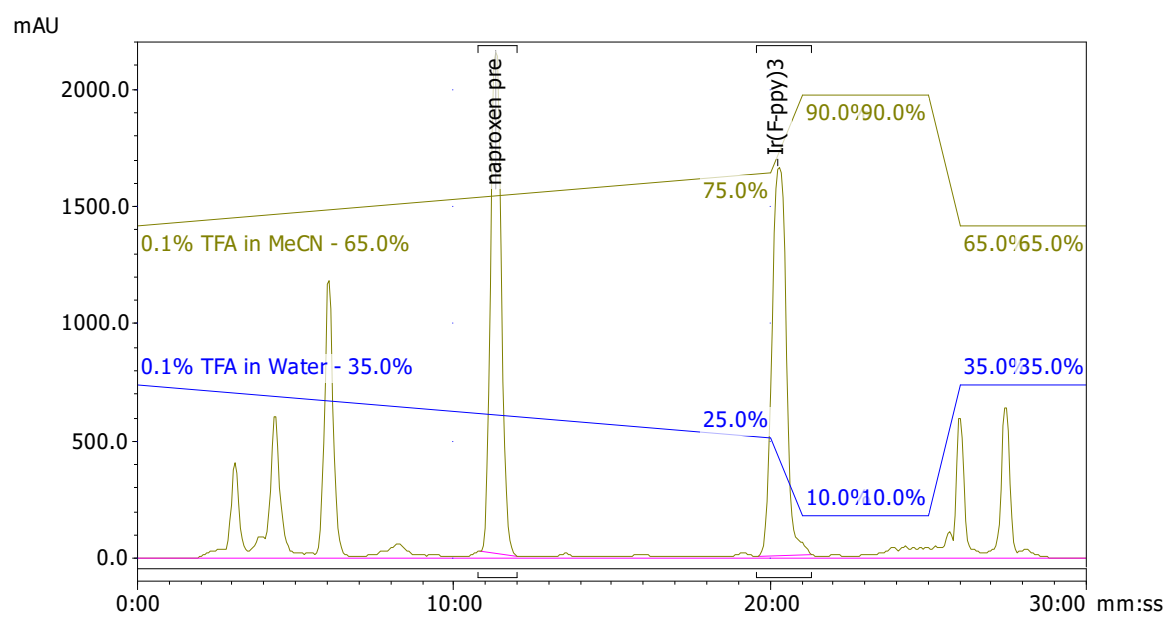

Figure S21. HPLC of Crude Reaction Mixture with 2-(1-(fluoro- ${ }^{18}$ F)ethyl)-6-methoxynaphthalene $\left(\left[{ }^{18} \mathbf{F}\right] 2\right)$ with Coinjection (2).

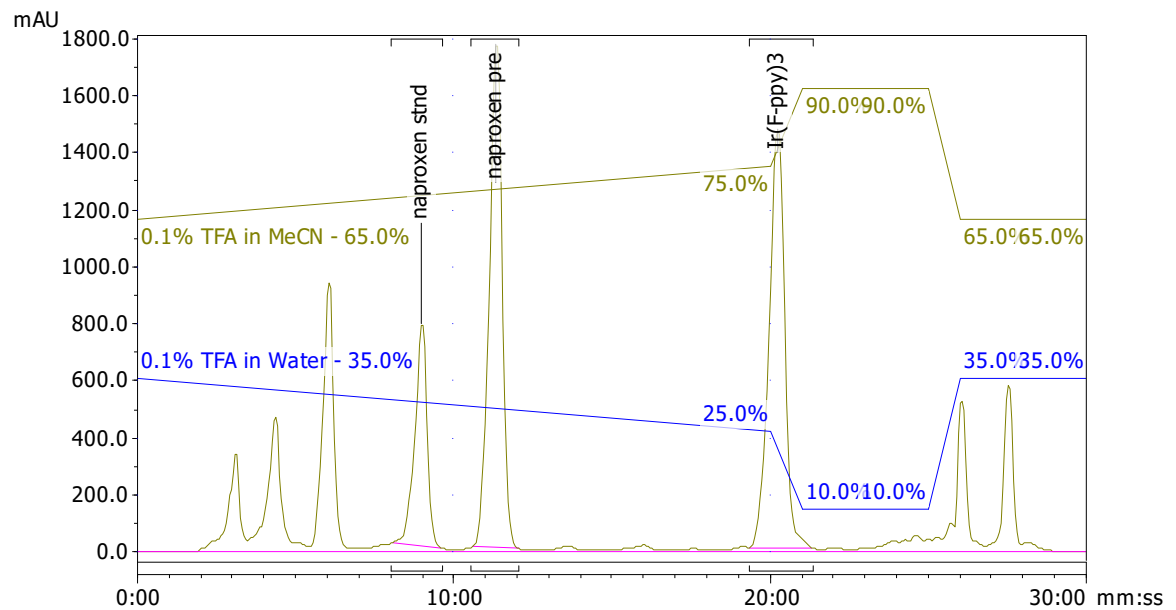

Figure S22. Sample Radio-TLC of 2-(1-(fluoro-18F)ethyl)-6-methoxynaphthalene ([$\left.\left.{ }^{18} \mathrm{~F}\right] 2\right)$. Region 1: Inorganic ("free" and unincorporated) $\left[{ }^{18} \mathrm{~F}\right] \mathrm{F}^{-}$at baseline of plate; Region 2: 2-(1-(fluoro- $\left.{ }^{18} \mathrm{~F}\right)$ ethyl)-6-methoxynaphthalene $\left(\left[{ }^{18} \mathbf{F}\right] 2\right)$. 


\begin{tabular}{lrrrrrrrr} 
Reg & $\begin{array}{r}(\mathrm{mm}) \\
\text { Start }\end{array}$ & $\begin{array}{r}(\mathrm{mm}) \\
\text { Stop }\end{array}$ & $\begin{array}{r}(\mathrm{mm}) \\
\text { Centroid }\end{array}$ & RF & $\begin{array}{r}\text { Region } \\
\text { Counts }\end{array}$ & $\begin{array}{r}\text { Region } \\
\text { CPM }\end{array}$ & $\begin{array}{r}\% \text { of } \\
\text { Total }\end{array}$ & $\begin{array}{r}\% \text { of } \\
\text { ROI }\end{array}$ \\
\hline Rgn 1 & 1.0 & 22.5 & 12.3 & 0.123 & 80423.0 & 26807.7 & 35.30 & 38.90 \\
Rgn 2 & 57.7 & 89.5 & 69.9 & 0.699 & 126323.0 & 42107.7 & 55.44 & 61.10 \\
\hline 2 Peaks & & & & 206746.0 & 68915.3 & 90.74 & 100.00
\end{tabular}

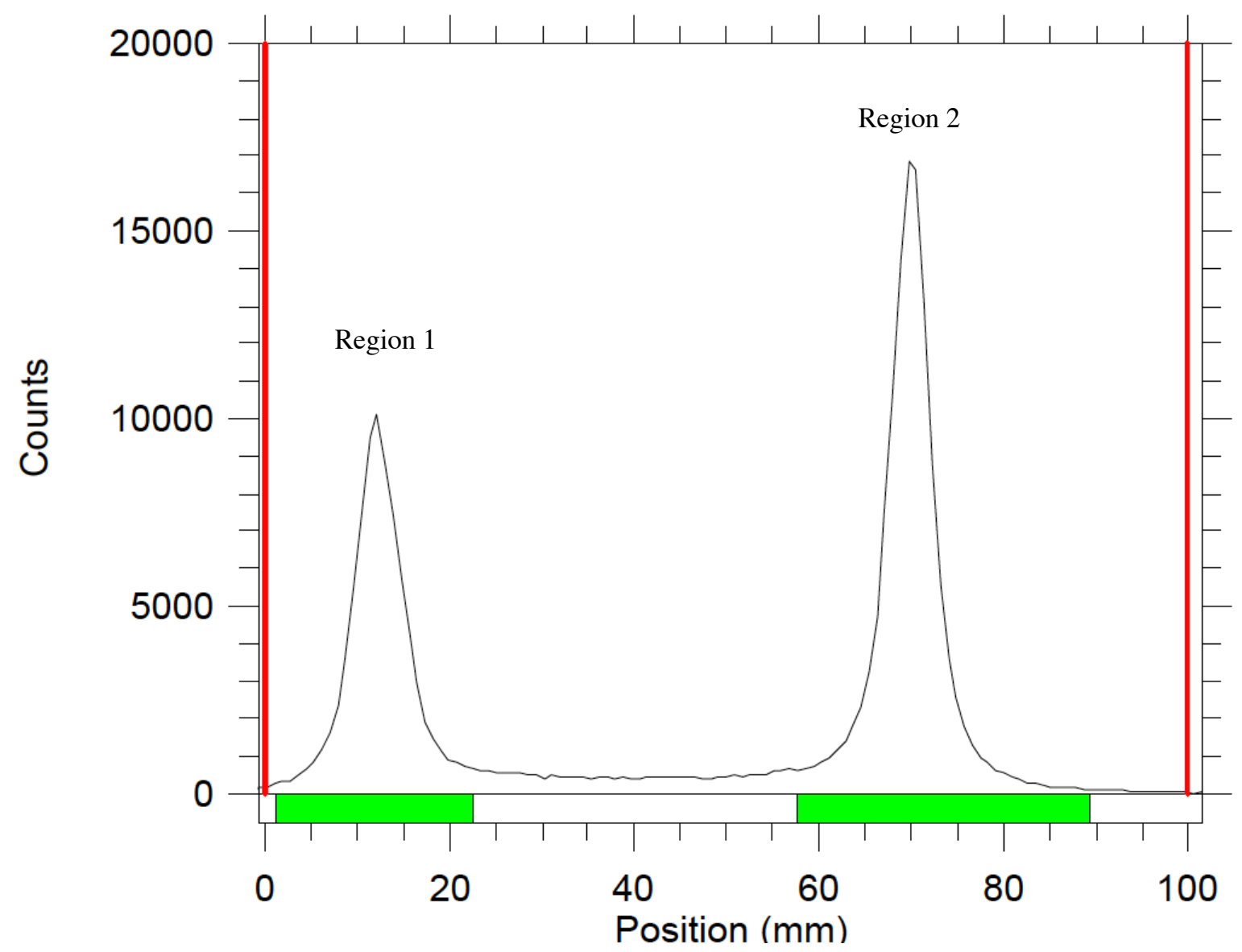

Figure S23. Sample Radio-TLC of the $[18 \mathrm{~F}] \mathrm{F}^{-} / \mathrm{K}_{222} / \mathrm{K}_{2} \mathrm{CO}_{3}$ (unincorporated $\left[{ }^{18} \mathrm{~F}\right] \mathrm{F}^{-}, 50 \%$ EtOAc/hexanes eluent). 

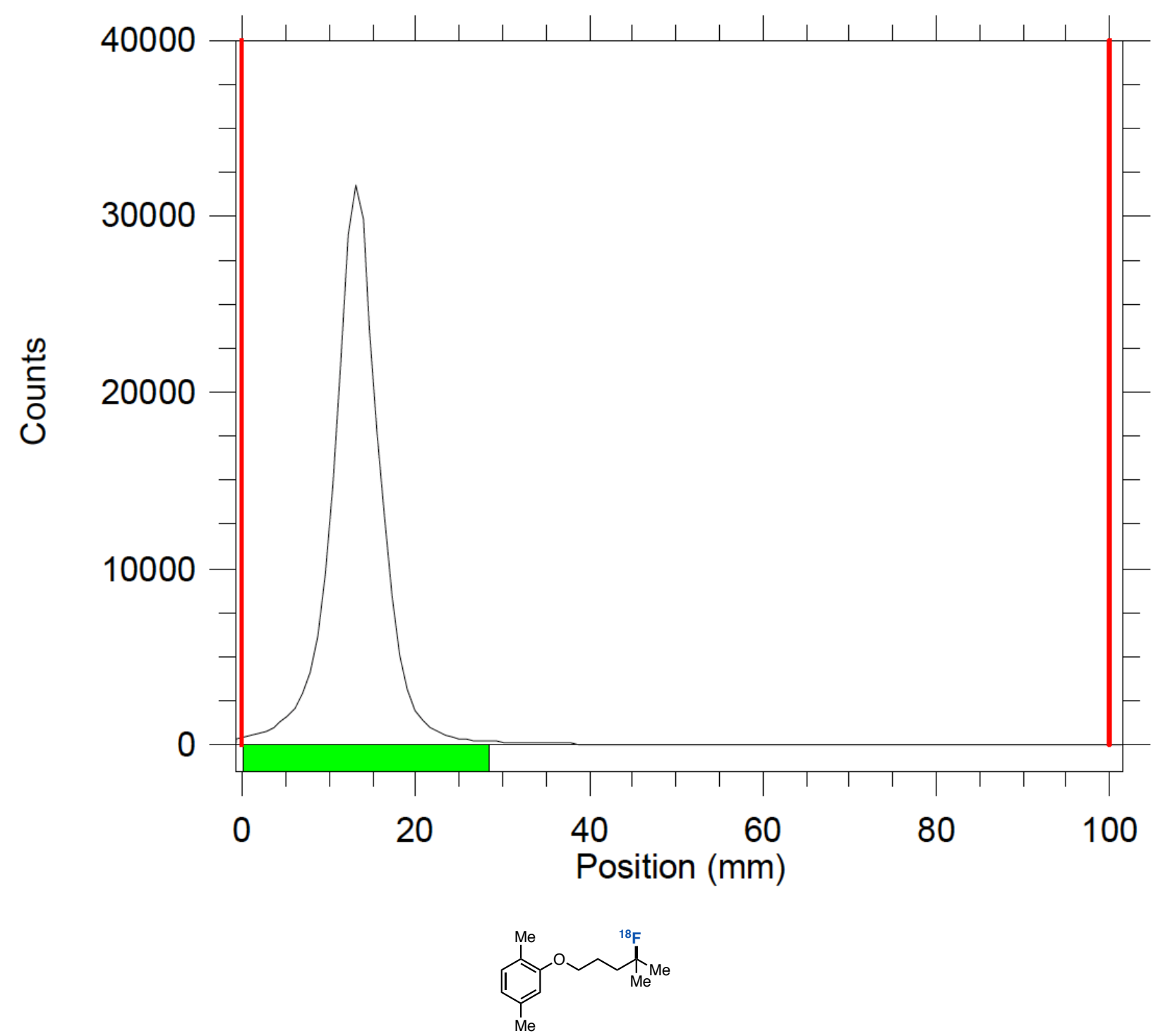

2-(1-(fluoro- ${ }^{18}$ F)ethyl)-6-methoxynaphthalene $\left(\left[{ }^{18} \mathrm{~F}\right] 30\right)$

A vial was charged with 1,3-dioxoisoindolin-2-yl 5-(2,5-dimethylphenoxy)-2,2-dimethylpentanoate (30.5 mg, 0.0800 mmol, 1 equiv.) and $3.0 \mathrm{~mL}$ of MeCN. The solid was dissolved and $200 \mu \mathrm{L}$ aliquots was added to 5 separate vials. Similarly, a separate vial was charged with $\operatorname{Ir}(\mathrm{dF}-\mathrm{ppy})_{3}(5.22 \mathrm{mg}, 0.00684 \mathrm{mmol}, 0.18$ equiv. $)$. MeCN $(1.400 \mathrm{~mL})$ was added to the vial and used to suspend the photocatalyst. Aliquots of $200 \mu \mathrm{L}$ of photocatalyst solution and of phthalimide ester solution were then added to separate vials to have vials with $400 \mu \mathrm{L}$ of photocatalyst and phthalimide ester. The vials were septa capped and subjected to the general procedure for radiochemistry. This procedure was repeated to furnish additional replicates.

Note: we found $\operatorname{Ir}(\mathrm{F}-\mathrm{ppy})_{3}$ was less effective for this substrate under radiochemical conditions. 
Table S27. Replicates for Generation of 2-((4-(fluoro- $\left.{ }^{18} F\right)-4$-methylpentyl)oxy)-1,4-dimethylbenzene $\left(\left[{ }^{18} \mathbf{F}\right] \mathbf{3 0}\right)$.

\begin{tabular}{cccc}
\hline Entry & Activity at EOS $(\mathbf{m C i})$ & RCC-TLC $(\%, \mathbf{5 0} \%$ EtOAc in hexanes) & RCC-HPLC (\%) \\
\hline 1 & 14.50 & 10 & 15 \\
2 & 9.84 & 10 & 14 \\
3 & 6.05 & 7 & 17 \\
4 & 3.83 & 11 & 14 \\
5 & 5.75 & 6 & 16 \\
\hline
\end{tabular}

Figure S24. HPLC of Authentic Materials: 2-((4-fluoro-4-methylpentyl)oxy)-1,4-dimethylbenzene (30, 7:08 min) and 1,3-dioxoisoindolin-2-yl 5-(2,5-dimethylphenoxy)-2,2-dimethylpentanoate (41, 8:45 $\mathrm{min})$ at $254 \mathrm{~nm}$.

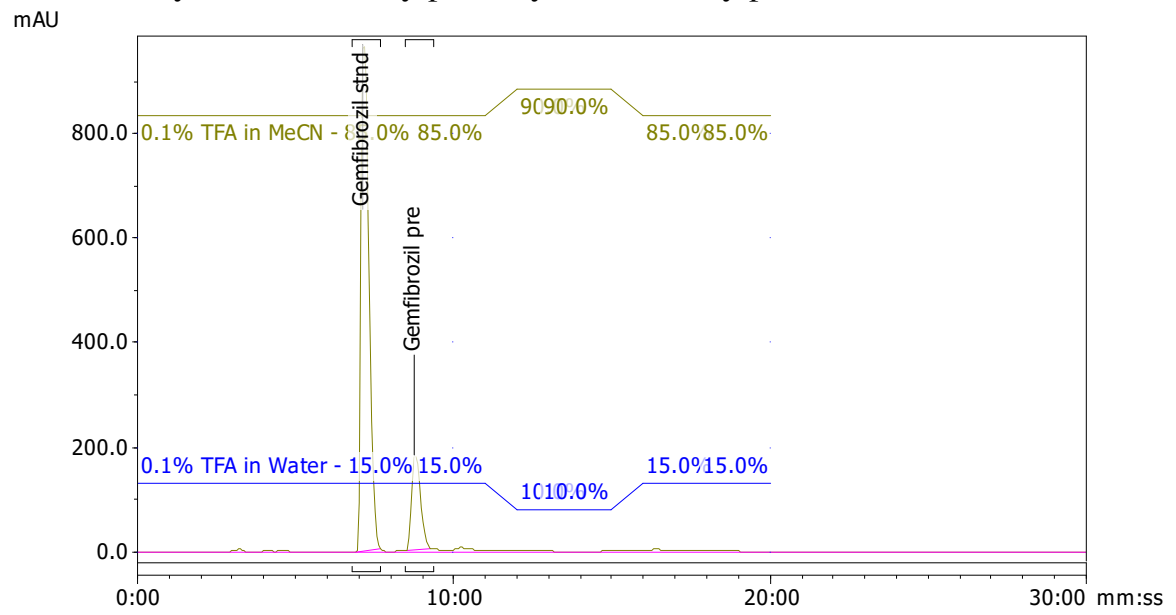

Figure S25. HPLC of Authentic Ir(dF-ppy)3 (14.33 min) at $254 \mathrm{~nm}$.

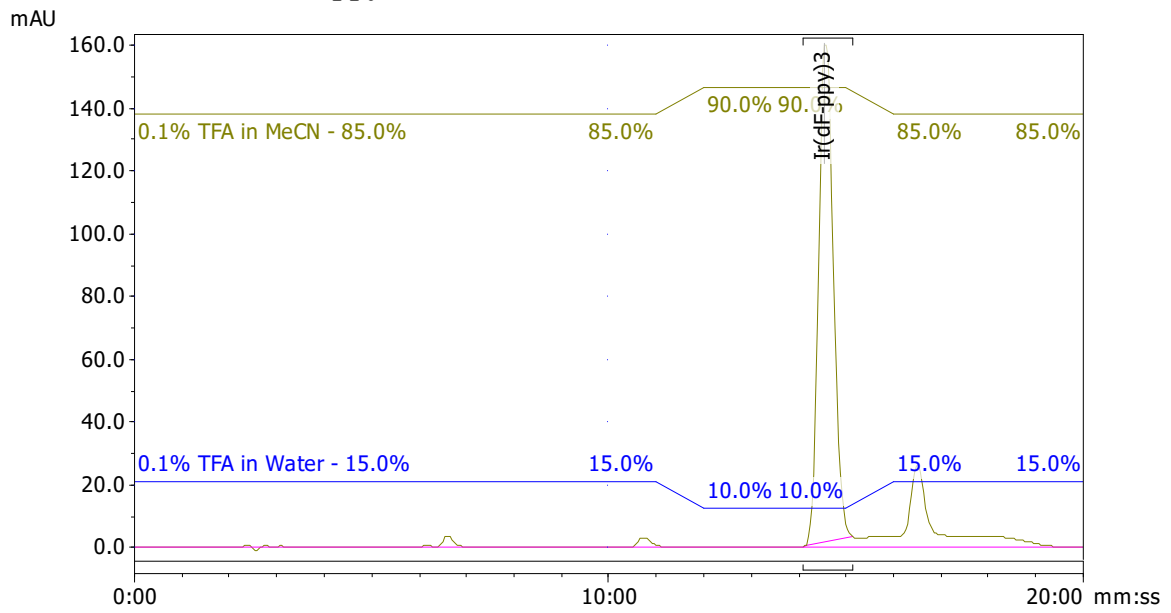


Figure S26. Sample HPLC of Crude Reaction Mixture with 2-((4-(fluoro- $\left.{ }^{18} F\right)-4$-methylpentyl)oxy)-1,4dimethylbenzene $\left(\left[{ }^{18} \mathbf{F}\right] \mathbf{3 0}\right.$, Posi-RAM).

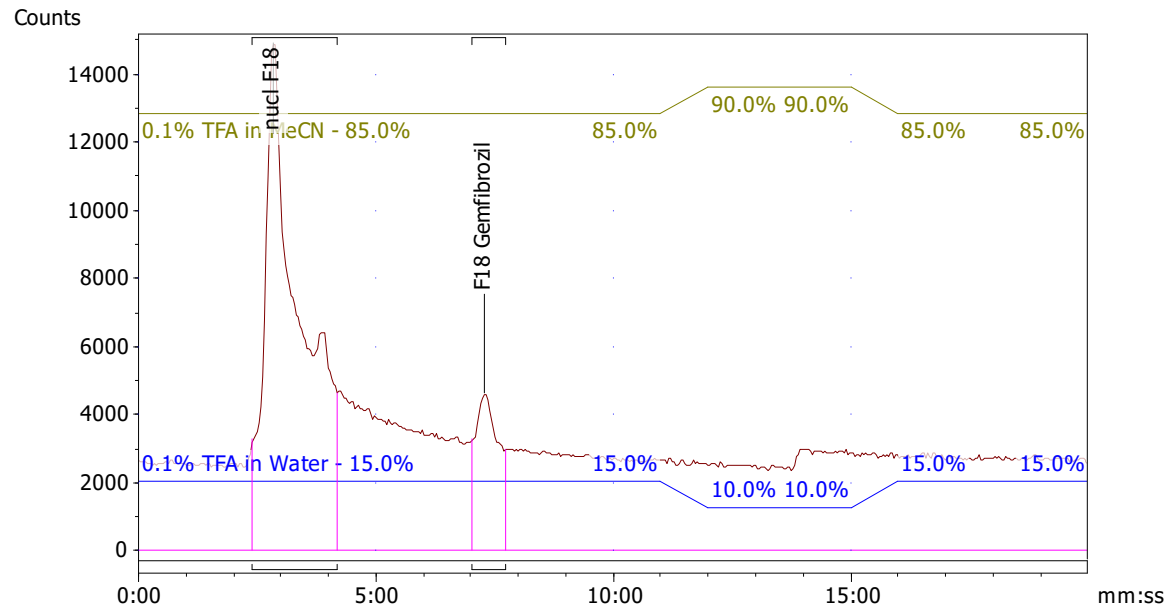

Figure S27. Sample HPLC of Crude 2-((4-(fluoro- $\left.{ }^{18} F\right)-4$-methylpentyl)oxy)-1,4-dimethylbenzene ([$\left.\left.{ }^{18} \mathbf{F}\right] \mathbf{3 0}, 254 \mathrm{~nm}\right)$. $\mathrm{mAU}$

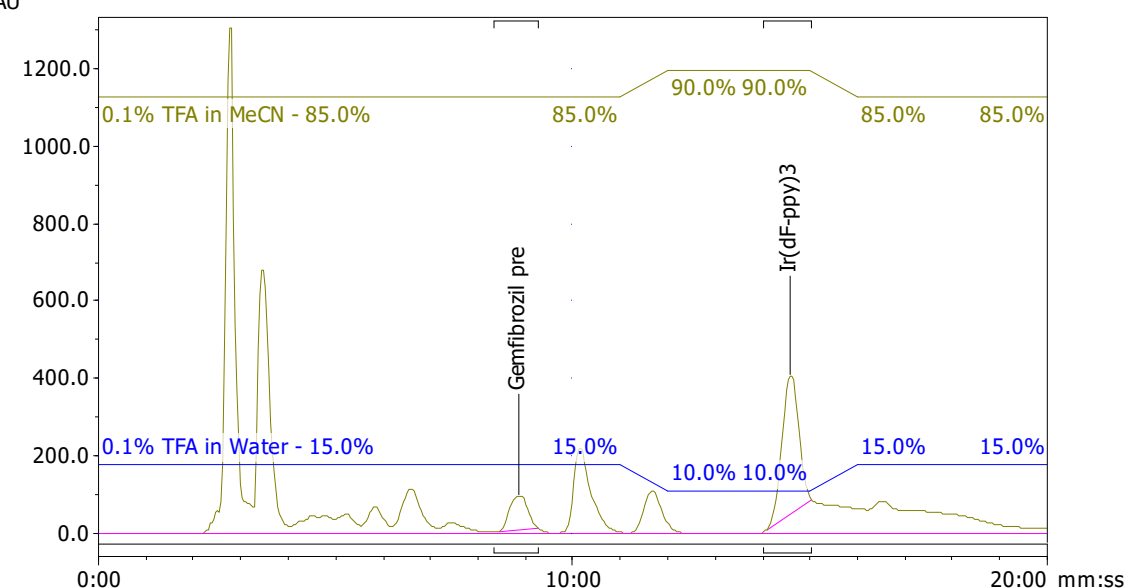

Figure S28. Sample HPLC of Crude 2-((4-(fluoro- $\left.{ }^{18} F\right)$-4-methylpentyl)oxy)-1,4-dimethylbenzene ([$\left.\left.{ }^{18} \mathbf{F}\right] \mathbf{3 0}\right)$ with 2((4-fluoro-4-methylpentyl)oxy)-1,4-dimethylbenzene (30) Coinjection (254 nm).

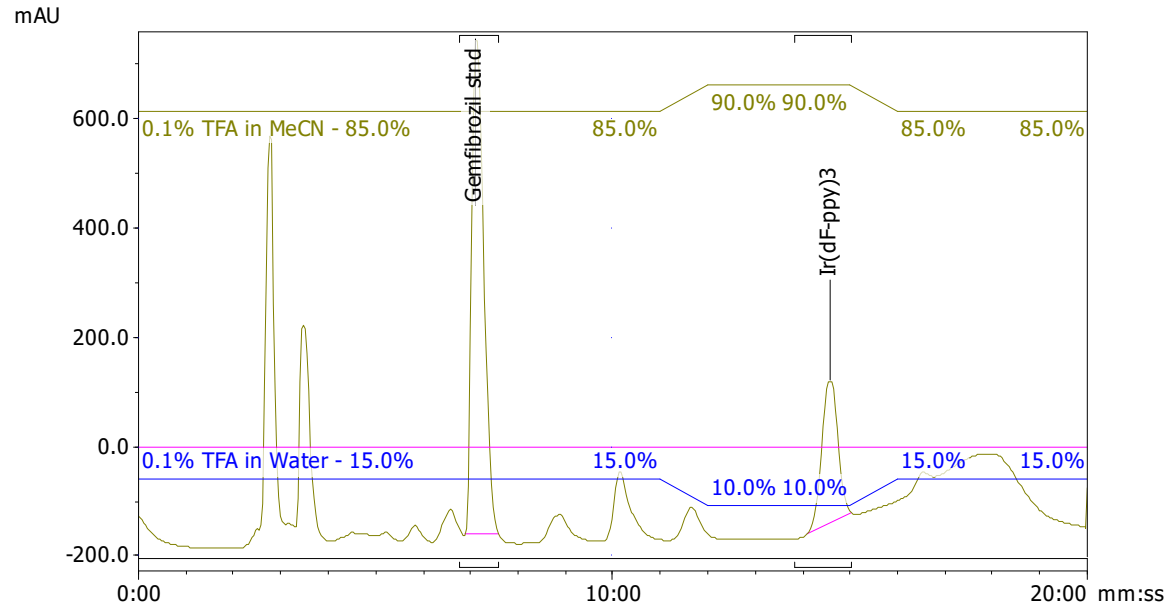


Figure S29. Sample Radio-TLC of 2-((4-(fluoro-18F)-4-methylpentyl)oxy)-1,4-dimethylbenzene $\left(\left[{ }^{18} \mathrm{~F}\right] 2\right)$. Region 1: Inorganic ("free" and unincorporated) $\left[{ }^{18} \mathrm{~F}\right] \mathrm{F}^{-}$at baseline of plate; Region 2: 2-((4-(fluoro- $\left.{ }^{18} \mathrm{~F}\right)-4$-methylpentyl)oxy)1,4-dimethylbenzene $\left(\left[{ }^{18} \mathbf{F}\right] \mathbf{3 0}\right)$.

\begin{tabular}{lrrrrrrrr} 
Reg & $\begin{array}{r}(\mathrm{mm}) \\
\text { Start }\end{array}$ & $\begin{array}{r}(\mathrm{mm}) \\
\text { Stop }\end{array}$ & $\begin{array}{r}(\mathrm{mm}) \\
\text { Centroid }\end{array}$ & RF & $\begin{array}{r}\text { Region } \\
\text { Counts }\end{array}$ & $\begin{array}{r}\text { Region } \\
\text { CPM }\end{array}$ & $\begin{array}{r}\% \text { of } \\
\text { Total }\end{array}$ & $\begin{array}{r}\% \text { of } \\
\text { ROI }\end{array}$ \\
\hline Rgn 1 & 1.9 & 30.2 & 14.7 & 0.147 & 214150.0 & 71383.3 & 86.39 & 89.26 \\
Rgn 2 & 67.1 & 89.5 & 79.0 & 0.790 & 25772.0 & 8590.7 & 10.40 & 10.74 \\
\hline 2 Peaks & & & & 239922.0 & 79974.0 & 96.79 & 100.00
\end{tabular}
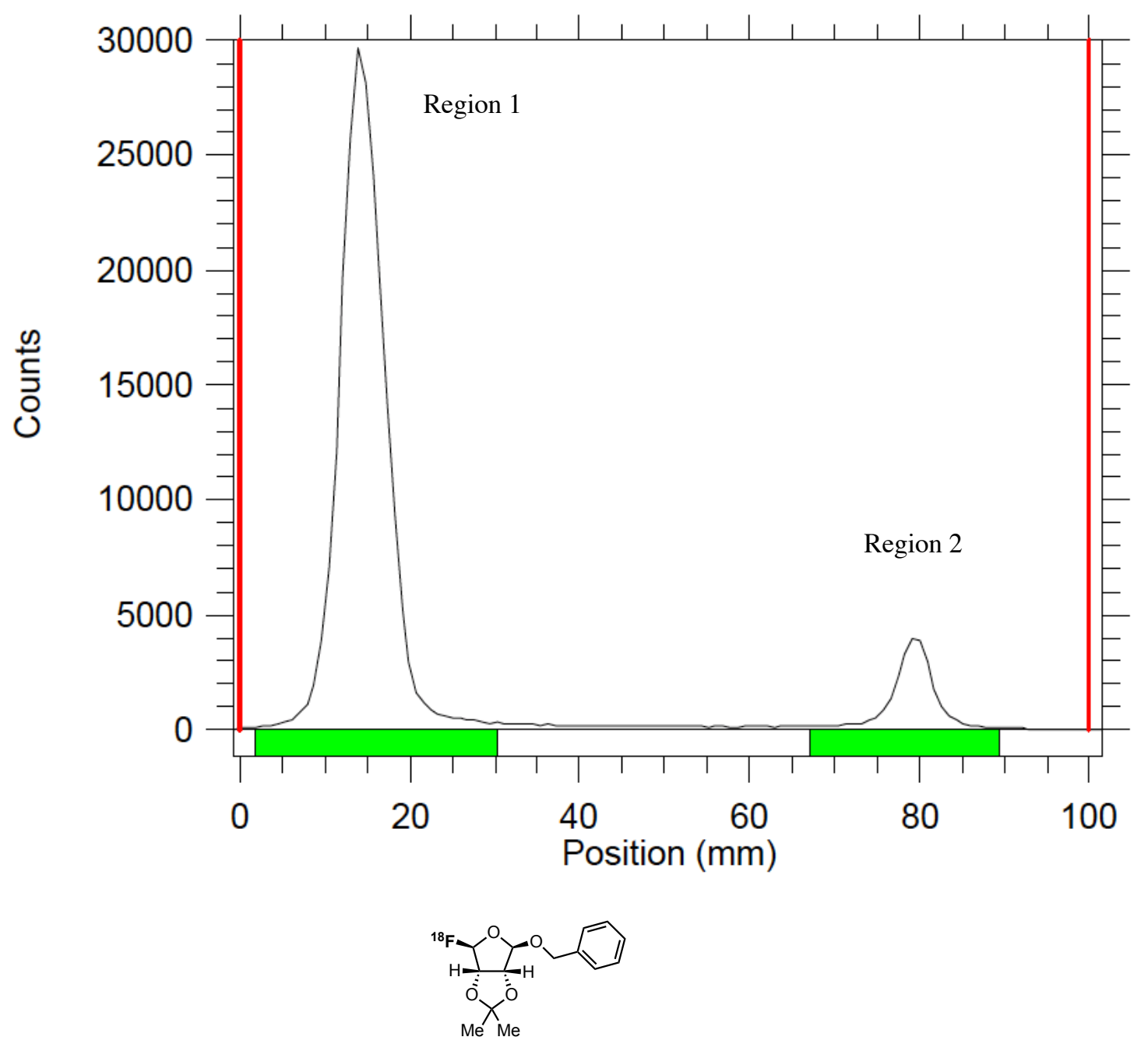

$(3 \mathrm{a} R, 4 R, 6 R, 6 \mathrm{a} S)$-4-(benzyloxy)-6-(fluoro- $\left.{ }^{-18} F\right)$-2,2-dimethyltetrahydrofuro[3,4- $\left.d\right][1,3]$ dioxole $\left(\left[{ }^{18} \mathrm{~F}\right] 34\right)$

A vial was charged with 1,3-dioxoisoindolin-2-yl (3aS,4S,6R,6aR)-6-(benzyloxy)-2,2-dimethyltetrahydrofuro[3,4d] [1,3]dioxole-4-carboxylate ( $35.1 \mathrm{mg}, 0.0800 \mathrm{mmol}, 1$ equiv.) and $3.0 \mathrm{~mL}$ of MeCN. The solid was dissolved and $200 \mu \mathrm{L}$ aliquots was added to 5 separate vials. Similarly, a separate vial was charged with $5.03 \mathrm{mg}$ of $\operatorname{Ir}(\mathrm{F}-\mathrm{ppy})_{3}(5.03$ $\mathrm{mg}, 0.00710 \mathrm{mmol}, 0.19 \mathrm{mmol})$. MeCN $(1.400 \mathrm{~mL})$ was added to the vial and used to suspend the photocatalyst. Aliquots of $200 \mu \mathrm{L}$ of photocatalyst solution and of phthalimide ester solution were then added to separate vials to have vials with $400 \mu \mathrm{L}$ of photocatalyst and phthalimide ester. The vials were septa capped and then subjected to the general procedure for radiochemistry. (3a $R, 4 R, 6 R, 6 \mathrm{a} S$ )-4-(benzyloxy)-6-(fluoro- ${ }^{-18} F$ )-2,2-dimethyltetrahydrofuro[3,4$d][1,3]$ dioxole was determined to be obtained by co-spotting in the course of radio TLC with authentic $(3 \mathrm{a} R, 4 R, 6 R, 6 \mathrm{a} S)$-4-(benzyloxy)-6-(fluoro- $\left.{ }^{18} F\right)$-2,2-dimethyltetrahydrofuro[3,4- $\left.d\right][1,3]$ dioxole. This procedure was repeated to furnish additional replicates. 
Note: This substrate appeared to undergo decomposition (likely acetal deprotection) under HPLC conditions thereby prohibiting identity confirmation by HPLC coinjection.

Table S28. Replicates for Generation of (3aR,4R,6R,6aS)-4-(benzyloxy)-6-(fluoro- $\left.{ }^{18} F\right)-2,2-$ dimethyltetrahydrofuro[ $[3,4-d][1,3]$ dioxole $\left(\left[{ }^{18} \mathbf{F}\right] \mathbf{3 4}\right)$.

\begin{tabular}{ccc}
\hline Entry & Activity at EOS $(\mathbf{m C i})$ & RCC-TLC (\%, 50\% EtOAc in hexanes) \\
\hline 1 & 1.41 & 65 \\
2 & 11.16 & 25 \\
3 & 4.79 & 39 \\
4 & 4.74 & 33 \\
5 & 2.25 & 49 \\
\hline
\end{tabular}

Figure S30. Sample Radio-TLC of 2-((4-(fluoro-18F)-4-methylpentyl)oxy)-1,4-dimethylbenzene ([$\left.\left.{ }^{18} \mathbf{F}\right] \mathbf{3 4}\right)$. Region 1: Inorganic ("free" and unincorporated) $\left[{ }^{18} \mathrm{~F}\right] \mathrm{F}^{-}$at baseline of plate; Region 2 : $(3 \mathrm{a} R, 4 R, 6 R, 6 \mathrm{a} S)$-4-(benzyloxy)-6-(fluoro$\left.{ }^{18} F\right)$-2,2-dimethyltetrahydrofuro[3,4- $\left.d\right][1,3]$ dioxole $\left(\left[{ }^{18} \mathbf{F}\right] \mathbf{3 4}\right)$.

\begin{tabular}{lrrrrrrrr} 
Reg & $\begin{array}{c}(\mathrm{mm}) \\
\text { Start }\end{array}$ & $\begin{array}{r}(\mathrm{mm}) \\
\text { Stop }\end{array}$ & $\begin{array}{r}(\mathrm{mm}) \\
\text { Centroid }\end{array}$ & RF & $\begin{array}{r}\text { Region } \\
\text { Counts }\end{array}$ & $\begin{array}{r}\text { Region } \\
\text { CPM }\end{array}$ & $\begin{array}{r}\% \text { of } \\
\text { Total }\end{array}$ & $\begin{array}{r}\% \text { of } \\
\text { ROI }\end{array}$ \\
\hline Rgn 1 & 4.5 & 21.6 & 13.0 & 0.130 & 103756.0 & 34585.3 & 43.57 & 51.81 \\
Rgn 2 & 56.8 & 79.2 & 65.2 & 0.652 & 96508.0 & 32169.3 & 40.53 & 48.19 \\
\hline 2 Peaks & & & & 200264.0 & 66754.7 & 84.10 & 100.00
\end{tabular}

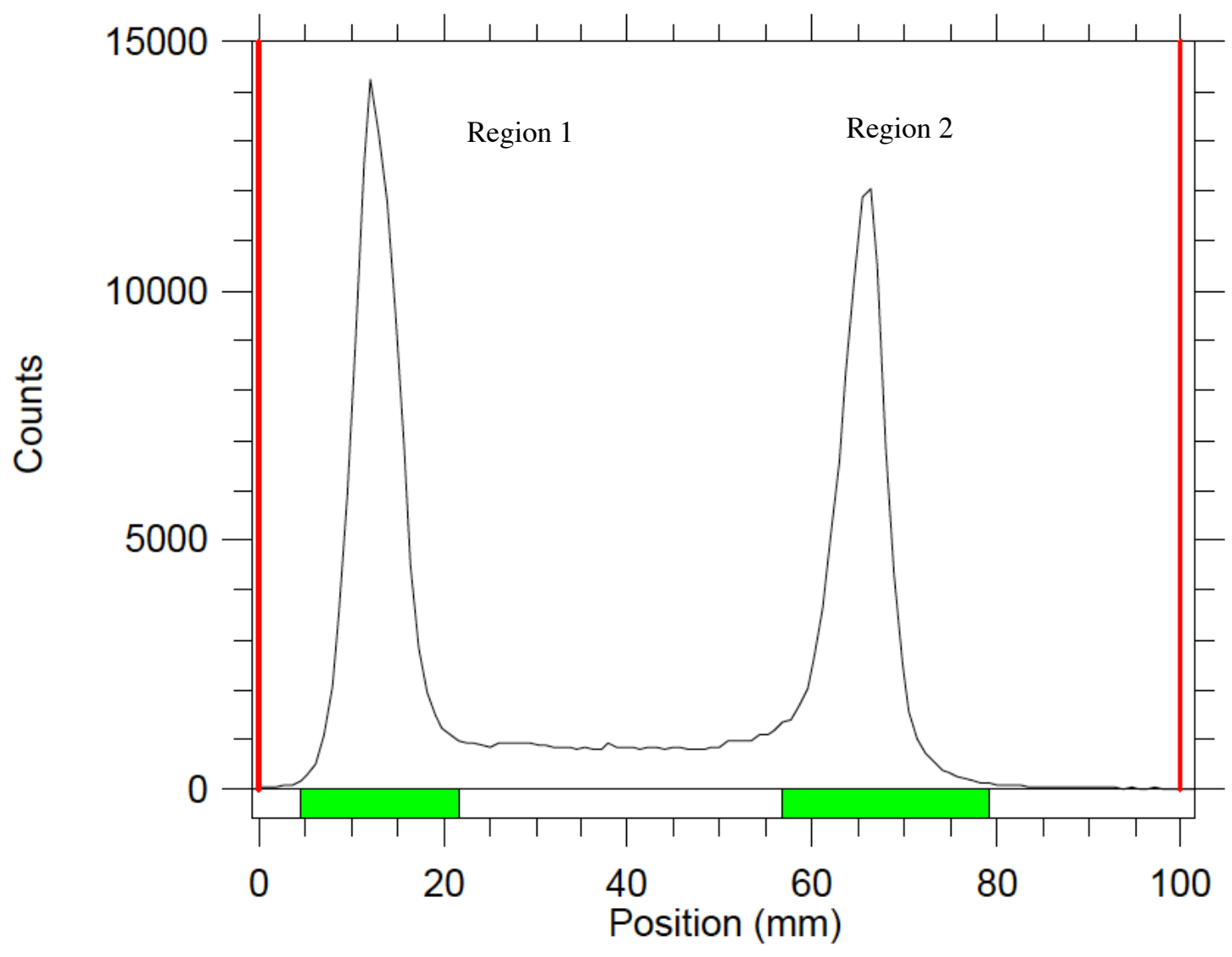


VIII. NMR Spectra 
$500 \mathrm{MHz}{ }^{1} \mathrm{H}-\mathrm{NMR}$ of 1,3-dioxoisoindolin-2-yl 2-(6-methoxynaphthalen-2-yl)propanoate (1) in $\mathrm{CDCl}_{3}$
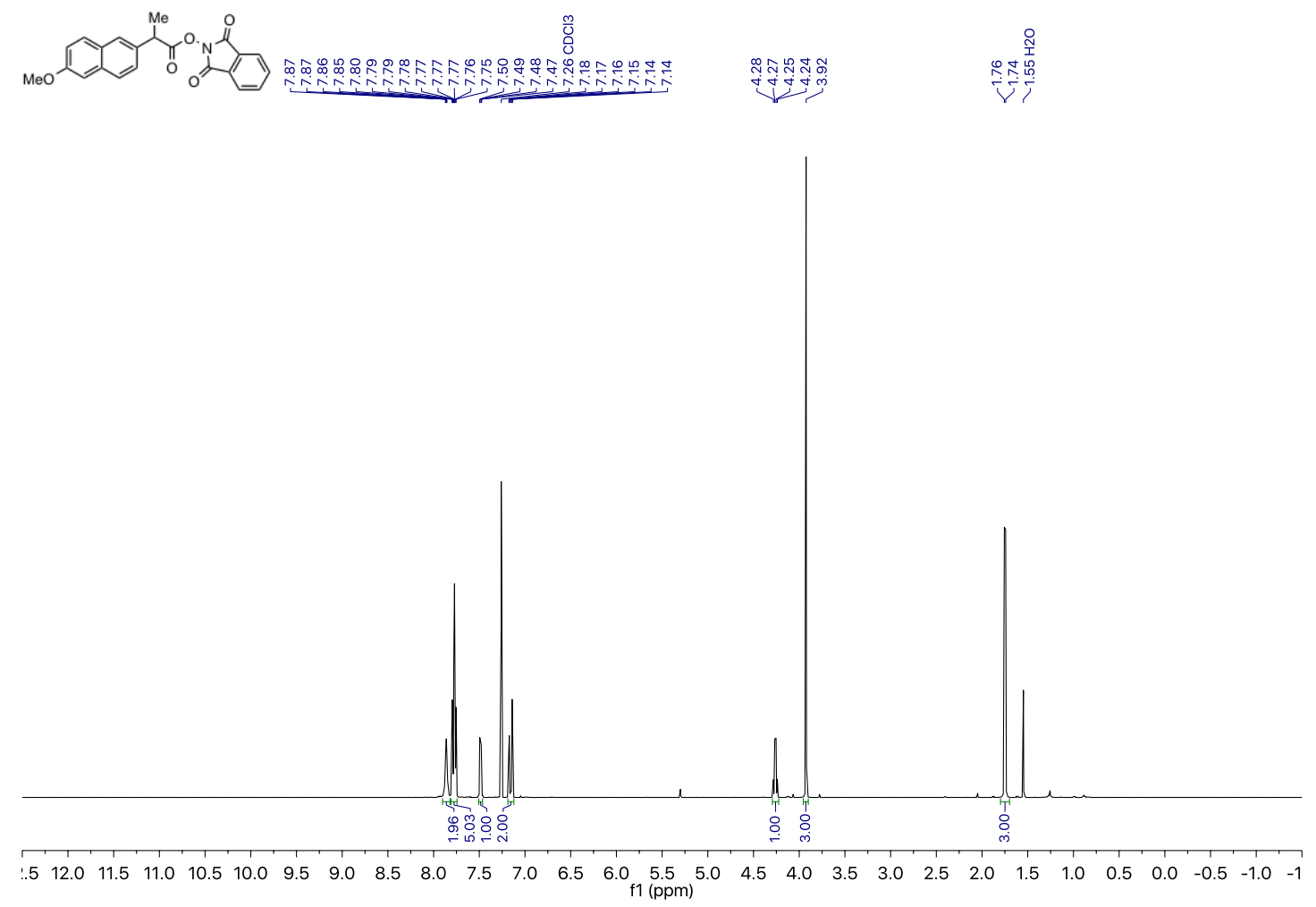

$126 \mathrm{MHz}{ }^{13} \mathrm{C}-\mathrm{NMR}$ of 1,3-dioxoisoindolin-2-yl 2-(6-methoxynaphthalen-2-yl)propanoate (1) in $\mathrm{CDCl}_{3}$
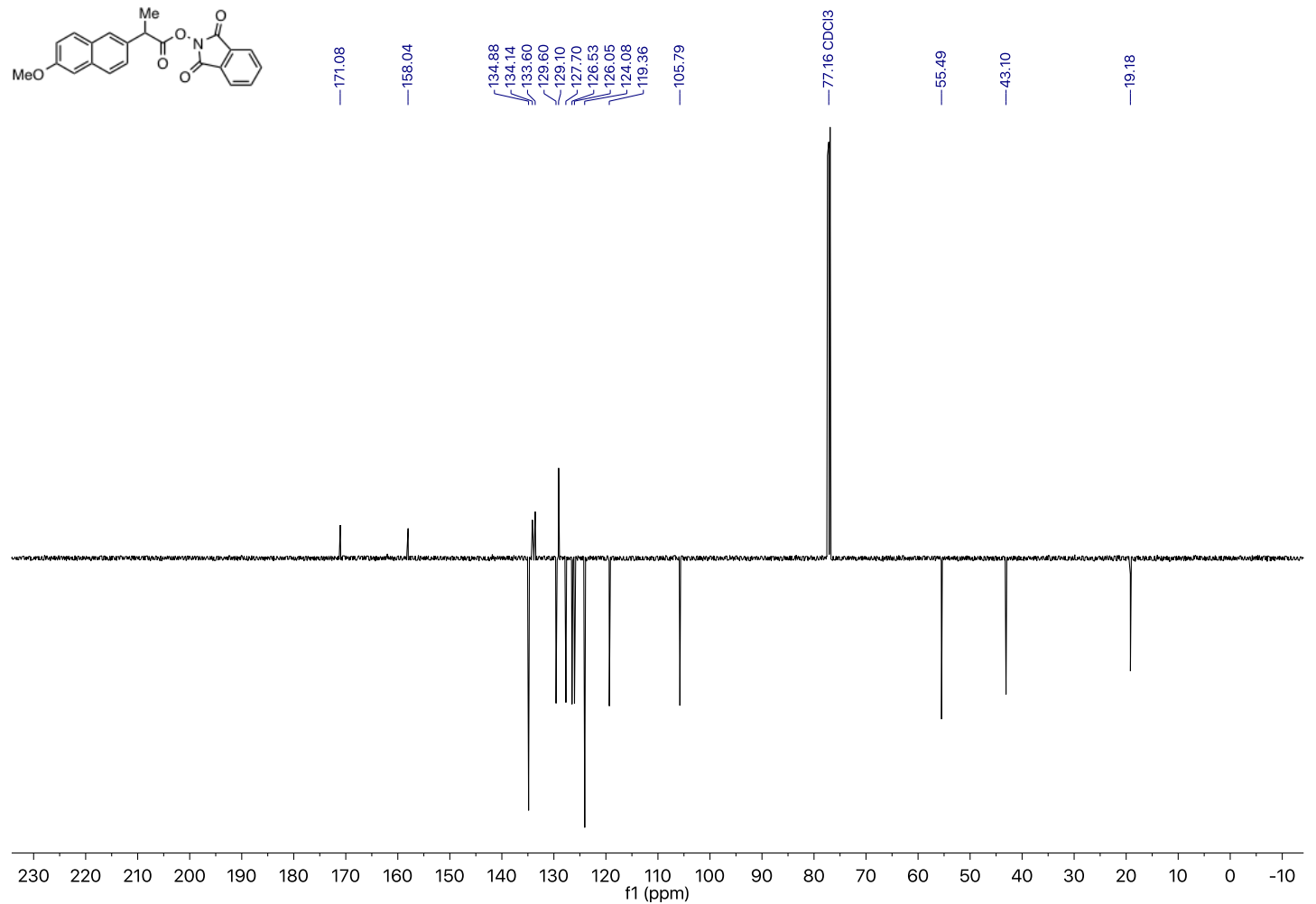
$500 \mathrm{MHz}{ }^{1} \mathrm{H}-\mathrm{NMR}$ of 4,5,6,7-tetrachloro-1,3-dioxoisoindolin-2-yl 2-(6-methoxynaphthalen-2-yl)propanoate (S1) in $\mathrm{CDCl}_{3}$

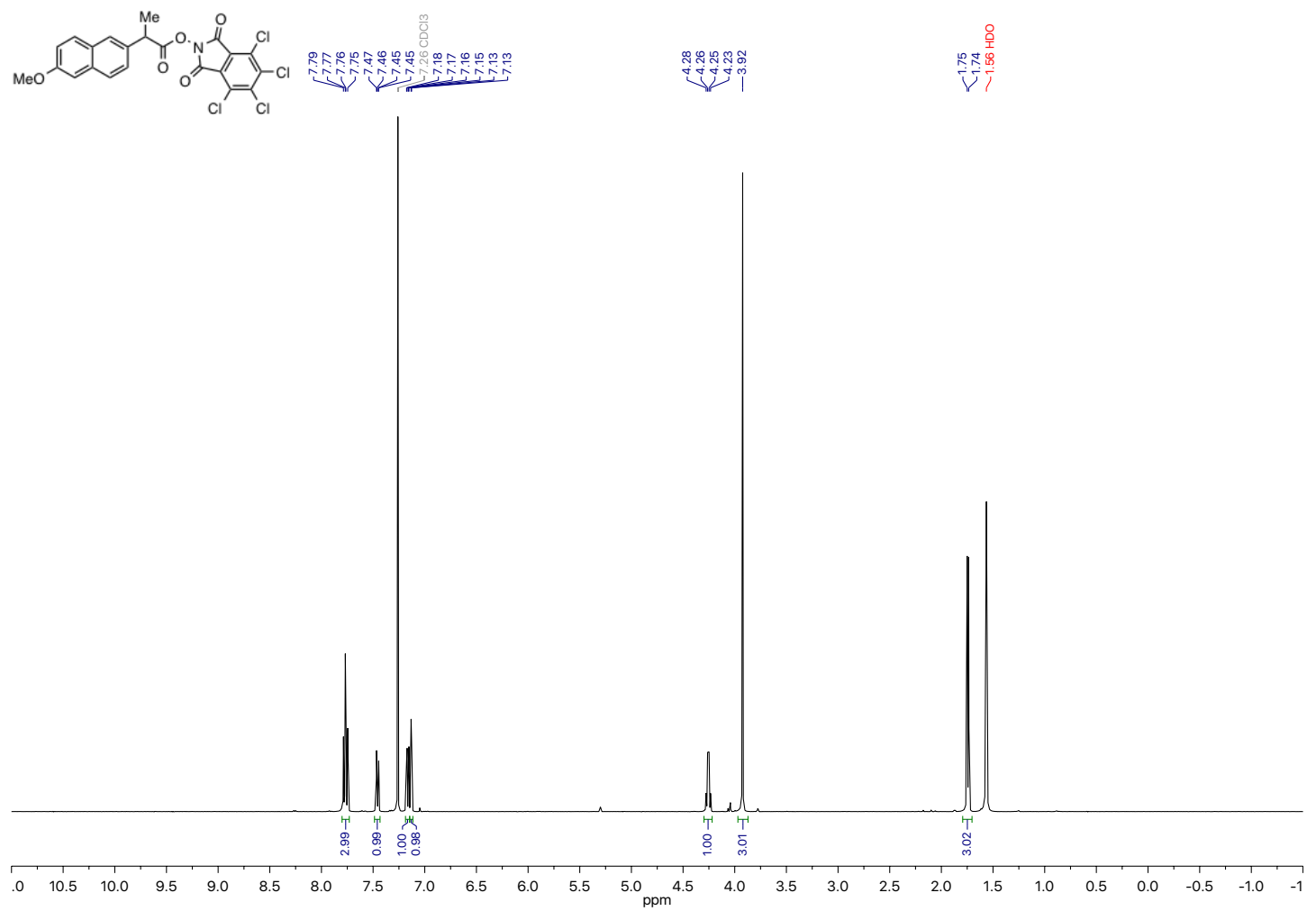


$126 \mathrm{MHz}{ }^{13} \mathrm{C}-\mathrm{NMR}$ of 4,5,6,7-tetrachloro-1,3-dioxoisoindolin-2-yl 2-(6-methoxynaphthalen-2-yl)propanoate (S1) in $\mathrm{CDCl}_{3}$

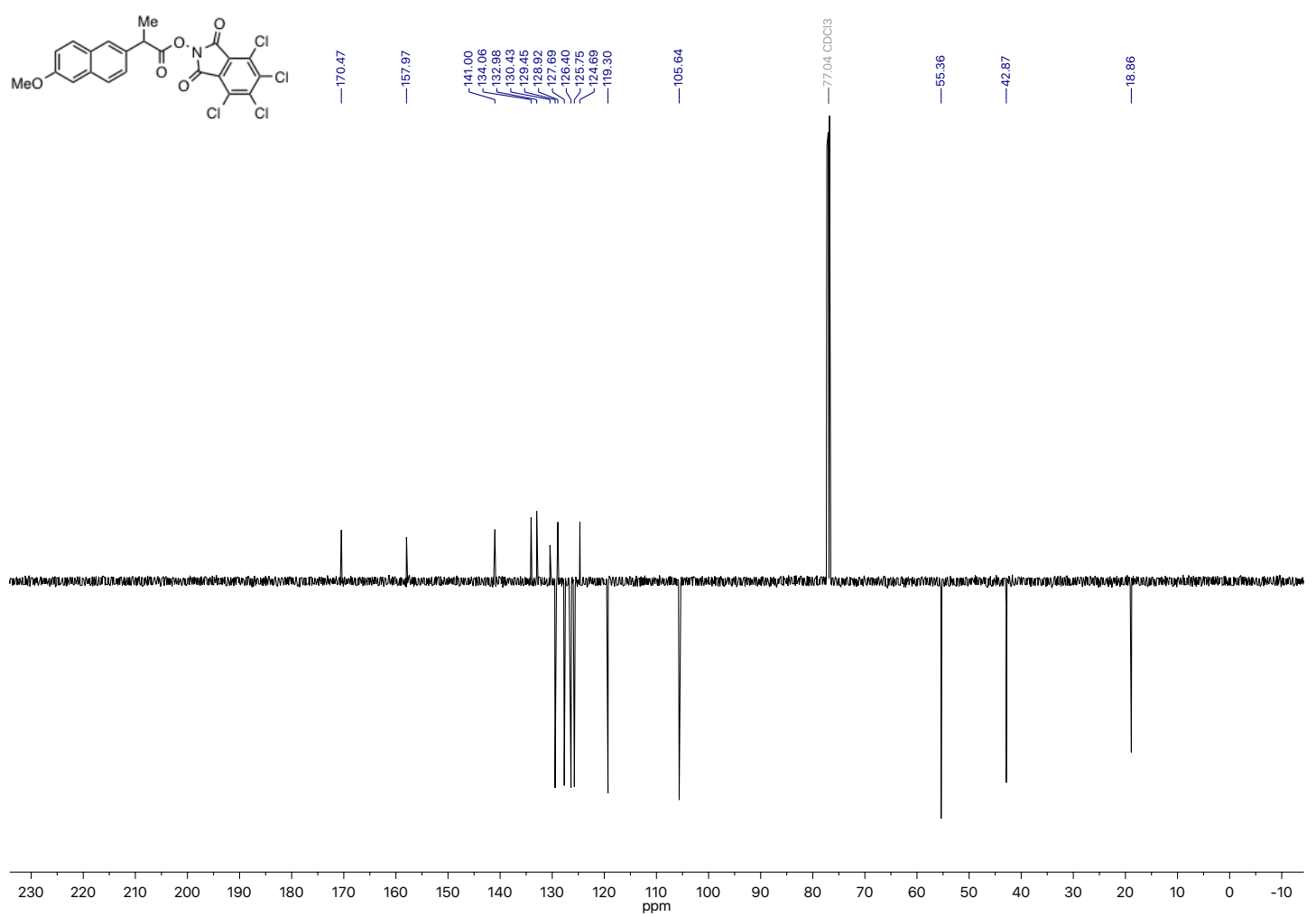


$500 \mathrm{MHz}{ }^{1} \mathrm{H}-\mathrm{NMR}$ of 2-(1-fluoroethyl)-6-methoxynaphthalene (2) in $\mathrm{CDCl}_{3}$

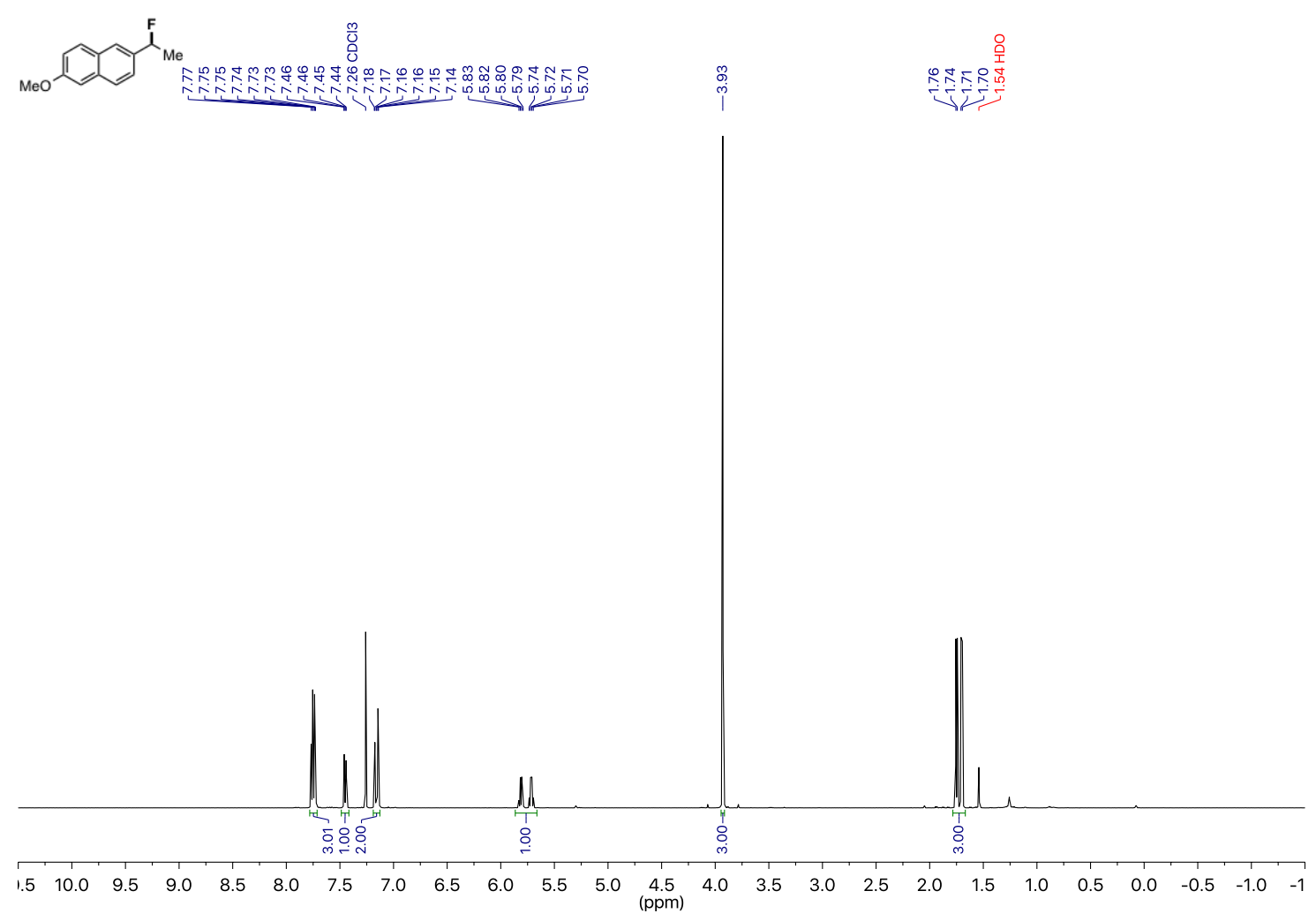

$126 \mathrm{MHz}{ }^{13} \mathrm{C}$-NMR of 2-(1-fluoroethyl)-6-methoxynaphthalene (2) in $\mathrm{CDCl}_{3}$
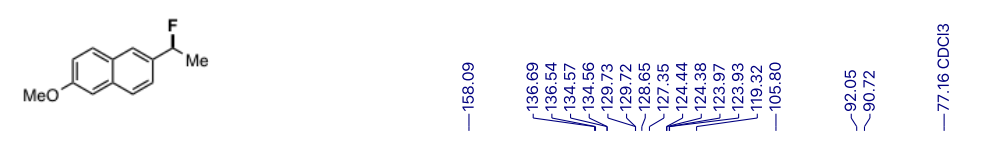

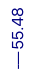
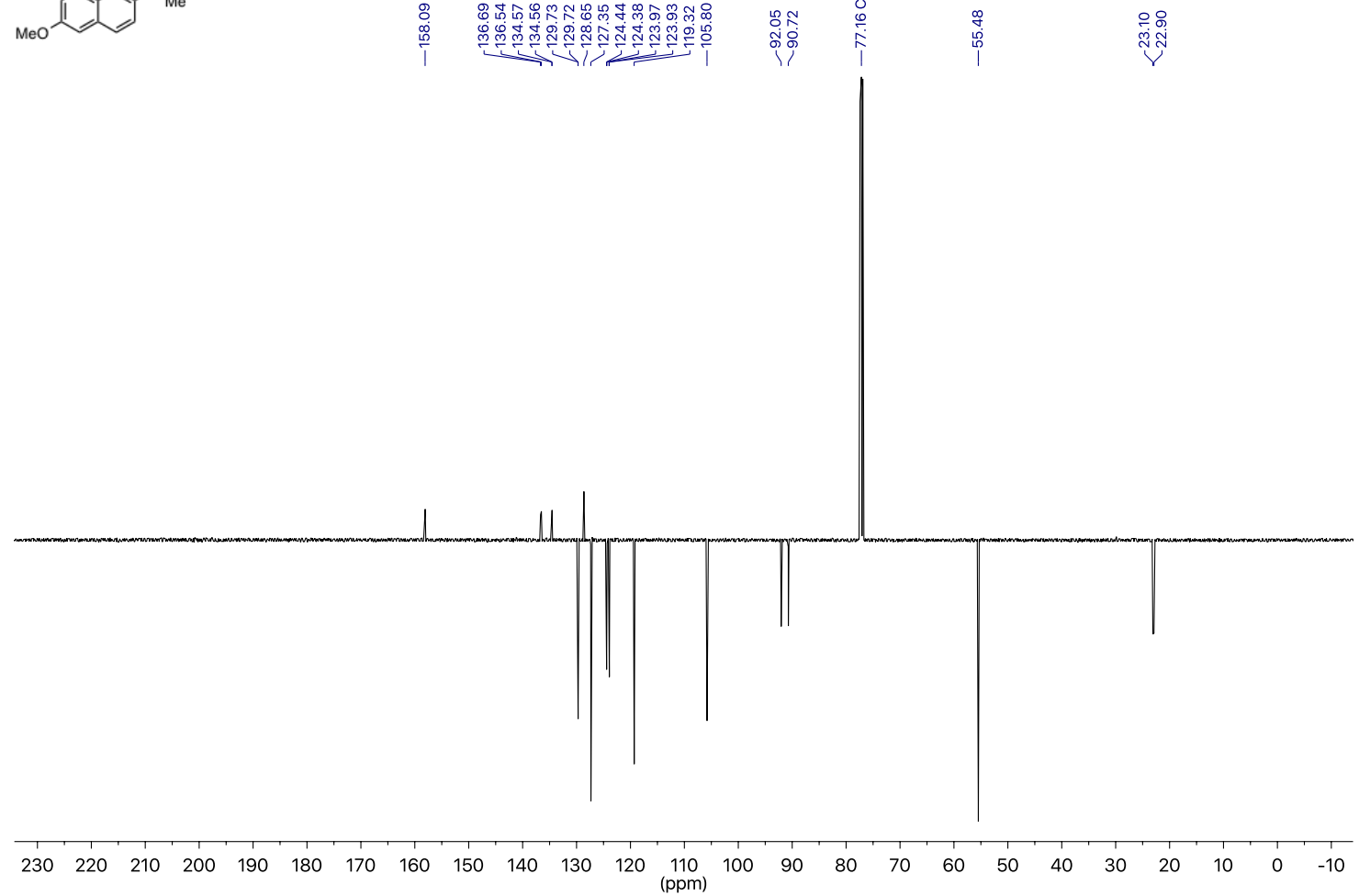
$376 \mathrm{MHz}{ }^{19} \mathrm{~F}-\mathrm{NMR}$ of 2-(1-fluoroethyl)-6-methoxynaphthalene (2) in $\mathrm{CDCl}_{3}$
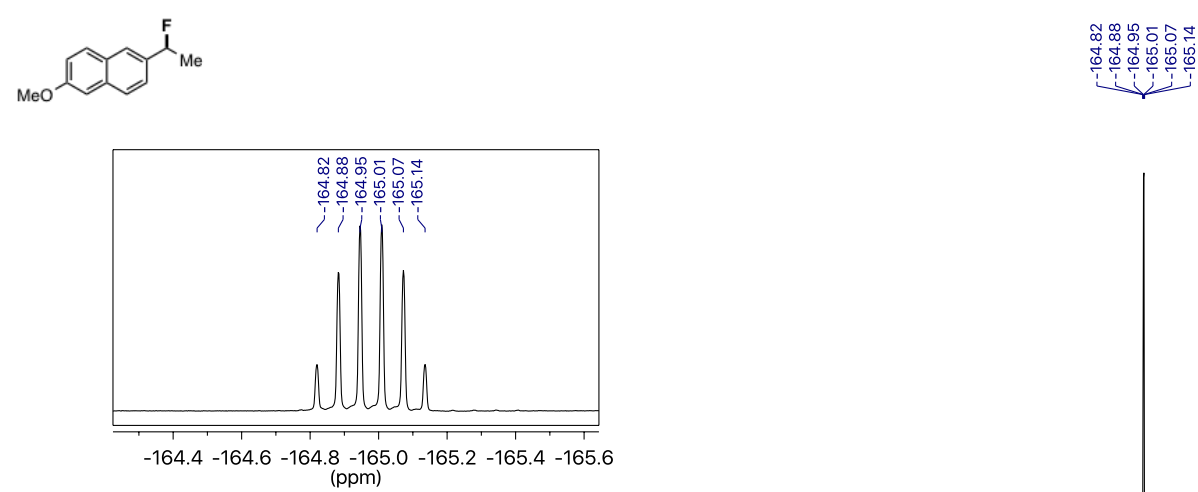

i

(ppm)

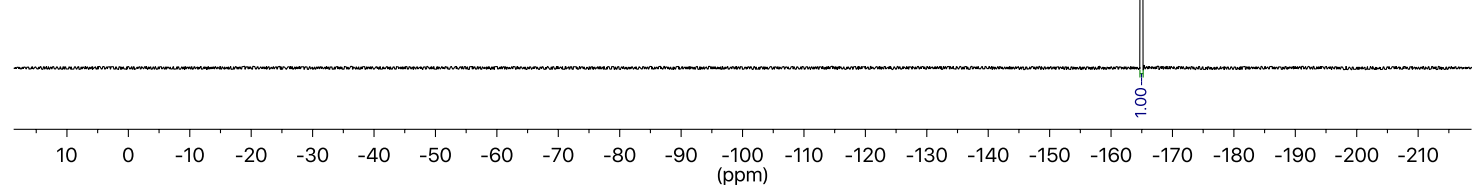


$500 \mathrm{MHz}{ }^{1} \mathrm{H}-\mathrm{NMR}$ of 1,3-dioxoisoindolin-2-yl 2-(4-(tert-butyl)phenyl)acetate (S2) in $\mathrm{CDCl}_{3}$

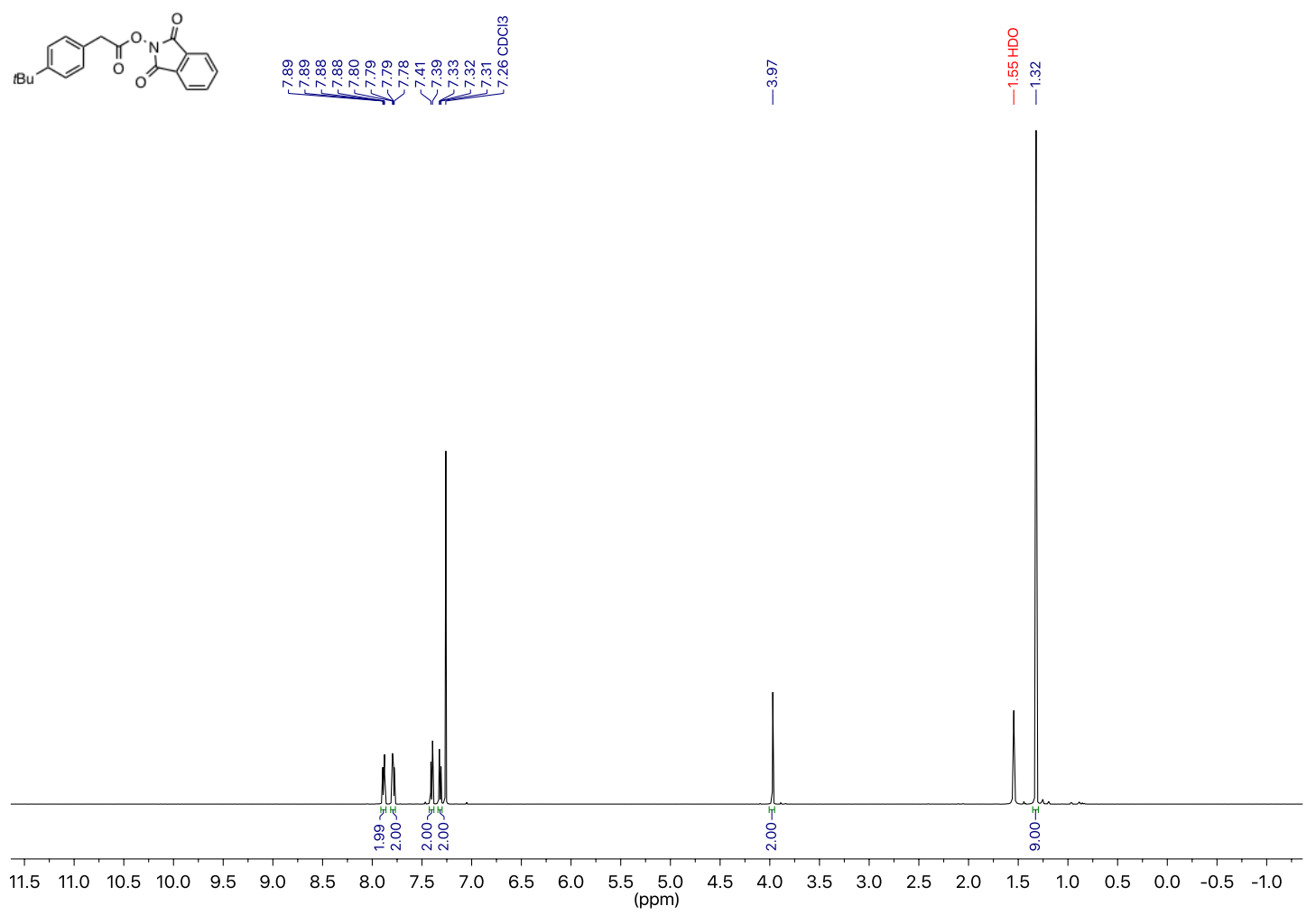

$126 \mathrm{MHz}{ }^{13} \mathrm{C}-\mathrm{NMR}$ of 1,3-dioxoisoindolin-2-yl 2-(4-(tert-butyl)phenyl)acetate (S2) in $\mathrm{CDCl}_{3}$
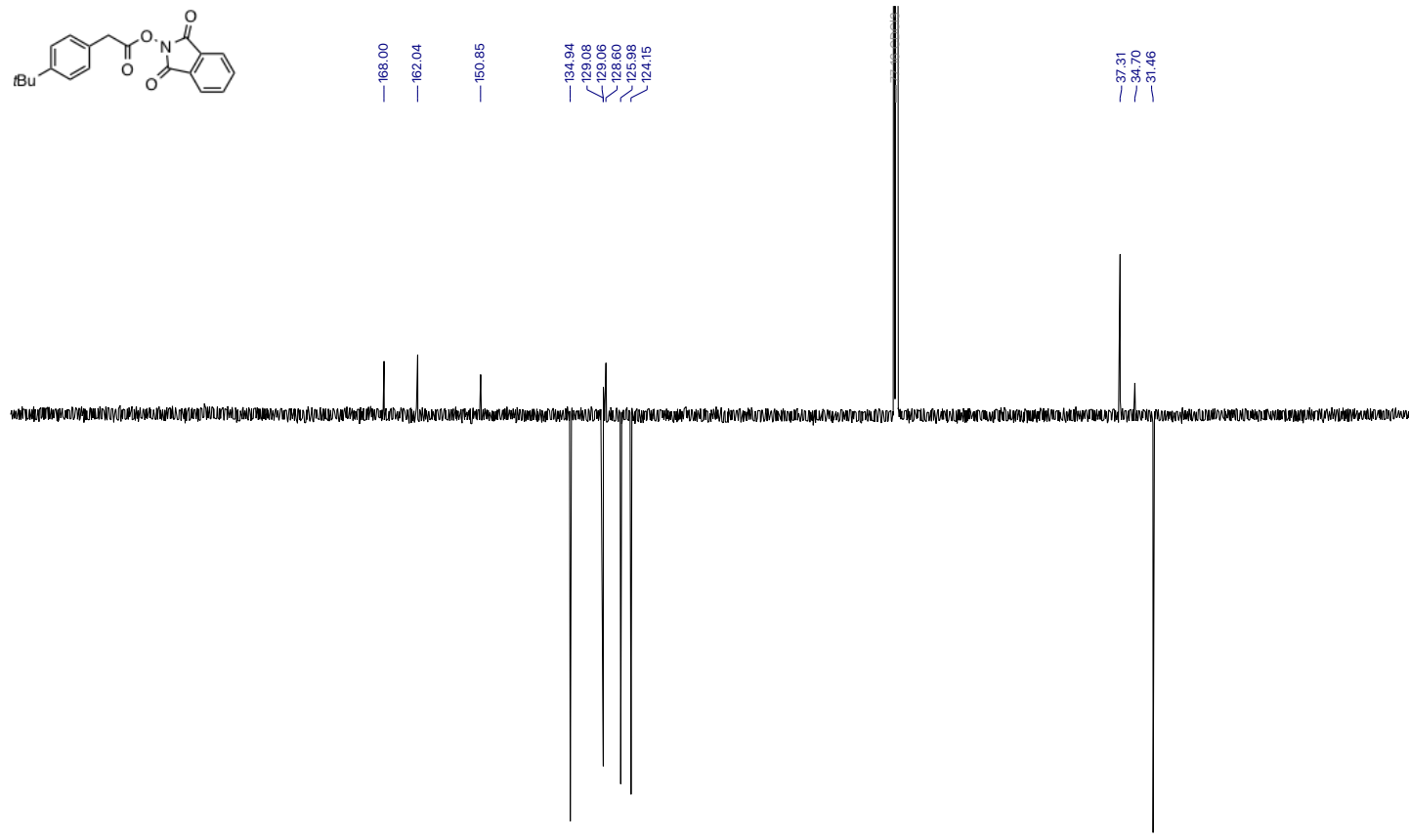

\begin{tabular}{rlllllllllllllllllllllllllllll}
\hline & 130 & 220 & 210 & 200 & 190 & 180 & 170 & 160 & 150 & 140 & 130 & 120 & $\begin{array}{c}110 \\
(\mathrm{pm})\end{array}$ & 100 & 90 & 80 & 70 & 60 & 50 & 40 & 30 & 20 & 10 & 0 & -10
\end{tabular} 
$500 \mathrm{MHz}{ }^{1} \mathrm{H}-\mathrm{NMR}$ of 1-(tert-butyl)-4-(fluoromethyl)benzene (7) in $\mathrm{CDCl}_{3}$

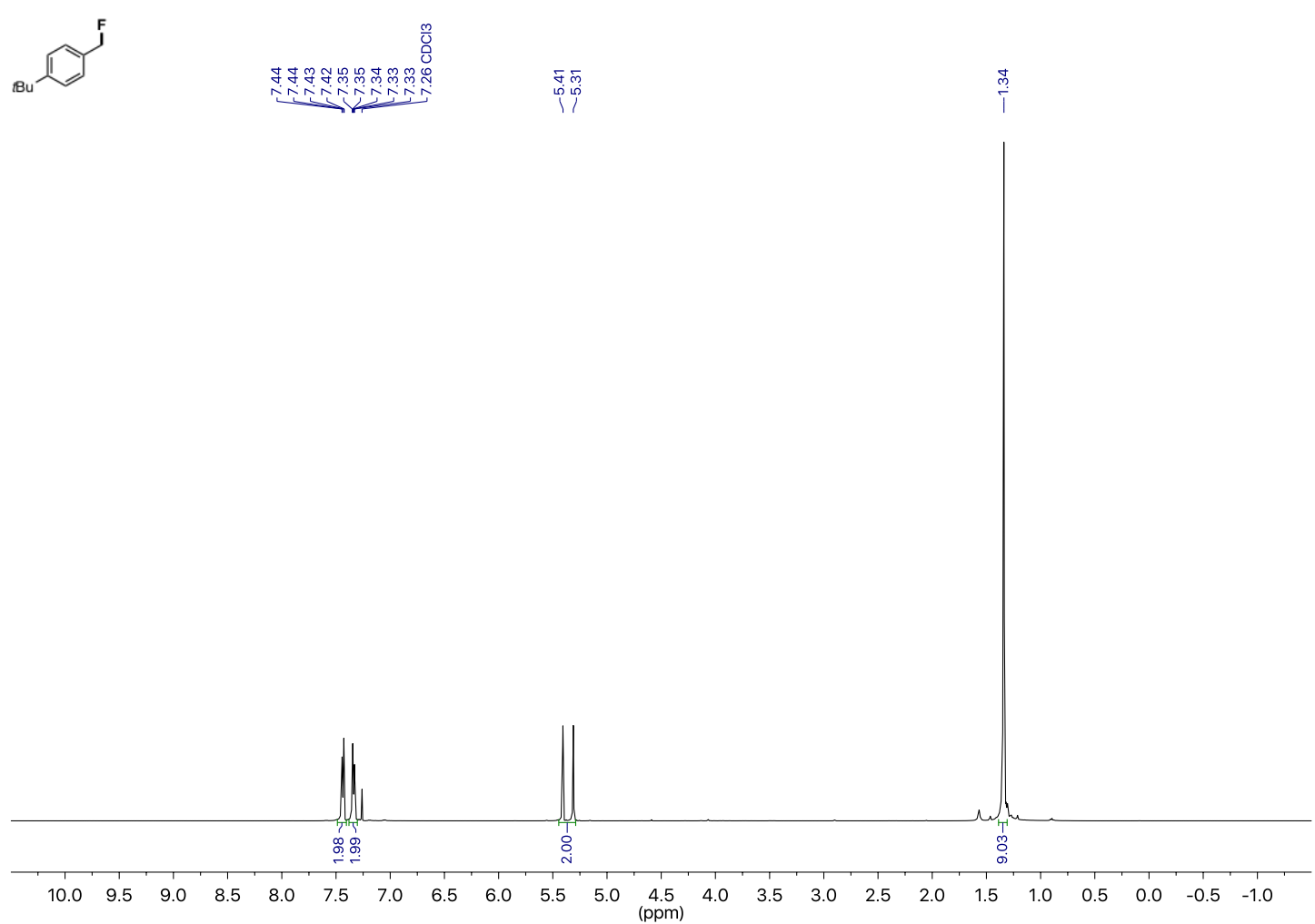

$126 \mathrm{MHz}{ }^{13} \mathrm{C}$-NMR of 1-(tert-butyl)-4-(fluoromethyl)benzene (7) in $\mathrm{CDCl}_{3}$

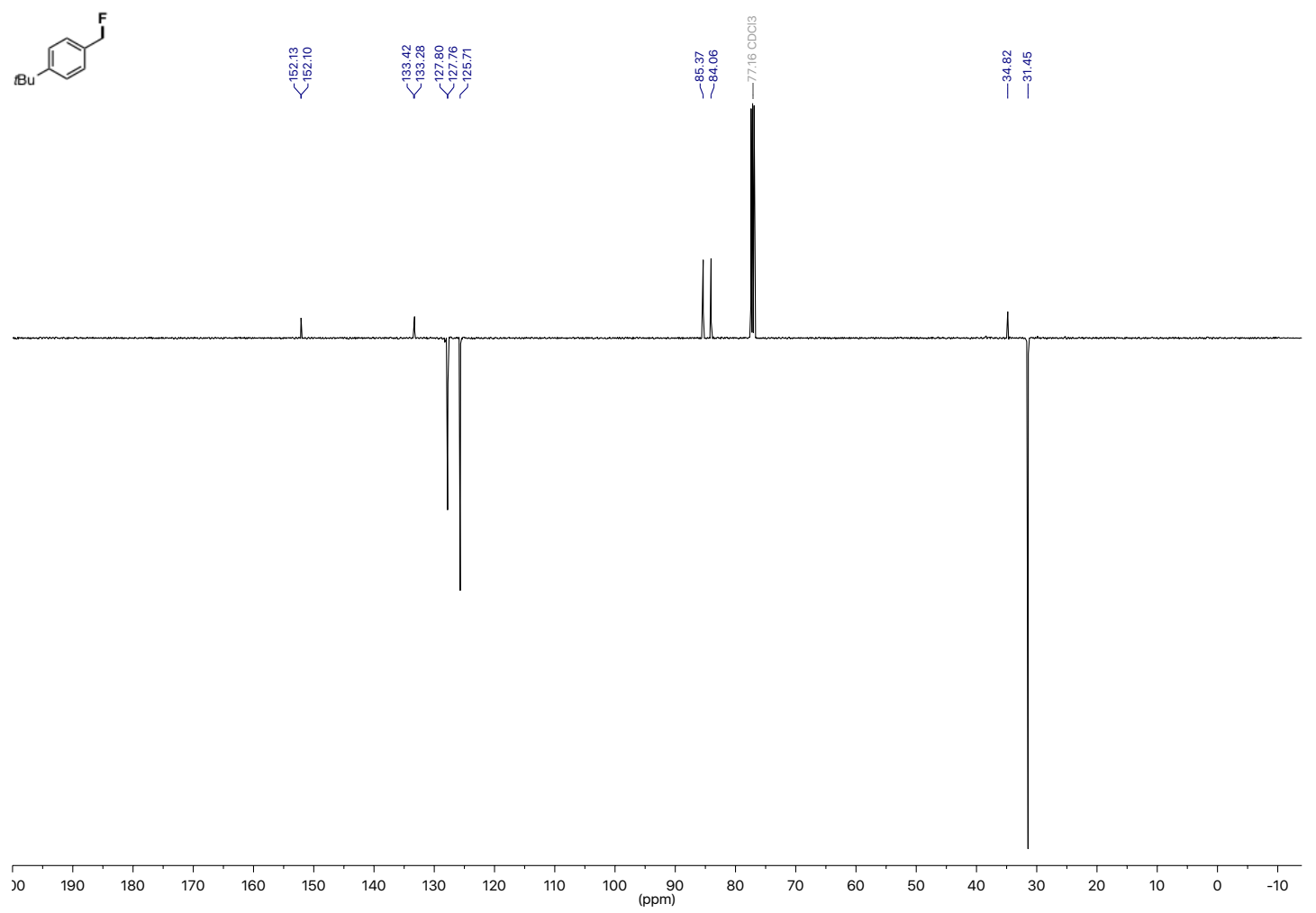


$282 \mathrm{MHz}{ }^{19} \mathrm{~F}-\mathrm{NMR}$ of 1-(tert-butyl)-4-(fluoromethyl)benzene (7) in $\mathrm{CDCl}_{3}$

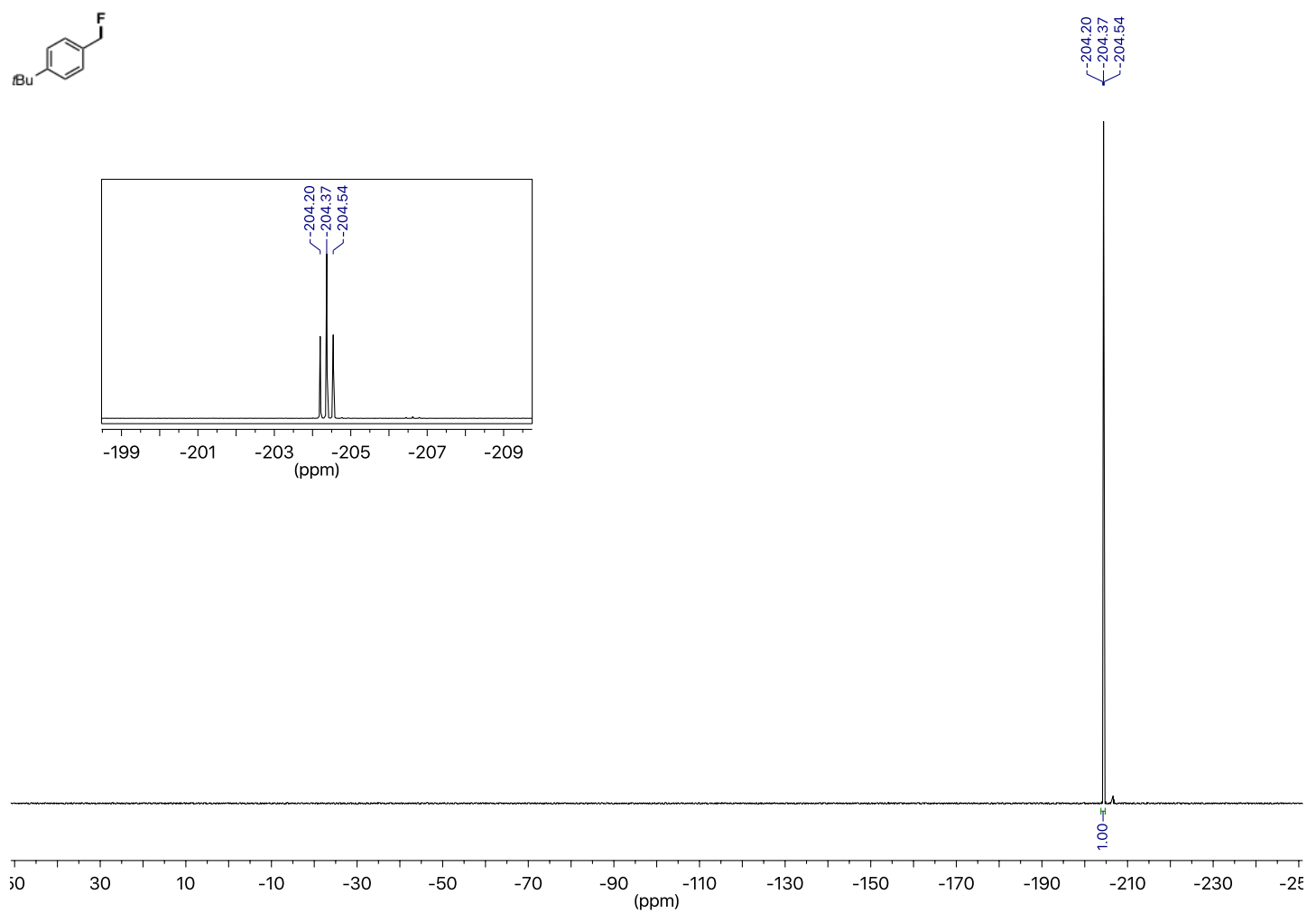

$400 \mathrm{MHz}{ }^{1} \mathrm{H}-\mathrm{NMR}$ of 1,3-dioxoisoindolin-2-yl 2-([1,1'-biphenyl]-4-yl)acetate (S3) in $\mathrm{CDCl}_{3}$

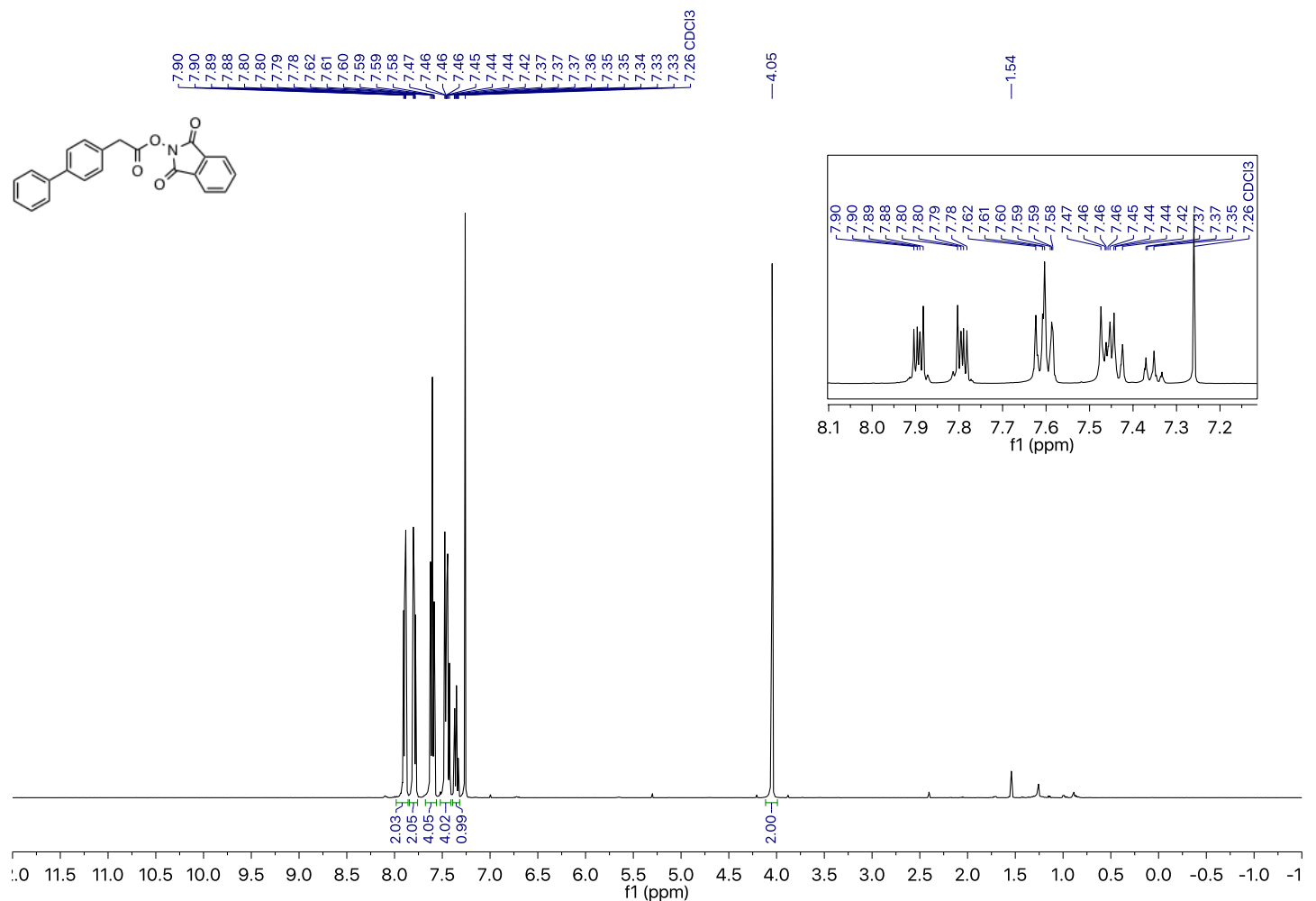


$101 \mathrm{MHz}{ }^{13} \mathrm{C}-\mathrm{NMR}$ of 1,3-dioxoisoindolin-2-yl 2-([1,1'-biphenyl]-4-yl)acetate (S3) in $\mathrm{CDCl}_{3}$

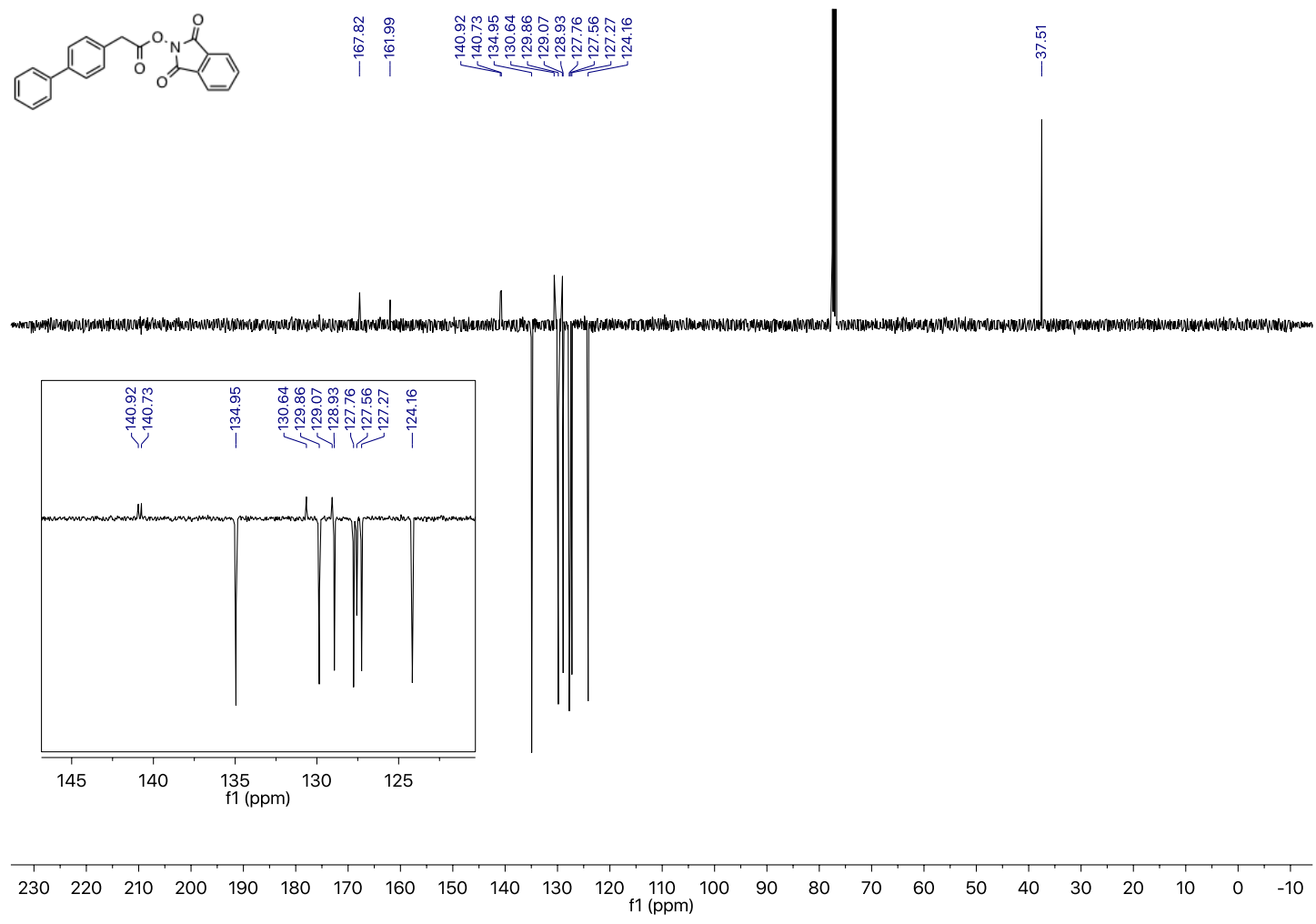

$500 \mathrm{MHz}{ }^{1} \mathrm{H}-\mathrm{NMR}$ of 4-(fluoromethyl)-1,1'-biphenyl (8) in $\mathrm{CDCl}_{3}$
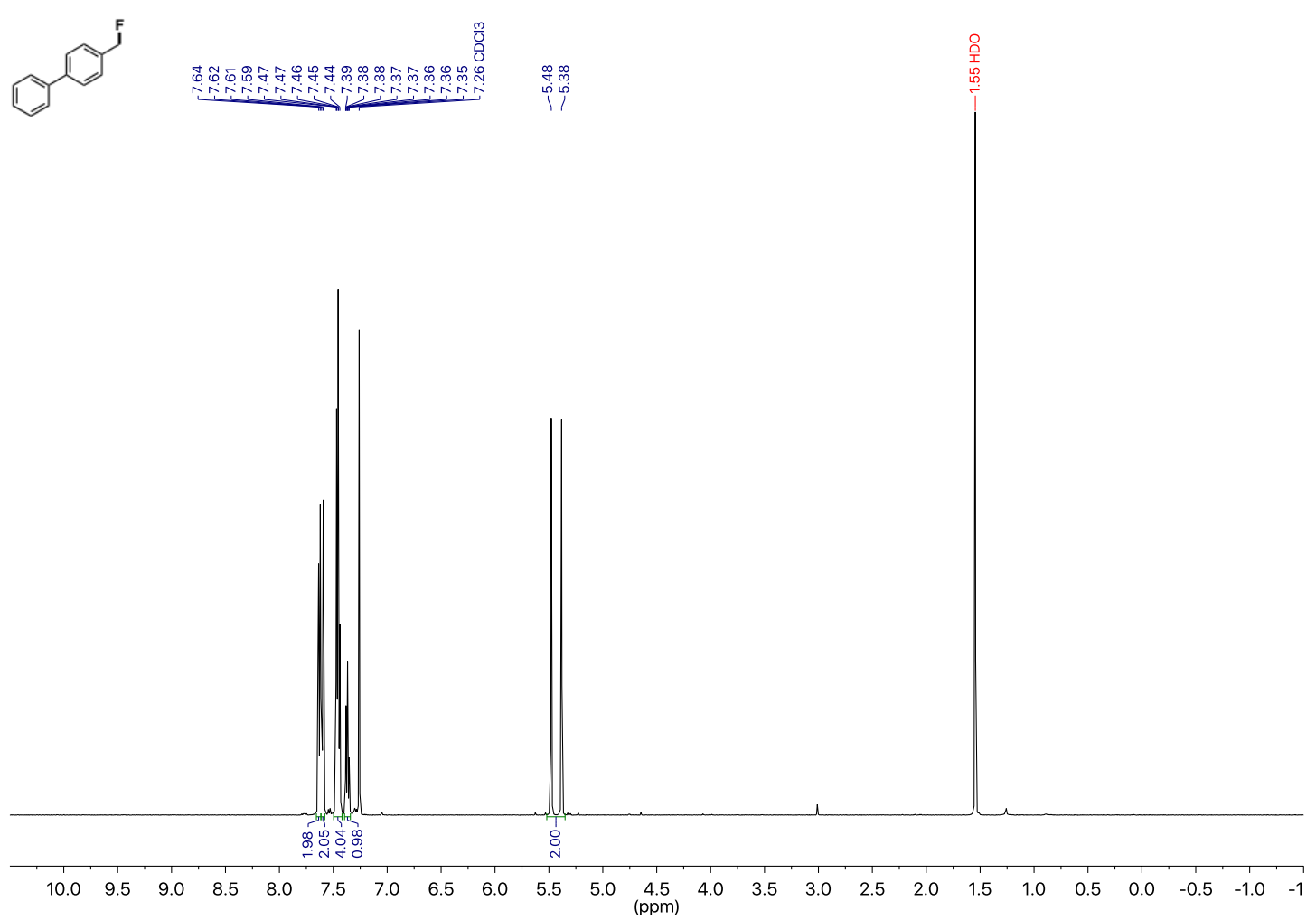
$126 \mathrm{MHz}{ }^{13} \mathrm{C}-\mathrm{NMR}$ of 4-(fluoromethyl)-1,1'-biphenyl (8) in $\mathrm{CDCl}_{3}$

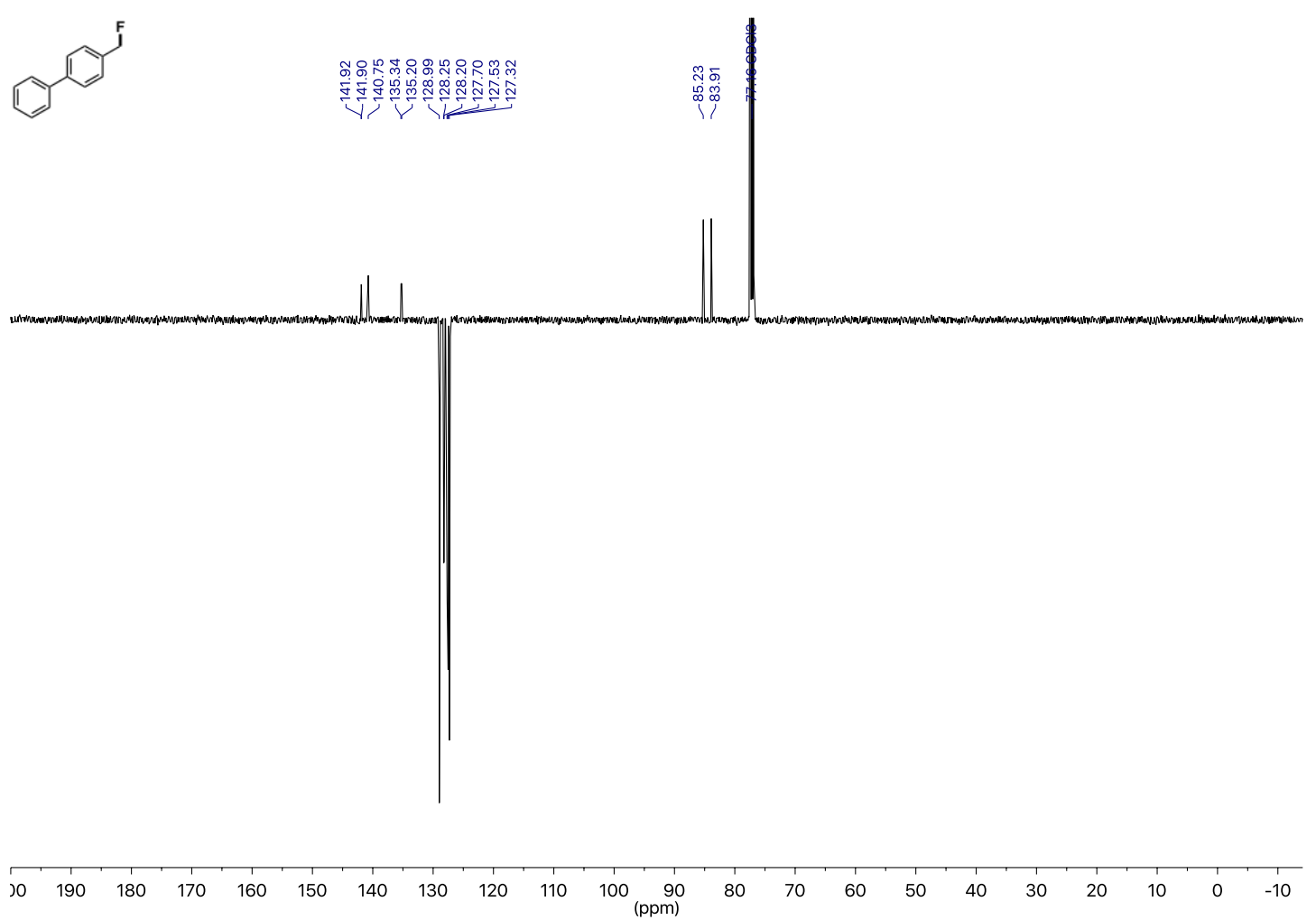

$376 \mathrm{MHz}{ }^{19} \mathrm{~F}-\mathrm{NMR}$ of 4-(fluoromethyl)-1,1'-biphenyl (8) in $\mathrm{CDCl}_{3}$
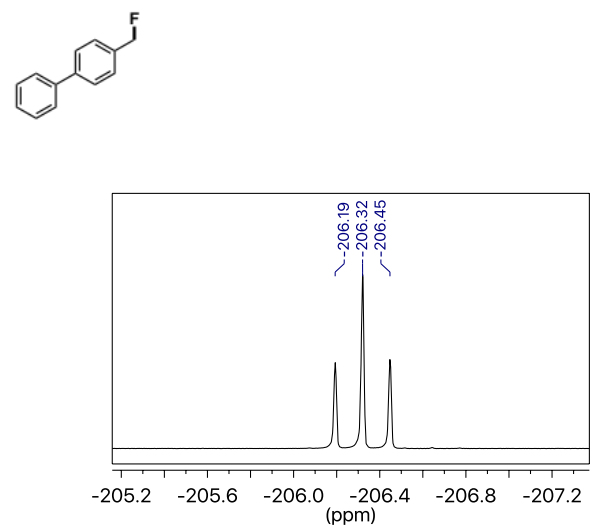

$\begin{array}{llllllllllllllllllllllll}10 & 0 & -10 & -20 & -30 & -40 & -50 & -60 & -70 & -80 & -90 & \begin{array}{c}-100 \\ (\mathrm{ppm})\end{array} & -110 & -120 & -130 & -140 & -150 & -160 & -170 & -180 & -190 & -200 & -210\end{array}$ 
$500 \mathrm{MHz}{ }^{1} \mathrm{H}-\mathrm{NMR}$ of 1,3-dioxoisoindolin-2-yl 2-(4-iodophenyl)acetate (S4) in $\mathrm{CDCl}_{3}$

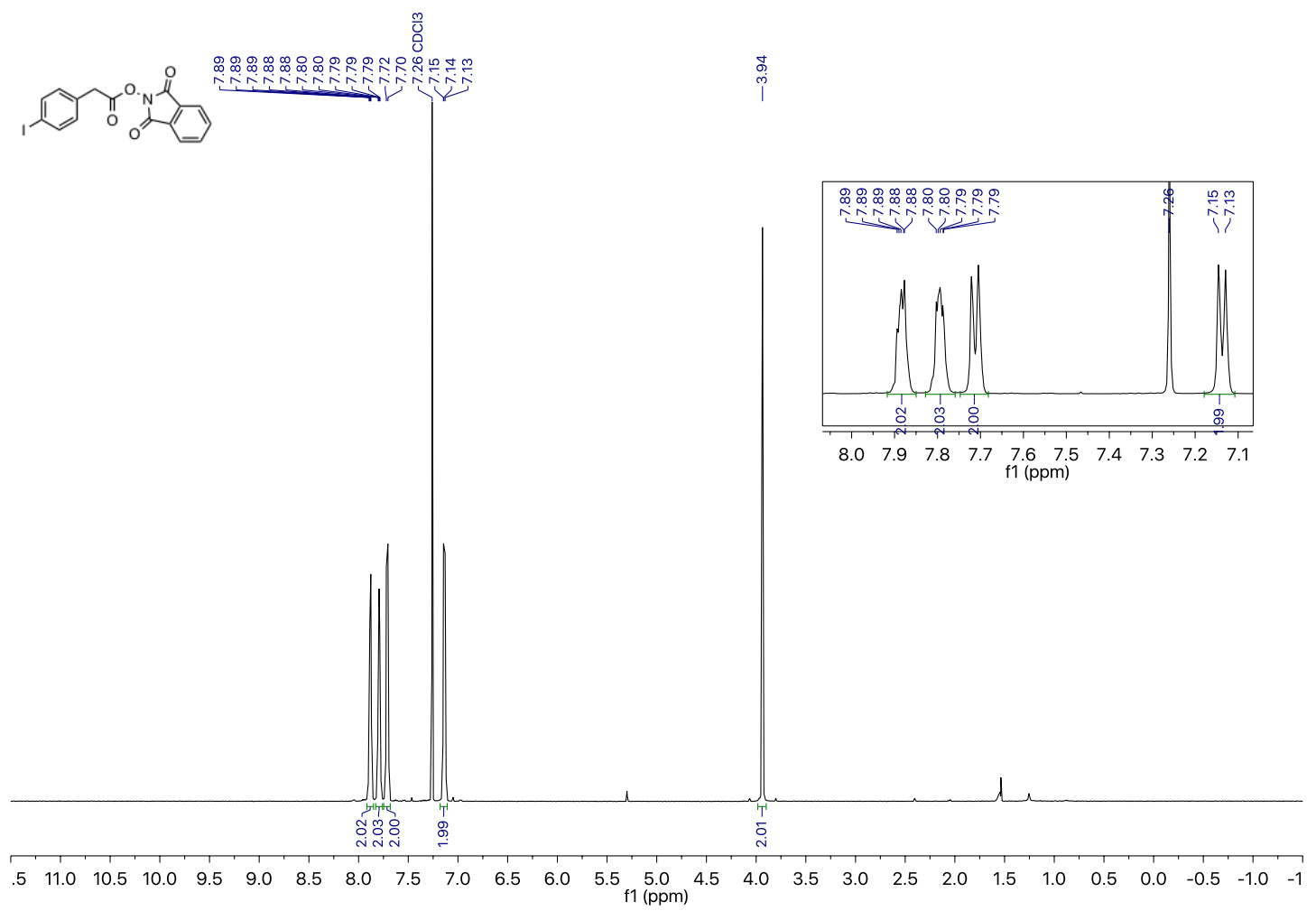

$126 \mathrm{MHz}^{13} \mathrm{C}$-NMR of 1,3-dioxoisoindolin-2-yl 2-(4-iodophenyl)acetate (S4) in $\mathrm{CDCl}_{3}$
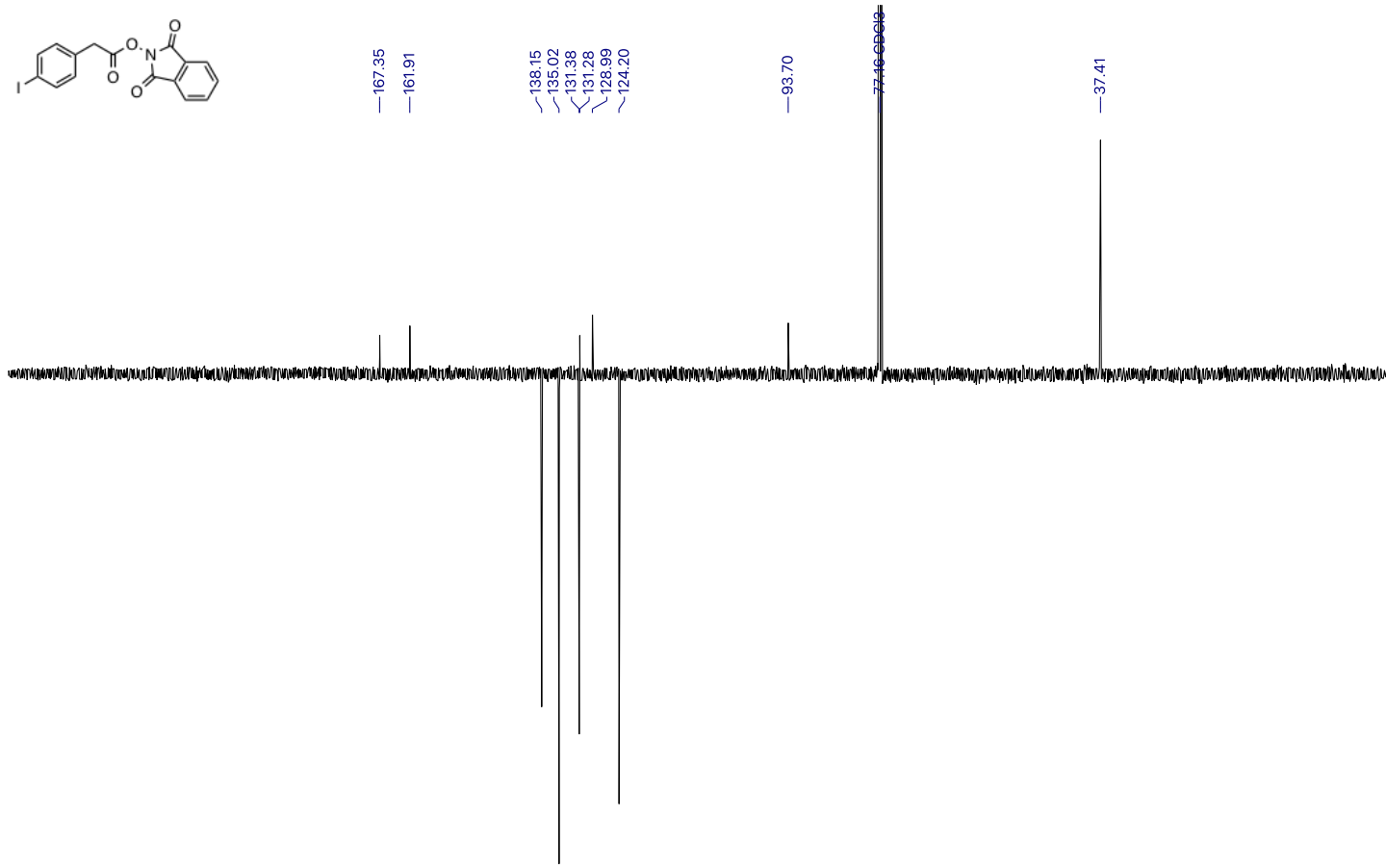

$\begin{array}{llllllllllllllllllllllllll}230 & 220 & 210 & 200 & 190 & 180 & 170 & 160 & 150 & 140 & 130 & 120 & \begin{array}{c}110 \\ \mathrm{f}(\mathrm{ppm})\end{array} & 100 & 90 & 80 & 70 & 60 & 50 & 40 & 30 & 20 & 10 & 0 & -10\end{array}$ 
$500 \mathrm{MHz}{ }^{1} \mathrm{H}-\mathrm{NMR}$ of 1,3-dioxoisoindolin-2-yl 2-(4-(benzyloxy)phenyl)acetate (S5) in $\mathrm{CD}_{2} \mathrm{Cl}_{2}$

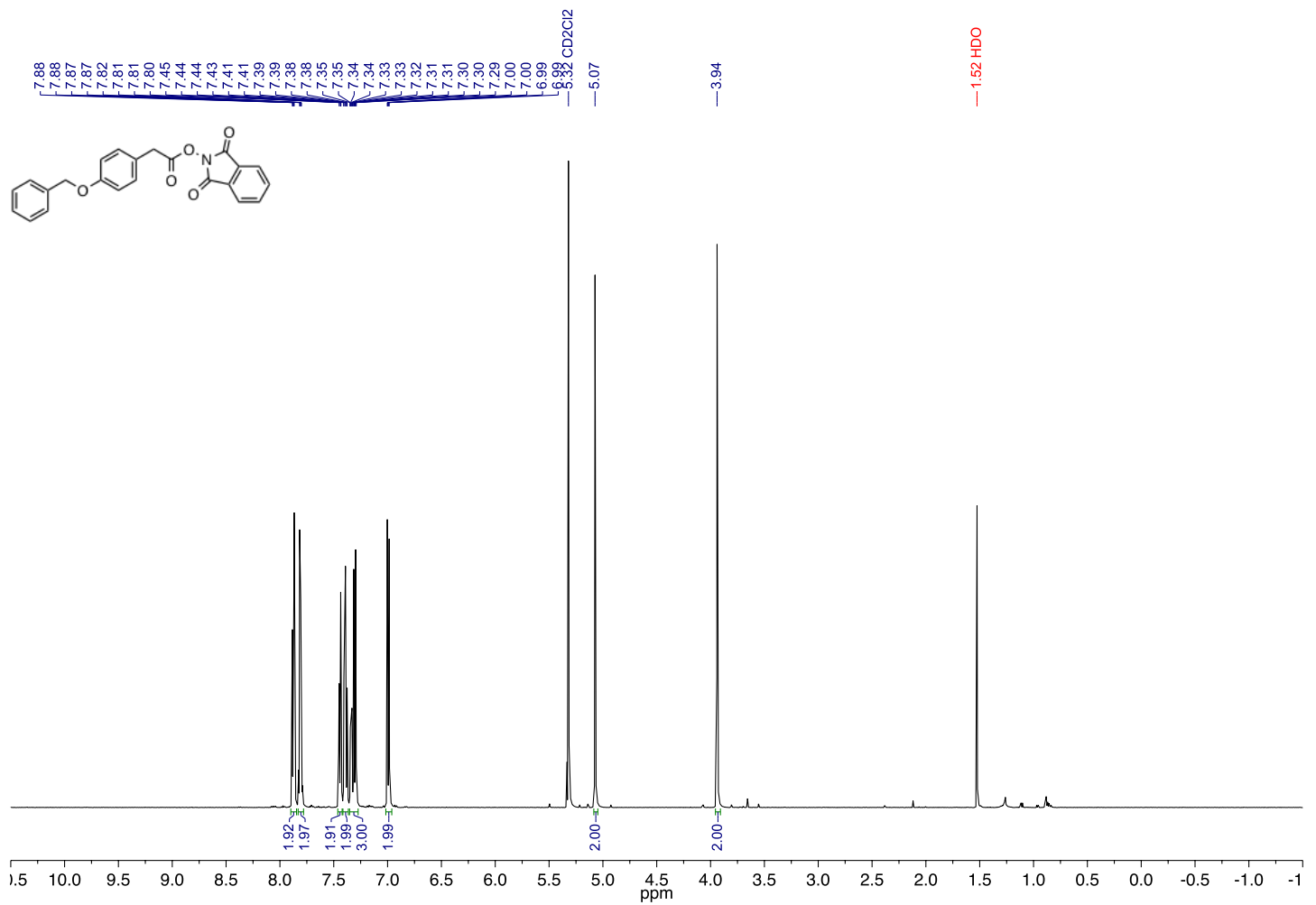

$126 \mathrm{MHz}{ }^{1} \mathrm{H}-\mathrm{NMR}$ of 1,3-dioxoisoindolin-2-yl 2-(4-(benzyloxy)phenyl)acetate (S5) in $\mathrm{CD}_{2} \mathrm{Cl}_{2}$
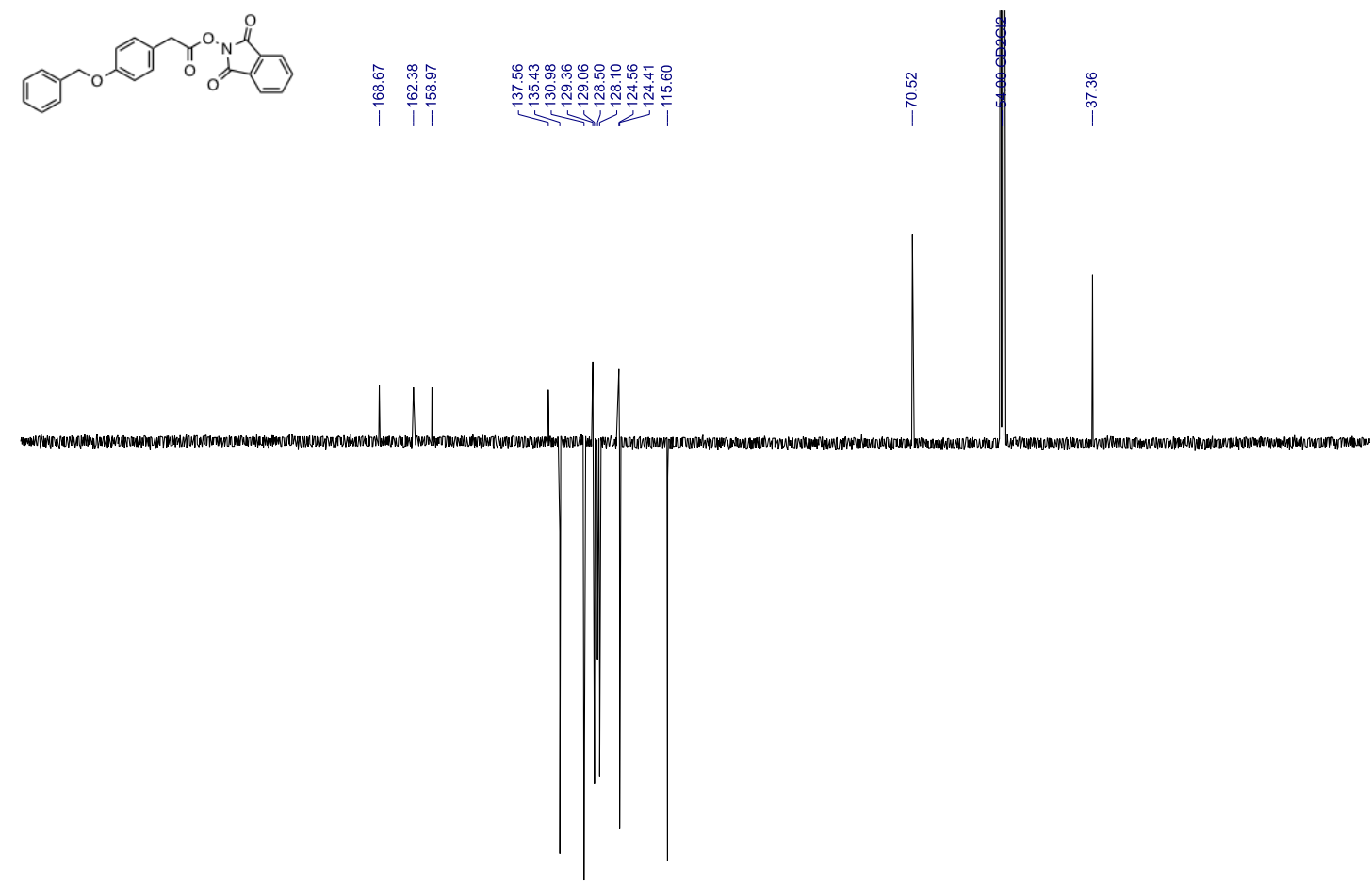

\begin{tabular}{llllllllllllllllllllllllllllllllllll}
\hline 230 & 220 & 210 & 200 & 190 & 180 & 170 & 160 & 150 & 140 & 130 & 120 & 110 & 100 & 90 & 80 & 70 & 60 & 50 & 40 & 30 & 20 & 10 & 0 & -10
\end{tabular} 


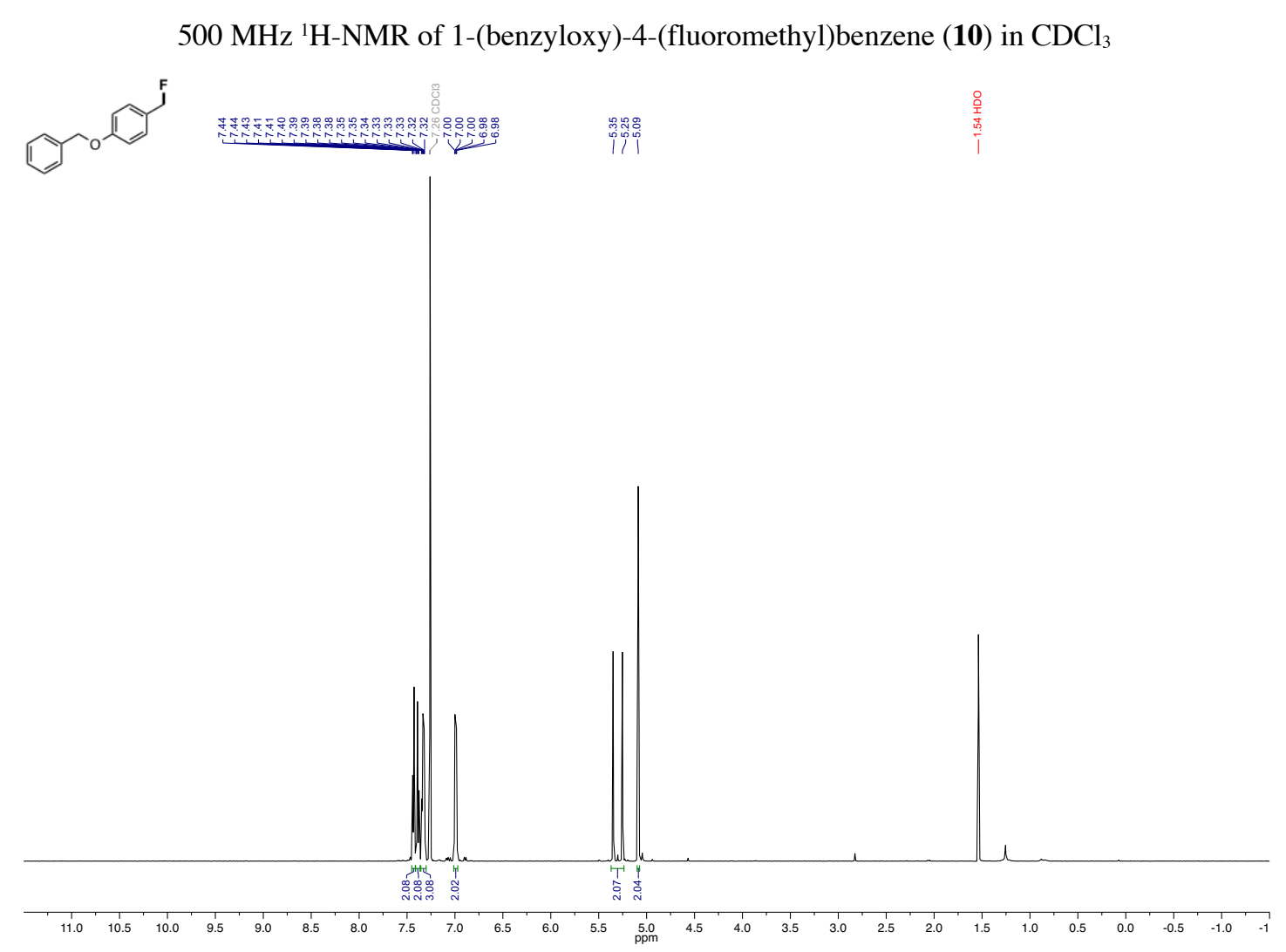

$126 \mathrm{MHz}$ 1H-NMR of 1-(benzyloxy)-4-(fluoromethyl)benzene (10) in CDCl3

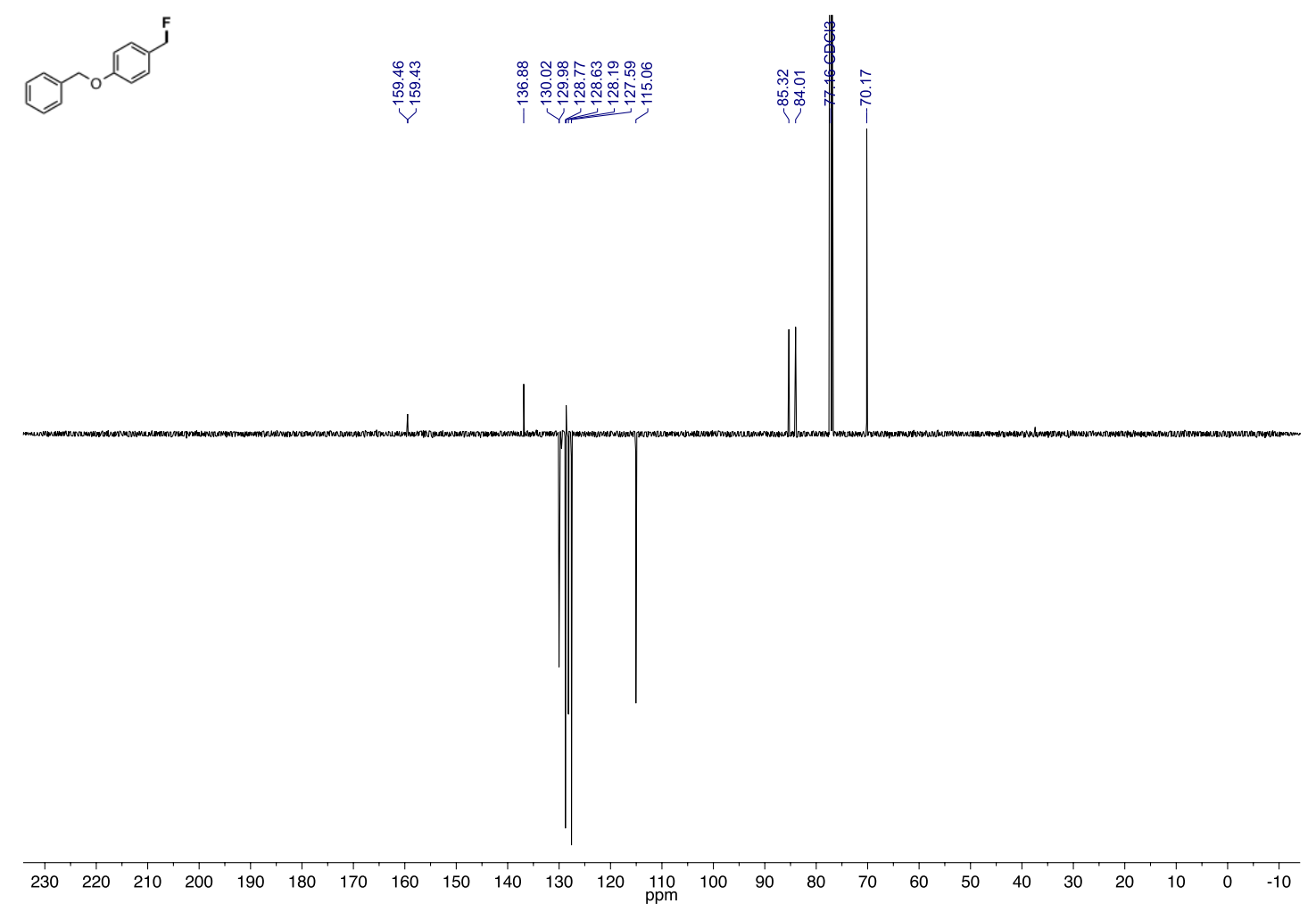


$282 \mathrm{MHz}{ }^{19} \mathrm{~F}-\mathrm{NMR}$ of 1-(benzyloxy)-4-(fluoromethyl)benzene (10) in $\mathrm{CDCl}_{3}$
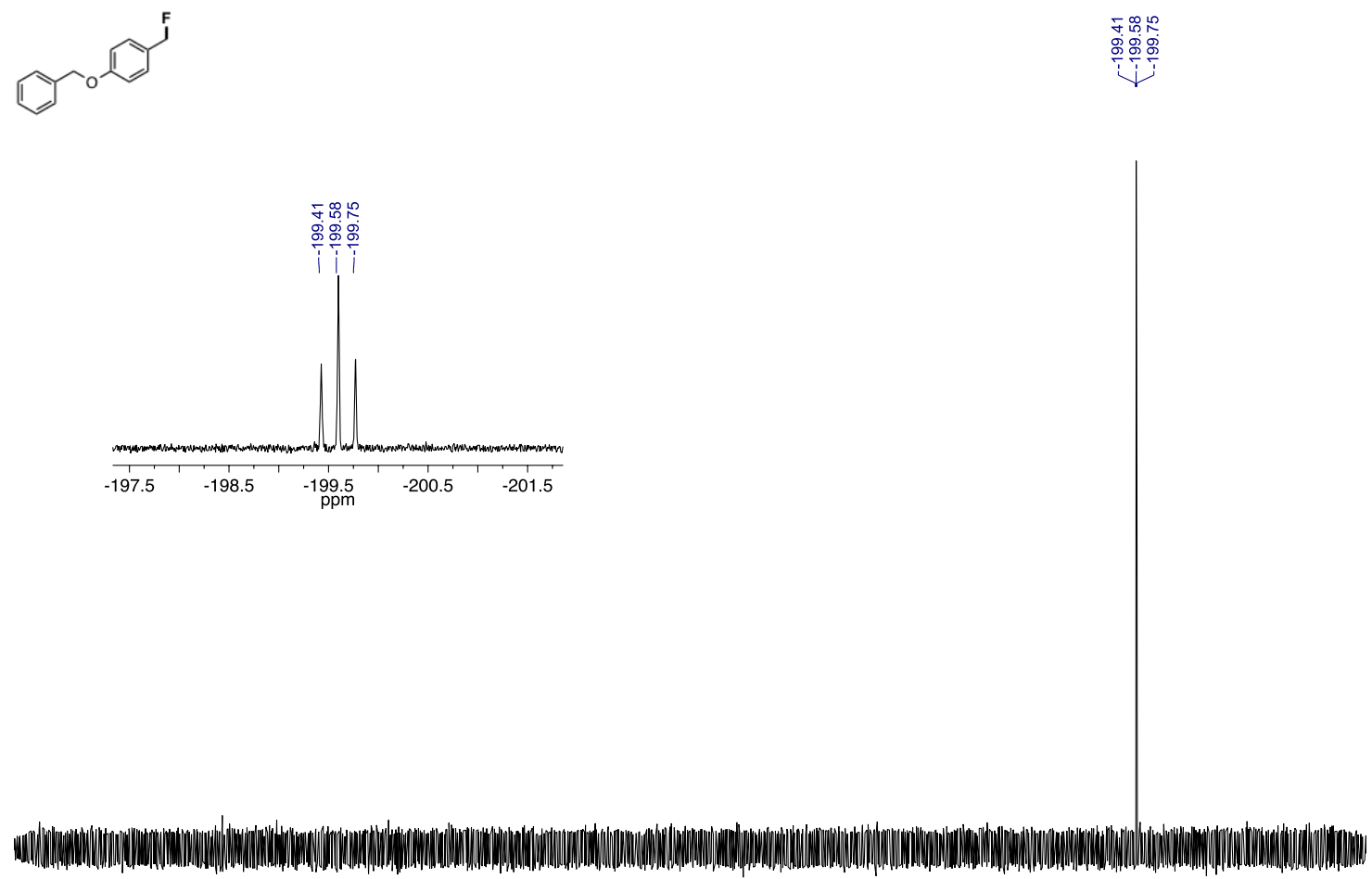

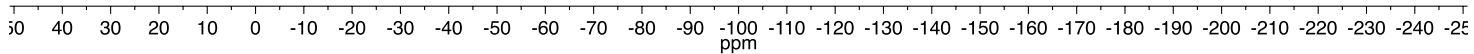

$500 \mathrm{MHz}{ }^{1} \mathrm{H}-\mathrm{NMR}$ of 1,3-dioxoisoindolin-2-yl 2-(4-(methylthio)phenyl)acetate (S6) in $\mathrm{CD}_{2} \mathrm{Cl}_{2}$
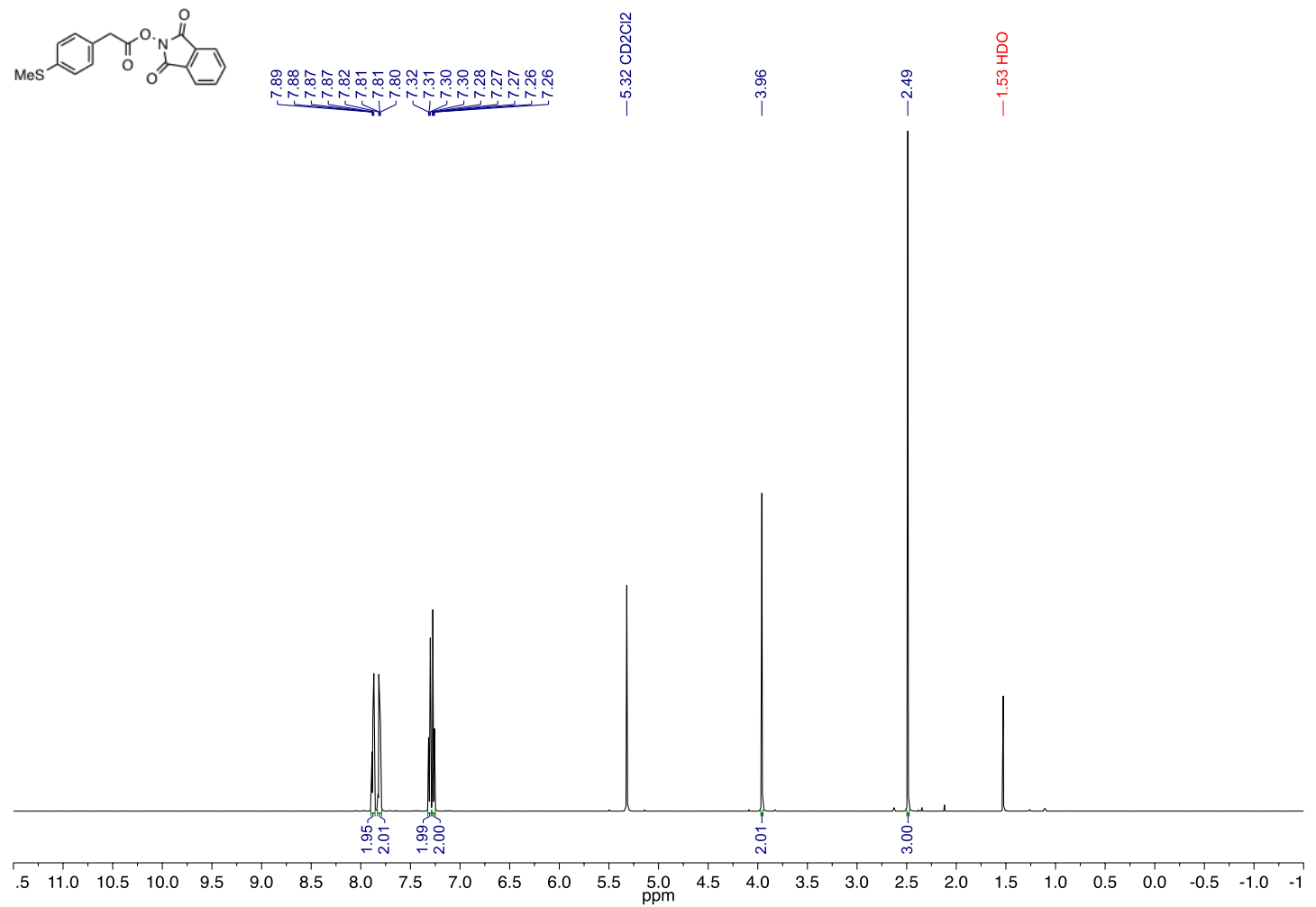
$126 \mathrm{MHz}{ }^{13} \mathrm{C}-\mathrm{NMR}$ of 1,3-dioxoisoindolin-2-yl 2-(4-(methylthio)phenyl)acetate (S6) in $\mathrm{CD}_{2} \mathrm{Cl}_{2}$

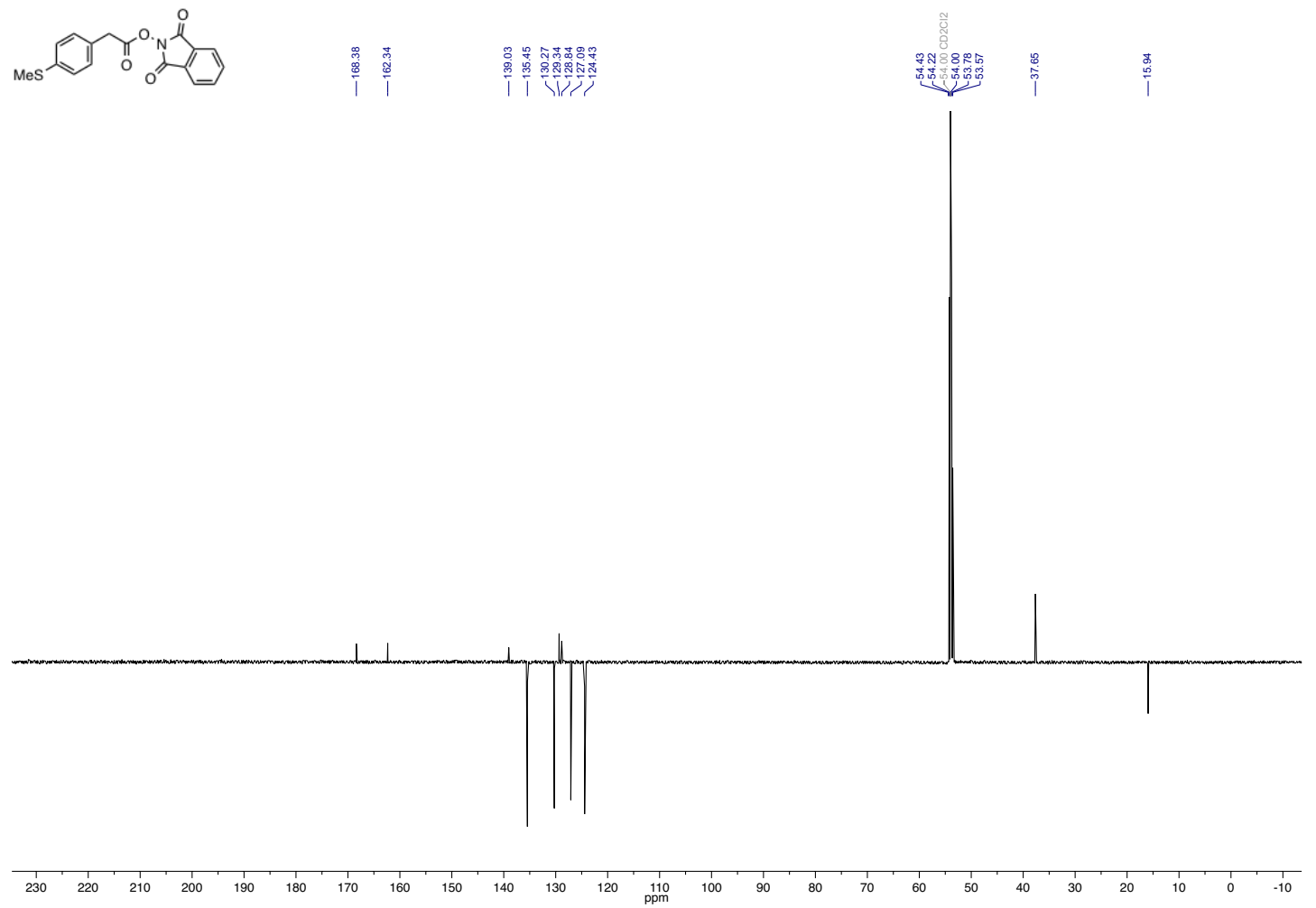

$500 \mathrm{MHz}{ }^{1} \mathrm{H}-\mathrm{NMR}$ of (4-(fluoromethyl)phenyl)(methyl)sulfane (11) in $\mathrm{CD}_{2} \mathrm{Cl}_{2}$
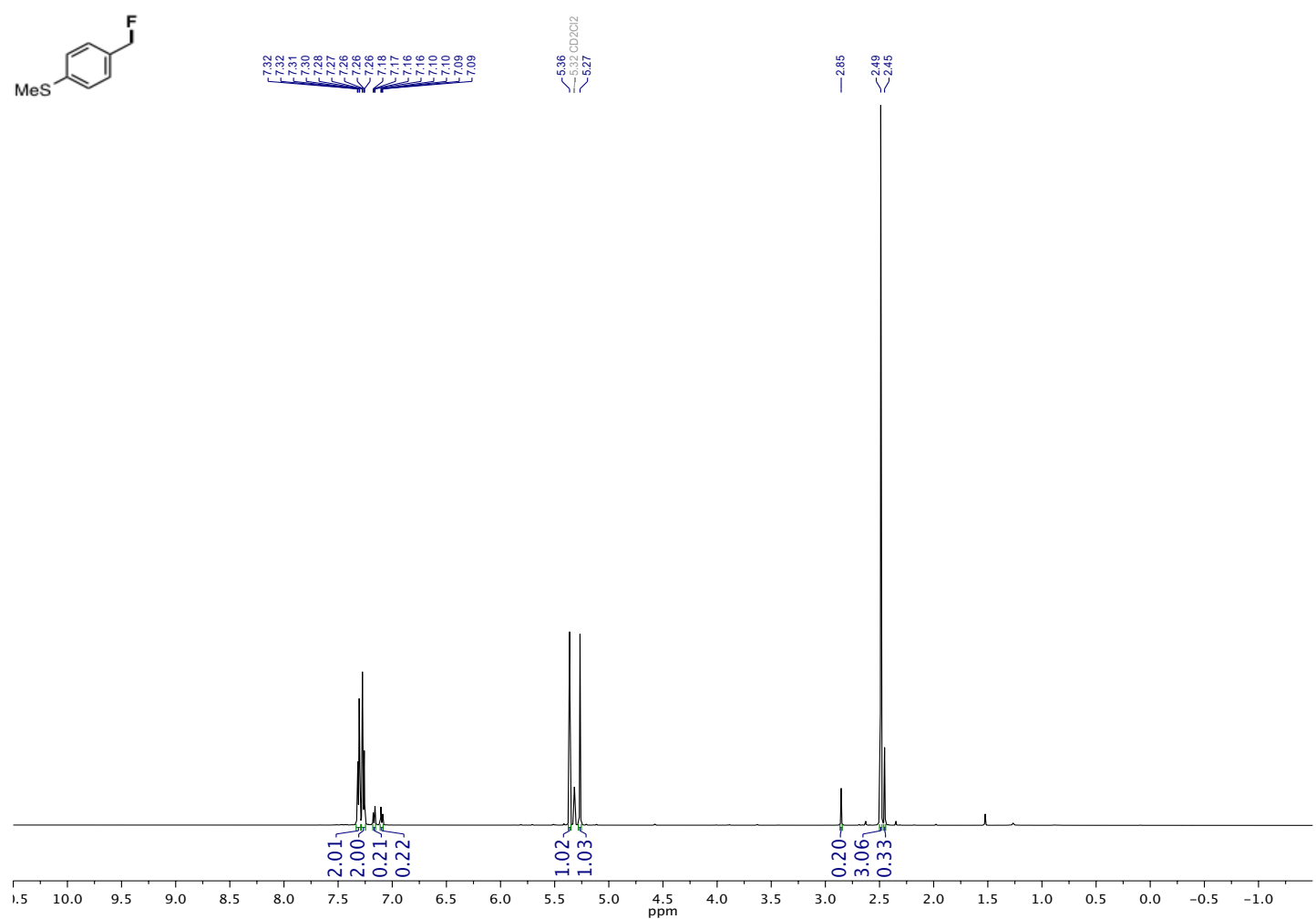
$126 \mathrm{MHz}{ }^{13} \mathrm{C}$-NMR of (4-(fluoromethyl)phenyl)(methyl)sulfane (11) in $\mathrm{CD}_{2} \mathrm{Cl}_{2}$
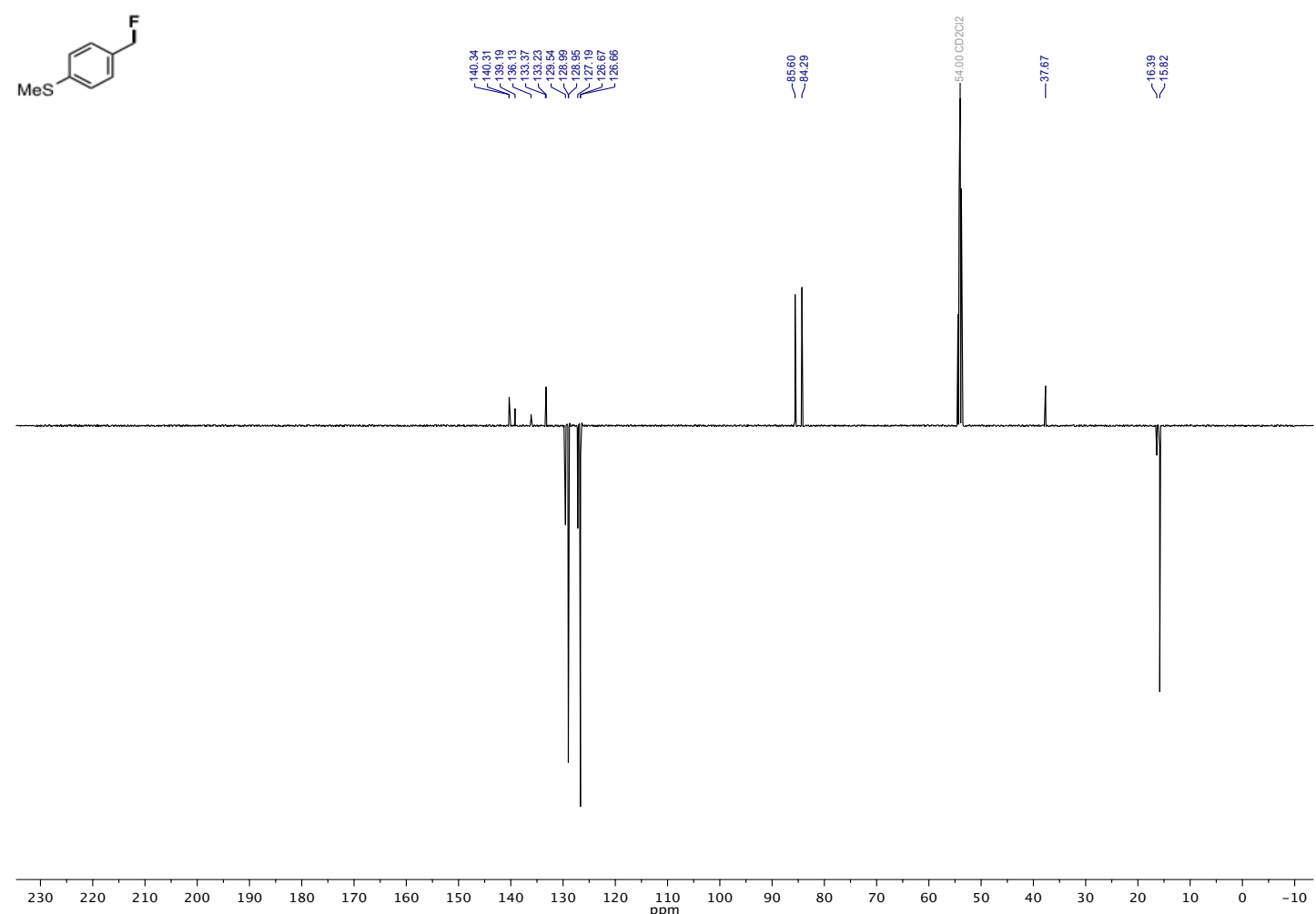

$282 \mathrm{MHz}{ }^{19} \mathrm{~F}-\mathrm{NMR}$ of (4-(fluoromethyl)phenyl)(methyl)sulfane (11) in $\mathrm{CD}_{2} \mathrm{Cl}_{2}$
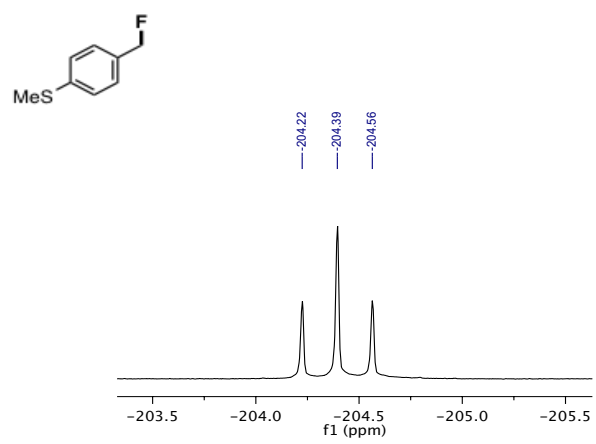

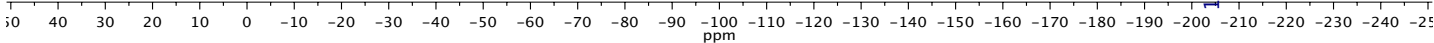


$500 \mathrm{MHz}{ }^{1} \mathrm{H}-\mathrm{NMR}$ of 1,3-dioxoisoindolin-2-yl 2-(4-acetamidophenyl)acetate (S7) in $\mathrm{CDCl}_{3}$

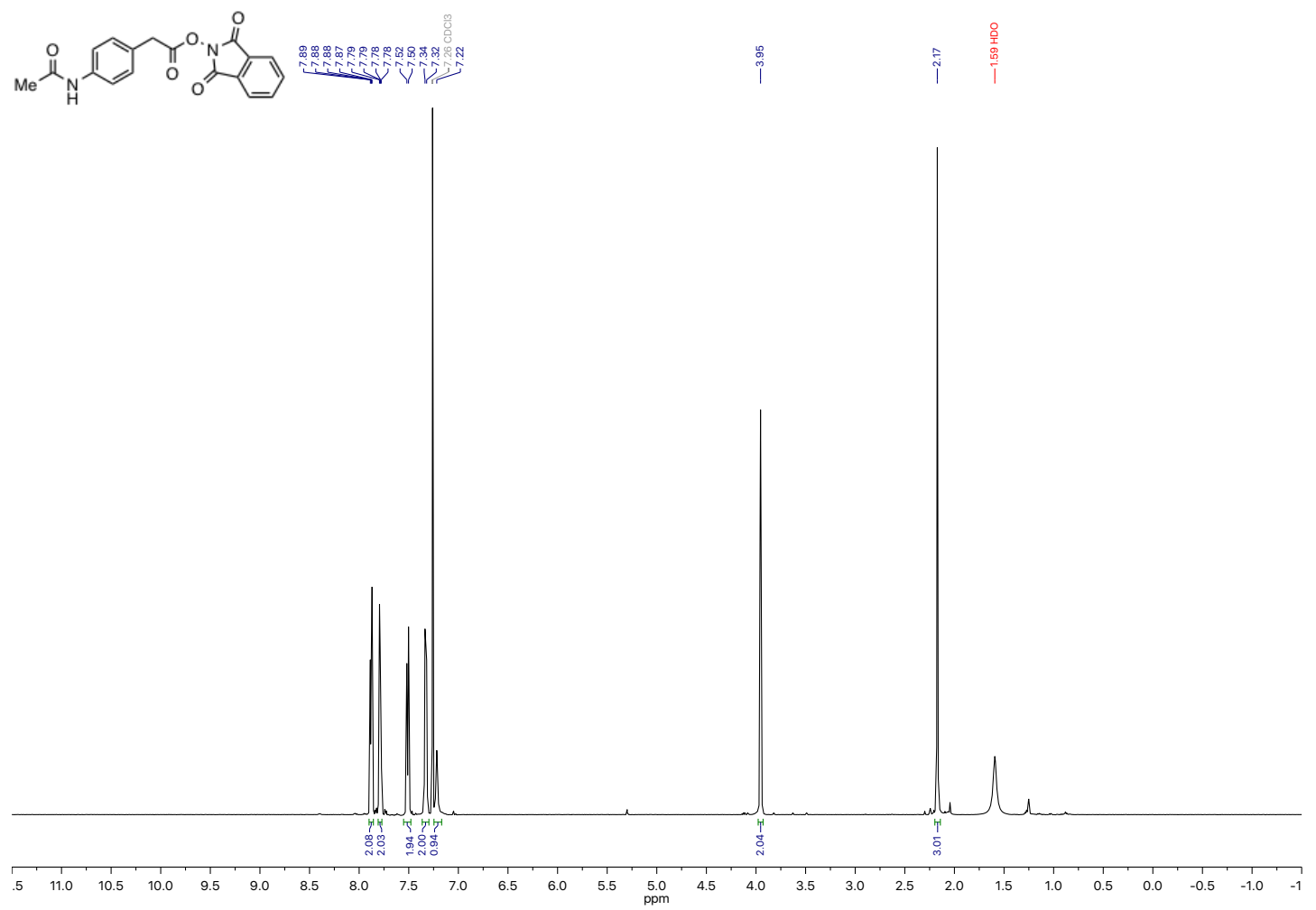

$126 \mathrm{MHz}{ }^{13} \mathrm{C}-\mathrm{NMR}$ of 1,3-dioxoisoindolin-2-yl 2-(4-acetamidophenyl)acetate (S7) in $\mathrm{CDCl}_{3}$
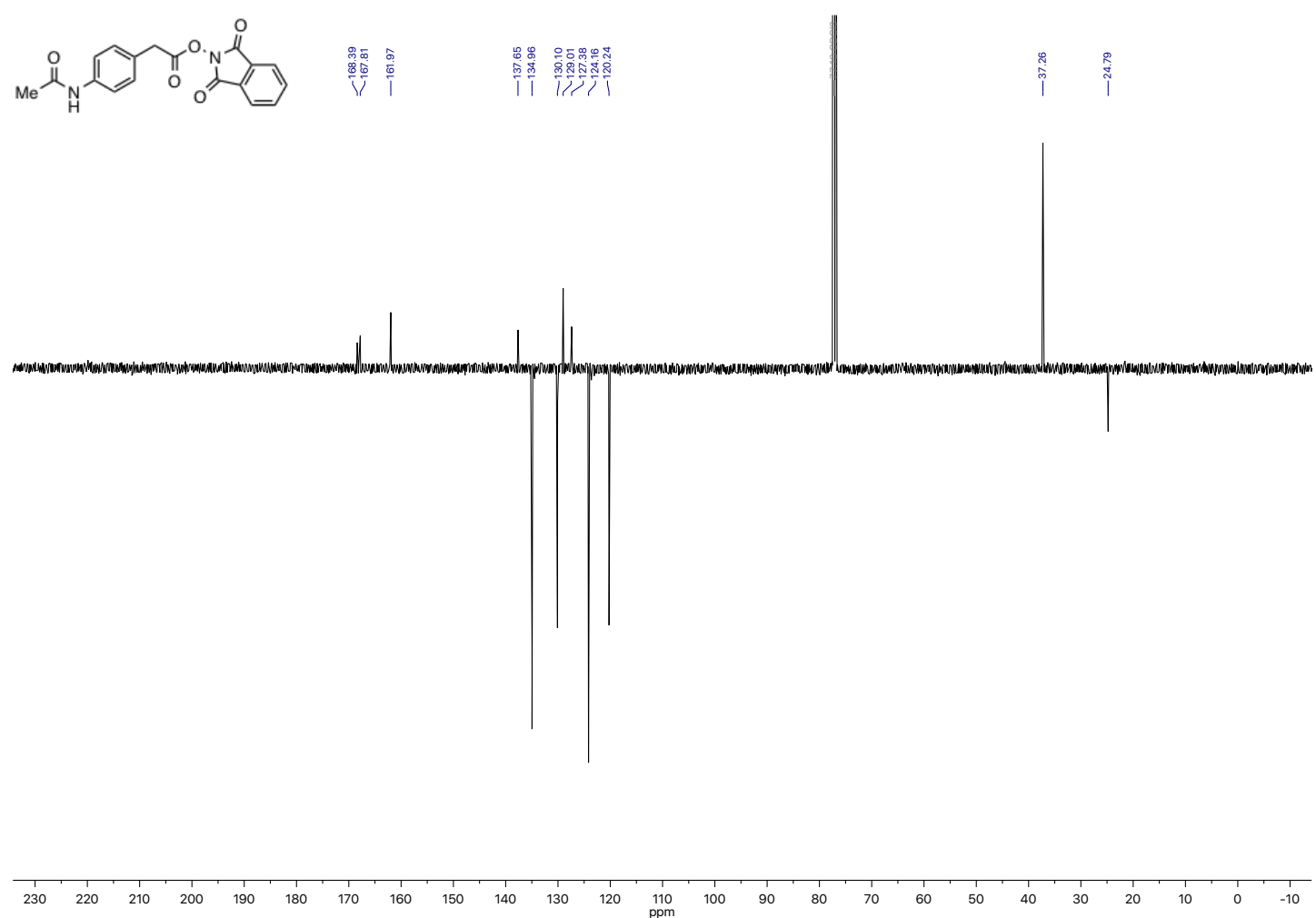
$500 \mathrm{MHz}{ }^{1} \mathrm{H}-\mathrm{NMR}$ of $\mathrm{N}$-(4-(fluoromethyl)phenyl)acetamide (12) in $\mathrm{CDCl}_{3}$
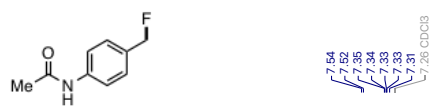

|
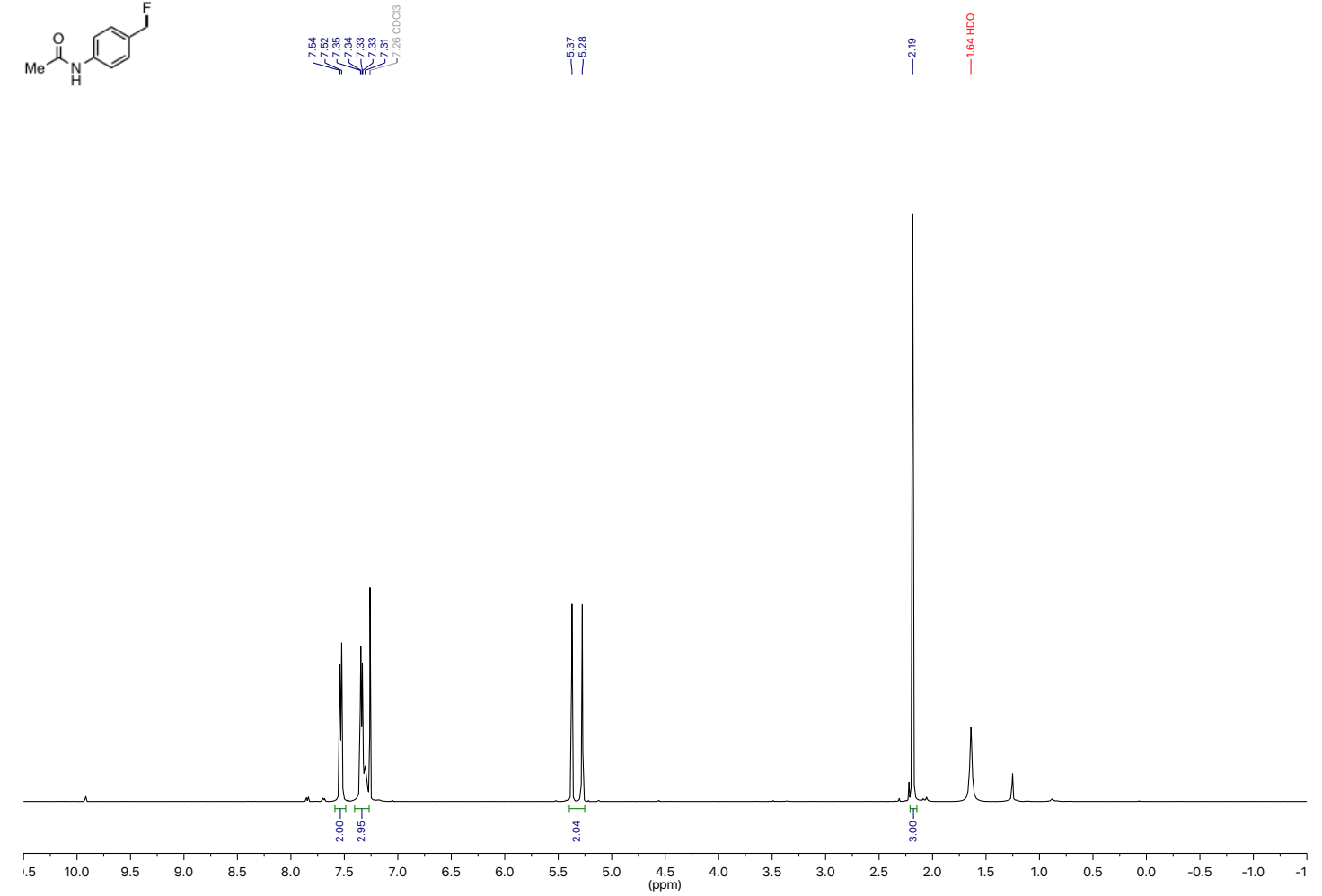

$126 \mathrm{MHz}{ }^{13} \mathrm{C}$-NMR of $N$-(4-(fluoromethyl)phenyl)acetamide (12) in $\mathrm{CDCl}_{3}$

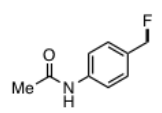

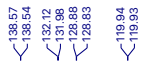

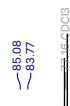
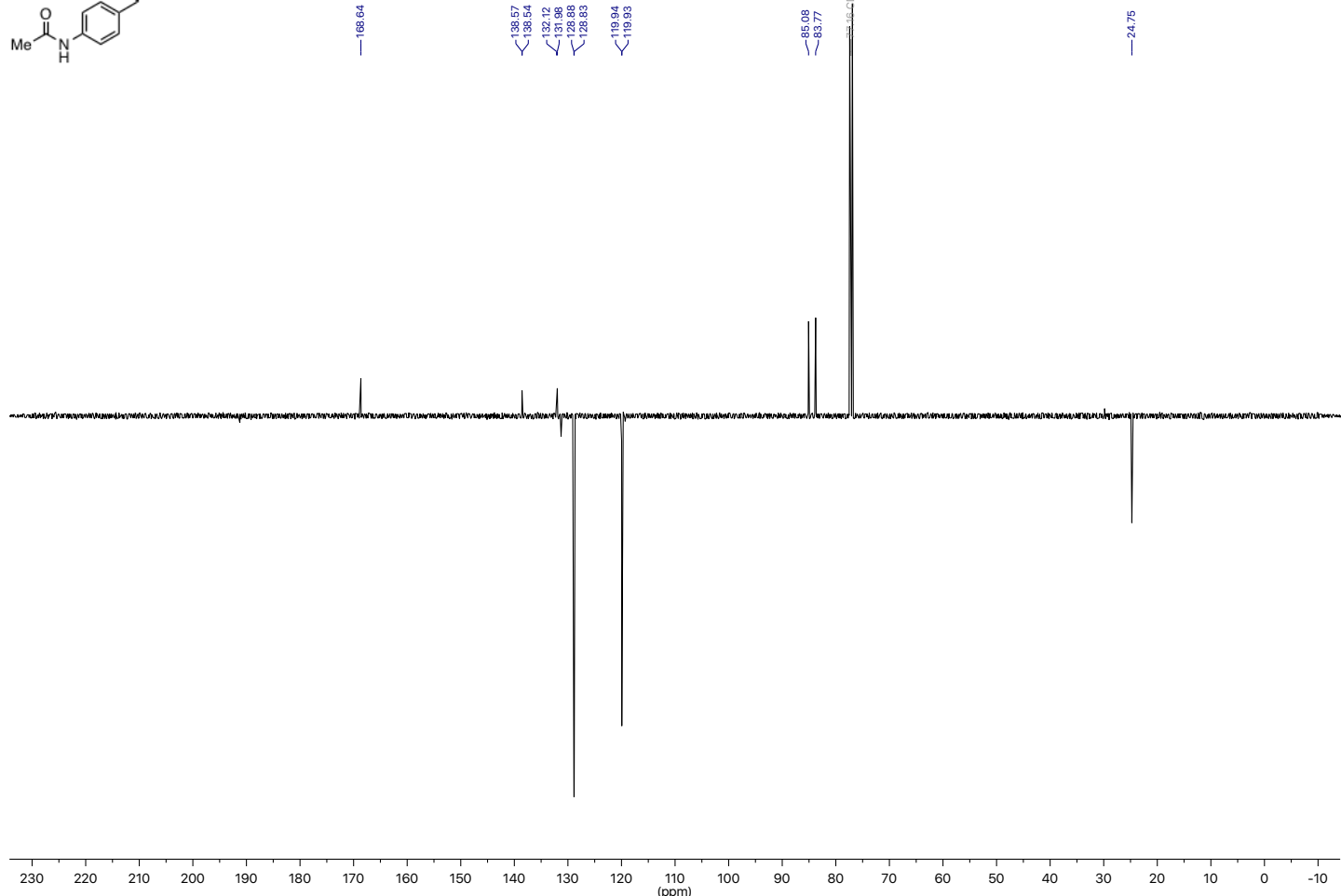

$120 \underset{\substack{110 \\(\mathrm{ppm})}}{1100}$ 
$376 \mathrm{MHz}{ }^{19} \mathrm{~F}-\mathrm{NMR}$ of $N$-(4-(fluoromethyl)phenyl)acetamide (12) in $\mathrm{CDCl}_{3}$<smiles>CCC1CCC(NC(C)=O)C1</smiles>
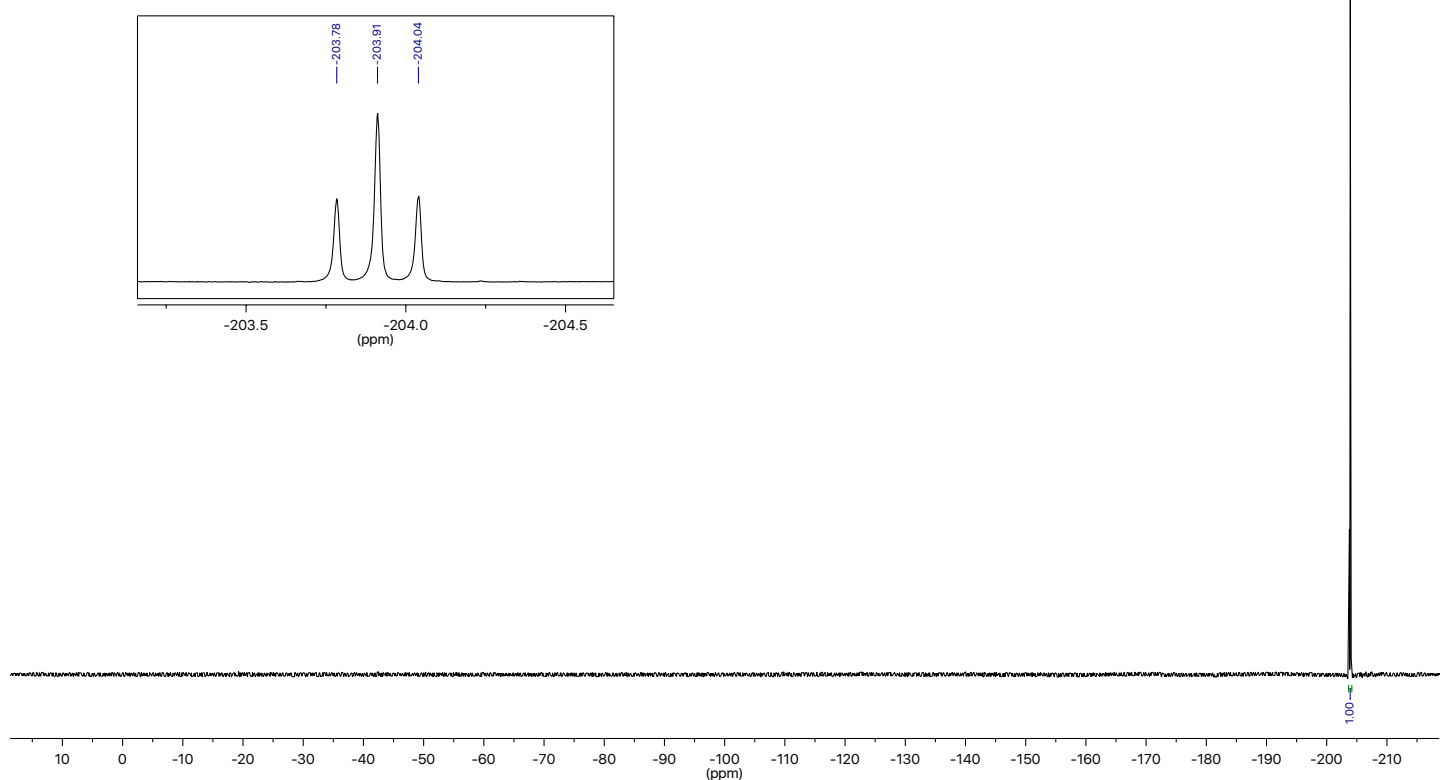

$500 \mathrm{MHz}{ }^{1} \mathrm{H}-\mathrm{NMR}$ of 1,3-dioxoisoindolin-2-yl 2-(4-(trifluoromethoxy)phenyl)acetate (S8) in $\mathrm{CDCl}_{3}$
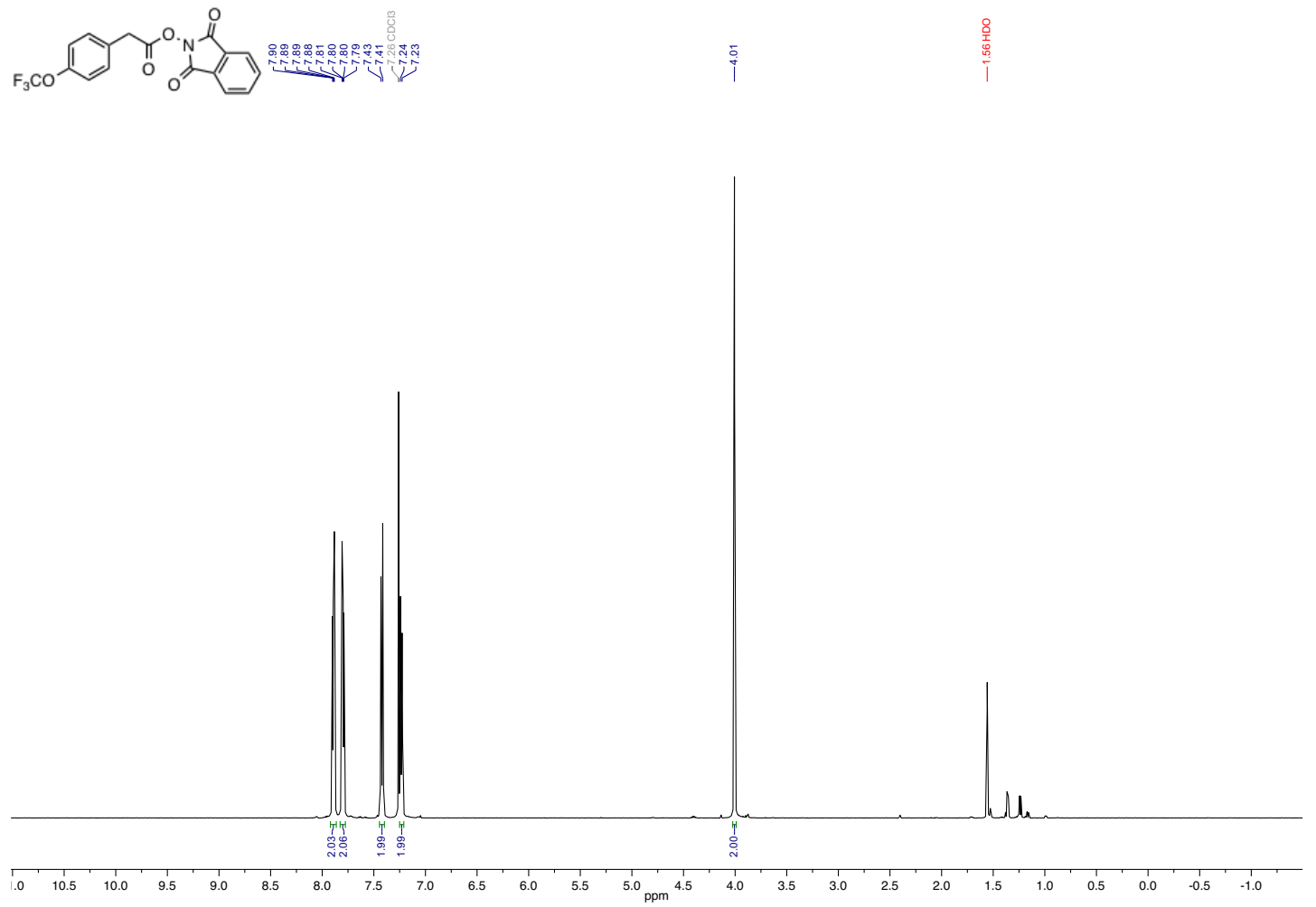
$126 \mathrm{MHz}{ }^{13} \mathrm{C}-\mathrm{NMR}$ of 1,3-dioxoisoindolin-2-yl 2-(4-(trifluoromethoxy)phenyl)acetate (S8) in $\mathrm{CDCl}_{3}$

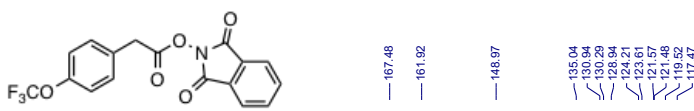
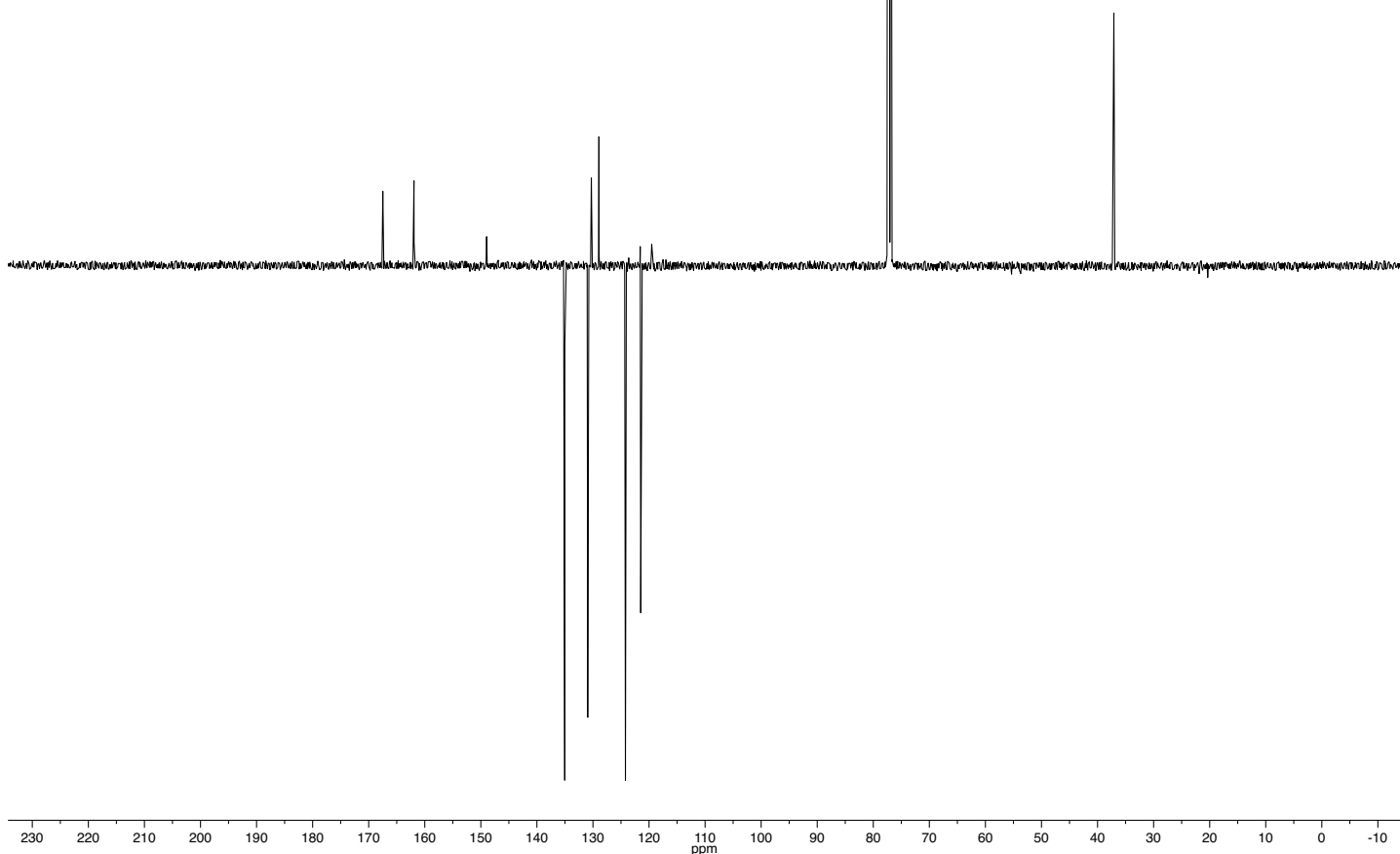

$282 \mathrm{MHz}{ }^{19} \mathrm{~F}-\mathrm{NMR}$ of 1,3-dioxoisoindolin-2-yl 2-(4-(trifluoromethoxy)phenyl)acetate (S8) in $\mathrm{CDCl}_{3}$
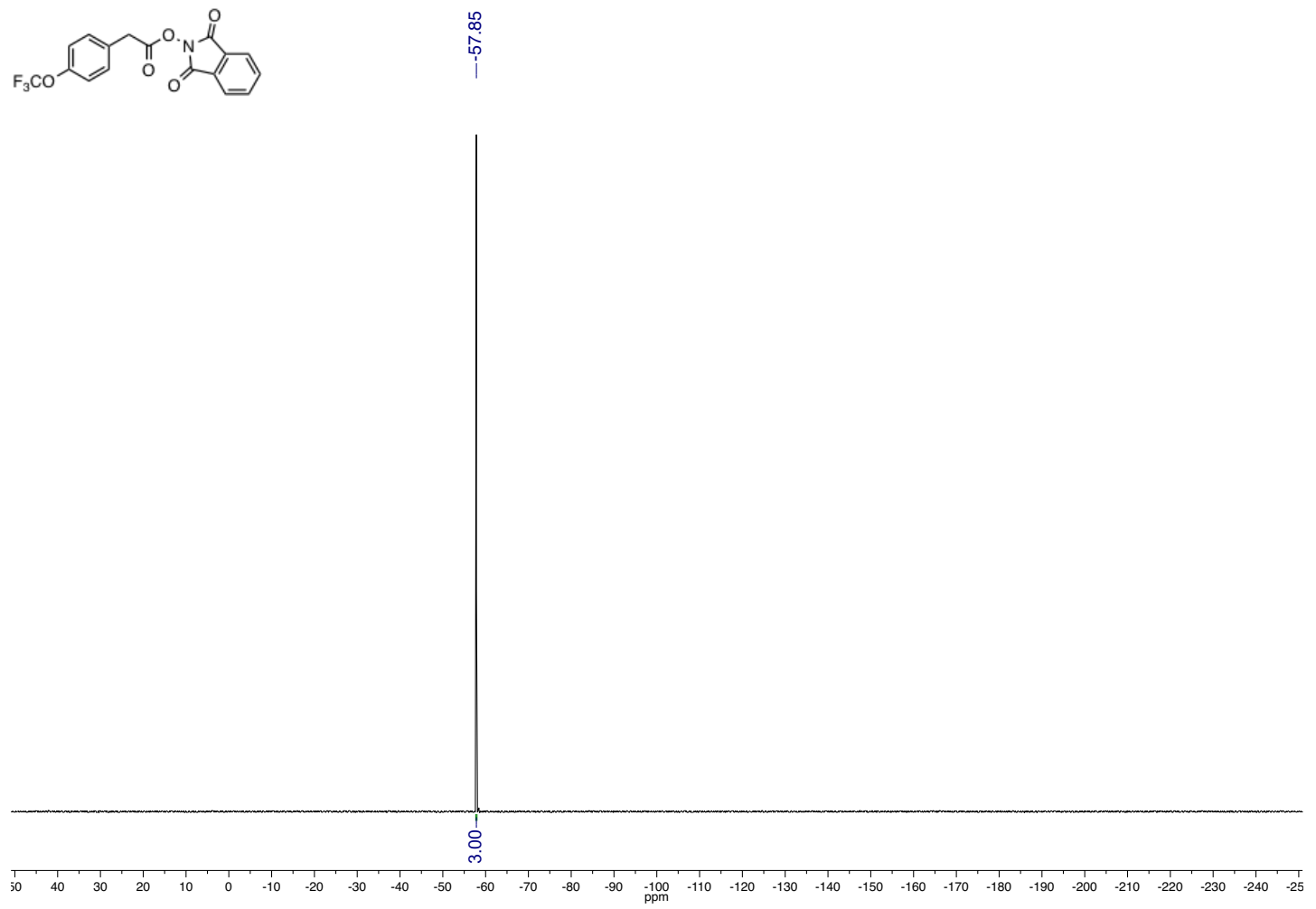
$282 \mathrm{MHz}{ }^{19} \mathrm{~F}-\mathrm{NMR}$ of 1-(fluoromethyl)-4-(trifluoromethoxy)benzene (13) in $\mathrm{CDCl}_{3}$

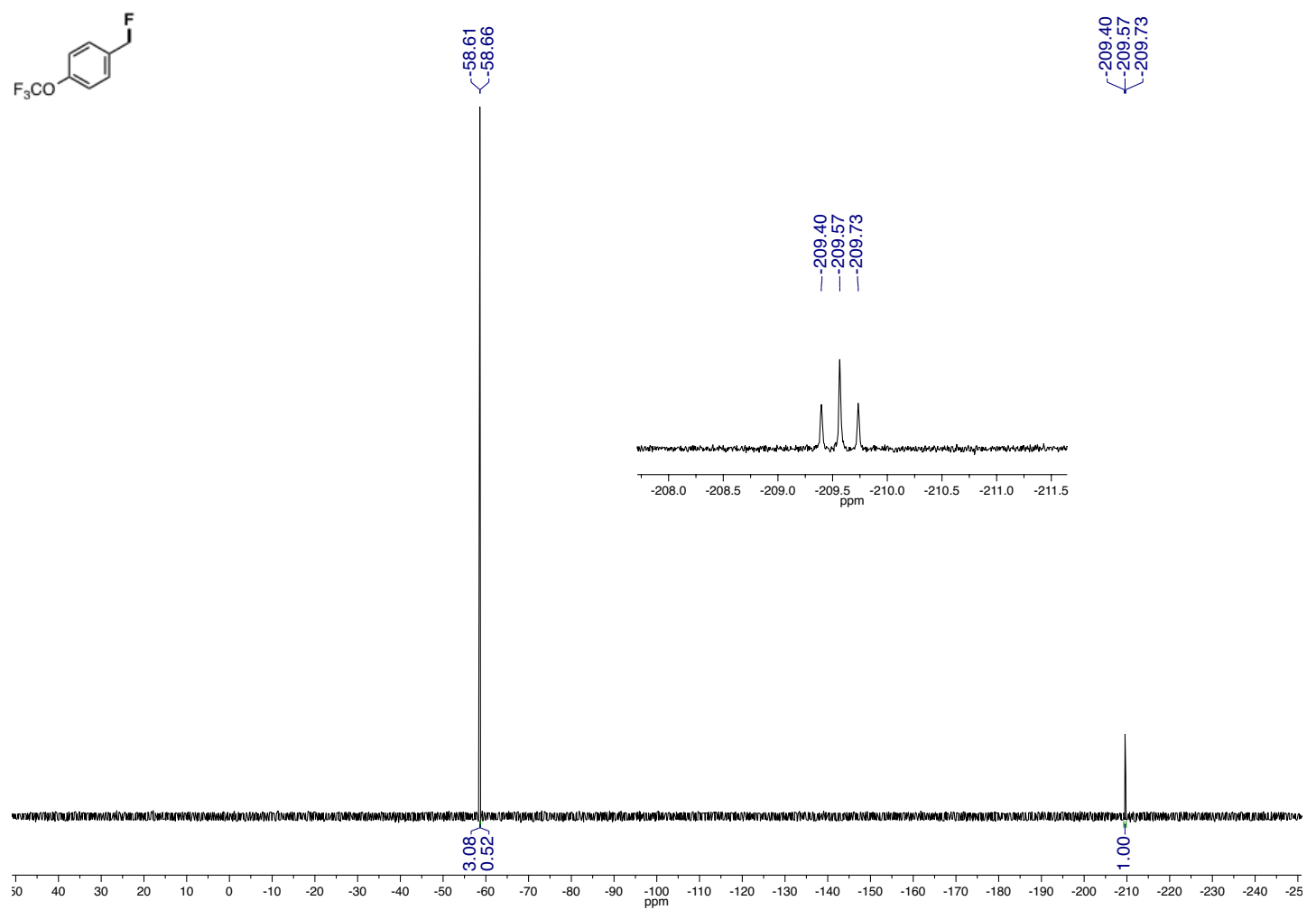

$500 \mathrm{MHz}{ }^{1} \mathrm{H}-\mathrm{NMR}$ of 1,3-dioxoisoindolin-2-yl 2-(4-(trifluoromethyl)phenyl)acetate (S9) in $\mathrm{CDCl}_{3}$
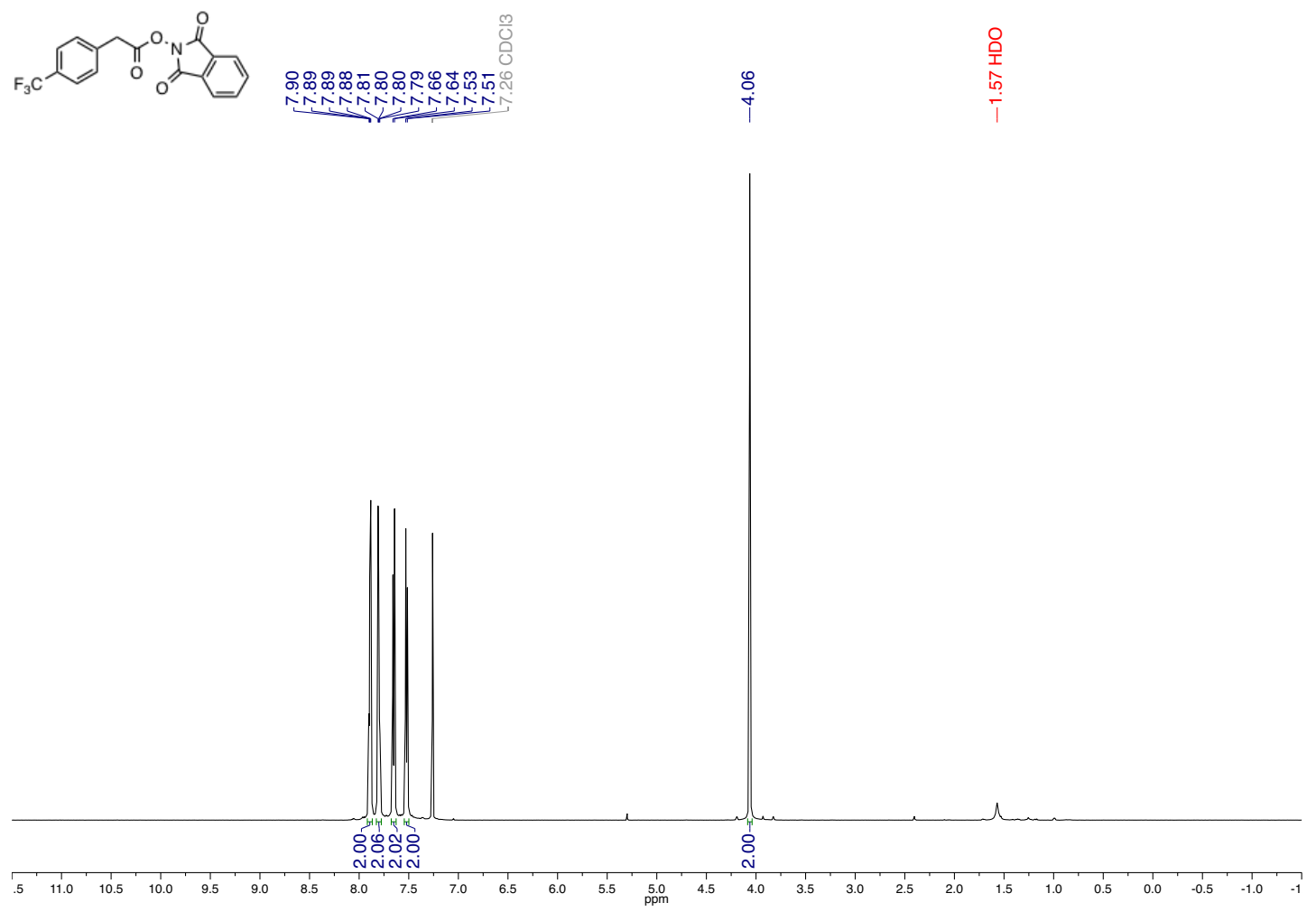
$126 \mathrm{MHz}{ }^{13} \mathrm{C}$-NMR of 1,3-dioxoisoindolin-2-yl 2-(4-(trifluoromethyl)phenyl)acetate (S9) in $\mathrm{CDCl}_{3}$

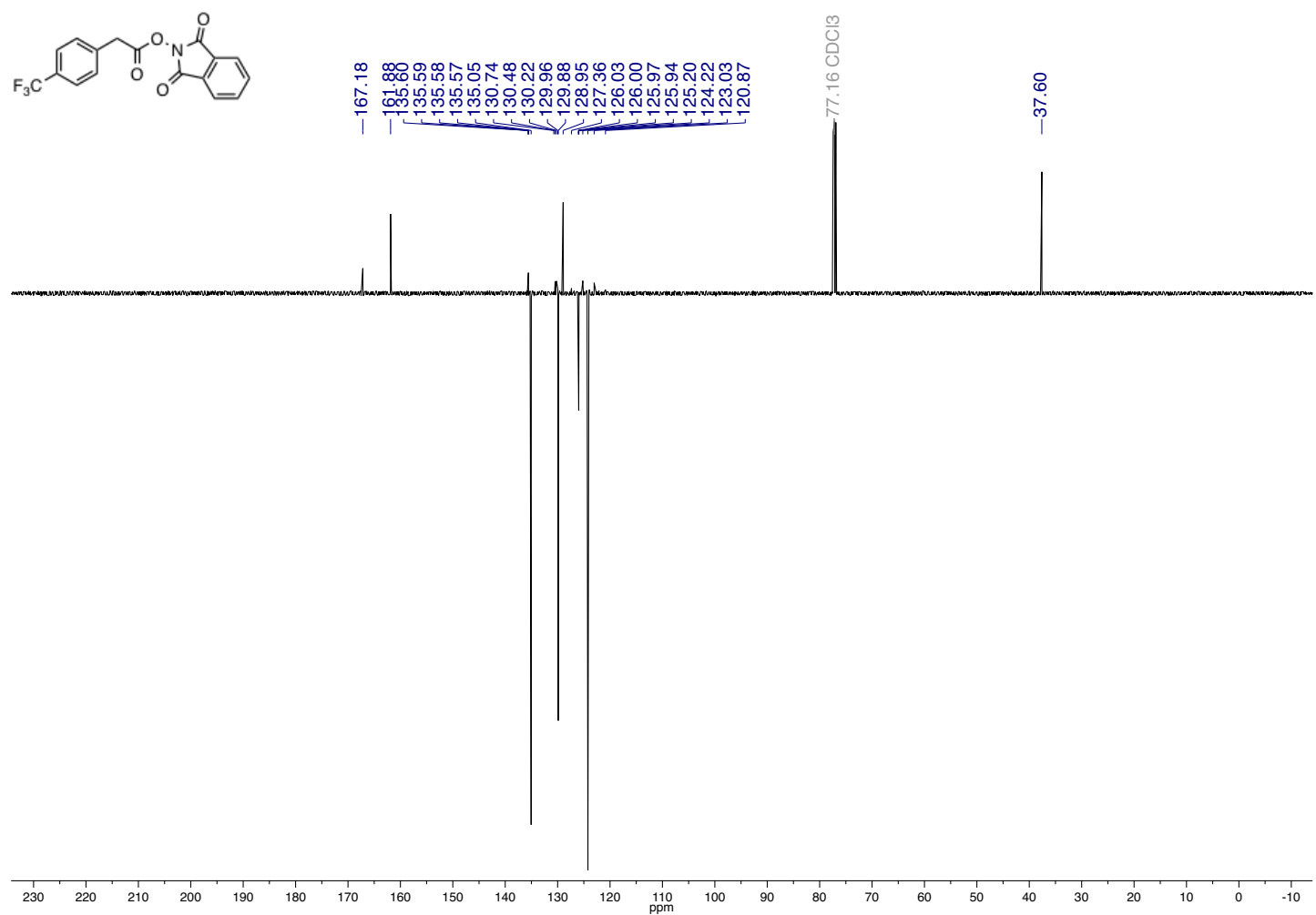

$282 \mathrm{MHz}^{19} \mathrm{~F}-\mathrm{NMR}$ of 1,3-dioxoisoindolin-2-yl 2-(4-(trifluoromethyl)phenyl)acetate (S9) in $\mathrm{CDCl}_{3}$

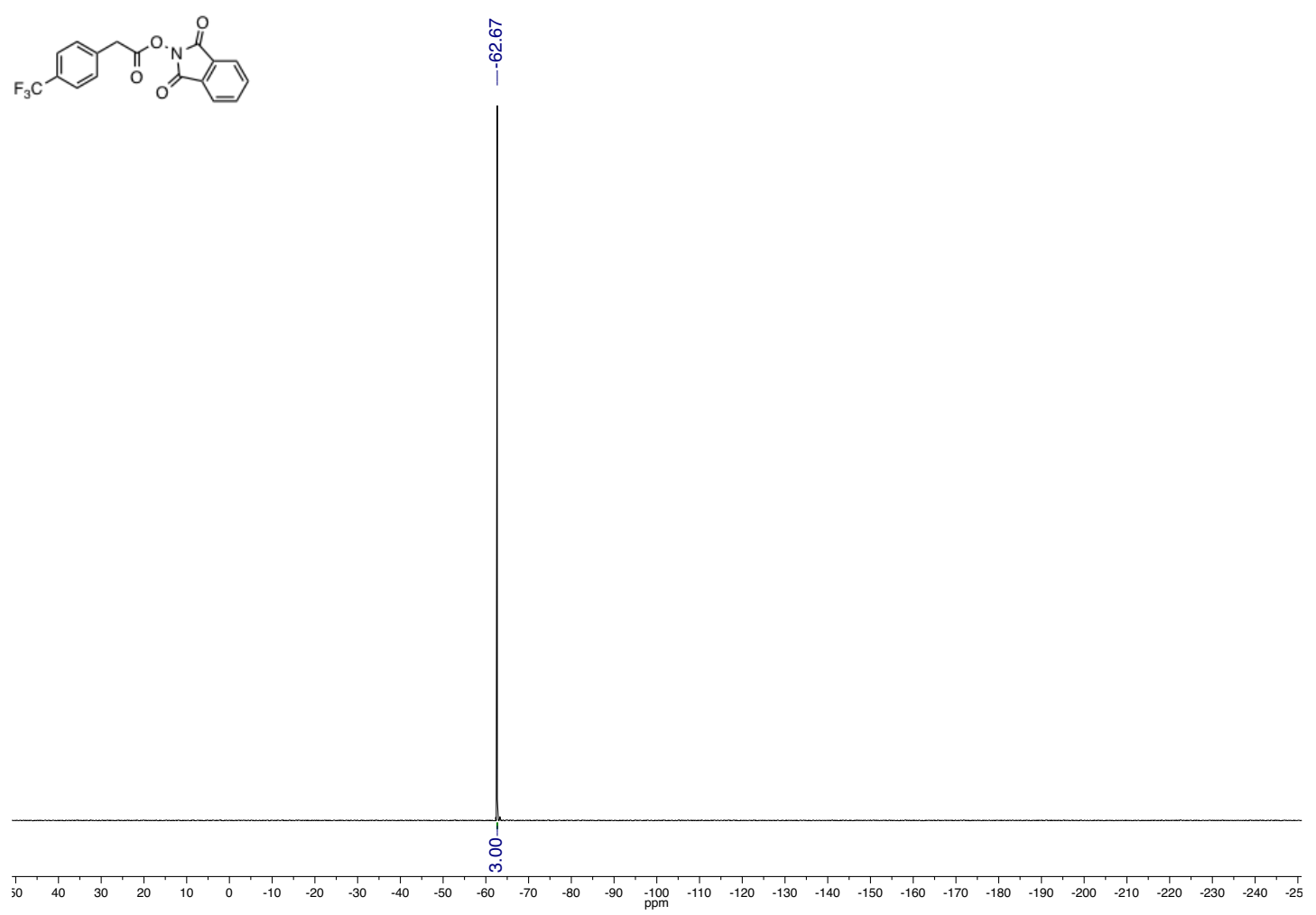


$282 \mathrm{MHz}{ }^{19} \mathrm{~F}-\mathrm{NMR}$ of 1-(fluoromethyl)-4-(trifluoromethyl)benzene (14) in $\mathrm{CDCl}_{3}$

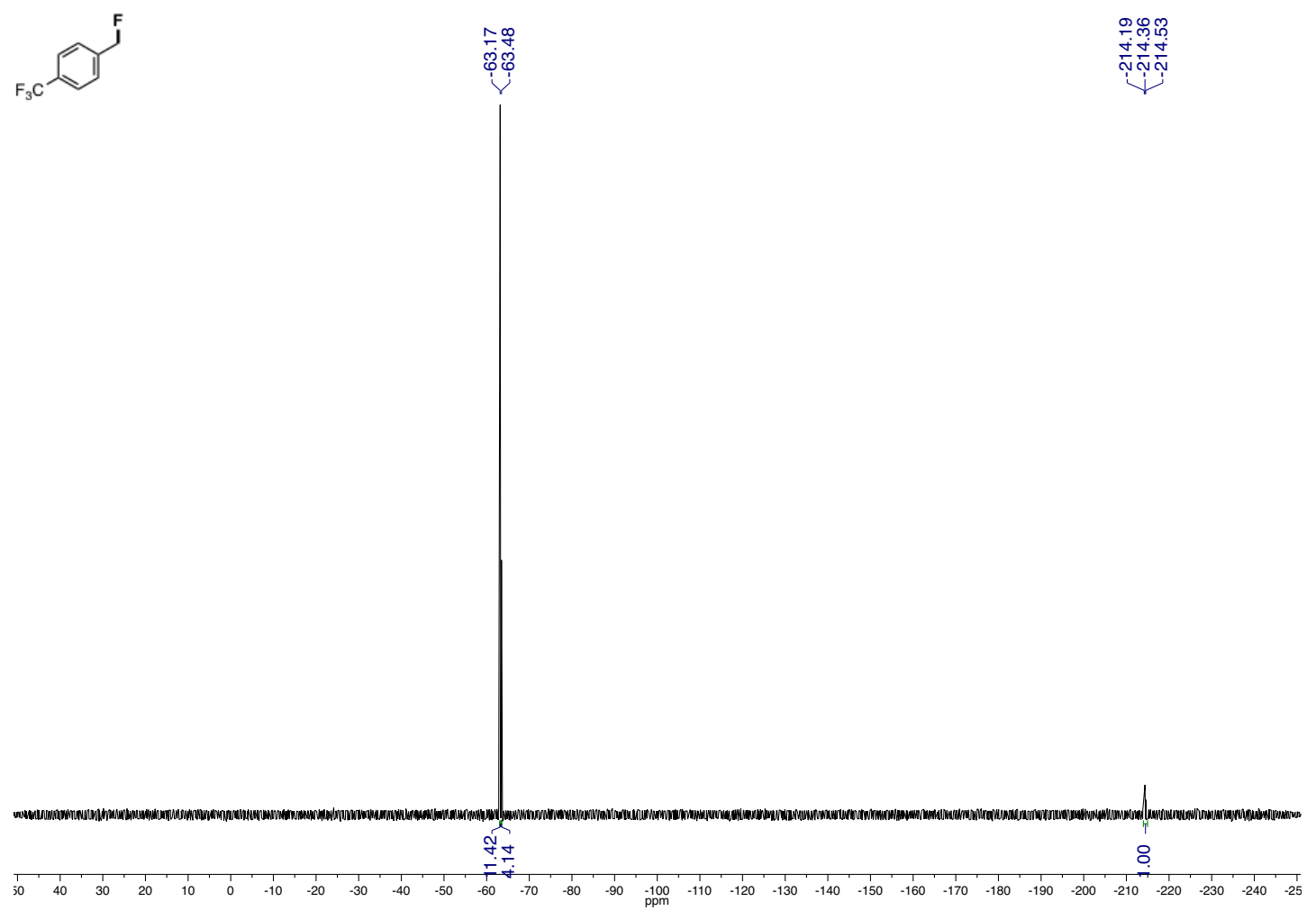

$500 \mathrm{MHz}{ }^{1} \mathrm{H}-\mathrm{NMR}$ of 1,3-dioxoisoindolin-2-yl 2-(benzo[d][1,3]dioxol-5-yl)acetate (S10) in $\mathrm{CDCl}_{3}$
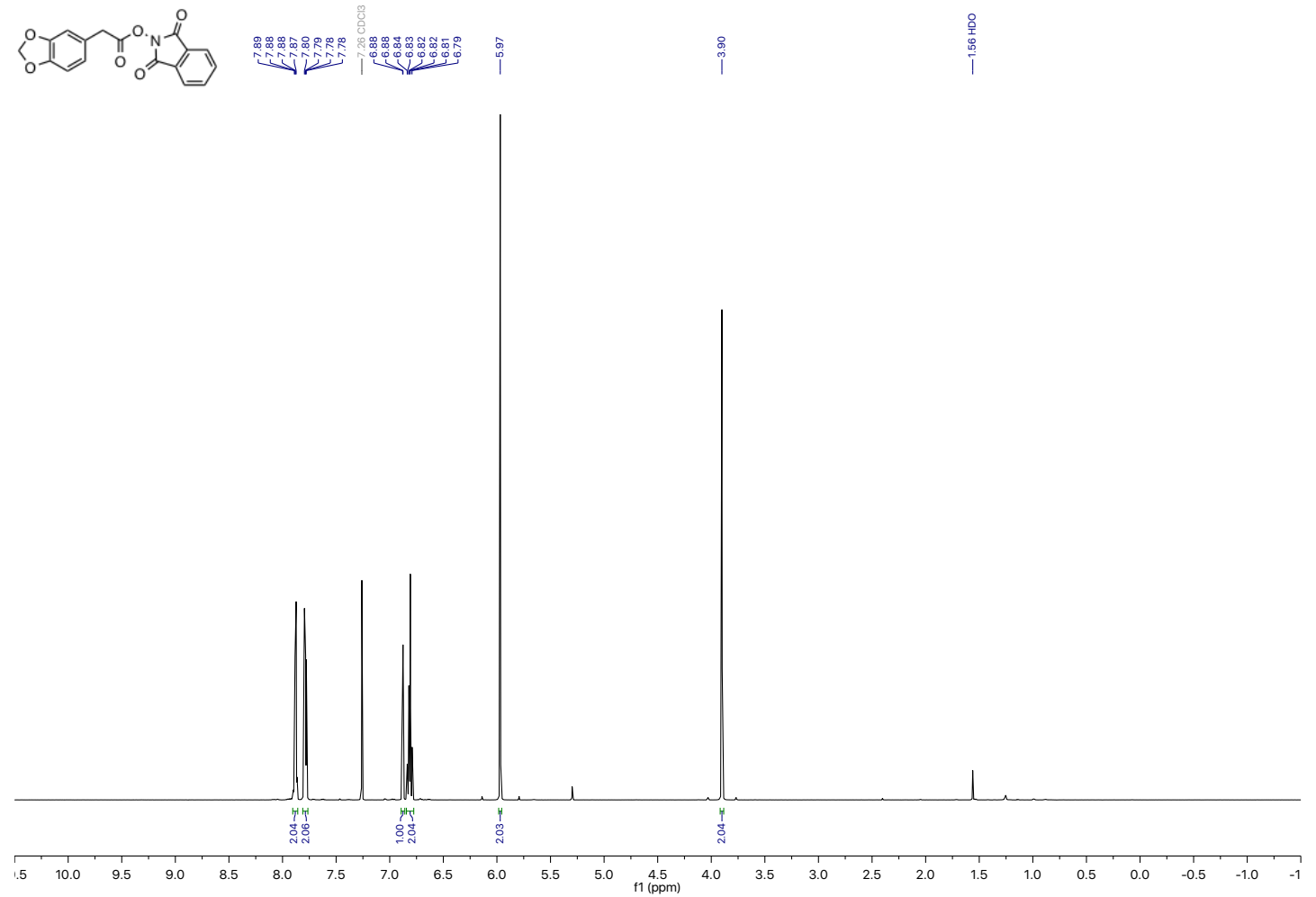
$126 \mathrm{MHz}{ }^{13} \mathrm{C}-\mathrm{NMR}$ of 1,3-dioxoisoindolin-2-yl 2-(benzo[d][1,3]dioxol-5-yl)acetate (S10) in $\mathrm{CDCl}_{3}$

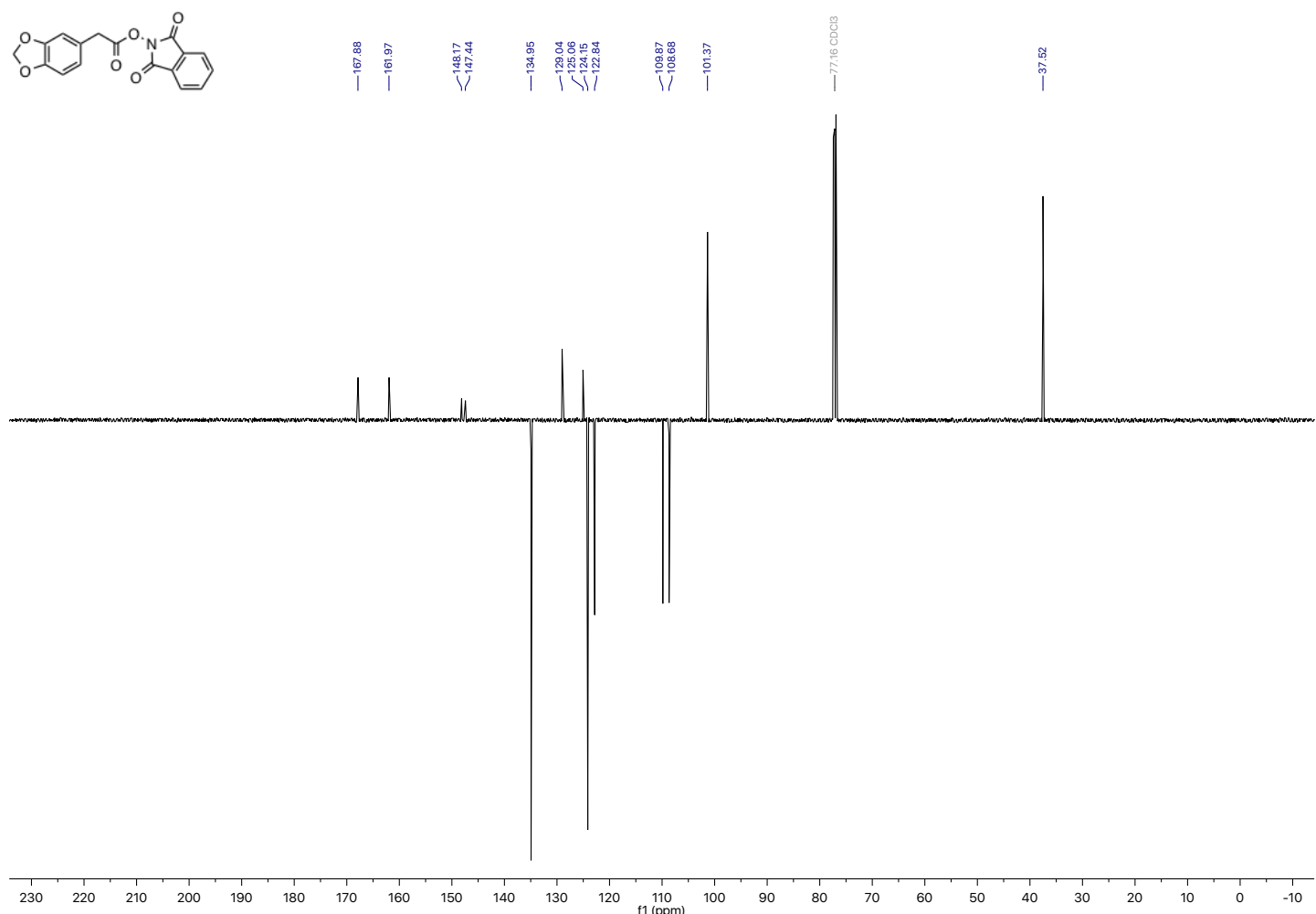

$500 \mathrm{MHz}{ }^{1} \mathrm{H}-\mathrm{NMR}$ of 5-(fluoromethyl)benzo[d][1,3]dioxole (15) in $\mathrm{CD}_{2} \mathrm{Cl}_{2}$
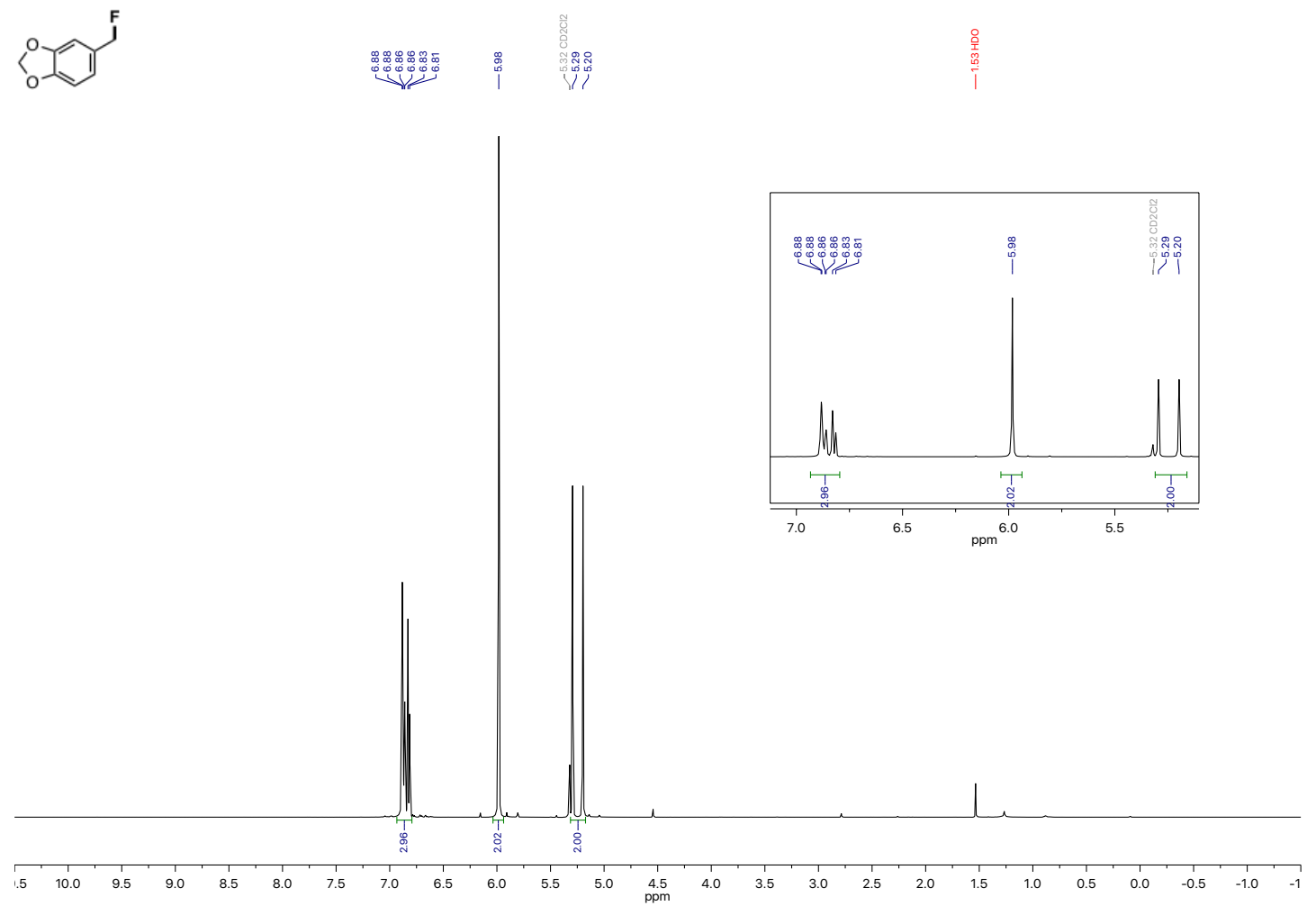


\section{$126 \mathrm{MHz}{ }^{13} \mathrm{C}$-NMR of 5-(fluoromethyl)benzo[d][1,3]dioxole (15) in $\mathrm{CD}_{2} \mathrm{Cl}_{2}$}

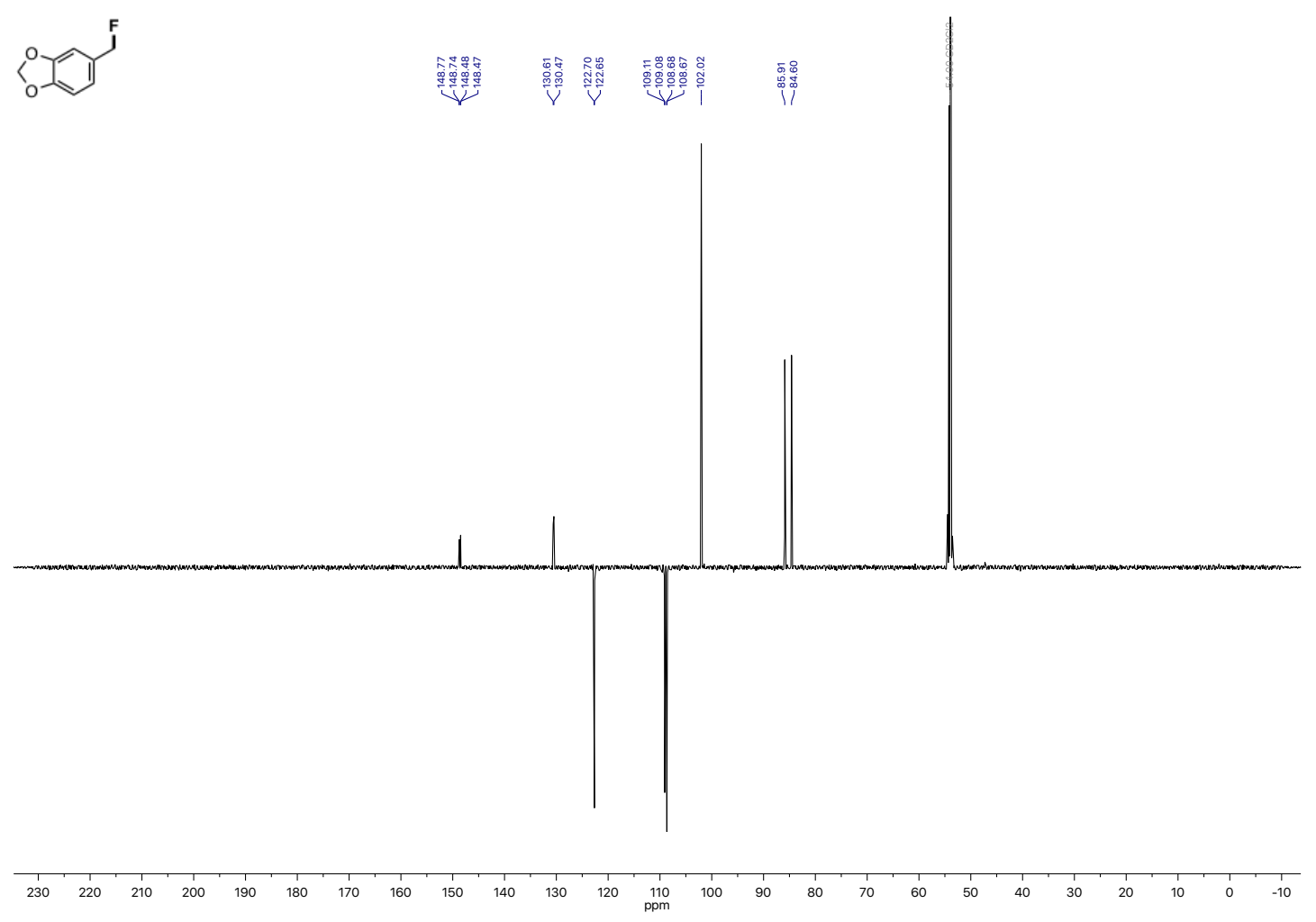

$376 \mathrm{MHz}{ }^{19} \mathrm{~F}-\mathrm{NMR}$ of 5-(fluoromethyl)benzo[d][1,3]dioxole (15) in $\mathrm{CD}_{2} \mathrm{Cl}_{2}$
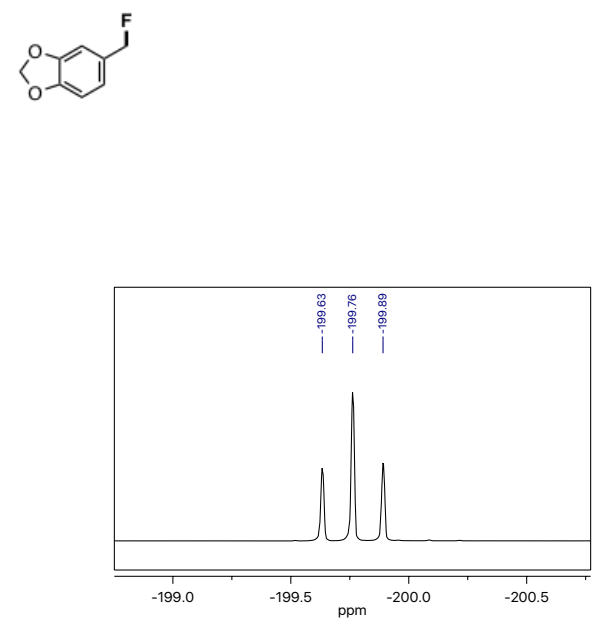

$\stackrel{1}{\stackrel{2}{2}}$

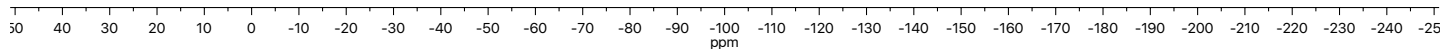


$500 \mathrm{MHz}{ }^{1} \mathrm{H}-\mathrm{NMR}$ of 1,3-dioxoisoindolin-2-yl 2-(4-bromothiophen-2-yl)acetate (S11) in $\mathrm{CDCl}_{3}$
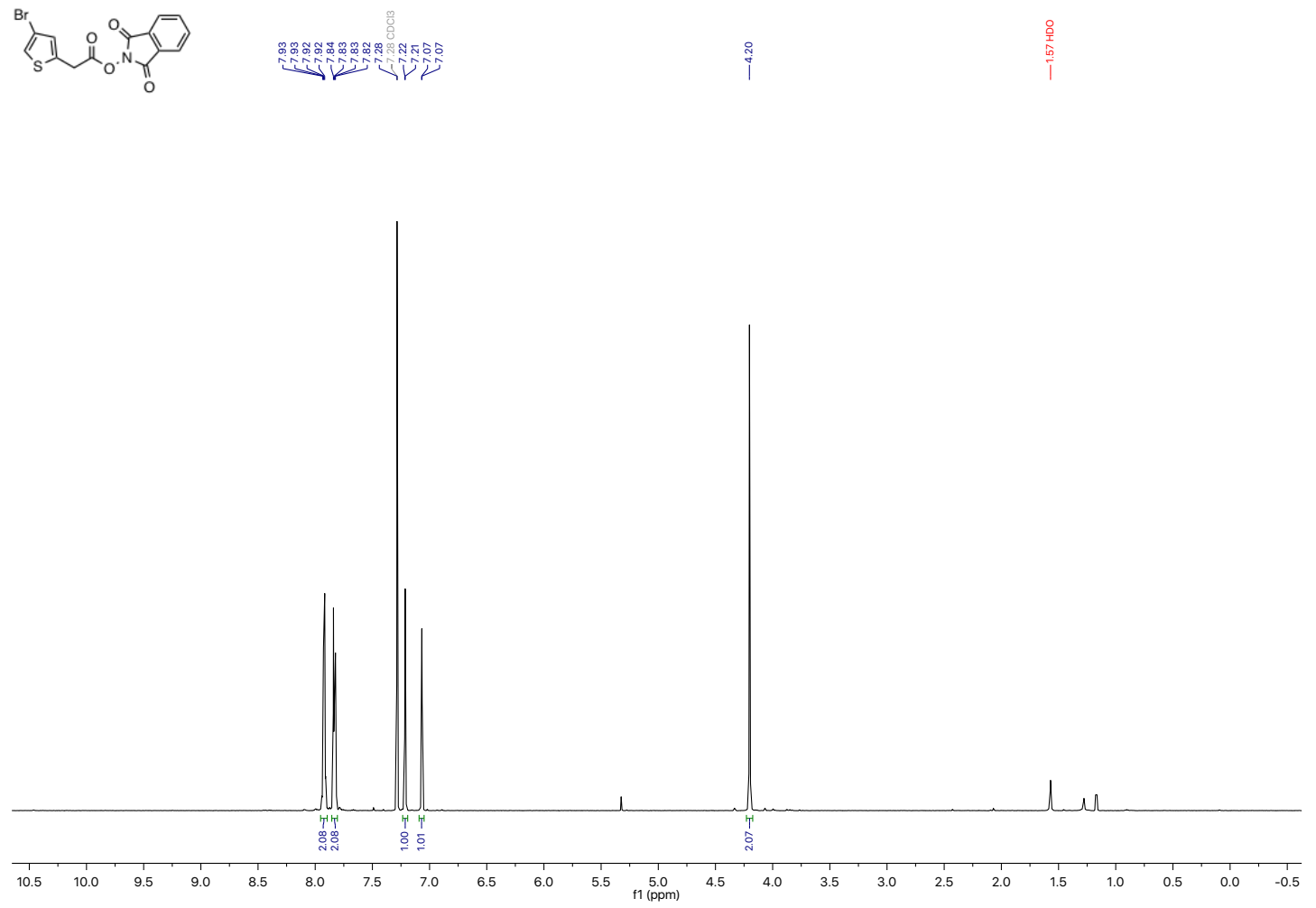

$126 \mathrm{MHz}{ }^{13} \mathrm{C}-\mathrm{NMR}$ of 1,3-dioxoisoindolin-2-yl 2-(4-bromothiophen-2-yl)acetate (S11) in $\mathrm{CDCl}_{3}$
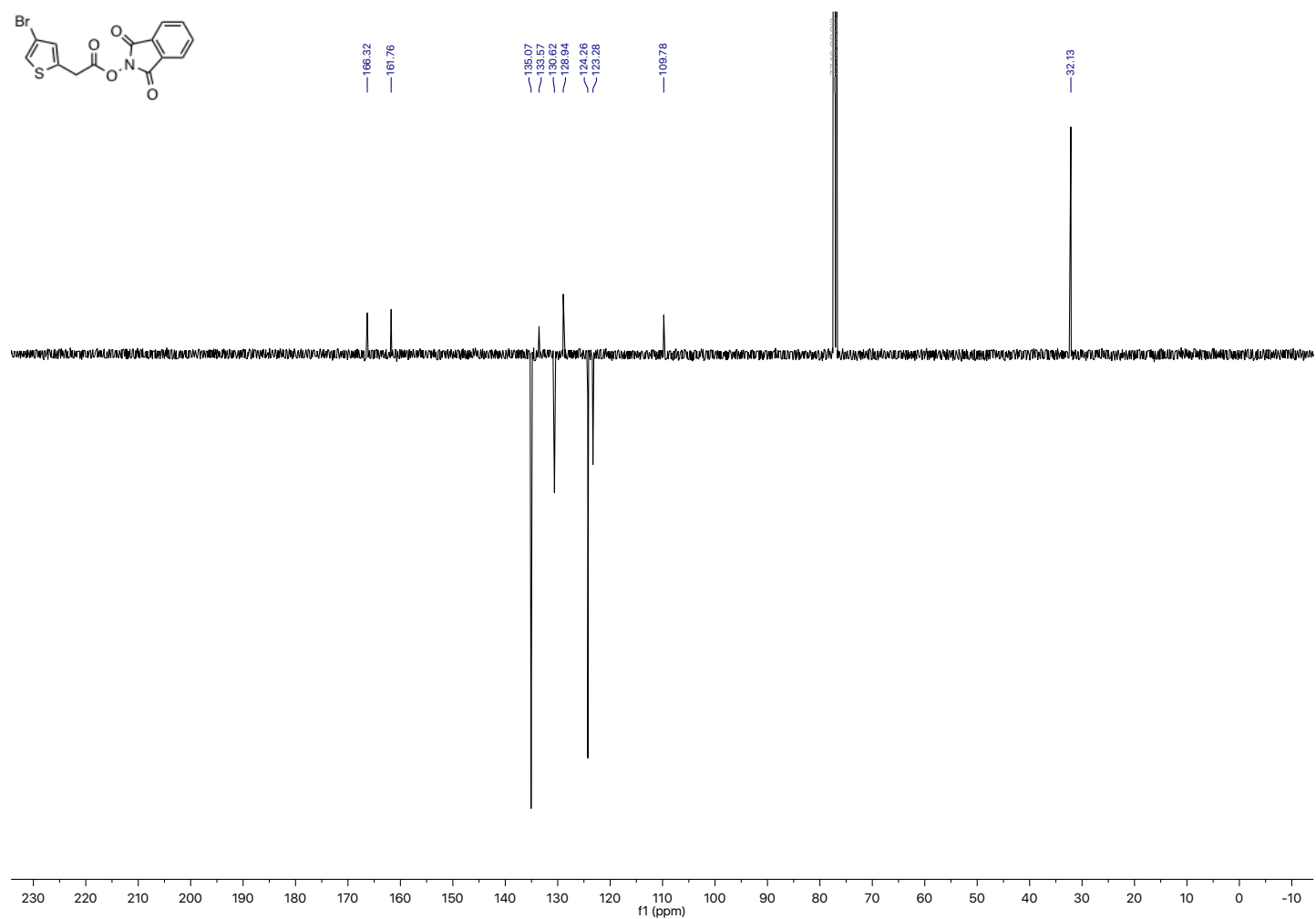
$282 \mathrm{MHz}{ }^{19} \mathrm{~F}-\mathrm{NMR}$ of 4-bromo-2-(fluoromethyl)thiophene (16) in $\mathrm{CDCl}_{3}$<smiles>[B]C1C[C+](CF)C1</smiles>

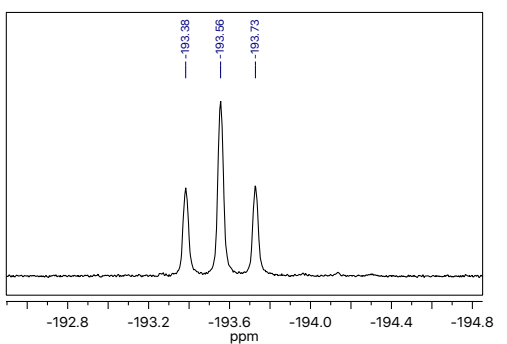

$\begin{array}{lllllllllllllllllllllllllllllllllllll}50 & 40 & 30 & 20 & 10 & 0 & -10 & -20 & -30 & -40 & -50 & -60 & -70 & -80 & -90 & -100 & -110 & -120 & -130 & -140 & -150 & -160 & -170 & -180 & -190 & -200 & -210 & -220 & -230 & -240 & -25\end{array}$

$500 \mathrm{MHz}{ }^{1} \mathrm{H}-\mathrm{NMR}$ of 1,3-dioxoisoindolin-2-yl 2-cyclopentyl-2-(4-(trifluoromethyl)phenyl)acetate (S12) in $\mathrm{CDCl}_{3}$

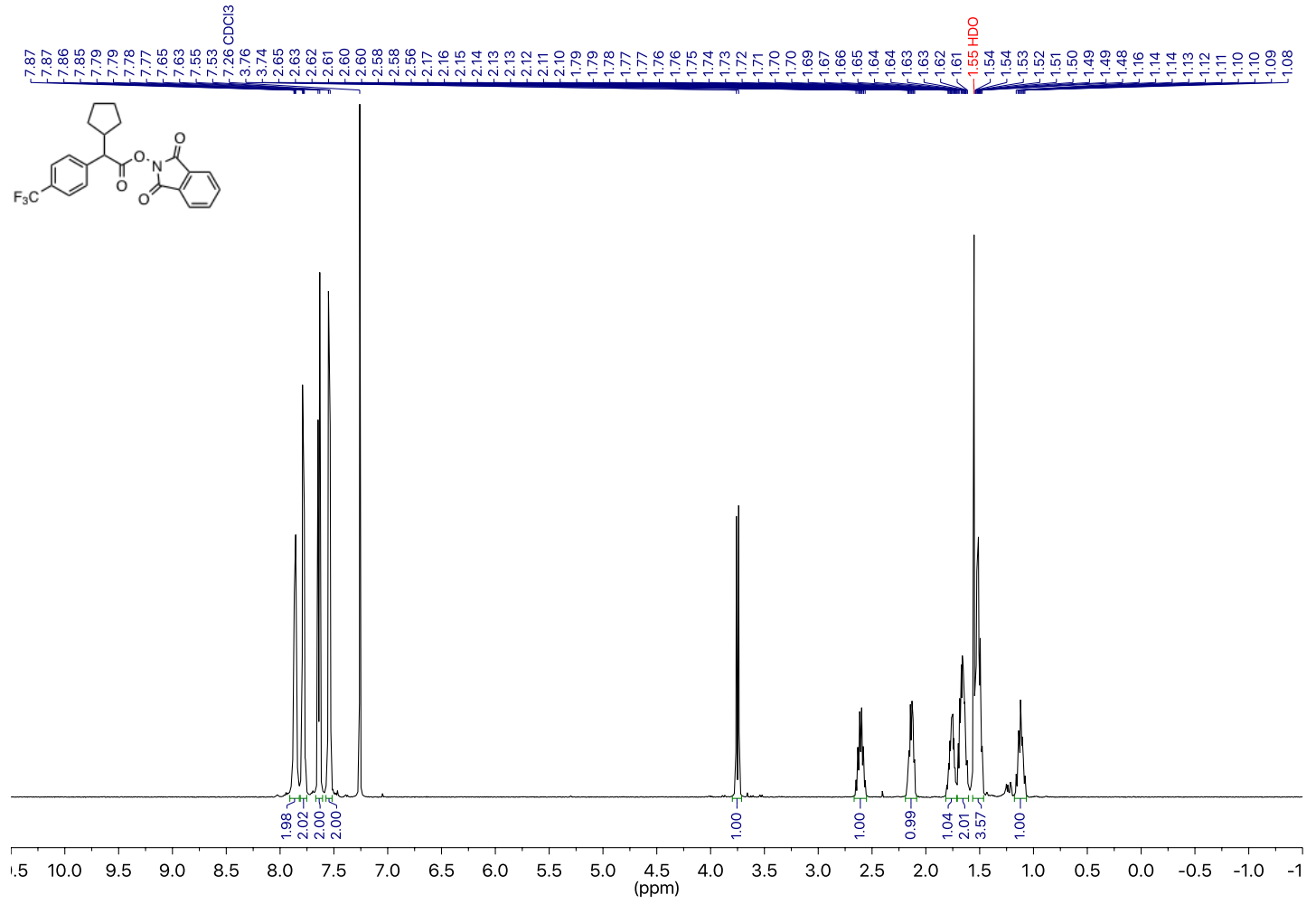


$126 \mathrm{MHz}{ }^{13} \mathrm{C}-\mathrm{NMR}$ of 1,3-dioxoisoindolin-2-yl 2-cyclopentyl-2-(4-(trifluoromethyl)phenyl)acetate (S12) in $\mathrm{CDCl}_{3}$

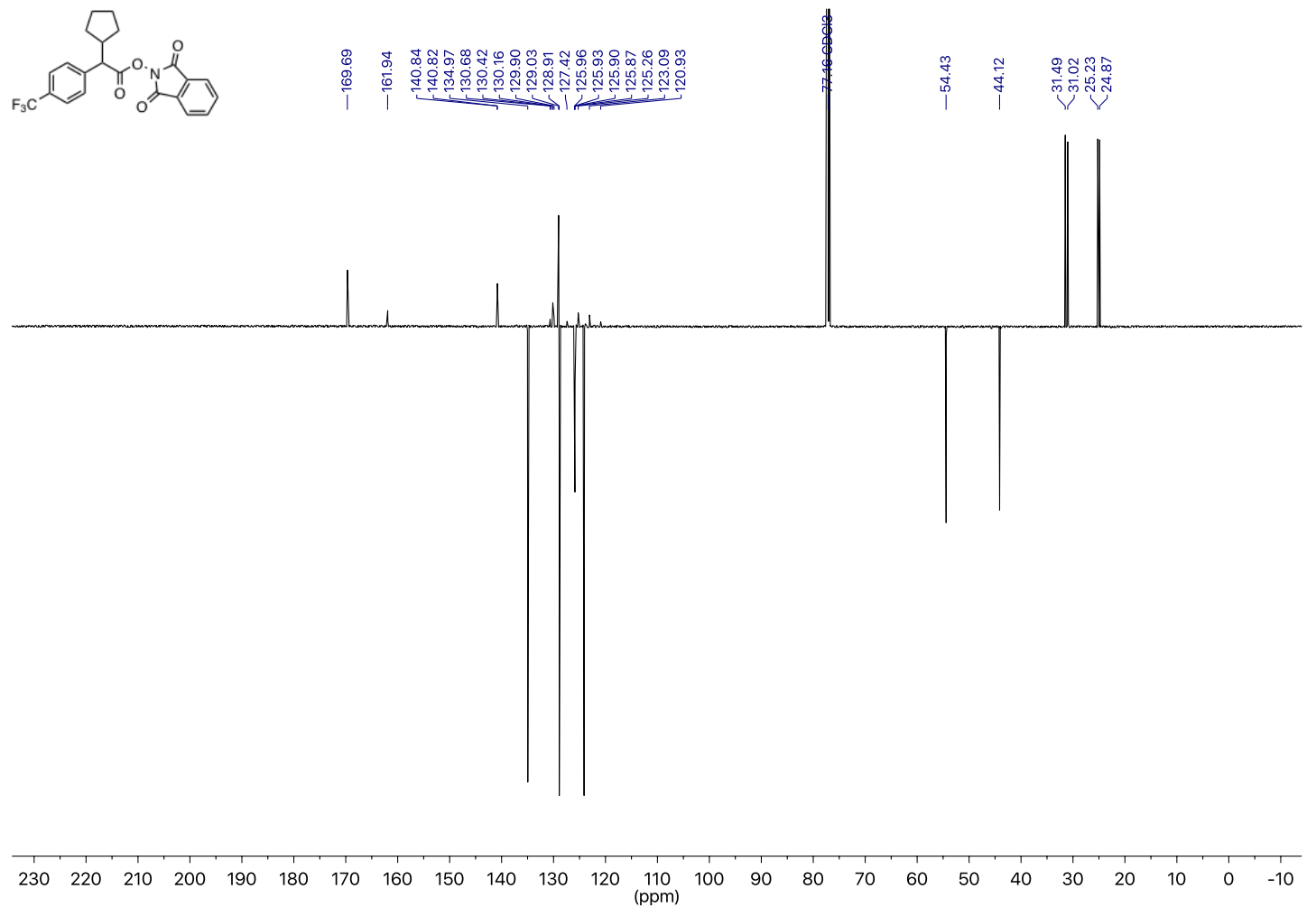

$282 \mathrm{MHz}{ }^{19} \mathrm{~F}-\mathrm{NMR}$ of 1,3-dioxoisoindolin-2-yl 2-cyclopentyl-2-(4-(trifluoromethyl)phenyl)acetate (S12) in $\mathrm{CDCl}_{3}$

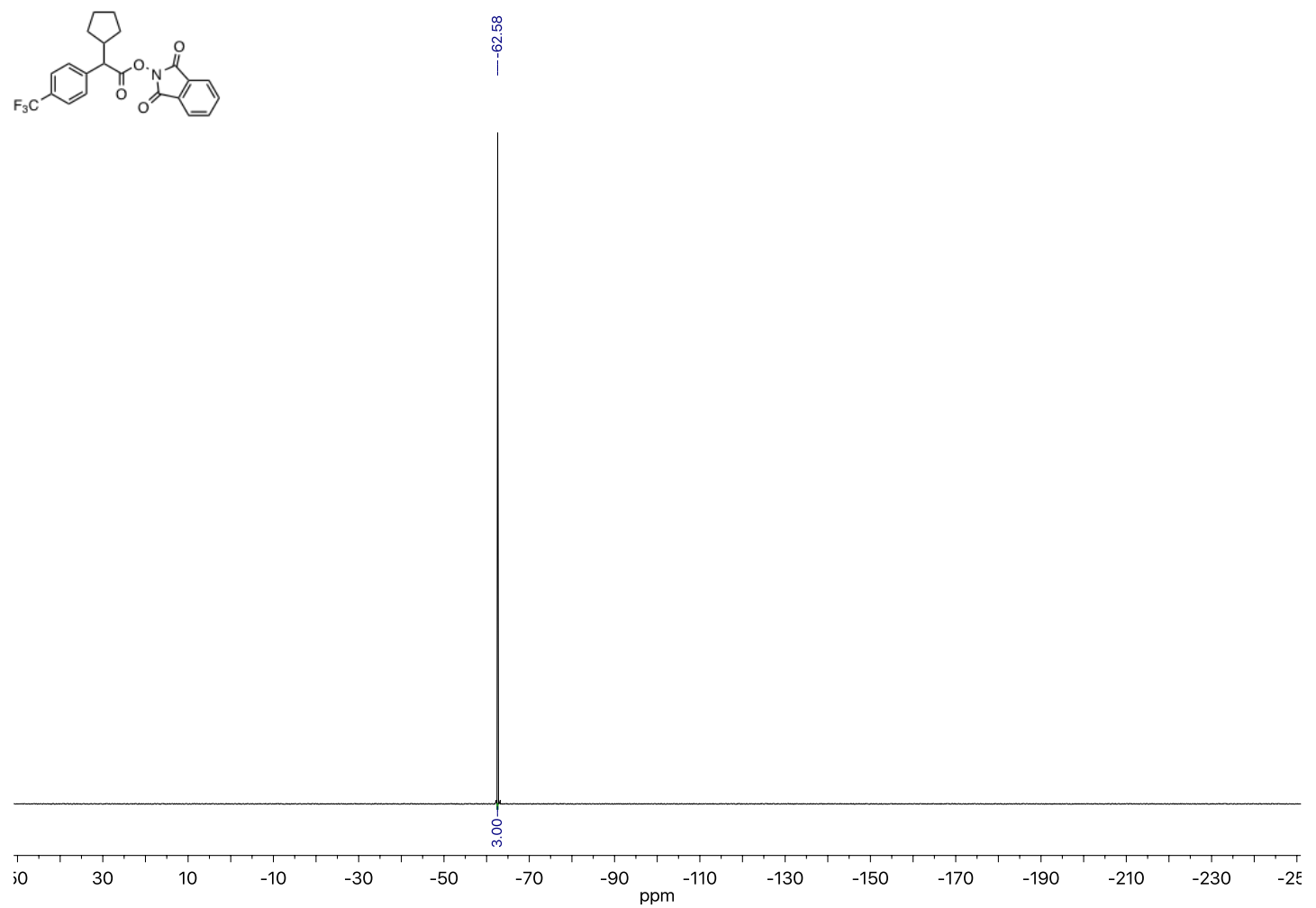


$500 \mathrm{MHz}{ }^{1} \mathrm{H}-\mathrm{NMR}$ of 1-(cyclopentylfluoromethyl)-4-(trifluoromethyl)benzene (17) in $\mathrm{CDCl}_{3}$

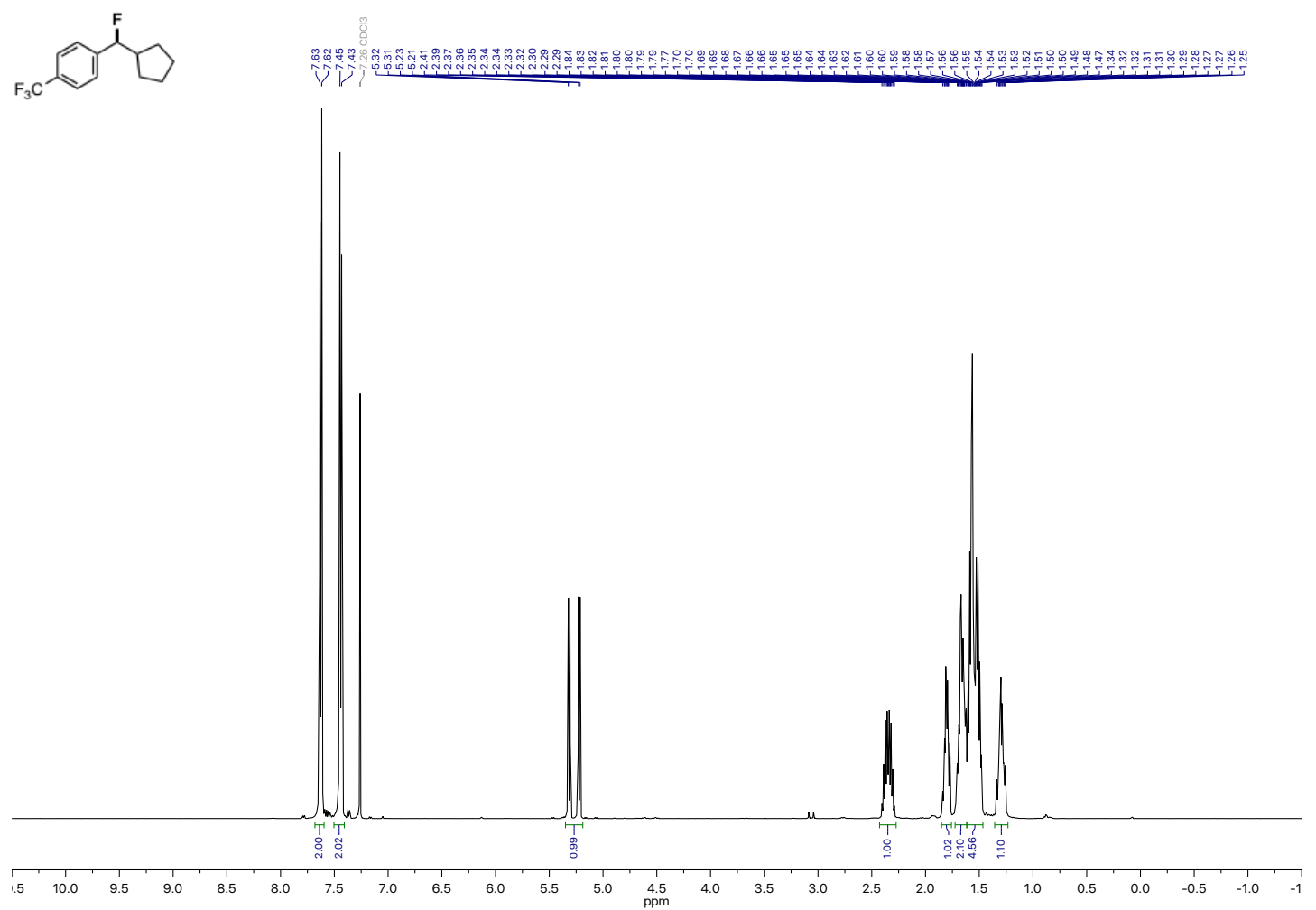

$126 \mathrm{MHz}{ }^{13} \mathrm{C}-\mathrm{NMR}$ of 1-(cyclopentylfluoromethyl)-4-(trifluoromethyl)benzene (17) in $\mathrm{CDCl}_{3}$
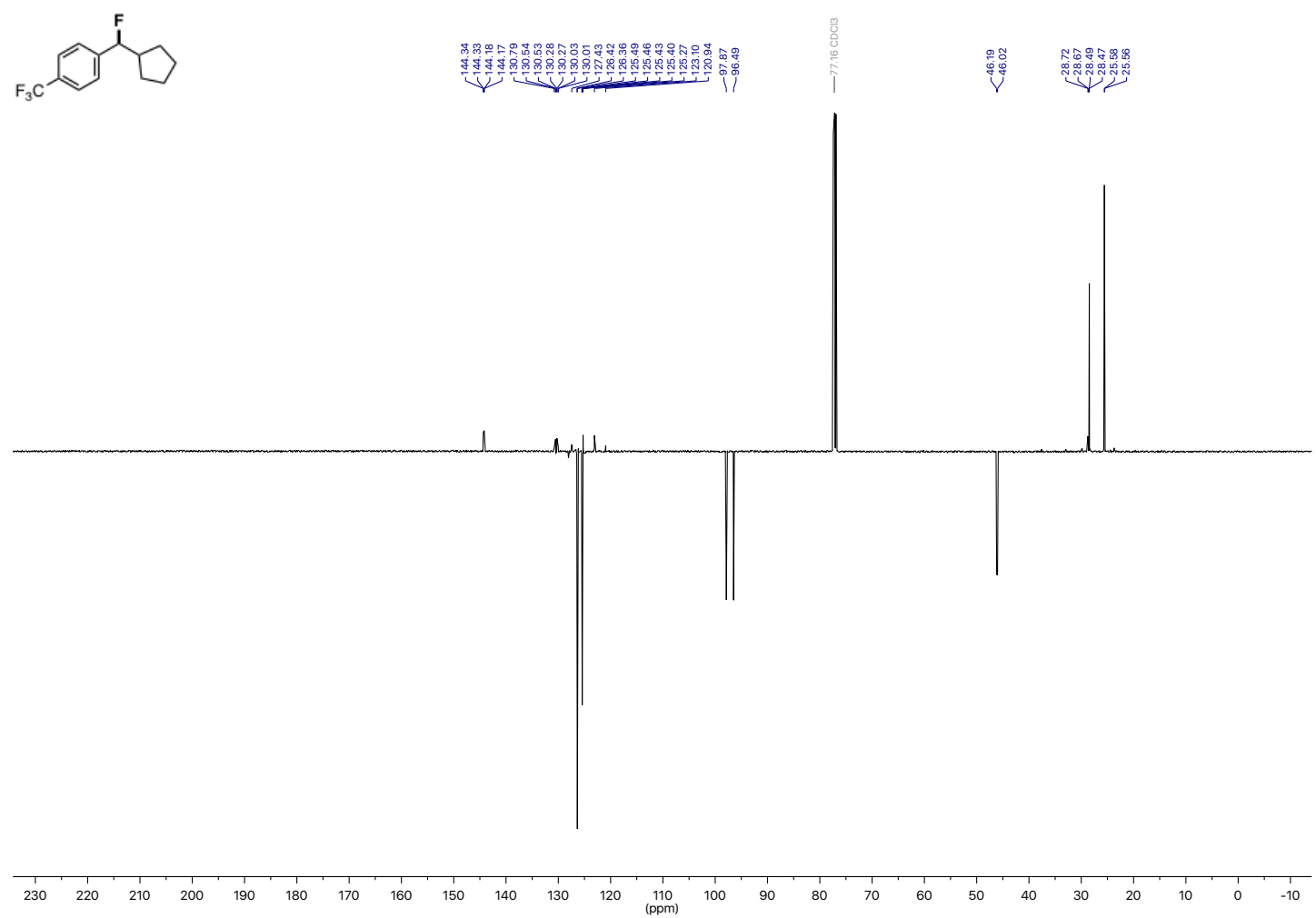
$376 \mathrm{MHz}{ }^{19} \mathrm{~F}-\mathrm{NMR}$ of 1-(cyclopentylfluoromethyl)-4-(trifluoromethyl)benzene (17) in $\mathrm{CDCl}_{3}$
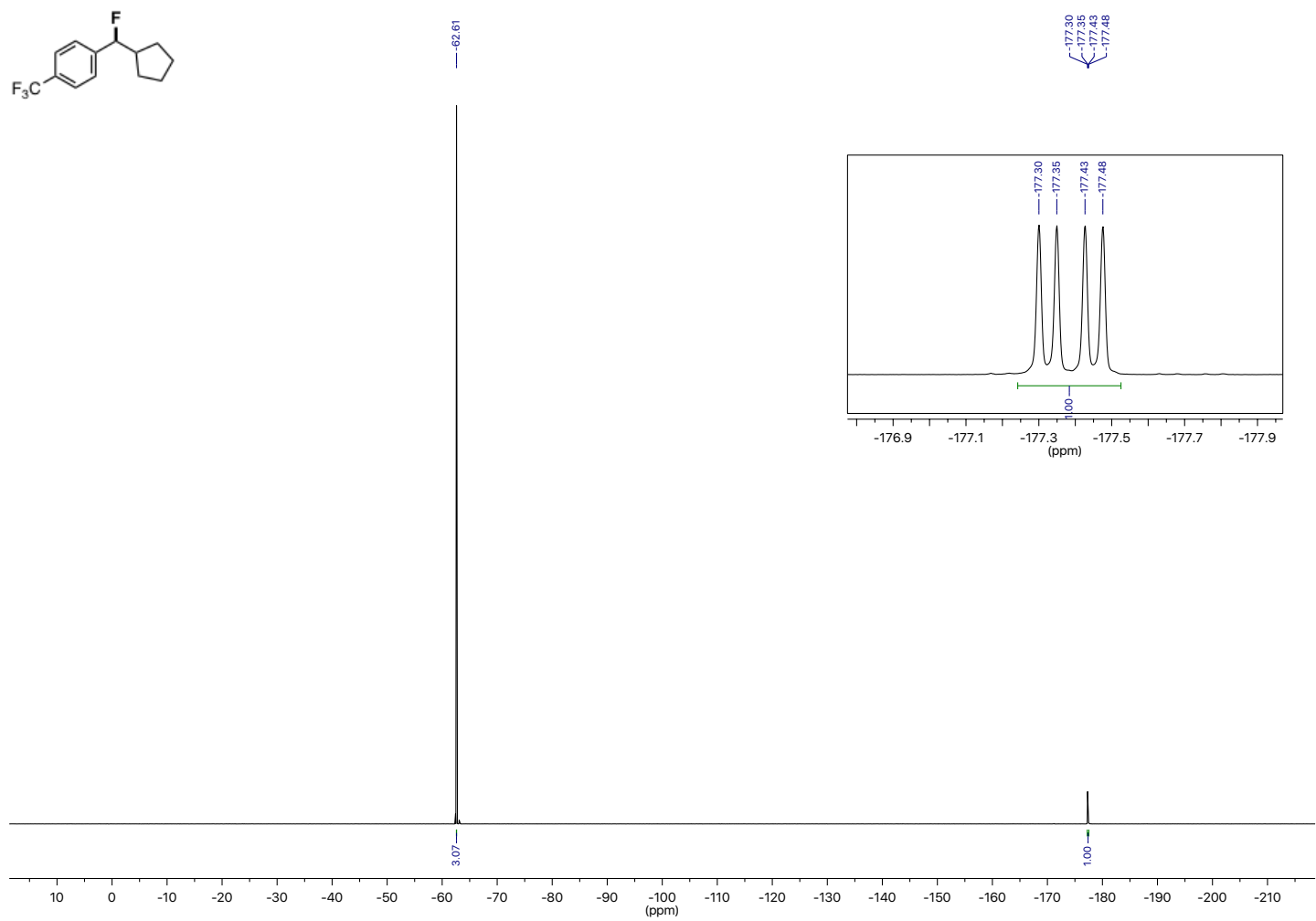

$500 \mathrm{MHz}{ }^{1} \mathrm{H}-\mathrm{NMR}$ of 1,3-dioxoisoindolin-2-yl 2-(6-chloropyridin-3-yl)propanoate (S13) in $\mathrm{CD}_{2} \mathrm{Cl}_{2}$
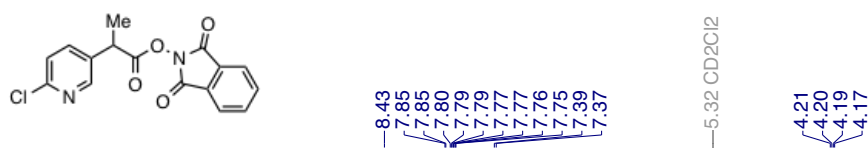

$\frac{800}{4}$

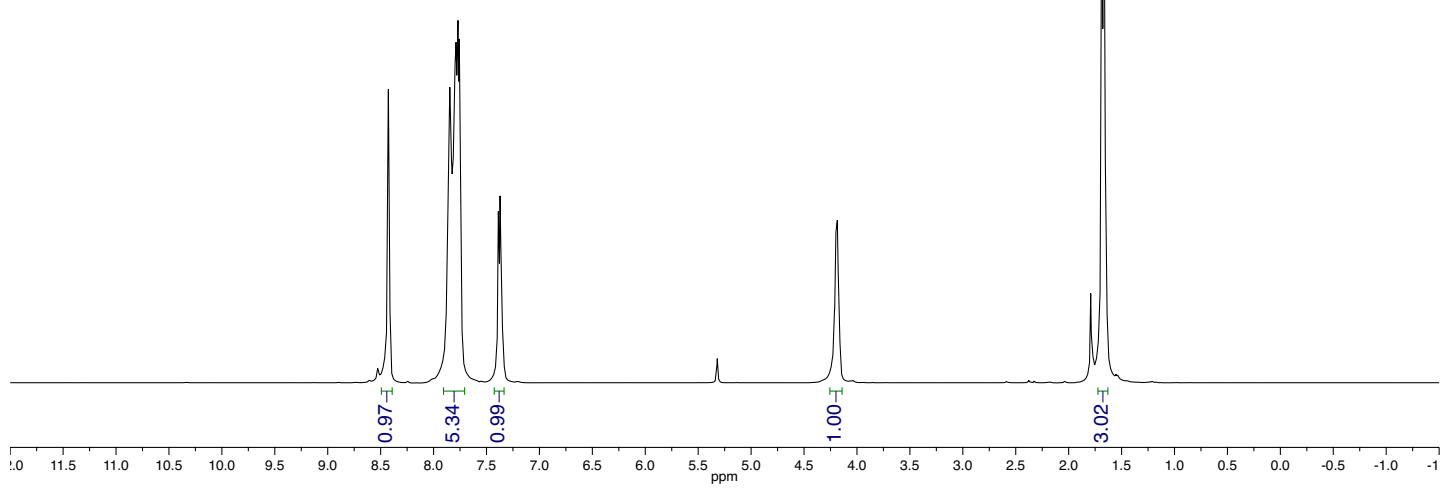


$126 \mathrm{MHz}{ }^{13} \mathrm{C}-\mathrm{NMR}$ of 1,3-dioxoisoindolin-2-yl 2-(6-chloropyridin-3-yl)propanoate (S13) in $\mathrm{CD}_{2} \mathrm{Cl}_{2}$
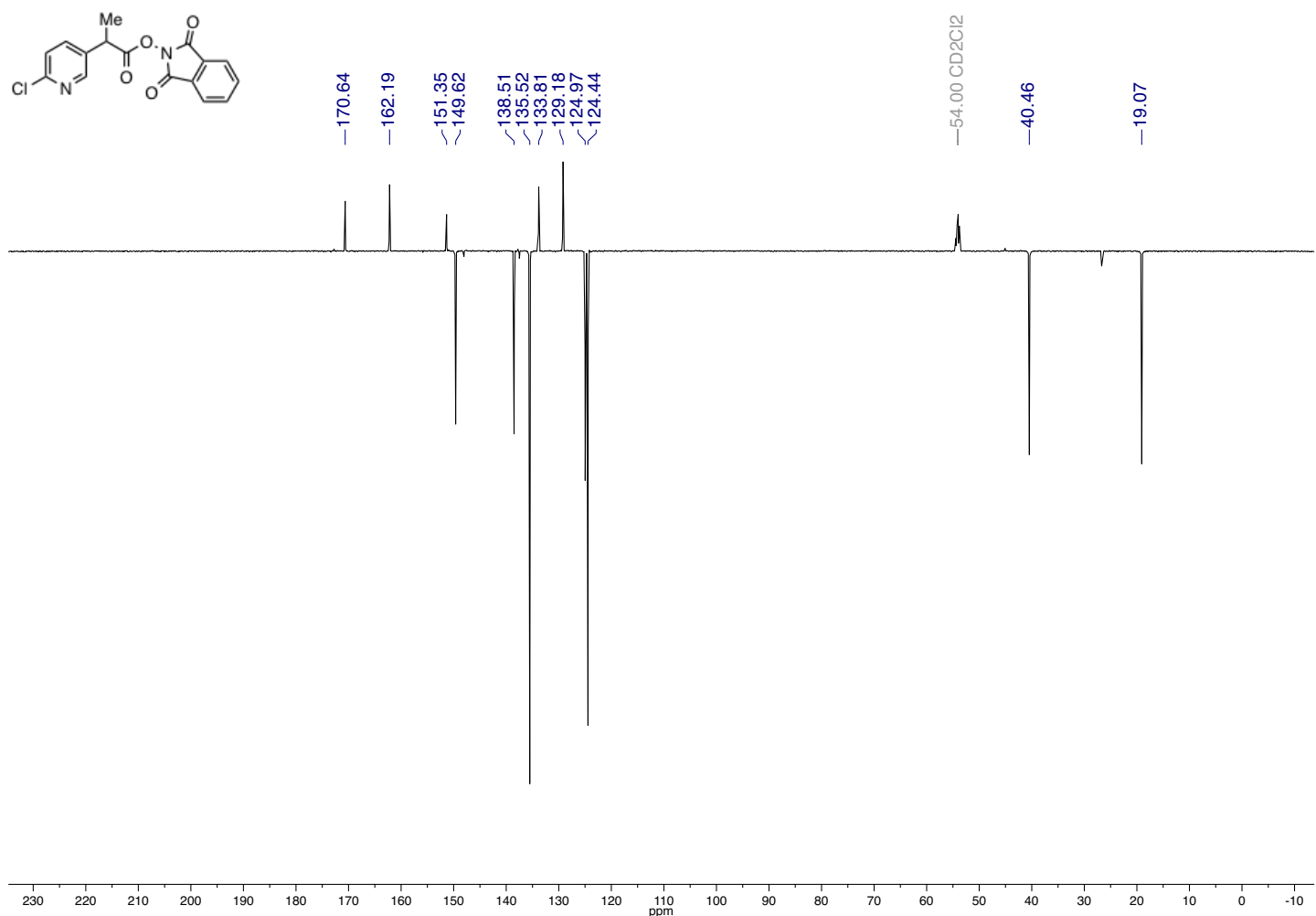

$500 \mathrm{MHz}{ }^{1} \mathrm{H}-\mathrm{NMR}$ of 2-chloro-5-(1-fluoroethyl)pyridine (18) in $\mathrm{CD}_{2} \mathrm{Cl}_{2}$
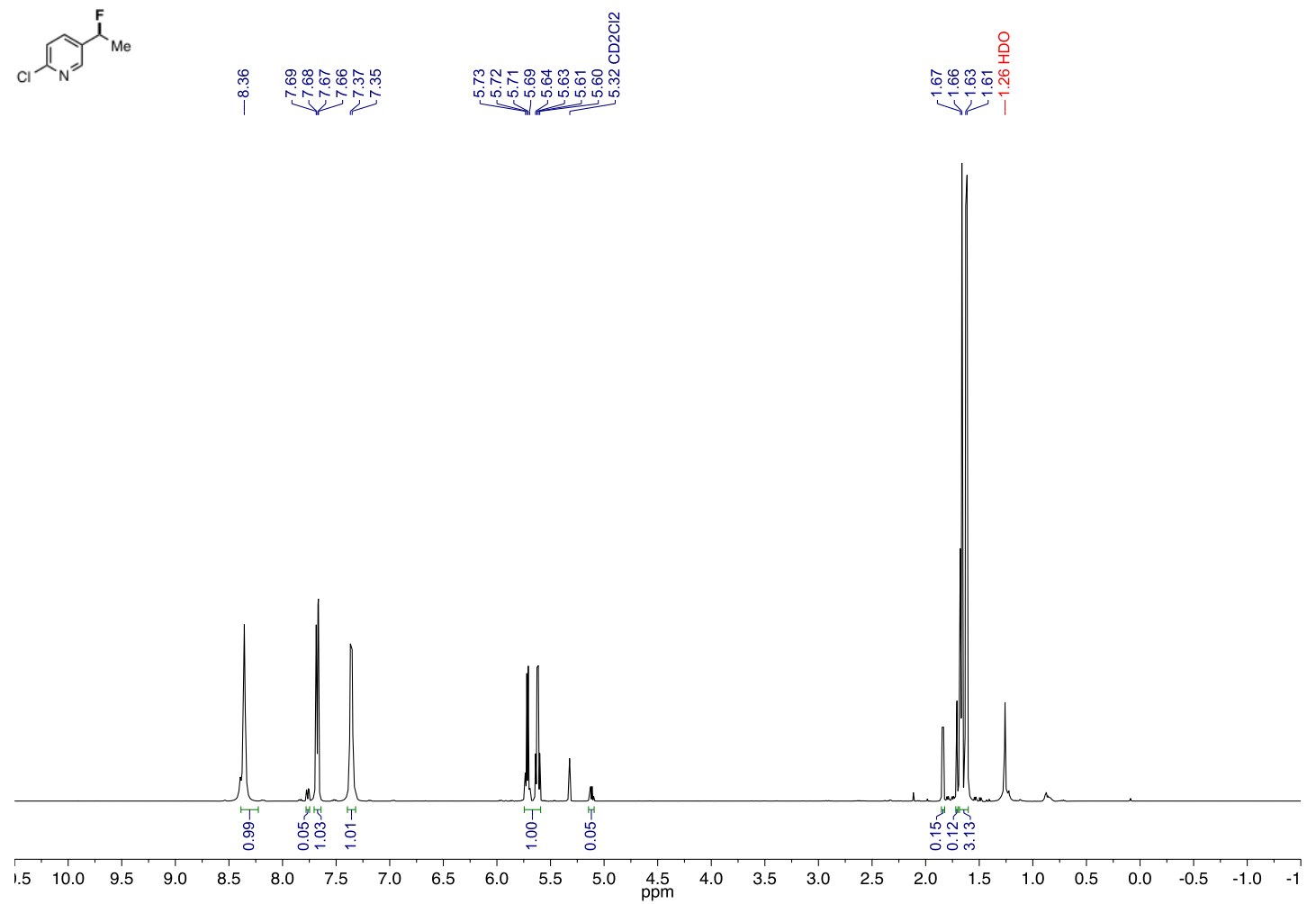
$126 \mathrm{MHz}{ }^{13} \mathrm{C}$-NMR of 2-chloro-5-(1-fluoroethyl)pyridine (18) in $\mathrm{CD}_{2} \mathrm{Cl}_{2}$

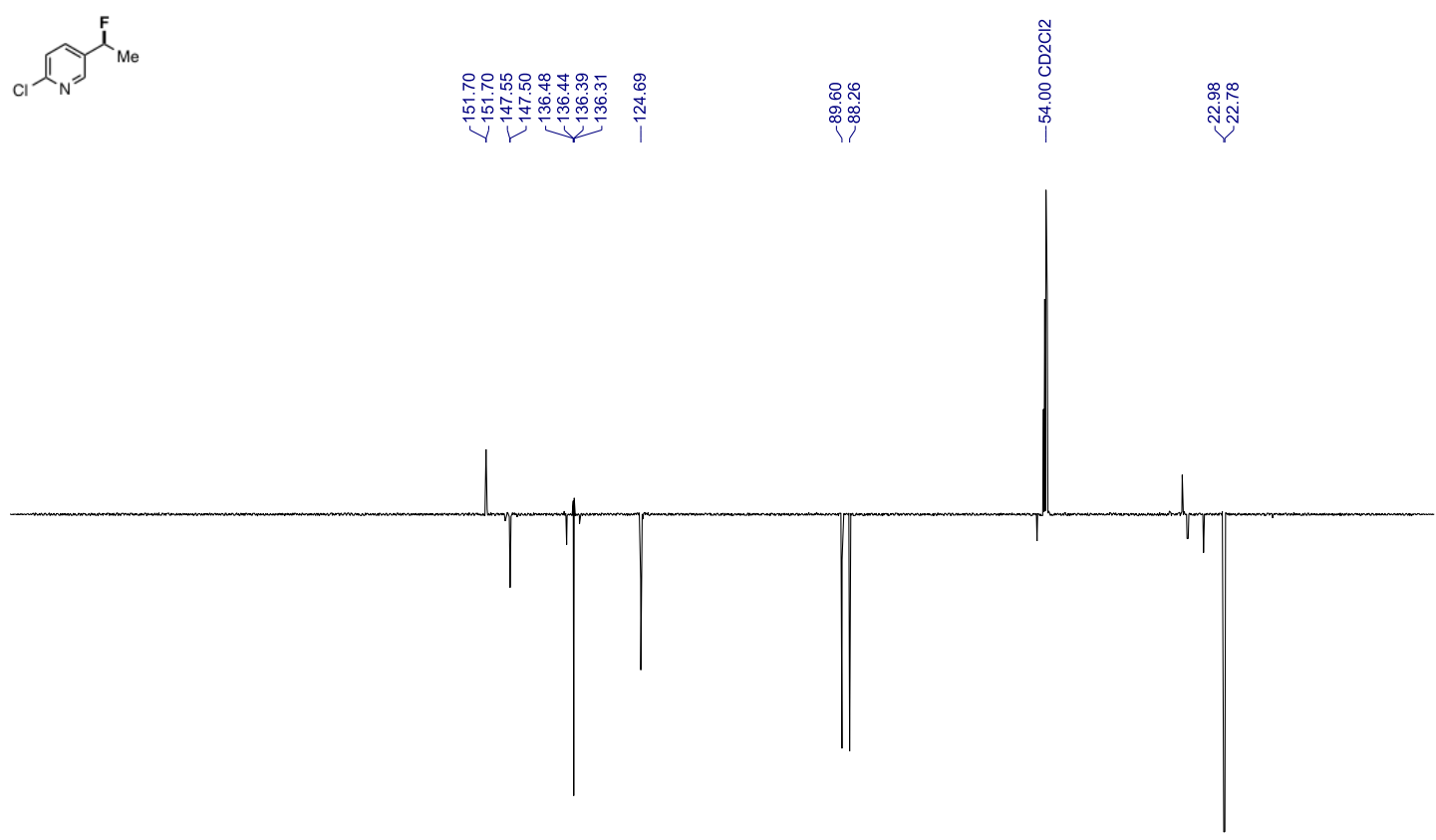

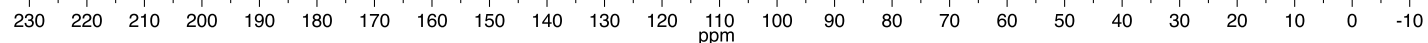

$282 \mathrm{MHz}{ }^{19} \mathrm{~F}$-NMR of 2-chloro-5-(1-fluoroethyl)pyridine (18) in $\mathrm{CD}_{2} \mathrm{Cl}_{2}$

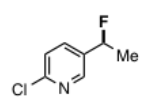

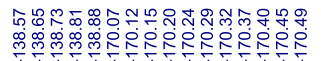
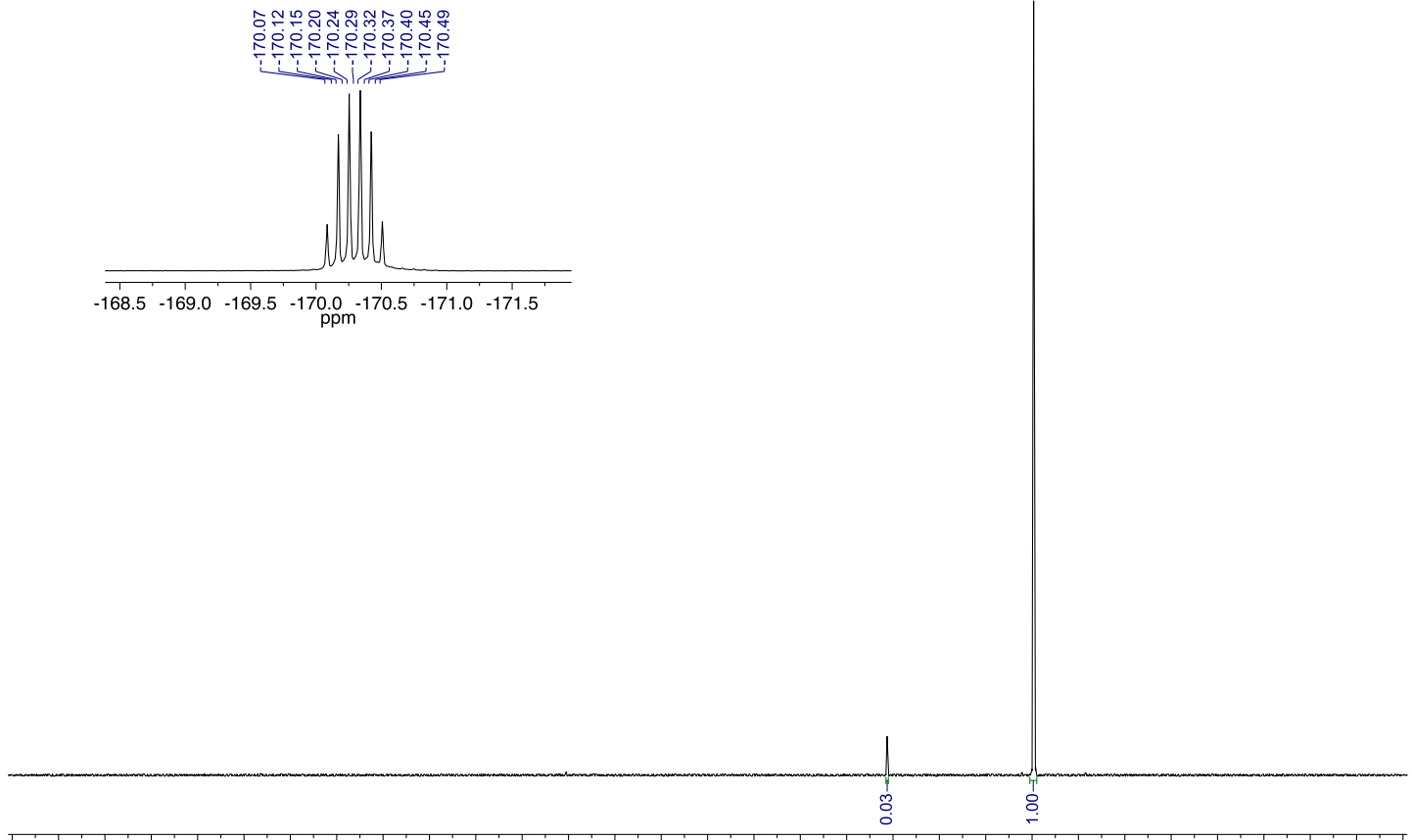

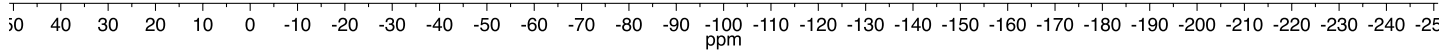


$500 \mathrm{MHz}{ }^{1} \mathrm{H}-\mathrm{NMR}$ of 1,3-dioxoisoindolin-2-yl 4-(dimethylamino)-2-(naphthalen-2-yl)-4-oxobutanoate (S15) in $\mathrm{CDCl}_{3}$

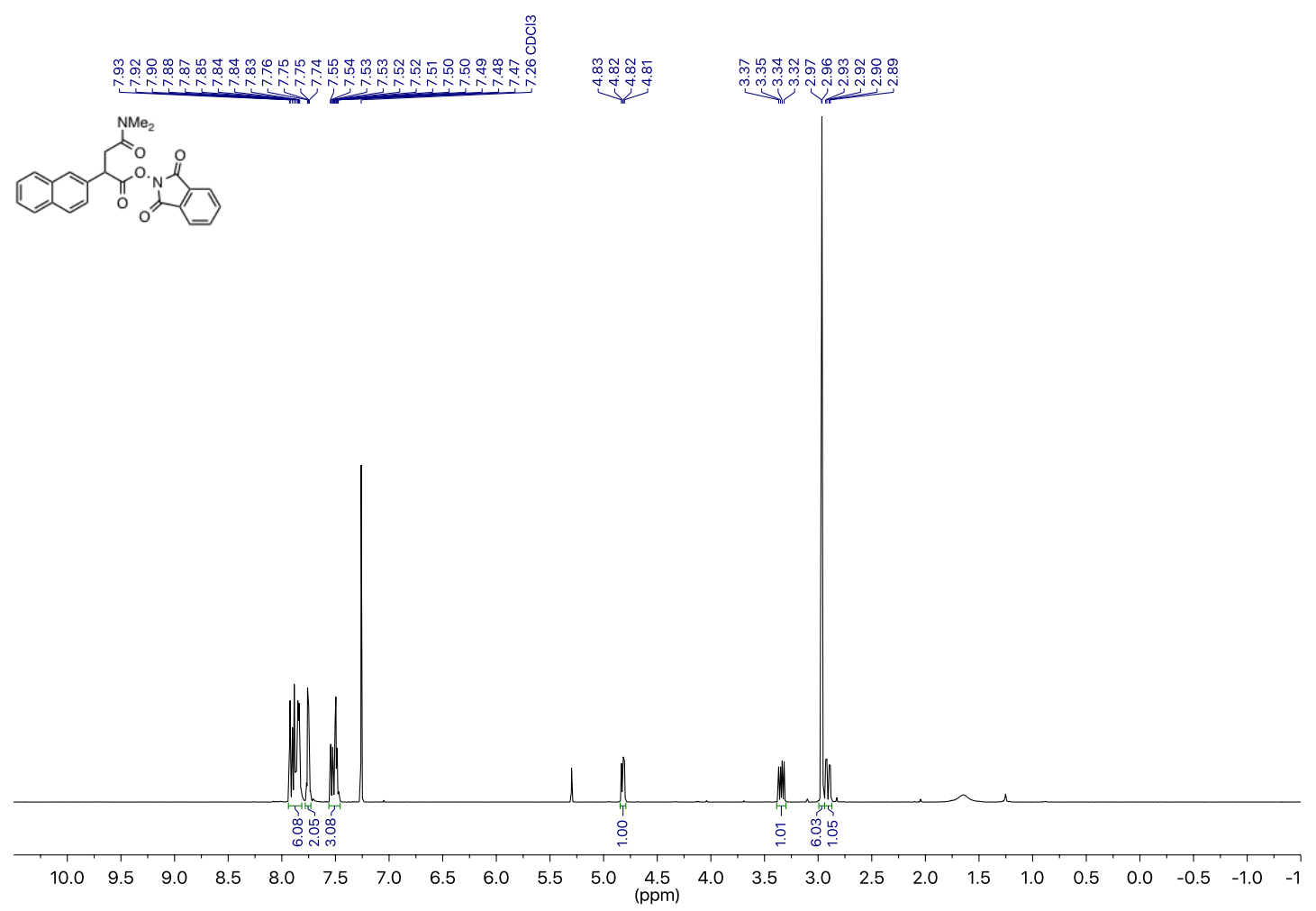


$126 \mathrm{MHz}{ }^{13} \mathrm{C}-\mathrm{NMR}$ of 1,3-dioxoisoindolin-2-yl 4-(dimethylamino)-2-(naphthalen-2-yl)-4-oxobutanoate (S14) in $\mathrm{CDCl}_{3}$

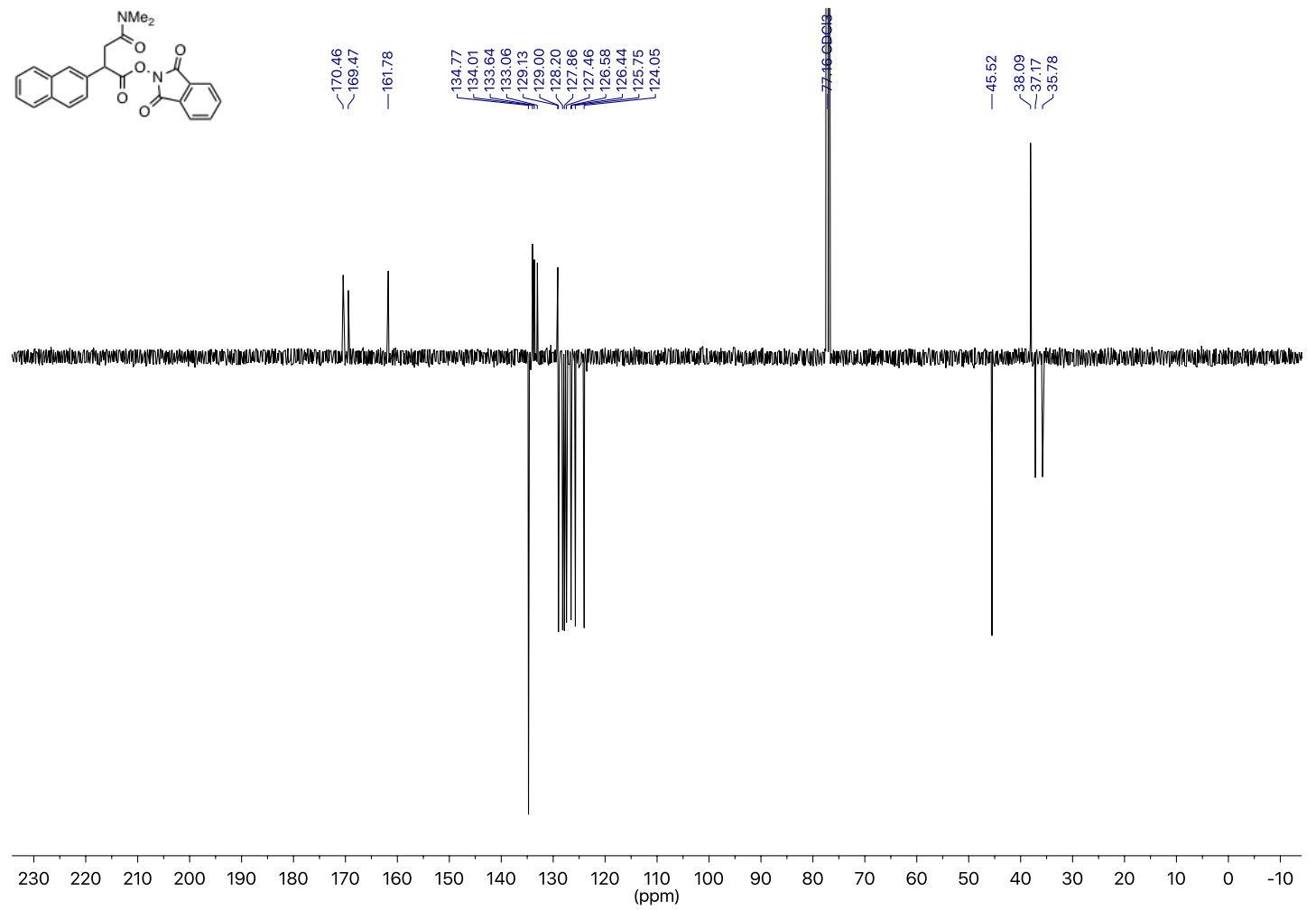


$500 \mathrm{MHz}{ }^{1} \mathrm{H}-\mathrm{NMR}$ of ( \pm )-3-fluoro- $N, N$-dimethyl-3-(naphthalen-2-yl)propenamide (19) in $\mathrm{CDCl}_{3}$

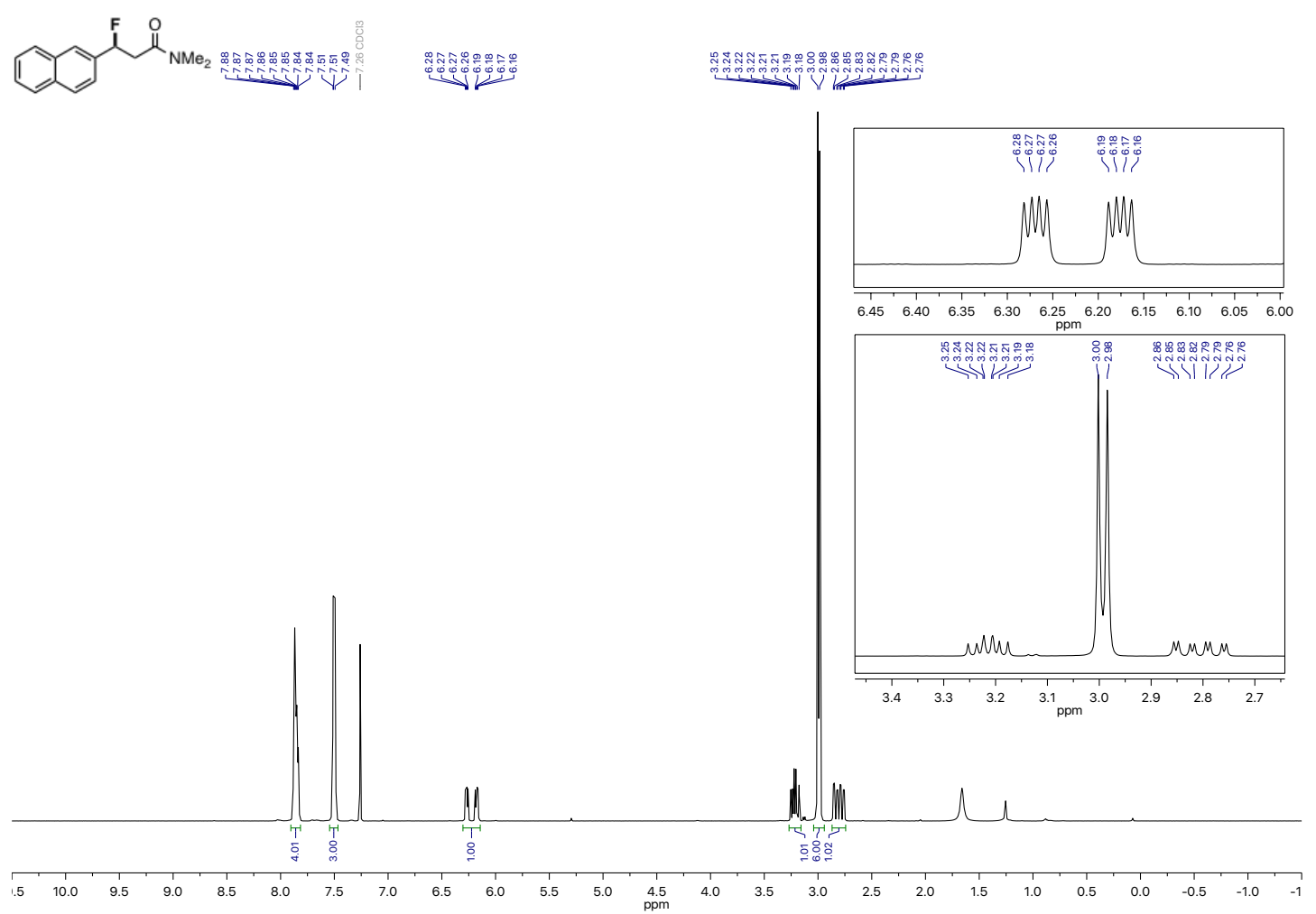

$126 \mathrm{MHz}{ }^{13} \mathrm{C}-\mathrm{NMR}$ of 3-fluoro- $N, N$-dimethyl-3-(naphthalen-2-yl)propenamide (19) in $\mathrm{CDCl}_{3}$

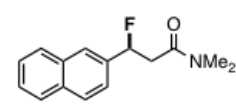

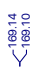
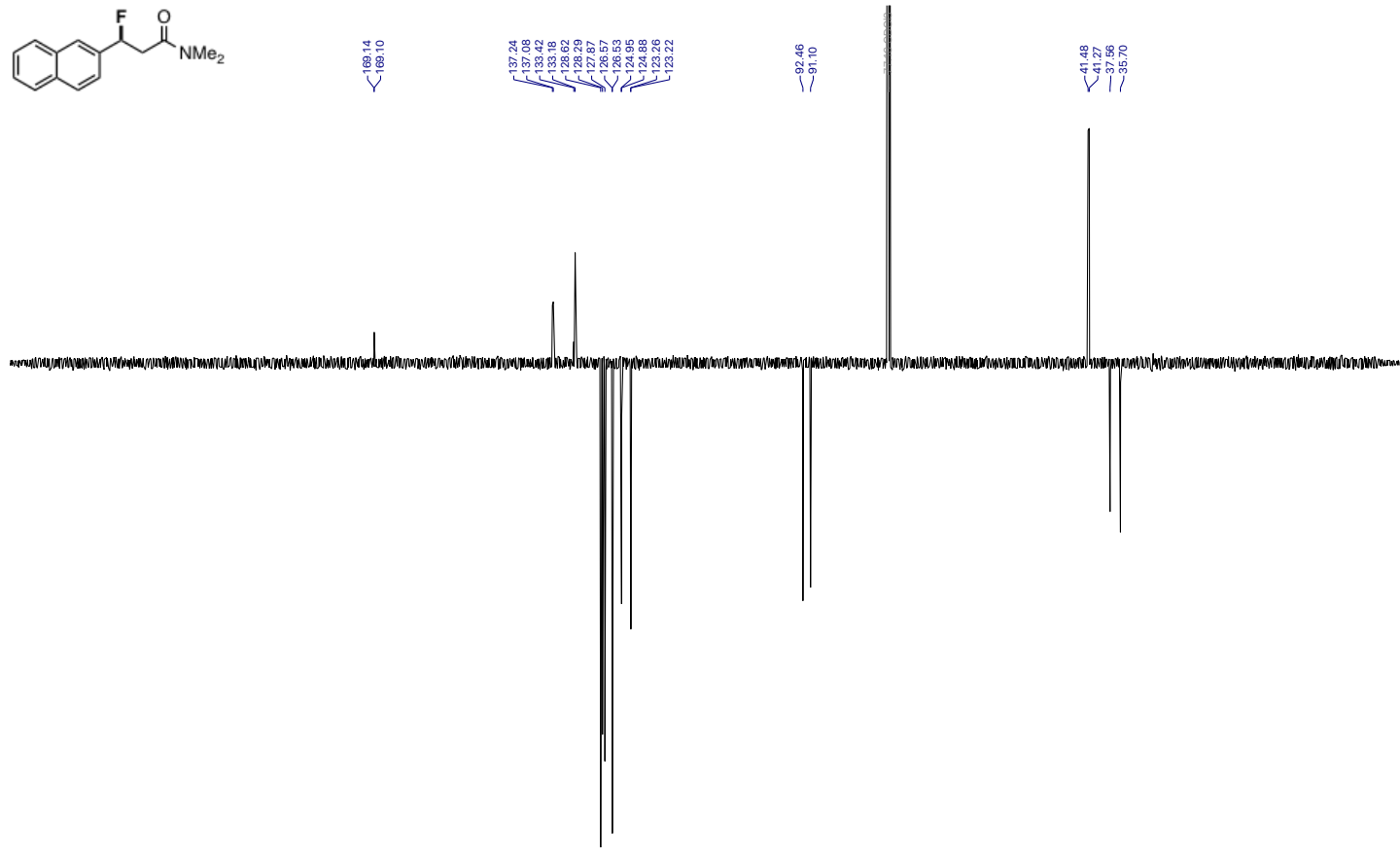

$\begin{array}{lllllllllll}230 & 220 & 210 & 200 & 190 & 180 & 170 & 160 & 150 & 140 & 130\end{array}$ 
$282 \mathrm{MHz}{ }^{19} \mathrm{~F}-\mathrm{NMR}$ of ( \pm )-3-fluoro- $N, N$-dimethyl-3-(naphthalen-2-yl)propenamide (19) in $\mathrm{CDCl}_{3}$
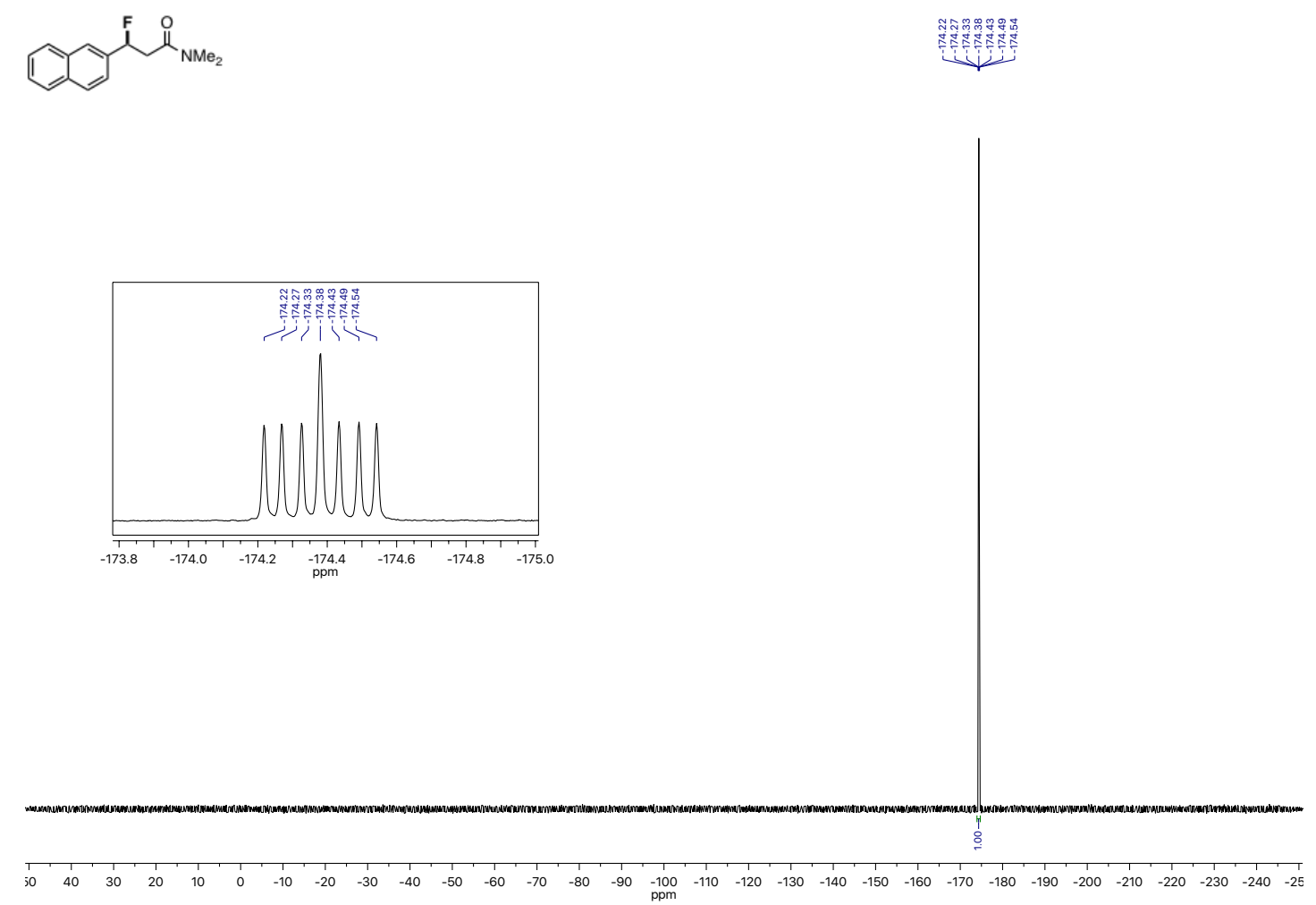

$500 \mathrm{MHz}$ 1H-NMR of 1,3-dioxoisoindolin-2-yl 2-(4-(azidomethyl)phenyl)propanoate (S15) in $\mathrm{CDCl}_{3}$
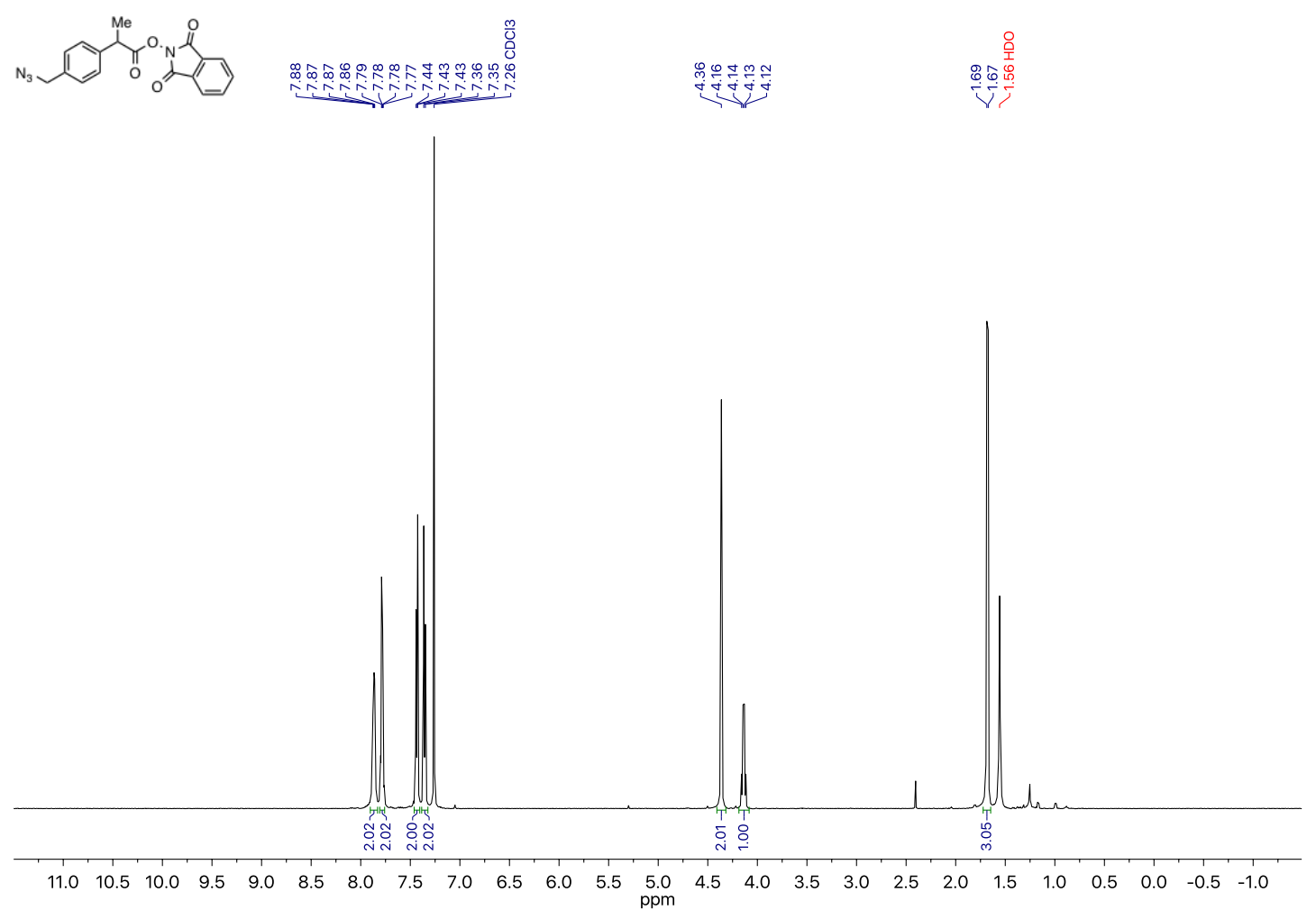
$126 \mathrm{MHz}{ }^{13} \mathrm{C}-\mathrm{NMR}$ of 1,3-dioxoisoindolin-2-yl 2-(4-(azidomethyl)phenyl)propanoate (S15) in $\mathrm{CDCl}_{3}$ N

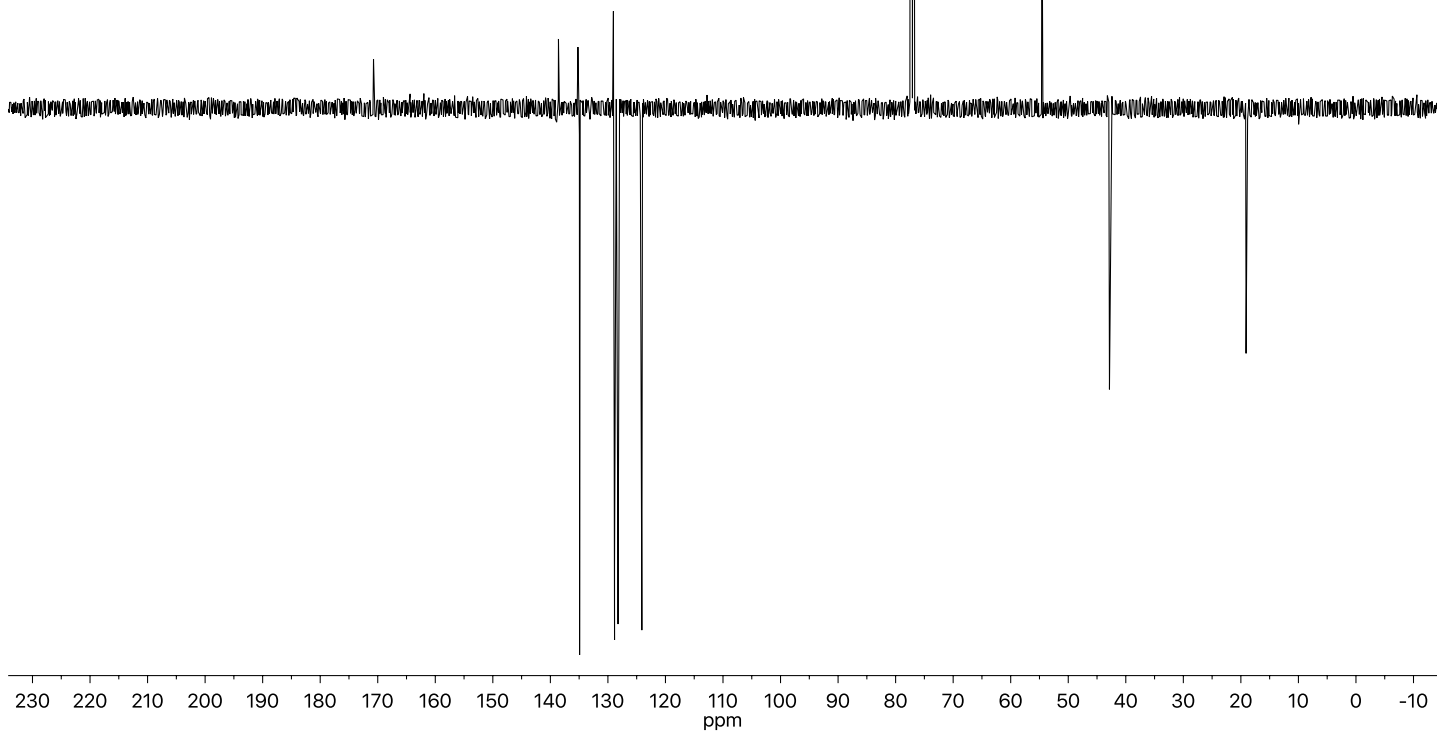

$500 \mathrm{MHz}{ }^{1} \mathrm{H}$-NMR of 1-(azidomethyl)-4-(1-fluoroethyl)benzene (20) in $\mathrm{CDCl}_{3}$<smiles>C[C](C)c1ccc(CN)cc1</smiles>
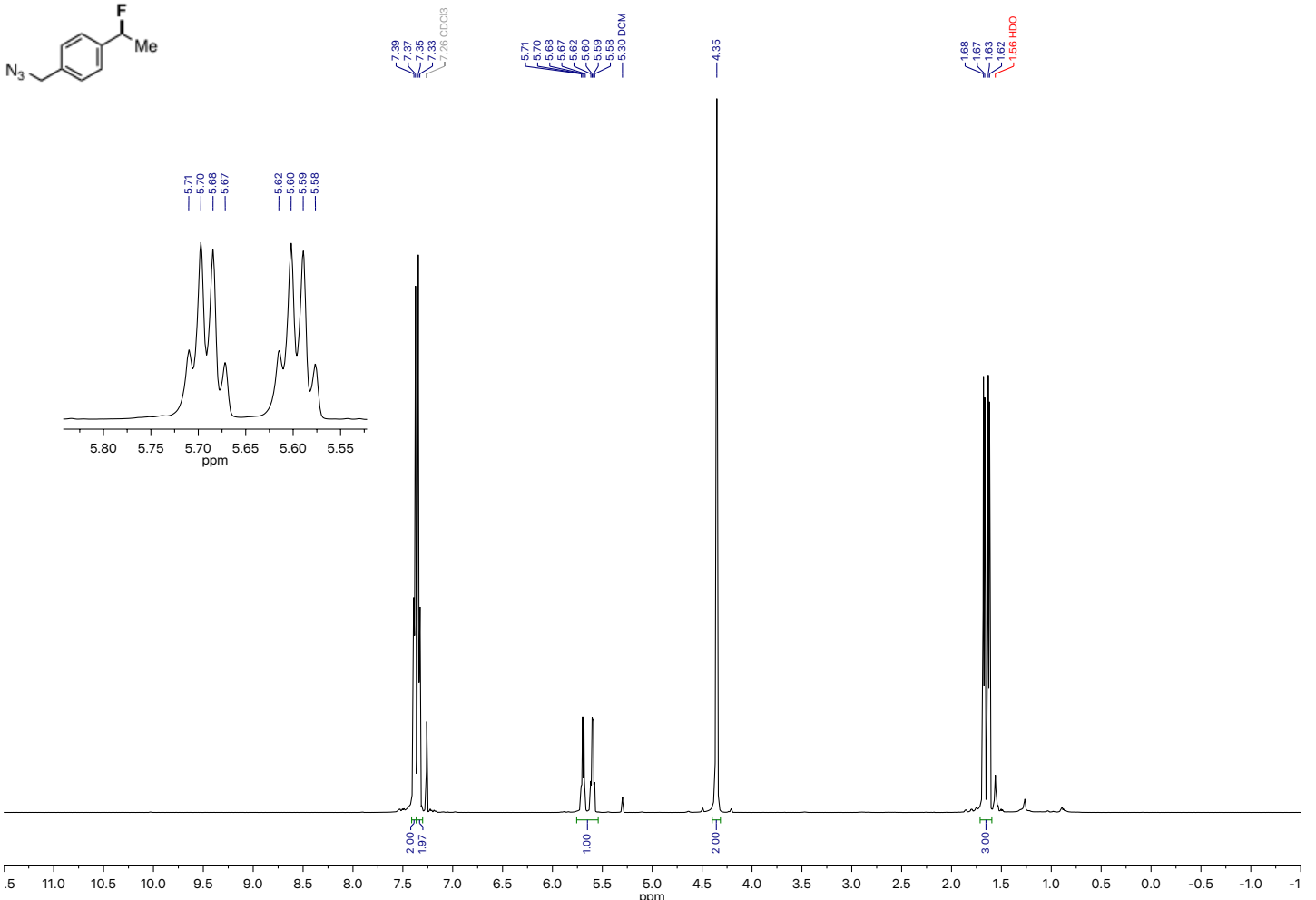
$126 \mathrm{MHz}{ }^{13} \mathrm{C}-\mathrm{NMR}$ of 1-(azidomethyl)-4-(1-fluoroethyl)benzene (20) in $\mathrm{CDCl}_{3}$

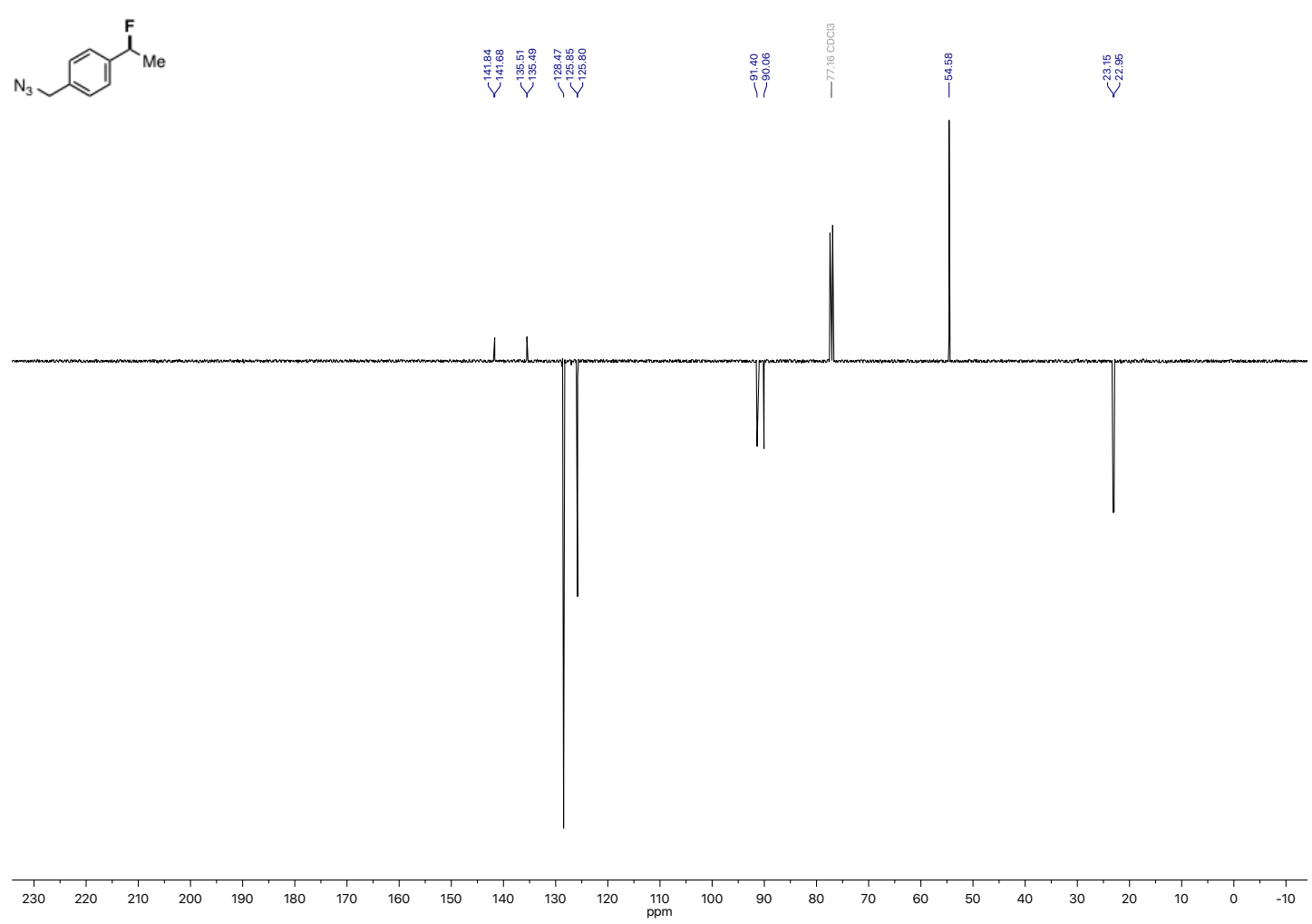

$376 \mathrm{MHz}{ }^{19} \mathrm{~F}-\mathrm{NMR}$ of 1-(azidomethyl)-4-(1-fluoroethyl)benzene (20) in $\mathrm{CDCl}_{3}$
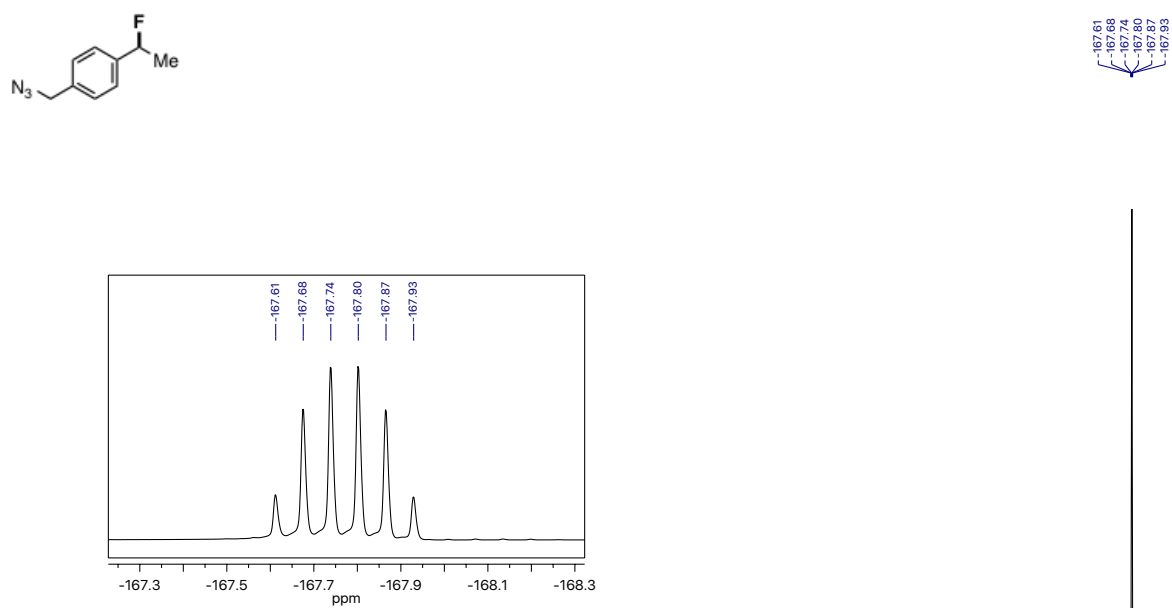

'T-'

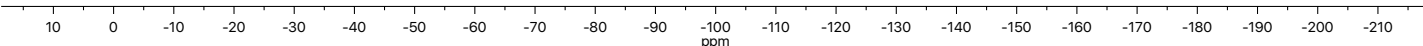


$500 \mathrm{MHz}{ }^{1} \mathrm{H}-\mathrm{NMR}$ of 1,3-dioxoisoindolin-2-yl 2-(3-benzoylphenyl)propanoate (S16) in $\mathrm{CDCl}_{3}$

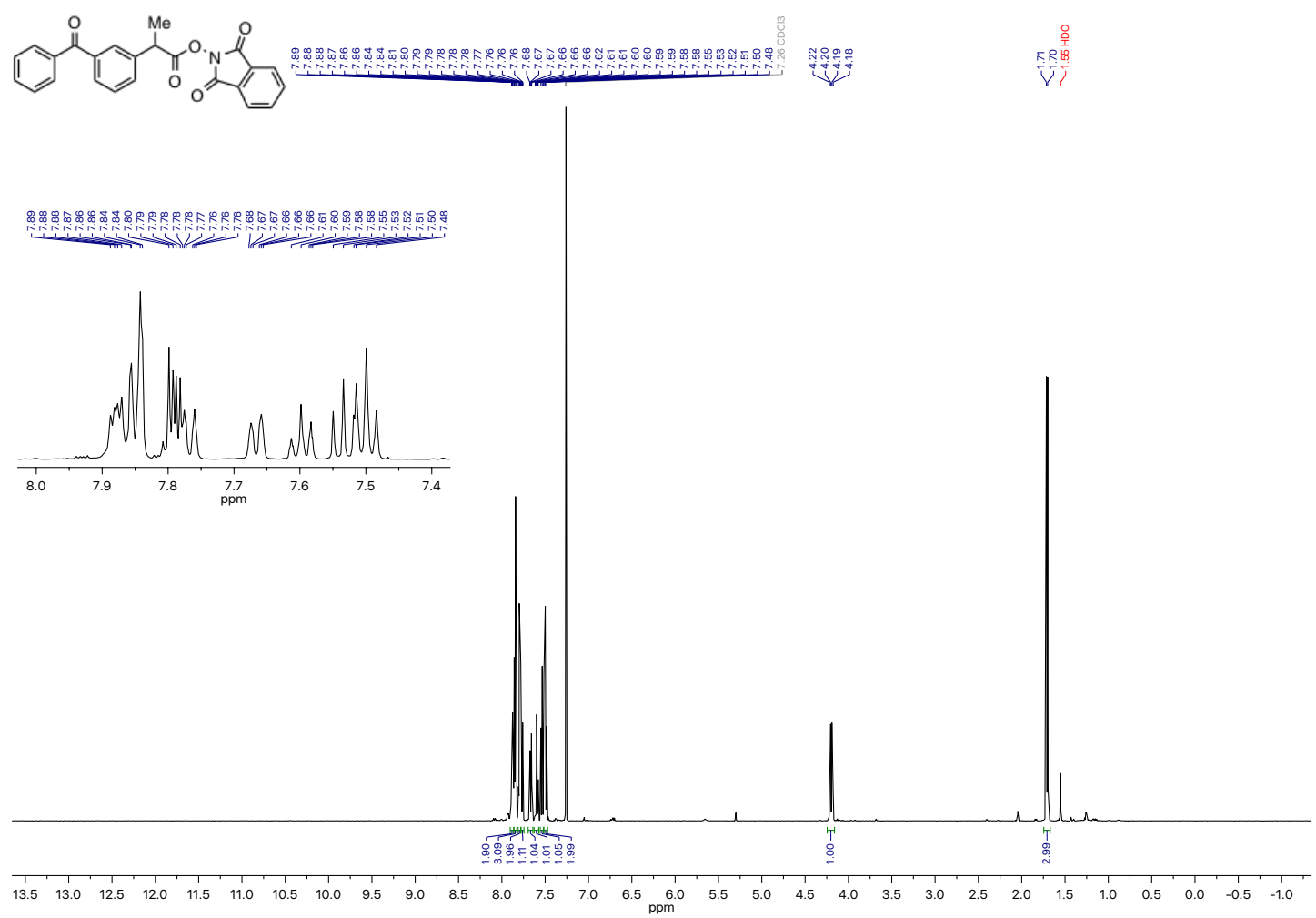

$126 \mathrm{MHz}{ }^{13} \mathrm{C}$-NMR of 1,3-dioxoisoindolin-2-yl 2-(3-benzoylphenyl)propanoate (S16) in $\mathrm{CDCl}_{3}$

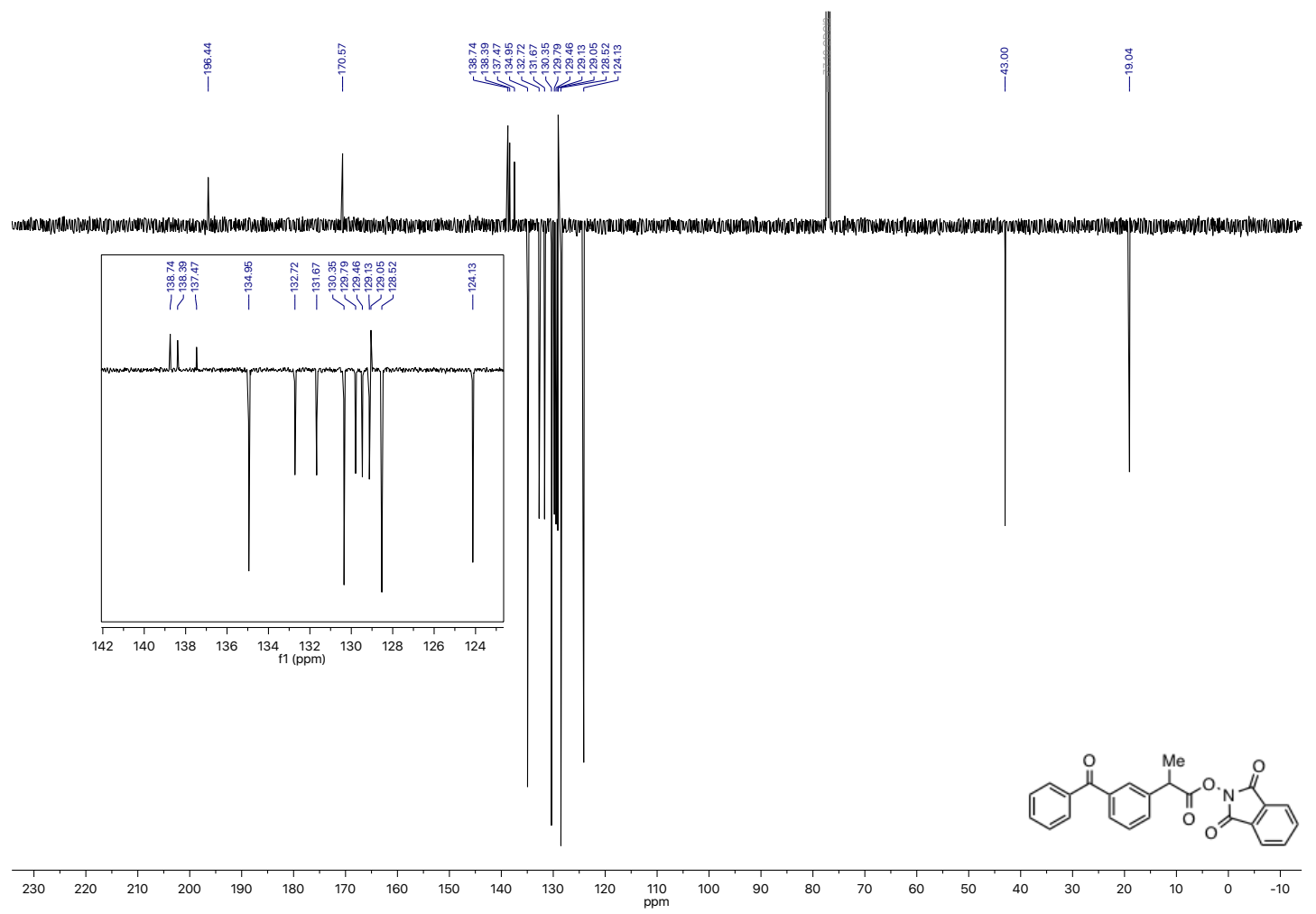


$500 \mathrm{MHz}{ }^{1} \mathrm{H}-\mathrm{NMR}$ of (3-(1-fluoroethyl)phenyl)(phenyl)methanone (21) in $\mathrm{CDCl}_{3}$

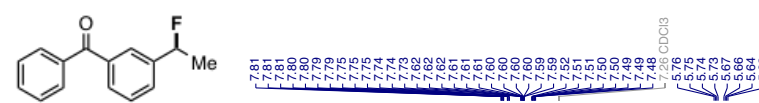

ن|

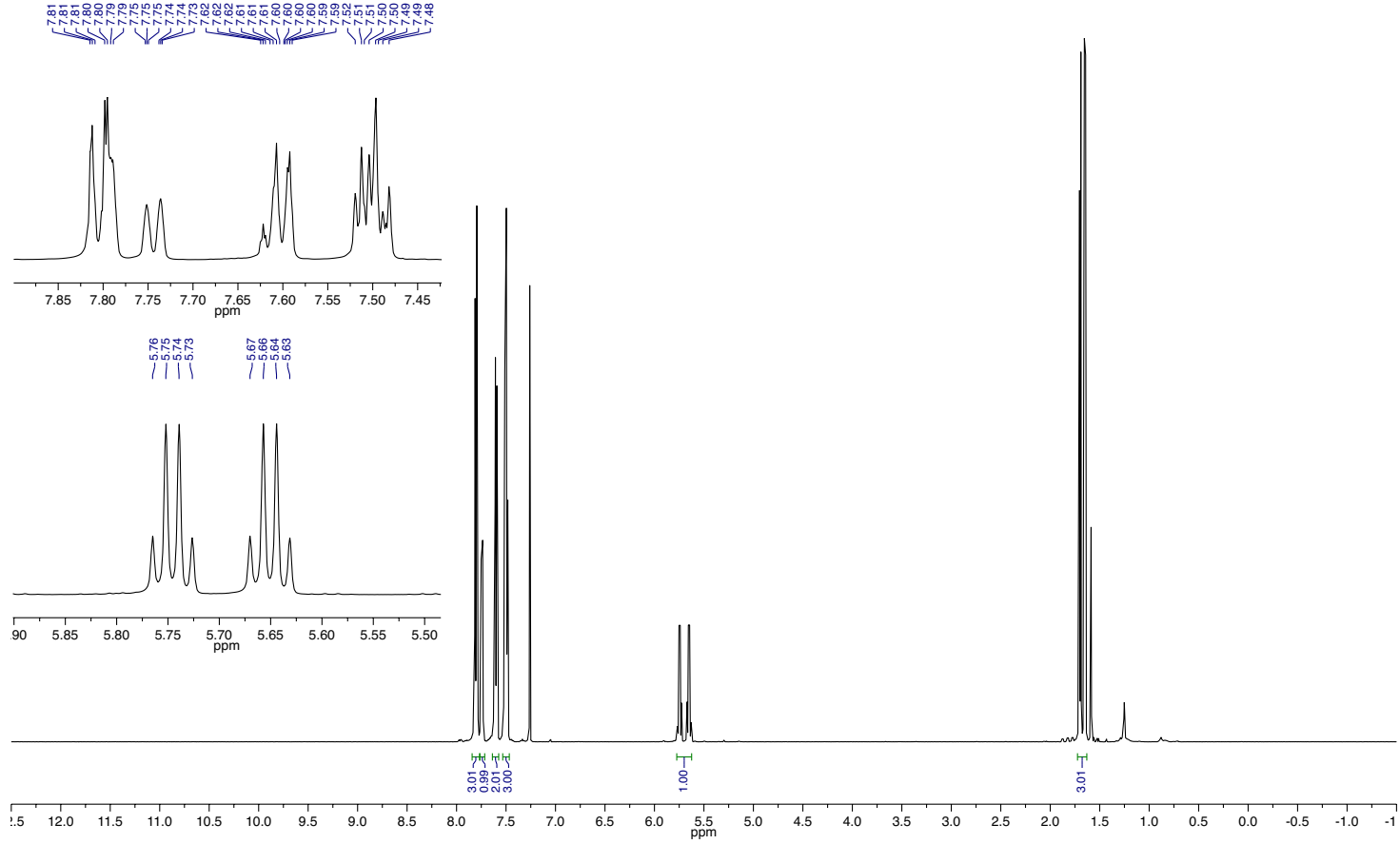

$126 \mathrm{MHz}{ }^{13} \mathrm{C}$-NMR of (3-(1-fluoroethyl)phenyl)(phenyl)methanone (21) in $\mathrm{CDCl}_{3}$
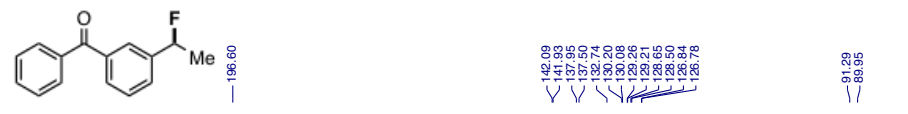

疎跑

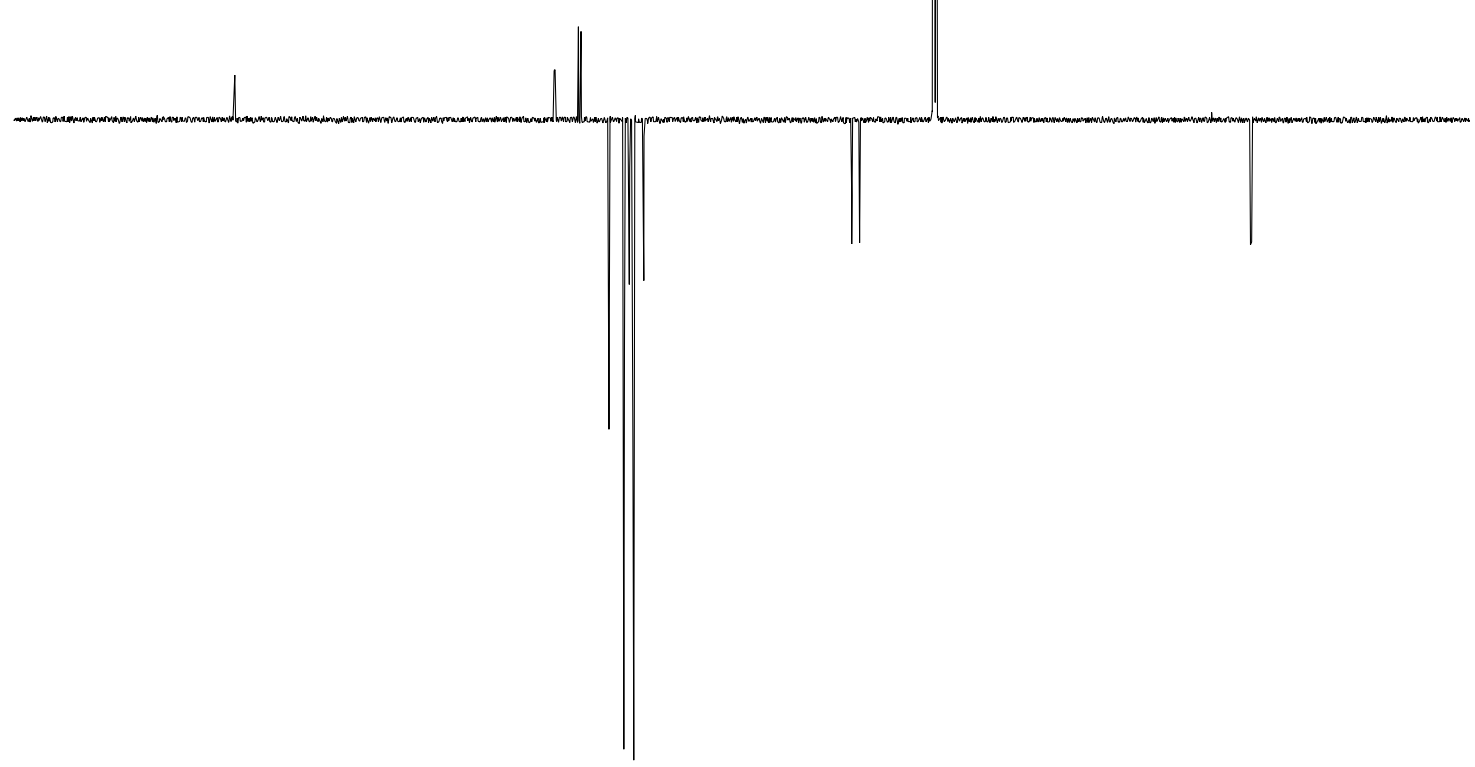

$\begin{array}{llllllllllll}230 & 220 & 210 & 200 & 190 & 180 & 170 & 160 & 150 & 140 & 130 & 120\end{array}$ 
$376 \mathrm{MHz}{ }^{19} \mathrm{~F}-\mathrm{NMR}$ of (3-(1-fluoroethyl)phenyl)(phenyl)methanone (21) in $\mathrm{CDCl}_{3}$
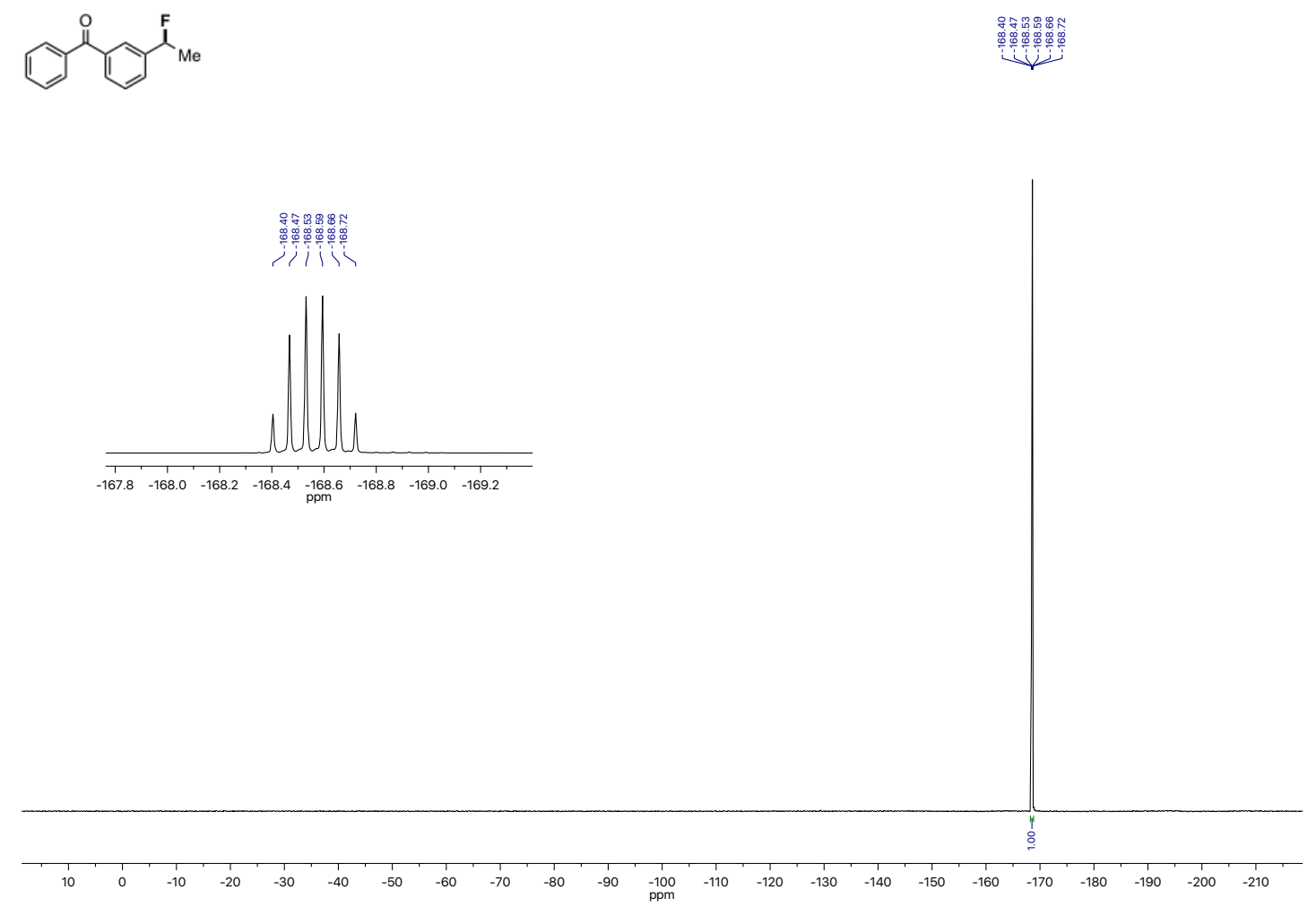

$500 \mathrm{MHz}{ }^{1} \mathrm{H}-\mathrm{NMR}$ of 1,3-dioxoisoindolin-2-yl 2-methyl-2-phenylpropanoate (S17) in $\mathrm{CDCl}_{3}$
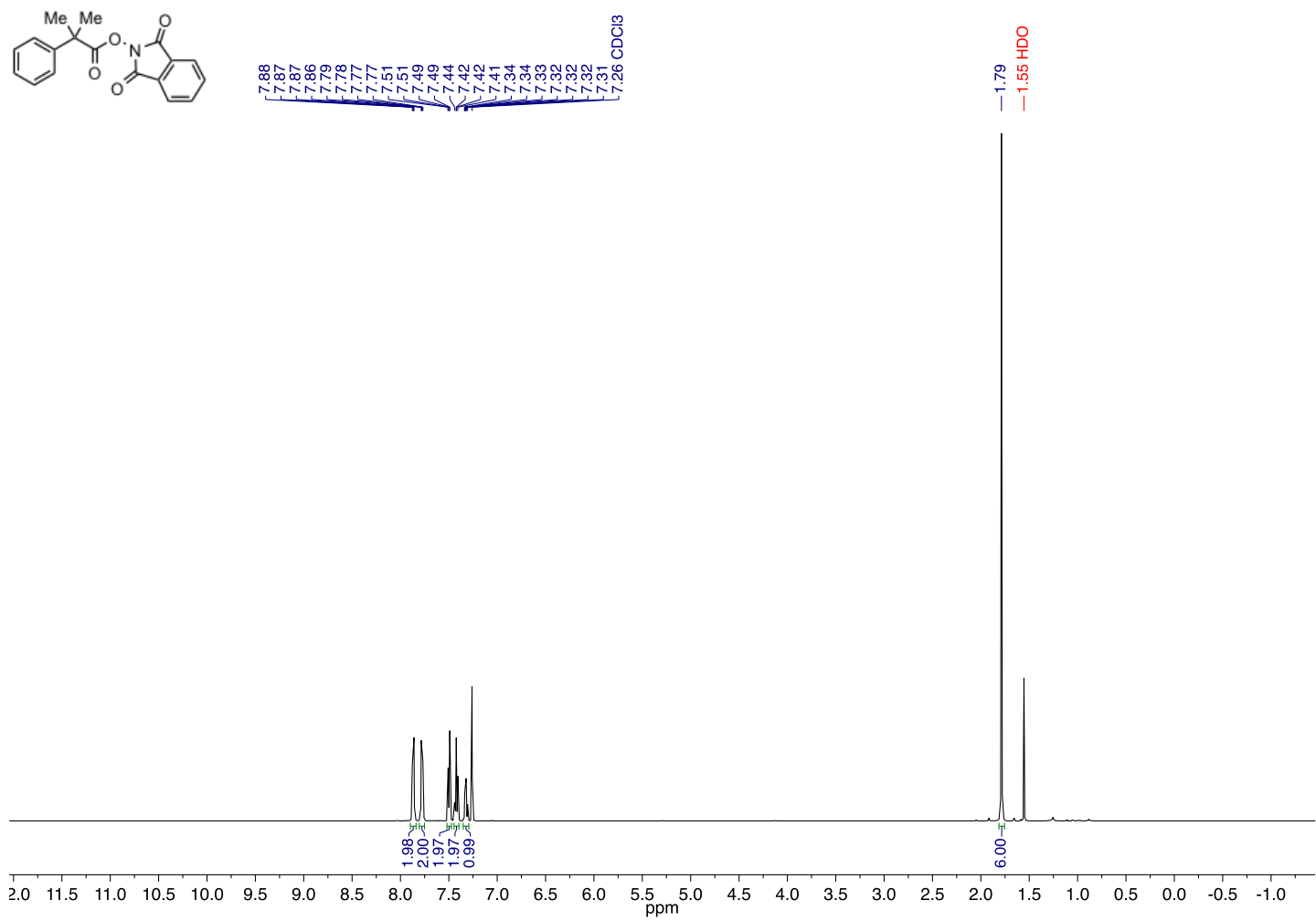
$126 \mathrm{MHz}{ }^{13} \mathrm{C}-\mathrm{NMR}$ of 1,3-dioxoisoindolin-2-yl 2-methyl-2-phenylpropanoate (S17) in $\mathrm{CDCl}_{3}$

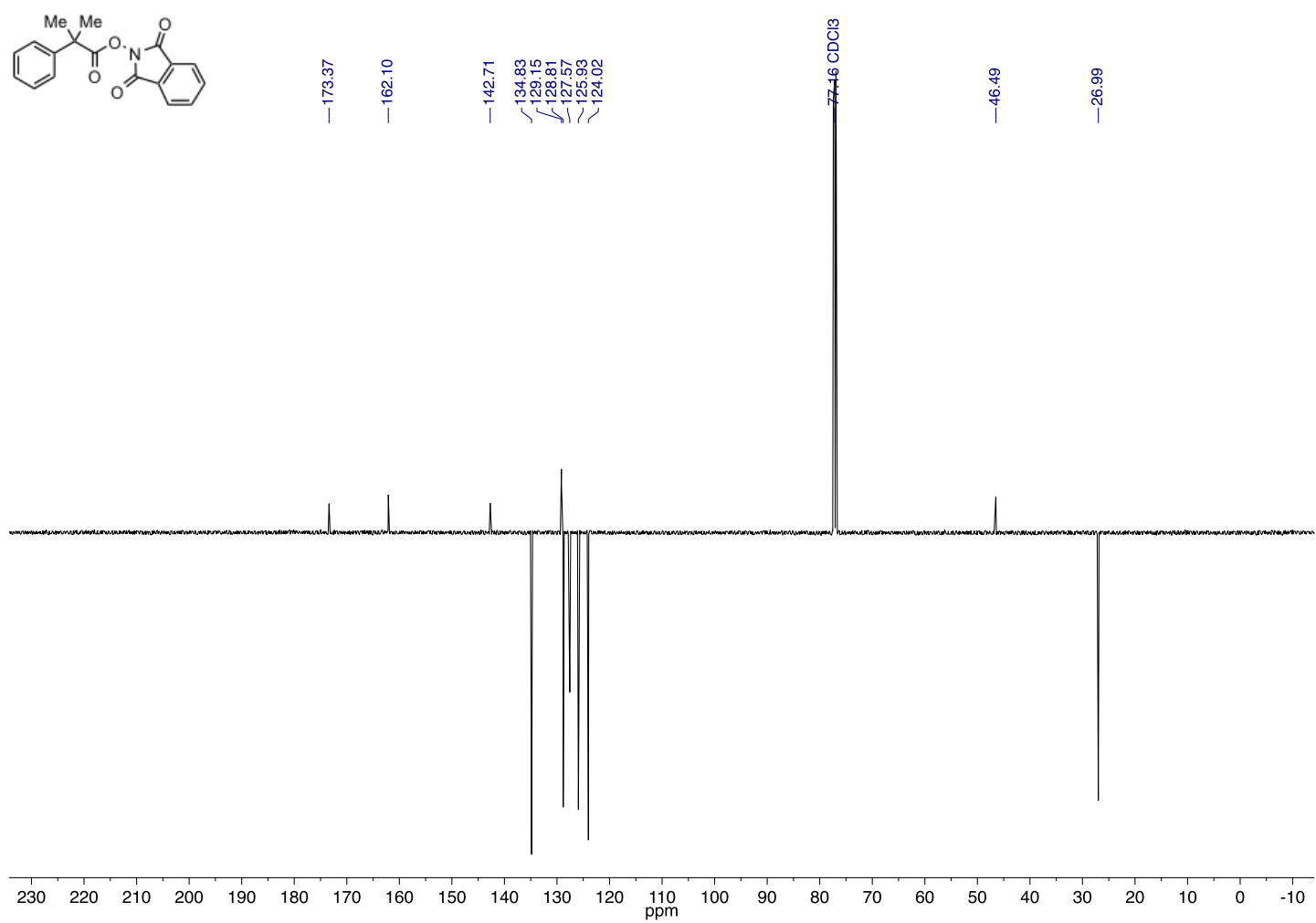

$500 \mathrm{MHz}{ }^{1} \mathrm{H}-\mathrm{NMR}$ of (2-fluoropropan-2-yl)benzene (22) in $\mathrm{CDCl}_{3}$

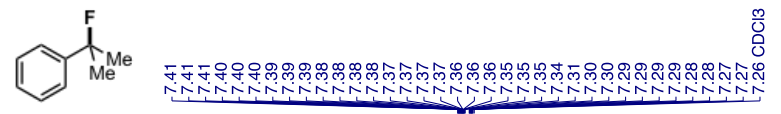

ำ

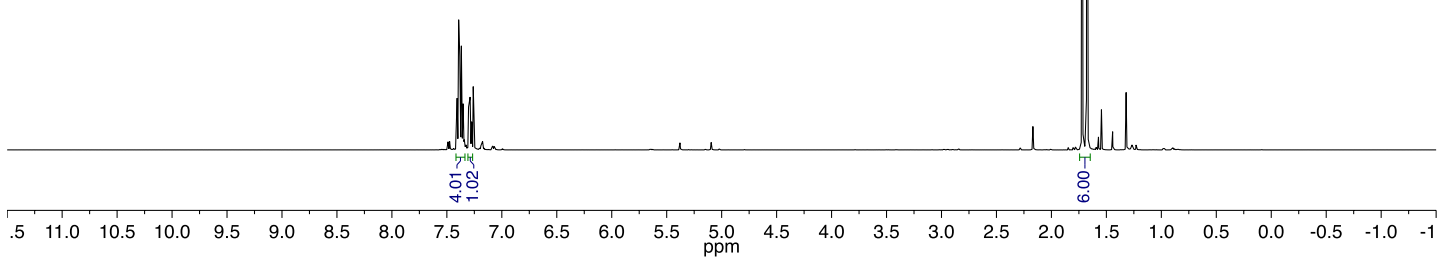


$126 \mathrm{MHz}{ }^{13} \mathrm{C}$-NMR of (2-fluoropropan-2-yl)benzene (22) in $\mathrm{CDCl}_{3}$
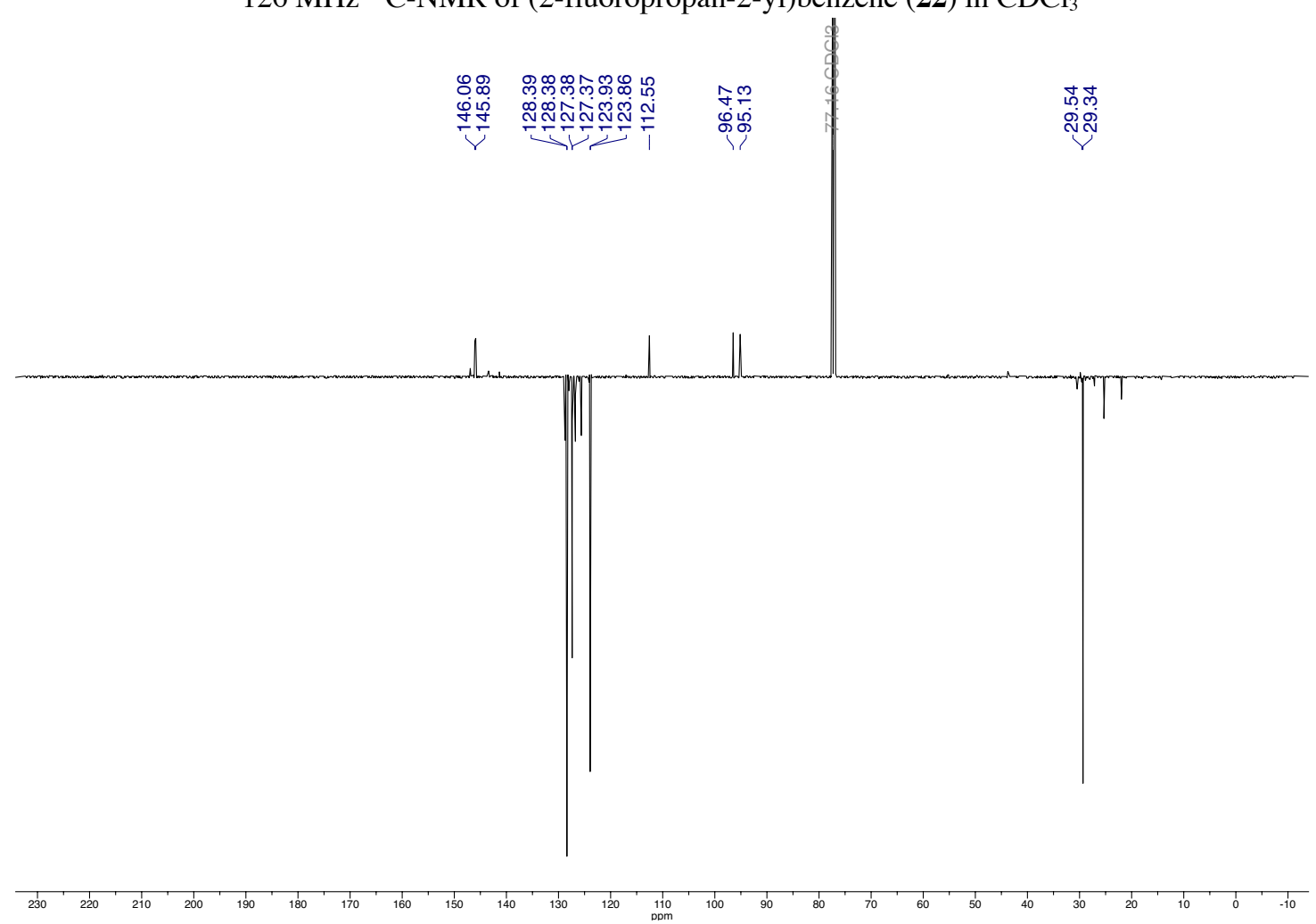

$282 \mathrm{MHz}{ }^{19} \mathrm{~F}-\mathrm{NMR}$ of (2-fluoropropan-2-yl)benzene (22) in $\mathrm{CDCl}_{3}$
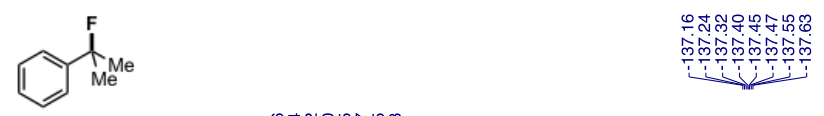

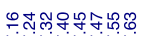

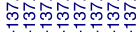

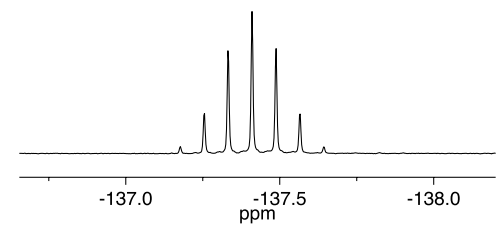

$\stackrel{1}{8}$

$104-106-108-110-112-114-116-118-120-122-124-126-128-130-132-134-136-138-140-142-144-146-148-150-152-154-156-158-160-162-164-166-168$ 
$500 \mathrm{MHz}{ }^{1} \mathrm{H}-\mathrm{NMR}$ of 1,3-dioxoisoindolin-2-yl 1-(4-chlorophenyl)cyclohexane-1-carboxylate (S18) in $\mathrm{CDCl}_{3}$

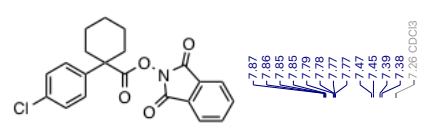
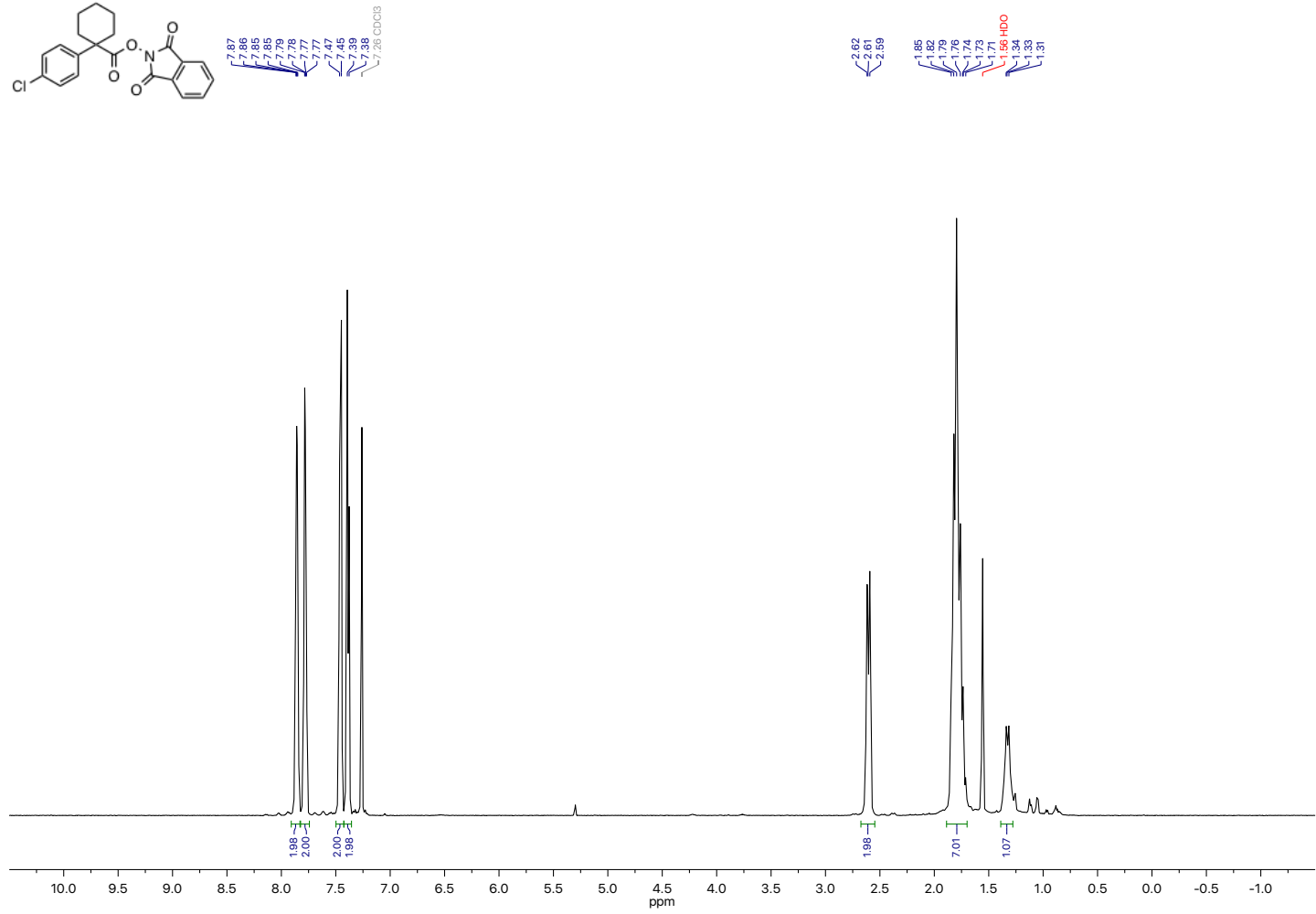

$126 \mathrm{MHz}{ }^{13} \mathrm{C}-\mathrm{NMR}$ of 1,3-dioxoisoindolin-2-yl 1-(4-chlorophenyl)cyclohexane-1-carboxylate (S18) in $\mathrm{CDCl}_{3}$

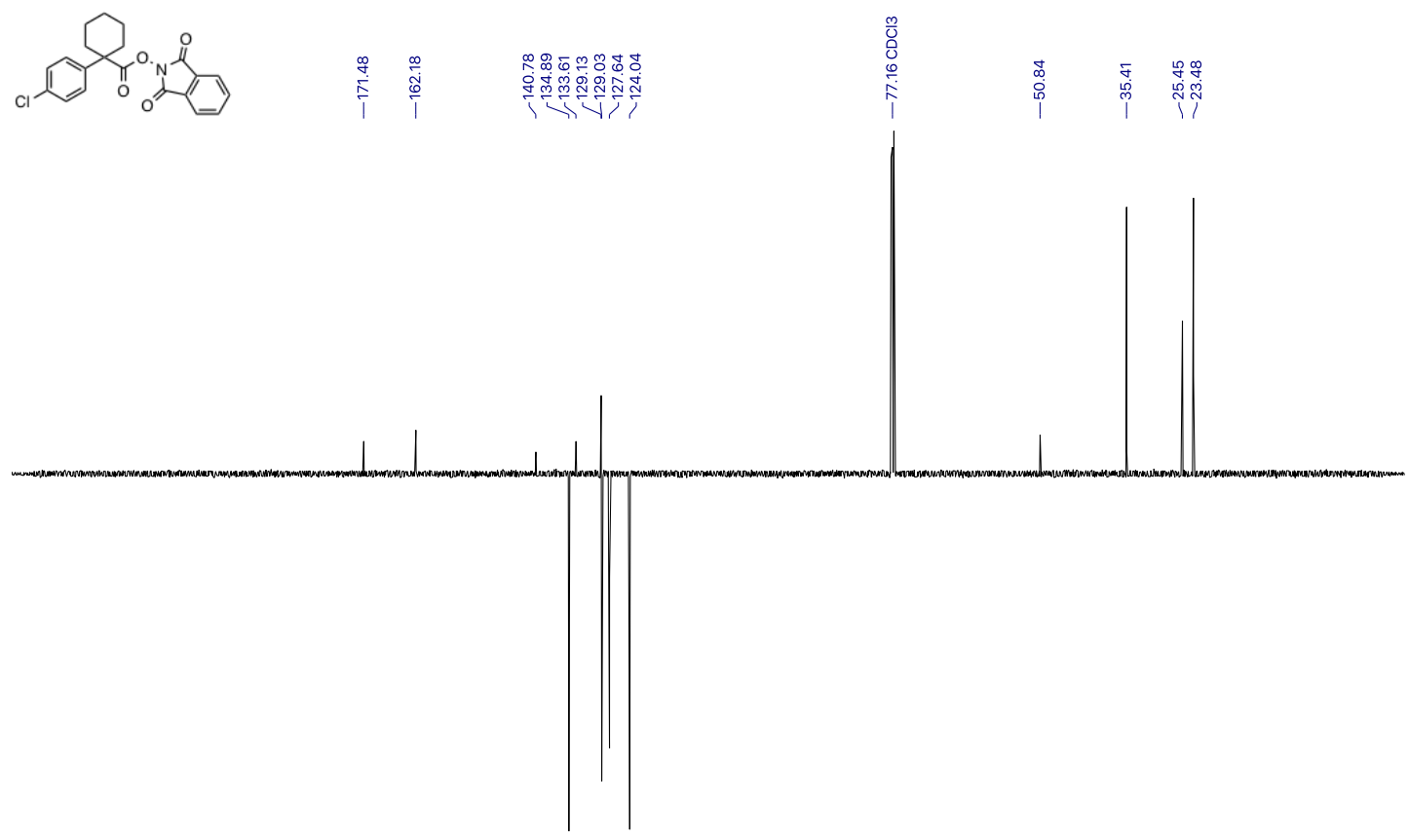

\begin{tabular}{llllllllllllllllllllllllllllllll}
\hline 230 & 220 & 210 & 200 & 190 & 180 & 170 & 160 & 150 & 140 & 130 & 120 & 110 & 100 & 90 & 80 & 70 & 60 & 50 & 40 & 30 & 20 & 10 & 0 & -10
\end{tabular} 
$500 \mathrm{MHz}{ }^{1} \mathrm{H}-\mathrm{NMR}$ of 1-chloro-4-(1-fluorocyclohexyl)benzene (23) in $\mathrm{CDCl}_{3}$

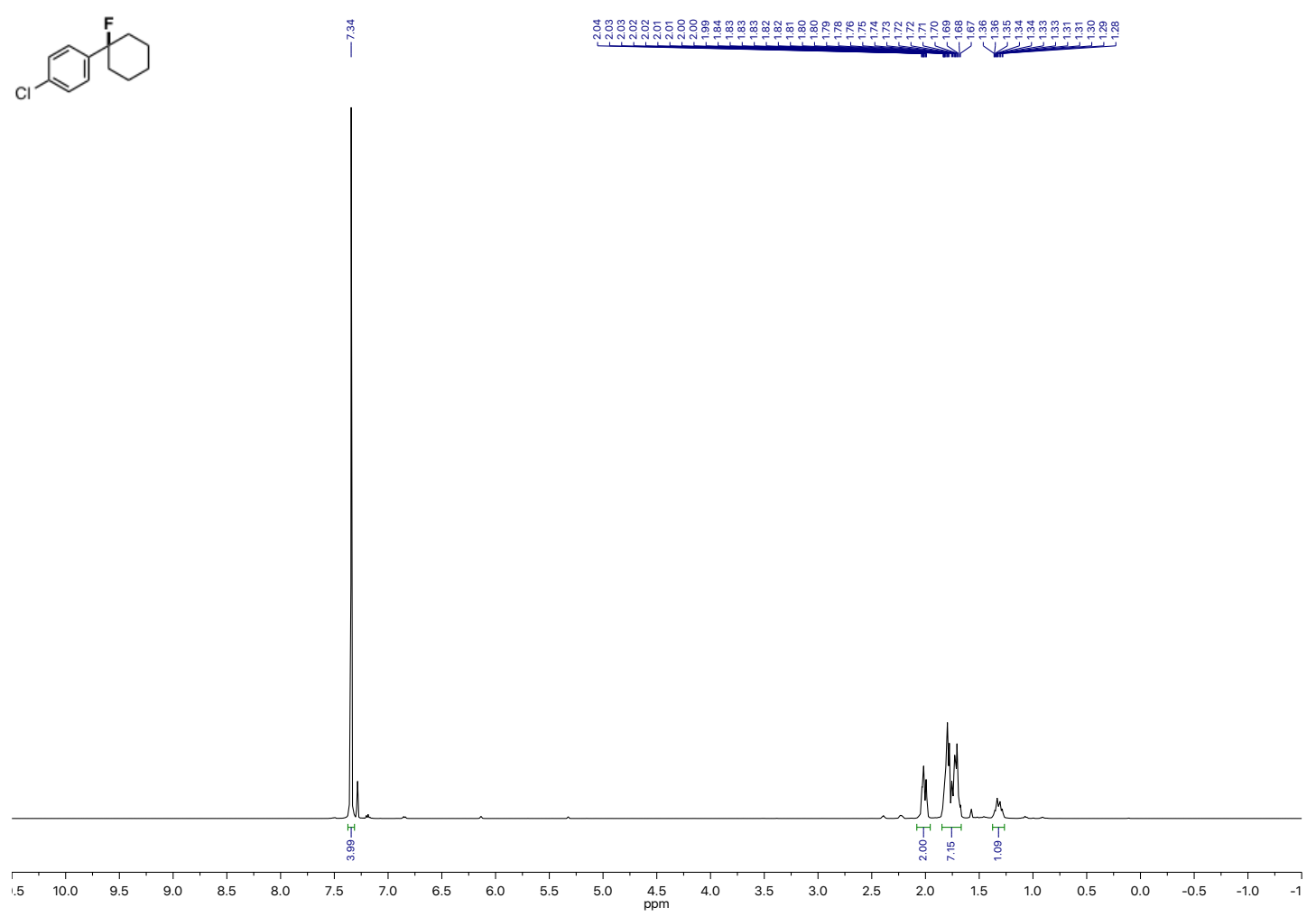

$126 \mathrm{MHz}{ }^{13} \mathrm{C}-\mathrm{NMR}$ of 1-chloro-4-(1-fluorocyclohexyl)benzene (23) in $\mathrm{CDCl}_{3}$
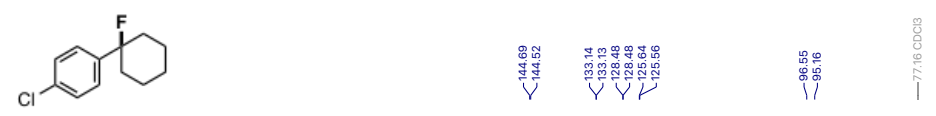

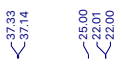
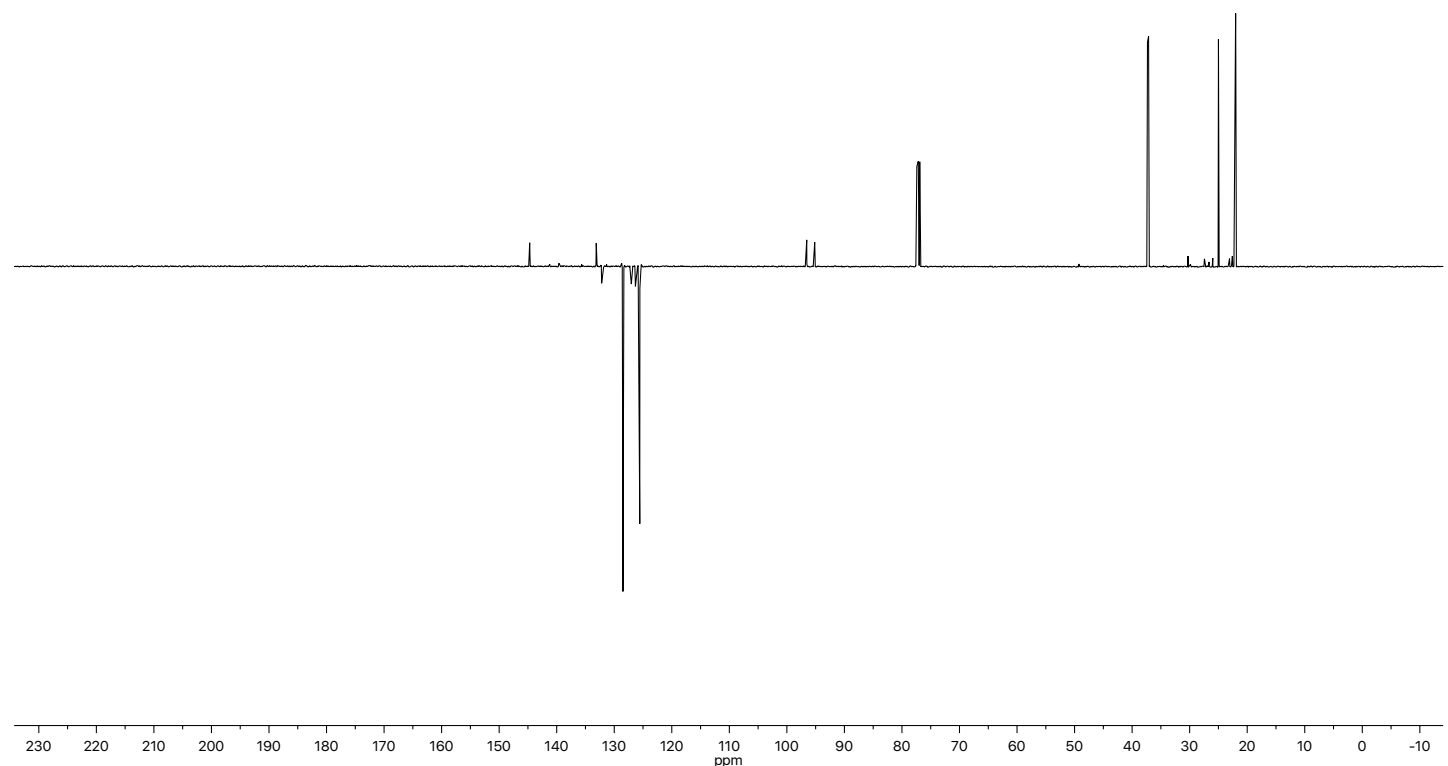
$376 \mathrm{MHz}{ }^{19} \mathrm{~F}-\mathrm{NMR}$ of 1-chloro-4-(1-fluorocyclohexyl)benzene (23) in $\mathrm{CDCl}_{3}$<smiles>Clc1ccc([Te])c2c1CCC2</smiles>
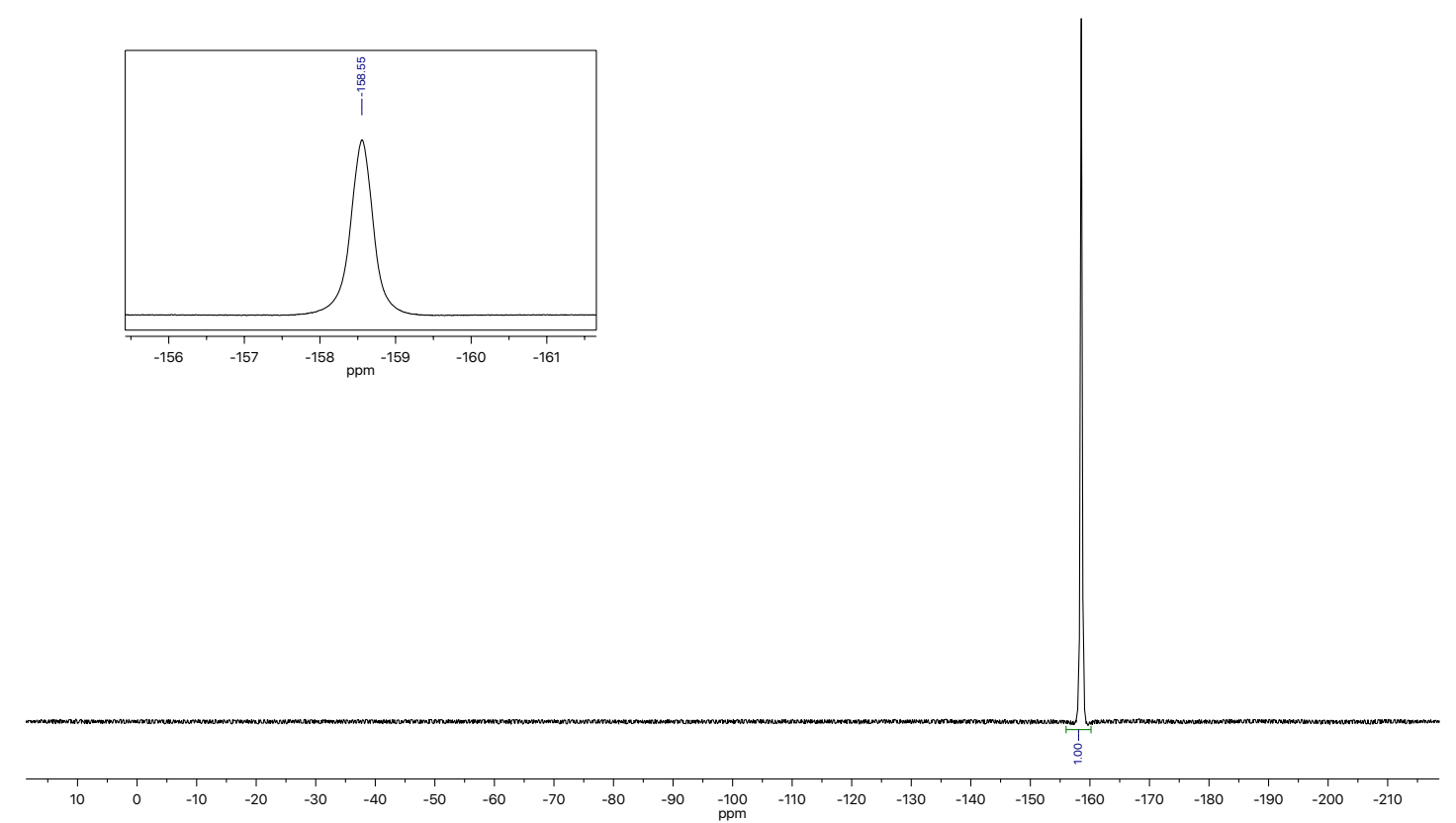

$500 \mathrm{MHz}{ }^{1} \mathrm{H}-\mathrm{NMR}$ of 1,3-dioxoisoindolin-2-yl 1-(4-chlorophenyl)cyclopentane-1-carboxylate (S19) in $\mathrm{CDCl}_{3}$

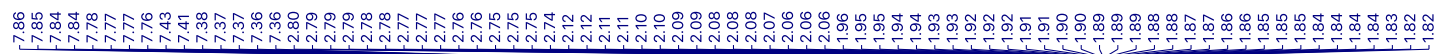

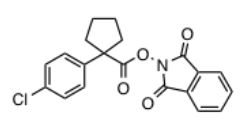

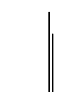


$126 \mathrm{MHz}{ }^{13} \mathrm{C}-\mathrm{NMR}$ of 1,3-dioxoisoindolin-2-yl 1-(4-chlorophenyl)cyclopentane-1-carboxylate (S19) in $\mathrm{CDCl}_{3}$

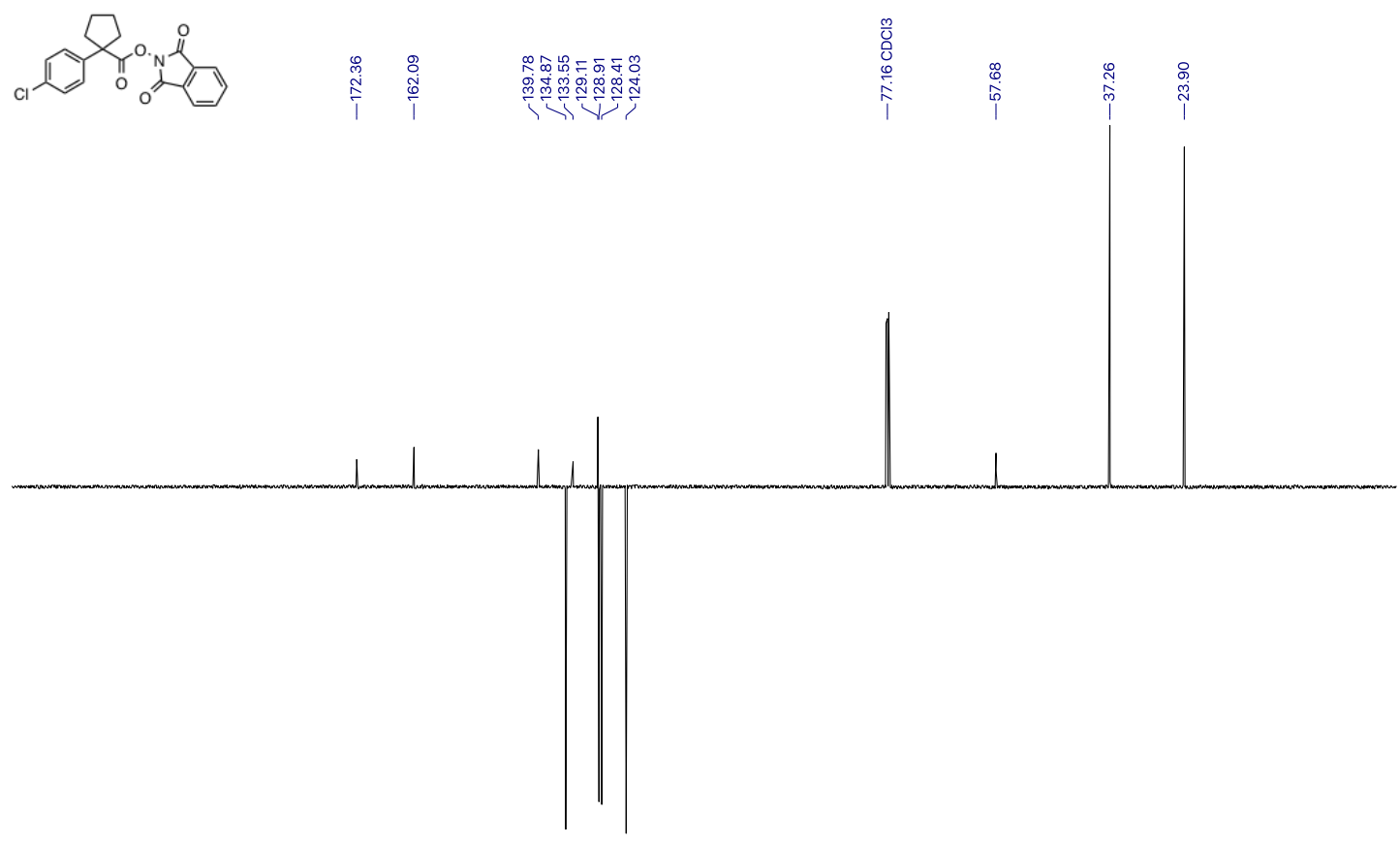

$\begin{array}{llllllllllllllllllllllllllllll}230 & 220 & 210 & 200 & 190 & 180 & 170 & 160 & 150 & 140 & 130 & 120 & \begin{array}{c}110 \\ \mathrm{ppm}\end{array} & 100 & 90 & 80 & 70 & 60 & 50 & 40 & 30 & 20 & 10 & 0 & -10\end{array}$

$470 \mathrm{MHz}{ }^{19} \mathrm{~F}-\mathrm{NMR}$ of 1-chloro-4-(1-fluorocyclopentyl)benzene (24) in $\mathrm{CDCl}_{3}$<smiles>[2H][C+]1CCCC1</smiles>

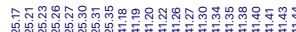

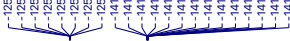

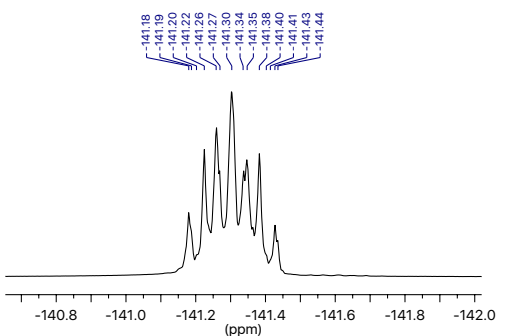


$500 \mathrm{MHz}{ }^{1} \mathrm{H}-\mathrm{NMR}$ of 1,3-dioxoisoindolin-2-yl 1-(4-chlorophenyl)cyclobutane-1-carboxylate (S20) in $\mathrm{CDCl}_{3}$

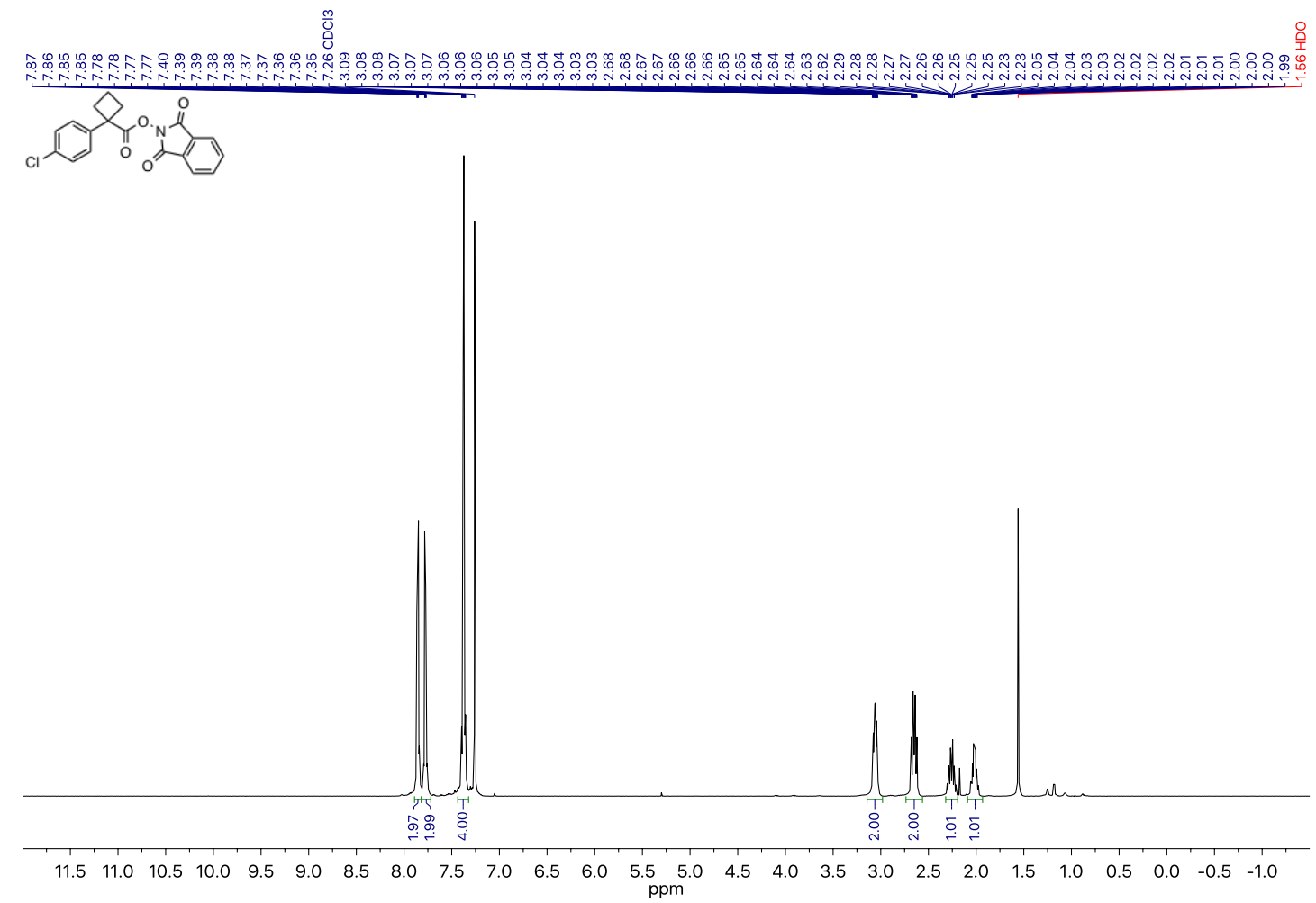


$126 \mathrm{MHz}{ }^{13} \mathrm{C}-\mathrm{NMR}$ of 1,3-dioxoisoindolin-2-yl 1-(4-chlorophenyl)cyclobutane-1-carboxylate (S20) in $\mathrm{CDCl}_{3}$
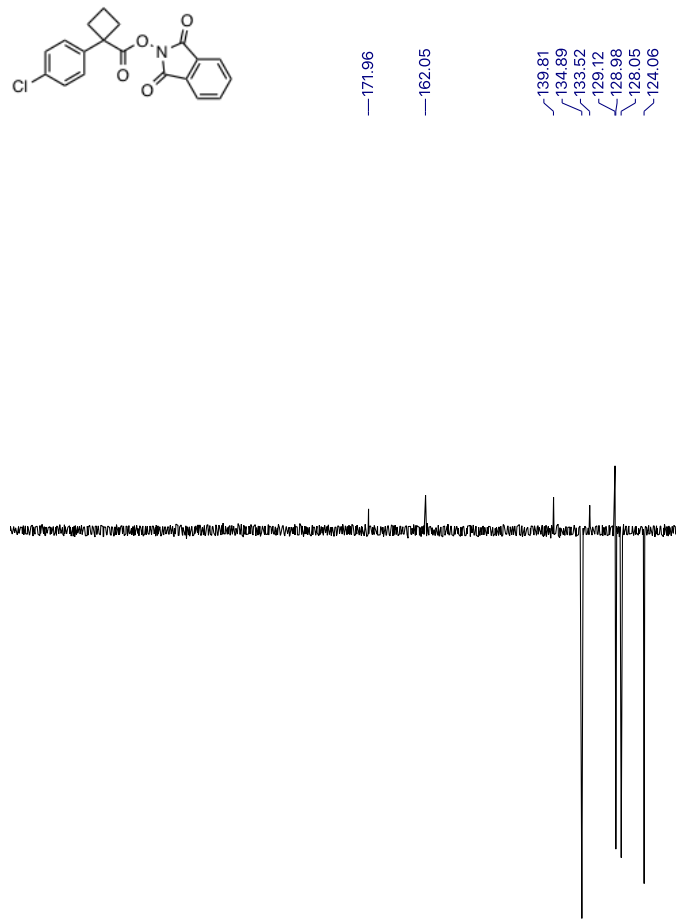

$\begin{array}{llllllllllllll}230 & 220 & 210 & 200 & 190 & 180 & 170 & 160 & 150 & 140 & 130 & 120 & \begin{array}{c}110 \\ \mathrm{ppm}\end{array} & 100\end{array}$

$500 \mathrm{MHz}{ }^{1} \mathrm{H}-\mathrm{NMR}$ of 1-chloro-4-(1-fluorocyclobutyl)benzene (25) in $\mathrm{CDCl}_{3}$
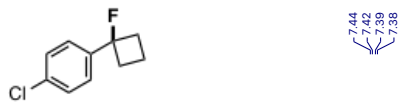

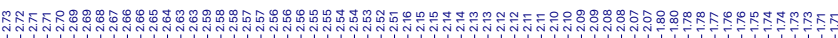

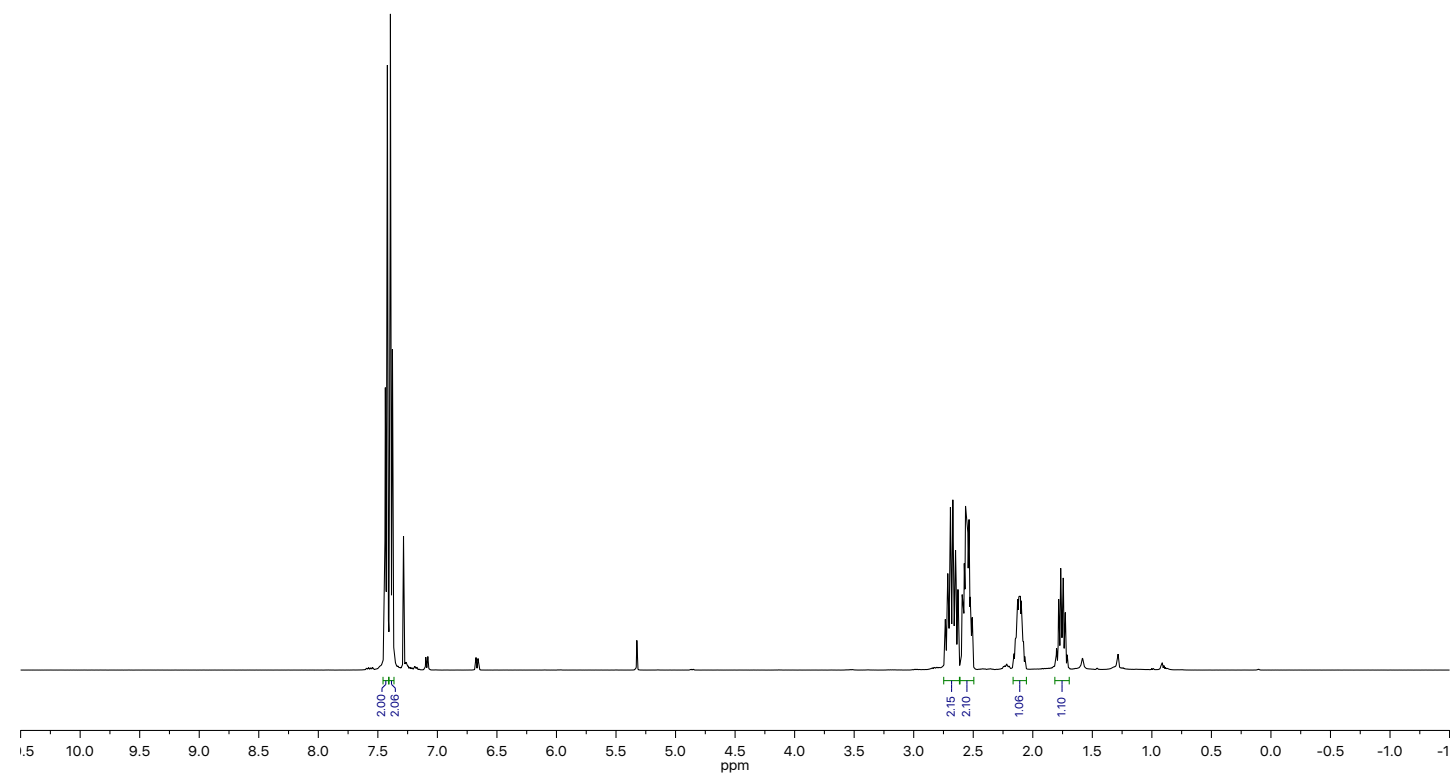


$126 \mathrm{MHz}{ }^{13} \mathrm{C}-\mathrm{NMR}$ of 1-chloro-4-(1-fluorocyclobutyl)benzene (25) in $\mathrm{CDCl}_{3}$
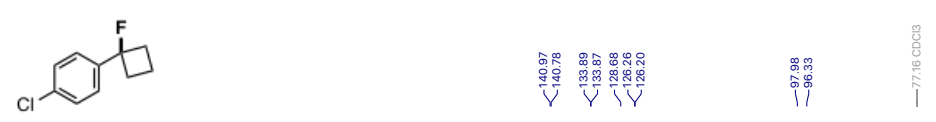

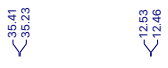
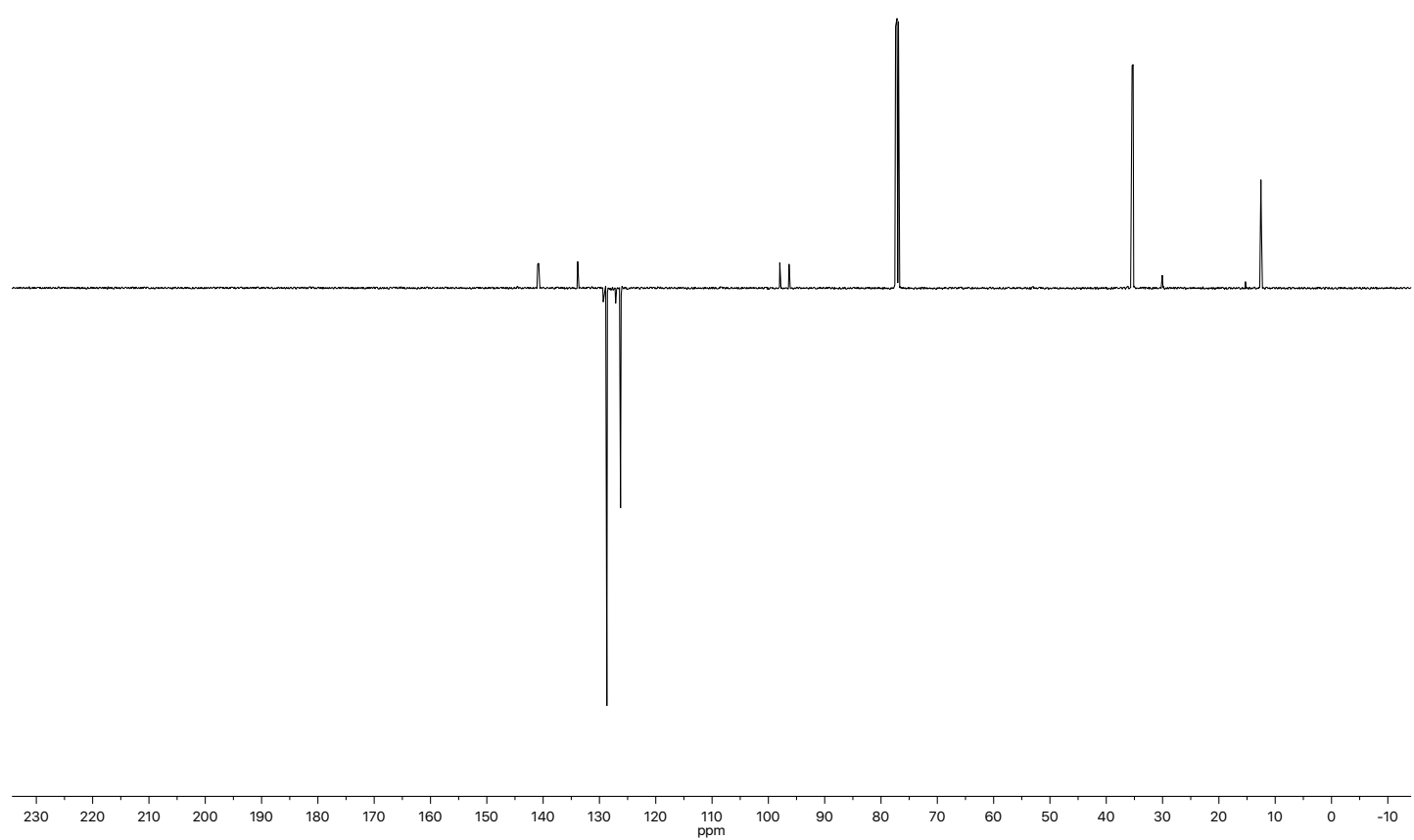

$470 \mathrm{MHz}{ }^{19} \mathrm{~F}-\mathrm{NMR}$ of 1-chloro-4-(1-fluorocyclobutyl)benzene (25) in $\mathrm{CDCl}_{3}$
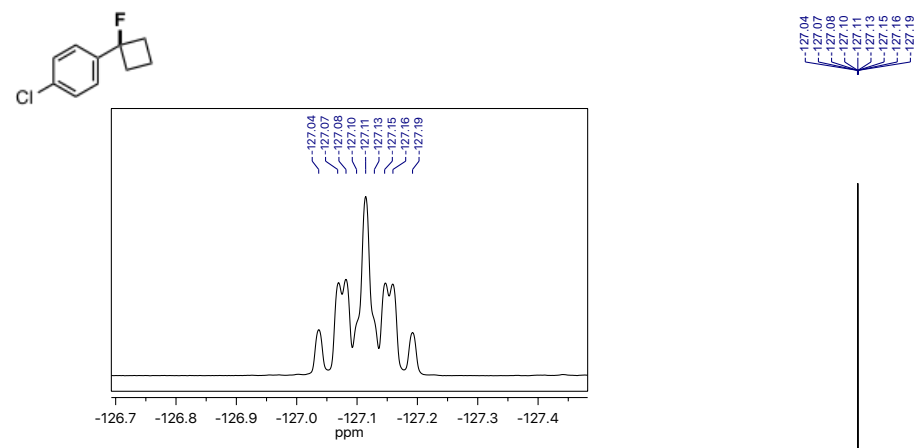

Then

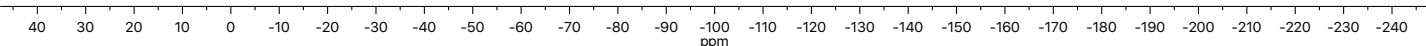


$400 \mathrm{MHz}{ }^{1} \mathrm{H}-\mathrm{NMR}$ of 1,3-dioxoisoindolin-2-yl 4-(6-chloropyridin-3-yl)tetrahydro-2H-pyran-4-carboxylate (S21) in $\mathrm{CDCl}_{3}$

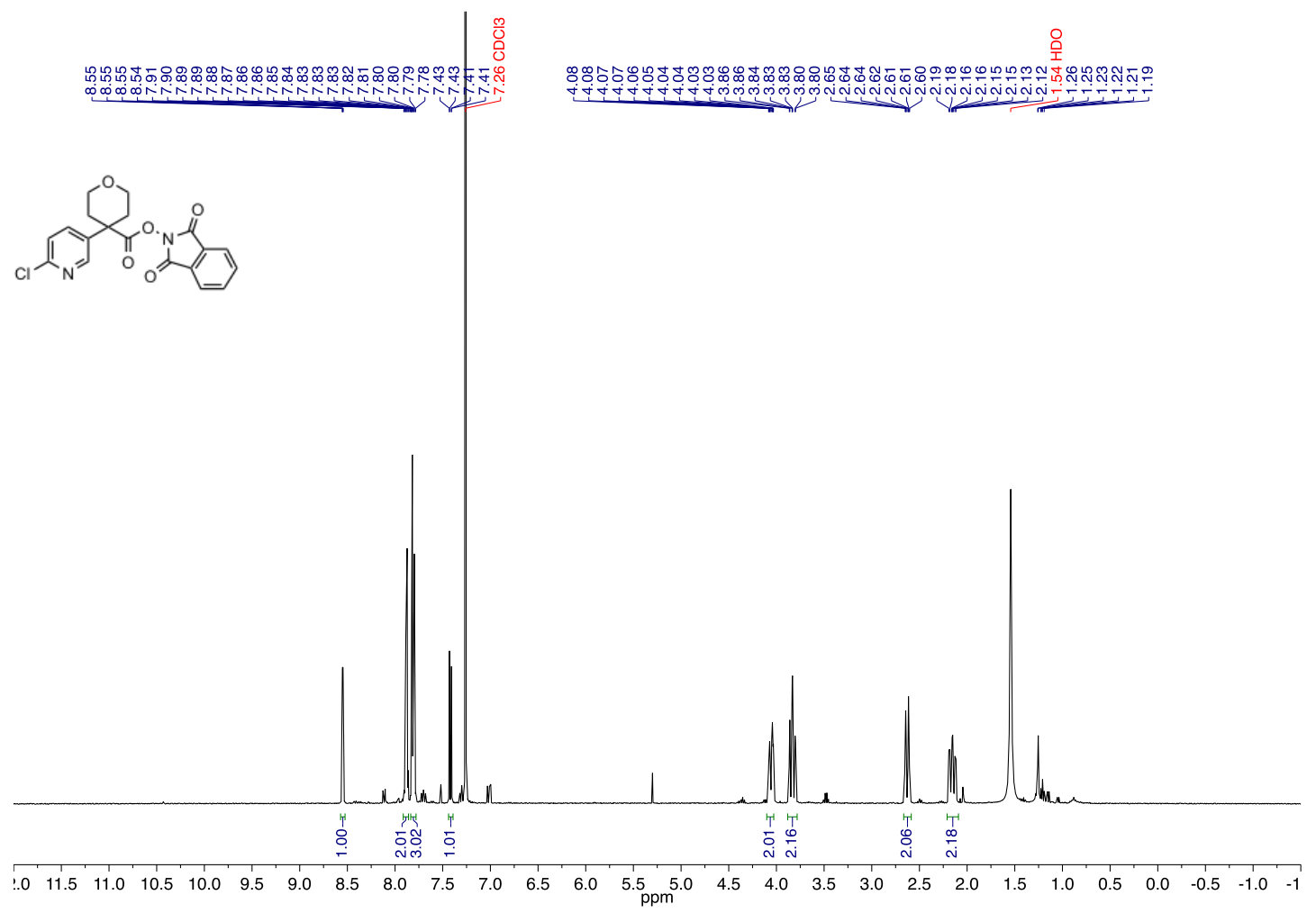


$126 \mathrm{MHz}{ }^{13} \mathrm{C}-\mathrm{NMR}$ of 1,3-dioxoisoindolin-2-yl 4-(6-chloropyridin-3-yl)tetrahydro-2H-pyran-4-carboxylate (S21) in $\mathrm{CDCl}_{3}$

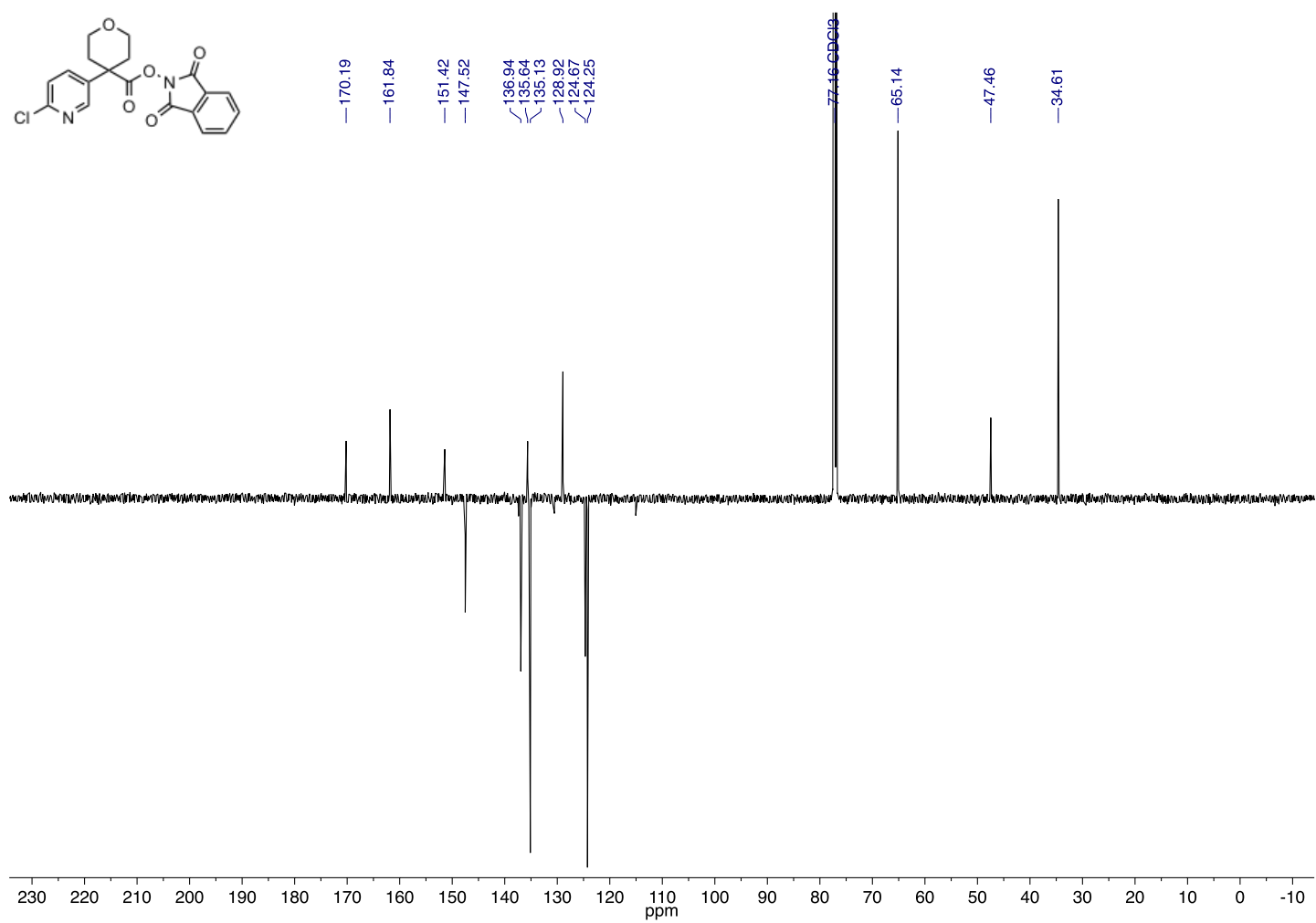

$500 \mathrm{MHz}{ }^{1} \mathrm{H}-\mathrm{NMR}$ of 2-chloro-5-(4-fluorotetrahydro-2 $\mathrm{H}$-pyran-4-yl)pyridine (26) in $\mathrm{CDCl}_{3}$
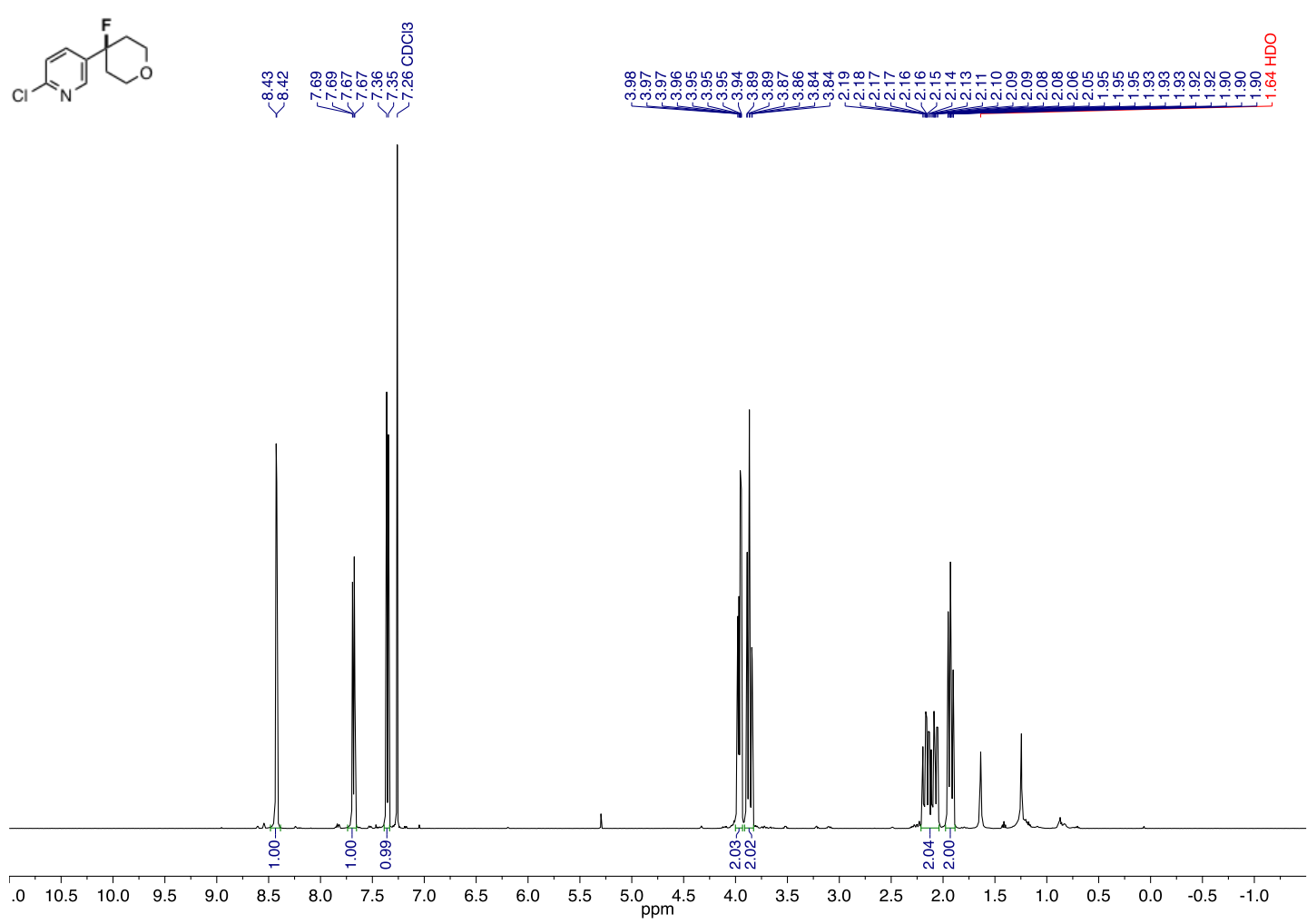
$126 \mathrm{MHz}{ }^{13} \mathrm{C}-\mathrm{NMR}$ of 2-chloro-5-(4-fluorotetrahydro-2 $\mathrm{H}$-pyran-4-yl)pyridine (26) in $\mathrm{CDCl}_{3}$

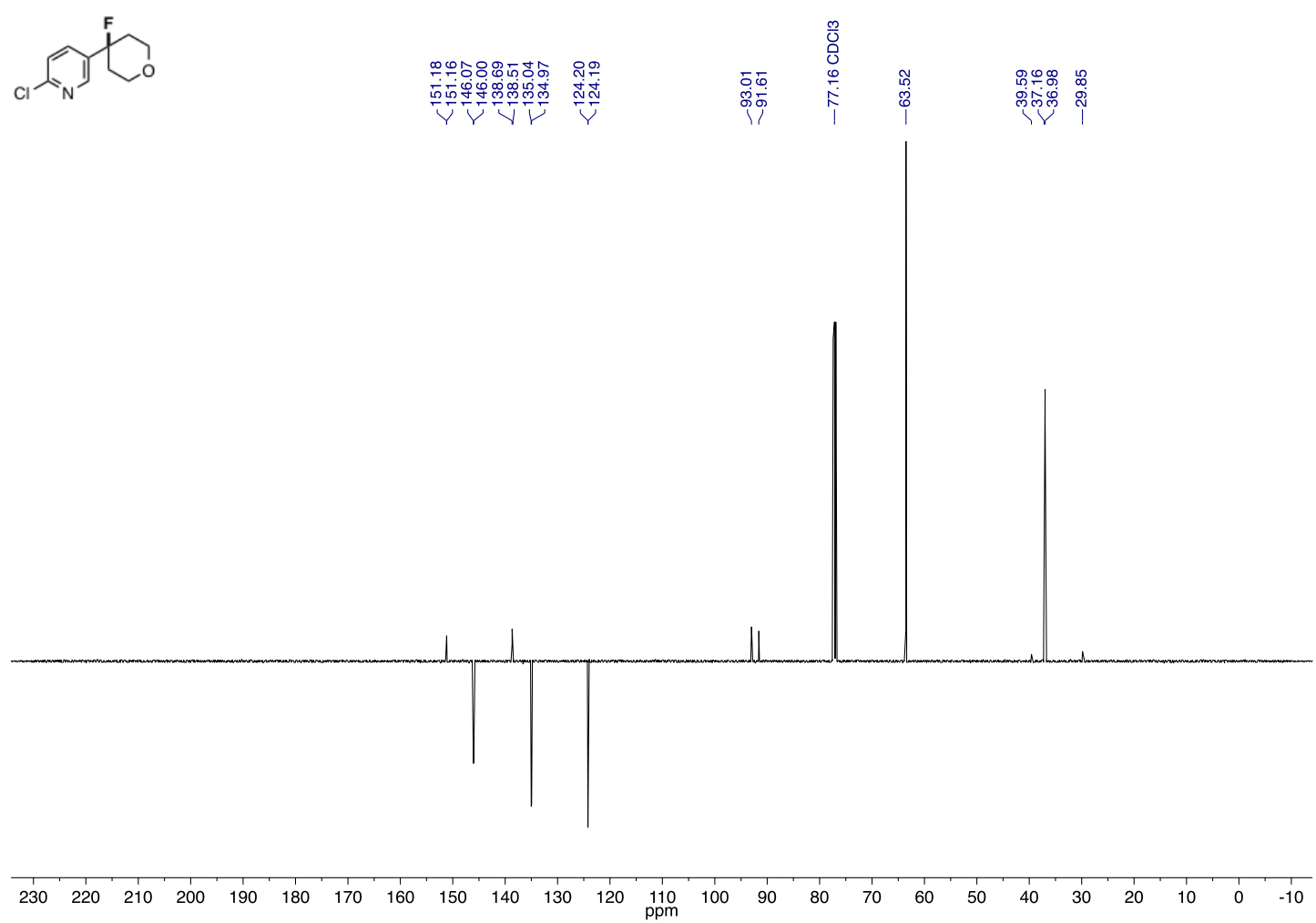

$282 \mathrm{MHz}{ }^{19} \mathrm{~F}-\mathrm{NMR}$ of 2-chloro-5-(4-fluorotetrahydro- $2 \mathrm{H}$-pyran-4-yl)pyridine (26) in $\mathrm{CDCl}_{3}$
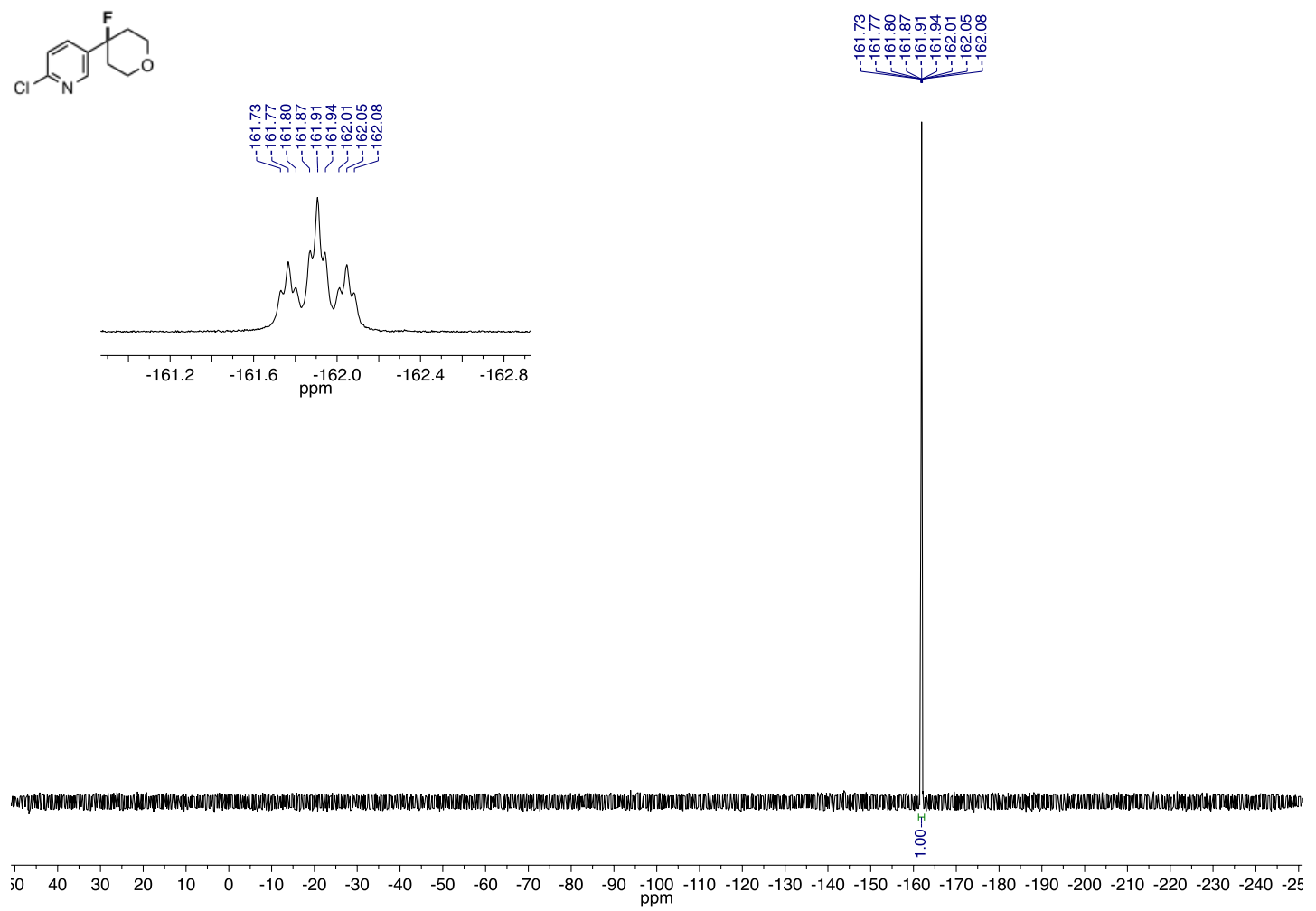
$500 \mathrm{MHz}{ }^{1} \mathrm{H}-\mathrm{NMR}$ of 1,3-dioxoisoindolin-2-yl adamantane-1-carboxylate (S22) in $\mathrm{CDCl}_{3}$
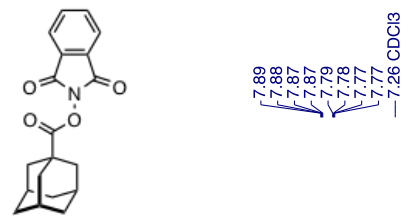

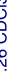

2

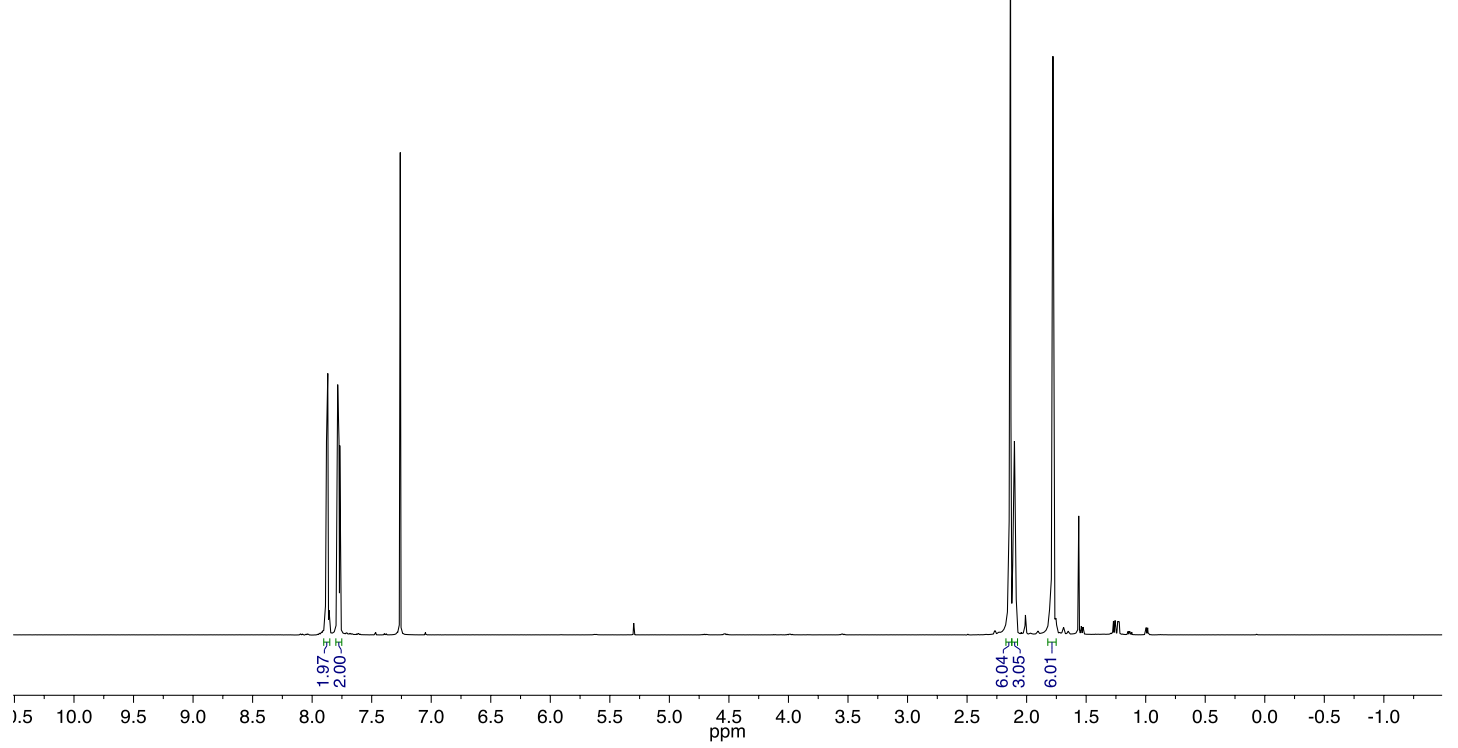

$126 \mathrm{MHz}{ }^{13} \mathrm{C}$-NMR of 1,3-dioxoisoindolin-2-yl adamantane-1-carboxylate (S22) in $\mathrm{CDCl}_{3}$
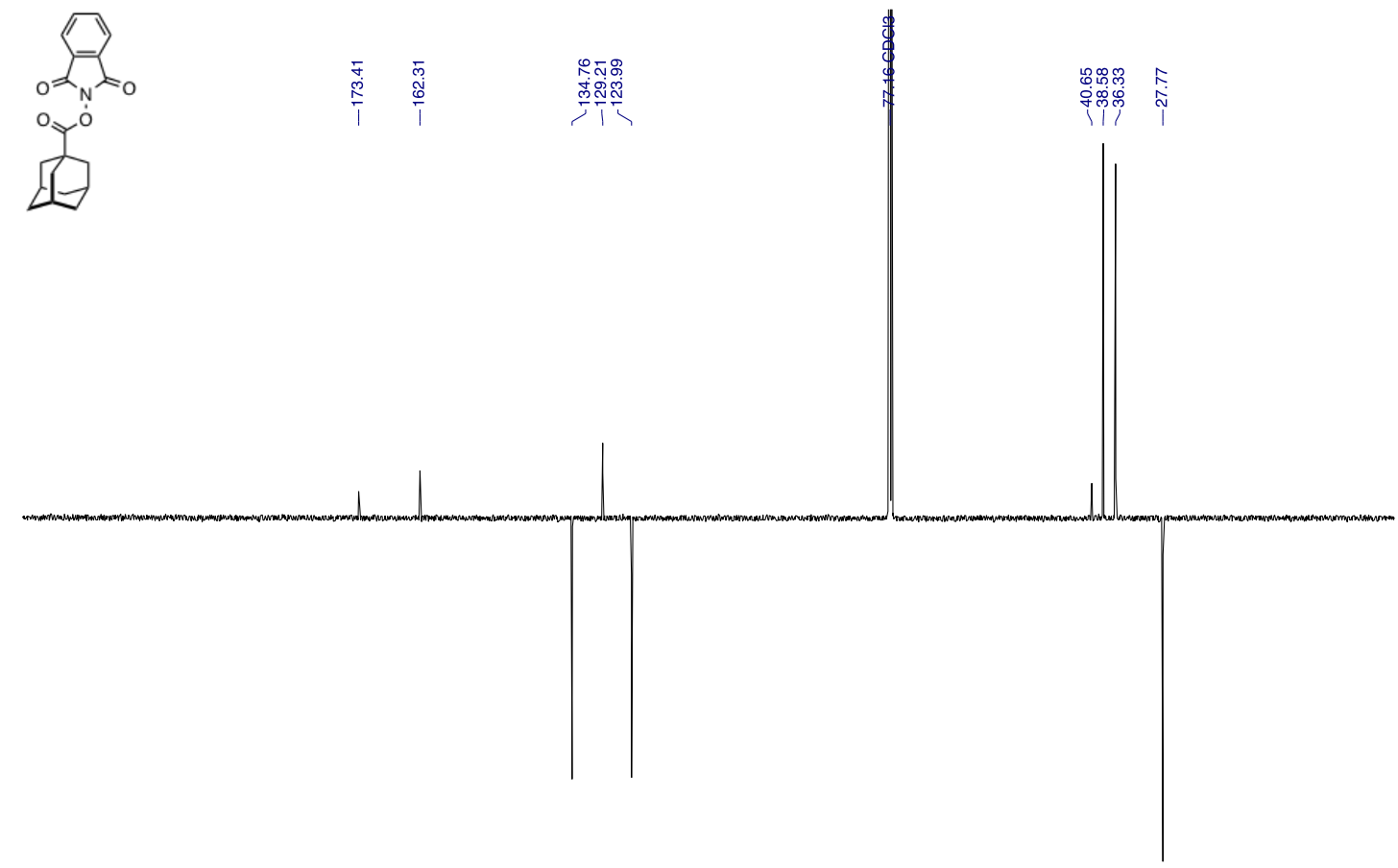

\begin{tabular}{llllllllllllllllllllllllllll}
\hline 230 & 220 & 210 & 200 & 190 & 180 & 170 & 160 & 150 & 140 & 130 & 120 & $\begin{array}{c}110 \\
\mathrm{ppm}\end{array}$ & 100 & 90 & 80 & 70 & 60 & 50 & 40 & 30 & 20 & 10 & 0 & -10
\end{tabular} 
$500 \mathrm{MHz}{ }^{1} \mathrm{H}-\mathrm{NMR}$ of 1,3-dioxoisoindolin-2-yl 3-(4-(tert-butyl)phenyl)-2,2-dimethylpropanoate (S23) in $\mathrm{CDCl}_{3}$
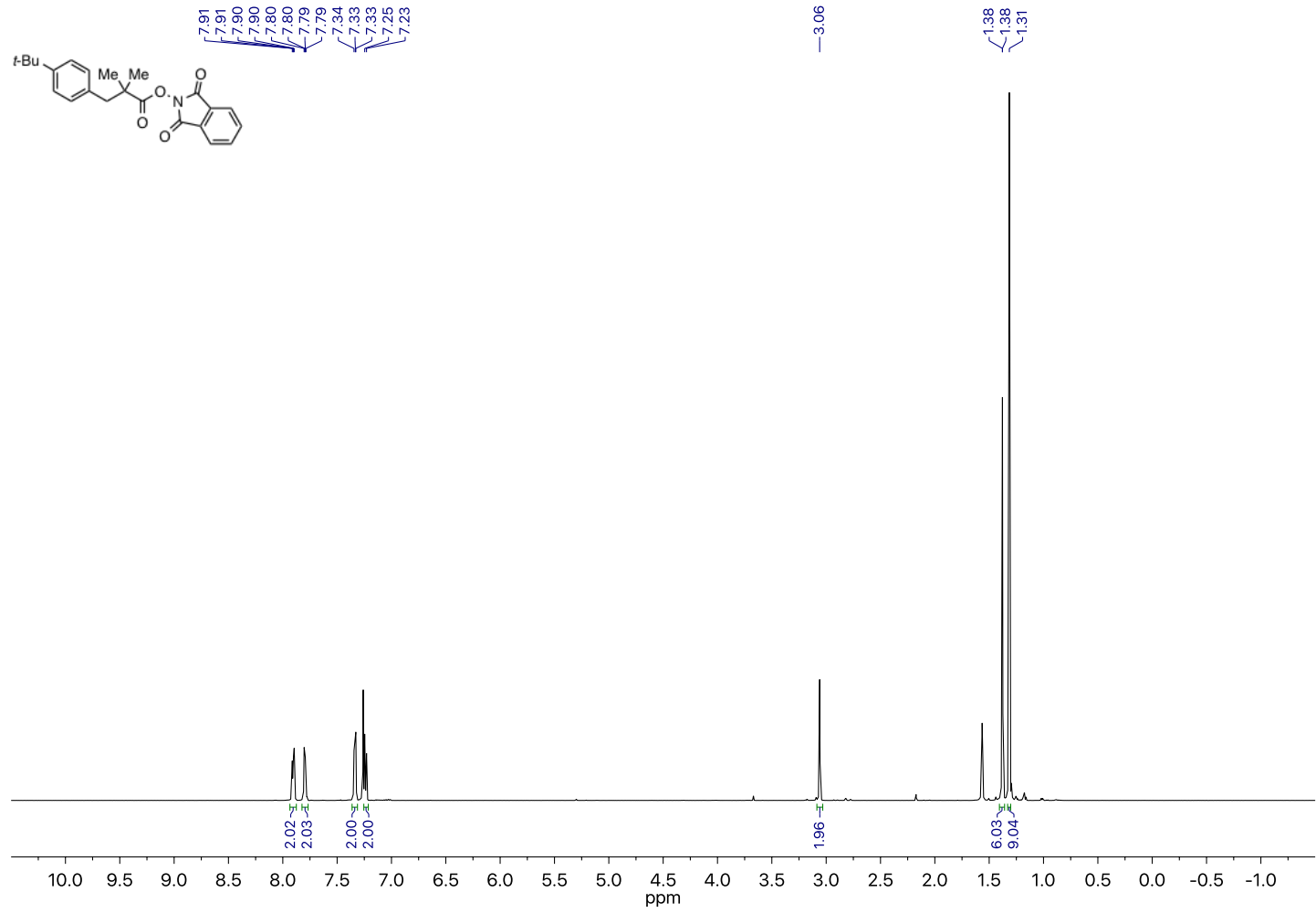

$126 \mathrm{MHz}{ }^{13} \mathrm{C}-\mathrm{NMR}$ of 1,3-dioxoisoindolin-2-yl 3-(4-(tert-butyl)phenyl)-2,2-dimethylpropanoate (S23) in $\mathrm{CDCl}_{3}$

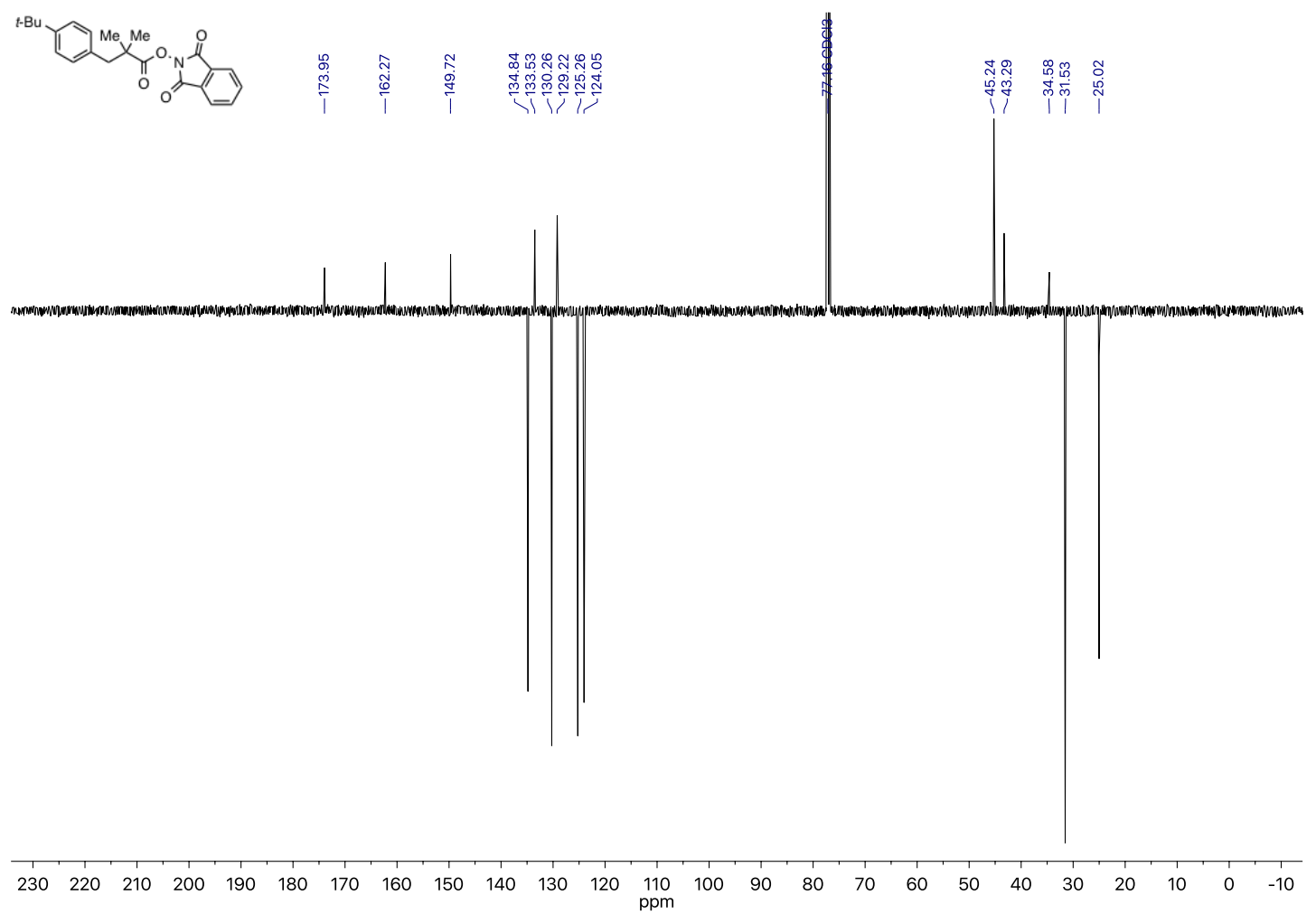


$500 \mathrm{MHz}$ 1H-NMR of 1-(tert-butyl)-4-(2-fluoro-2-methylpropyl)benzene (28) in CDCl3

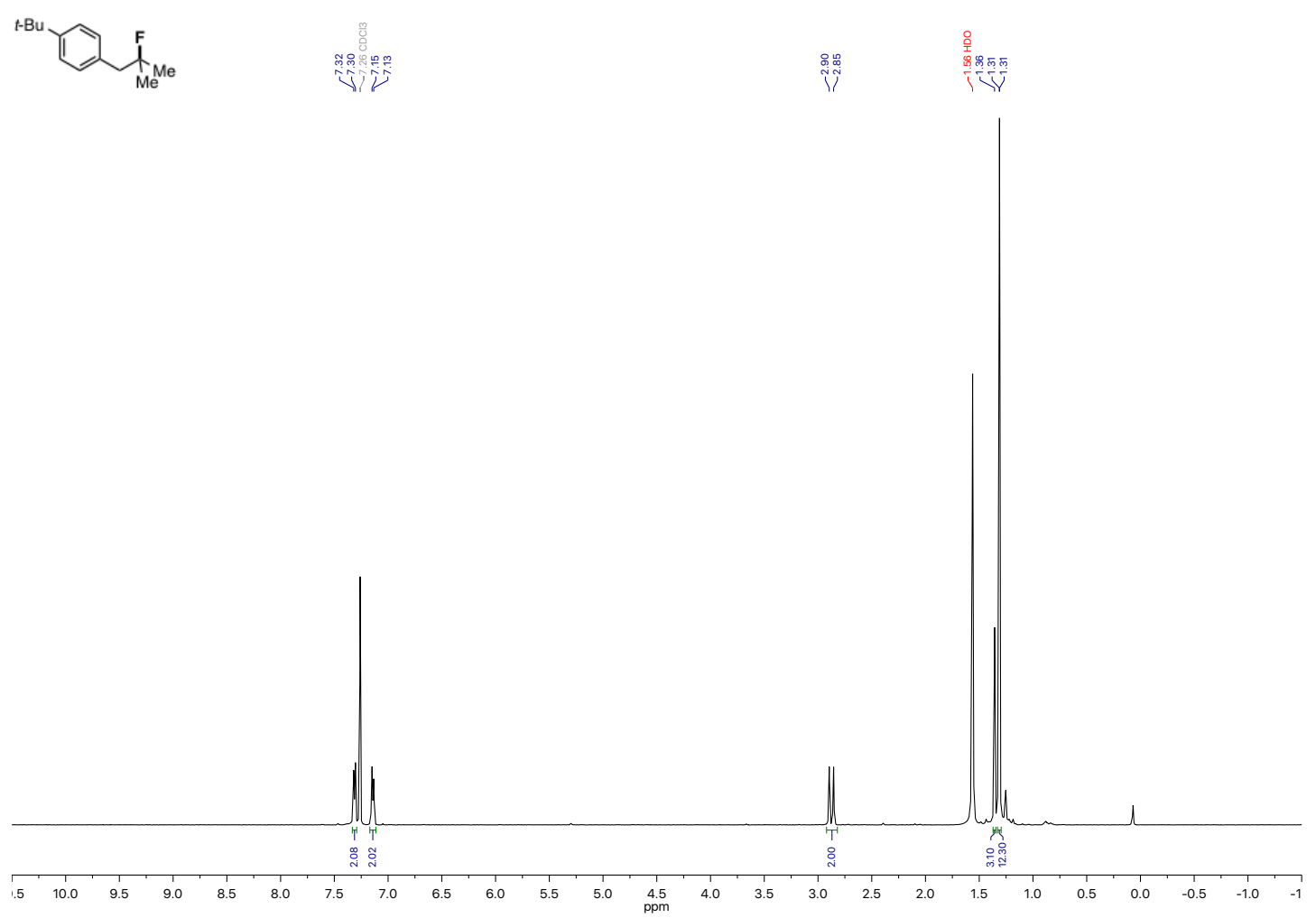

$126 \mathrm{MHz}{ }^{13} \mathrm{C}$-NMR of 1-(tert-butyl)-4-(2-fluoro-2-methylpropyl)benzene (28) in $\mathrm{CDCl}_{3}$
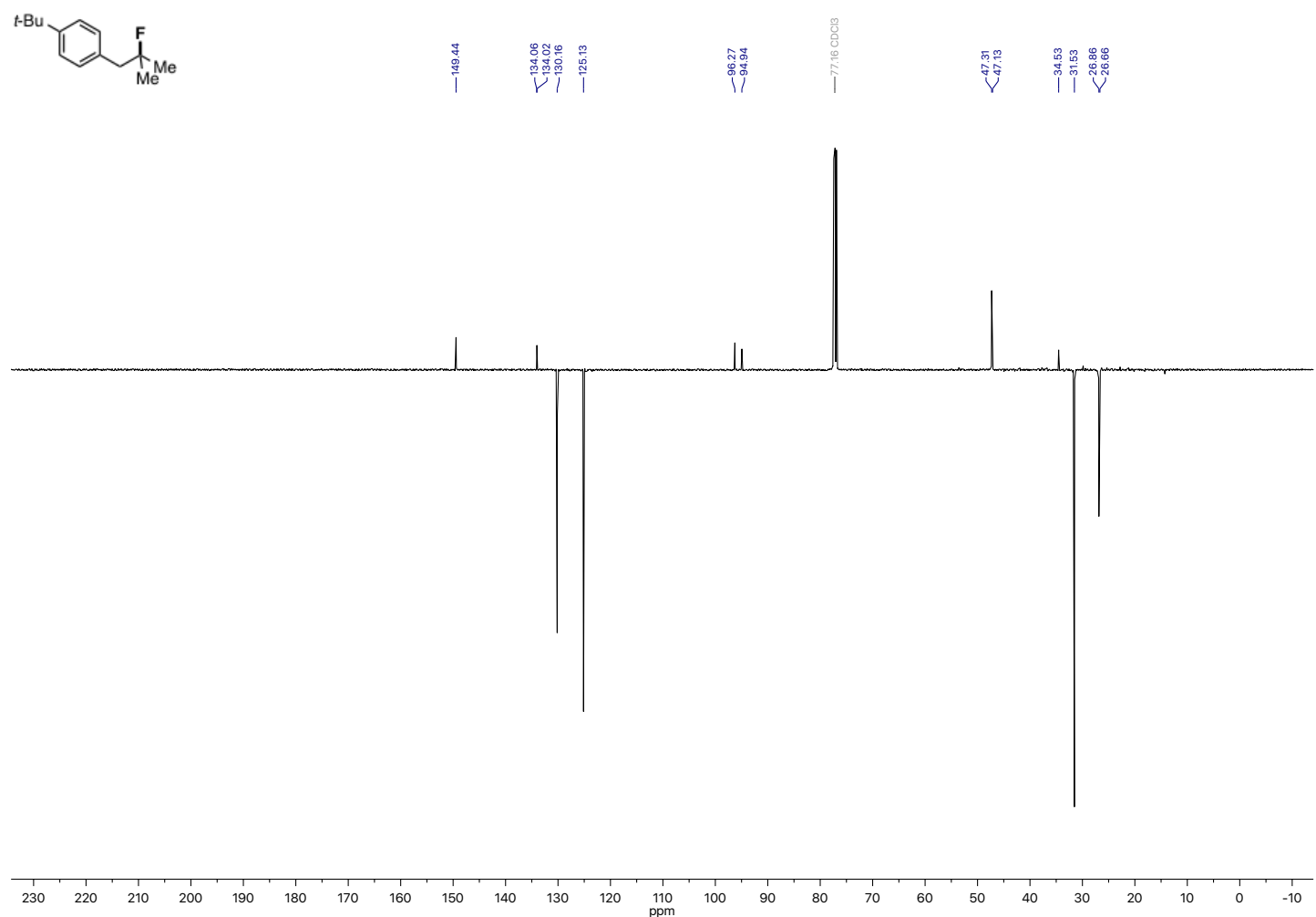
$376 \mathrm{MHz}{ }^{19} \mathrm{~F}-\mathrm{NMR}$ of 1-(tert-butyl)-4-(2-fluoro-2-methylpropyl)benzene (28) in $\mathrm{CDCl}_{3}$
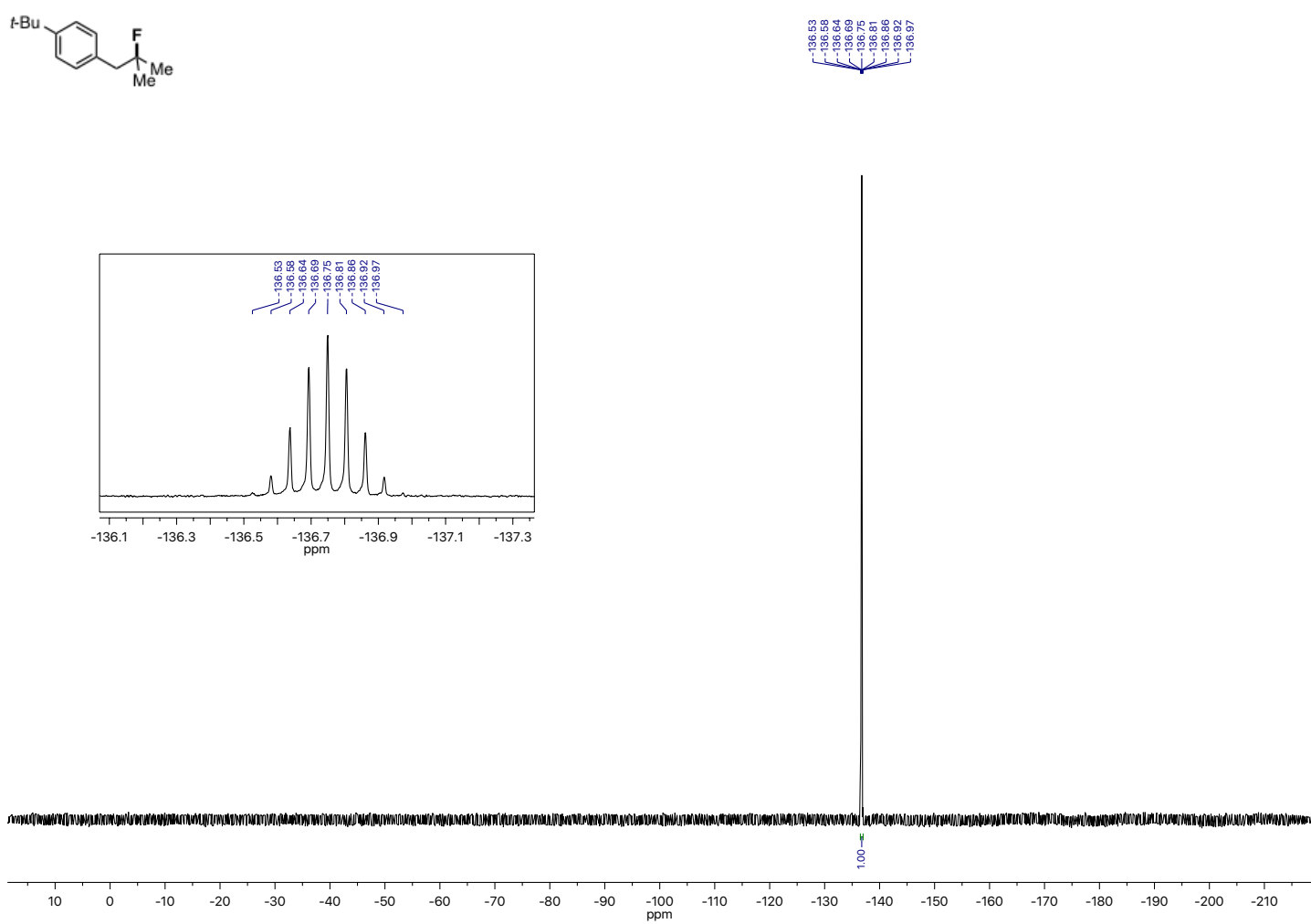

$500 \mathrm{MHz}$ 1H-NMR of 1,3-dioxoisoindolin-2-yl 3-(4-cyanophenyl)-2,2-dimethylpropanoate (S24) in CDCl3
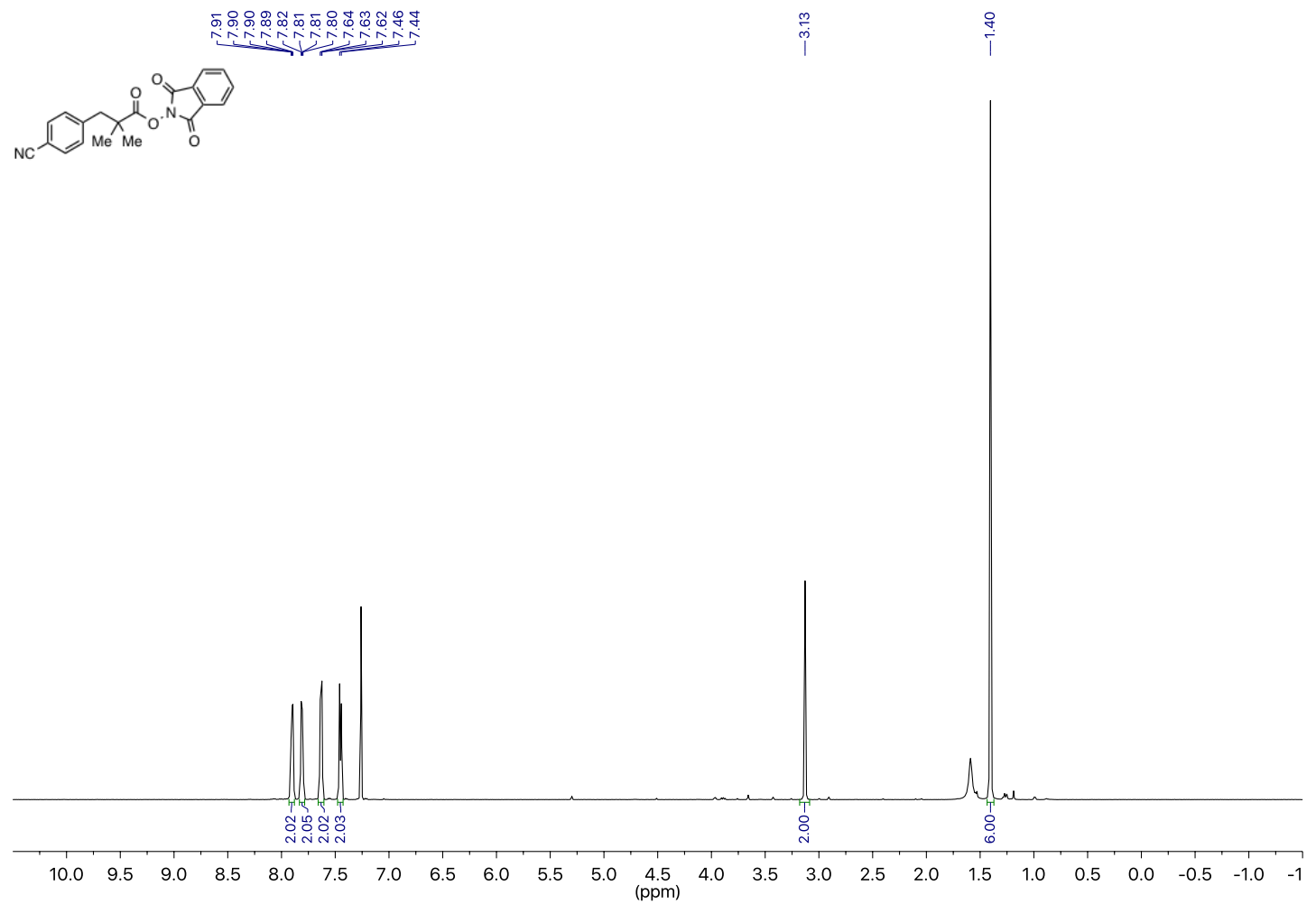
$126 \mathrm{MHz}{ }^{13} \mathrm{C}$-NMR of 1,3-dioxoisoindolin-2-yl 3-(4-cyanophenyl)-2,2-dimethylpropanoate (S24) in $\mathrm{CDCl}_{3}$

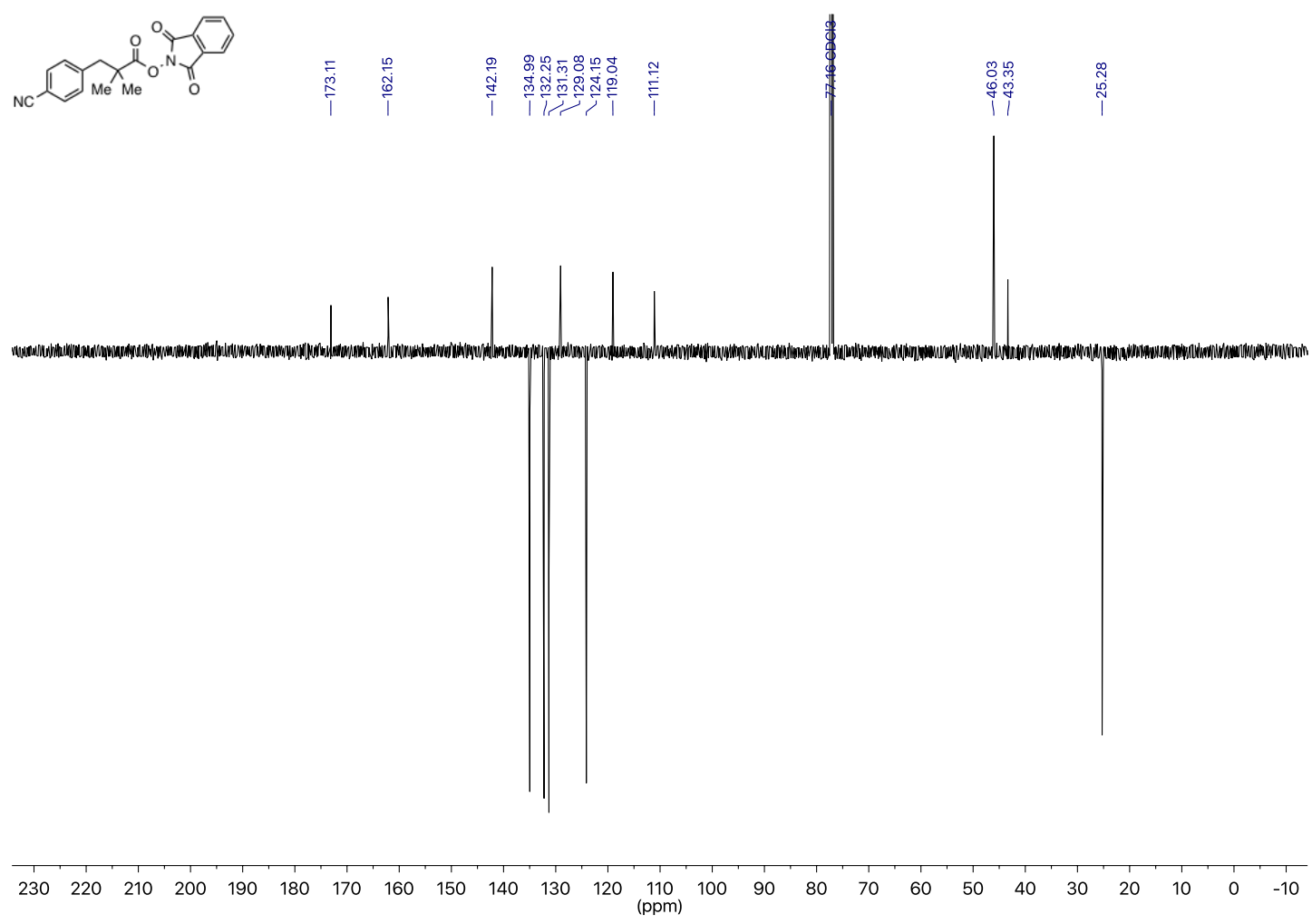

$500 \mathrm{MHz}{ }^{1} \mathrm{H}-\mathrm{NMR}$ of 4-(2-fluoro-2-methylpropyl)benzonitrile (29) in $\mathrm{CDCl}_{3}$
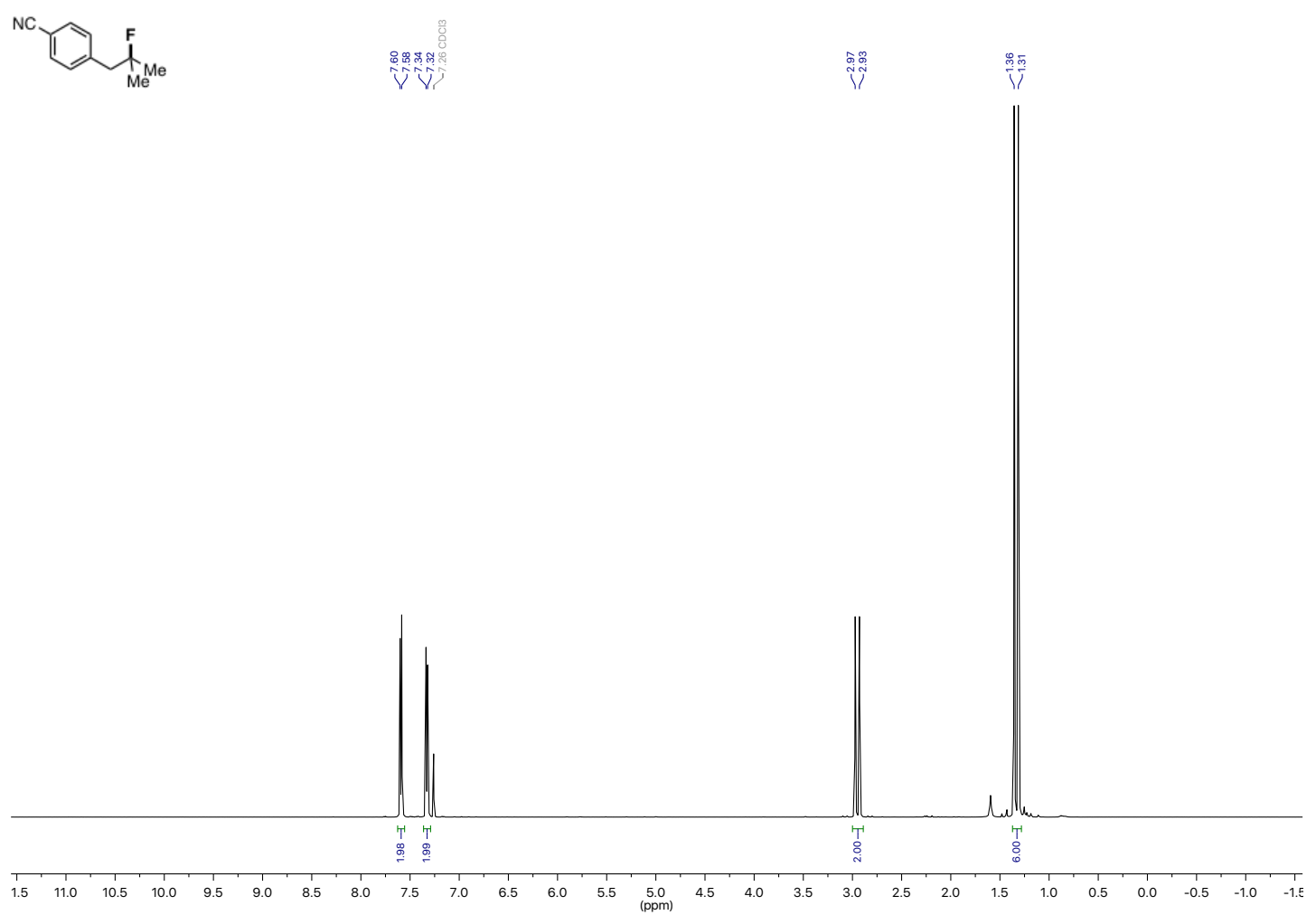
$126 \mathrm{MHz}{ }^{13} \mathrm{C}-\mathrm{NMR}$ of 4-(2-fluoro-2-methylpropyl)benzonitrile (29) in $\mathrm{CDCl}_{3}$

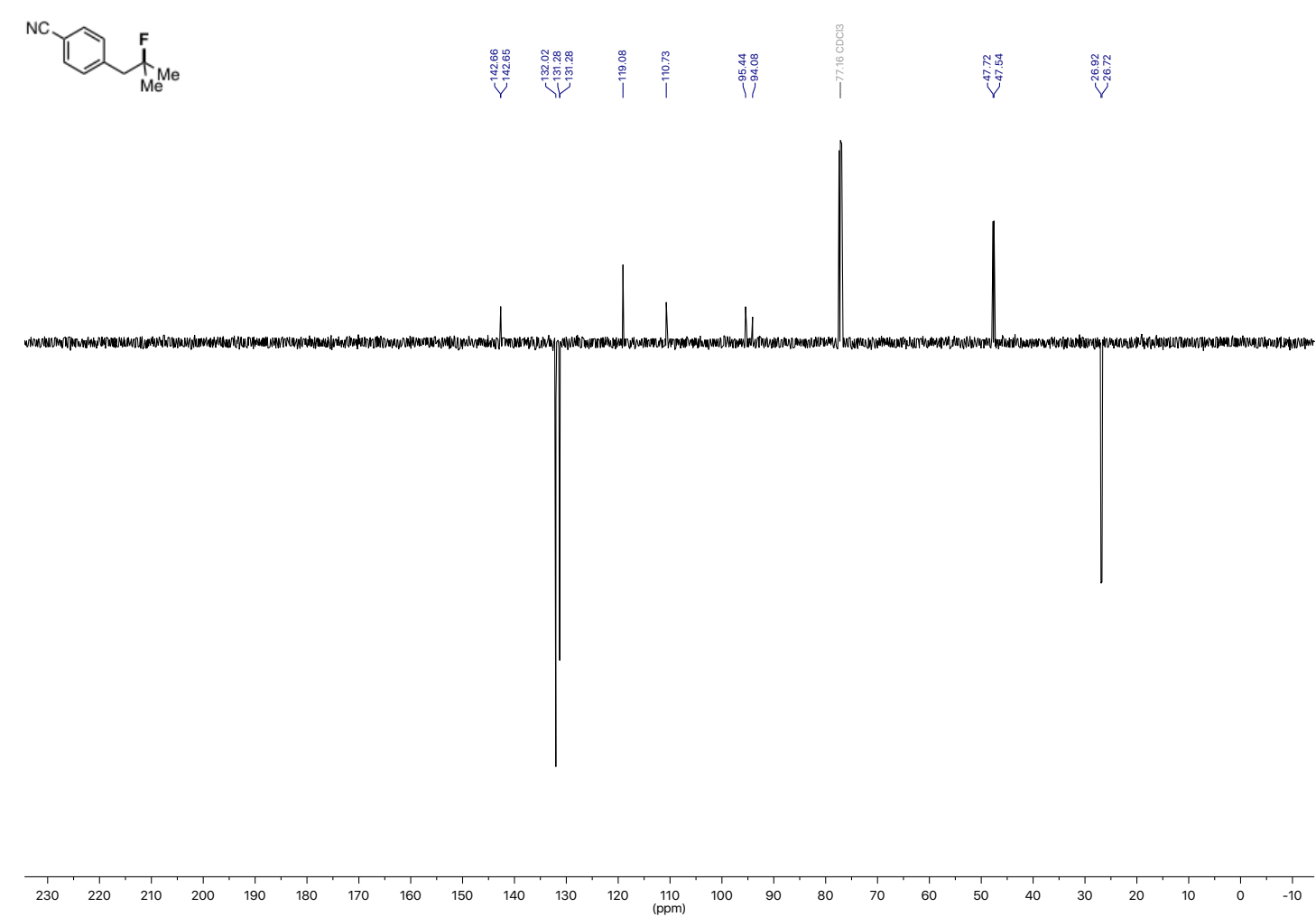

$126 \mathrm{MHz}{ }^{13} \mathrm{C}-\mathrm{NMR}$ of 4-(2-fluoro-2-methylpropyl)benzonitrile (29) in $\mathrm{CDCl}_{3}$

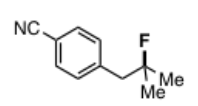

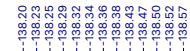

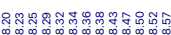

gas.

Dijilisiliti

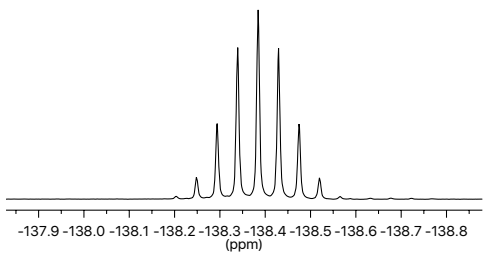

$\stackrel{!}{!}$

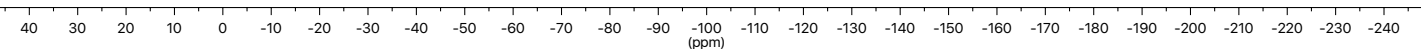


$500 \mathrm{MHz}{ }^{1} \mathrm{H}-\mathrm{NMR}$ of 1,3-dioxoisoindolin-2-yl 5-(2,5-dimethylphenoxy)-2,2-dimethylpentanoate (41) in $\mathrm{CDCl}_{3}$
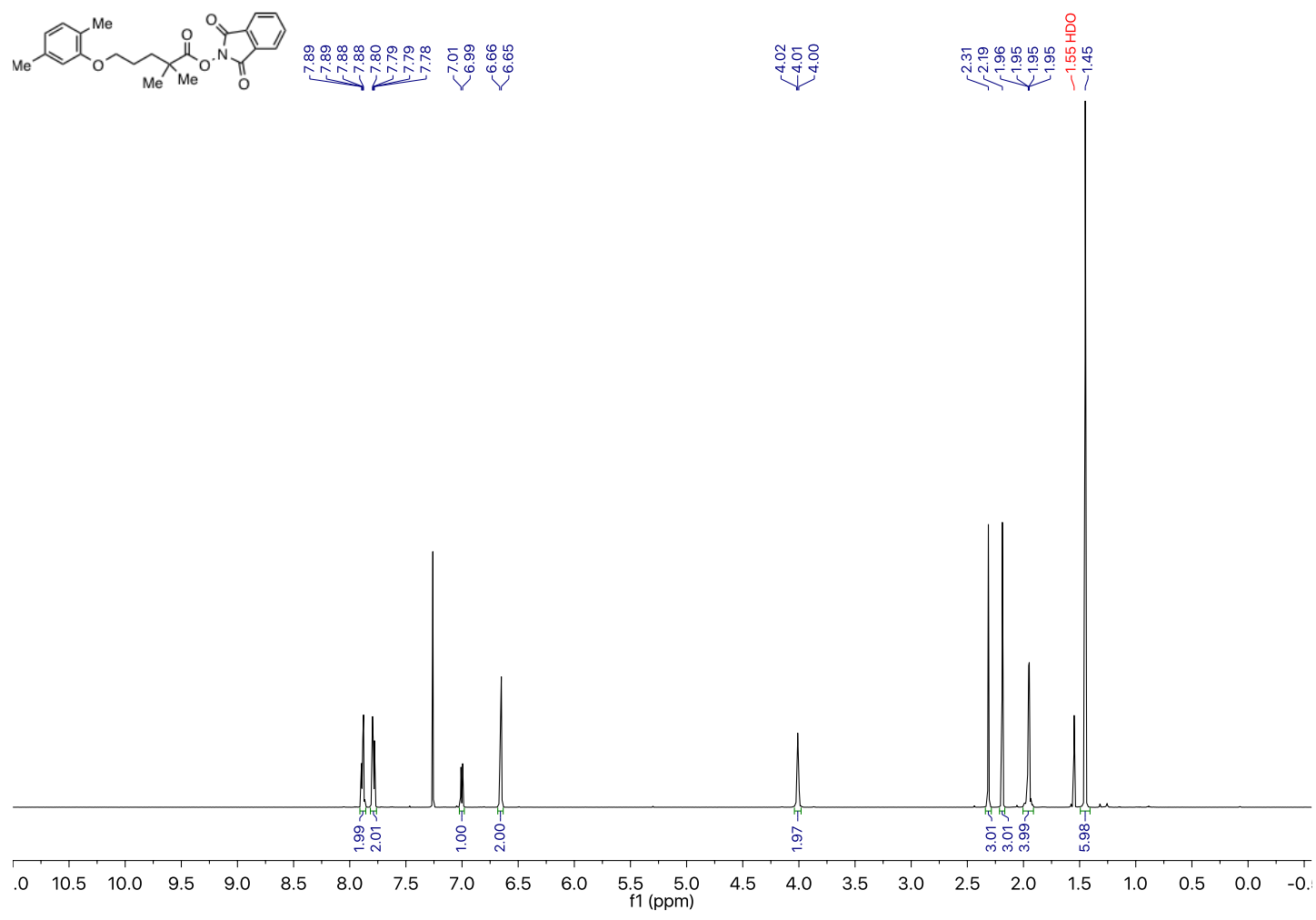

$126 \mathrm{MHz}{ }^{13} \mathrm{C}$-NMR of 1,3-dioxoisoindolin-2-yl 5-(2,5-dimethylphenoxy)-2,2-dimethylpentanoate (41) in $\mathrm{CDCl}_{3}$

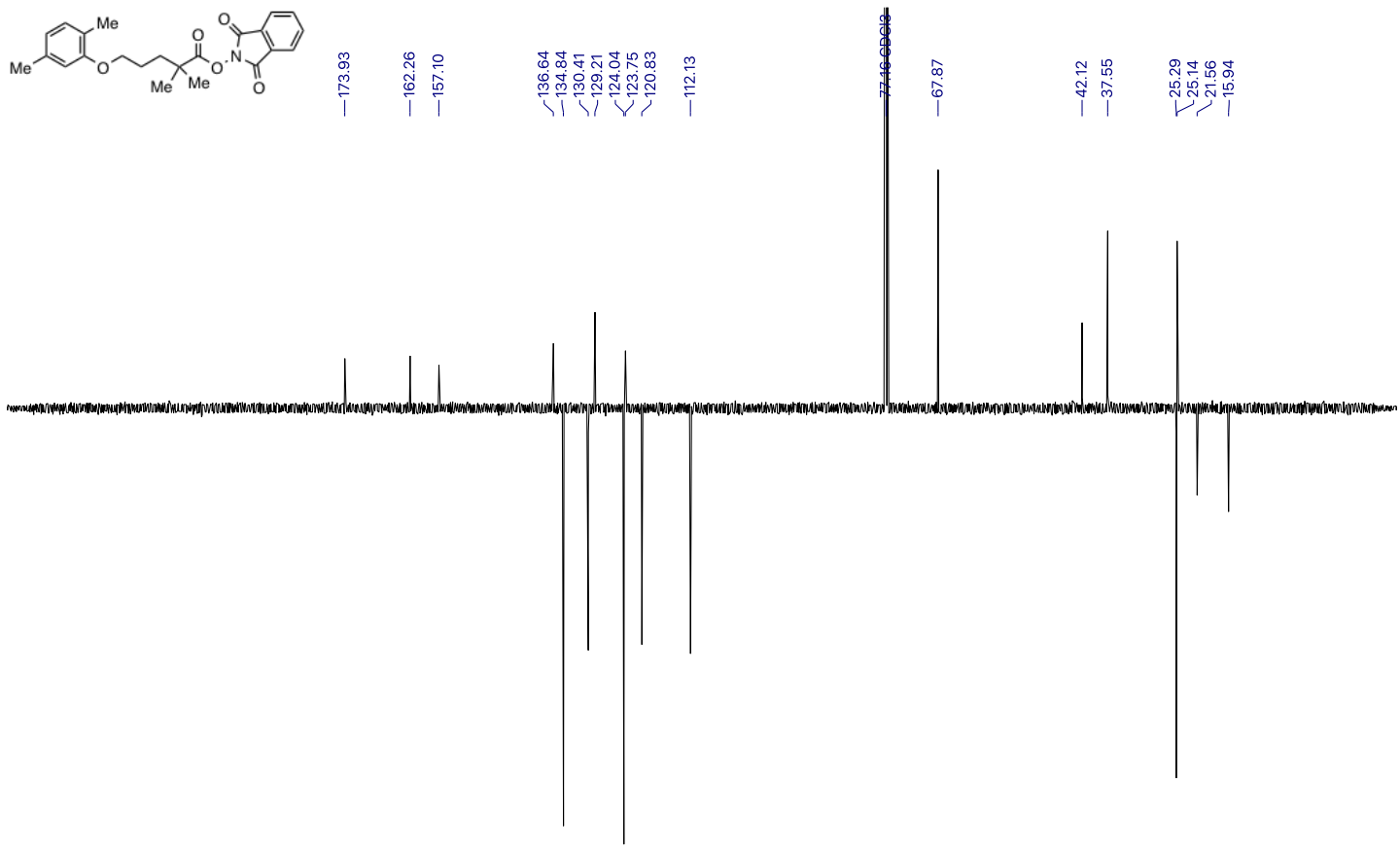

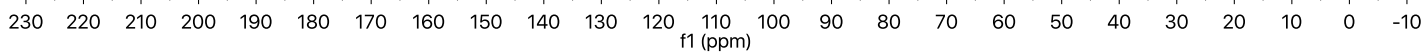


$500 \mathrm{MHz}{ }^{1} \mathrm{H}-\mathrm{NMR}$ of 2-((4-fluoro-4-methylpentyl)oxy)-1,4-dimethylbenzene (30) in $\mathrm{CDCl}_{3}$

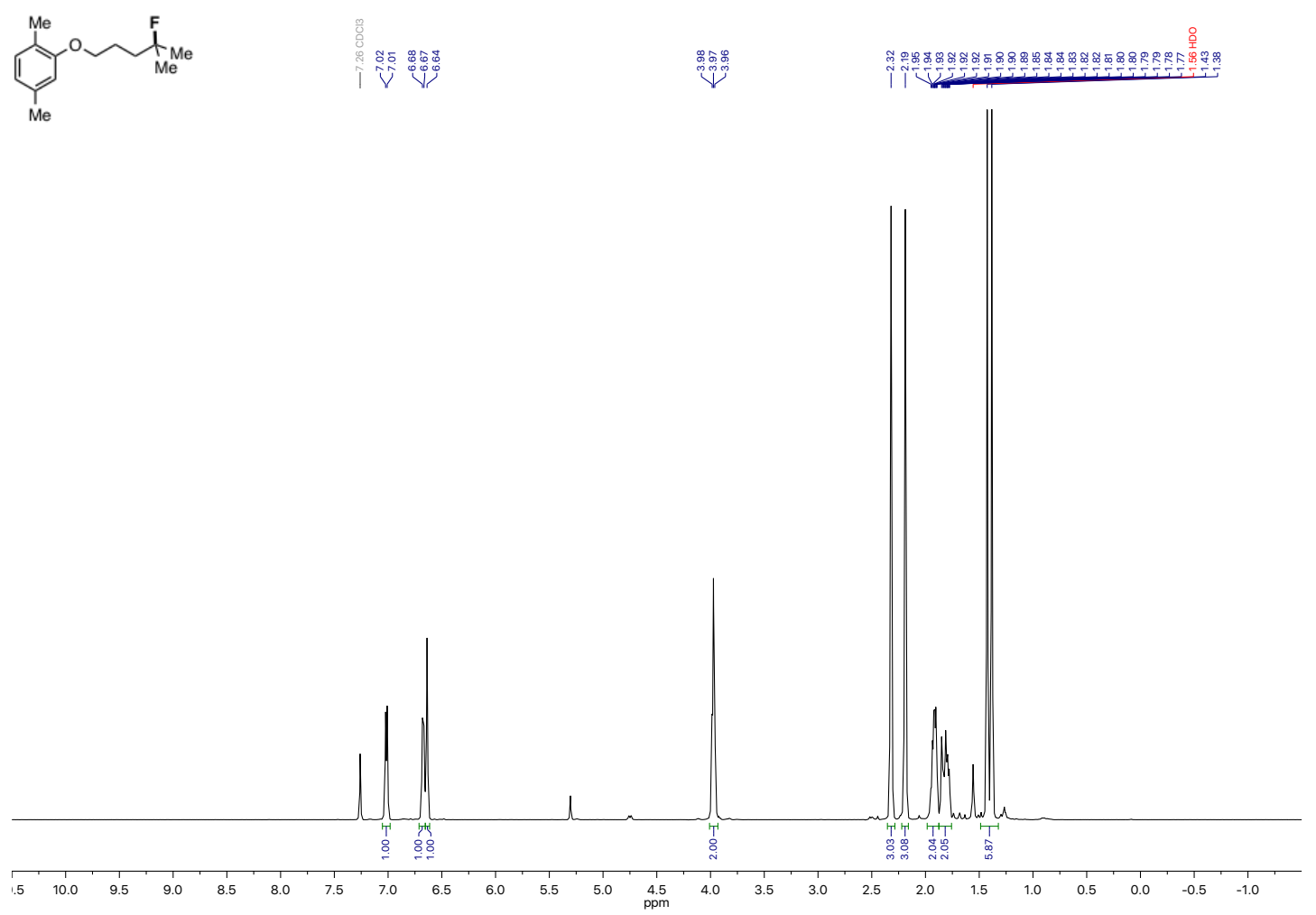

$126 \mathrm{MHz}{ }^{13} \mathrm{C}-\mathrm{NMR}$ of 2-((4-fluoro-4-methylpentyl)oxy)-1,4-dimethylbenzene (30) in $\mathrm{CDCl}_{3}$

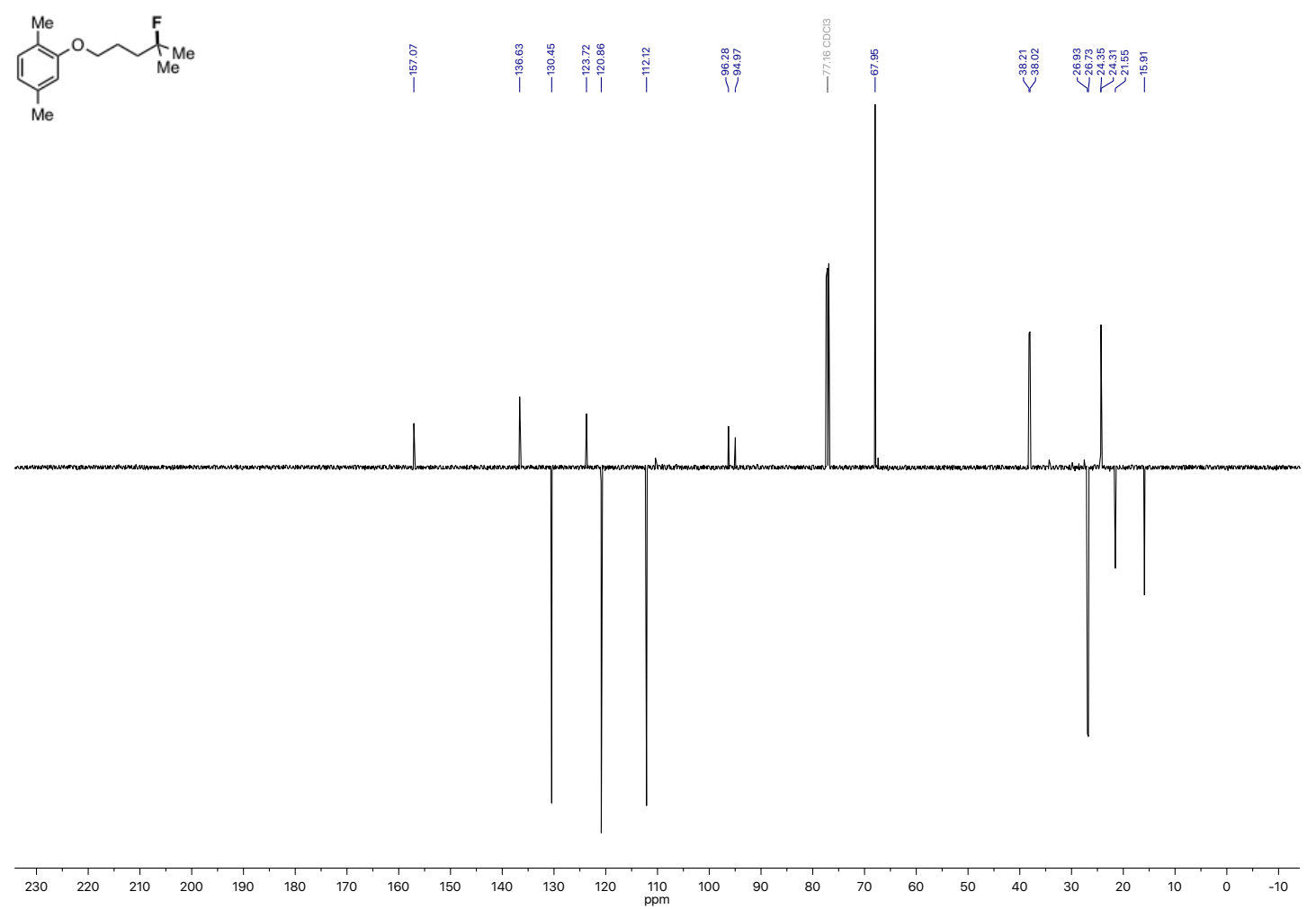


$376 \mathrm{MHz}{ }^{19} \mathrm{~F}-\mathrm{NMR}$ of 2-((4-fluoro-4-methylpentyl)oxy)-1,4-dimethylbenzene (30) in $\mathrm{CDCl}_{3}$
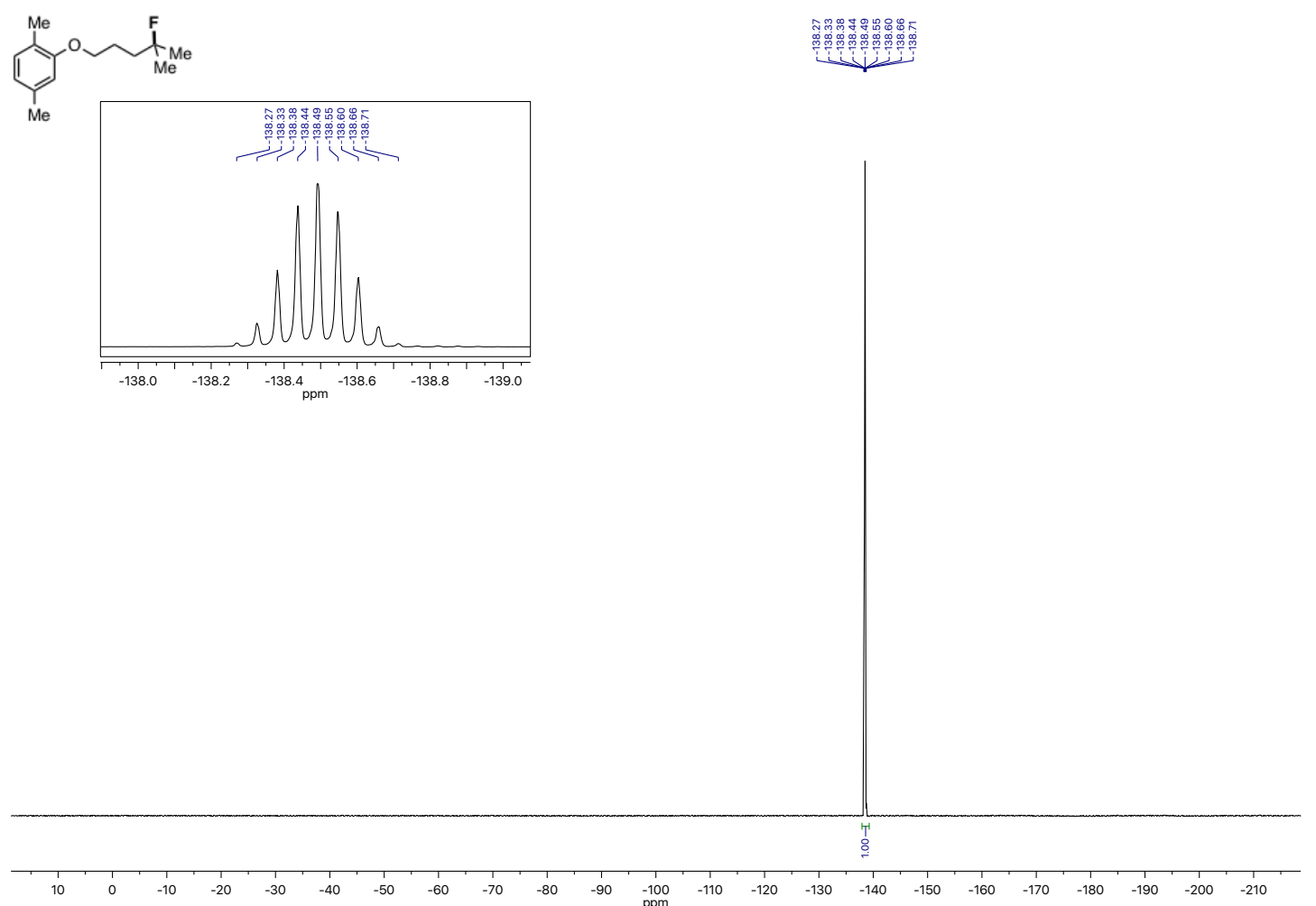
$500 \mathrm{MHz}{ }^{1} \mathrm{H}-\mathrm{NMR}$ of 1,3-dioxoisoindolin-2-yl ( $\left.2 S, 4 \mathrm{a} S, 6 \mathrm{a} S, 6 \mathrm{~b} R, 12 \mathrm{a} S, 12 \mathrm{~b} R, 14 \mathrm{~b} R\right)-2,4 \mathrm{a}, 6 \mathrm{a}, 6 \mathrm{~b}, 9,9,12 \mathrm{a}$-heptamethyl10,13-dioxo-1,2,3,4,4a ,5,6,6a,6b,7,8,8a,9,10,11,12,12a,12b,13,14b-icosahydropicene-2-carboxylate (S25) in $\mathrm{CDCl}_{3}$

$\frac{\infty}{0}$

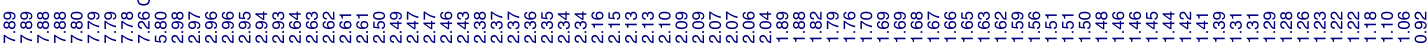
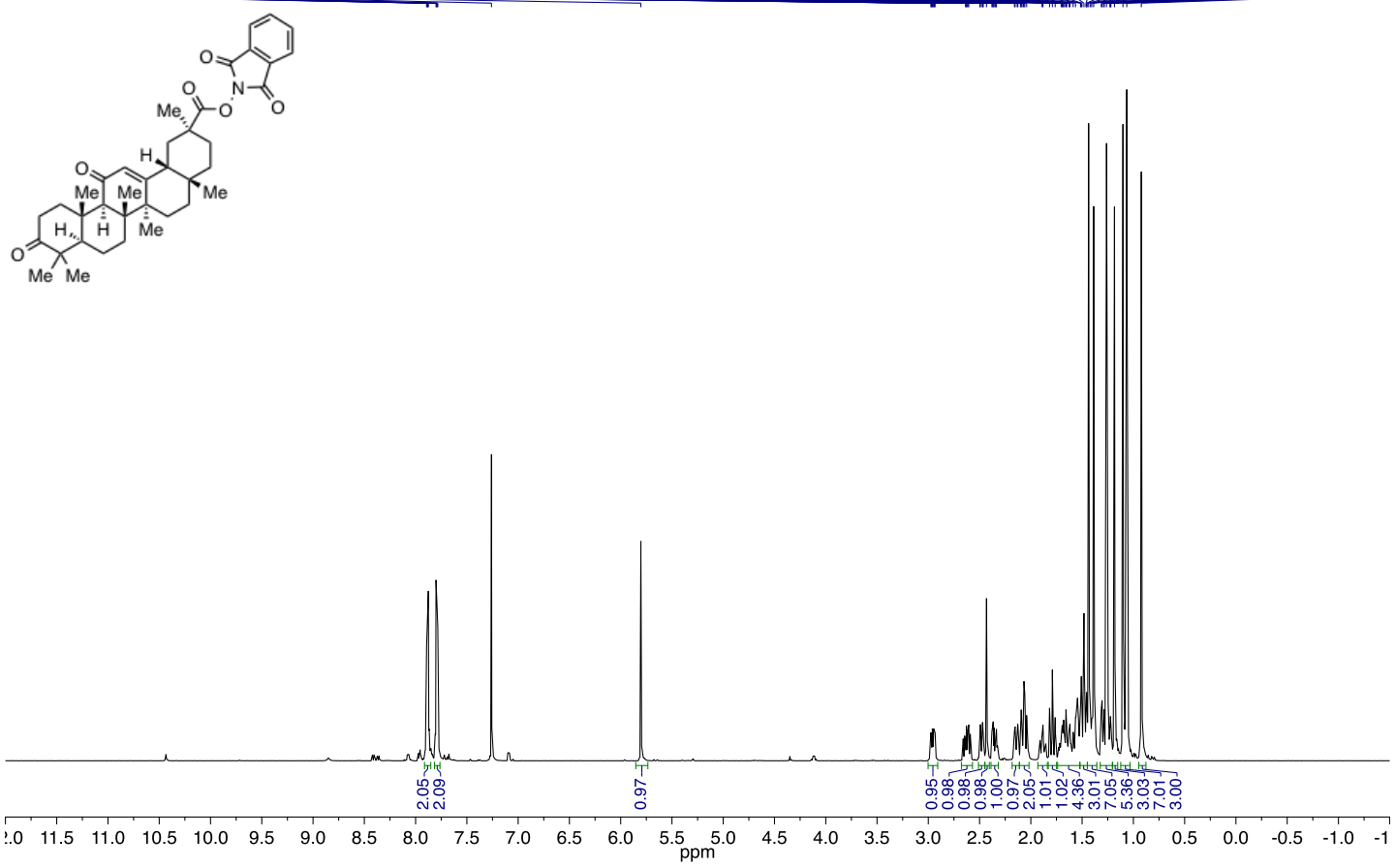
$126 \mathrm{MHz}{ }^{13} \mathrm{C}-\mathrm{NMR}$ of 1,3-dioxoisoindolin-2-yl (2S,4aS,6aS,6b $\left.R, 12 \mathrm{a} S, 12 \mathrm{~b} R, 14 \mathrm{~b} R\right)$-2,4a,6a,6b,9,9,12a-heptamethyl10,13-dioxo-1,2,3,4,4a ,5,6,6a,6b,7,8,8a,9,10,11,12,12a,12b,13,14b-icosahydropicene-2-carboxylate (S25) in $\mathrm{CDCl}_{3}$

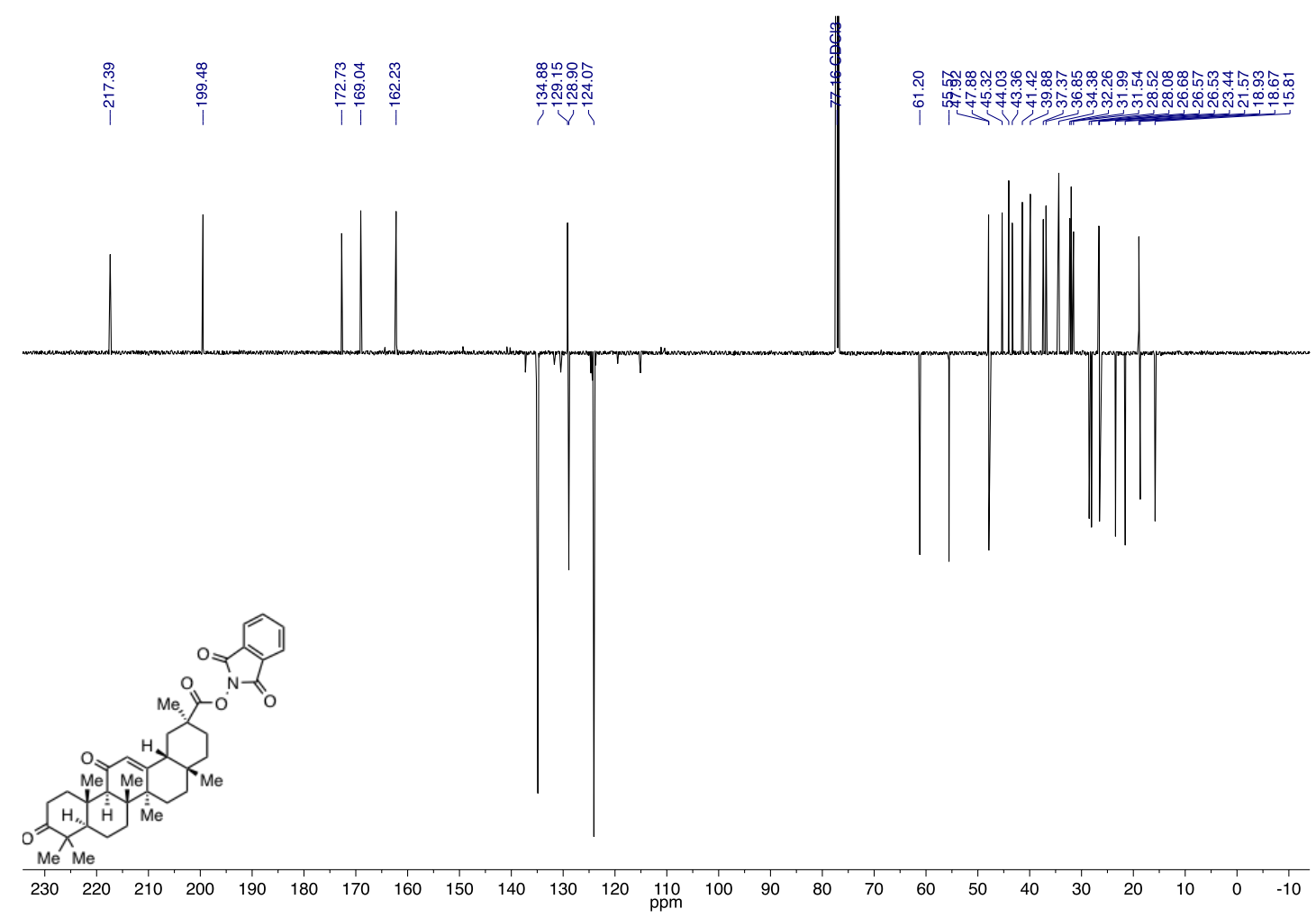


$500 \mathrm{MHz}{ }^{1} \mathrm{H}-\mathrm{NMR}$ of $(4 \mathrm{a} R, 6 \mathrm{a} R, 6 \mathrm{~b} S, 8 \mathrm{a} S, 12 \mathrm{a} R, 14 \mathrm{a} R, 14 \mathrm{~b} S)$-11-fluoro-4,4,6a $, 6 \mathrm{~b}, 8 \mathrm{a}, 11,14 \mathrm{~b}$-heptamethyl$1,2,4,4 \mathrm{a}, 5,6,6 \mathrm{a}, 6 \mathrm{~b}, 7,8,8 \mathrm{a}, 9,10,11,12,12 \mathrm{a}, 14 \mathrm{a}, 14 \mathrm{~b}$-octadecahydropicene-3,14-dione (31) in $\mathrm{CDCl}_{3}$

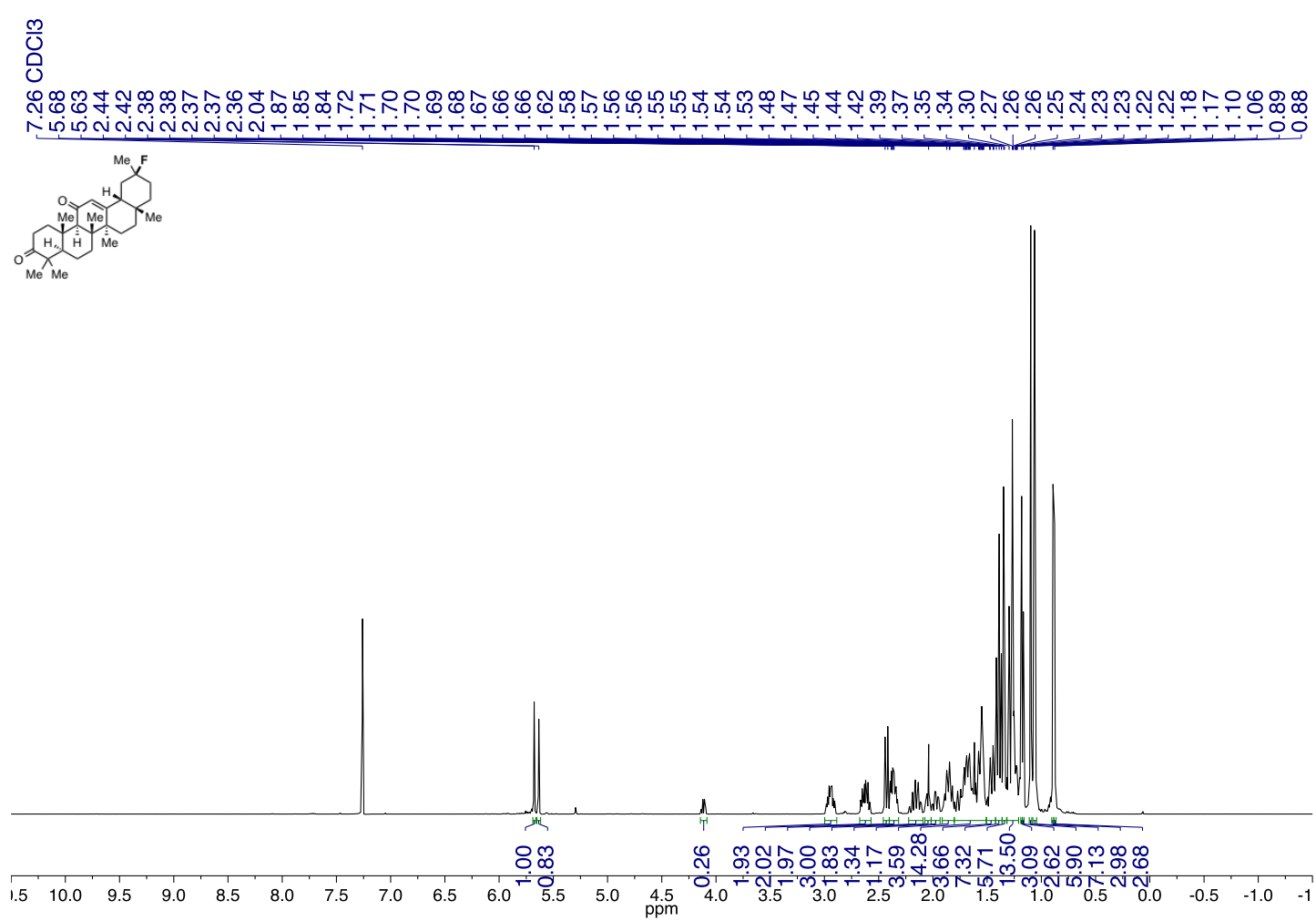


$126 \mathrm{MHz}{ }^{13} \mathrm{C}-\mathrm{NMR}$ of $(4 \mathrm{a} R, 6 \mathrm{a} R, 6 \mathrm{~b} S, 8 \mathrm{a} S, 12 \mathrm{a} R, 14 \mathrm{a} R, 14 \mathrm{~b} S)$-11-fluoro-4,4,6a,6b,8a, 11,14b-heptamethyl$1,2,4,4 \mathrm{a}, 5,6,6 \mathrm{a}, 6 \mathrm{~b}, 7,8,8 \mathrm{a}, 9,10,11,12,12 \mathrm{a}, 14 \mathrm{a}, 14 \mathrm{~b}$-octadecahydropicene-3,14-dione (31) in $\mathrm{CDCl}_{3}$

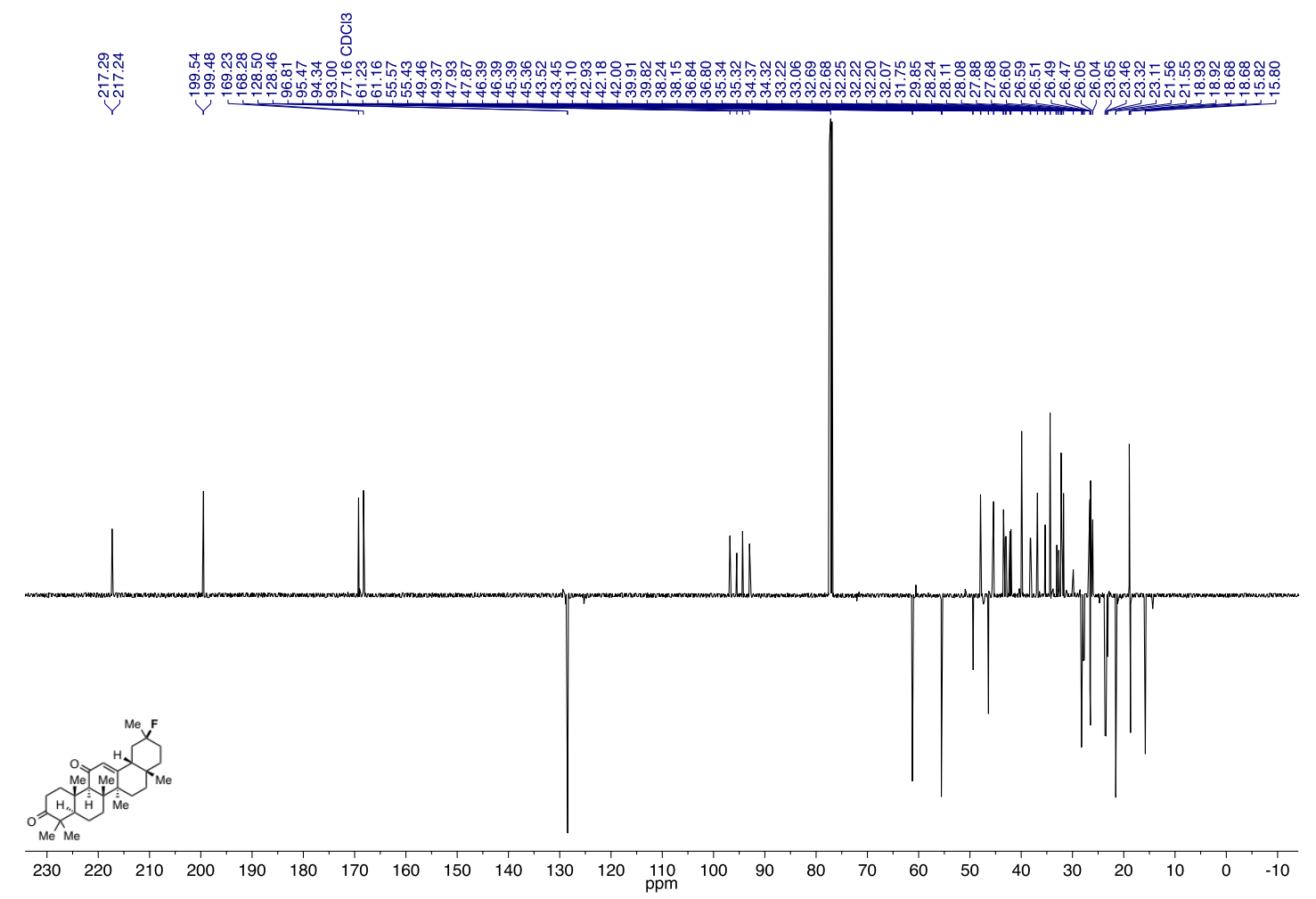


$282 \mathrm{MHz}{ }^{19} \mathrm{~F}-\mathrm{NMR}$ of $(4 \mathrm{a} R, 6 \mathrm{a} R, 6 \mathrm{~b} S, 8 \mathrm{a} S, 12 \mathrm{a} R, 14 \mathrm{a} R, 14 \mathrm{~b} S)$-11-fluoro-4,4,6a,6b,8a, 11,14b-heptamethyl$1,2,4,4 \mathrm{a}, 5,6,6 \mathrm{a}, 6 \mathrm{~b}, 7,8,8 \mathrm{a}, 9,10,11,12,12 \mathrm{a}, 14 \mathrm{a}, 14 \mathrm{~b}$-octadecahydropicene-3,14-dione (31) in $\mathrm{CDCl}_{3}$

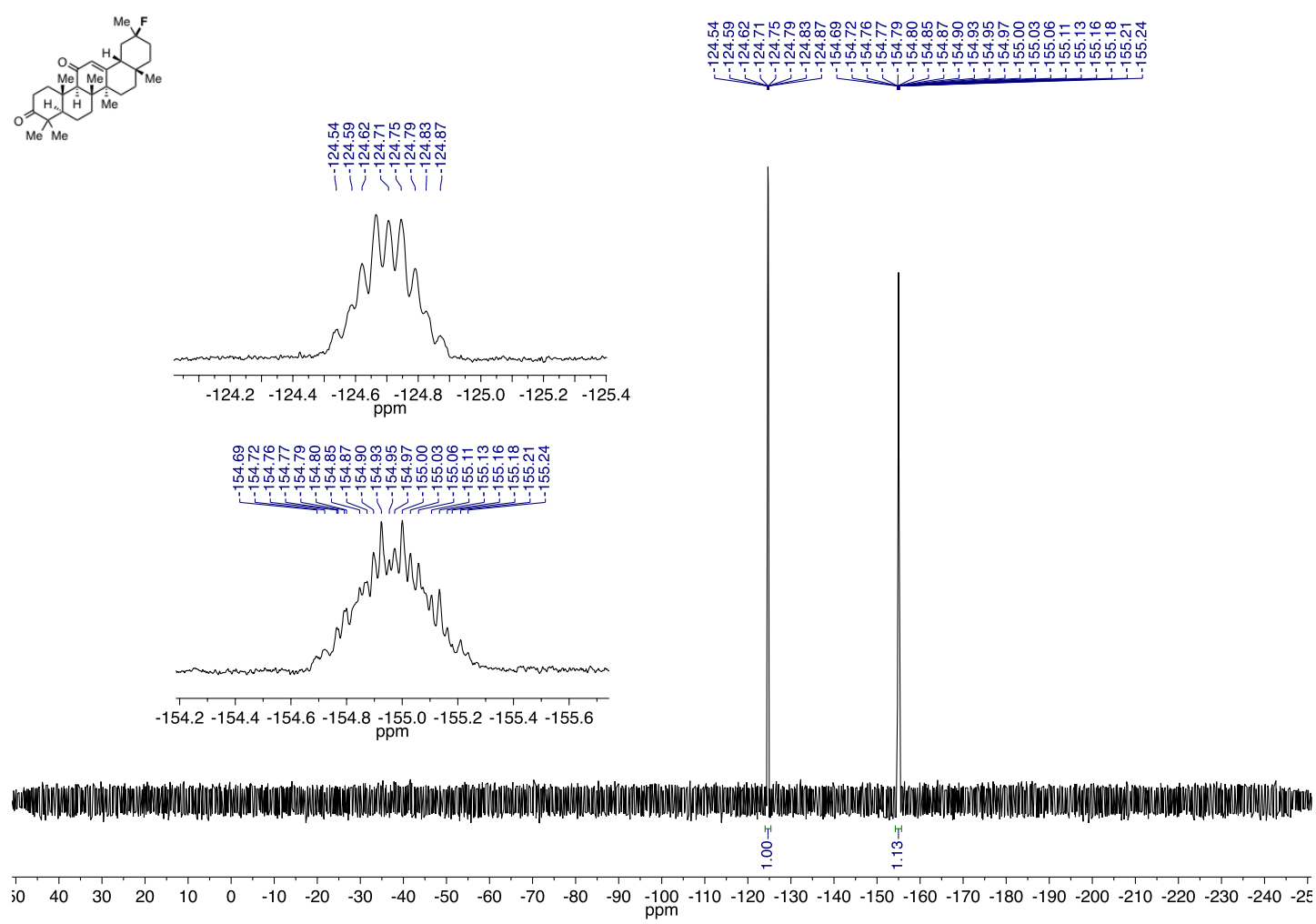

$500 \mathrm{MHz}{ }^{1} \mathrm{H}-\mathrm{NMR}$ of 1,3-dioxoisoindolin-2-yl 2-(4-(tert-butyl)phenoxy)acetate (S26) in $\mathrm{CDCl}_{3}$

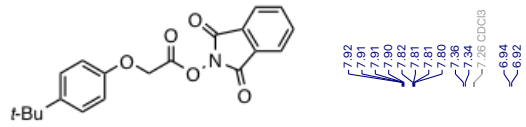

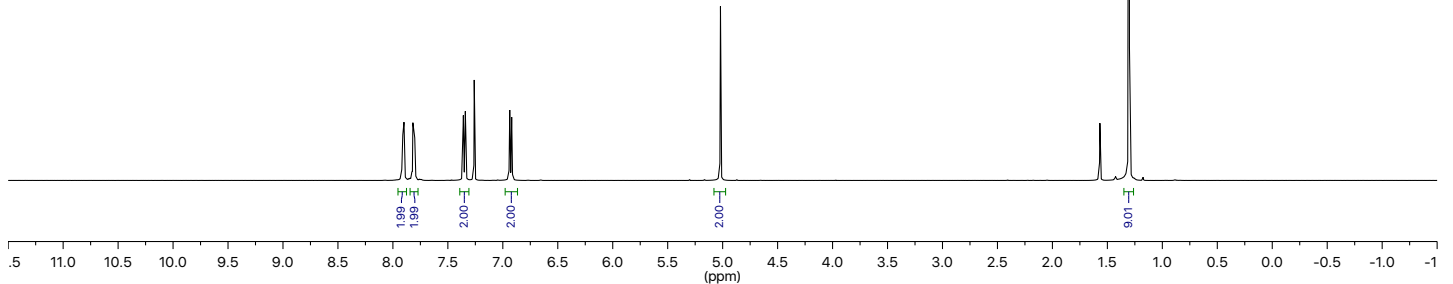


$126 \mathrm{MHz}{ }^{13} \mathrm{C}-\mathrm{NMR}$ of 1,3-dioxoisoindolin-2-yl 2-(4-(tert-butyl)phenoxy)acetate (S26) in $\mathrm{CDCl}_{3}$
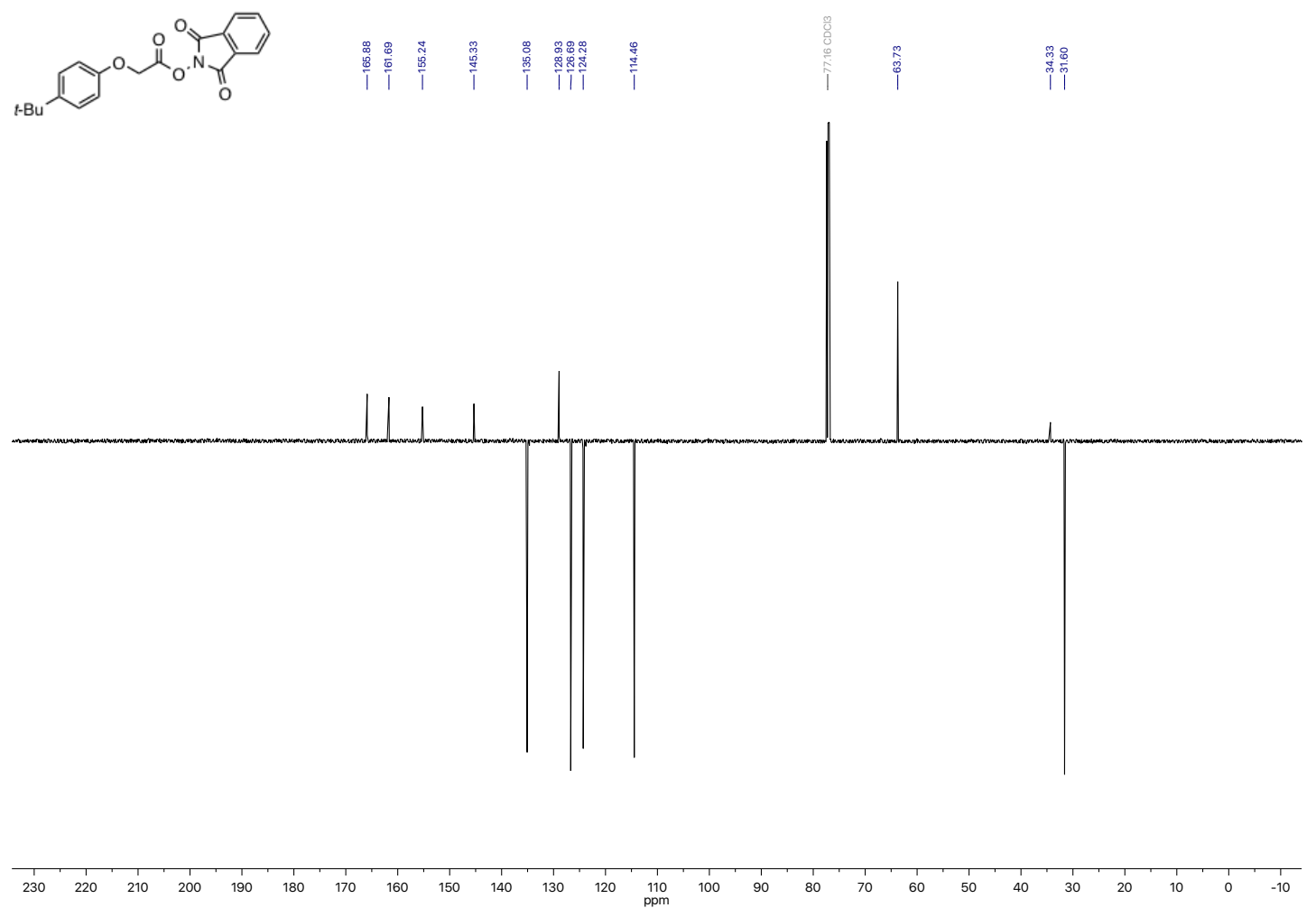

$126 \mathrm{MHz}{ }^{13} \mathrm{C}-\mathrm{NMR}$ of 1,3-dioxoisoindolin-2-yl 2-(4-(tert-butyl)phenoxy)acetate (32) in $\mathrm{CDCl}_{3}$

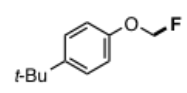
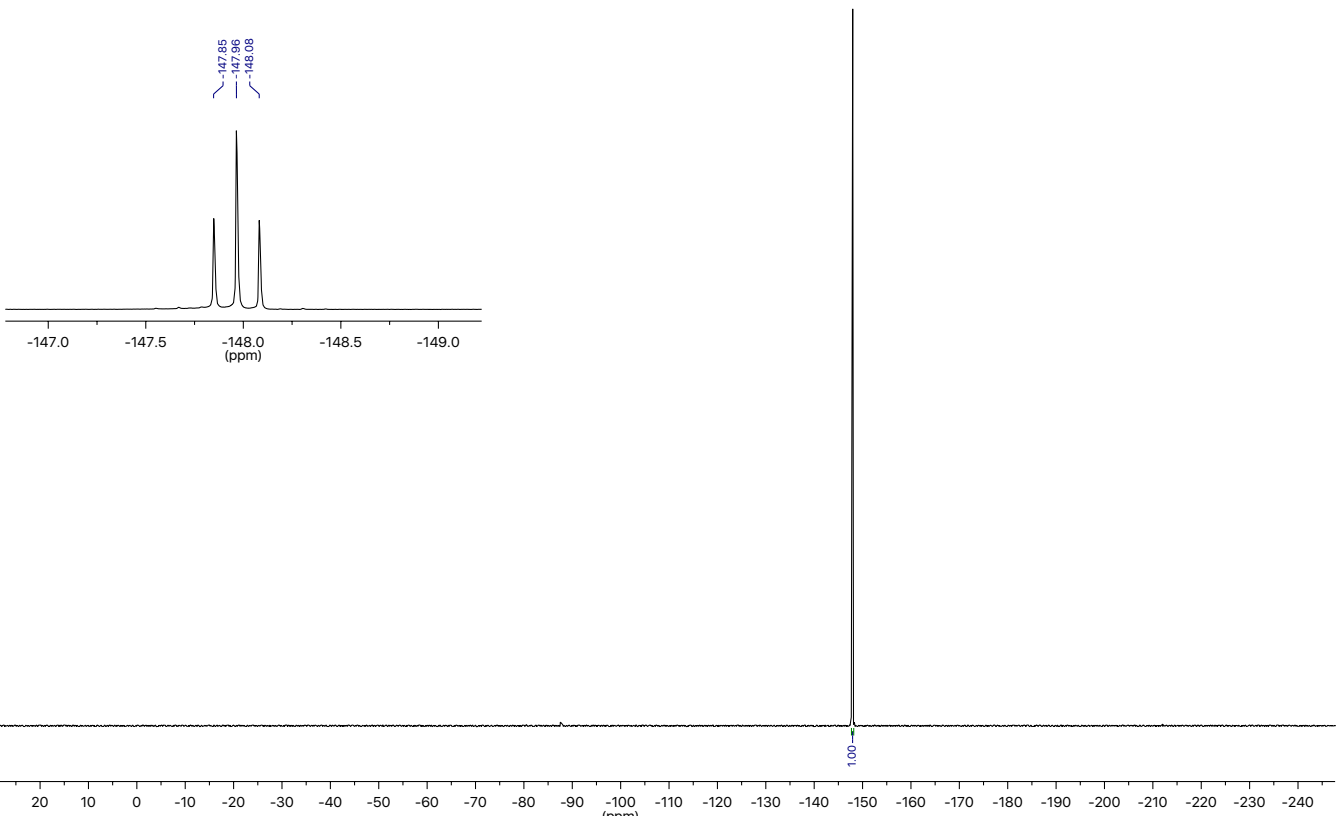
$500 \mathrm{MHz}{ }^{1} \mathrm{H}-\mathrm{NMR}$ of 1,3-dioxoisoindolin-2-yl 2-((4-bromophenyl)thio)acetate (S27) in $\mathrm{CDCl}_{3}$

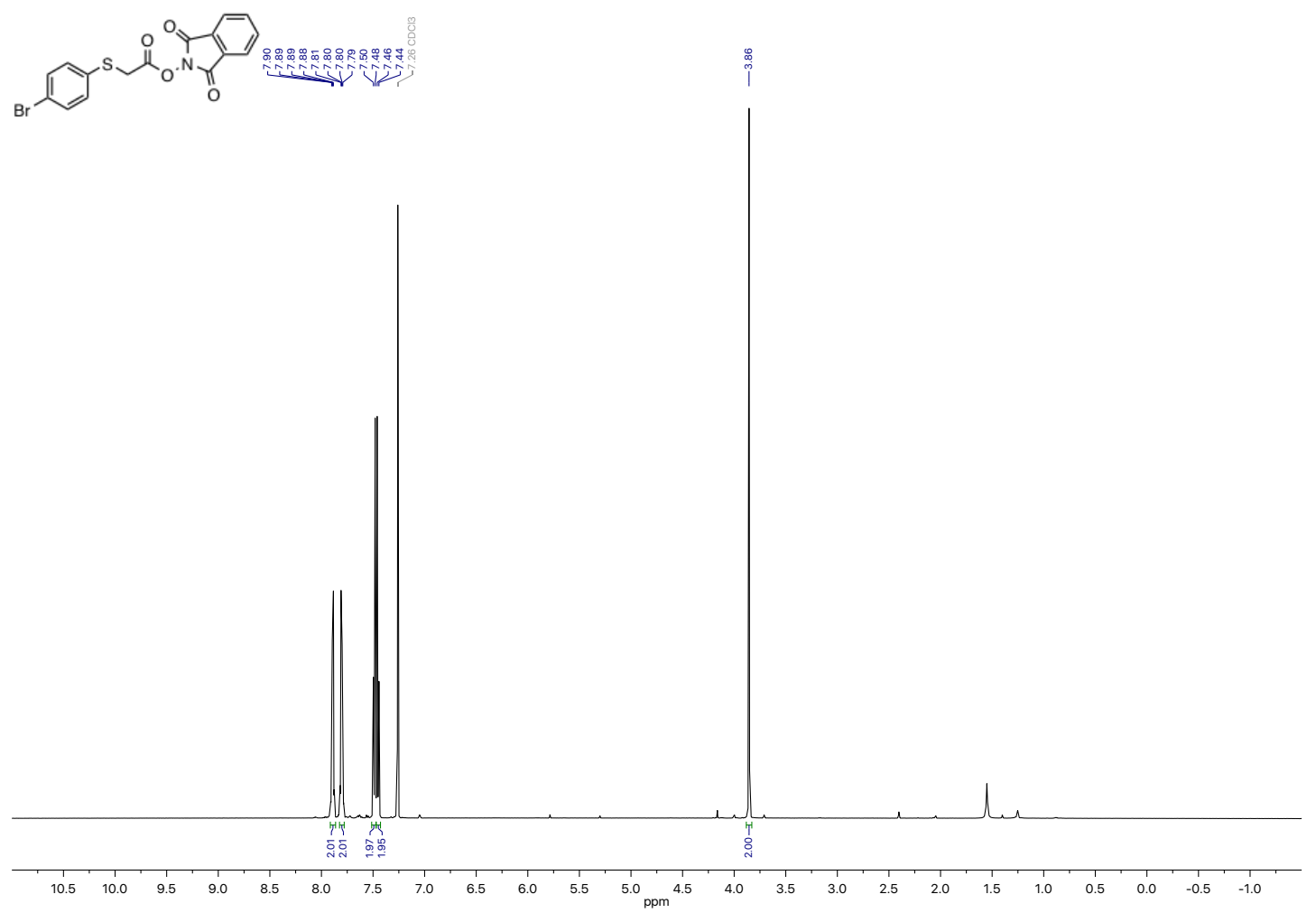

$126 \mathrm{MHz}{ }^{13} \mathrm{C}$-NMR of 1,3-dioxoisoindolin-2-yl 2-((4-bromophenyl)thio)acetate (S27) in $\mathrm{CDCl}_{3}$
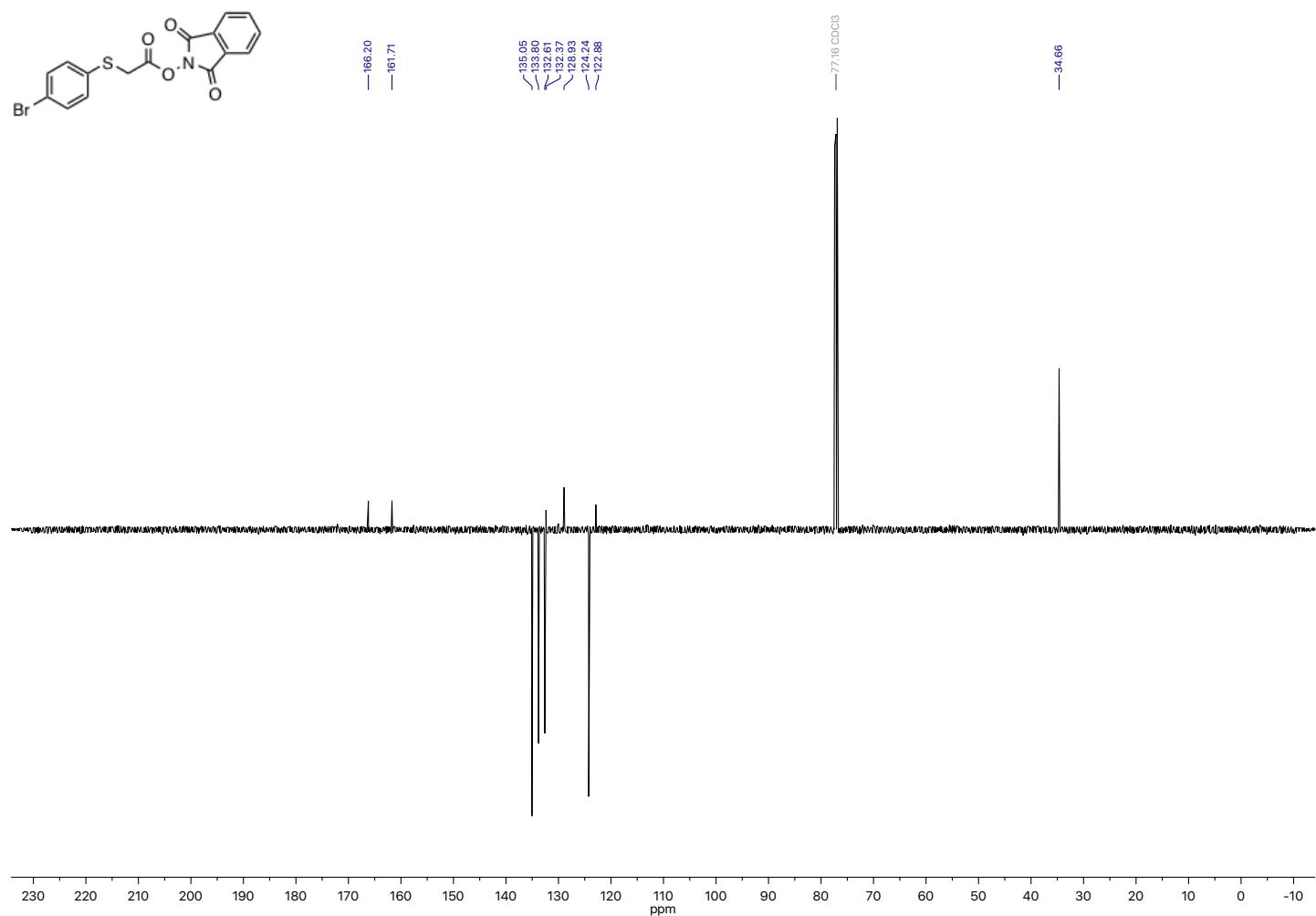
$500 \mathrm{MHz}{ }^{1} \mathrm{H}-\mathrm{NMR}$ of (4-bromophenyl)(fluoromethyl)sulfane (33) in $\mathrm{CDCl}_{3}$

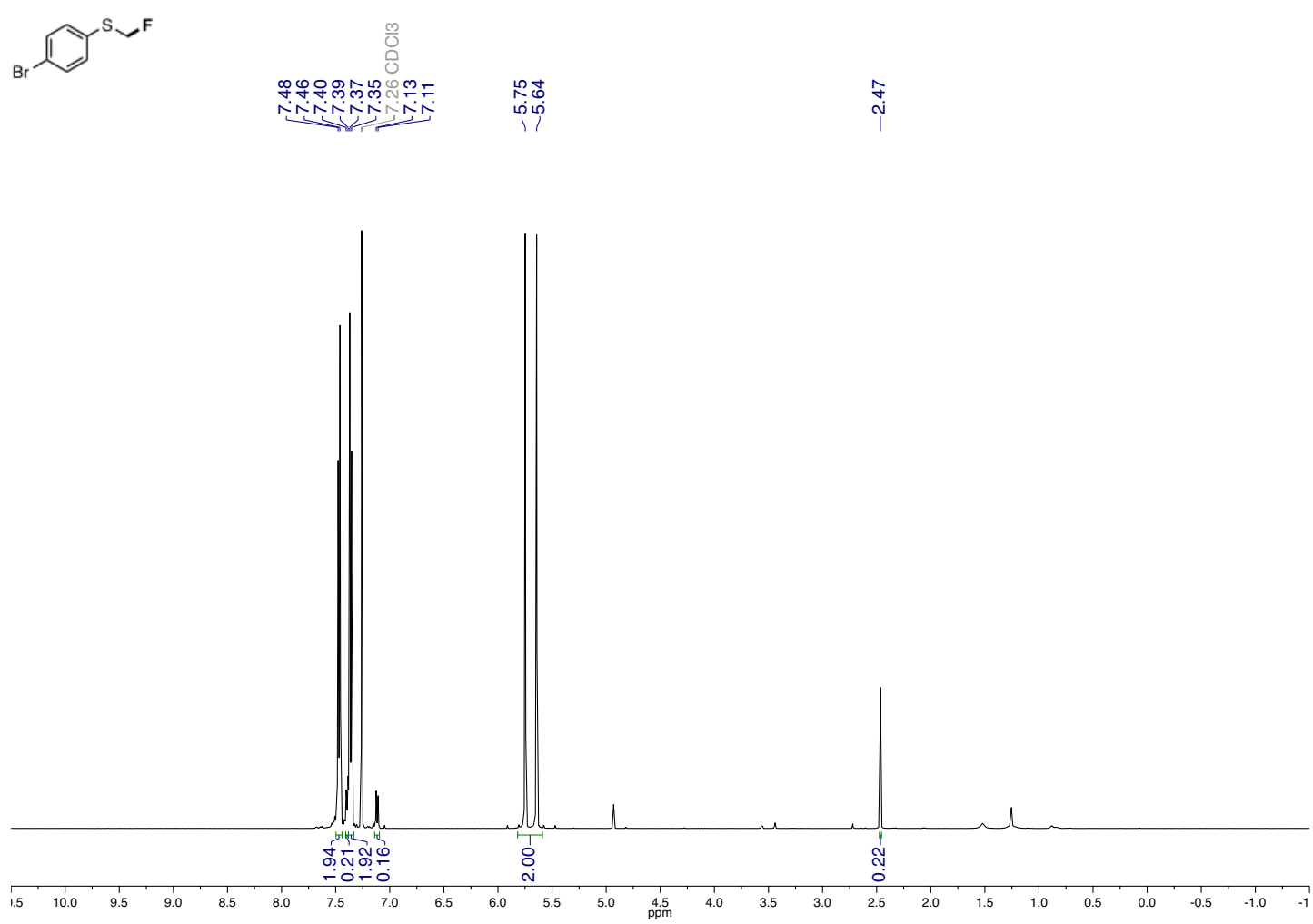

$126 \mathrm{MHz}{ }^{13} \mathrm{C}$-NMR of (4-bromophenyl)(fluoromethyl)sulfane (33) in $\mathrm{CDCl}_{3}$
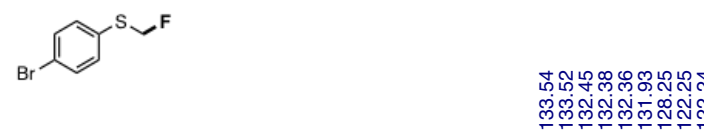

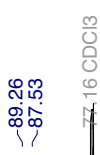
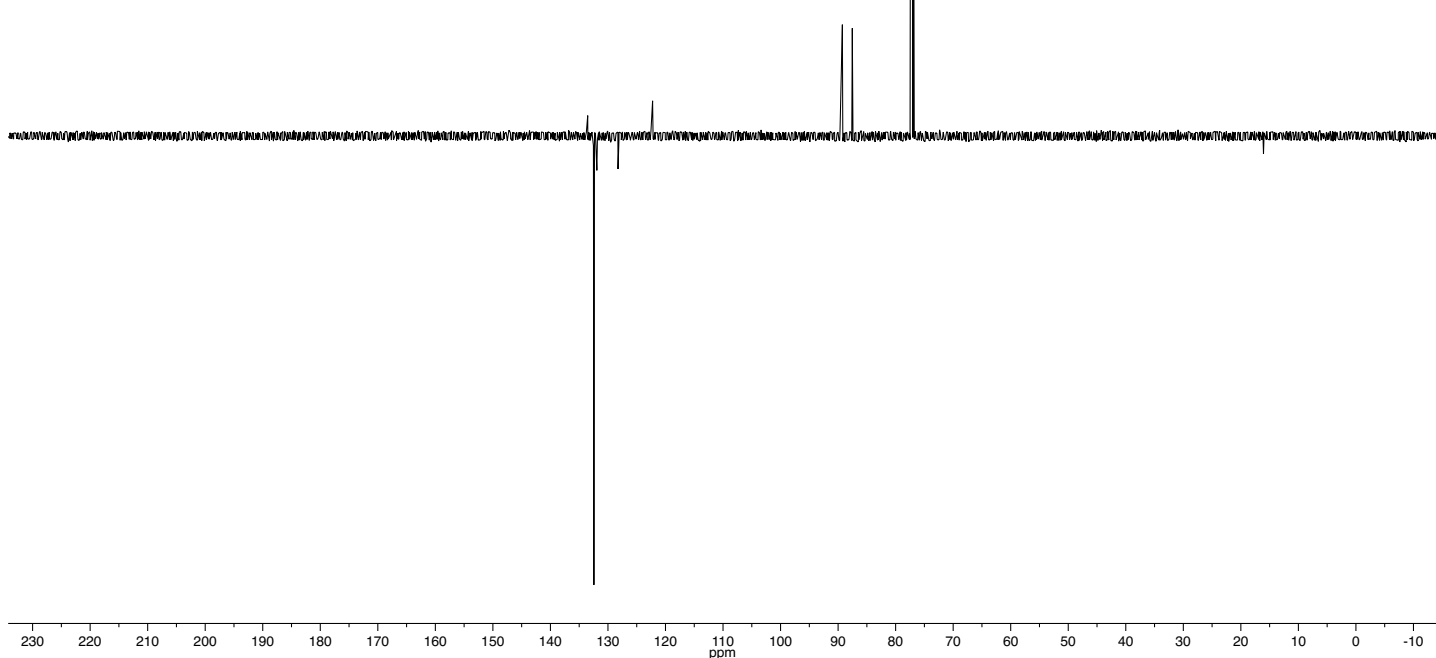
$282 \mathrm{MHz}{ }^{19} \mathrm{~F}-\mathrm{NMR}$ of (4-bromophenyl)(fluoromethyl)sulfane (33) in $\mathrm{CDCl}_{3}$

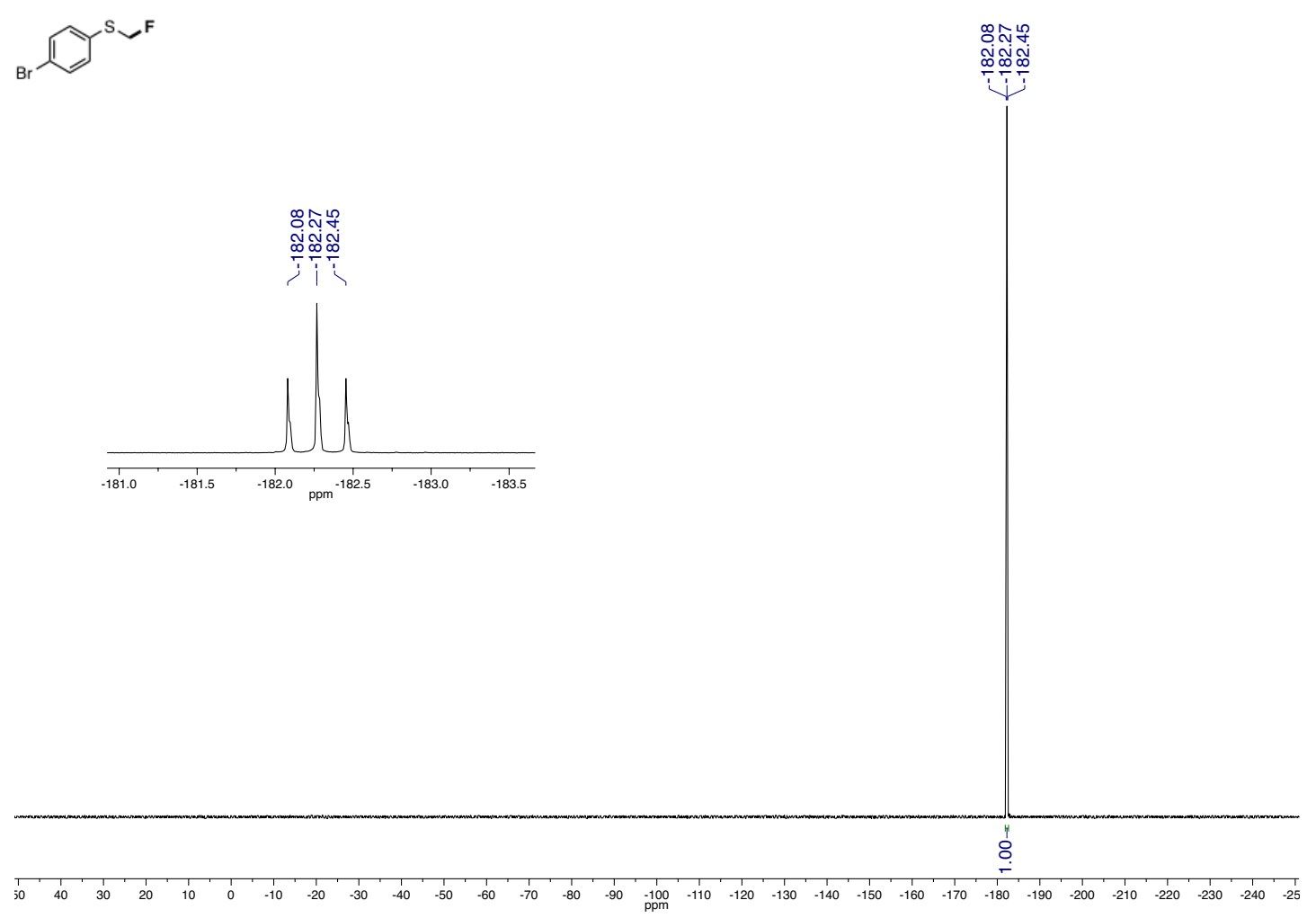


$500 \mathrm{MHz}$ 1H-NMR of 1,3-dioxoisoindolin-2-yl (3aS,4S,6R,6aR)-6-(benzyloxy)-2,2-dimethyltetrahydrofuro[3,4$d][1,3]$ dioxole-4-carboxylate (S28) in $\mathrm{CDCl} 3$

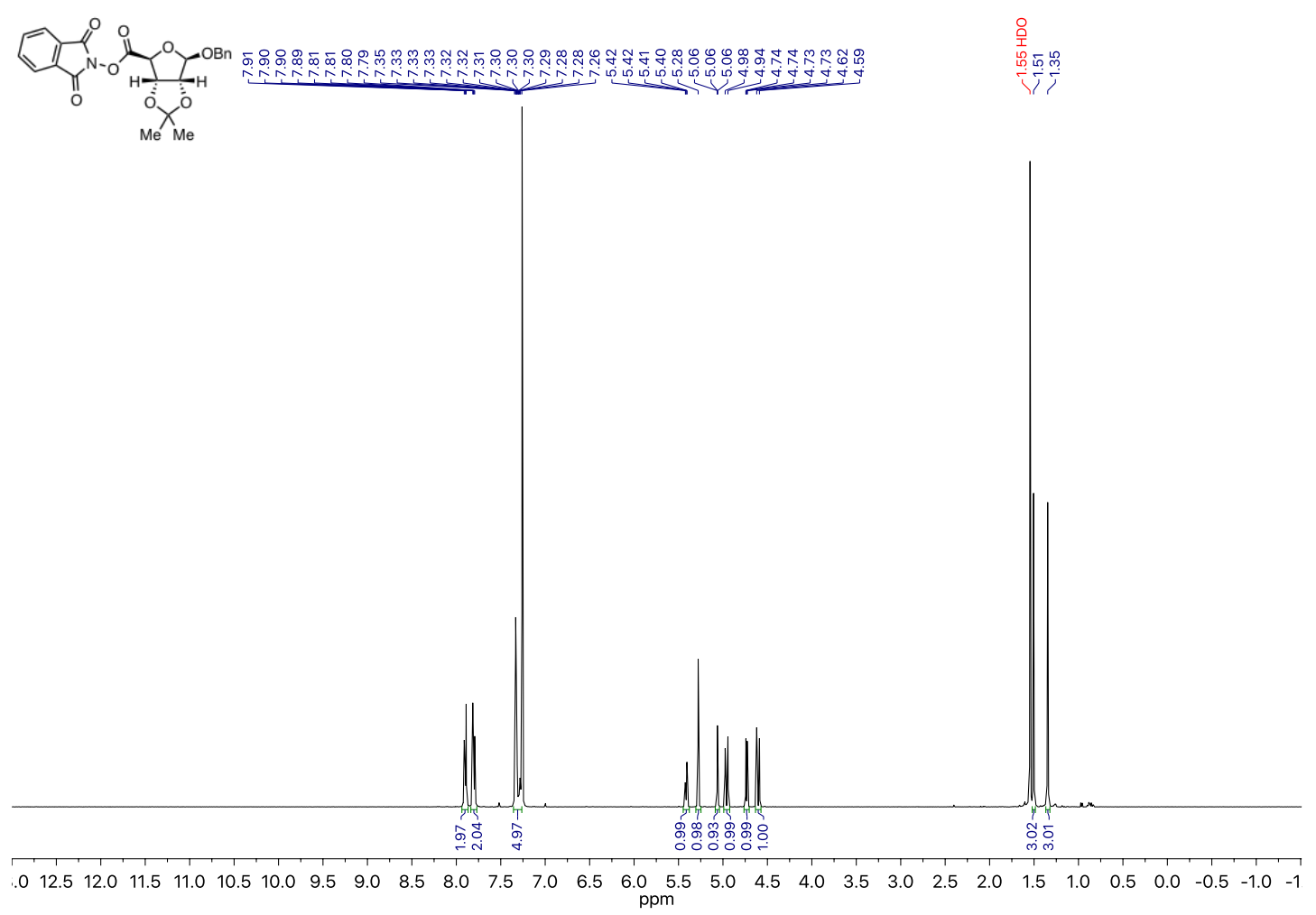


$126 \mathrm{MHz}{ }^{13} \mathrm{C}-\mathrm{NMR}$ of 1,3-dioxoisoindolin-2-yl (3aS,4S,6R,6aR)-6-(benzyloxy)-2,2-dimethyltetrahydrofuro[3,4d] [1,3]dioxole-4-carboxylate (S28) in $\mathrm{CDCl}_{3}$
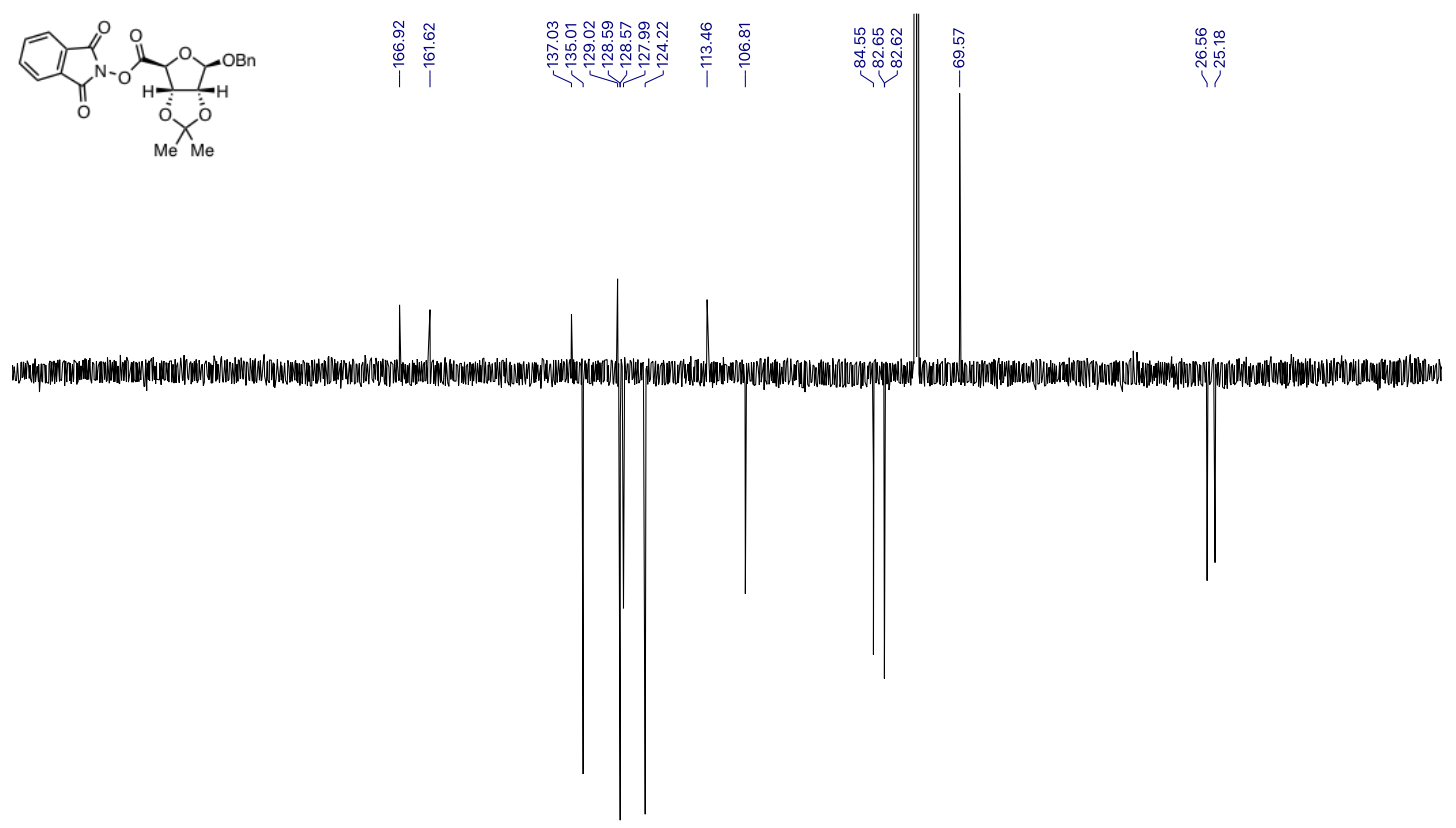

$\begin{array}{lllllllllllllllllllllllllllllll}230 & 220 & 210 & 200 & 190 & 180 & 170 & 160 & 150 & 140 & 130 & 120 & \begin{array}{c}110 \\ (\mathrm{ppm})\end{array} & 100 & 90 & 80 & 70 & 60 & 50 & 40 & 30 & 20 & 10 & 0 & -10\end{array}$ 
$500 \mathrm{MHz}{ }^{1} \mathrm{H}-\mathrm{NMR}$ of $(3 \mathrm{a} R, 4 R, 6 R, 6 \mathrm{a} S)$-4-(benzyloxy)-6-fluoro-2 2-dimethyltetrahydrofuro[3,4- $\left.d\right][1,3]$ dioxole (34) in $\mathrm{CDCl}_{3}$

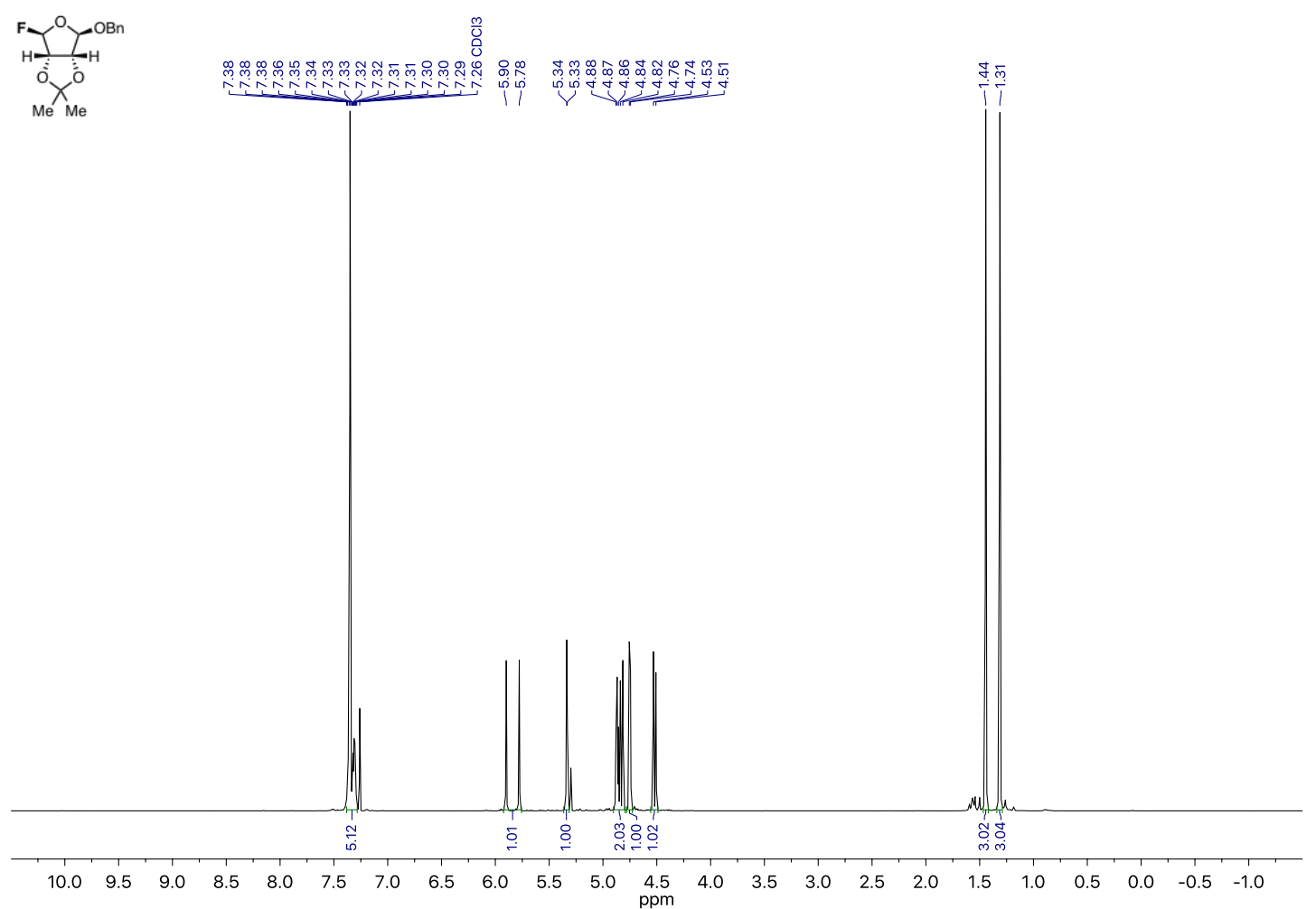


$126 \mathrm{MHz}{ }^{13} \mathrm{C}-\mathrm{NMR}$ of $(3 \mathrm{a} R, 4 R, 6 R, 6 \mathrm{a} S)$-4-(benzyloxy)-6-fluoro-2,2-dimethyltetrahydrofuro[3,4- $\left.d\right][1,3]$ dioxole (34) in $\mathrm{CDCl}_{3}$

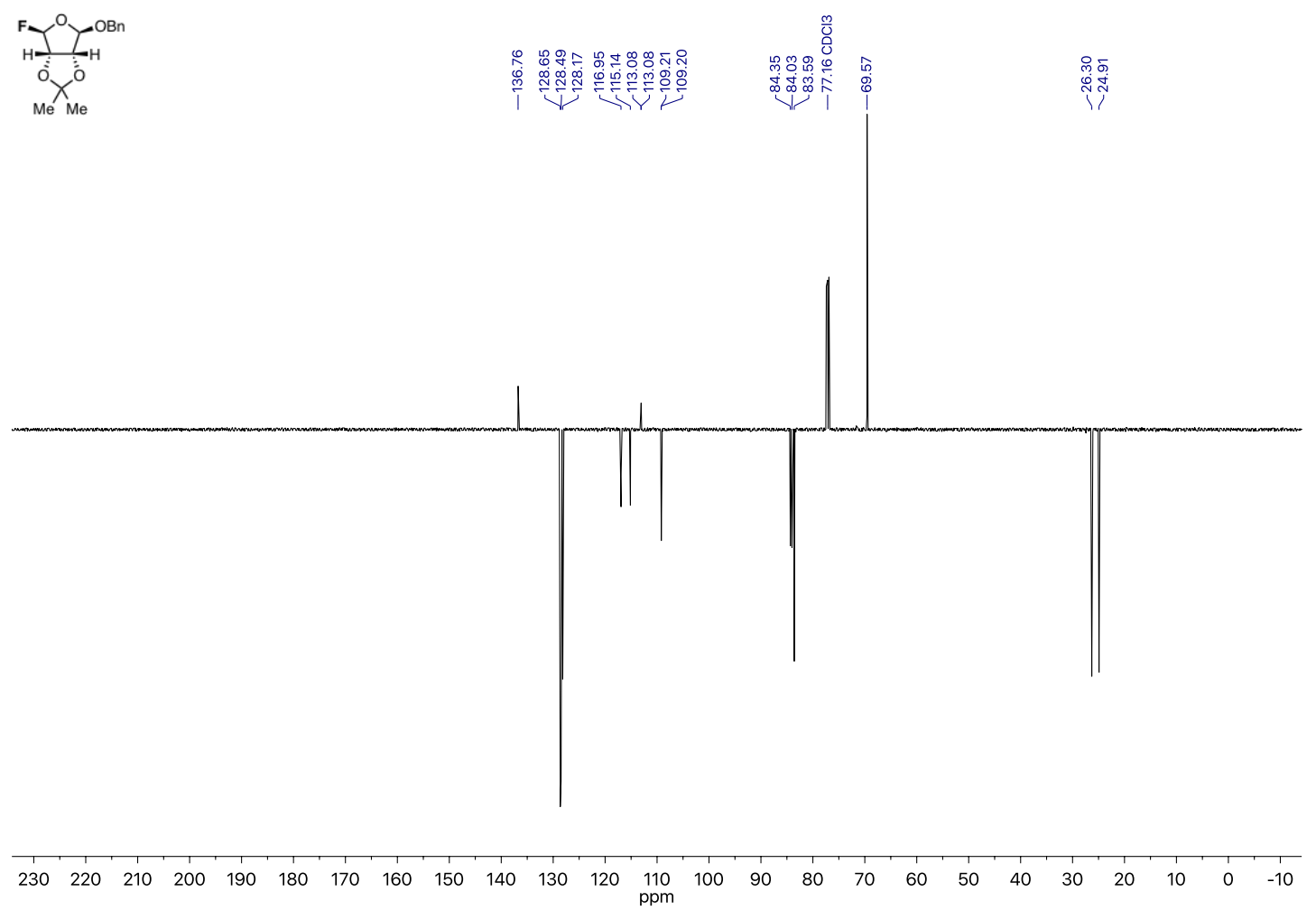


$470 \mathrm{MHz}{ }^{19} \mathrm{~F}-\mathrm{NMR}$ of $(3 \mathrm{a} R, 4 R, 6 R, 6 \mathrm{a} S)$-4-(benzyloxy)-6-fluoro-2,2-dimethyltetrahydrofuro[3,4- $\left.d\right][1,3]$ dioxole (34) in $\mathrm{CDCl}_{3}$
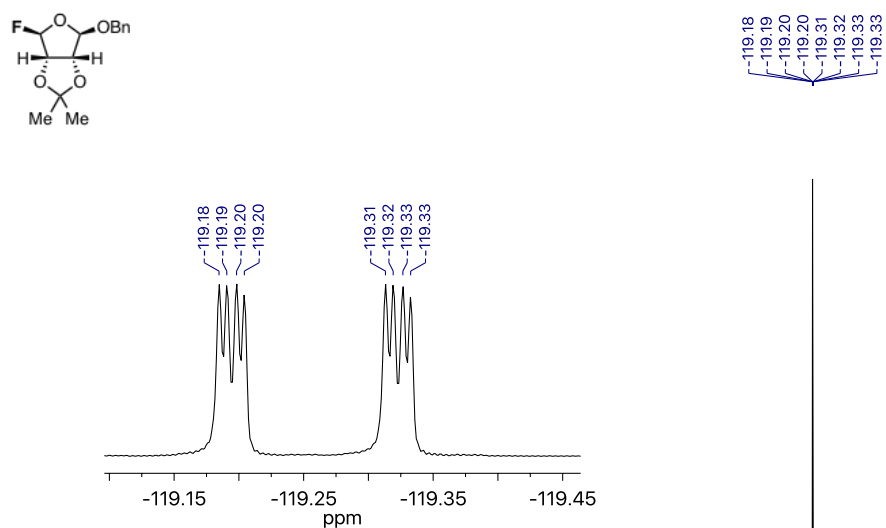

\section{$\stackrel{8}{8}$}

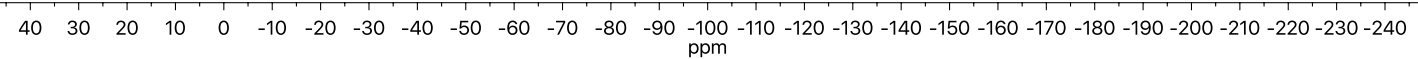


$500 \mathrm{MHz}{ }^{1} \mathrm{H}-\mathrm{NMR}$ of 1,3-dioxoisoindolin-2-yl 2-(4-(4-chlorobenzoyl)phenoxy)-2-methylpropanoate (S29) in $\mathrm{CDCl}_{3}$
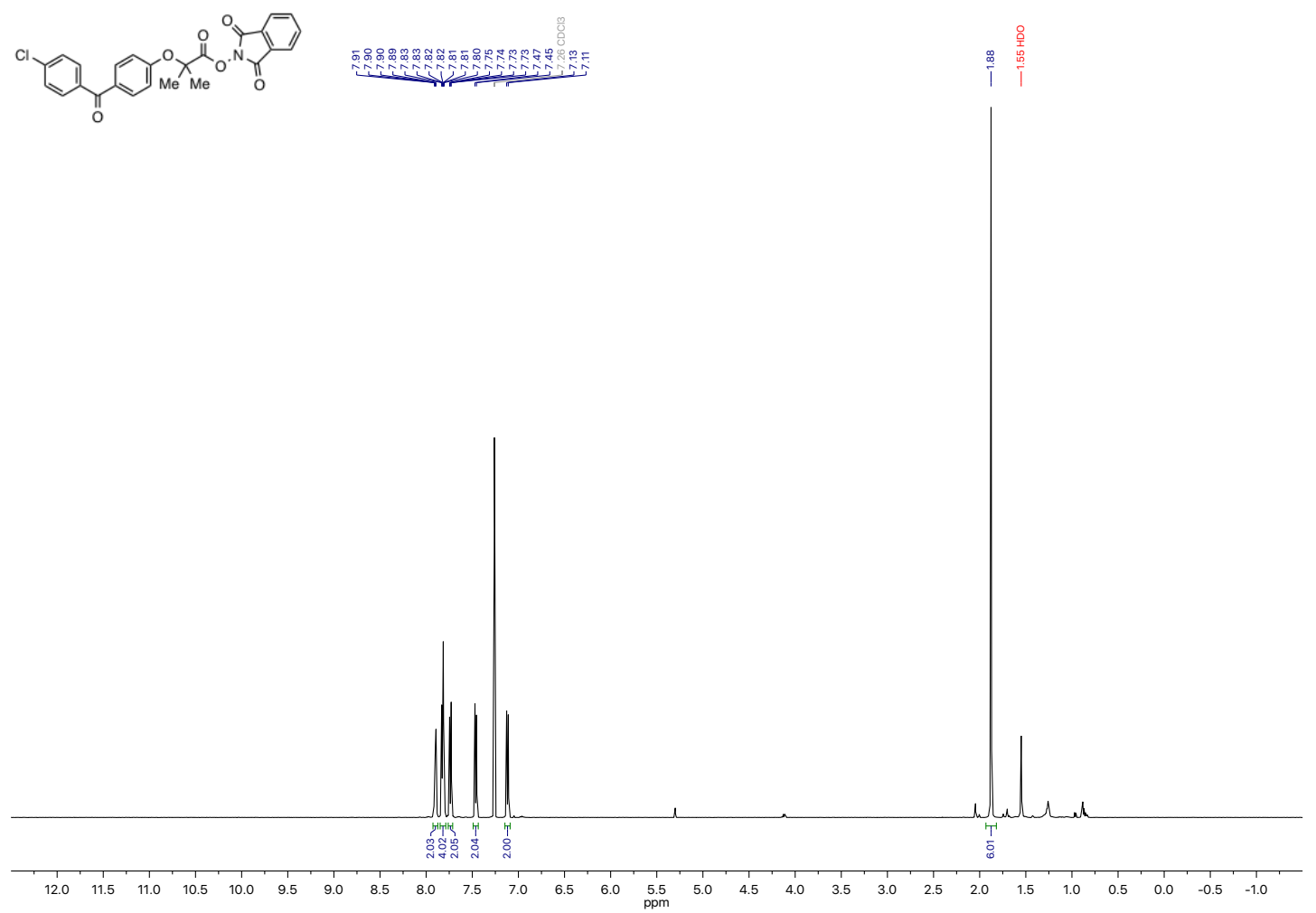
$126 \mathrm{MHz}{ }^{13} \mathrm{C}-\mathrm{NMR}$ of 1,3-dioxoisoindolin-2-yl 2-(4-(4-chlorobenzoyl)phenoxy)-2-methylpropanoate (S29) in $\mathrm{CDCl}_{3}$

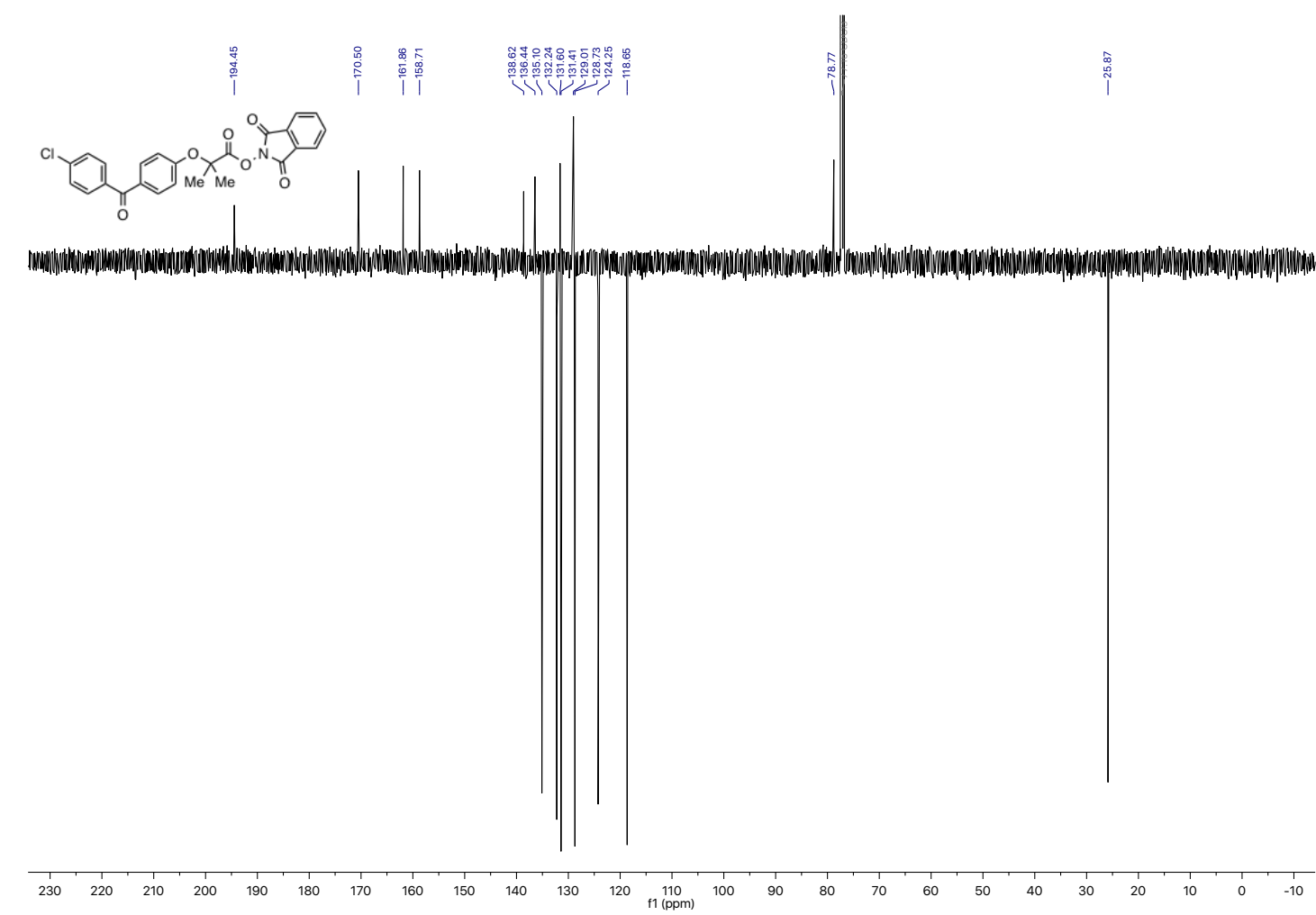

$500 \mathrm{MHz}{ }^{1} \mathrm{H}-\mathrm{NMR}$ of (4-chlorophenyl)(4-((2-fluoropropan-2-yl)oxy)phenyl)methanone (35) in $\mathrm{CDCl}_{3}$
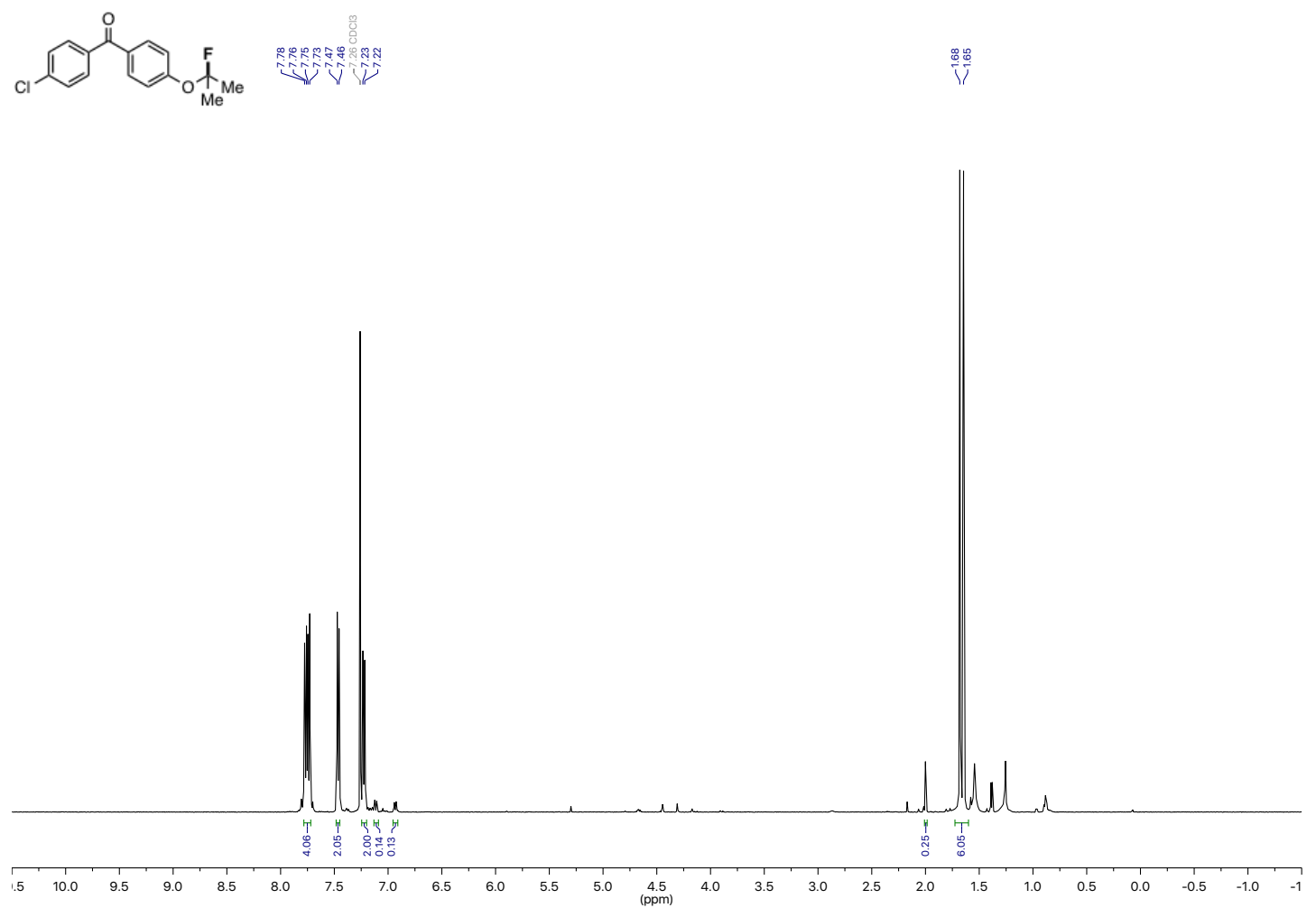
$126 \mathrm{MHz}{ }^{13} \mathrm{C}-\mathrm{NMR}$ of (4-chlorophenyl)(4-((2-fluoropropan-2-yl)oxy)phenyl)methanone (35) in $\mathrm{CDCl}_{3}$

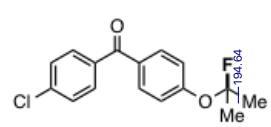

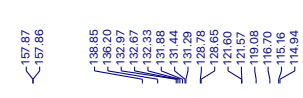

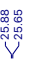

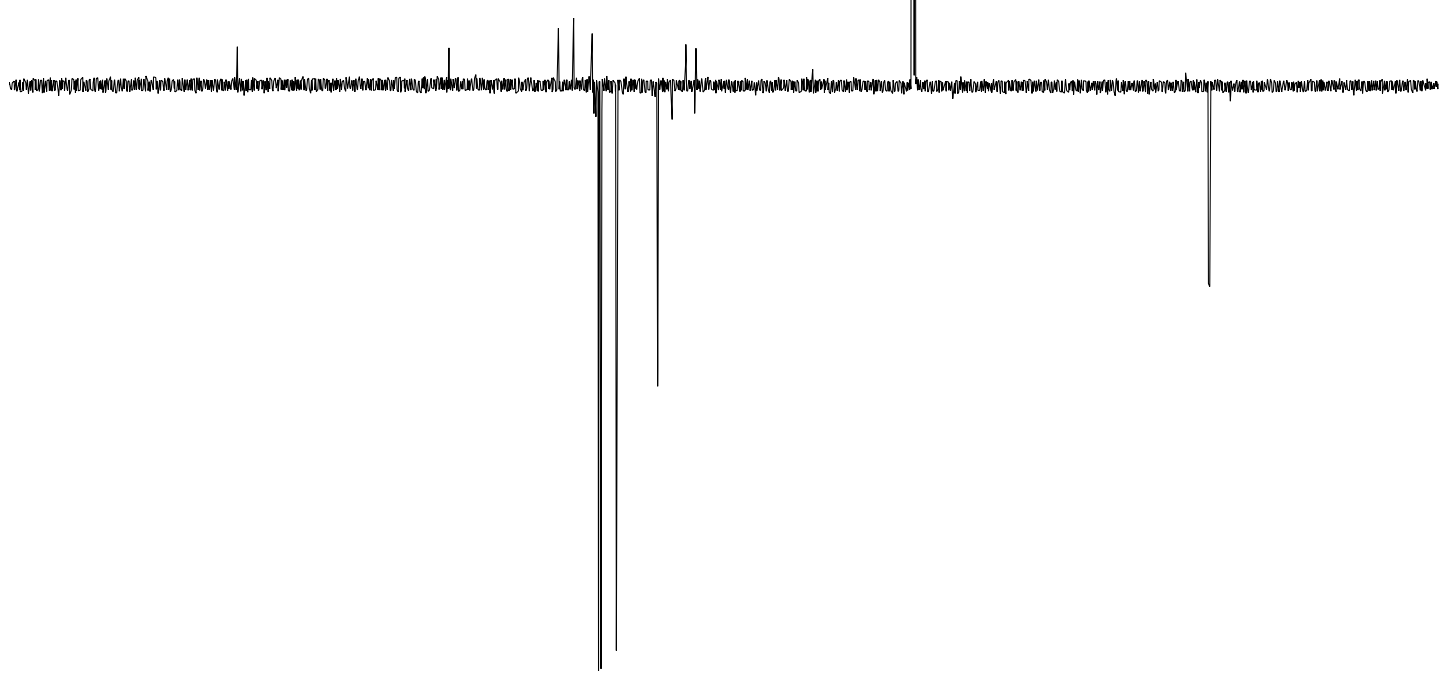

$\begin{array}{llllllllllll}230 & 220 & 210 & 200 & 190 & 180 & 170 & 160 & 150 & 140 & 130 & 120\end{array}$

$470 \mathrm{MHz}^{19} \mathrm{~F}-\mathrm{NMR}$ of (4-chlorophenyl)(4-((2-fluoropropan-2-yl)oxy)phenyl)methanone (35) in $\mathrm{CDCl}_{3}$<smiles>CN(C)Oc1ccc(C(=O)c2ccc(Cl)cc2)cc1</smiles>
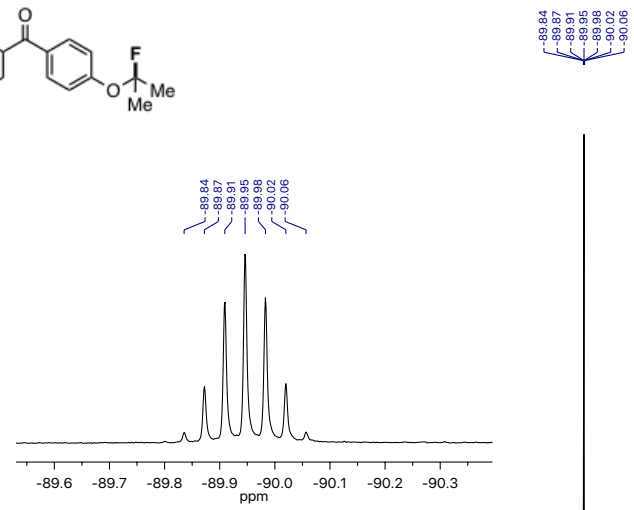

$\stackrel{1}{8}$

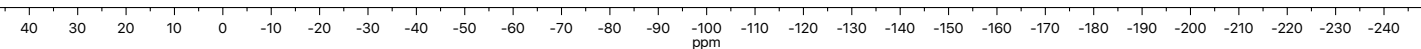


$500 \mathrm{MHz}{ }^{1} \mathrm{H}-\mathrm{NMR}$ of 1,3-dioxoisoindolin-2-yl 2-(4-(4-cyano-2-fluorophenoxy)phenoxy)propanoate (S30) in $\mathrm{CDCl}_{3}$

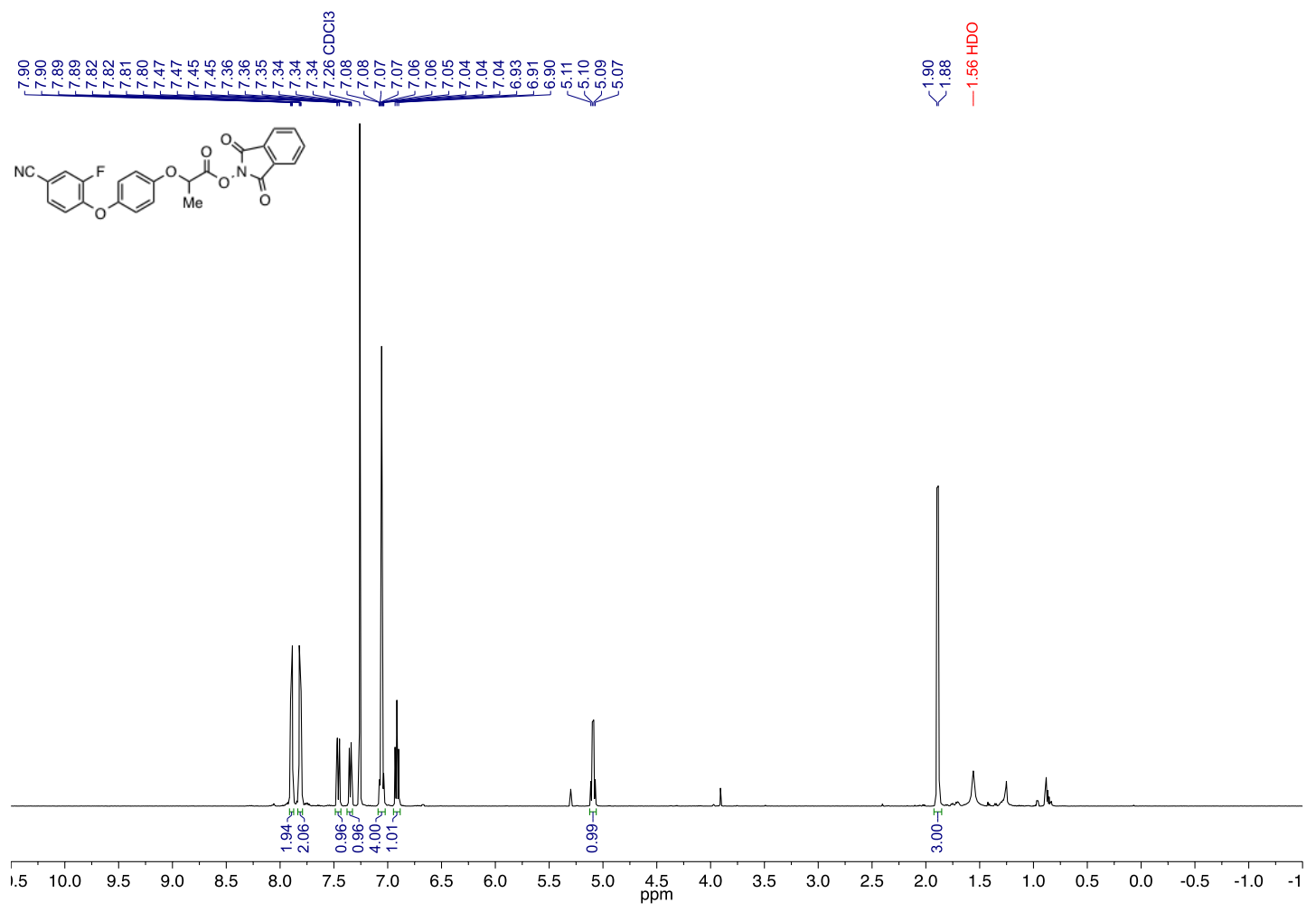

$126 \mathrm{MHz}{ }^{13} \mathrm{C}-\mathrm{NMR}$ of 1,3-dioxoisoindolin-2-yl 2-(4-(4-cyano-2-fluorophenoxy)phenoxy)propanoate (S30) in $\mathrm{CDCl}_{3}$

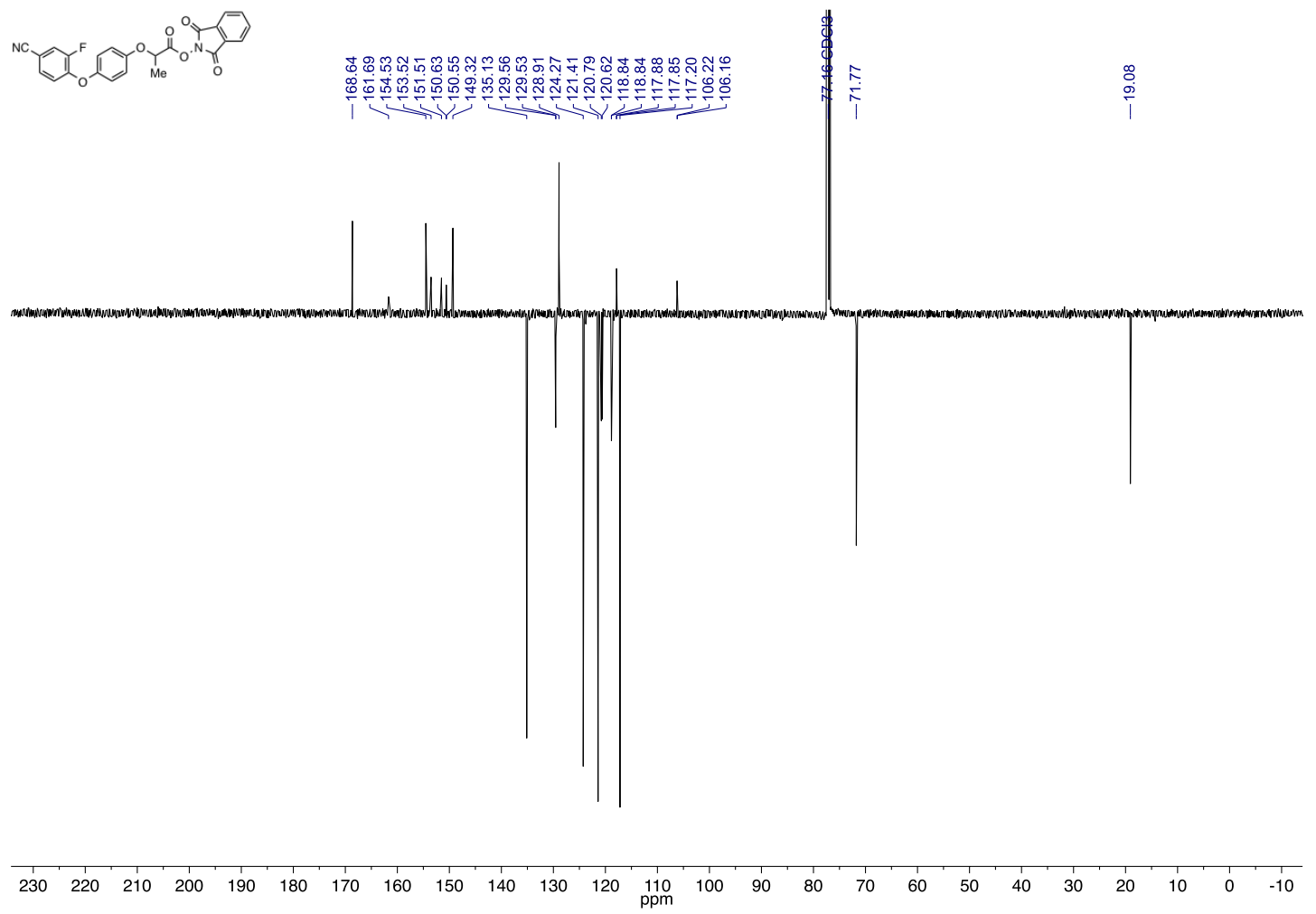


$282 \mathrm{MHz}{ }^{19} \mathrm{~F}-\mathrm{NMR}$ of 1,3-dioxoisoindolin-2-yl 2-(4-(4-cyano-2-fluorophenoxy)phenoxy)propanoate (S30) in $\mathrm{CDCl}_{3}$
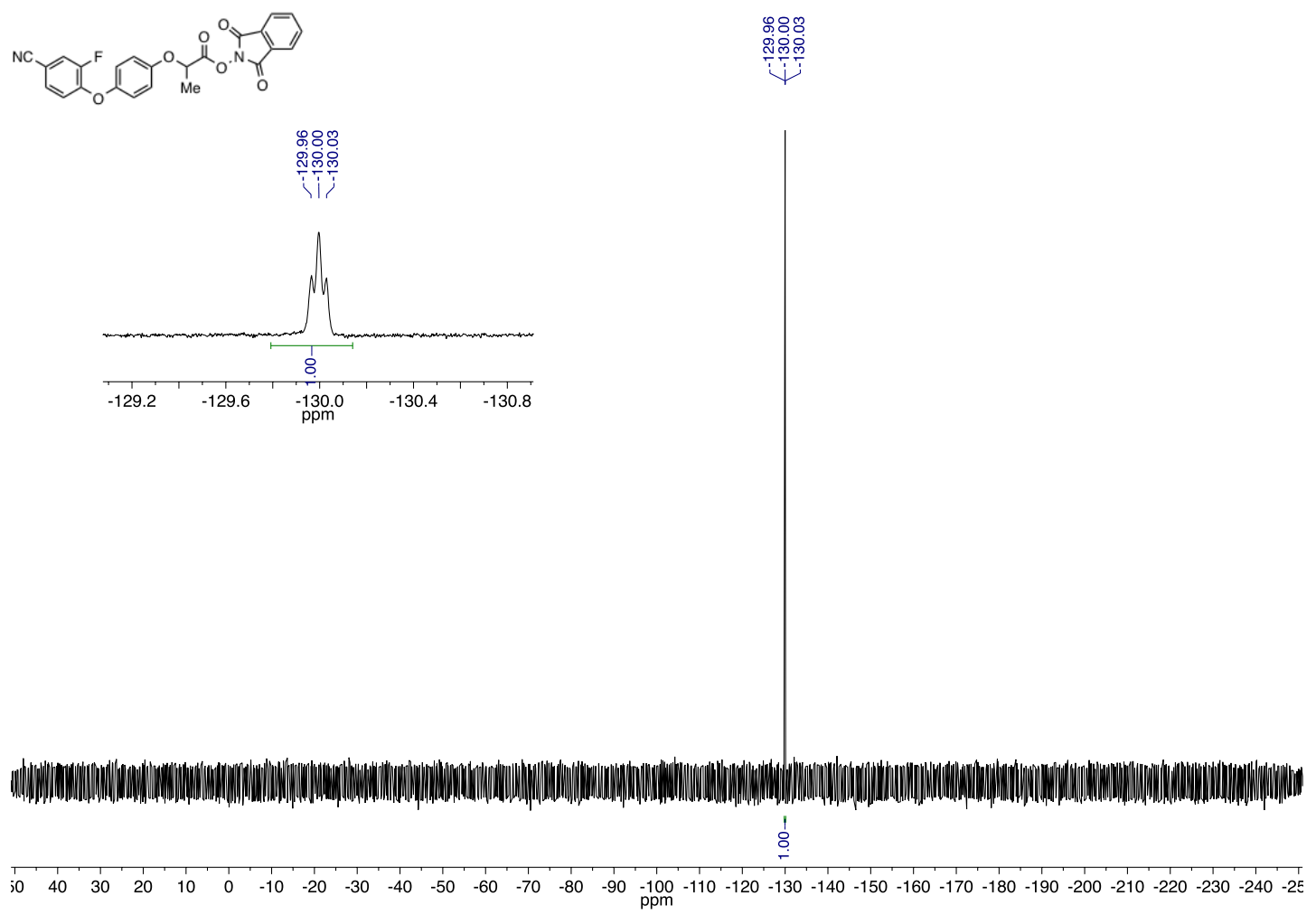

$500 \mathrm{MHz}{ }^{1} \mathrm{H}-\mathrm{NMR}$ of 3-fluoro-4-(4-(1-fluoroethoxy)phenoxy)benzonitrile (36) in $\mathrm{CD}_{2} \mathrm{Cl}_{2}$

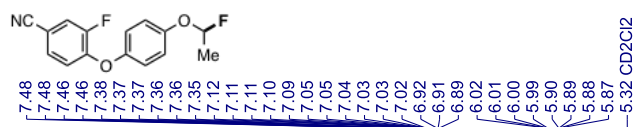

\section{$\sqrt{10}$}

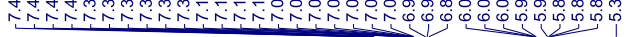

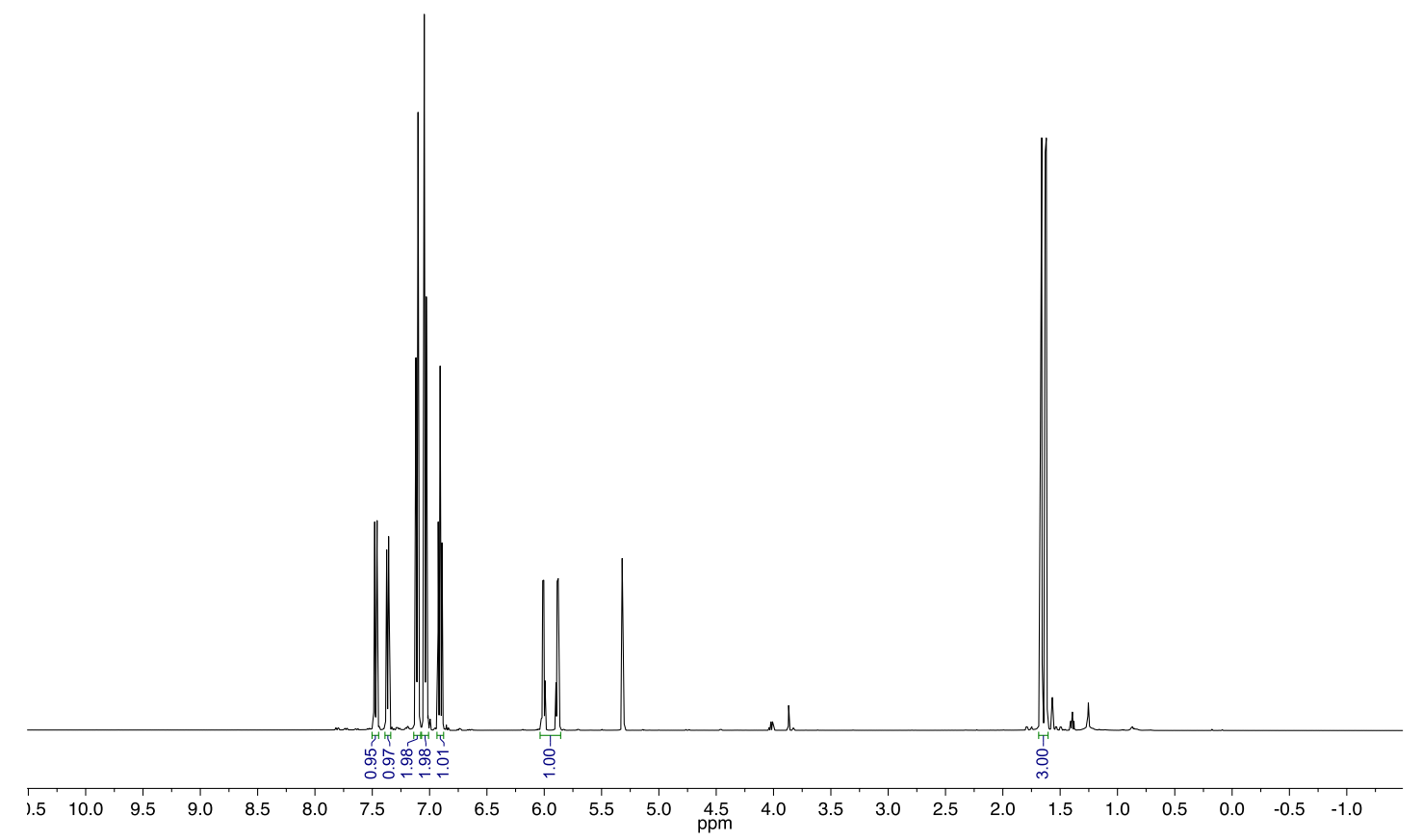


$126 \mathrm{MHz}{ }^{13} \mathrm{C}-\mathrm{NMR}$ of 3-fluoro-4-(4-(1-fluoroethoxy)phenoxy)benzonitrile (36) in $\mathrm{CD}_{2} \mathrm{Cl}_{2}$

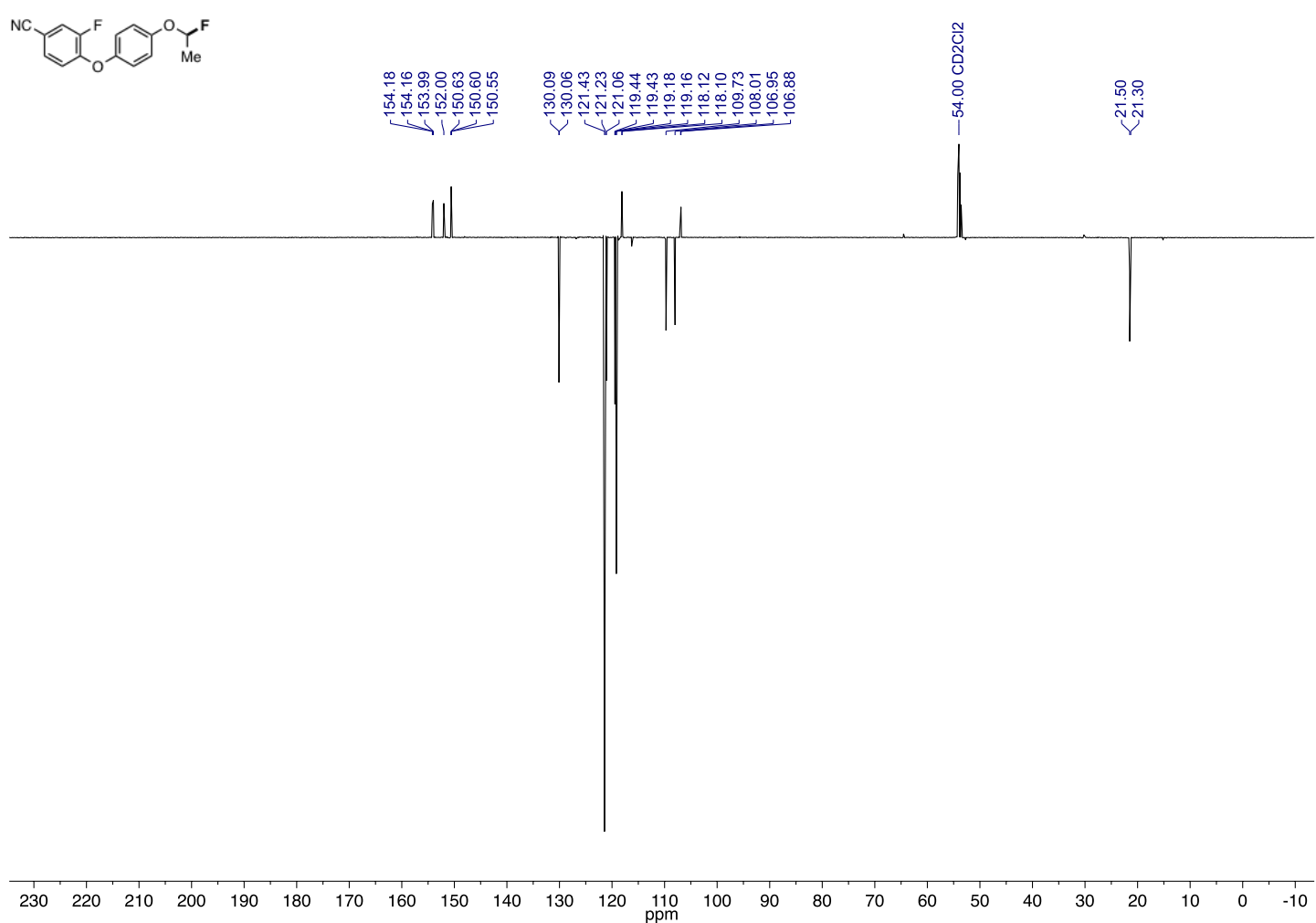

$282 \mathrm{MHz}{ }^{19} \mathrm{~F}-\mathrm{NMR}$ of 3-fluoro-4-(4-(1-fluoroethoxy)phenoxy)benzonitrile (36) in $\mathrm{CD}_{2} \mathrm{Cl}_{2}$

${ }^{\mathrm{NC}} \mathrm{C}_{\mathrm{O}}^{\mathrm{F}} \mathrm{Y}_{\mathrm{Me}}^{\mathrm{F}}$

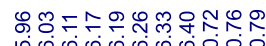

ம்

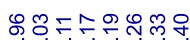

읻은은은

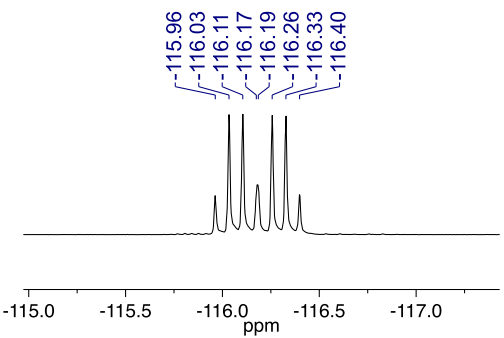

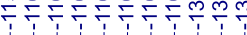

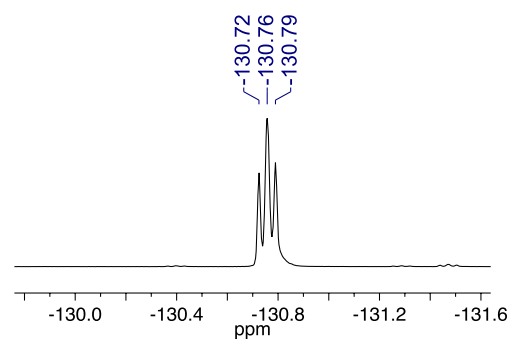

$\stackrel{0}{\circ} \stackrel{-10}{0}$

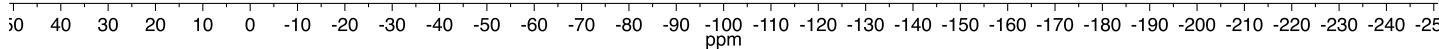


$500 \mathrm{MHz}{ }^{1} \mathrm{H}-\mathrm{NMR}$ 1,3-dioxoisoindolin-2-yl 2-([1,1'-biphenyl]-4-yl)-2-fluoroacetate (S31) in $\mathrm{CDCl}_{3}$

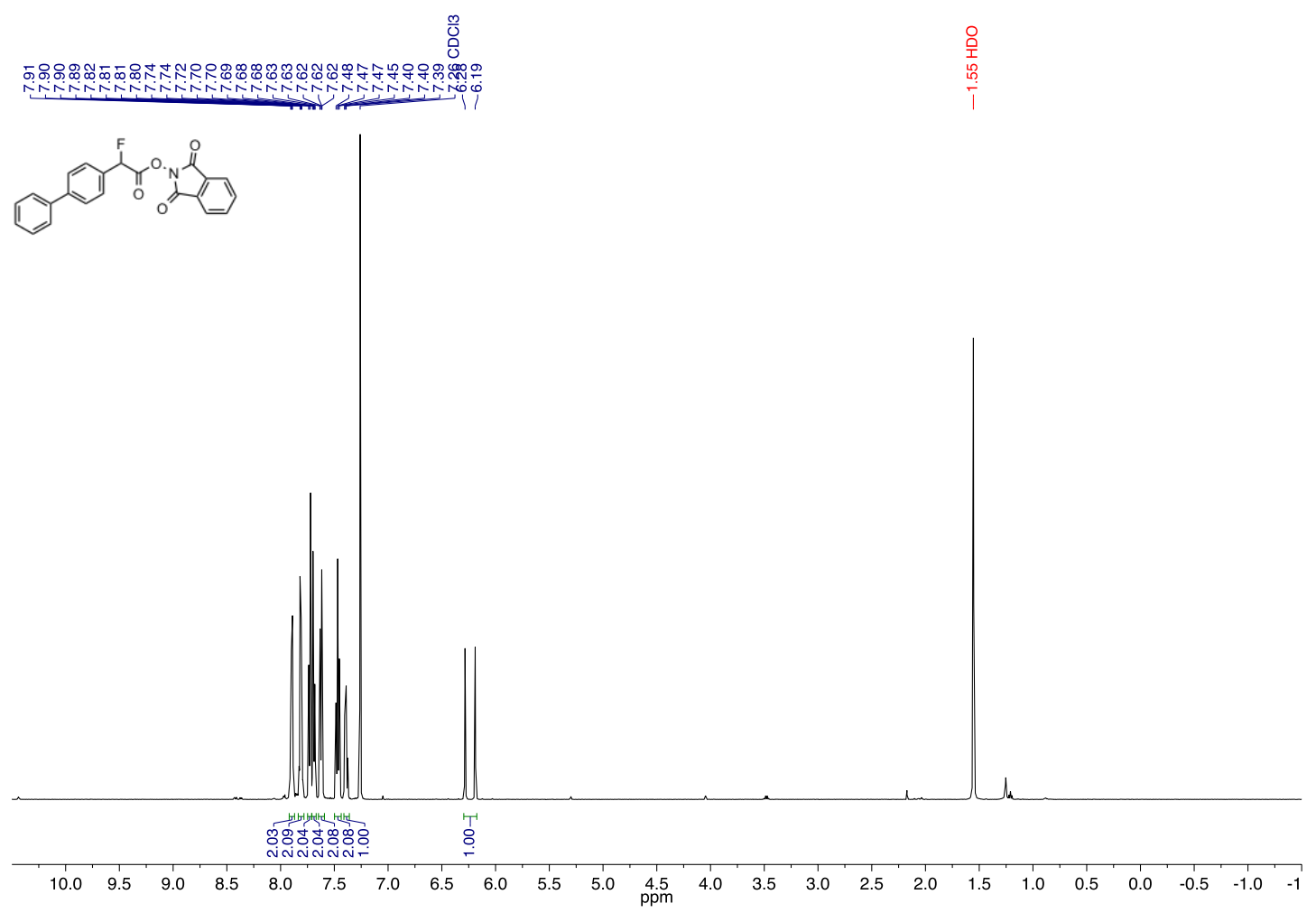

$126 \mathrm{MHz}{ }^{13} \mathrm{C}-\mathrm{NMR}$ 1,3-dioxoisoindolin-2-yl 2-([1,1'-biphenyl]-4-yl)-2-fluoroacetate (S31) in $\mathrm{CDCl}_{3}$

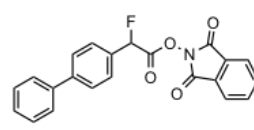

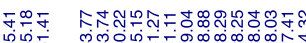

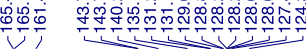

ר.

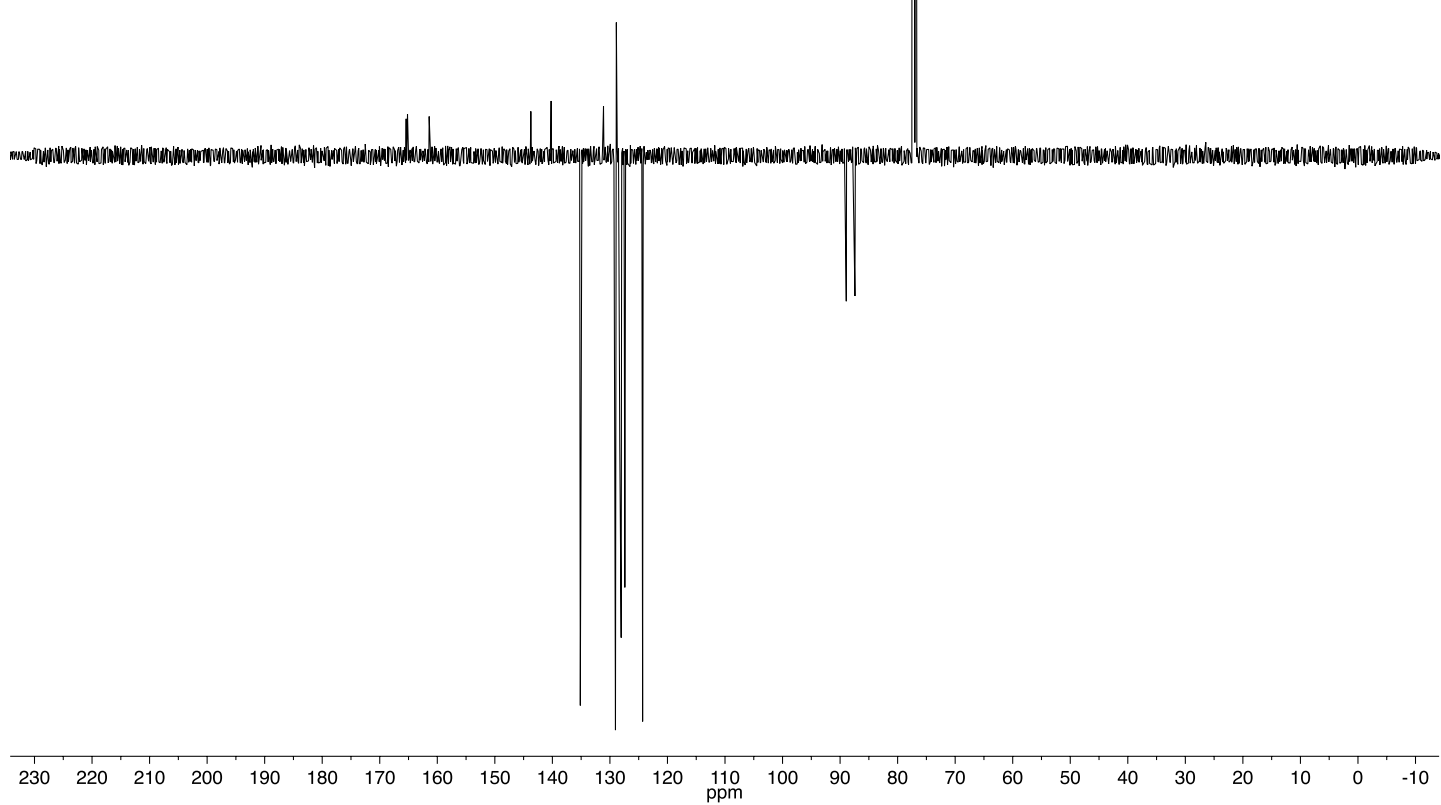


$282 \mathrm{MHz}{ }^{19} \mathrm{~F}-\mathrm{NMR}$ 1,3-dioxoisoindolin-2-yl 2-([1,1'-biphenyl]-4-yl)-2-fluoroacetate (S31) in $\mathrm{CDCl}_{3}$

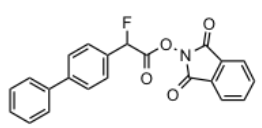

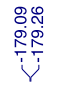
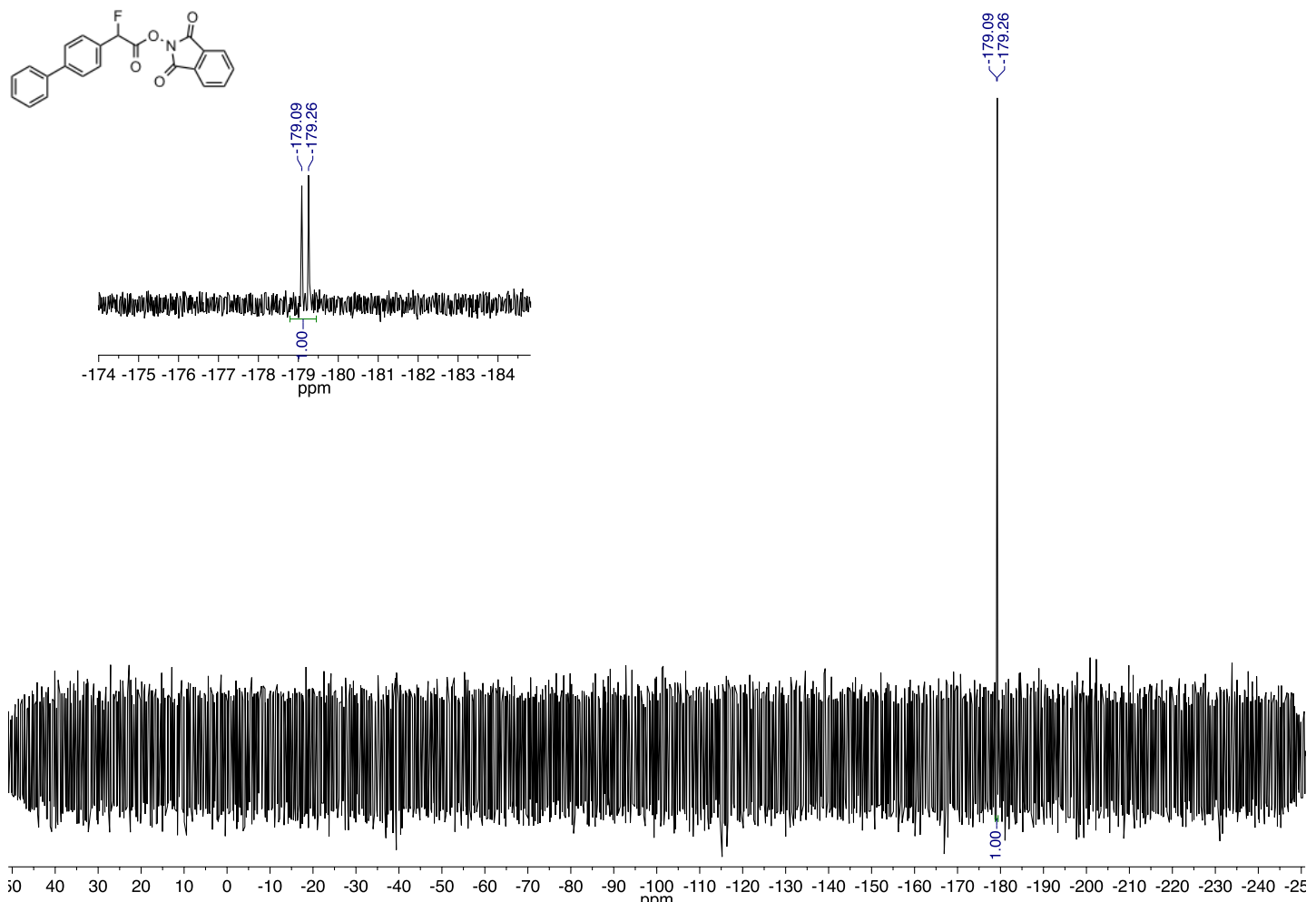

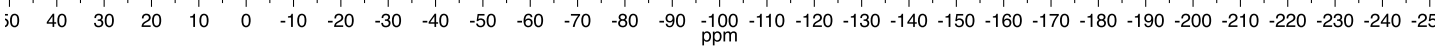

$500 \mathrm{MHz}{ }^{1} \mathrm{H}-\mathrm{NMR}$ 4-(difluoromethyl)-1,1'-biphenyl (37) in $\mathrm{CDCl}_{3}$

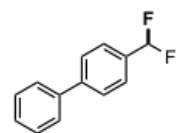

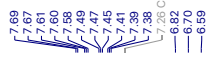

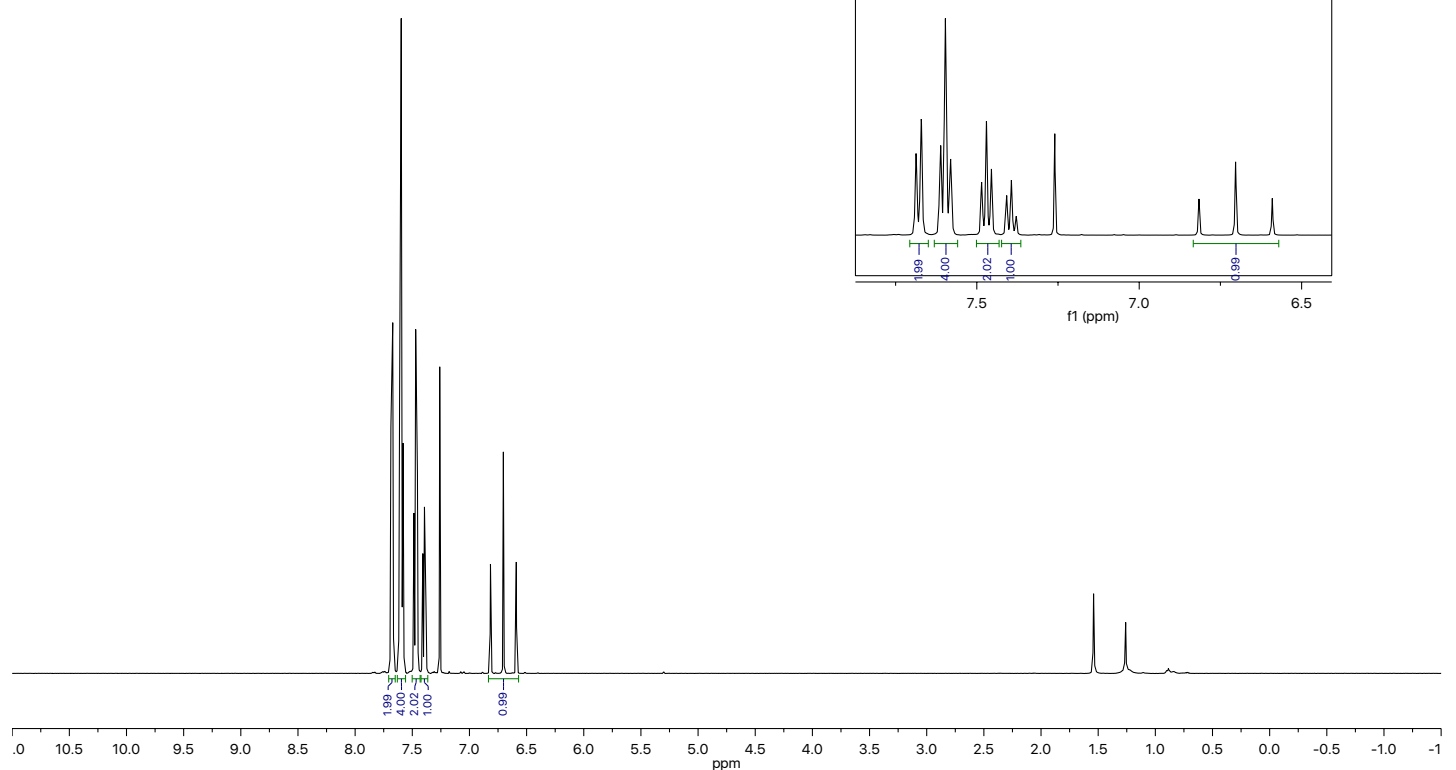


$126 \mathrm{MHz}{ }^{13} \mathrm{C}-\mathrm{NMR}$ 4-(difluoromethyl)-1,1'-biphenyl (37) in $\mathrm{CDCl}_{3}$

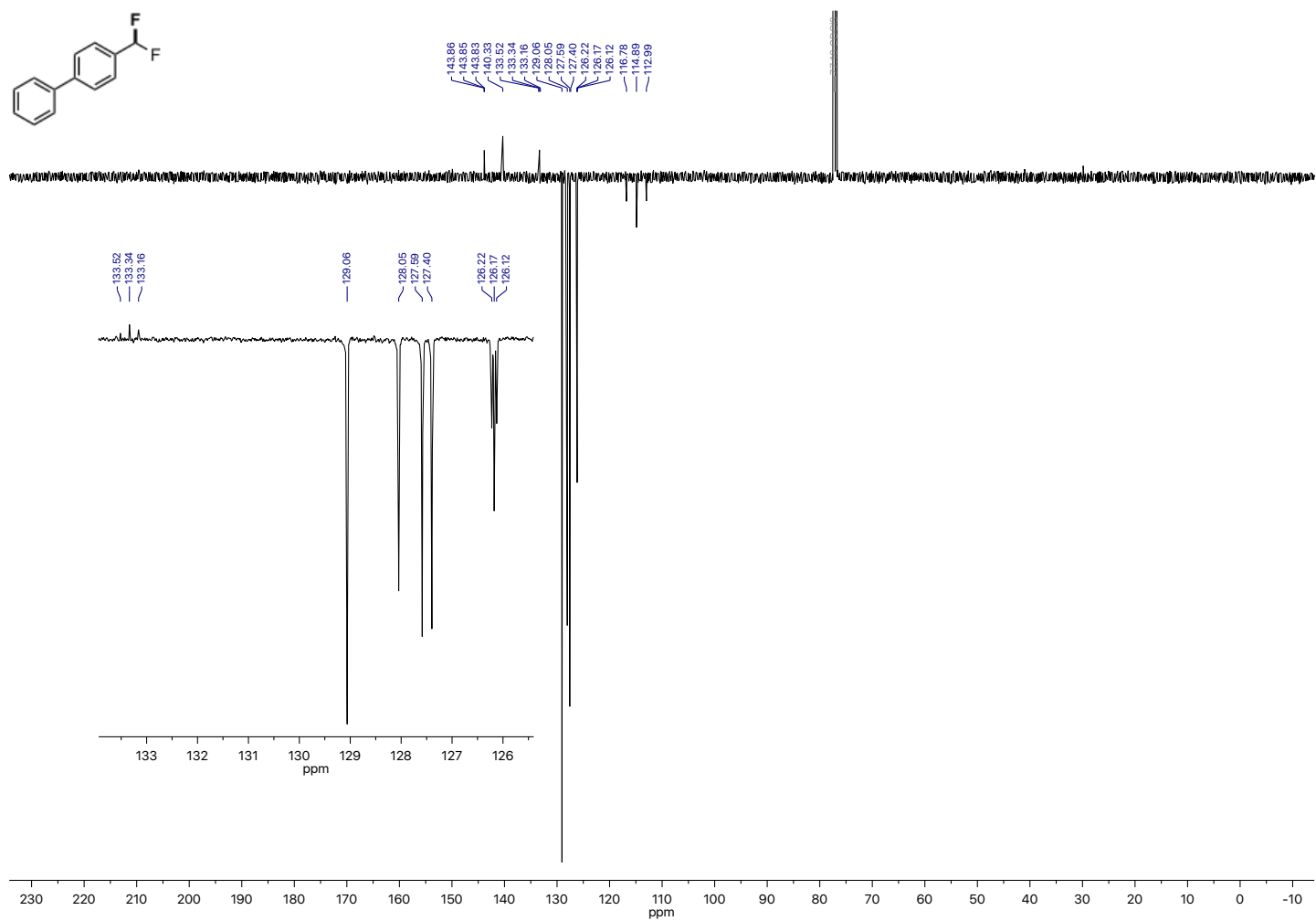


$126 \mathrm{MHz}{ }^{19} \mathrm{~F}-\mathrm{NMR}$ 4-(difluoromethyl)-1,1'-biphenyl (37) in $\mathrm{CDCl}_{3}$

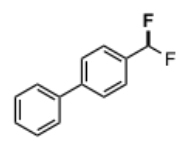

ทำ
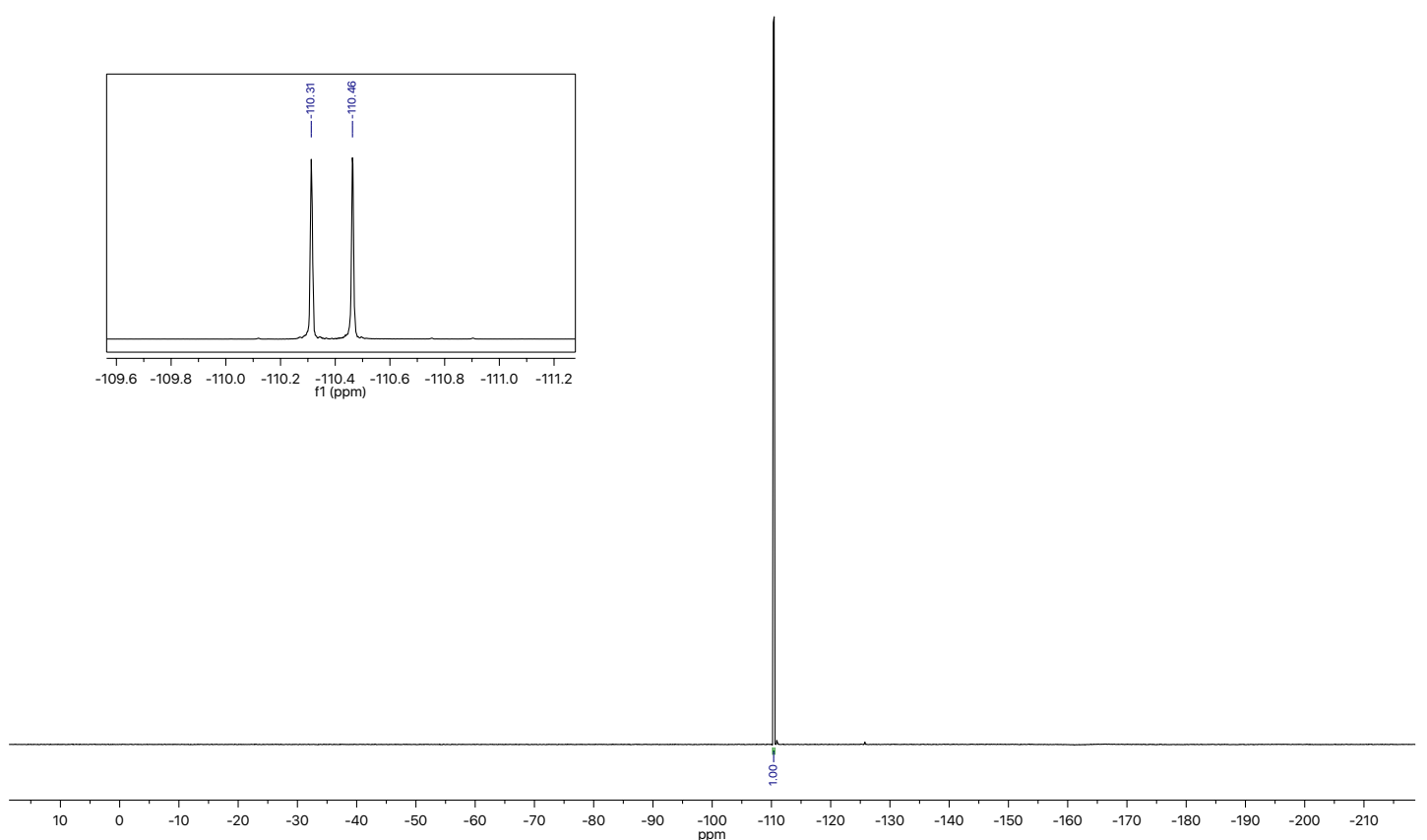

$500 \mathrm{MHz}{ }^{1} \mathrm{H}-\mathrm{NMR}$ of 1,3-dioxoisoindolin-2-yl 3,3,3-trifluoro-2-methoxy-2-phenylpropanoate (S32) in $\mathrm{CDCl}_{3}$
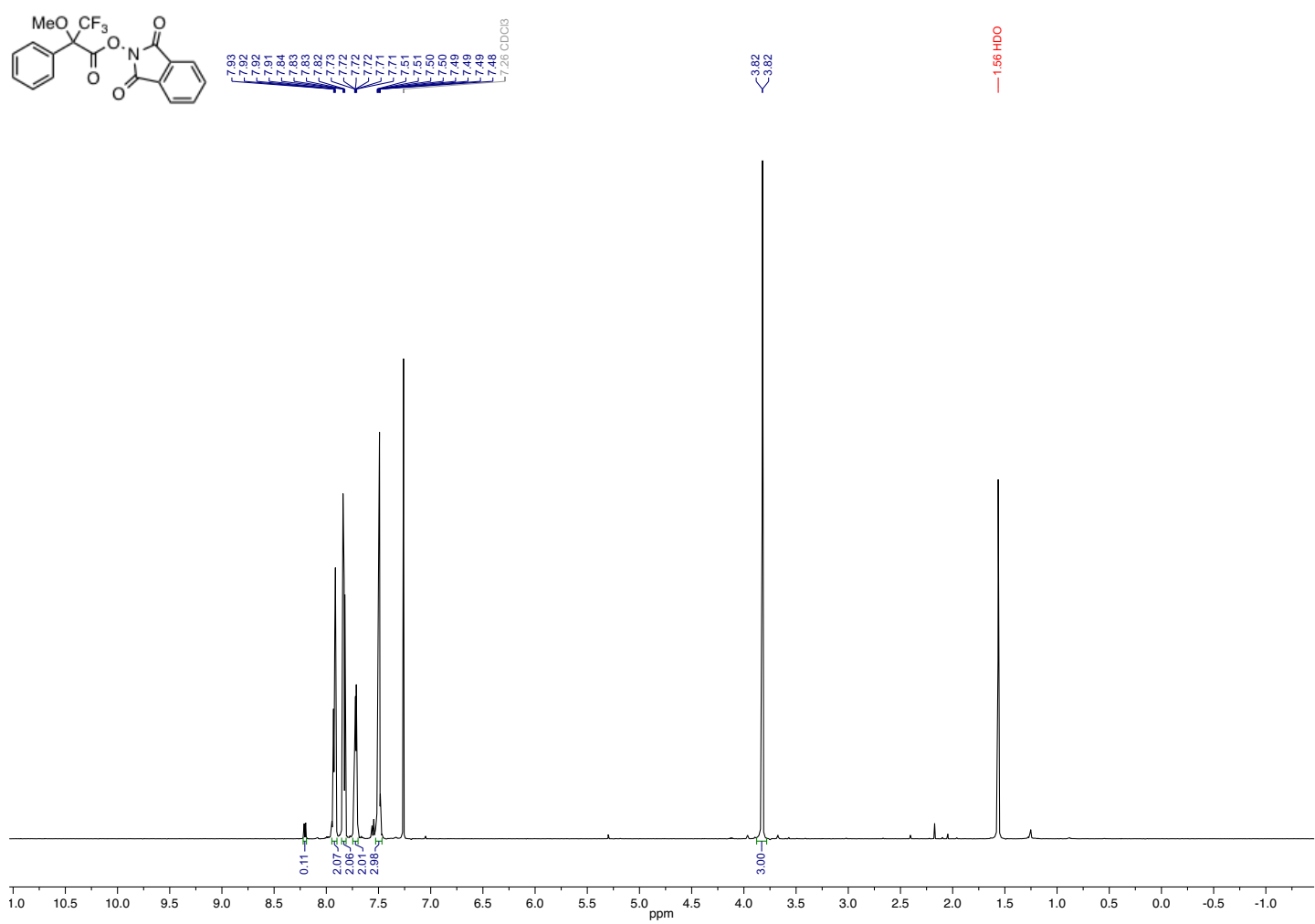
$126 \mathrm{MHz}{ }^{13} \mathrm{C}-\mathrm{NMR}$ of 1,3-dioxoisoindolin-2-yl 3,3,3-trifluoro-2-methoxy-2-phenylpropanoate (S32) in $\mathrm{CDCl}_{3}$

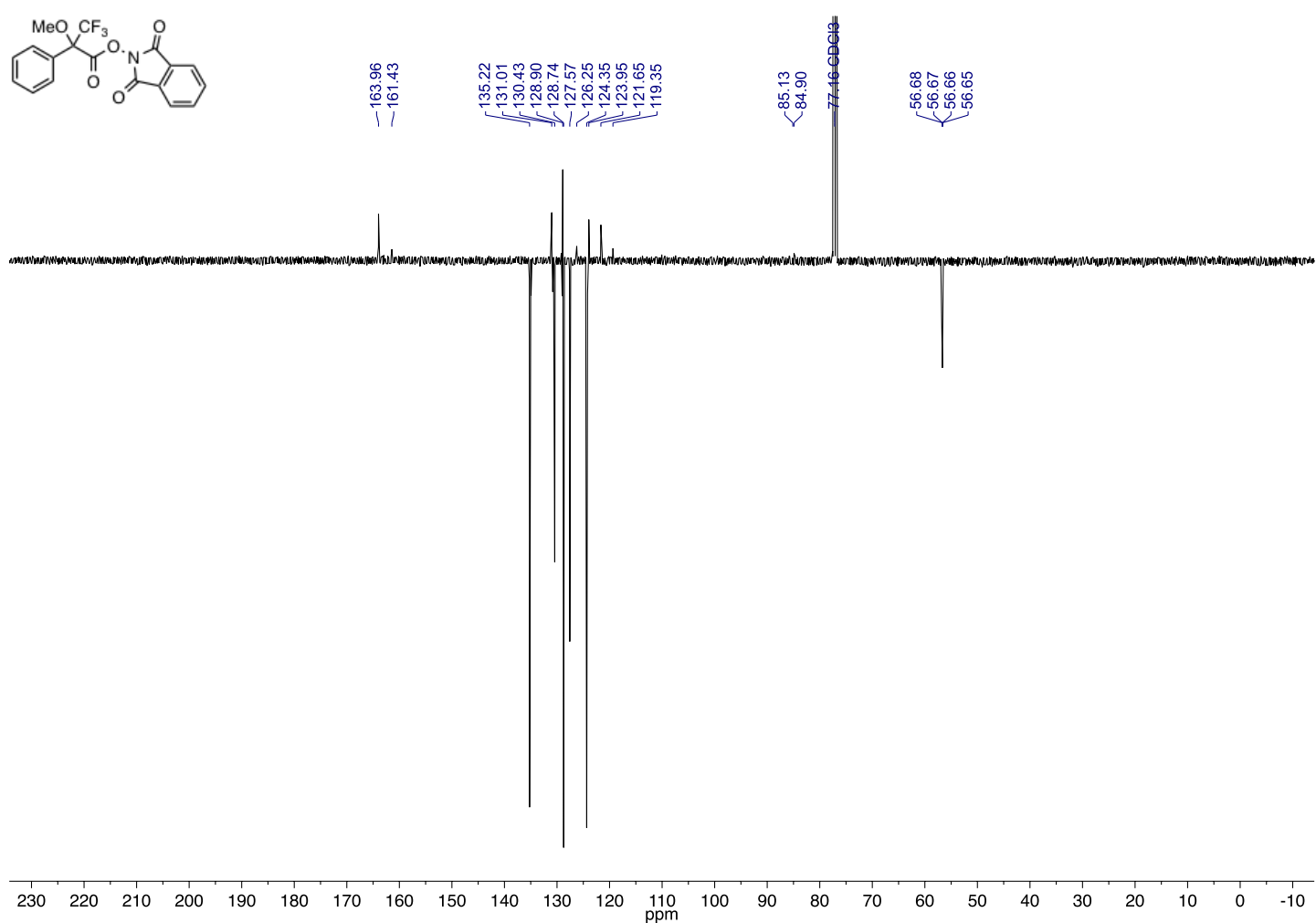

$282 \mathrm{MHz}{ }^{19} \mathrm{~F}-\mathrm{NMR}$ of 1,3-dioxoisoindolin-2-yl 3,3,3-trifluoro-2-methoxy-2-phenylpropanoate (S32) in $\mathrm{CDCl}_{3}$

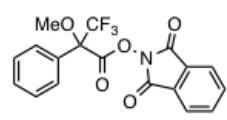

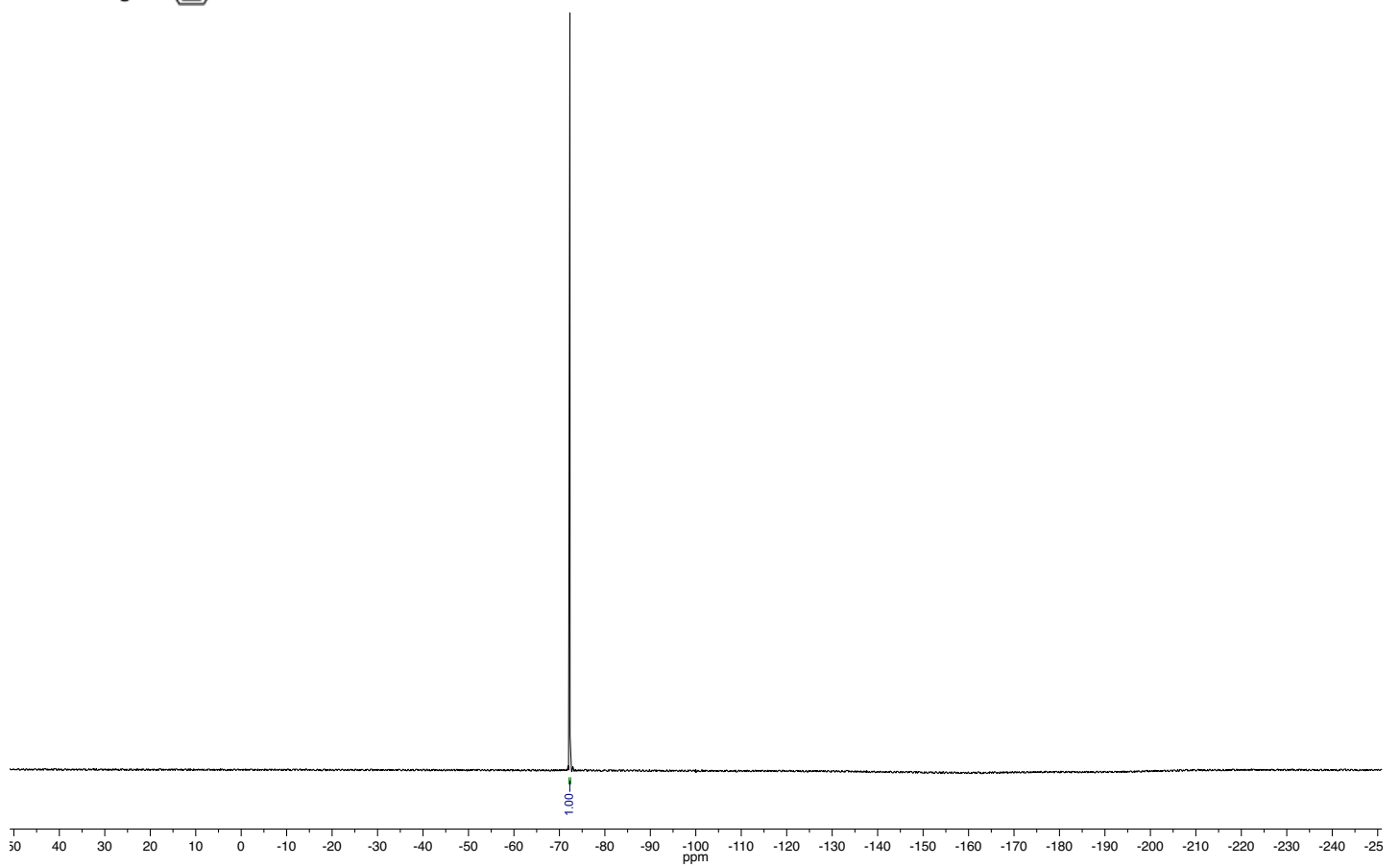


$500 \mathrm{MHz}{ }^{1} \mathrm{H}-\mathrm{NMR}$ of 1,3-dioxoisoindolin-2-yl 9-fluoro-9H-fluorene-9-carboxylate (S33) in $\mathrm{CDCl}_{3}$

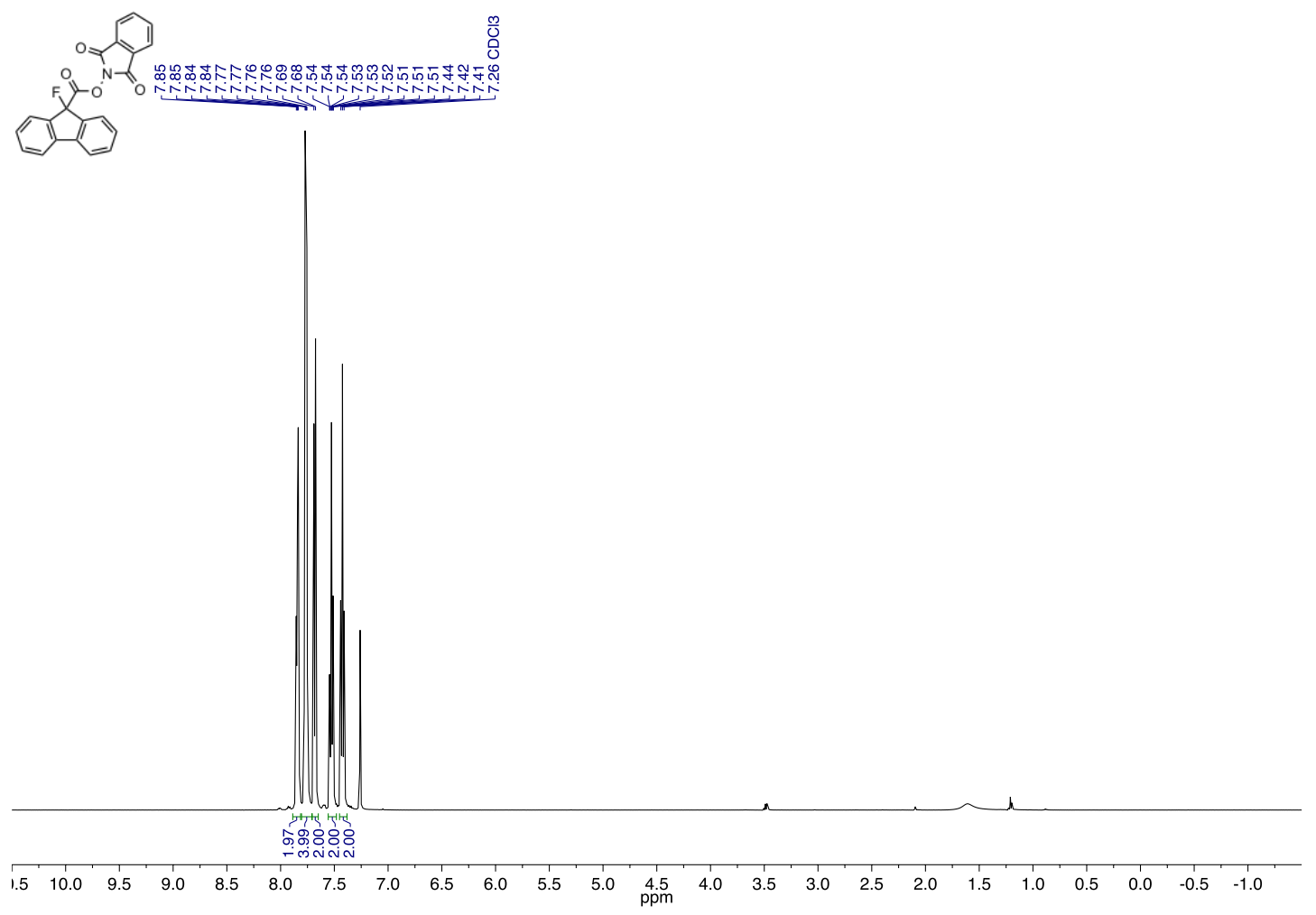

$126 \mathrm{MHz}{ }^{13} \mathrm{C}$-NMR of 1,3-dioxoisoindolin-2-yl 9-fluoro-9H-fluorene-9-carboxylate $(\mathbf{S 3 3})$ in $\mathrm{CDCl}_{3}$

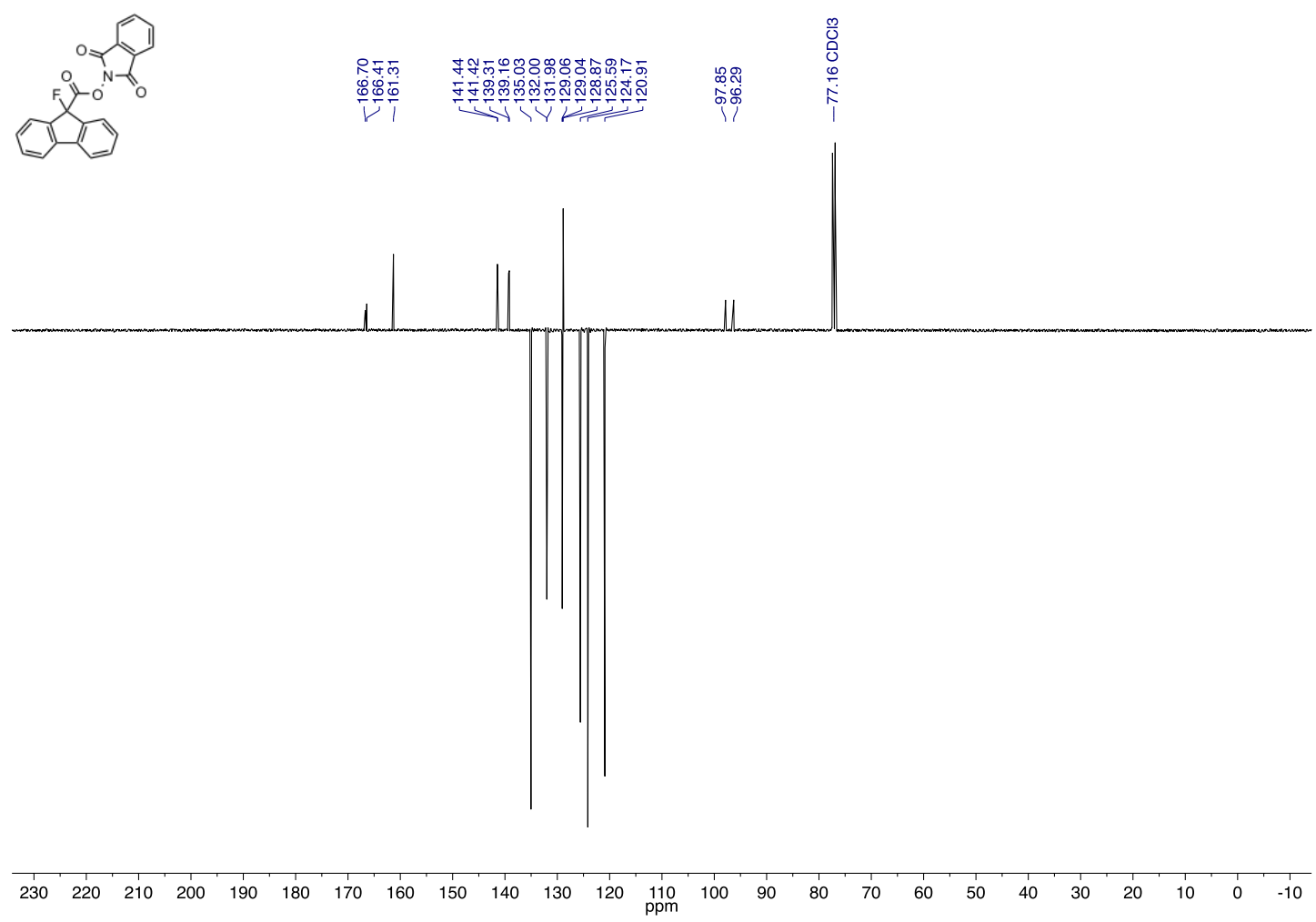


$282 \mathrm{MHz}{ }^{19} \mathrm{~F}-\mathrm{NMR}$ 1,3-dioxoisoindolin-2-yl 9-fluoro-9H-fluorene-9-carboxylate (S33) in $\mathrm{CDCl}_{3}$

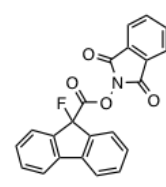

$500 \mathrm{MHz}{ }^{1} \mathrm{H}-\mathrm{NMR}$ of 9,9-difluoro-9 $\mathrm{H}$-fluorene (39) in $\mathrm{CDCl}_{3}$

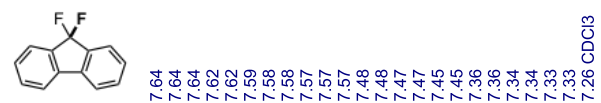

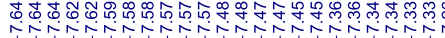

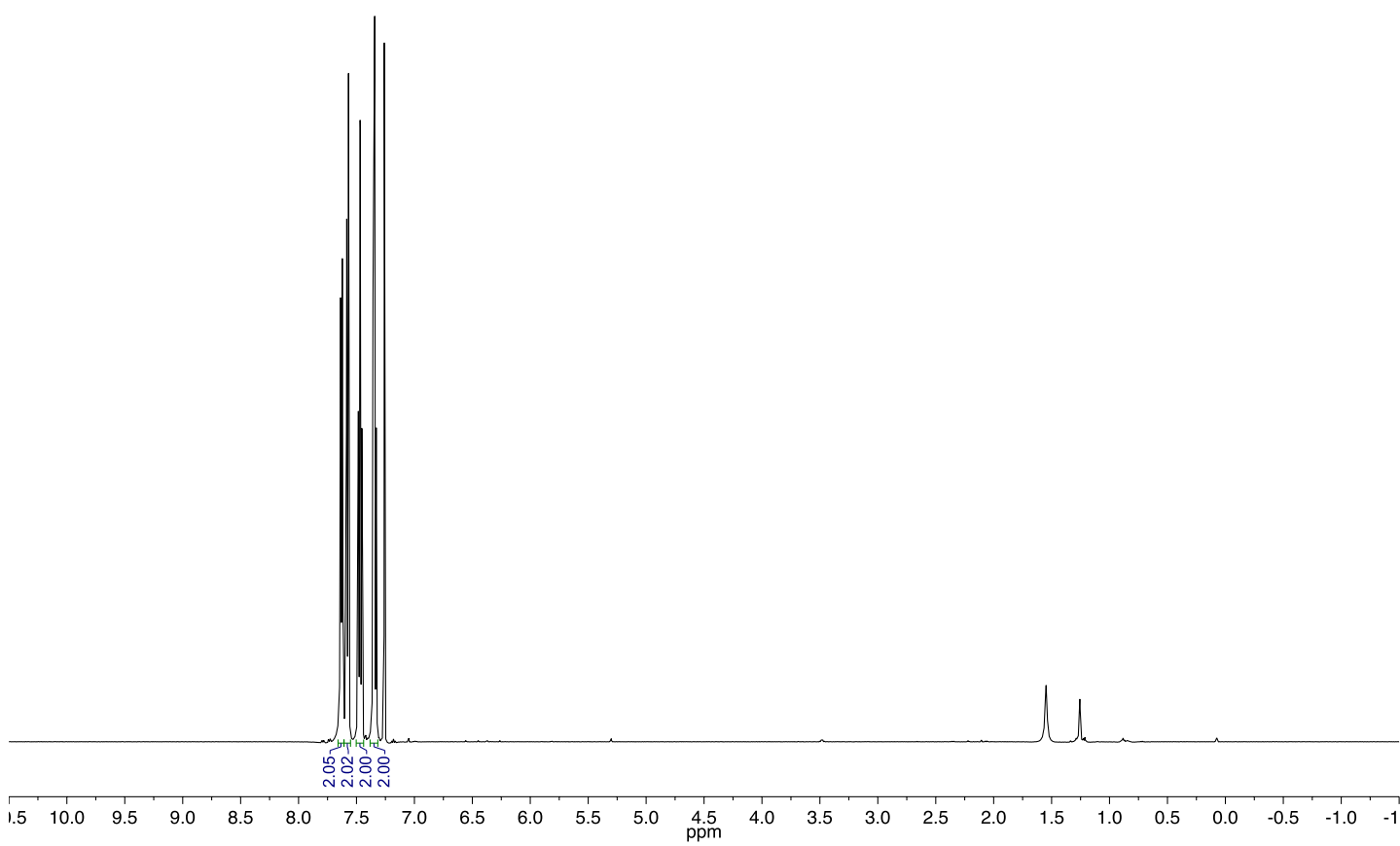


$126 \mathrm{MHz}{ }^{13} \mathrm{C}$-NMR of 9,9-difluoro-9H-fluorene (39) in $\mathrm{CDCl}_{3}$

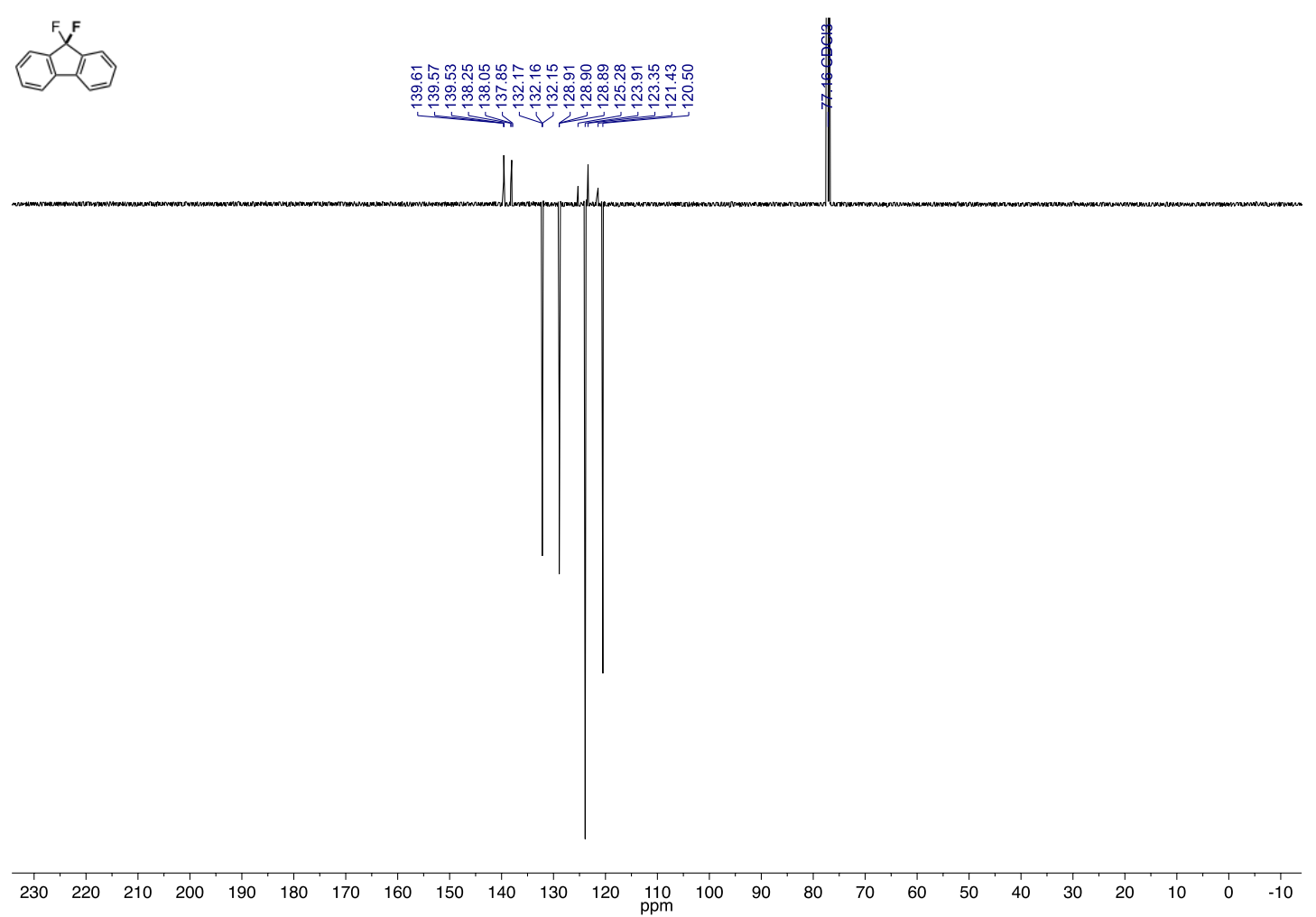

$282 \mathrm{MHz}{ }^{19} \mathrm{~F}-\mathrm{NMR}$ of 9,9-difluoro-9H-fluorene (39) in $\mathrm{CDCl}_{3}$
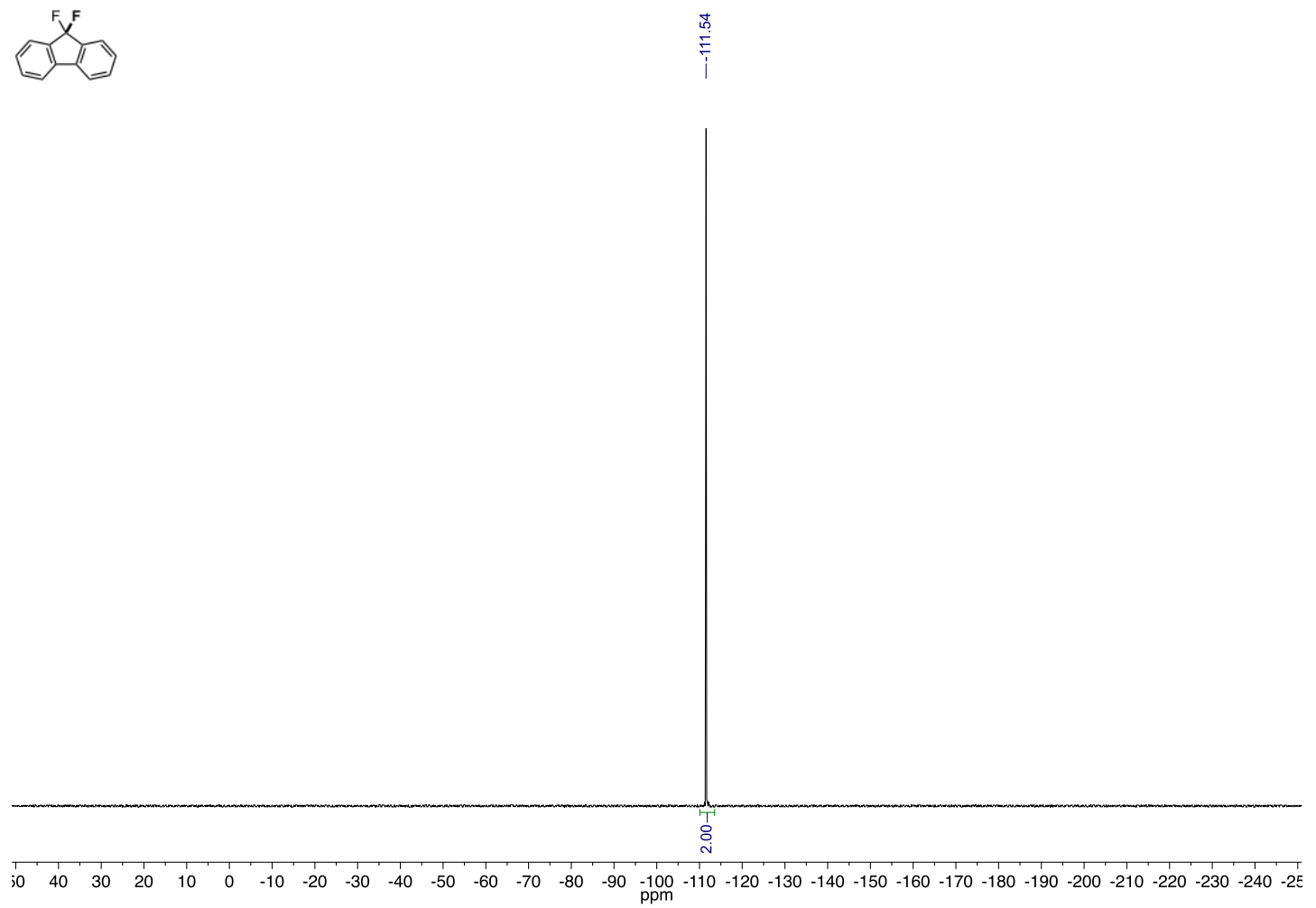
$500 \mathrm{MHz}{ }^{1} \mathrm{H}-\mathrm{NMR}$ of 1,3-dioxoisoindolin-2-yl (2-phenylpropan-2-yl) oxalate (S34) in $\mathrm{CDCl}_{3}$
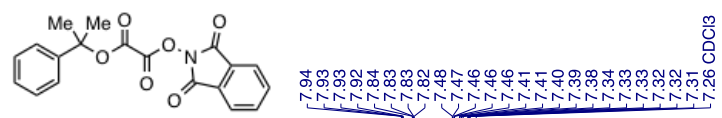

궁

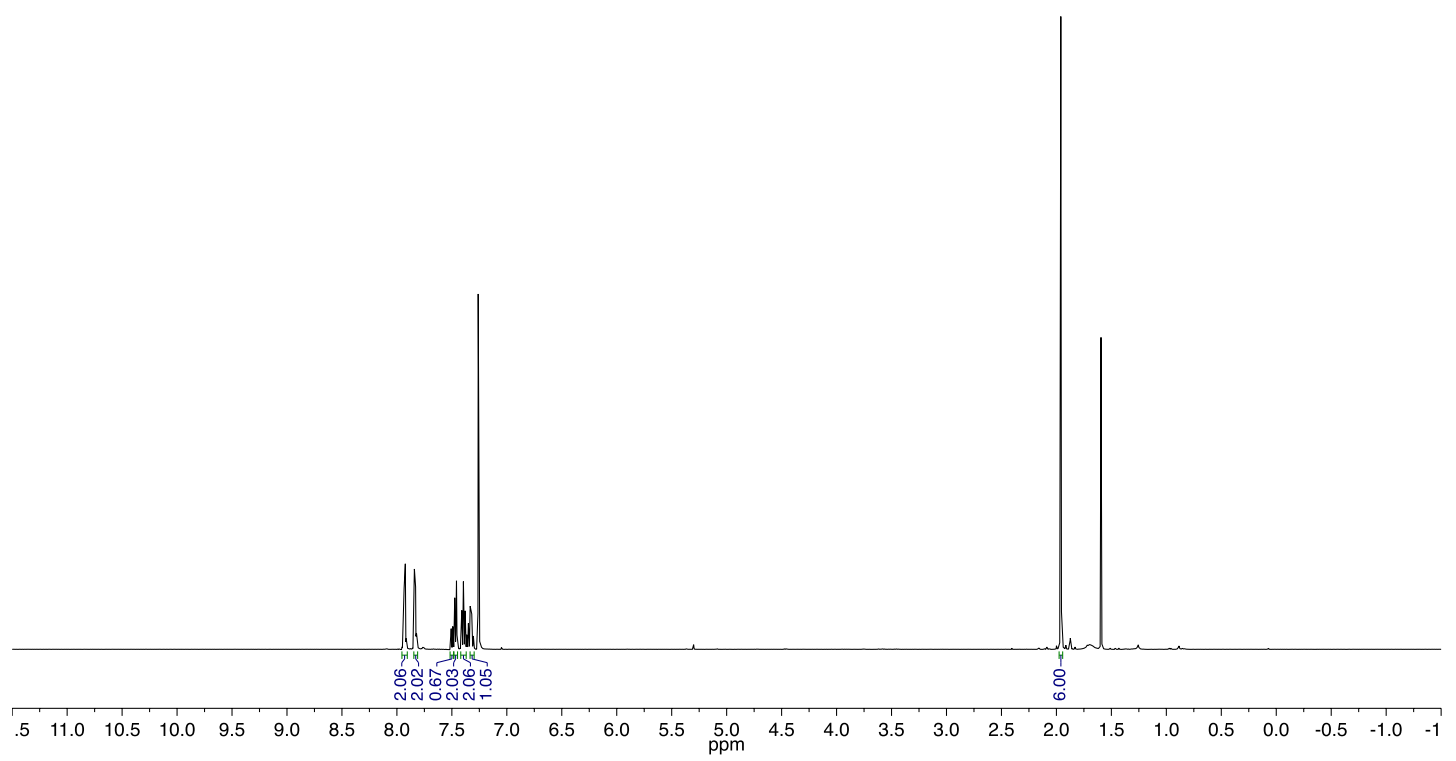

$126 \mathrm{MHz}{ }^{13} \mathrm{C}$-NMR of 1,3-dioxoisoindolin-2-yl (2-phenylpropan-2-yl) oxalate (S34) in $\mathrm{CDCl}_{3}$<smiles>CC(C)(OC(=O)C(=O)ON1C(=O)c2ccccc2C1=O)c1ccccc1</smiles>

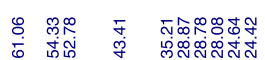

广

$\begin{array}{cc} & \frac{m}{0} \\ & 0 \\ 0 & 0 \\ \infty & 0 \\ \infty & 0 \\ 0 & 1 \\ 1 & 1\end{array}$

৫ํำ

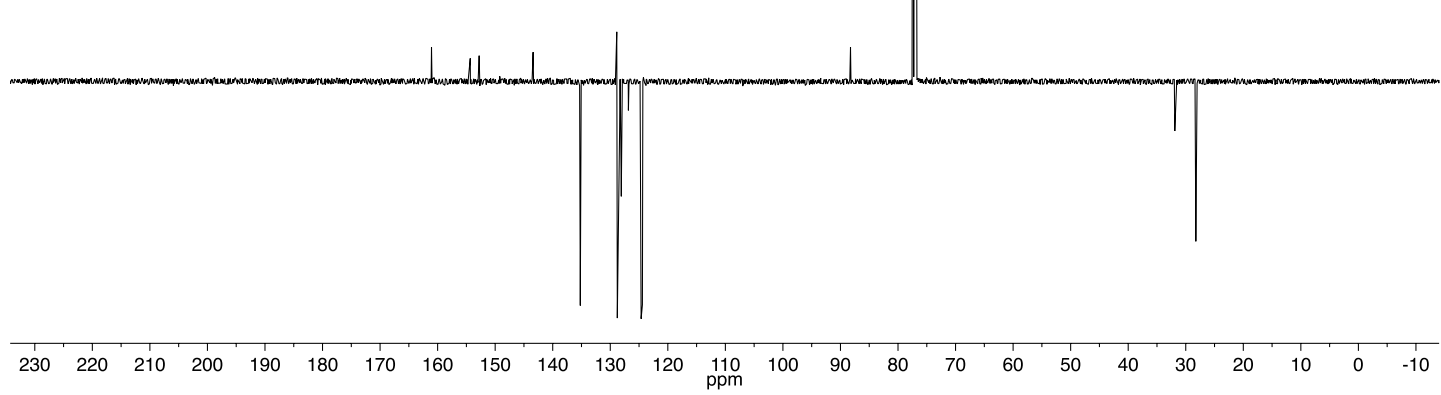


$500 \mathrm{MHz}{ }^{1} \mathrm{H}-\mathrm{NMR}$ of 1,3-dioxoisoindolin-2-yl (2-methyl-4-phenylbutan-2-yl) oxalate (S35) in $\mathrm{CDCl}_{3}$
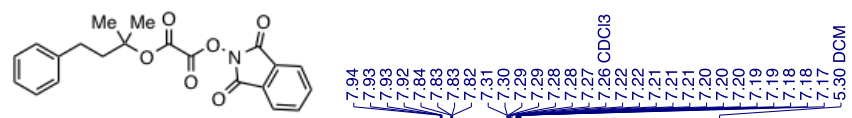

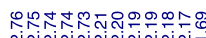
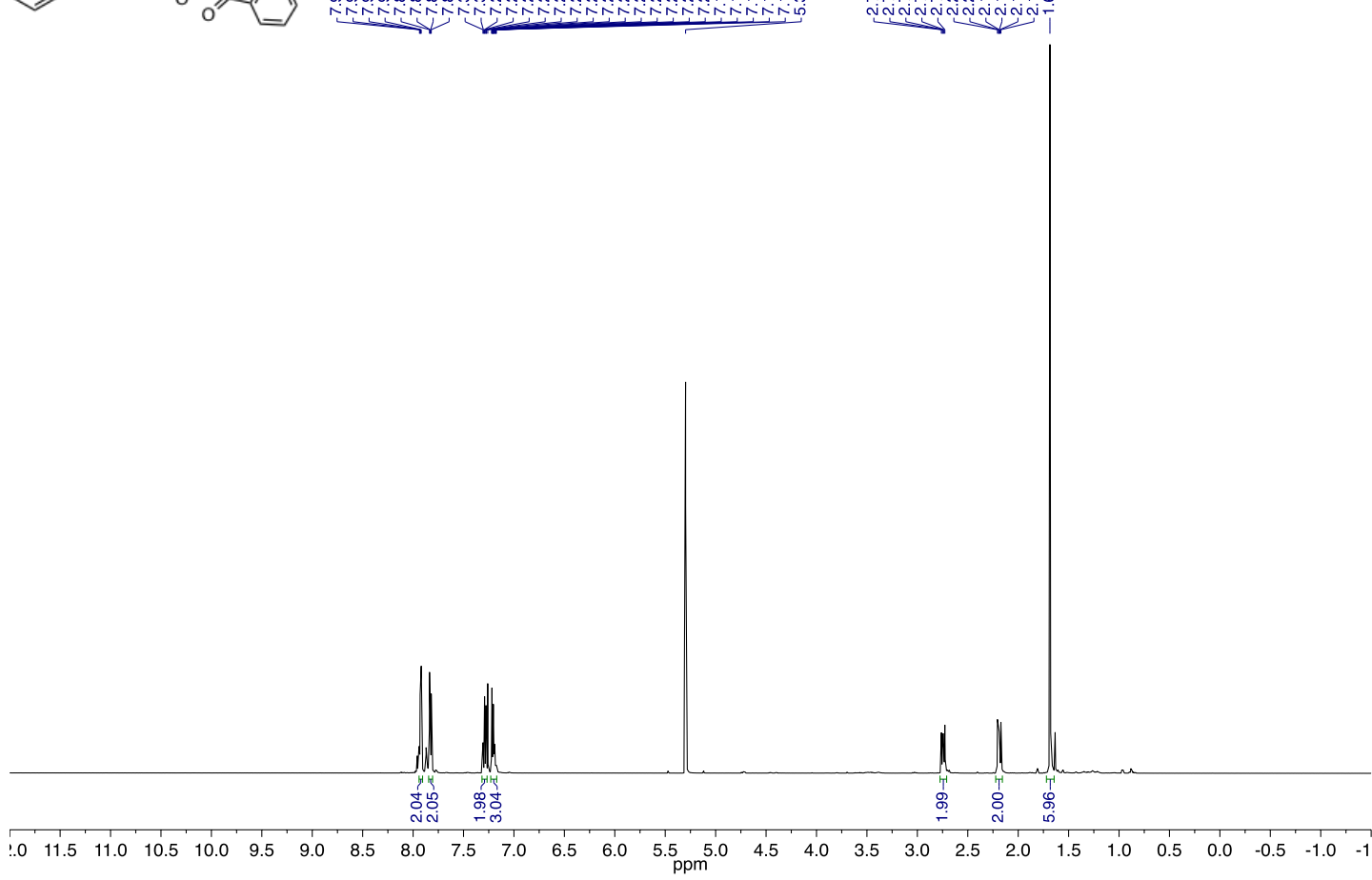

$126 \mathrm{MHz}{ }^{13} \mathrm{C}-\mathrm{NMR}$ of 1,3-dioxoisoindolin-2-yl (2-methyl-4-phenylbutan-2-yl) oxalate (S35) in $\mathrm{CDCl}_{3}$

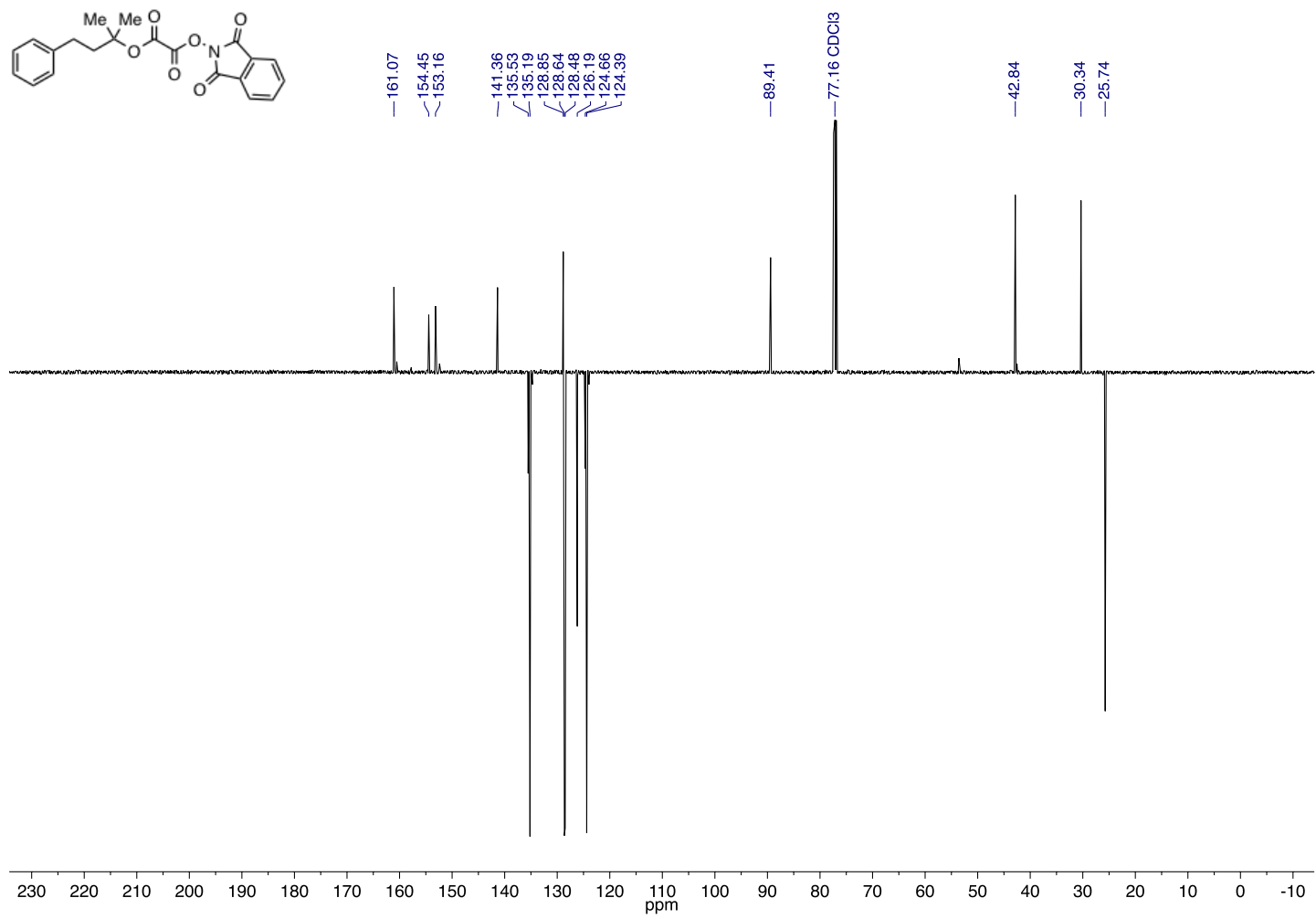


$500 \mathrm{MHz}{ }^{1} \mathrm{H}$-NMR of (3-fluoro-3-methylbutyl)benzene (40) in $\mathrm{CDCl}_{3}$
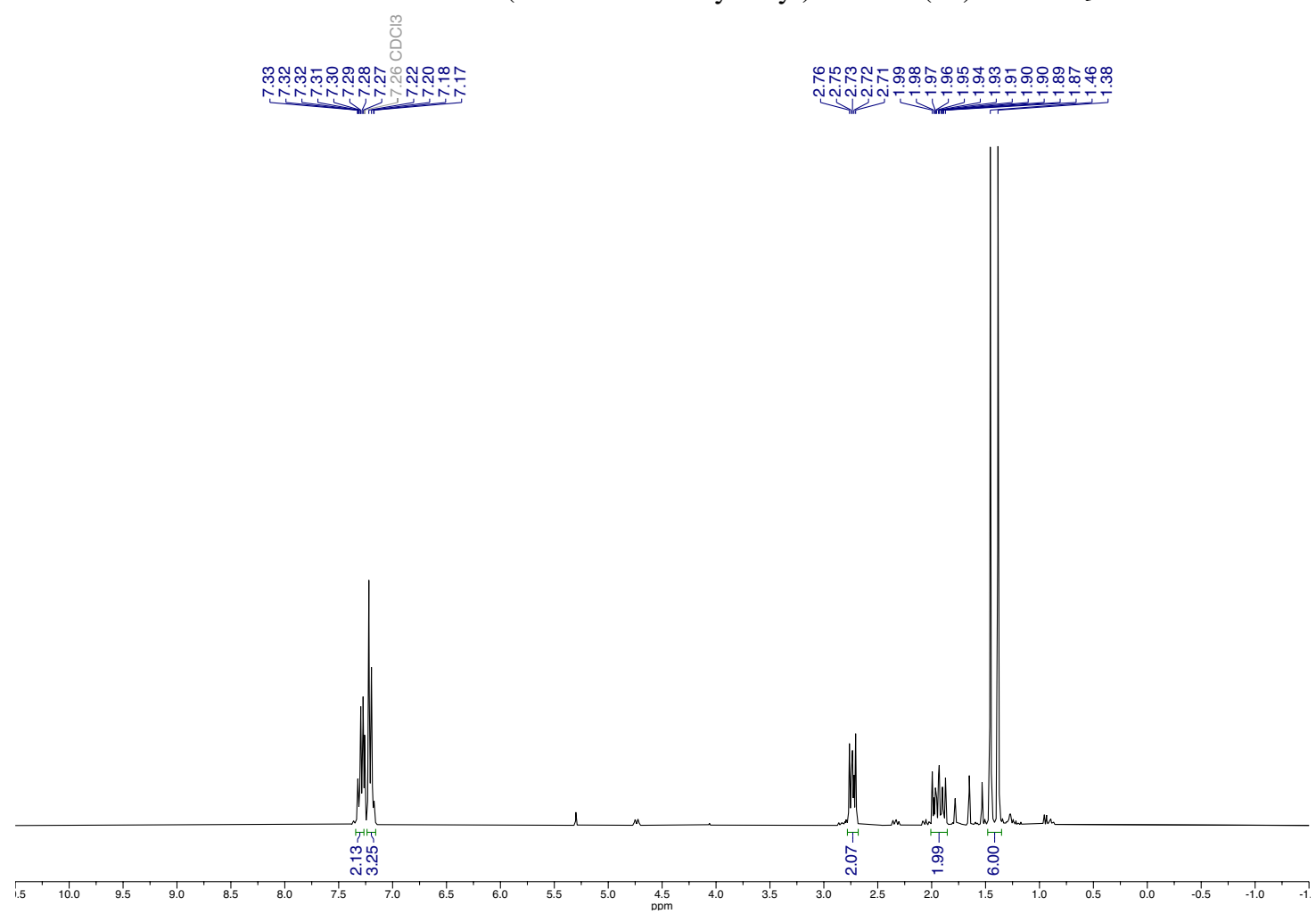

$75 \mathrm{MHz}{ }^{19} \mathrm{~F}-\mathrm{NMR}$ of (3-fluoro-3-methylbutyl)benzene (40) in $\mathrm{CDCl}_{3}$

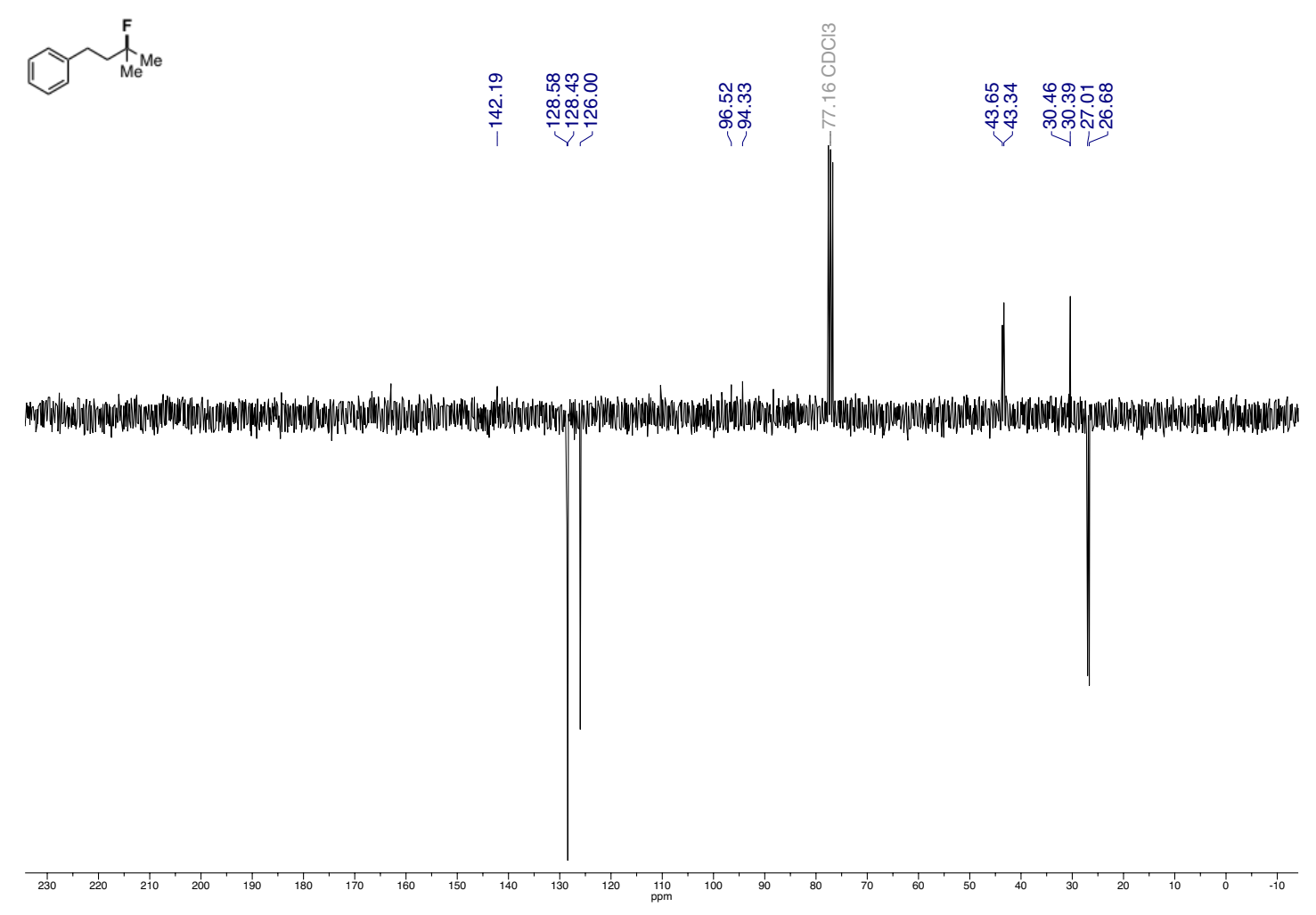


$282 \mathrm{MHz}{ }^{19} \mathrm{~F}-\mathrm{NMR}$ of (3-fluoro-3-methylbutyl)benzene (40) in $\mathrm{CDCl}_{3}$

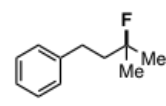
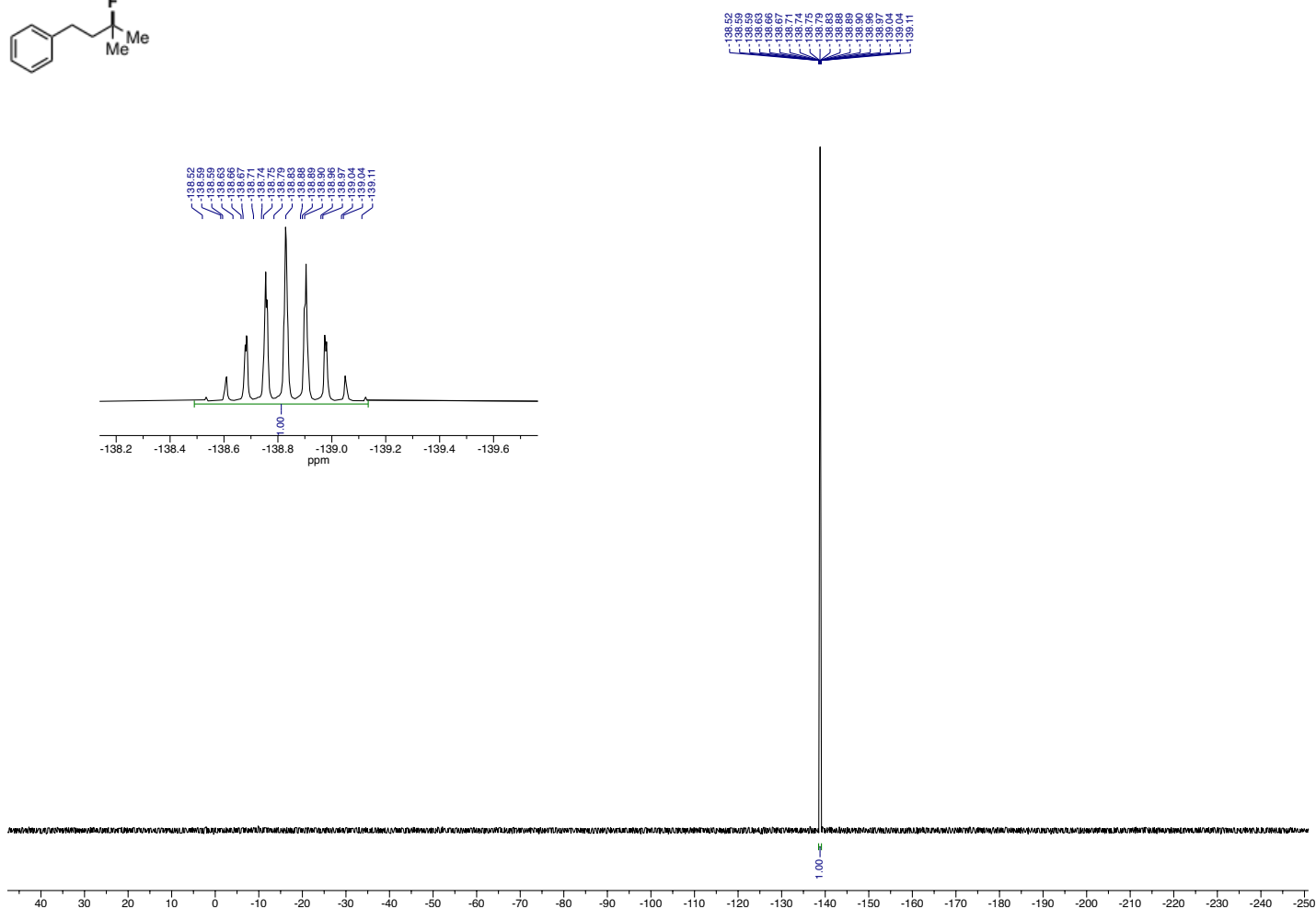

$500 \mathrm{MHz}{ }^{1} \mathrm{H}-\mathrm{NMR}$ of 2-methoxy-6-(1-(2,4,6-trimethoxyphenyl)ethyl)naphthalene (45) in $\mathrm{CDCl}_{3}$
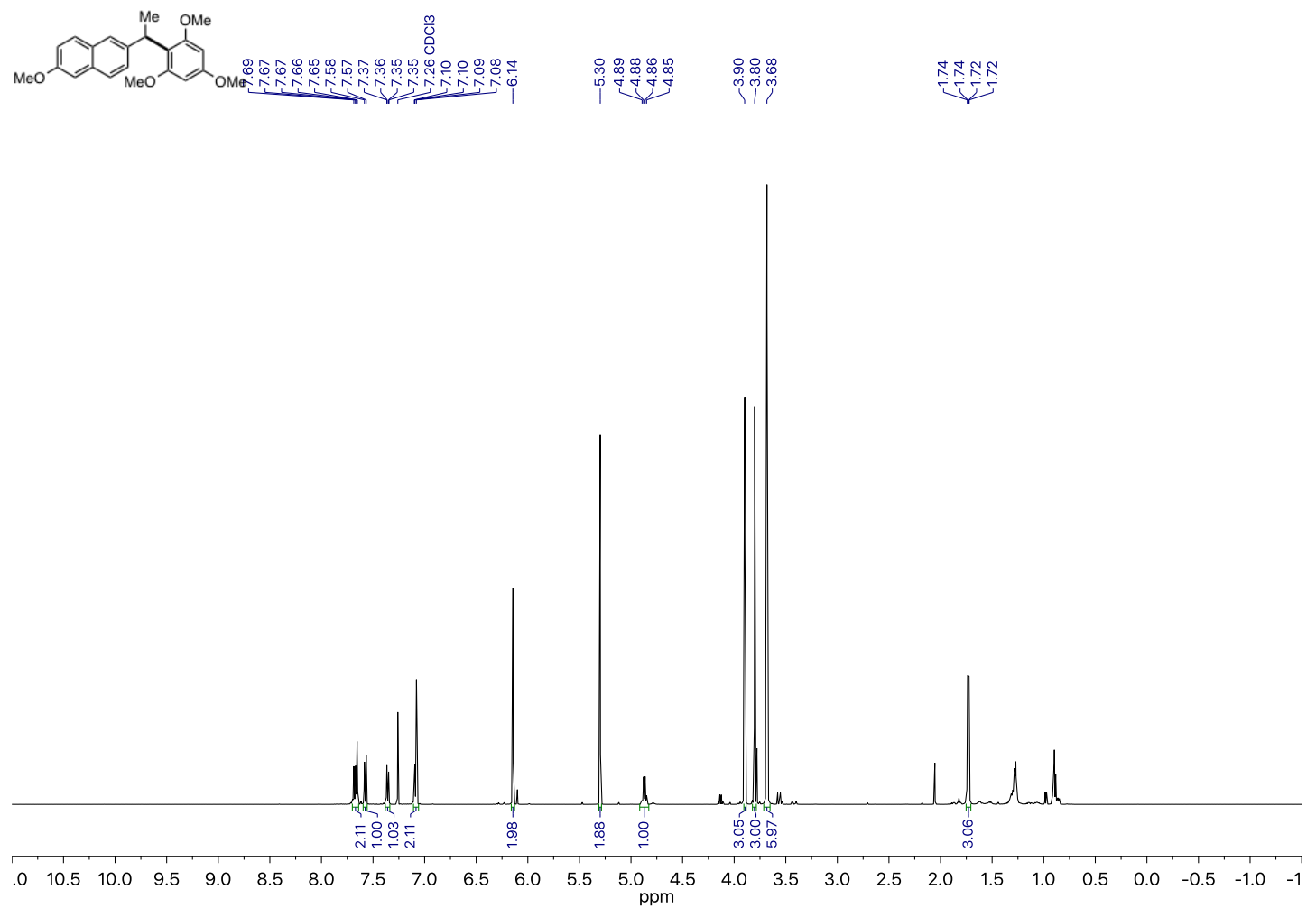
$126 \mathrm{MHz}{ }^{13} \mathrm{C}-\mathrm{NMR}$ of 2-methoxy-6-(1-(2,4,6-trimethoxyphenyl)ethyl)naphthalene (45) in $\mathrm{CDCl}_{3}$
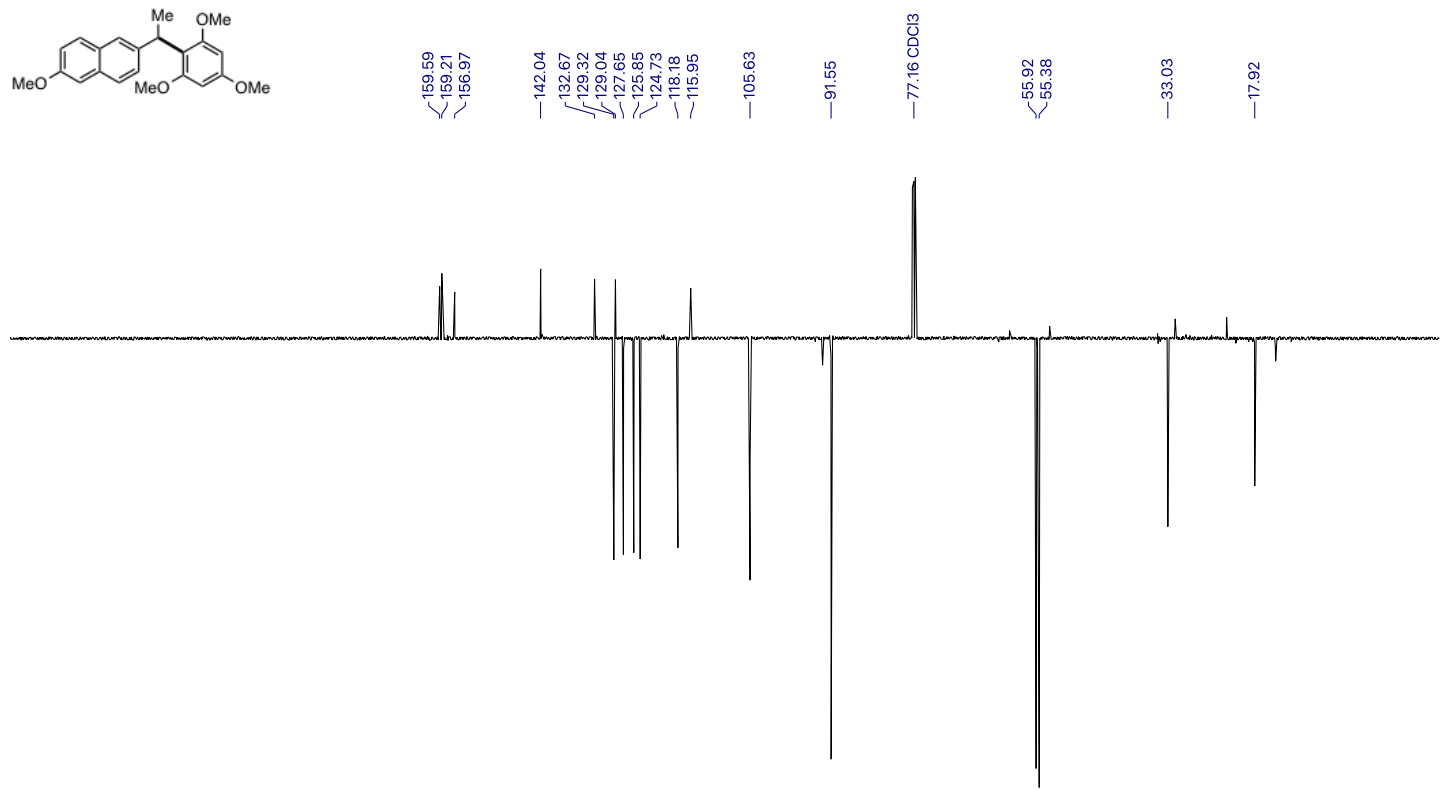

$\begin{array}{llllllllllllllllllllllllllllllll}230 & 220 & 210 & 200 & 190 & 180 & 170 & 160 & 150 & 140 & 130 & 120 & \begin{array}{c}110 \\ \mathrm{ppm}\end{array} & 100 & 90 & 80 & 70 & 60 & 50 & 40 & 30 & 20 & 10 & 0 & -10\end{array}$

$500 \mathrm{MHz}{ }^{1} \mathrm{H}-\mathrm{NMR}$ of 2-methoxy-6-(1-phenoxyethyl)naphthalene (46) in $\mathrm{CDCl}_{3}$
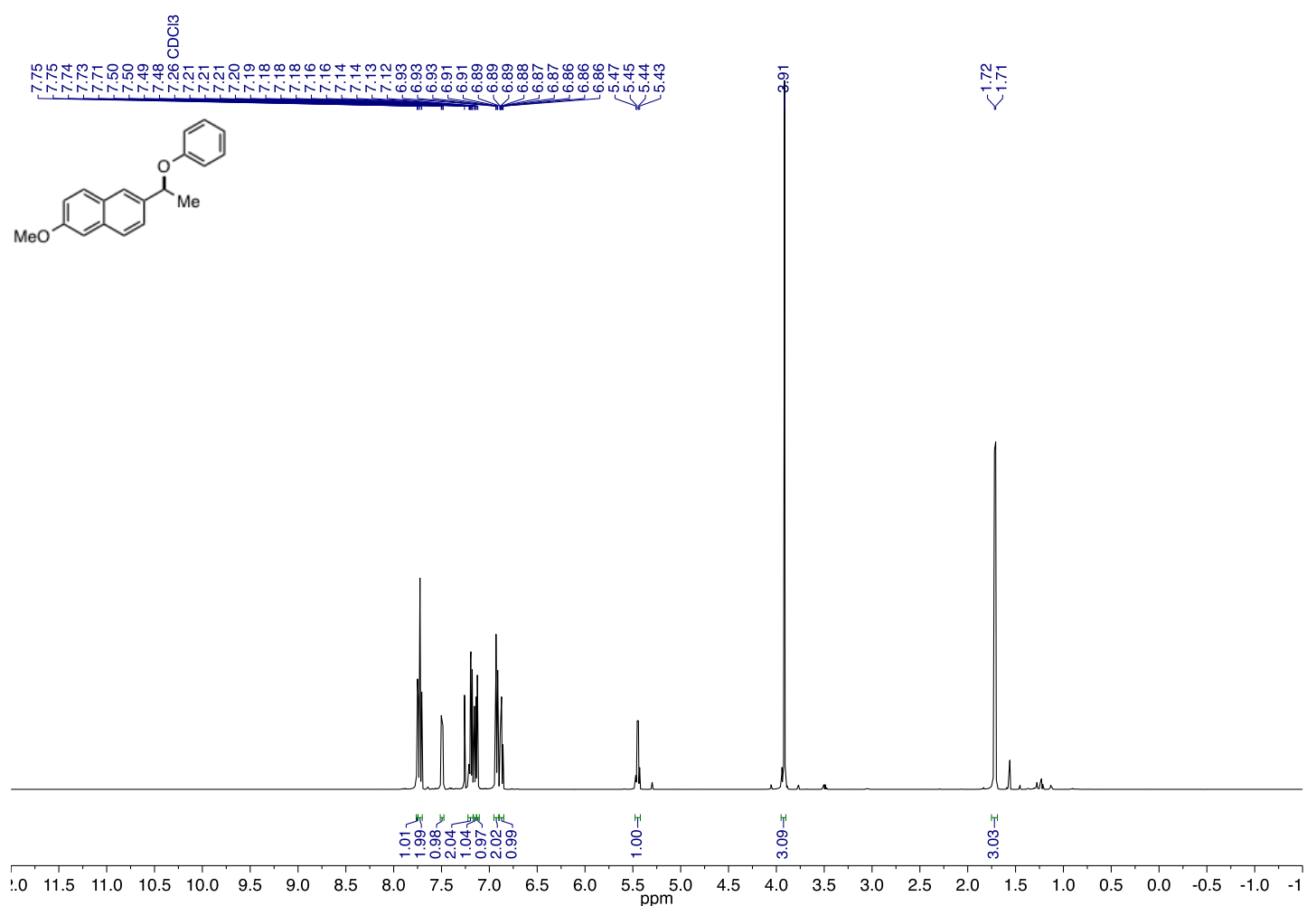
$126 \mathrm{MHz}{ }^{1} \mathrm{H}-\mathrm{NMR}$ of 2-methoxy-6-(1-phenoxyethyl)naphthalene (46) in $\mathrm{CDCl}_{3}$

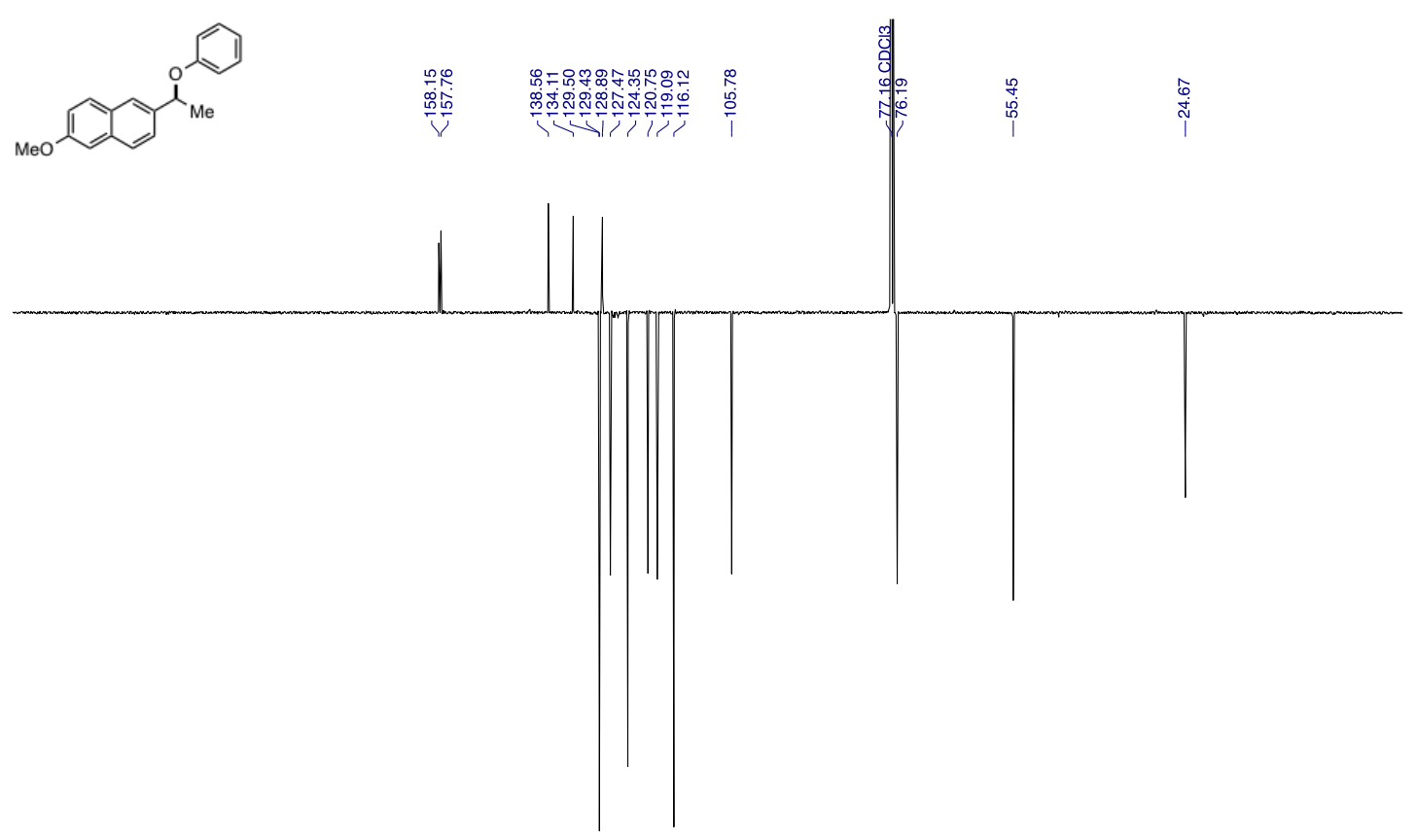

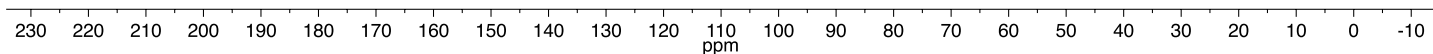

$500 \mathrm{MHz}{ }^{1} \mathrm{H}$-NMR of 2-methoxy-6-(1-methoxyethyl)naphthalene (47) in $\mathrm{CDCl}_{3}$

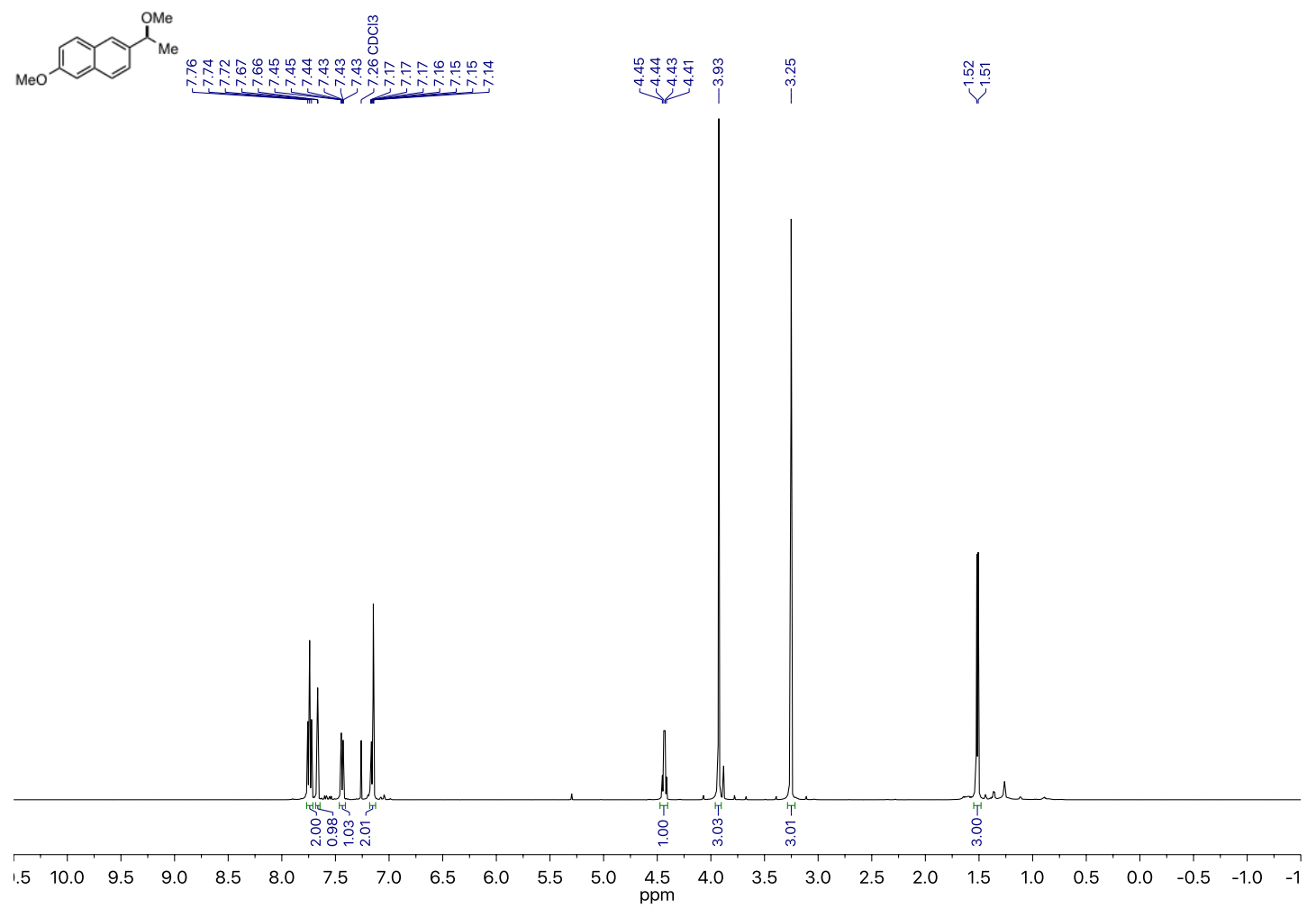


$126 \mathrm{MHz}{ }^{13} \mathrm{C}-\mathrm{NMR}$ of 2-methoxy-6-(1-methoxyethyl)naphthalene (47) in $\mathrm{CDCl}_{3}$
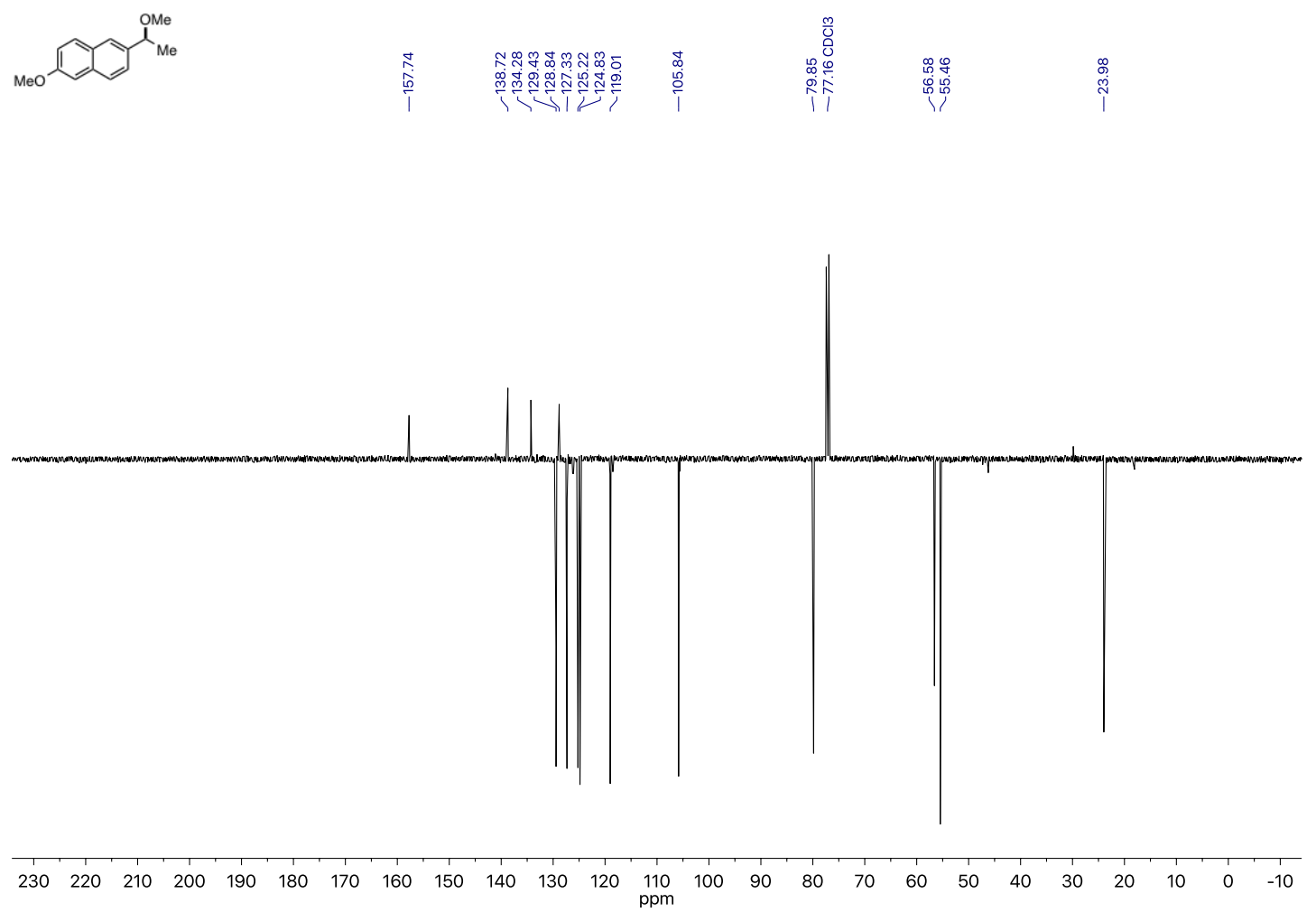

$500 \mathrm{MHz}{ }^{1} \mathrm{H}-\mathrm{NMR}$ of 2-(1-((1,1,1,3,3,3-hexafluoropropan-2-yl)oxy)ethyl)-6-methoxynaphthalene (48) in $\mathrm{CDCl}_{3}$

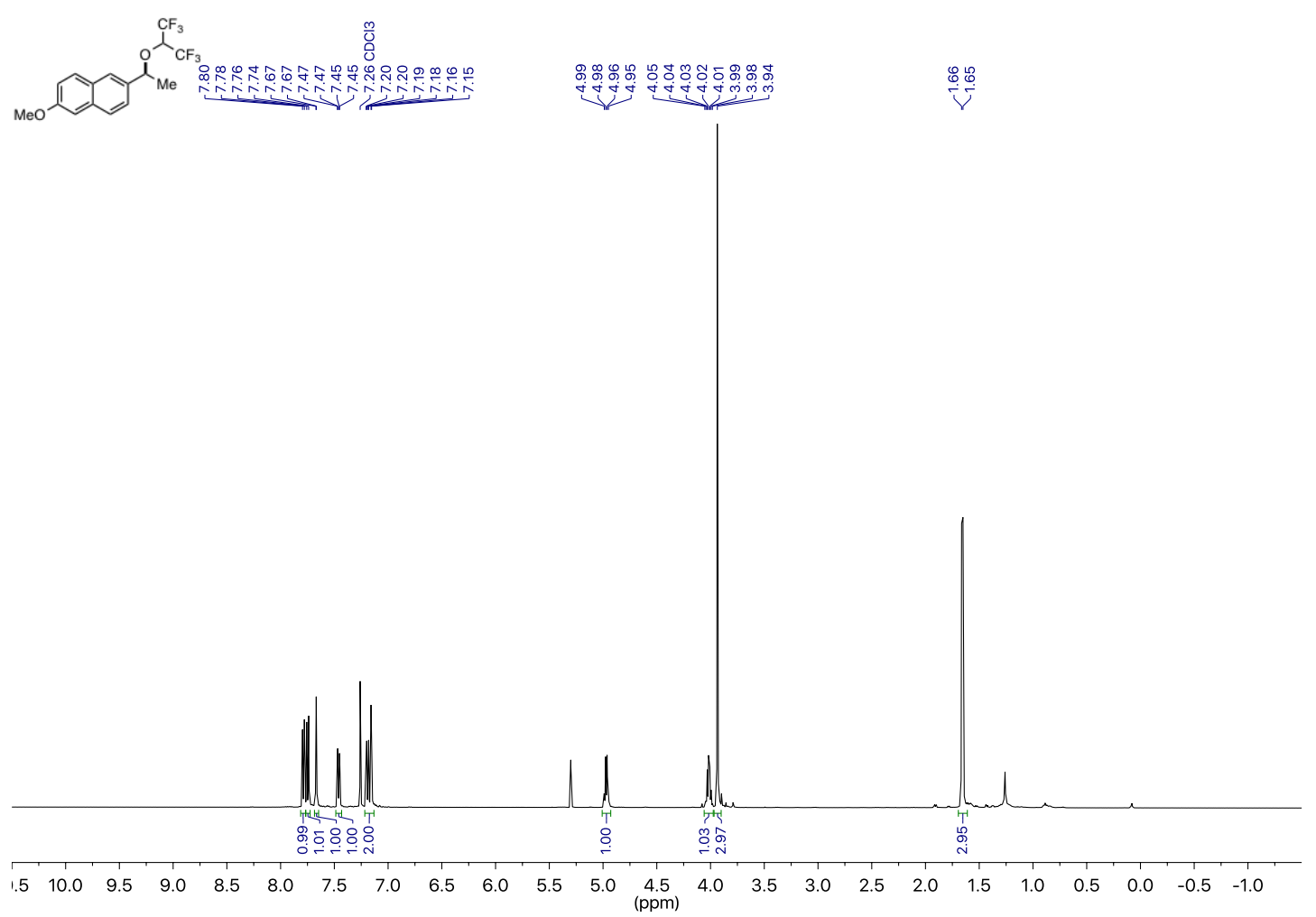


$126 \mathrm{MHz}{ }^{13} \mathrm{C}-\mathrm{NMR}$ of 2-(1-((1,1,1,3,3,3-hexafluoropropan-2-yl)oxy)ethyl)-6-methoxynaphthalene (48) in $\mathrm{CDCl}_{3}$

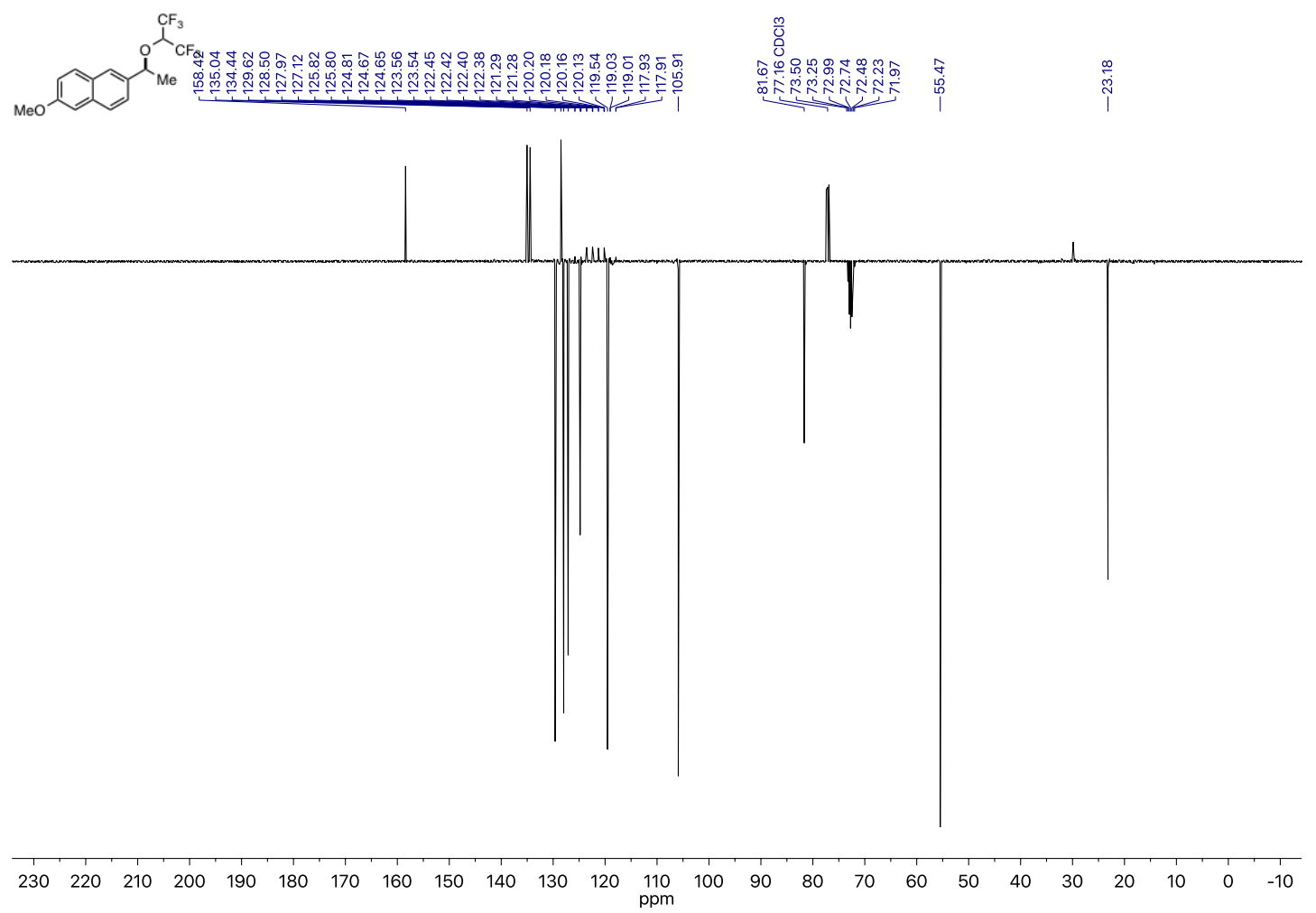

$282 \mathrm{MHz}{ }^{19} \mathrm{~F}-\mathrm{NMR}$ of 2-(1-((1,1,1,3,3,3-hexafluoropropan-2-yl)oxy)ethyl)-6-methoxynaphthalene (48) in $\mathrm{CDCl}_{3}$
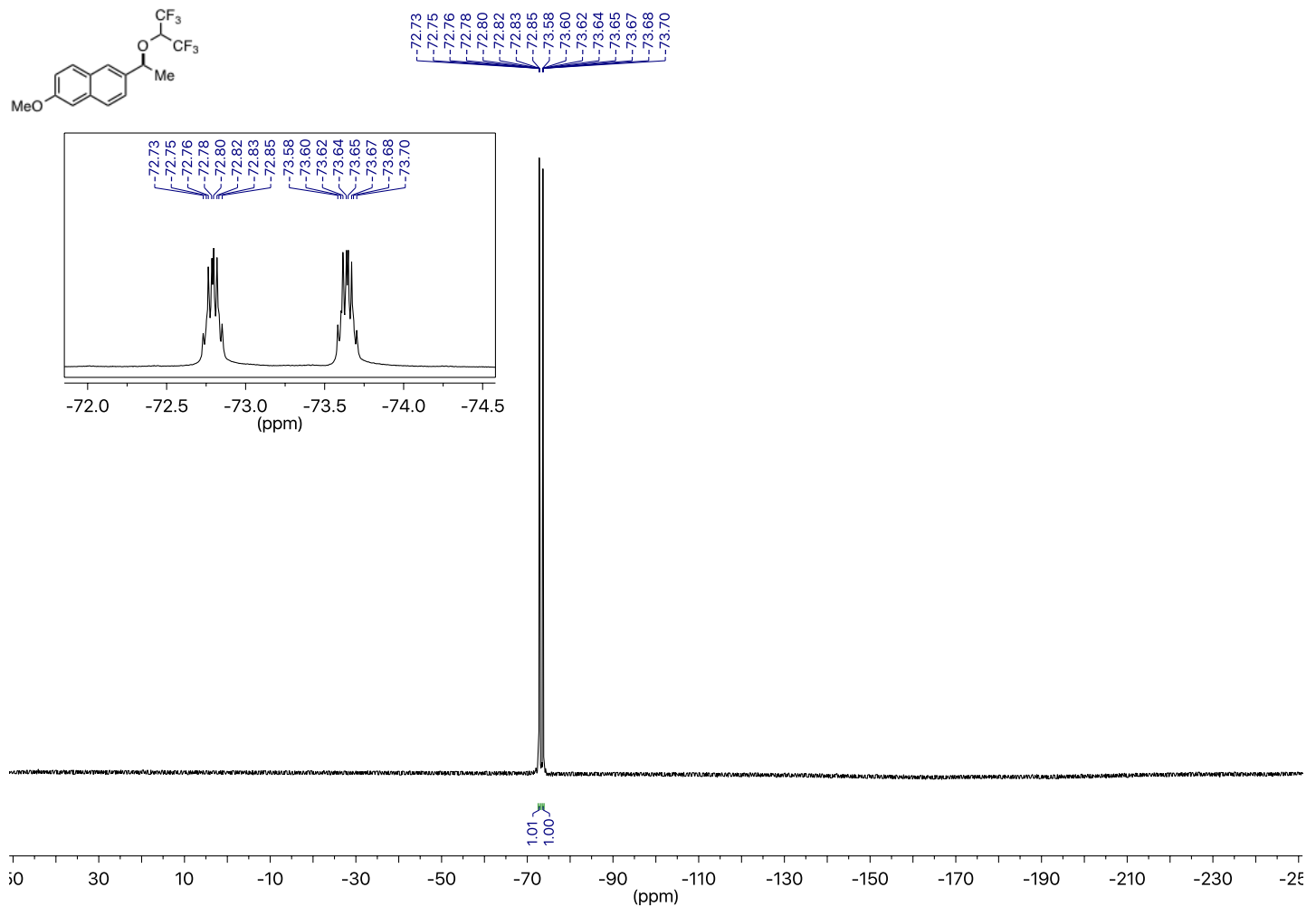


\section{References}

(1) Shields, B. J.; Doyle, A. G. Direct C(sp3)-H Cross Coupling Enabled by Catalytic Generation of ChlorineRadicals. J. Am. Chem. Soc. 2016, 138, 12719.

(2) Huihui, K. M. M.; Caputo, J. A.; Melchor, Z.; Olivares, A. M.; Spiewak, A. M.; Johnson, K. A.; Kim, S.;

DiBenedetto, T. A.; Kim, S.; Ackerman, L. K. G.; Weix, D. J. Decarboxylative Cross-Electrophile Coupling of N Hydroxyphthalimide Esters with Aryl Iodides. J. Am. Chem. Soc. 2016, 138, 5016.

(3) Zhao, W.; Wurz, R. P.; Peters, J. C.; Fu, G. C. Photoinduced, Copper-Catalyzed Decarboxylative C-N Coupling to Generate Protected Amines: An Alternative to the Curtius Rearrangement. J. Am. Chem. Soc. 2017, 139 (35), 12153.

(4) Wang, D.; Zhu, N.; Chen, P.; Lin, Z.; Liu, G. Enantioselective Decarboxylative Cyanation Employing Cooperative Photoredox Catalysis and Copper Catalysis. J. Am. Chem. Soc. 2017, 139 (44), 15632.

(5) Büll, C.; Heise, T.; van Hilten, N.; Pijnenborg, J. F. A.; Bloemendal, V. R. L. J.; Gerrits, L.; Kers-Rebel, E. D.; Ritschel, T.; den Brok, M. H.; Adema, G. J.; Boltje, T. J. Steering Siglec-Sialic Acid Interactions on Living Cells Using Bioorthogonal Chemistry. Angew. Chem. Int. Ed. 2017, 56 (12), 3309.

(6) Pratsch, G.; Lackner, G. L.; Overman, L. E. Constructing Quaternary Carbons from N-(Acyloxy)Phthalimide Precursors of Tertiary Radicals Using Visible-Light Photocatalysis. J. Org. Chem. 2015, 80 (12), 6025.

(7) Subba Rao, G. S. R.; Kondaiah, P.; Singh, S. K.; Ravanan, P.; Sporn, M. B. Chemical Modifications of Natural Triterpenes-Glycyrrhetinic and Boswellic Acids: Evaluation of Their Biological Activity. Tetrahedron 2008, 64 (51), 11541.

(8) Zarei, M.; Mohamadzadeh, M. 3-Thiolated 2-Azetidinones: Synthesis and in Vitro Antibacterial and Antifungal Activities. Tetrahedron 2011, 67 (32), 5832.

(9) Pearson, A. D.; Williams, R. M. Synthetic Studies towards Zetekitoxin AB: Preparation of 4,5-Epi-11-HydroxySaxitoxinol. Tetrahedron 2014, 70 (43), 7942.

(10) Zhao, M.; Li, J.; Mano, E.; Song, Z.; Tschaen, D. M.; Grabowski, E. J. J.; Reider, P. J. Oxidation of Primary Alcohols to Carboxylic Acids with Sodium Chlorite Catalyzed by TEMPO and Bleach. J. Org. Chem. 1999, 64 (7), 2564.

(11) Mizuta, S.; Stenhagen, I. S. R.; O’Duill, M.; Wolstenhulme, J.; Kirjavainen, A. K.; Forsback, S. J.; Tredwell, M.; Sandford, G.; Moore, P. R.; Huiban, M.; Luthra, S. K.; Passchier, J.; Solin, O.; Gouverneur, V. Catalytic Decarboxylative Fluorination for the Synthesis of Tri- and Difluoromethyl Arenes. Org. Lett. 2013, 15 (11), 2648. (12) Lackner, G. L.; Quasdorf, K. W.; Overman, L. E. Direct Construction of Quaternary Carbons from Tertiary Alcohols via Photoredox-Catalyzed Fragmentation of Tert-Alkyl $N$-Phthalimidoyl Oxalates. J. Am. Chem. Soc. 2013, 135 (41), 15342.

(13) Chen, J.; Lin, J. H.; Xiao, J. C. Halogenation through Deoxygenation of Alcohols and Aldehydes. Org. Lett. 2018, 20 (10), 3061.

(14) Kwan, E. E.; Zeng, Y.; Besser, H. A.; Jacobsen, E. N. Concerted Nucleophilic Aromatic Substitutions. Nat. Chem. 2018, 10 (9), 917.

(15) Xiang, M.; Xin, Z. K.; Chen, B.; Tung, C. H.; Wu, L. Z. Exploring the Reducing Ability of Organic Dye (Acr+Mes) for Fluorination and Oxidation of Benzylic C(sp3)-H Bonds under Visible Light Irradiation. Org. Lett. 2017, 19 (11), 3009.

(16) Huang, X.; Liu, W.; Hooker, J. M.; Groves, J. T. Targeted Fluorination with the Fluoride Ion by ManganeseCatalyzed Decarboxylation. Angew. Chem. Int. Ed. 2015, 54 (17), 5241.

(17) Pieber, B.; Shalom, M.; Antonietti, M.; Seeberger, P. H.; Gilmore, K. Continuous Heterogeneous Photocatalysis in Serial Micro-Batch Reactors. Angew. Chem. Int. Ed. 2018, 57 (31), 9976.

(18) Patrick, T. B.; Johri, K. K.; White, D. H.; Bertrand, W. S.; Mokhtar, R.; Kilbourn, M. R.; Welch, M. J. Replacement of the Carboxylic Acid Function with Fluorine. Can. J. Chem. 1986, 64 (1), 138.

(19) González-Esguevillas, M.; Miró, J.; Jeffrey, J. L.; MacMillan, D. W. C. Photoredox-Catalyzed Deoxyfluorination of Activated Alcohols with Selectfluor ${ }^{\circledR}$. Tetrahedron 2019, 75, 4222.

(20) Teegardin, K.; Day, J. I.; Chan, J.; Weaver, J. Advances in Photocatalysis: A Microreview of Visible Light Mediated Ruthenium and Iridium Catalyzed Organic Transformations. Org. Process Res. Dev. 2016, 20 (7), 1156. (21) Tlahuext-Aca, A.; Garza-Sanchez, R. A.; Glorius, F. Multicomponent Oxyalkylation of Styrenes Enabled by Hydrogen-Bond-Assisted Photoinduced Electron Transfer. Angew. Chem. Int. Ed. 2017, 56 (13), 3708.

(22) Cismesia, M. A.; Yoon, T. P. Characterizing Chain Processes in Visible Light Photoredox Catalysis. Chem. Sci. 2015, 6 (10), 5426 . 
(23) Zhu, Q.; Gentry, E. C.; Knowles, R. R. Catalytic Carbocation Generation Enabled by the Mesolytic Cleavage of Alkoxyamine Radical Cations. Angew. Chem. Int. Ed. 2016, 55 (34), 9969.

(24) Hatchard, C. G.; Parker, C. A. A New Sensitive Chemical Actinometer. II. Potassium Ferrioxalate as a Standard Chemical Actinometer. Proc. R. Soc. Lond. A. Math. Phys. Sci. 1956, 253 (1203), 518.

(25) Okada, K.; Okamoto, K.; Oda, M. A New and Practical Method of Decarboxylation: Photosensitized Decarboxylation of $N$-Acyloxyphthalimides via Electron-Transfer Mechanism. J. Am. Chem. Soc. 1988, 110 (26), 8736.

(26) Wayner, D. D. M.; Houmam, A. Redox Properties of Free Radicals. Acta Chem. Scand. 1998, 377.

(27) Wayner, D. D. M.; McPhee, D. J.; Griller, D. Oxidation and Reduction Potentials of Transient Free Radicals. J. Am. Chem. Soc. 1988, 110 (1), 132.

(28) Gray, E. E.; Nielsen, M. K.; Choquette, K. A.; Kalow, J. A.; Graham, T. J. A.; Doyle, A. G. Nucleophilic (Radio)Fluorination of $\alpha$-Diazocarbonyl Compounds Enabled by Copper-Catalyzed H-F Insertion. J. Am. Chem. Soc. 2016, 138 (34), 10802.

(29) Cresswell, A. J.; Davies, S. G.; Roberts, P. M.; Thomson, J. E. Beyond the Balz-Schiemann Reaction: The Utility of Tetrafluoroborates and Boron Trifluoride as Nucleophilic Fluoride Sources. Chem. Rev. 2015, 115 (2), 566. 
